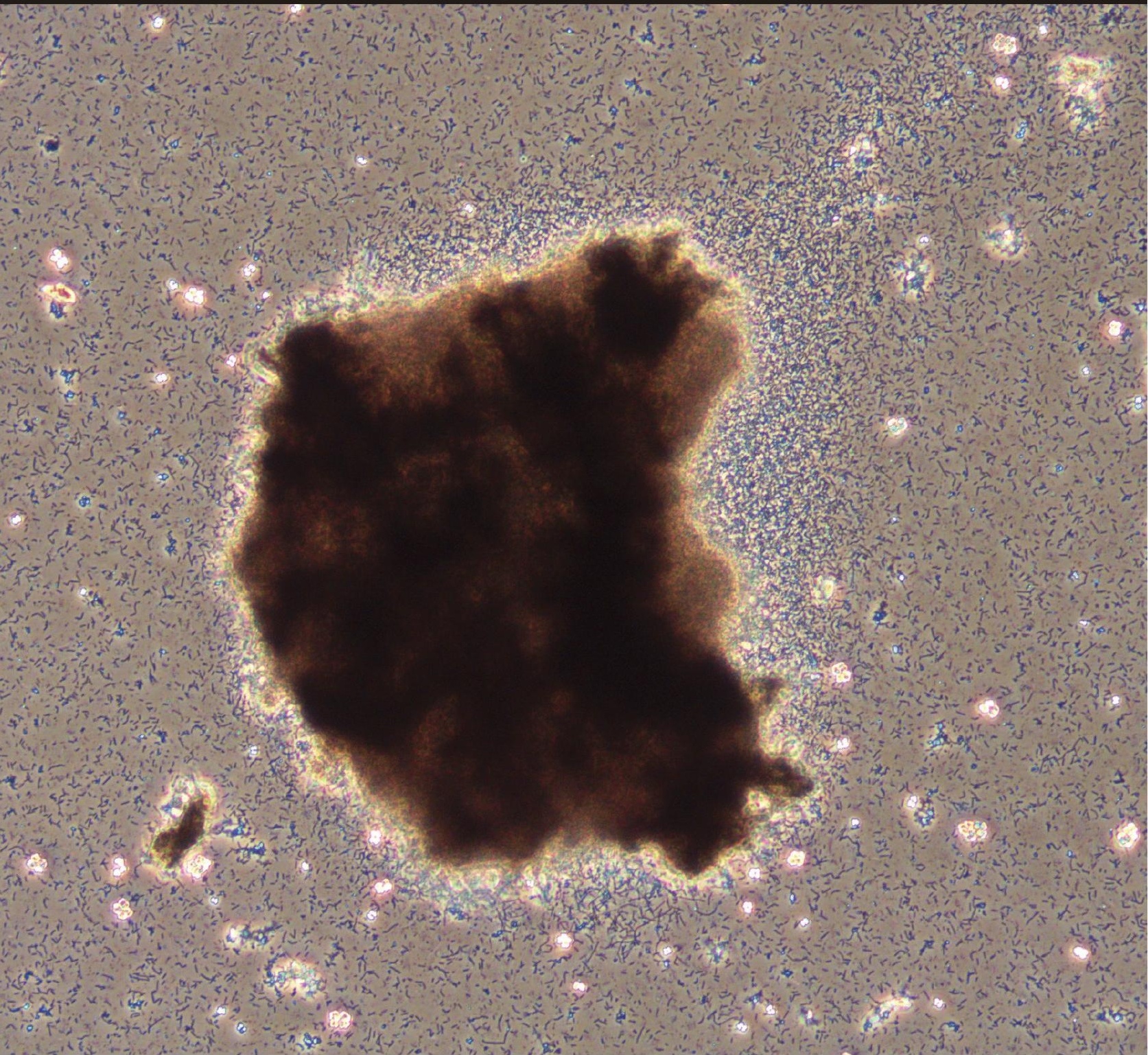

\title{
Open Culture Chain Elongation for Branched Carboxylate Formation
}

Kasper Daniël de Leeuw 


\section{Propositions}

1. The concentrations of $\mathrm{n}$ - and isobutyrate during methanol-based chain elongation at low $\mathrm{pH}$ are determined by the thermodynamic equilibrium of isomerization.

(This thesis)

2. The reduction of carboxylates to their corresponding alcohols, coupled to ethanol oxidation can be done by both chain elongators and by a syntrophic community.

(This thesis)

3. The greatest obstacle to discovery is not ignorance It is the illusion of knowledge. (Steven Hawking)

4. Science filters personal perception of truth to absolute truths, however a "truth" is meaningless without context.

5. Free will gradually emerges when an individual entity can both understand and manipulate its own environment.

6. A conscious observation requires the expression of language.

7. Overcoming cognitive dissonance is necessary to reach compromises.

Propositions belonging to the thesis, entitled

"Open Culture Chain Elongation for Branched Carboxylate Formation"

Kasper Daniël de Leeuw

Wageningen, November 3, 2020 

Open Culture Chain Elongation for Branched Carboxylate Formation 


\section{Thesis committee}

\section{Promotor}

Prof. Dr C. J. N. Buisman

Professor of Biological Recovery \& Reuse Technology

Wageningen University \& Research

\section{Co-promotors}

Dr D.P.B.T.B. Strik

Associate Professor, Environmental Technology

Wageningen University \& Research

\section{Other members}

Prof. Dr D. Machado de Sousa, Wageningen University \& Research

Prof. Dr L. Angenent, Max Planck Institute for Developmental Biology, Tübingen, Germany

Dr R. Kleerebezem, Delft University of Technology

Dr T.I.M. Grootscholten, Cosun Research and Development, Dinteloord

This research was conducted under the auspices of the Graduate School for SocioEconomic and Natural Sciences of the Environment (SENSE). 


\title{
Open Culture Chain Elongation for Branched Carboxylate Formation
}

\author{
Kasper Daniël de Leeuw
}

\author{
Thesis \\ submitted in fulfilment of the requirements for the degree of doctor \\ at Wageningen University \\ by the authority of the Rector Magnificus, \\ Prof. Dr A.P.J. Mol, \\ in the presence of the \\ Thesis Committee appointed by the Academic Board \\ to be defended in public \\ on Monday 3 November 2020 \\ at 4 p.m. in the Aula.
}


Kasper de Leeuw

Open Culture Chain Elongation for Branched Carboxylate Formation

262 pages.

PhD thesis, Wageningen University, Wageningen, NL (2020)

With references, with summary in English

ISBN: 978-94-6395-529-4

DOI: https://doi.org/10.18174/530424 


\section{Table of contents}

Chapter 1

Chapter 2

Chapter 3

Chapter 4

Chapter 5

Chapter 6
General Introduction 7

Methanol-based Chain Elongation of Acetate to $\mathrm{n}$ - and Isobutyrate

Methanol-based Chain Elongation of Propionate to n-Valerate. 43

Ethanol-based Chain Elongation of Isobutyrate to Isocapraote.

Alcohol Formation and Ethanol-based Branched Chain Elongation.....

General Discussion

Summary.

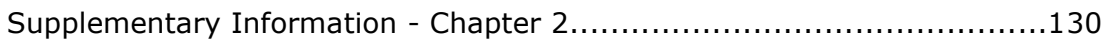

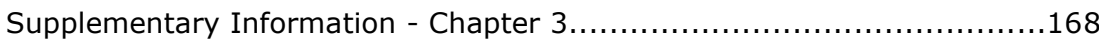

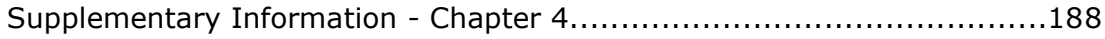

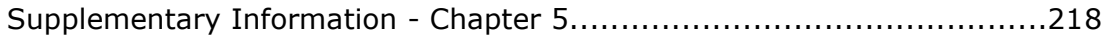

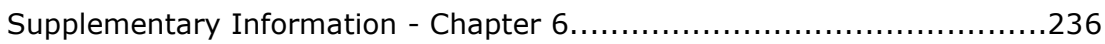

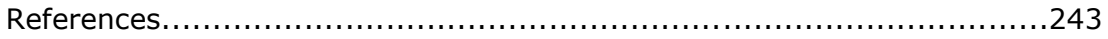

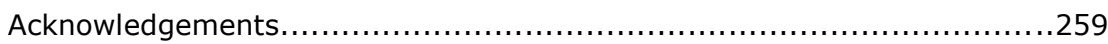





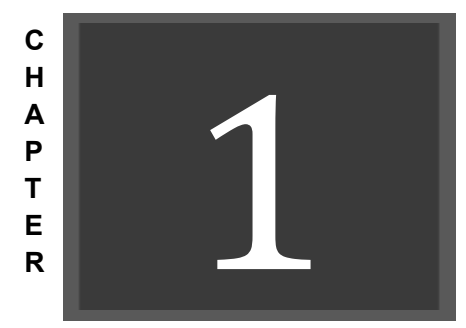

General Introduction 


\section{Introduction}

\section{The "circular economy"}

In this thesis research on open culture chain elongation fermentation is presented that can aid in the development of a "circular economy". However, before diving into the technological specifics, it is warranted to discuss what is meant with a "circular economy", without simply using the term as a buzzword to grab attention. A research performed in 2017 analyzed 114 different definitions for circular economy and distinguished differences between the formulated definitions ${ }^{1}$. In most cases the definitions of circular economy state that it should lead to economic prosperity by replacing the take-make-dispose system with a system of recycling and increasing the valorization of waste streams ${ }^{2}$. In fewer cases the definitions included that a circular economy should promote maintaining and improving environmental quality, whereas the impact of a circular economy on promoting social equity and on maintaining a suitable environment for future generations was barely mentioned. The research eventually came up with the following aggregated definition to incorporate all these aspects: "Circular economy is an economic system that is based on business models which replace the 'end-of-life' concept with reducing, alternatively reusing, recycling and recovering materials in production/distribution and consumption processes, thus operating at the micro level (products, companies, consumers), meso level (ecoindustrial parks) and macro level (city, region, nation and beyond), with the aim to accomplish sustainable development, which implies creating environmental quality, economic prosperity and social equity, to the benefit of current and future generations. "I

The systemic innovations that are necessary for a circular economy to truly be implemented on a global scale are not only of technological nature ${ }^{3}$. The regulatory barriers, market barriers and cultural barriers are numerous and act as a resistances that can significantly slow down adoption of circular economy practices, even though required technologies might already exist ${ }^{4}$. Moreover, a growing wealth inequality has been argued to be a significant cause of environmental degradation ${ }^{5-8}$.

Environmental degradation, which should be prevented at all cost within a circular economy framework, can be partly attributed to an absence of sufficient incentivization mechanisms in the form of regulatory measures ${ }^{9}$. A strong type of incentivization is deemed necessary to resolve conflicts between economical stakeholders and environmentalist stakeholders in a game theory-derived way that creates an environment that selects decisions which lead to a long-term sustainable outcome ${ }^{9}$. Although human conflict resolutions are undoubtedly much more complex, a parallel can be drawn from game theory ${ }^{10}$ to the principle in biotechnological mixed culture engineering of using a strict environmental pressure in a 
system to select for a functionality. This biotechnological principle is implied by the Baas Becking hypothesis:" Everything is everywhere, but the environment selects ${ }^{11}$." It is clear that when discussing how to achieve a circular economy it should be included how the global environment would select for authorities and companies that make decisions in favor of a long-term sustainability.

Moreover, irrevocably intertwined with the "economy" in "circular economy" is the concept of money itself. The current fiat currencies are, among others, created via a continuous expansion of debt by issuance from commercial and central banks ${ }^{12}$. To what degree this financial system can exist without infinite consumer growth and facilitate a sustainable economy is up for debate ${ }^{13}, 14$. Necessary bail-out measures in differing forms of quantitative easing by central banks now have become more unconventional by including large scale asset purchases in order to prevent an economic collapse ${ }^{15}$. Aside from creating moral hazard ${ }^{16}$, these unconventional measures could have further aggravated wealth inequality ${ }^{17}$ and, arguably, with it the strain on the environment.

Some propose that in order to achieve a sustainable economy it is necessary to reform the economic environment on various levels. Examples of reform include new economic instruments such as the concept of an obligatory "precycling insurance", where manufacturers are responsible to price in the complete recycling costs of their goods ${ }^{18}$. It would create incentive towards innovation and counter planned obsolescence strategies. Another, rather radical, alternative approach would be to replace the centralized money issuance by more incentive-driven decentralized governance structures. With the rise of blockchain technologies, decentralized governance structures can be organized while being able to facilitate transparency, harness democratic principles and temper corrupted practices ${ }^{14,19-21}$.

How to create an environment that selects for decision making to favor the development of a circular economy, however, is a very complex endeavor and fortunately does not fall any further into the scope of this thesis. Inside the scope of this thesis is a much simpler research on how to use mixed culture biotechnology principles to enrich microbiomes that can be used to recycle organic residue streams via production of useful biochemicals. 


\section{Chain elongation fermentation within a circular economy}

This research contributes to the transition towards a circular economy by addressing a technological aspect that could help increase efficiency and sustainability within our ecosystem. More specifically the research is focused on exploring the potential of chain elongation fermentation to widen its product spectrum and thereby its application range. Chain elongation is an emerging biological resource recovery method that can be utilized to recycle for example complex organic residues via production of short and medium chain carboxylates (SCCs and MCCs) ${ }^{22}$. It is an attractive alternative to biogas fermentation due to the wide application potential and higher economic value of carboxylates compared to methane ${ }^{23}$. SCCs and MCCs are suitable for large scale implementation in the industry as building blocks for lubricants, plasticizers, plastics, feed additives and eventually - if production costs go down sufficiently - for biofuel precursors 22,24 . The many potential applications of SCCs and MCCs require a market and manufacturing shift for biobased SCC and MCCs to become important platform chemicals ${ }^{25}$. ChainCraft is an example of such development by being the one of the first companies to have started up a full scale demonstration plant in 2020 that produces medium chain carboxylate salts from GFT (Vegetables, Fruit and Garden)-waste with the purpose to sell the product as feed additive in the agricultural industry ${ }^{26}$.

\section{Chain elongation fermentation within open-culture anaerobic fermentation}

Anaerobic fermentation processes that are used to valorize organic residue streams often employ enriched open cultures. These enriched cultures are acquired by designing the environment of the reactors in such a way that a selection pressure causes the desired metabolisms to become dominant. Such systems have several advantages: (i) they inherently cannot be contaminated which removes the necessity of costly sterilization, (ii) they are able to convert complex substrate mixes due to the functional abundance within open-culture microbiomes and (iii) they often allow for continuous operation which reduces overall complexity and costs of the process ${ }^{27}$. Typically, when anaerobic microbiomes convert organic carbon matter in the absence of a strong external electron acceptor, a large part of the energy within the chemical bonds of the compounds is remained in the end-product. As a result the energy yield within the metabolism of anaerobic fermentative bacteria is relatively low, leading to low biomass yields per formed product and subsequently to high product yields ${ }^{28}$. Consequently, anaerobic fermentation can be used to efficiently convert complex mixtures of carbohydrates, proteins and fats into useful energy-rich chemicals ${ }^{25}$. 


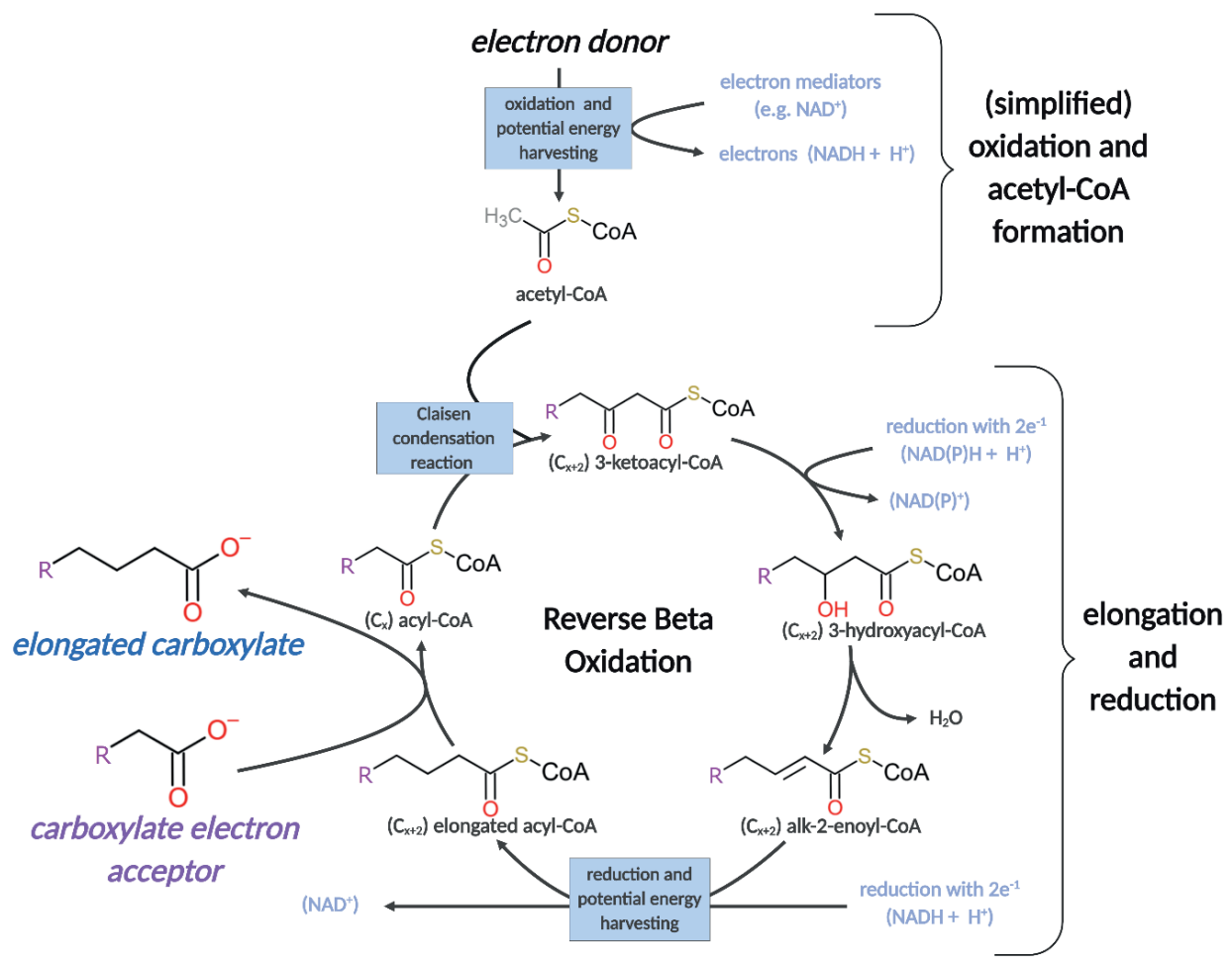

Figure 1. A generalized scheme for chain elongation is depicted that utilizes the reverse beta oxidation pathway ${ }^{29}$. The mechanism for oxidation and acetyl-CoA formation is dependent on the used electron donor. ATP formation during oxidation and acetyl-CoA formation prior to the reverse beta-oxidation could occur but is excluded in this simplified scheme. The amounts of electrons and acetyl-CoA intermediates that are released during the oxidation are dependent on the carbon content and degree of reduction of the electron donor. In the case where methanol is used as electron donor, acetyl-CoA formation would require the utilization of the Wood-Ljungdahl pathway ${ }^{30}$. Ethanolderived acetyl-CoA requires oxidation via dehydrogenases ${ }^{31}$.

Chain elongation bioprocesses utilize a specific niche within anaerobic microbial conversions, that with the right selective pressure can be made dominant within microbiomes. They combine an electron donor (such as ethanol, lactate, methanol and glucose) with a short or medium chain carboxylate as electron acceptor within their energy metabolism ${ }^{32}$. Typically the chain elongation is executed via what is known as the reverse beta-oxidation pathway ${ }^{33}$. In Figure $\mathbf{1}$ a generalized scheme for chain elongation metabolic route is shown. Within the metabolism a carboxylate electron acceptor is elongated with an acetyl-CoA unit in a Claisen condensation reaction that is catalyzed by a thiolase ${ }^{34}$. The acetyl-CoA elongation unit is usually derived from oxidation of the electron donor, whereas electrons from this oxidation are used to reduce the elongation product after condensation. It results in an elongated carboxylate as metabolic end-product that has been extended by two hydrated carbon atoms. 


\section{Formation of the branched carboxylates via isomerization and elongation - expanding the product spectrum}

In 2017 it was discovered that using methanol as electron donor in food-waste chain elongation fermentations can lead to the formation of branched carboxylates, in particular isobutyrate ${ }^{35}$. It opened up the possibility to investigate the potential of enriched microbiomes to produce branched carboxylates via open culture chain elongation. A robust fermentation process to produce branched carboxylates at high selectivities can bring to the industry biochemicals with enhanced chemical performance properties such as better oxidative stabilities, lower melting points and different viscosities ${ }^{36-38}$. Hypothetically, isobutyrate is formed after chain elongation of acetate to n-butyrate via subsequent isomerization by butyryl-CoA:isobutyryl-CoA mutase (see Figure $\mathbf{2}$ ). This isomerization step has been hypothesized to help overcome the toxic effect of $n$-butyrate at high concentrations ${ }^{35}$. Methanol based chain elongation is one of the bioprocesses that has been researched in this thesis for its capacity to produce branched carboxylates.

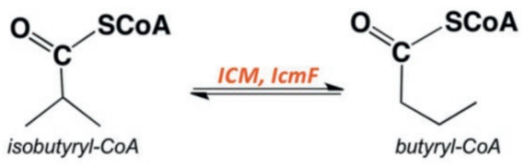

Figure 2. The figure shows the carboxylate group rearrangement as it occurs during isomerization of $\mathrm{n}$-butyrate to isobutyate. The figure is taken from Cracan and Banerjee (2012) (39 $^{39}$ and adapted to show the $n$-butyrate-isobutyrate specific isomerization reaction performed by butyrylCoA:isobutyryl-CoA mutase (ICM, IcmF).

A mechanism by which medium chain branched carboxylates could hypothetically be formed is by extension of smaller branched carboxylates within the reverse beta-oxidation. In this scheme the branched groups of the electron acceptor are maintained throughout the elongation process. To predict what product can be expected from this chain elongation process is it helpful to study the catalytic mechanism of extension: the thiolase driven Claisen condensation reaction ${ }^{34}$. Figure 3 shows that during condensation the carboxylate electron acceptor (green) is elongated with acetyl-CoA (blue). The keton group on the third carbon atom is then targeted for subsequent reduction within the reverse beta oxidation route. As a result the branched groups of the chain elongation product will be located at the end of the molecule opposing the carboxylate group. 
A prerequisite for this process is that the involved enzymatic machinery of the chain elongating microbes have sufficient affinity for branched carboxylates. Additionally a high enough throughput through this alternative metabolism would be required in order for microbes that utilize this route to be competitive enough to maintain their dominance in an open reactor. Isocaproate formation was observed in trace amount in a study that focused on ethanol based chain elongation of carboxylates from acidified supermarket waste ${ }^{40}$. The potential of ethanol based chain elongation to produce branched medium chain carboxylates is further investigated in this thesis.

(a) Acetyl-transfer reaction

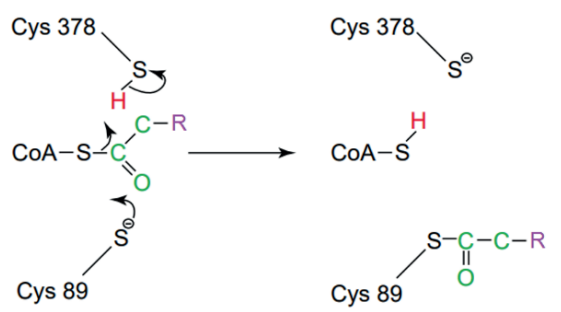

(b) Claisen condensation reaction

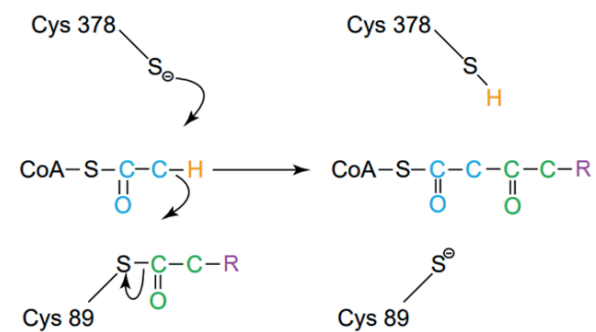

Figure 3. This scheme shows the catalytic process of the Claisen condensation reaction as it occurs within the thiolase catalytic site. The green acyl-CoA compound is the initial carboxylate electron acceptor; the blue acetyl-CoA is the unit that is used to elongate the electron acceptor by two carbon atom. The figure is taken from Haapalainen et al (2006) ${ }^{34}$ and adapted by adding an R-group to include alternative carboxylate electron acceptors. 


\section{Selection pressure in open systems - engineering microbiomes for chain elongation bioprocesses}

Enriching open culture microbiomes towards an efficient ethanol and/or methanol based chain elongation functionality requires operation at conditions that impose a strict selective pressure. This requires a multifaceted approach to incorporate all aspects in a reactor that can influence bacterial proliferation. One of the most important selection pressure tools is the choice of carbon source. For the investigated chain elongation processes, the limited carbon source range already significantly narrows down the possible microbial conversions. Still, there are anaerobic microbes with different energy metabolisms that will compete for the available substrates, which could lessen the yield and rate of chain elongation. By performing a thermodynamic state analysis of the possible conversions within the microbiome, insight can be gained in what bioprocesses are feasible within the system ${ }^{28}$. Table 1 shows an overview of possible (ethanol- and methanol-based) chain elongation conversions and alternative bioprocesses that could occur within the microbiome. The reaction Gibbs energy of these conversions is calculated for standard biological conditions, as well as for two hydrogen partial pressures ( $1 \mathrm{kPa}$ and $1 \mathrm{~Pa}$ ). A short description of methanol-based and ethanol-based chain elongation is given in Box 1 and Box 2 . 


\section{Methanol-based chain elongation}

Methanol-based chain elongation was previously described as acidogenesis with methanol as substrate ${ }^{41-44}$. It is known that microorganisms, which anaerobically metabolize methanol within their energy-providing pathway, utilize the Wood-Ljungdahl pathway (see Figure B1). Methanol-based chain elongation can facilitate $n$-caproate formation, but only up to a low concentration $(\sim 10 \mathrm{mM})$ relative to other types of chain elongation ${ }^{45}$. Besides $\mathrm{n}$-caproate formation via n-butyrate elongation, $\mathrm{n}$-valerate can also be formed via propionate elongation. During open-culture fermentation at high n-butyrate concentrations, also isobutyrate formation was observed ${ }^{35}$. Methanol based chain elongation of acetate experimentally followed this stoichiometry $35,43,46,47$ :

$2 \mathrm{CH}_{3} \mathrm{OH}+\mathrm{CH}_{3} \mathrm{COO}^{-} \rightarrow \mathrm{C}_{3} \mathrm{H}_{7} \mathrm{COO}^{-}+2 \mathrm{H}_{2} \mathrm{O}$

$$
\Delta_{r} \mathrm{G}^{01}=-106.8 \mathrm{~kJ}
$$

Besides the elongation of existing carboxylates, $\mathrm{CO}_{2}$ can also be utilized as electron acceptor which results in methanolotrophic acetate formation (Figure B1B). In cases where sufficient methanol and $\mathrm{CO}_{2}$ is provided methanol chain elongation to butyrate can also occur ${ }^{44}$. However, it can be argued that in this instance the butyrate formation is simply the result of methanolotrophic acetate formation follow by methanol-based chain elongation of acetate.

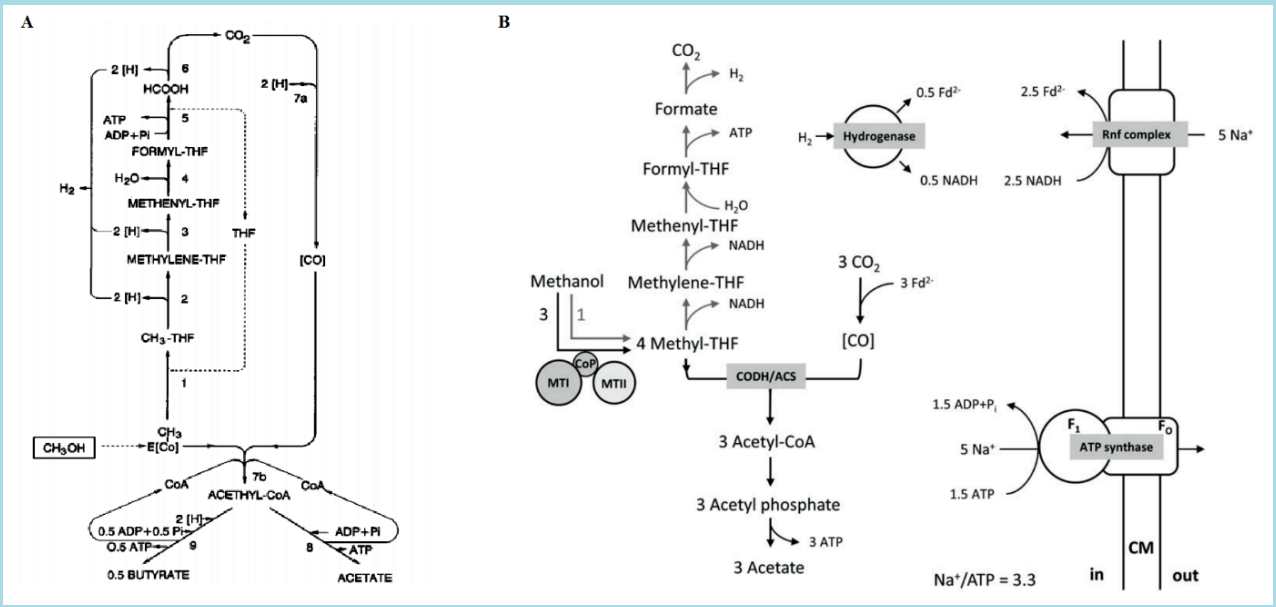

Figure B1. A) Pathway in methanol-utilizing acidogenis bacteria as was suggested back in 1986 (taken from Heijthuijsen and Hansen 1986) ${ }^{42}$. B) Pathway for methanol metabolism by A. Woodii. (taken from Kremp et al. (2018) that includes electron bifurcation mechanisms ${ }^{30}$. A complete verified pathway for methanol-based chain elongators that includes the elongation and reduction of longer carboxylates in detail, has not yet been elucidated. 
Box 2

\section{Ethanol-based chain elongation}

Clostridium kluyveri is a well-known chain elongator that uses ethanol as electron donor. Its activity was first described in 1937 by Barker ${ }^{48}$. Later in 2008 its full genome was sequenced and its metabolic pathway unraveled ${ }^{31}$. As shown in Figure 1, chain elongation consists of two stages: (i) the oxidation and acetyl-CoA formation and (ii) the elongation and reduction. In the case of ethanol based chain elongation an additional part of the ethanol is oxidized towards acetate and two dihydrogen molecules, while performing ATP synthesis via substrate level phosphorylation (Figure B2). Bifurcating and confurcating enzymes allow redirection of electron flows to different electron carriers with different redox potentials to maximize energy conservation (NAD(P)H, Ferrodoxin, FAD) 49,50 . This allows $C$. kluyveri to have a versatile metabolism and it was proposed that $C$. kluyveri can adjust the stoichiometry of chain elongation to maximize the ATP yield and maintain chain elongation during varying conditions ${ }^{22}$. Within this thesis the stoichiometry of ethanol based chain elongation was generalized as reverse beta-oxidation combined with ethanol oxidation coupled in a $5(n=5)$ to $1(p=1)$, or $4(n=4)$ to $1(p=1)$ ratio (reaction 1 for acetate and 2 for longer carboxylates):

$$
\begin{aligned}
& \left(n_{\mathrm{C} 2}+p\right) \mathrm{CH}_{3} \mathrm{CH}_{2} \mathrm{OH}+\left(n_{\mathrm{C} 2}-p\right) \mathrm{CH}_{3} \mathrm{COO}^{-} \rightarrow n_{\mathrm{C} 2} \mathrm{C}_{3} \mathrm{H}_{7} \mathrm{COO}^{-}+2 \mathrm{pH}_{2}+\left(n_{\mathrm{C} 2}-p\right) \mathrm{H}_{2} \mathrm{O}+p \mathrm{H}^{+} \\
& \left(n_{\mathrm{Ci}}+p\right) \mathrm{CH}_{3} \mathrm{CH}_{2} \mathrm{OH}+n_{\mathrm{Ci}} \mathrm{C}_{x} \mathrm{H}_{2 x+1} \mathrm{COO}^{-} \rightarrow n_{\mathrm{Ci}} \mathrm{C}_{x+2} \mathrm{H}_{2(x+2)+1} \mathrm{COO}^{-}+2 p \mathrm{H}_{2}+\left(n_{\mathrm{Ci}}-p\right) \mathrm{H}_{2} \mathrm{O}+p \mathrm{H}^{+}+p \mathrm{CH}_{3} \mathrm{COO}^{-}
\end{aligned}
$$
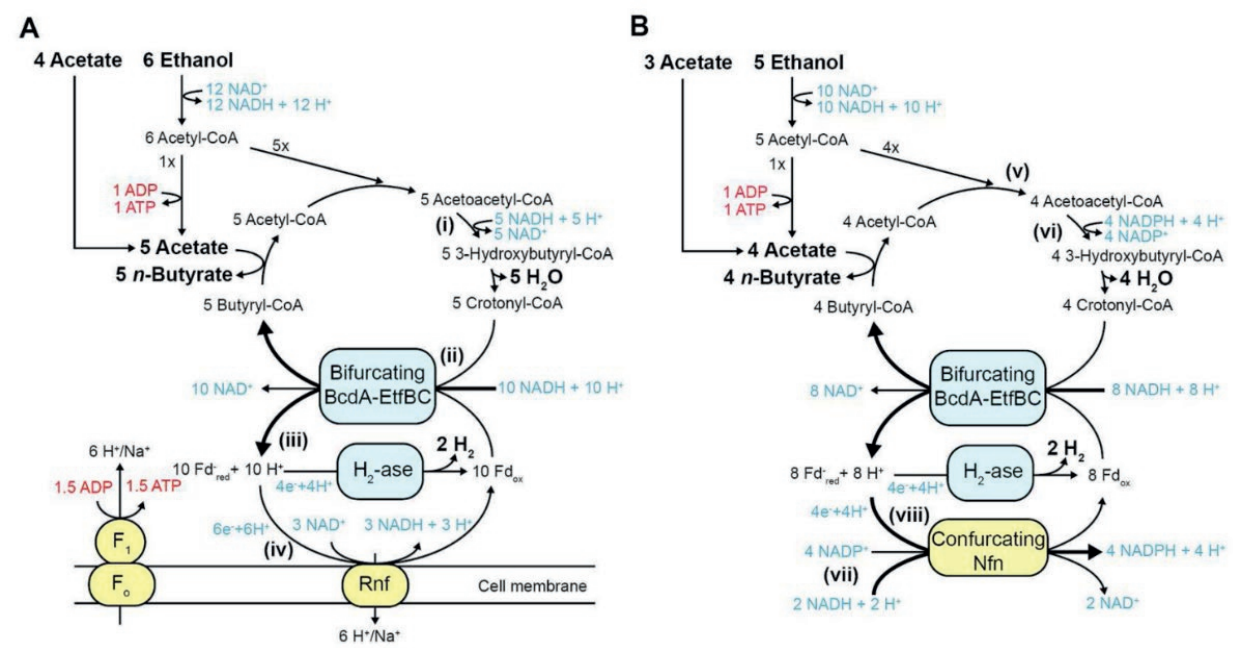

Figure B2. The figure shows two proposed stoichiometries for ethanol based acetate elongation that are adjusted to maximize ATP yield and allow chain elongation to proceed under different conditions: A) At high substrate concentrations relative to product concentrations the resulting $\Delta_{\mathrm{r}} \mathrm{G}^{\prime}<-184.5$ kJ reaction ${ }^{-1}$, which allows ATP synthesis via both SLP (1 ATP) and electromotive force-driven ATPase (1.5 ATP). B) At low substrate to product concentration ratios, the reduction of 3-ketoacyl-CoA requires $\mathrm{NAPDH}$ instead of $\mathrm{NADH}$ to sufficiently drive the reaction and a single ATP is formed only via $\operatorname{SLP}\left(\Delta_{\mathrm{r}} \mathrm{G}^{0^{\prime}} \approx-77 \mathrm{~kJ}\right.$ reaction $\left.{ }^{-1}\right)$. The figure was taken from Angenent et al $(2016)^{22}$. 
The hydrogen partial pressure is an important parameter that determines the feasibility of competing reactions such as ethanol and methanol oxidation (substrate competition) and beta oxidation (product degradation). These bioconversions generally require a syntrophic partner to keep the hydrogen pressure low enough for the reaction to be energetically favorable ${ }^{51}$. Syntophic partners include hydrogenotrophic acetogenesis (reaction 9), hydrogenotrophic carboxylate reduction (reaction 10) and hydrogenotrophic methanogenesis (reaction 11). Maintaining a sufficiently high hydrogen partial pressure is one strategy to minimize substrate competition and product degradation within a chain elongation microbiome. Because hydrogen is produced within ethanol based chain elongation, this chain elongation process already helps create a favorable environment for itself. However, a too high hydrogen partial pressure (>80kPa) is known to also affect the rate of ethanol based chain elongation ${ }^{22}$. In addressing the feasibility of reactions from a thermodynamic standpoint it must always be considered that macro-level parameters may differ from what is happening at the micro-level. Environmental niches can be exist within biofilms, which could allow locally different hydrogen partial pressures during syntrophic interactions. Sufficient mixing could prevent biofilm formation and with it the syntrophic growth. In some cases, however, biofilm formation can be desired to help increase the volumetric conversion rate of a bioprocess ${ }^{52}$. This would pose additional challenges to maintain a stringent selection pressure and will be discussed in later chapters.

In general, the rate by which a conversion can take place will affect the degree by which the converting microbe establishes their relative abundance within a microbiome. For instance there are many reactions that require bicarbonate as electron acceptor (reactions 2, 3 6, 7 and 9) and by limiting the bicarbonate supply, the rate of such reactions can be lowered. As a result the microbes performing these bioconversions can be prevented from becoming dominant within the microbiome. Note that in some conversions, for example acetotrophic and methylotrophic methanogenesis, bicarbonate is produced. Therefore, limiting these types of methanogenesis in a chain elongation system would have an added effect of preventing other substrate-competing bioconversions that require bicarbonate. However, limiting the bicarbonate availability too much will also affect the growth of ethanol based chain elongator: Clostridium kluyveri, because it requires bicarbonate for biomass formation ${ }^{53}$. For methanol based chain elongation bicarbonate can be a direct substrate for the formation of acetate and butyrate ${ }^{43}$. Though, recent research has also shown that methanol based chain elongation of acetate, without the utilization of bicarbonate, is possible ${ }^{45}$. Bicarbonate dosing in this case should be dependent on the desired methanol based chain elongation conversions.

Another important selection pressure tool is the possibility to enforce a certain minimum growth rate by setting the hydraulic retention time of a reactor during continuous operation 
54, 55. As a result, suspended microorganisms that cannot grow as fast or faster than the dilution rate will be washed out the reactor. Note that in cases where biofilms are growing in a reactor, this selection pressure tool becomes less effective as hydraulic retention time is then decoupled from solid retention time. In the continuous reactor experiments that are presented in this thesis, the hydraulic retention time was always selected with the aim to promote the growth of chain elongation microbes and simultaneously minimize the accumulation of methanogens.

Additionally, what helps to promote successful acidogenic fermentation in general is an environment with high carbon source concentrations $\left(>10 \mathrm{~g} \mathrm{~L}^{-1}\right)^{56}$. Previous studies showed that a resulting high MCC product concentration inhibits ethanol oxidation and increases the selectivity of ethanol based chain elongation 57 . In the case of methanol, high concentrations ( $>1 \mathrm{~g} \mathrm{~L}^{-1}$ ) have been observed to trigger methanol based chain elongation within methanogenic systems while reducing methanogenesis 58 .

Finally, a low pH can amplify the toxic effect of high carboxylate concentrations. When the reactor $\mathrm{pH}$ approaches the $\mathrm{pK}_{\mathrm{a}}$ values of carboxylates, protonated carboxylic acids will start to diffuse into the bacterial cells creating an energy draining futile cycle that significantly increases the maintenance requirement for microbes ${ }^{59}$. This will put more strain on the whole microbiome and thereby more stringently select for the most adapted microbe. This strategy is utilized in the chapters about methanol-based chain elongation. 
\begin{tabular}{ll} 
n \\
0 \\
$\frac{1}{0}$ \\
$\frac{1}{0}$ \\
$\frac{1}{3}$ \\
$\frac{5}{3}$ \\
\hline
\end{tabular}

ㄷํㅇ

든

든

เั

'm

노는.

究

$\checkmark \frac{0}{3}$

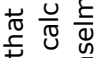

들

넨

วิे

บे 융

घं

Йं

$\circlearrowleft$ ठ

을 을

음 중

닌

㒖

는 으 일

등 하

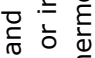

도

음 苋

ơ u

드

든 壳

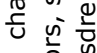

Ð 응

त ญ

$\frac{1}{1} \frac{0}{\pi}$

등ㅎํ 믐

足完

$\stackrel{\oplus}{\omega} \varepsilon$

प्र

त $\frac{5}{2}$

1)

齐产文

40

ㅇำ

歈言

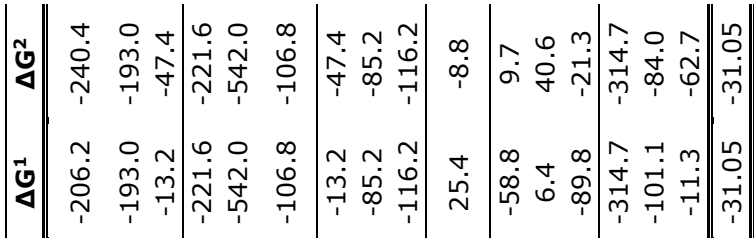

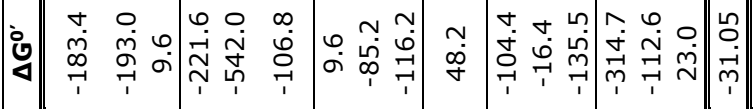

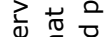

ठำ

- $\rightarrow$ 은

o

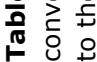

(1)

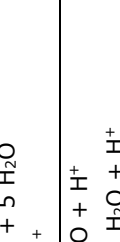

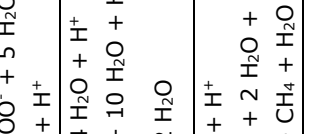

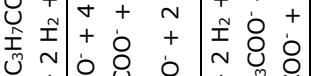

in +8 \& 8

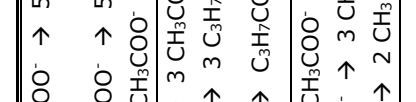

䍃

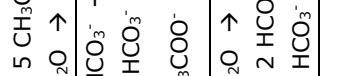

$+\quad+$ II

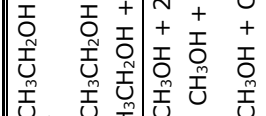

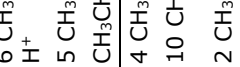

I + +

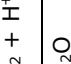

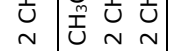

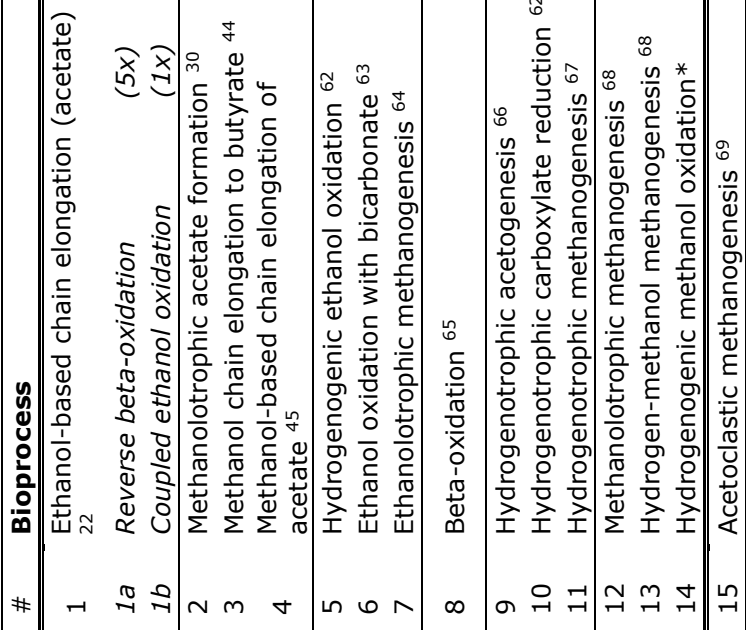

ก

종미

气

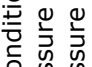

ठํㅐ

오 흔

元 $\frac{\pi}{2}$

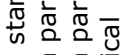

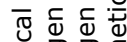

क응 뭉

음 空坌空

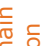




\section{Outline of this thesis}

This thesis presents proof of principles to produce branched carboxylates via the use of enriched microbiomes that perform chain elongation fermentations. Two different types of chain elongation fermentations were investigated: (i) ethanol-based chain elongation and (ii) methanol-based chain elongation. The results show research on how to obtain alternative products using enriched mixed culture anaerobic digestion techniques.

In Chapter 2 a long-term continuous reactor experiment shows how to enrich a microbiome for the production of $\mathrm{n}-\mathrm{C}_{4}$ and $\mathrm{i}-\mathrm{C}_{4}$ via methanol-based chain elongation of acetate. The reactor $\mathrm{pH}$ is identified as an important parameter with which the product spectrum can be changed from almost solely $n-C_{4}$ formation to the formation of $\mathrm{i}-\mathrm{C}_{4}$ and $\mathrm{n}-\mathrm{C}_{4}$ in varying ratios. The reactor experiments were complemented with two batch series that showed that the formation of $n-C_{4}$ and $i-C_{4}$ is coupled to the thermodynamics of isomerization. The slightly lower Gibbs formation energy of $\mathrm{i}-\mathrm{C}_{4}$ compared to $\mathrm{n}-\mathrm{C}_{4}$ steers the broth concentrations towards a ratio of $0.69 \mathrm{i}-\mathrm{C}_{4}$ to $0.31 \mathrm{n}-\mathrm{C}_{4}$. The dominant microorganisms in the reactor were related to Eubacterium limosum for methanol based chain elongation of acetate to $\mathrm{n}-\mathrm{C}_{4}$ at a high $\mathrm{pH}(6.75)$ and related to Clostridium luticellarii for methanol based chain elongation of acetate to $i-C_{4}$ and $n-C_{4}$ at a low pH (5.2).

The potential of methanol-based chain elongation is further explorer in Chapter 3. Two continuous reactor experiments show successful elongation of propionate to n-valerate via methanol-based chain elongation. One of the initial goals of this research was to investigate if $\mathrm{i}-\mathrm{C}_{5}$ could be produced from propionate in a similar fashion as how $\mathrm{i}-\mathrm{C}_{4}$ and $\mathrm{n}-\mathrm{C}_{4}$ are formed during methanol-based chain elongation of acetate. This, however, did not occur and propionate was elongated to only $n-C_{5}$ without any significant isomerization. The most dominant microorganism within the valerate producing enriched microbiome was found to be the same as in the $\mathrm{i}-\mathrm{C}_{4}$ producing microbiome: related to Clostridium luticellarii.

The research presented in Chapter 4 shows successful formation of branched medium chain carboxylates. During a continuous reactor experiment an enriched microbiome utilized ethanol-based chain elongation to elongate isobutyrate $\left(i-C_{4}\right)$ to isocaproate $\left(i-C_{6}\right)$. The results are explained from the perspective of the known metabolic pathway of Clostridium kluyverii, called the reverse beta-oxidation pathway, and from the perspective of known ethanol oxidation routes that directly compete with chain elongation microbes for ethanol as substrate. Microbial community analyses were performed to confirm that the most dominant microorganism within the enriched microbiome is 
strongly related to Clostridium kluyverii. Additionally, it shows data that support the stoichiometric analysis in relation to competing biochemical routes.

The possibilities and limitations of this enriched microbiome are further investigated in Chapter 5. A continuous reactor experiment explores the effect of $\mathrm{CO}_{2}$ dosage on branched chain elongation during acetate limitation. Higher alcohol formation was observed to be thermodynamically coupled to ethanol oxidation that led to acetate formation. Additionally multiple series of batch experiments showed the likely elongation of $\mathrm{i}-\mathrm{C}_{5}$ to $\mathrm{i}-\mathrm{C}_{7}$, albeit in very small quantities. This chapter shows that the elongation of branched electron acceptors in the acquired enriched microbiomes is closely coupled to the elongation of straight chain carboxylates in the sense that the first only occurs as side-process to the latter, which is known as co-metabolism.

A general discussion of the experimental chapters is presented in Chapter 6. Some content of the research chapters is reevaluated in scope of additional literature research. Process performances are discussed; also various applications and follow-up research topics are presented. 


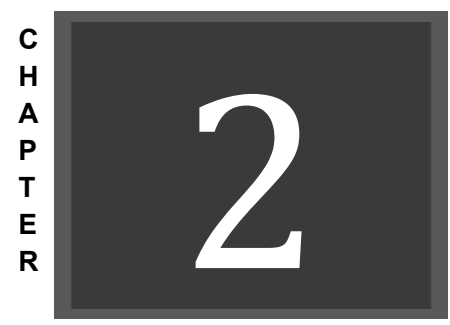

\section{Methanol-based Chain Elongation with Acetate to n-butyrate and isobutyrate at varying selectivities dependent on $\mathrm{pH}$}

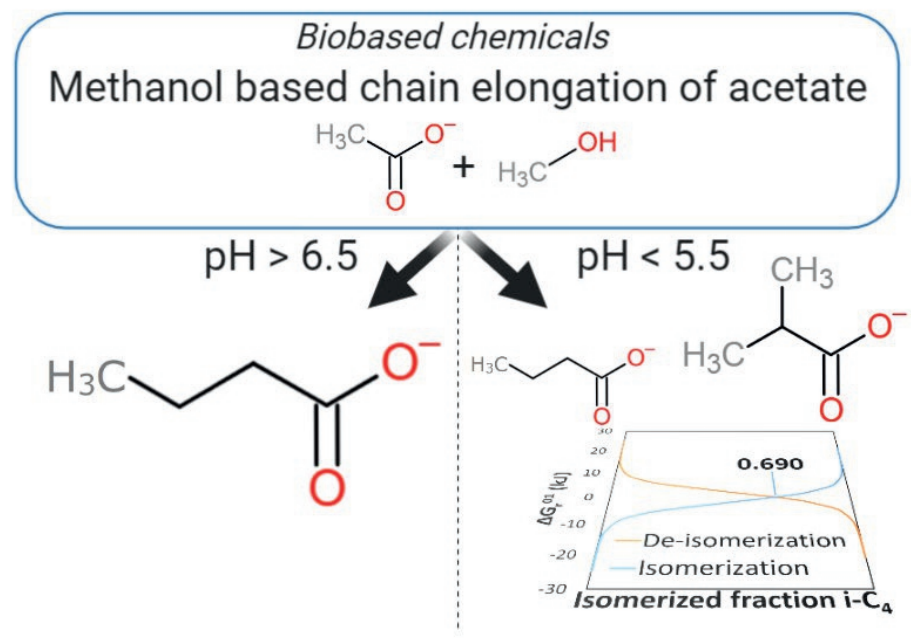

This chapter is published as:

Kasper D. de Leeuw, Sanne M. de Smit, Sabine van Oossanen, Marinus J. Moerland Cees J.N. Buisman, David P.B.T.B. Strik, 2020, Methanol based chain elongation with acetate to $\mathrm{n}$-butyrate and isobutyrate at varying selectivities dependent on $\mathrm{pH}$

ACS Sustainable Chem. Eng. 2020, 8, 22, 8184-8194

https://doi.org/10.1021/acssuschemeng.0c00907 


\section{Abstract}

Biomass fermentation technologies offer alternative methods to produce platform chemicals that currently originate from fossil sources. This research showed that an enriched microbiome was capable to produce isobutyrate $\left(i-C_{4}\right)$ from acetate via methanol based chain elongation. A long term continuous reactor experiment showed that the selectivity for $\mathrm{i}-\mathrm{C}_{4}$ and/or $\mathrm{n}$-butyrate ( $\left.\mathrm{n}-\mathrm{C}_{4}\right)$ could be reversibly adjusted by changing the reactor $\mathrm{pH}$. A reactor $\mathrm{pH}$ of 6.75 led to formation of (carbon per total carbon of products) $0.78 \mathrm{n}-\mathrm{C}_{4}$ and $0.024 \mathrm{i}-\mathrm{C}_{4}$, whereas a reactor $\mathrm{pH}$ of 5.2 led to a selectivity of $0.24 \mathrm{n}-\mathrm{C}_{4}$ and $0.65 \mathrm{i}-\mathrm{C}_{4}$. This shift in product spectrum was also represented by a shift in microbial composition. The results suggest that an Eubacterium genus is responsible for the formation of $\mathrm{n}-\mathrm{C}_{4}$, whereas a Clostridium luticellarii strain is responsible for the formation of a mixture of $\mathrm{i}-\mathrm{C}_{4}$ and $\mathrm{n}-\mathrm{C}_{4}$. The formation of $\mathrm{n}$ - and $\mathrm{i}-\mathrm{C}_{4}$ at a low $\mathrm{pH}$ was observed to be coupled according to the thermodynamics of isomerization. At a reactor $\mathrm{pH}$ of 5.5 and 5.2 the product ratio of $\mathrm{i}-\mathrm{C}_{4}: \mathrm{n}-\mathrm{C}_{4}$ approached $0.69 \mathrm{i}-\mathrm{C}_{4}: 0.31 \mathrm{n}-\mathrm{C}_{4}$, which is the theoretical ratio that would be achieved when determined by the equilibrium of isomerization. Various batch experiments at pH 5.5 and 5.2 confirmed that addition of either $\mathrm{n}-\mathrm{C}_{4}$ or $\mathrm{i}-\mathrm{C}_{4}$ at the start of the batch would directly lead to the formation of the other butyrate component. Moreover, batch experiments performed at $\mathrm{pH} 6.5$ produced mainly $\mathrm{n}-\mathrm{C}_{4}$ and led to the development of a completely different microbiome. The imposed $\mathrm{pH}$ is a strong selection pressure that can facilitate changes in product selectivities for $n-C_{4}$ and $i-C_{4}$ during methanol based chain elongation of acetate.

Keywords: Chain elongation, Selection pressure, Open-culture fermentation, Biobased chemicals, Methanol, n-Butyrate, Isobutyrate 


\section{Introduction}

The necessity to transition towards a circular economy has driven researchers to explore new biomass fermentation processes for alternative production of currently oil-derived and palm-derived chemicals22, 70-72. Microbial chain elongation fermentations have potential to become an alternative and sustainable technology for acquiring platform chemicals, such as short chain carboxylates (SCCs) and medium chain carboxylates (MCCs) ${ }^{25,73,74}$. Biomass residues and $\mathrm{CO}_{2}$ are used as favorable substrates for these bioprocesses. The carbon streams can be more efficiently recycled which could help lessen environmental harmful expansion of agricultural areas. Microbiomes that catalyze chain elongation processes have been enriched using different selection pressures, for which the electron donor is an important factor ${ }^{22,75}$. Methanol is an exceptionally interesting electron donor, because it can be electrochemically synthesized from $\mathrm{CO}_{2}$ or thermochemically produced from lignocellulosic biomass ${ }^{45,}$, 76-78.

Recently it has been shown that isobutyrate (i- $\left.\mathrm{C}_{4}\right)$ can be formed during a methanol based microbial chain elongation ${ }^{35}, 79$. It was observed that a high concentration of n-butyrate ( $n$ $\mathrm{C}_{4}$ ) is necessary to stimulate isomerization towards $\mathrm{i}-\mathrm{C}_{4}$ within the methanol based chain elongation microbiome. It was also shown that addition of methanol to $\mathrm{n}$ - $\mathrm{C}_{4}$-rich fermented supermarket waste residue induced the formation of $\mathrm{i}-\mathrm{C}_{4}$. The precise metabolic route for the biochemical $\mathrm{i}-\mathrm{C}_{4}$ formation has not been fully elucidated, but it was suggested that it takes place via methanol chain elongation and subsequent isomerization of $n-C_{4}$ to $i-C_{4}$. Alternatively $\mathrm{i}_{-} \mathrm{C}_{4}$ can be formed via microbial electrosynthesis, however, for now its formation was only observed as a side product ${ }^{80,81}$. Also biological production of $\mathrm{i}-\mathrm{C}_{4}$ from sugars has been shown using metabolically engineered strains ${ }^{82}, 83$. Currently, $\mathrm{i}-\mathrm{C}_{4}$ is produced chemically from fossil-based feedstocks and other compounds such as alkali metal bases or metal alcoholates ${ }^{84}$. Isobutyrate can be used in the production processes of transparent thermoplastics, emulsifiers, vitamins, antibiotics and organic solvents 82,85 , ${ }^{86}$. It has a market size of around $2.7 \cdot 10^{6}$ ton/year ${ }^{83}$. The newly discovered methanol chain elongation bioprocess within a mixed culture prospects an alternative route towards $\mathrm{i}-\mathrm{C}_{4}$ production ${ }^{35}$. By using only acetate and methanol as sole substrates and by using a mixed culture, a versatile organic residue based fermentation process can be developed ${ }^{27}$. Methanol based chain elongation experimentally complied to the following stoichiometry ${ }^{35}$, 43, 46:

2 methanol + acetate $\rightarrow$ n-butyrate $+2 \mathrm{H}_{2} \mathrm{O} \quad \Delta \mathrm{Gr}^{01}=-106.8 \mathrm{~kJ}$

The objectives of this study were to further elucidate the selection pressure involved in enriching the $\mathrm{i}-\mathrm{C}_{4}$ forming microbiome and to develop this microbiome without supplying exogenous $n-C_{4}$. A long term continuous reactor system was operated to study varying $\mathrm{pH}$ 
levels (from 6.75 to 5.2 ). The necessity for high $n-C_{4}$ concentrations in earlier studies was hypothesized as a toxic effect on the microbiome that induced isomerization ${ }^{35}$. Hypothetically a similar selection pressure could be achieved by high amounts of in situ butyrate formation from acetate and methanol. Additionally, lowering the $\mathrm{pH}$ and thus increasing the amount of undissociated acids in the broth could further amplify the toxicity of the formed butyrates towards microorganisms ${ }^{59,87}$. We show with this experimental work that at $\mathrm{pH} 5.2$ the microbiome produced $\mathrm{i}-\mathrm{C}_{4}$ with a $65 \%$ carbon selectivity from acetate and methanol as sole substrates. A selection pressure tool is presented that can be used to form i- $\mathrm{C}_{4}$ with a mixed culture from simple molecules such as acetate and methanol.

In addition to the continuous reactor experiment, a series of batch experiments was performed to gain insight into the microbial activity and microbiome enrichment under varying starting $\mathrm{pH}$ levels, different $\mathrm{n}-/ \mathrm{i}-\mathrm{C}_{4}$ starting concentrations and different substrate (acetate and methanol) concentrations. These batch series give insight into how the i- $\mathrm{C}_{4}$ production is coupled to catabolic conversion of the chain elongation substrates and how the isomer formation specificity is affected.

\section{Material and Methods}

This research aimed to investigate what selection pressure is needed for a methanol chain elongation microbiome to produce isobutyrate $\left(i-C_{4}\right)$ and/or $n$-butyrate $\left(n-C_{4}\right)$ from acetate $\left(\mathrm{C}_{2}\right)$ and methanol $(\mathrm{MeOH})$. A continuous reactor was operated throughout seven phases to investigate the effect of different $\mathrm{pH}$ levels ( $\mathrm{pH} 5.2,5.5,6.0$ and 6.75). Moreover, $\mathrm{CO}_{2}$ addition was removed (phase $\mathrm{I} \rightarrow \mathrm{II}$ ), a slight increase and consecutive decrease of acetate in the influent was executed (II $\rightarrow \mathrm{III} \rightarrow \mathrm{IV}$ ) and the vitamin B12 supply was dropped by a factor of $30(\mathrm{III} \rightarrow \mathrm{IV})$. A description of the operating conditions during the seven phases is shown in Table 1. The reactor system was characterized by analysis of the steady state mass balances for the total amount of carbon and electrons in the liquid and gas phase.

In addition to the continuous reactor, batch experiments were performed to investigate (i) the immediate effect of two different $\mathrm{pH}$ levels (5.2 and 6.5) on product formation and microbial composition; (ii) whether initial $\mathrm{n}-\mathrm{C}_{4}$ or $\mathrm{i}-\mathrm{C}_{4}$ addition affects the product selectivity; (iii) how the absence or presence of carbon sources affect the (de-)isomerization of $\mathrm{n}$ - and $\mathrm{i}-\mathrm{C}_{4}$ and (iv) how different substrate concentrations affect the methanol chain elongation process. An overview of the batch experiments is given in Table 2. 


\section{Medium}

The synthetic growth medium contained the following macro nutrients $\left(\mathrm{g} \mathrm{L}^{-1}\right): \mathrm{NH}_{4} \mathrm{H}_{2} \mathrm{PO}_{4}$ $3.60 ; \mathrm{MgCl}_{2} .6 \mathrm{H}_{2} \mathrm{O} 0.33 ; \mathrm{MgSO}_{4} .7 \mathrm{H}_{2} \mathrm{O} 0.20 ; \mathrm{CaCl}_{2} .2 \mathrm{H}_{2} \mathrm{O} 0.20 ; \mathrm{KCl} 0.20$ and yeast extract 1.0. Micro nutrients were used as described in Phillips et al. (1993) (designed basal medium Pfennig trace metals and B-vitamins) ${ }^{88}$. The B-vitamin composition of this medium differed in phase I, II, and III. The vitamin $B_{12}$ cobalamin concentration in these phases was 30 times higher than in the following phases $\left(0.375 \mathrm{mg} \mathrm{L}^{-1}\right.$ compared to $\left.0.0125 \mathrm{mg} \mathrm{L}^{-1}\right)$. The higher vitamin concentration was initially used in concordance to the earlier performed research on methanol based formation $\mathrm{i}-\mathrm{C}_{4}{ }^{35}$. The vitamin concentration was lowered to rule out the necessity of these high amounts. The carbon sources during the whole study were acetate and methanol. For the continuous reactor experiment in phase I, II, IV, V VI and VII the concentrations were $150 \mathrm{mM}\left(8.9 \mathrm{~g} \mathrm{~L}^{-1}\right)$ acetate and $300 \mathrm{mM}\left(9.6 \mathrm{~g} \mathrm{~L}^{-1}\right)$ methanol and during phase III the acetate concentration was temporarily increased to 180 $\mathrm{mM}$ (10.8 $\left.\mathrm{g} \mathrm{L}^{-1}\right)$. The starting concentrations for the batch experiments ranged from 50 $\mathrm{mM}$ and $25 \mathrm{mM}$ to respectively $300 \mathrm{mM}$ and $200 \mathrm{mM}$ for methanol and acetate. See Table 2 for the exact starting concentrations for each batch.

\section{Inoculum}

The reactor was inoculated with an undefined anaerobic culture that originated from a reactor that performed methanol chain elongation from $\mathrm{n}-\mathrm{C}_{4}$ to $\mathrm{n}$-caproate and also formed $\mathrm{i}-\mathrm{C}_{4}{ }^{35}$. It's complete $1 \mathrm{~L}$ broth was used at the start of this experiment. The batches of the first series (I-VIII, Table 2) were inoculated with suspended biomass from the continuous reactor during the end of phase VII. The batches of the second series were inoculated with biomass that originated from batch III and was subsequently kept active with a repeated batch. A more detailed description of the inoculation is described in the Supplementary Information section 'Materials \& Methods'.

\section{Reactor set-up and operation}

The reactor experiment was performed for 489 days in a lab-scale continuous up flow anaerobic reactor setup ( $1 \mathrm{~L}$ working volume) as described in an earlier chain elongation research $^{89}$. Table 1 summarizes the operating parameters (HRT, $\left.\mathrm{pH}, \mathrm{CO}_{2}\right)$. The temperature was kept constant at $35^{\circ} \mathrm{C}$. The medium inflow was set at $25 \mathrm{ml} \mathrm{hour}^{-1}$, which led to an HRT of around 40-46 hours. During the first four phases the HRT slowly increased due to diminished flow rate caused by clogging of the influent tubes. This problem was solved from phase $V$ and onwards. The aim of the chosen HRT value was to provide sufficient retention for methanol based chain elongation organisms, while simultaneously suppressing suspended methanogenic growth ${ }^{45}$, 90. Biofilms were allowed to grow in the reactor; periodically (approximately once per month) sludge-like biomass that 
accumulated in the $\mathrm{pH}$ control unit was removed. The reactor $\mathrm{pH}$ was regulated by automatic titration using $1 \mathrm{M} \mathrm{KOH}$ as base. In the first phase $\left(120 \mathrm{mln} \mathrm{day}^{-1}\right) \mathrm{CO}_{2}$ was supplied, because earlier experiments showed successful methanol based chain elongation under $\mathrm{CO}_{2}$ supplementation ${ }^{45}, 46$. However, $\mathrm{CO}_{2}$ can act as electron acceptor for acetateproducing methylotrophs which compete for methanol as substrate and reduce selectivity towards butyrates ${ }^{43}$. Additionally, sufficient $\mathrm{CO}_{2}$ was produced in the reactor itself, via possibly yeast extract conversion and/or methanogenesis. Therefore its supply was stopped from phase II and onwards. The reactor was partially mixed via internal recirculation at $150 \mathrm{ml} \mathrm{min}^{-1}$. A schematic overview of the reactor setup is shown in Figure S1.

Table 1. The overview shows a short description of each phase as well as the phase duration, the steady state period per phase, the $\mathrm{pH}$ set points and the set HRTs.

\begin{tabular}{|c|c|c|c|c|c|c|c|}
\hline & Phase I & Phase II & Phase III & Phase IV & Phase V & Phase VI & Phase VII \\
\hline Description & $\begin{array}{l}\text { Changed } \\
\text { pH set } \\
\text { point } 5.5 \\
\mathrm{CO}_{2} \text { flow } \\
\text { set to } 120 \\
\mathrm{mLn} \text { day }^{-1}\end{array}$ & $\begin{array}{l}\text { no } \mathrm{CO} 2 \\
\text { input }\end{array}$ & $\begin{array}{l}\text { Changed } \\
\text { pH set } \\
\text { point from } \\
5.5 \text { to } 6, \\
\text { increased } \\
\text { acetate } \\
\text { from } 9.0 \text { to } \\
10.8 \mathrm{~g} \mathrm{~L}^{-1}\end{array}$ & $\begin{array}{l}\text { Decreased } \\
\text { acetate from } \\
10.8 \text { to } 9.0 \\
\mathrm{~g} \mathrm{~L}^{-1} \text {, } \\
\text { decreased } \\
\text { vitB12 from } \\
362.5 \mu \mathrm{g} \mathrm{L}^{-1} \\
\text { to } 12.5 \mu \mathrm{g} \mathrm{L}^{-1}\end{array}$ & $\begin{array}{l}\text { Changed } \mathrm{pH} \\
\text { set point } \\
\text { from } 6.0 \text { to } \\
6.75\end{array}$ & $\begin{array}{l}\text { Changed } \\
\text { pH set } \\
\text { point from } \\
6.75 \text { to } \\
5.50\end{array}$ & $\begin{array}{l}\text { Changed } \mathrm{pH} \\
\text { set point } \\
\text { from } 5.50 \text { to } \\
5.20\end{array}$ \\
\hline Phase period & $\begin{array}{l}\text { day } 1 \text { to } \\
48\end{array}$ & $\begin{array}{l}\text { day } 48 \text { to } \\
111\end{array}$ & $\begin{array}{l}\text { day } 111 \text { to } \\
\text { day } 142\end{array}$ & $\begin{array}{l}\text { day } 142 \text { to } \\
181\end{array}$ & $\begin{array}{l}\text { day } 181 \text { to } \\
327\end{array}$ & $\begin{array}{l}\text { day } 327 \text { to } \\
399\end{array}$ & $\begin{array}{l}\text { day } 399 \text { to } \\
489\end{array}$ \\
\hline $\begin{array}{l}\text { Steady state } \\
\text { period }\end{array}$ & $\begin{array}{l}\text { day } 40 \text { to } \\
48\end{array}$ & $\begin{array}{l}\text { day } 94 \text { to } \\
111\end{array}$ & $\begin{array}{l}\text { day } 123 \text { to } \\
135\end{array}$ & $\begin{array}{l}\text { day } 166 \text { to } \\
181\end{array}$ & $\begin{array}{l}\text { day } 311 \text { to } \\
327\end{array}$ & $\begin{array}{l}\text { day } 345 \text { to } \\
364\end{array}$ & $\begin{array}{l}\text { day } 472 \text { to } \\
489\end{array}$ \\
\hline $\begin{array}{l}\text { Temperature } \\
\left({ }^{\circ} \mathrm{C}\right)\end{array}$ & 35 & 35 & 35 & 35 & 35 & 35 & 35 \\
\hline $\mathrm{CO}_{2}$ inflow & $\begin{array}{l}120 \mathrm{mLn} \\
\text { day }^{-1}\end{array}$ & - & - & - & - & - & - \\
\hline $\mathrm{pH}$ set point & 5.5 & 5.5 & 6.0 & 6.0 & 6.75 & 5.5 & 5.2 \\
\hline $\begin{array}{l}\text { Set HRT } \\
\text { (hrs) }\end{array}$ & 40 & 40 & 40 & 40 & 40 & 40 & 40 \\
\hline
\end{tabular}

\section{Steady state characterization}

During the seven phases a steady state was assumed when the following criteria were met:

(i) for at least 5 times the HRT no setup change had occurred and (ii) main metabolite concentrations and corresponding conversion rates were relatively constant. Relatively constant was defined as when the confidence interval was less than $20 \%$ of the steady state average values. Confidence intervals were determined with an a of 0.01 and are indicated with a \pm . 


\section{Batch experiments}

At the end of the seventh phase a series of 8 batch experiments was carried out in duplo to investigate how the formation of $\mathrm{n}-\mathrm{C}_{4}$ and $\mathrm{i}-\mathrm{C}_{4}$ is coupled to methanol based acetate elongation under various conditions (see Table $\mathbf{2}$ ).

Table 2. Overview of the performed batches.

\begin{tabular}{|c|c|c|c|c|c|c|}
\hline Inoculum & Methanol mM & Acetate $\mathrm{mM}$ & $\mathrm{pH}$ & $\mathrm{i}-\mathrm{C}_{4} \mathrm{mM}$ & $\mathrm{n}-\mathrm{C}_{4} \mathrm{mM}$ & Batch Code \\
\hline \multirow{4}{*}{$\begin{array}{l}\text { Phase VII } \\
\text { (suspended cells) }\end{array}$} & 50 & 25 & 5.2 & & 50 & I \\
\hline & 50 & 25 & 6.5 & & 50 & II \\
\hline & 50 & 25 & 5.2 & 50 & & III \\
\hline & 50 & 25 & 6.5 & 50 & & IV \\
\hline \multirow{4}{*}{$\begin{array}{l}\text { Phase VII } \\
\text { (suspended cells) }\end{array}$} & - & - & 5.2 & & 50 & v \\
\hline & - & - & 6.5 & & 50 & VI \\
\hline & - & - & 5.2 & 50 & & VII \\
\hline & - & - & 6.5 & 50 & & VIII \\
\hline \multirow{4}{*}{$\begin{array}{l}\text { Repeated batch } \\
\text { (suspended cells) }\end{array}$} & 300 & 150 & 5.5 & 0 & - & A \\
\hline & 300 & 150 & 5.5 & 5 & - & B \\
\hline & 300 & 150 & 5.5 & 20 & - & C \\
\hline & 300 & 150 & 5.5 & 50 & - & D \\
\hline none & 300 & 150 & 5.5 & 0 & - & E \\
\hline $\begin{array}{l}\text { Repeated batch } \\
\text { (suspended cells) }\end{array}$ & 0 & 0 & 5.5 & 0 & - & $\mathrm{F}$ \\
\hline \multirow{6}{*}{$\begin{array}{l}\text { Repeated batch } \\
\text { (suspended cells) }\end{array}$} & 50 & 25 & 5.5 & 50 & - & G \\
\hline & 100 & 50 & 5.5 & 50 & - & $\mathrm{H}$ \\
\hline & 200 & 200 & 5.5 & 50 & - & J \\
\hline & 300 & 150 & 5.5 & 50 & - & K \\
\hline & 50 & 25 & 5.2 & 50 & - & $\begin{array}{l}\text { L (III } \\
\text { repeat) }\end{array}$ \\
\hline & 0 & 0 & 5.5 & 50 & - & M \\
\hline $\begin{array}{l}\text { Eubacterium } \\
\text { limosum }\end{array}$ & 300 & 150 & 5.5 & - & - & EL batch \\
\hline
\end{tabular}

Firstly, batches were performed at pH 6.5 (batch II, IV, VI and VIII) and at pH 5.2 (batch I, III, V and VII). Secondly the effect of initially present chain elongation product on formation selectivity was investigated: either $50 \mathrm{mM} \mathrm{n}-\mathrm{C}_{4}$ (batch I, II, V and VI) or $50 \mathrm{mM}$ $\mathrm{i}_{-} \mathrm{C}_{4}$ (batch III, IV, VII and VIII) was added. Thirdly (V to VIII), a control series was performed without methanol and acetate in the medium, because it was unclear if $\mathrm{i}-\mathrm{C}_{4}$ was formed via a secondary isomerization coupled to degradation ${ }^{91-95}$ or via a catabolically (and seemingly irreversible) driven formation. After this first series the microbiome from batch III was used for a second series of 12 batch experiments in duplicate (batches $A$ to M, Table 2) to determine how different substrate concentrations affect the methanol based 
chain elongation. In addition to these batches a pure culture Eubacterium limosum $2 \mathrm{~A}$ (DSM 2593) was used as inoculum to perform a batch at pH 5.5 and methanol and acetate as substrate. The aim was to check if this culture was able to also form $\mathrm{i}-\mathrm{C}_{4}$ under these conditions, as it is know that $E$. limosum can form $n-C_{4}{ }^{96}$. The protocols for the batch experiments are described in the Supplementary Information section M\&M (and Table S1 to Table S6).

\section{Sampling and measurement}

Continuous reactor samples were taken approximately two or three times per week. Batch samples were taken three times per week in the beginning and less frequently as the batch progressed. The $\mathrm{pH}$ of the samples was checked using an external $\mathrm{pH}$ meter, which allowed periodic recalibration of the internal $\mathrm{pH}$-controller in the continuous system. The liquid samples were analyzed for primary alcohols (methanol to hexanol), isobutanol and volatile fatty acids (acetic acid to n-caprylic acid) composition via gas chromatography by an earlier described method ${ }^{97}$. The headspace gas was analyzed every time a liquid sample was taken. The concentrations of $\mathrm{O}_{2}, \mathrm{~N}_{2}, \mathrm{CH}_{4}, \mathrm{H}_{2}$, and $\mathrm{CO}_{2}$ were determined via two gas chromatography systems as described earlier 45,74 . The headspace of the continuous reactor was connected to a gas meter ( $\mu$ Flow Bioprocess Control, Sweden) to measure gas production. The headspace pressure in the batches was measured (pressure meter GMH 3151).

\section{Microbial community analysis}

Biomass samples during the steady states in phase II, IV, V, VI and VII, as well as biomass samples taken at the end of batches (I to VIII) were used to analyze the microbial compositions. Biomass was acquired by spinning down approximately $25 \mathrm{~mL}$ of suspended broth (either from the continuous reactor, or at the end of a batch run) and subsequently snap freezing the pellet using liquid nitrogen and storing it at $-80^{\circ} \mathrm{C}$. In the case of the biofilm sample in phase VI $1 \mathrm{~mL}$ of biomass sludge was used for the DNA extraction. All biomass samples were taken in duplo and analyzed separately. DNA was extracted using a Powersoil DNA isolation kit. The isolated DNA was used as template for amplifying the V3-V4 region of 16S rRNA gene using the illumina library generation method described by Takahashi et al ${ }^{98}$. Exactly the same method for the 16S rRNA gene amplicon analysis was used as described earlier $89,99-101$. Sequences of frequently found OTUs were used for a NCBI BLAST query. Canoco 5 was used to make two redundancy triplots (Figure S21 and S22) to support discussed correlations. Sequencing data is submitted to the ENA database and can be found with the accession number PRJEB36205. 


\section{Results}

\section{Reactor $\mathrm{pH}$ influences formation selectivities of $\mathrm{n}-\mathrm{C}_{4}$ and i- $\mathrm{C}_{\mathbf{4}}$ during methanol based chain elongation of acetate.}

The continuous reactor system enriched microbiomes that performed methanol based chain elongation of acetate $\left(C_{2}\right)$ to isobutyrate $\left(i-C_{4}\right)$ and $n$-butyrate $\left(n-C_{4}\right)$ as main products in varying ratios dependent on reactor $\mathrm{pH}$. Side products were $n$-caproate $\left(n-C_{6}\right)$ and methane $\left(\mathrm{CH}_{4}\right)$. Figure 1 shows the reactor broth concentrations of the main metabolites and Table $\mathbf{3}$ summarizes the performance of the reactor. The results from phase IV to VII are particularly important, because the only varied reactor parameter in the phases following phase IV was the broth $\mathrm{pH}$. During the shift from phase IV to $\mathrm{V}$ it took around 100 days for the $\mathrm{i}-\mathrm{C}_{4}$ formation rate to drop and stabilize at around $4 \mathrm{mmol} \mathrm{C \textrm {L } ^ { - 1 }}$ day $^{-1}$. Consequently $\mathrm{n}-\mathrm{C}_{4}$ formation increased with $86 \%$ to $142 \pm 13 \mathrm{mmol} \mathrm{C} \mathrm{L}^{-1}$ day $^{-1}$ or $3.1 \pm 0.3 \mathrm{~g} \mathrm{~L}^{-1}$ day $^{-1}$. With a concentration of $237 \pm 22 \mathrm{mM} \mathrm{C}$ or $5.2 \pm 0.5 \mathrm{~g} \mathrm{~L}^{-1}, \mathrm{n}-\mathrm{C}_{4}$ was the dominant product in phase $\mathrm{V}$ (78\% selectivity based on carbon atoms). The reactor $\mathrm{pH}$ set point was lowered to $\mathrm{pH} 5.5$ in phase VI to investigate the reversibility of using $\mathrm{pH}$ as a selection pressure to enrich an $\mathrm{i}-\mathrm{C}_{4}$ forming microbiome.

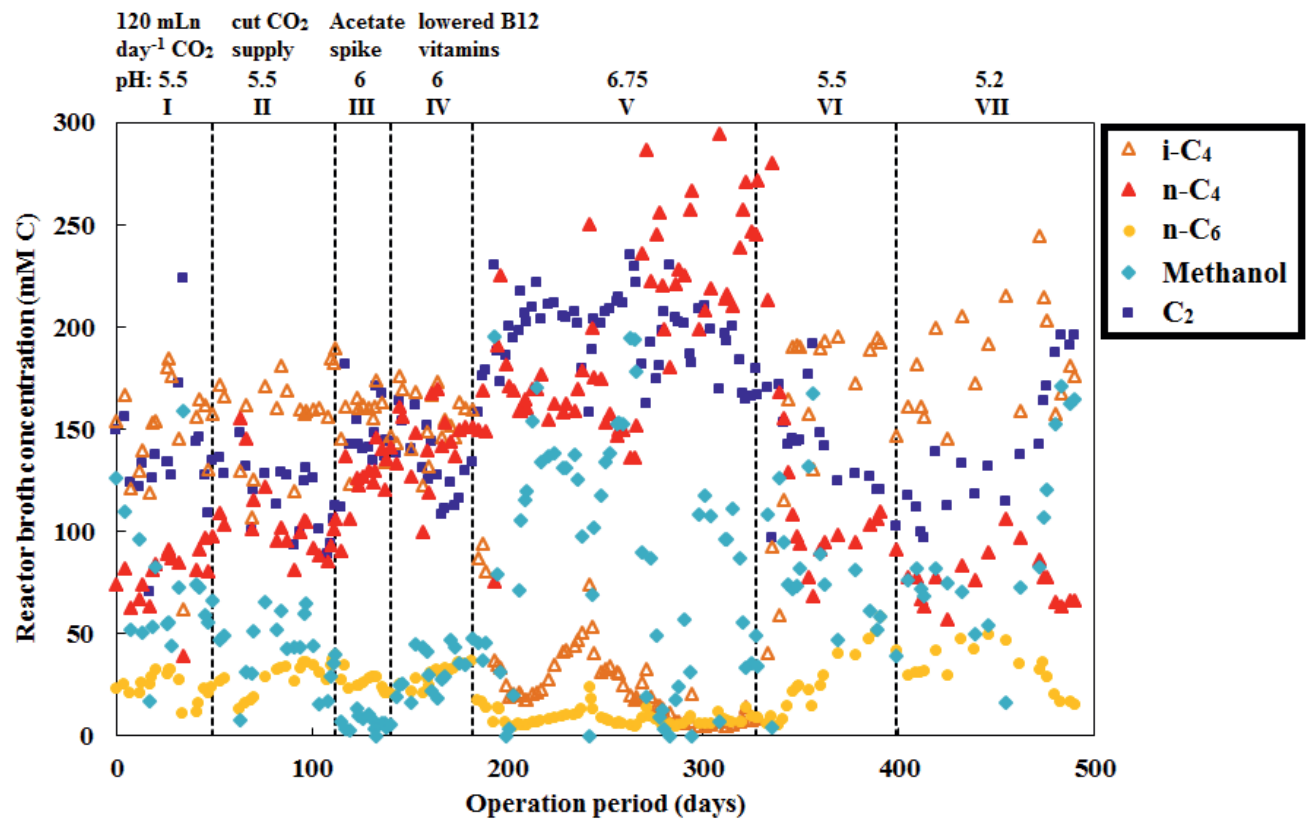

Figure 1. The graph shows the reactor broth concentrations of the main metabolites. A short summary of each phase is depicted above the graph. For phase VII the $\mathrm{pH}$ set point value 5.2 was stably maintained only for the last 50 days (10 measurement points) due to $\mathrm{pH}$ probe calibration issues. 
Table 3. This overview shows the measured steady state averaged values of $\mathrm{pH}, \mathrm{HRT}$, volumetric conversion rates, organic loading rate (OLR), $\mathrm{i}-\mathrm{C}_{4}$ to $\mathrm{n}-\mathrm{C}_{4}$ ratios, main product selectivites, percentages of unconverted substrate and carbon/electron balances. Yeast extract (YE) was assumed to be completely consumed (using $0.4148 \mathrm{~g} \mathrm{C} \mathrm{g}^{-1}$ ) ${ }^{102}$. Propionate and $\mathrm{b} / \mathrm{n}$-valerate are not shown $\left(<3 \mathrm{mmol} \mathrm{C} \mathrm{L}^{-1}\right.$ day $\left.^{-1}\right)$.

\begin{tabular}{|c|c|c|c|c|c|c|c|c|c|c|c|c|c|c|}
\hline & \multicolumn{2}{|c|}{ Phase I } & \multicolumn{2}{|c|}{ Phase II } & \multicolumn{2}{|c|}{ Phase III } & \multicolumn{2}{|c|}{ Phase IV } & \multicolumn{2}{|c|}{ Phase V } & \multicolumn{2}{|c|}{ Phase VI } & \multicolumn{2}{|c|}{ Phase VII } \\
\hline $\begin{array}{l}\text { Steady } \\
\text { state } \\
\text { period }\end{array}$ & \multicolumn{2}{|c|}{ day 40 to 48} & \multicolumn{2}{|c|}{$\begin{array}{l}\text { day } 94 \text { to } \\
111\end{array}$} & \multicolumn{2}{|c|}{$\begin{array}{l}\text { day } 123 \text { to } \\
135\end{array}$} & \multicolumn{2}{|c|}{$\begin{array}{l}\text { day } 166 \text { to } \\
181\end{array}$} & \multicolumn{2}{|c|}{$\begin{array}{l}\text { day } 217 \text { to } \\
233\end{array}$} & \multicolumn{2}{|c|}{$\begin{array}{l}\text { day } 345 \text { to } \\
364\end{array}$} & \multicolumn{2}{|c|}{$\begin{array}{l}\text { day } 472 \text { to } \\
489\end{array}$} \\
\hline $\mathrm{pH}$ & \multicolumn{2}{|c|}{$5.79 \pm 0.03$} & \multicolumn{2}{|c|}{$5.65 \pm 0.04$} & \multicolumn{2}{|c|}{$6.10 \pm 0.03$} & \multicolumn{2}{|c|}{$6.02 \pm 0.5$} & \multicolumn{2}{|c|}{$6.76 \pm 0.8$} & \multicolumn{2}{|c|}{$5.61 \pm 0.06$} & \multicolumn{2}{|c|}{$5.23 \pm 0.07$} \\
\hline HRT & \multicolumn{2}{|c|}{$42.8 \pm 2.2$} & \multicolumn{2}{|c|}{$45.6 \pm 5.2$} & \multicolumn{2}{|c|}{$45.5 \pm 1.0$} & \multicolumn{2}{|c|}{$46.3 \pm 1.9$} & \multicolumn{2}{|c|}{$40.9 \pm 0.2$} & \multicolumn{2}{|c|}{$40.2 \pm 0.8$} & \multicolumn{2}{|c|}{$41.5 \pm 0.9$} \\
\hline \multicolumn{15}{|c|}{ Volumetric C molar conversion rates and OLR $\left(\mathrm{mmol} \mathrm{C} \mathrm{L}^{-1}\right.$ day $\left.^{-1}\right)$} \\
\hline $\mathrm{i}-\mathrm{C}_{4}$ & 87 & \pm 12 & 90 & \pm 11 & 86 & \pm 3 & 80 & \pm 4 & 4 & \pm 2 & 106 & \pm 12 & 111 & \pm 14.5 \\
\hline$n-C_{4}$ & 49 & \pm 4 & 53 & \pm 6 & 69 & \pm 3 & 76 & \pm 2 & 142 & \pm 13 & 54 & \pm 7 & 42 & \pm 4 \\
\hline$n-C_{6}$ & 10 & \pm 3 & 19 & \pm 2 & 14 & \pm 1 & 17 & \pm 1 & 5.0 & \pm 1 & 14 & \pm 2 & 14 & \pm 4.4 \\
\hline $\mathrm{CH}_{4}$ & 31 & \pm 1 & 23 & \pm 3 & 31 & \pm 5 & 21 & \pm 1 & 22 & \pm 8 & 20 & \pm 5 & 2 & \pm 1.8 \\
\hline $\mathrm{CO}_{2}$ & 11 & \pm 1 & 9.1 & \pm 1 & 11 & \pm 1 & 6.2 & \pm 0.5 & 3.1 & \pm 0.9 & 8 & \pm 1.3 & 1 & \pm 0.4 \\
\hline $\mathrm{MeOH}$ & -129 & \pm 6 & -137 & \pm 14 & -154 & \pm 3 & -134 & \pm 3 & -134 & \pm 17 & -120 & \pm 18 & -93 & \pm 17 \\
\hline $\mathrm{C}_{2}$ & -91 & \pm 10 & -103 & \pm 16 & -110 & \pm 7 & -90 & \pm 4 & -67 & \pm 8 & -84 & \pm 11 & -68 & \pm 10 \\
\hline Yeast Extract & -14 & \pm 0.9 & -13.8 & \pm 1.3 & -14 & \pm 0.3 & -13.3 & \pm 0.2 & -15.3 & \pm 0.4 & -15.6 & \pm 0.3 & -15.0 & \pm 0.3 \\
\hline OLR & 347 & \pm 21 & 335 & \pm 33 & 361 & \pm 8 & 319 & \pm 4 & 367 & \pm 8 & 373 & \pm 6 & 359 & \pm 6 \\
\hline \multicolumn{15}{|c|}{ i-C4 to n-C4 ratios, selectivities and percentages of unconverted substrates } \\
\hline $\begin{array}{l}\mathrm{i}-\mathrm{C}_{4} \text { to } \mathrm{n}-\mathrm{C}_{4} \\
\text { ratios } \\
\text { (molar) }\end{array}$ & $0.64:$ & 0.36 & $0.63:$ & 0.37 & 0.55 & 0.45 & $0.51:$ & 0.49 & $0.03:$ & 0.97 & $0.66:$ & 0.34 & $0.73:$ & 0.27 \\
\hline $\begin{array}{l}\mathrm{i}-\mathrm{C}_{4} / \text { total } \\
\text { products } \\
\text { (carbon) }\end{array}$ & $46 \%$ & & $46 \%$ & & $40 \%$ & & $39 \%$ & & $2 \%$ & & $51 \%$ & & $65 \%$ & \\
\hline $\begin{array}{l}\mathrm{n}-\mathrm{C}_{4} / \text { total } \\
\text { products } \\
\text { (carbon) }\end{array}$ & $26 \%$ & & $27 \%$ & & $32 \%$ & & $37 \%$ & & $78 \%$ & & $26 \%$ & & $24 \%$ & \\
\hline $\begin{array}{l}\text { Unconverted } \\
\mathrm{C}_{2}\end{array}$ & $45 \%$ & & $38 \%$ & & $42 \%$ & & $41 \%$ & & $61 \%$ & & $53 \%$ & & $60 \%$ & \\
\hline $\begin{array}{l}\text { Unconverted } \\
\mathrm{MeOH}\end{array}$ & $22 \%$ & & $14 \%$ & & $3 \%$ & & $12 \%$ & & $24 \%$ & & $33 \%$ & & $46 \%$ & \\
\hline
\end{tabular}

Balances

\begin{tabular}{|c|c|c|c|c|c|c|c|c|c|c|c|c|c|c|}
\hline C-balance & $86 \%$ & $\pm 7 \%$ & $82 \%$ & $\pm 4 \%$ & $81 \%$ & $\pm 3 \%$ & $87 \%$ & $\pm 5 \%$ & $89 \%$ & $\pm 3 \%$ & $95 \%$ & $\pm 2 \%$ & $98 \%$ & $\pm 2 \%$ \\
\hline e-balance & $86 \%$ & $\pm 7 \%$ & $82 \%$ & $\pm 4 \%$ & $82 \%$ & $\pm 3 \%$ & $88 \%$ & $\pm 6 \%$ & $89 \%$ & $\pm 4 \%$ & $95 \%$ & $\pm 2 \%$ & $99 \%$ & $\pm 2 \%$ \\
\hline
\end{tabular}


It took 18 days (about 11 times the HRT) for the $\mathrm{i}-\mathrm{C}_{4}$ formation rate to return back to the levels achieved in phase I and II. In the last phase (VII) the pH set point was set to 5.2. This led to the highest achieved steady state average volumetric production rate for $\mathrm{i}-\mathrm{C}_{4}$, with $111 \pm 15 \mathrm{mmol} \mathrm{C} \mathrm{L}^{-1}$ day $^{-1}$ or $2.4 \pm 0.3 \mathrm{~g} \mathrm{~L}^{-1}$ day $^{-1}$, an average broth concentration of $191 \pm 28 \mathrm{mM} \mathrm{C}$ or $4.2 \pm 0.6 \mathrm{~g} \mathrm{~L}^{-1}$ and $65 \%$ selectivity (based on carbon atoms).

\section{Methanogenic activity was strongly sensitive to change between pH $\mathbf{5 . 6}$ and $\mathbf{5 . 2}$}

From day 374 in phase VI to day 455 in phase VII there were calibration issues of the automatic titration setup that led to increased hydroxide dosage and subsequently to a broth $\mathrm{pH}$ that was higher than the set point (See Figure S4). As a result, prior to the steady state in phase VII (at pH $5.23 \pm 0.07$ ), the $\mathrm{pH}$ rose slowly from pH 5.24 up to 5.64 on day 455. An increase and decrease in methane formation rate was observed concomitant with this $\mathrm{pH}$ rise and fall (Figure S4). During the steady state of phase VII the average methane formation rate had dropped with $90 \%$ compared to the steady state value in phase VI (at pH 5.61 $\pm 0.06)$. The $\mathrm{i}-\mathrm{C}_{4}$ formation rate and concentration did not change significantly compared to phase VI, whereas $\mathrm{n}-\mathrm{C}_{4}$ formation rate had dropped with $20 \%$.

\section{Cutting $\mathrm{CO}_{2}$ supply (II) and reducing vitamin $\mathrm{B} 12$ feed (IV) did not adversely affect the chain elongation performance}

In the second phase the $\mathrm{CO}_{2}$ addition was ceased, which led to a $26 \%$ decrease in $\mathrm{CH}_{4}$ formation and a $47 \%$ increase in $\mathrm{n}-\mathrm{C}_{6}$ formation. The steady state average $\mathrm{CO}_{2}$ partial pressure did drop from $23.7 \pm 1.4$ (phase I) to $17.2 \pm 1.3 \mathrm{kPa}$ (phase II). Gas headspace partial pressures are shown in Figure S5. Conversion of the main metabolites (substrates $\mathrm{MeOH}, \mathrm{C}_{2}$ and products $\mathrm{n}-\mathrm{C}_{4}$ and $\mathrm{i}-\mathrm{C}_{4}$ ) was not significantly affected by cutting $\mathrm{CO}_{2}$ dosage. Lowering the vitamin $\mathrm{B} 12$ concentration from 0.375 $\mathrm{mg} \mathrm{L}^{-1}$ to $0.0125 \mathrm{mg} \mathrm{L}^{-1}$ in phase IV also did not significantly affect the overall performance of the reactor (see Table 3 ). Confidence intervals of the conversion rates overlap for phase II and VI, where the only difference is the influent B12 concentration.

\section{Batch experiments show that at pH 5.2 i- $\mathrm{C}_{4}$ formation is caused by the presence of $n-C_{4}$ and vice versa}

The results of the first batch series are summarized in Figure 2 . The concentration profiles and gas headspace partial pressures for each separate batch are given in Figures S8 to S11 in the Supplementary Information section. The results show that at $\mathrm{pH} 5.2, \mathrm{i}-\mathrm{C}_{4}$ formation is triggered by the presence of $\mathrm{n}-\mathrm{C}_{4}(\mathrm{I}, \mathrm{V})$ and that $\mathrm{n}-\mathrm{C}_{4}$ 
formation is triggered by the presence of $\mathrm{i}^{-\mathrm{C}_{4}}$ (III, VII). Additionally, a $\mathrm{pH}$ of 5.2 induces $\mathrm{n}-\mathrm{C}_{6}$ formation, whereas at $\mathrm{pH} 6.5$ hardly any $\mathrm{n}-\mathrm{C}_{6}$ is formed. At $\mathrm{pH} 6.5$ there is mainly acetate and $n-\mathrm{C}_{4}$ formation from methanol and $\mathrm{CO}_{2}$ (II and IV). In batches $V$ to VIII substrate was limited to the amount that came from the inoculum. Here, methanol was quickly consumed in three batches (V, VI and VIII) and more slowly in the batch at $\mathrm{pH} 5.2$ with added $\mathrm{i}^{-\mathrm{C}_{4}}$ (VII). The batch with added $\mathrm{n}-\mathrm{C}_{4}$ at $\mathrm{pH} 6.5$ (VI) showed significant $\mathrm{n}-\mathrm{C}_{4}$ consumption ( $45 \%$ of the supplied $\mathrm{n}-\mathrm{C}_{4}$ was consumed) and acetate was formed together with methane. In contrast, the batch with added $\mathrm{i}-\mathrm{C}_{4}$ at $\mathrm{pH} 6.5$ (VIII) showed very little $\mathrm{i}-\mathrm{C}_{4}$ degradation ( 98\% remained) within the timeframe of the experiment ( 77 days). The substrate limited batches show that (de-)isomerization occurred at $\mathrm{pH} 5.2$ ( $\mathrm{V}$ and VII) as well as at pH 6.5 (VIII). However, the concentration profiles (Figure S9) of these batches show that (de-)isomerization activity occurred only during chain elongation activity when methanol was present, but not after methanol was depleted. This is emphasized by the calculated conversions that occurred after methanol was depleted (shown in Figure S7).

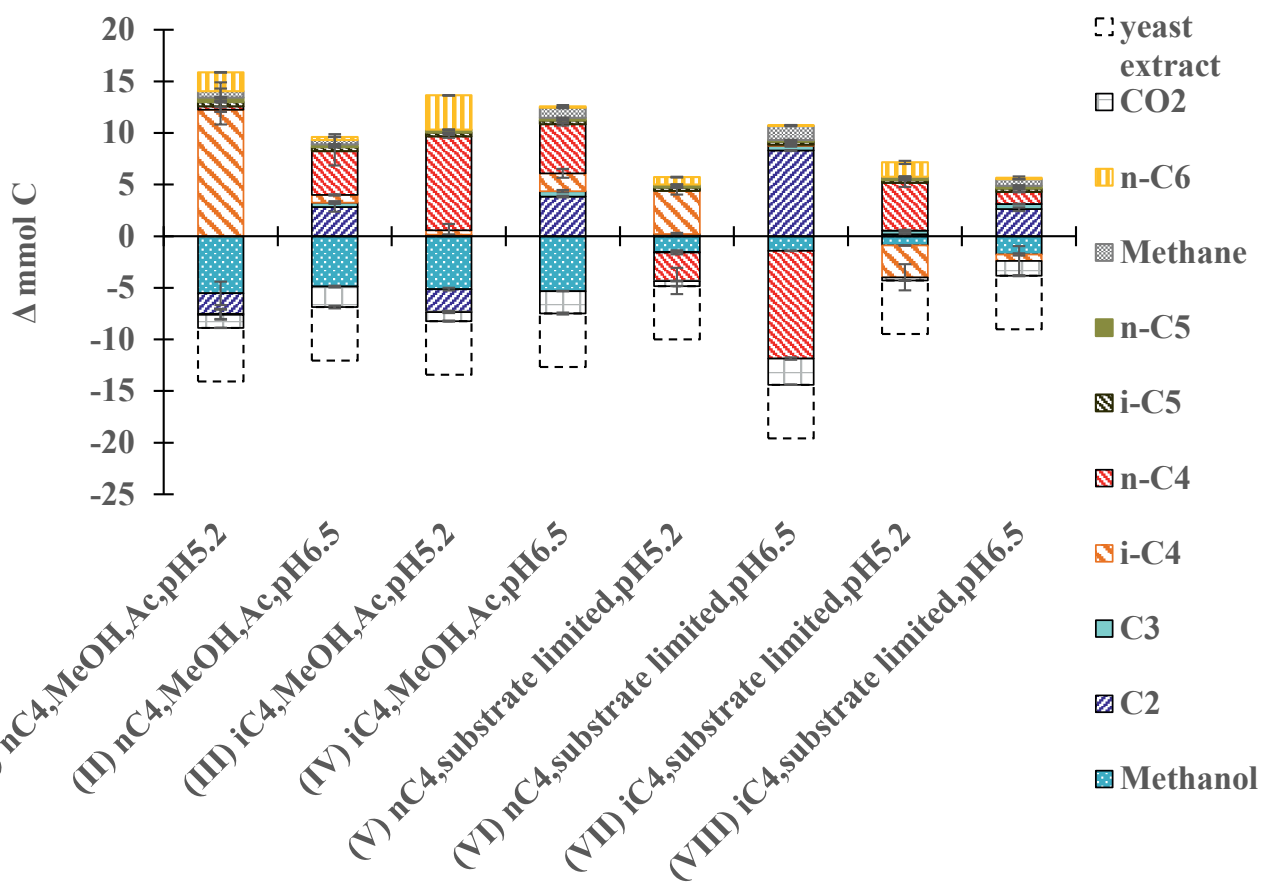

Figure 2. Formation and consumption of carbon containing components during the batch experiments. Error bars show the difference between the duplicate experiments. The yeast extract that was added to the medium is was assumed to be fully consumed ${ }^{102}$. The total formation and consumption after 77 days are shown. 


\section{Determining that $\mathrm{i}-\mathrm{C}_{4}$ and $\mathrm{n}-\mathrm{C}_{4}$ ratios meet thermodynamic equilibrium of isomerization}

A second batch series was performed (A-M, Table 2) at pH 5.5 to further investigate the impact of methanol, acetate and $\mathrm{i}-\mathrm{C}_{4}$. Batches $\mathrm{A}-\mathrm{F}$ started with different initial amounts of $\mathrm{i}-\mathrm{C}_{4}$ ( 0 to $50 \mathrm{mM}$ ) and batches $\mathrm{G}-\mathrm{M}$ started with different initial amounts of acetate and methanol ( $50 \mathrm{mMC}$ to $300 \mathrm{mMC}$ ) at $\mathrm{t}=0$. The results of each individual batch are shown in Figure S12-S19 (concentration profiles, as well as headspace partial pressure profiles). All batches showed $\mathrm{i}-\mathrm{C}_{4}$ and $\mathrm{n}-\mathrm{C}_{4}$ formation from acetate and methanol with a similar pattern as during the first series: presence of initial i$\mathrm{C}_{4}(\mathrm{~A}-\mathrm{F})$ caused $\mathrm{n}-\mathrm{C}_{4}$ to be formed first, followed by simultaneous formation of both butyrate species. The results of batches $A$ to $F$ and $G$ to $M$ are summarized in Figure 3A and $3 \mathbf{B}$ respectively, which show the ratios of $i-C_{4}$ and $n-C_{4}$ over total $C_{4}$ as measured during all batches throughout the operation period.
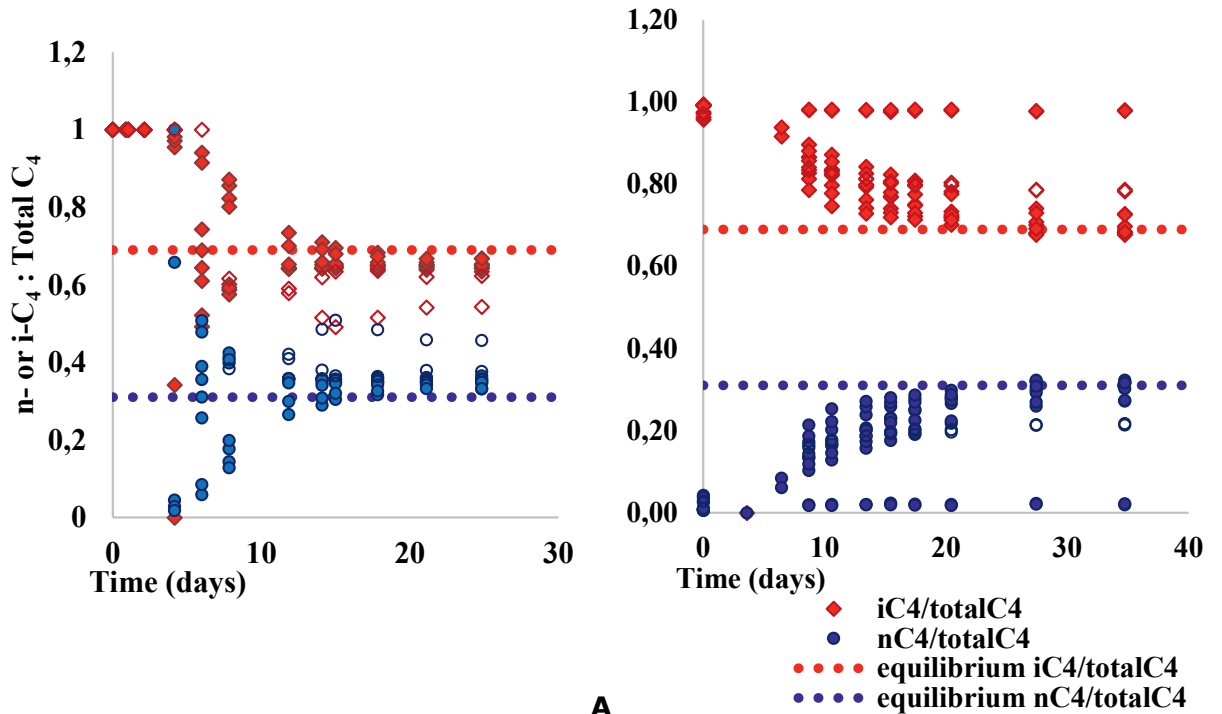

Figure 3. $A$ (batch $A$ to $E$, negative control $F$ excluded) and $B$ (batch $G$ to $L$ ) show the $i-C_{4}$ and $n$ $\mathrm{C}_{4}$ to total $\mathrm{C}_{4}$ ratios as measured during all batches throughout the operation period. The dotted lines represent the calculated equilibrium ratio using an isomerization $\Delta \mathrm{G}_{\mathrm{r}}$ of $-1.98 \mathrm{~kJ} 28,103$. The concentrations of $\mathrm{n}-\mathrm{C}_{4}$ and $\mathrm{i}-\mathrm{C}_{4}$ are interdependent and approach a ratio that is determined by equilibrium of isomerization as the batches progress. In $A$ the unfilled symbols represent the batch that was started without inoculum (but apparently was contaminated). In B the unfilled symbols represent the batch that started with $300 \mathrm{mMC}$ methanol, acetate and $50 \mathrm{mM} \mathrm{i}-\mathrm{C}_{4}$. The two series are highlighted in this way because they show a deviating behavior compared to the other batches (not seeming to approach the calculated equilibrium ratios).

In addition a horizontal line is added to the figure that represents the ratio at isomerization equilibrium conditions, which is calculated as shown on page S10 and S11 in the Supplemental Information. Eventually the concentrations of the butyrate 
species seem to approach this equilibrium in most cases. The progress of the $\mathrm{i}-\mathrm{C}_{4}$ and $\mathrm{n}-\mathrm{C}_{4}$ to total $\mathrm{C}_{4}$ ratios in the continuous reactor system are shown in Figure $\mathrm{S} 6$ and follow the same relation when operated at low $\mathrm{pH}(5.2-5.5)$.

\section{Microbial community analysis: key players in reactor are related to Clostridium Iuticellari and Eubacterium limosum}

The biomass from phase II, IV, V VI and VII of the continuous reactor, as well as biomass from batch I to VIII was harvested to be used for a 16S rRNA gene amplicon microbial community analysis. A heat map of the microbial community analysis data is shown in Table 4 (Table S7 in the Supplementary Information section shows the complete data). The results show that operating the reactor at increasing $\mathrm{pH}$ levels causes a gradual shift in Clostridiales composition from Clostridiaceae 1 (at pH 5.2) to Eubacteriaceae (at $\mathrm{pH}$ 6.75). The shift was reversed when reactor $\mathrm{pH}$ was decreased again. The batch experiments (I-VIII) that were inoculated with biomass from phase VII ( $\mathrm{pH}$ 5.2) show a selection towards different microbiomes at the end of the batch.

In the reactor at $\mathrm{pH} 6.75$ the most dominant genus is Eubacterium. The most abundant OTU (40\% relative abundance, Table S8) showed a slight similarity (94.8\% identity, $100 \%$ query cover) with Eubacterium limosum. Operating the reactor at $\mathrm{pH} 5.2$ caused an apparent complete washout of the Eubacterium order and strictly selected for Clostridium sensu stricto 12 dominance. The most abundant Clostridium sensu stricto 12 OTU (Table S9) showed a very high similarity $(99.7 \%$ identity, $100 \%$ query cover) with Clostridium luticellarii.

The batch series showed a microbial selection that was similar to the selection in the continuous reactor to some extent. $C$. luticellarii presence is maintained at $\mathrm{pH}$ 5.2 , albeit at a lower relative abundance than within the continuous reactor. At $\mathrm{pH}$ 6.5 the relative abundance of the Eubacterium order increased from $0 \%$ (phase VII used as inoculum) to $8 \%$ and $9 \%$ in batch II and IV respectively. In addition to these microbes, a large amount of other Clostridiales emerged in the batches among which the most abundant were Oxobacter (at pH 5.2), Family XI and Oscillibacter (at pH 6.5). 
Table 4. Heat map showing the relative abundances of all orders and for the Clostridiales also all observed genera.

\begin{tabular}{|c|c|c|c|c|c|c|c|c|c|c|c|c|c|c|c|}
\hline & \multirow{6}{*}{ Fami1ly } & \multirow{6}{*}{ Genus } & \multicolumn{5}{|c|}{ Reactor Phases } & \multicolumn{8}{|c|}{ Batches I-VIII } \\
\hline & & & III & IV: & $\mathrm{V}$ & VI & VII & 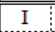 & III & III & IV & $\mathrm{V}$ & VI & VII & VIII \\
\hline & & & \multirow{4}{*}{$\begin{array}{l:l}\mathrm{pH} & \\
5.5 & \end{array}$} & \multirow{4}{*}{$\begin{array}{l:l}\mathrm{pH} & \\
6.0 & \end{array}$} & \multirow{4}{*}{$\begin{array}{r}\mathrm{pH} \\
6.75\end{array}$} & \multirow{4}{*}{$\begin{array}{l:l}\mathrm{pH} & \\
5.5 & \end{array}$} & \multirow{4}{*}{$\begin{array}{l}\mathrm{pH} \\
5.2\end{array}$} & \multicolumn{8}{|c|}{ Carbon source added I No carbon source added } \\
\hline & & & & & & & & $\mathrm{pH}$ & $\mathrm{pH}$ & $\mathrm{pH}$ & $\mathrm{pH}$ & $\mathrm{pH}$ & $\mathrm{pH}$ & $\mathrm{pH}$ & $\mathrm{pH}$ \\
\hline & & & & & & & & & & & & 5.2 & & 5.2 & \\
\hline & & & & & & & & $\mathrm{n}-\mathrm{C} 4$ & $\mathrm{n}-\mathrm{C} 4$ & $\mathrm{i}-\mathrm{C} 4$ & $\mathrm{i}-\mathrm{C} 4$ & $\mathrm{n}-\mathrm{C} 4$ & $\mathrm{n}-\mathrm{C} 4$ & $\mathrm{i}-\mathrm{C} 4$ & $\mathrm{i}-\mathrm{C} 4$ \\
\hline \multicolumn{2}{|c|}{ Methanomicrobiales (Archaea):Methanomicrobiaceae } & Methanoculleus & $1 \%$ & $5 \%$ & $3 \%$ & $3 \%$ & $0 \%$ & $0 \%$ & $1 \%$ & $0 \%$ & $0 \%$ & $0 \%$ & $7 \%$ & $0 \%$ & $4 \%$ \\
\hline Thermoplasmatales (Archaea) & Thermoplasmatales Incertae Sed & Candidatus Methanogranu & $14 \%$ & $8 \%$ & $4 \%$ & $14 \%$ & $6 \%$ & $0 \%$ & $11 \%$ & $0 \%$ & $4 \%$ & $0 \%$ & $4 \%$ & $0 \%$ & $2 \%$ \\
\hline \multirow{21}{*}{ Clostridiales } & \multirow{3}{*}{ Clostridiaceae 1} & $\begin{array}{ll}\text { Clostridium sensu stricto } 11 \\
\text {. }\end{array}$ & $37 \%$ & $19 \%$ & $0 \%$ & $27 \%$ & $61 \%$ & $12 \%$ & $0 \%$ & $20 \%$ & $0 \%$ & $21 \%$ & $0 \%$ & $51 \%$ & $0 \%$ \\
\hline & & Clostridium sensu stricto 1 & $0 \%$ & $0 \%$ & $3 \%$ & $0 \%$ & $0 \%$ & $0 \%$ & $0 \%$ & $0 \%$ & $1 \%$ & $0 \%$ & $1 \%$ & $0 \%$ & $3 \%$ \\
\hline & & Oxobacter & $0 \%$ & $0 \%$ & $0 \%$ & $0 \%$ & $0 \%$ & $27 \%$ & $0 \%$ & $17 \%$ & $1 \%$ & $0 \%$ & $2 \%$ & $2 \%$ & $0 \%$ \\
\hline & \multicolumn{2}{|c|}{ Clostridiales Incertae Si Proteiniborus } & $0 \%$ & $0 \%$ & $0 \%$ & $0 \%$ & $0 \%$ & $0 \%$ & $1 \%$ & $0 \%$ & $5 \%$ & $0 \%$ & $0 \%$ & $0 \%$ & $0 \%$ \\
\hline & Eubacteriaceae & Eubacterium & $3 \%$ & $23 \%$ & $40 \%$ & $6 \%$ & $0 \%$ & $0 \%$ & $8 \%$ & $0 \%$ & $9 \%$ & $0 \%$ & $1 \%$ & $2 \%$ & $2 \%$ \\
\hline & \multirow{2}{*}{ Family XI } & \multirow{2}{*}{$\begin{array}{l}\text { Sporanaerobacter } \\
\text { uncultured }\end{array}$} & $7 \%$ & $4 \%$ & $1 \%$ & $12 \%$ & $1 \%$ & $0 \%$ & $16 \%$ & $0 \%$ & $6 \%$ & $0 \%$ & $7 \%$ & $1 \%$ & $13 \%$ \\
\hline & & & $3 \%$ & $4 \%$ & $0 \%$ & $4 \%$ & $3 \%$ & $9 \%$ & $8 \%$ & $7 \%$ & $12 \%$ & $29 \%$ & $16 \%$ & $19 \%$ & $11 \%$ \\
\hline & Family XIII & uncultured & $0 \%$ & $0 \%$ & $0 \%$ & $0 \%$ & $0 \%$ & $0 \%$ & $0 \%$ & $0 \%$ & $0 \%$ & $0 \%$ & $0 \%$ & $0 \%$ & $0 \%$ \\
\hline & Gracilibacteraceae & Lutispora & $0 \%$ & $0 \%$ & $0 \%$ & $0 \%$ & $0 \%$ & $0 \%$ & $3 \%$ & $0 \%$ & $2 \%$ & $0 \%$ & $2 \%$ & $0 \%$ & $3 \%$ \\
\hline & Lachnospiraceae & Lachnoclostridium & $0 \%$ & $1 \%$ & $3 \%$ & $0 \%$ & $0 \%$ & $0 \%$ & $0 \%$ & $0 \%$ & $0 \%$ & $0 \%$ & $0 \%$ & $0 \%$ & $0 \%$ \\
\hline & Lactinospuraceá & Tyzzerella & $0 \%$ & $0 \%$ & $3 \%$ & $0 \%$ & $0 \%$ & $0 \%$ & $1 \%$ & $0 \%$ & $5 \%$ & $0 \%$ & $0 \%$ & $0 \%$ & $3 \%$ \\
\hline & Pepto & Desulfitobacterium & $1 \%$ & $0 \%$ & $0 \%$ & $0 \%$ & $0 \%$ & $1 \%$ & $0 \%$ & $0 \%$ & $0 \%$ & $0 \%$ & $0 \%$ & $0 \%$ & $10 \%$ \\
\hline & iceae & uncultured & $0 \%$ & $0 \%$ & $1 \%$ & $0 \%$ & $0 \%$ & $0 \%$ & $1 \%$ & $0 \%$ & $2 \%$ & $0 \%$ & $3 \%$ & $0 \%$ & $1 \%$ \\
\hline & & Caproiciproducens & $3 \%$ & $1 \%$ & $0 \%$ & $2 \%$ & $4 \%$ & $8 \%$ & $3 \%$ & $6 \%$ & $4 \%$ & $16 \%$ & $2 \%$ & $7 \%$ & $3 \%$ \\
\hline & & Hydrogenoanaerobacterina & $0 \%$ & $0 \%$ & $0 \%$ & $1 \%$ & $0 \%$ & $0 \%$ & $0 \%$ & $0 \%$ & $0 \%$ & $0 \%$ & $0 \%$ & $0 \%$ & $0 \%$ \\
\hline & Rum & Oscillibacter & $6 \%$ & $8 \%$ & 3\% & $5 \%$ & $2 \%$ & $3 \%$ & $14 \%$ & $4 \%$ & $16 \%$ & $1 \%$ & $1 \%$ & $1 \%$ & $2 \%$ \\
\hline & : $\sin$ & Ruminiclostridium 1 & $2 \%$ & $1 \%$ & $0 \%$ & $3 \%$ & $0 \%$ & $0 \%$ & $0 \%$ & $0 \%$ & $0 \%$ & $0 \%$ & $0 \%$ & $0 \%$ & $0 \%$ \\
\hline & & uncultured & $1 \%$ & $0 \%$ & $0 \%$ & $1 \%$ & $1 \%$ & $6 \%$ & $0 \%$ & $6 \%$ & $0 \%$ & $0 \%$ & $0 \%$ & $0 \%$ & $0 \%$ \\
\hline & & other & $1 \%$ & $1 \%$ & $0 \%$ & $0 \%$ & $0 \%$ & $0 \%$ & $0 \%$ & $0 \%$ & $0 \%$ & $0 \%$ & $0 \%$ & $0 \%$ & $0 \%$ \\
\hline & Syntrophomonadaceae & Syntrophomonas & $0 \%$ & $0 \%$ & $0 \%$ & $0 \%$ & $0 \%$ & $0 \%$ & $0 \%$ & $0 \%$ & $0 \%$ & $0 \%$ & $19 \%$ & $0 \%$ & $1 \%$ \\
\hline & 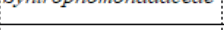 & uncultured & $0 \%$ & $0 \%$ & $0 \%$ & $0 \%$ & $0 \%$ & $0 \%$ & $0 \%$ & $0 \%$ & $0 \%$ & $0 \%$ & $1 \%$ & $0 \%$ & $4 \%$ \\
\hline Micrococcales & Microbacteriaceae & Pseudoclavibacter & $0 \%$ & $0 \%$ & $0 \%$ & $0 \%$ & $1 \%$ & $8 \%$ & $0 \%$ & $10 \%$ & $0 \%$ & $3 \%$ & $0 \%$ & $1 \%$ & $0 \%$ \\
\hline Coriobacteriales & Coriobacteriaceae & multiple & $1 \%$ & $0 \%$ & $0 \%$ & $1 \%$ & $2 \%$ & $2 \%$ & $0 \%$ & $1 \%$ & $0 \%$ & $1 \%$ & $0 \%$ & $0 \%$ & $1 \%$ \\
\hline Bacteroidales & multiple & multiple & $9 \%$ & $14 \%$ & $18 \%$ & $11 \%$ & $9 \%$ & $7 \%$ & $8 \%$ & $5 \%$ & $2 \%$ & $8 \%$ & $5 \%$ & $4 \%$ & $7 \%$ \\
\hline Sphingobacteriales & Lentimicrobiaceae & Lentimicrobium & $0 \%$ & $0 \%$ & $3 \%$ & $0 \%$ & $0 \%$ & $0 \%$ & $0 \%$ & $0 \%$ & $0 \%$ & $0 \%$ & $0 \%$ & $0 \%$ & $0 \%$ \\
\hline Bacillales & Sporolactobacillaceae & Sporolactobacillus & $1 \%$ & $0 \%$ & $0 \%$ & $0 \%$ & $0 \%$ & $0 \%$ & $0 \%$ & $6 \%$ & $0 \%$ & $1 \%$ & $0 \%$ & $0 \%$ & $0 \%$ \\
\hline Lactobacillales & Lactobacillaceae & multiple & $0 \%$ & $0 \%$ & $1 \%$ & $0 \%$ & $0 \%$ & $1 \%$ & $0 \%$ & $1 \%$ & $0 \%$ & $1 \%$ & $0 \%$ & $0 \%$ & $1 \%$ \\
\hline Erysipelotrichales & Erysipelotrichaceae & multiple & $1 \%$ & $1 \%$ & $1 \%$ & $1 \%$ & $4 \%$ & $2 \%$ & $0 \%$ & $2 \%$ & $0 \%$ & $1 \%$ & $1 \%$ & $1 \%$ & $0 \%$ \\
\hline Selenomonadales & multiple & multiple & $1 \%$ & $1 \%$ & $0 \%$ & $1 \%$ & $1 \%$ & $5 \%$ & $0 \%$ & $3 \%$ & $0 \%$ & $6 \%$ & $0 \%$ & $2 \%$ & $0 \%$ \\
\hline Burkholderiales & Alcaligenaceae & multiple & $2 \%$ & $2 \%$ & $0 \%$ & $1 \%$ & $1 \%$ & $2 \%$ & $1 \%$ & $4 \%$ & $0 \%$ & $5 \%$ & $1 \%$ & $2 \%$ & $0 \%$ \\
\hline Desulfovibrionales & Desulfovibrionaceae & multiple & $1 \%$ & $1 \%$ & $1 \%$ & $1 \%$ & $0 \%$ & $0 \%$ & $12 \%$ & $0 \%$ & $11 \%$ & $1 \%$ & $8 \%$ & $3 \%$ & $8 \%$ \\
\hline Enterobacteriales & Enterobacteriaceae & multiple & $0 \%$ & $0 \%$ & $3 \%$ & $0 \%$ & $0 \%$ & $0 \%$ & $0 \%$ & $0 \%$ & $0 \%$ & $0 \%$ & $4 \%$ & $0 \%$ & $0 \%$ \\
\hline Spirochaetales & Spirochaetaceae & multiple & $0 \%$ & $0 \%$ & $0 \%$ & $0 \%$ & $0 \%$ & $3 \%$ & $0 \%$ & $3 \%$ & $0 \%$ & $3 \%$ & $0 \%$ & $1 \%$ & $0 \%$ \\
\hline Synergistales & Synergistaceae & multiple & $0 \%$ & $0 \%$ & $0 \%$ & $0 \%$ & $0 \%$ & $0 \%$ & $5 \%$ & $0 \%$ & $6 \%$ & $0 \%$ & $6 \%$ & $0 \%$ & $10 \%$ \\
\hline Mollicutes RF9 & uncultured & uncultured & $0 \%$ & $0 \%$ & $0 \%$ & $0 \%$ & $0 \%$ & $0 \%$ & $0 \%$ & $0 \%$ & $5 \%$ & $0 \%$ & $0 \%$ & $0 \%$ & $0 \%$ \\
\hline$N B 1-n$ & uncultured & uncultured & $0 \%$ & $1 \%$ & $3 \%$ & $1 \%$ & $0 \%$ & $0 \%$ & $0 \%$ & $0 \%$ & $0 \%$ & $0 \%$ & $0 \%$ & $0 \%$ & $0 \%$ \\
\hline Sum of shown genera & & & $96 \%$ & $95 \%$ & $94 \%$ & $96 \%$ & $98 \%$ & 97\% & $95 \%$ & $97 \%$ & $94 \%$ & $\begin{array}{ll:} & 98 \% \\
\end{array}$ & $95 \%$ & $97 \%$ & $92 \%$ \\
\hline Other & & & $4 \%$ & $5 \%$ & $6 \%$ & $4 \%$ & $2 \%$ & $3 \%$ & $5 \%$ & $3 \%$ & $6 \%$ & $2 \%$ & $5 \%$ & $3 \%$ & $8 \%$ \\
\hline Total OTUs & & & $\begin{array}{l}1 \\
0 \\
6 \\
7 \\
7 \\
4\end{array}$ & $\begin{array}{l}1 \\
1 \\
1 \\
3 \\
4 \\
7\end{array}$ & $\begin{array}{l}1 \\
1 \\
4 \\
7 \\
7 \\
1\end{array}$ & $\begin{array}{l}9 \\
8 \\
5 \\
1 \\
7\end{array}$ & $\begin{array}{l}1 \\
7 \\
2 \\
2 \\
3 \\
7\end{array}$ & $\begin{array}{l}1 \\
3 \\
1 \\
1 \\
7 \\
0\end{array}$ & $\begin{array}{l}1 \\
3 \\
5 \\
2 \\
5 \\
2\end{array}$ & $\begin{array}{l}1 \\
0 \\
6 \\
0 \\
2 \\
5\end{array}$ & $\begin{array}{l}9 \\
8 \\
3 \\
5 \\
4\end{array}$ & {$\left[\begin{array}{l}9 \\
5 \\
3 \\
2 \\
7 \\
7\end{array}\right.$} & $\begin{array}{l}9 \\
6 \\
8 \\
8 \\
5\end{array}$ & $\begin{array}{l}1 \\
0 \\
6 \\
7 \\
8 \\
0 \\
\end{array}$ & $\begin{array}{l}1 \\
1 \\
9 \\
2 \\
4 \\
3\end{array}$ \\
\hline & & $0=10$ & & & & & & & & & & & & & \\
\hline
\end{tabular}




\section{Discussion}

\section{Methanol based chain elongation with acetate to n- butyrate and isobutyrate at varying (high) selectivities dependent on pH}

This study shows that the product spectrum of methanol based chain elongation can be adjusted using $\mathrm{pH}$ as a selection pressure tool. $\mathrm{A} \mathrm{pH}$ of around 6.75 in the continuous reactor facilitated the dominance of an unknown Eubacterium that resembled Eubacterium limosum, which is a known methanol based chain elongation microbe ${ }^{96}$. The results suggest that the microbiome dominated by Eubacterium does not form isobutyrate (i- $\left.\mathrm{C}_{4}\right)$ under these conditions. A pure culture experiment with $E$. limosum performed at pH 5.5 also showed no i- $\mathrm{C}_{4}$ formation, only $\mathrm{n}$-butyrate $\left(\mathrm{n}-\mathrm{C}_{4}\right)$ formation (Figure S20).

Lowering the $\mathrm{pH}$ in the reactor towards 5.5 changed the microbiome towards a state where a Clostridium luticellarii related species was dominant. At this $\mathrm{pH}$ there was still a small amount of Eubacteria present (6\%), and more so in the sampled biofilm that had grown within the $\mathrm{pH}$ control volume (See Figure S1 and Table S7). Further lowering the $\mathrm{pH}$ to 5.2 led to a steady state in phase VII without the Eubacterium genus ( $0 \%$ relative abundance) and with the OTU related to $C$. Iuticellarii at a $55.5 \%$ relative abundance. A pure culture of C. Iuticellarii has recently been shown to be able to form $\mathrm{i}-\mathrm{C}_{4}$ and $\mathrm{n}-\mathrm{C}_{4}$ under supplementation of acetate and methanol ${ }^{104}$.

\section{Suppressing methanogenesis at pH 5.2 increased chain elongation selectivity}

Shifting the reactor $\mathrm{pH}$ from 5.5 to 5.2 significantly lowered methanogenic activity as well as the relative abundances of the Archaea. The microbiome analysis showed two potential candidates for methanogenic activity: (i) hydrogenotrophic methanogenesis by the Methanoculleus genus (98.8\% identity, 99\% query cover, Methanoculleus palmolei DSM 4273) ${ }^{105}$ and (ii) methylotrophic methanogenesis by an unknown Candidatus methanogranum (closest similarity to Methanomassiliicoccus luminyensis, $87.76 \%$ identit, $99 \%$ query cover $)^{106,107}$. The decrease in methanogenic activity was simultaneous with a decrease in methanol consumption, suggesting that the observed methanogenesis at $\mathrm{pH} 5.5$ was of a methylotrophic nature ${ }^{108}$. This shift from pH 5.5 to 5.2 effectively lowered methanogenic activity, while maintaining chain elongation activity. Methylotrophic methanogenensis can occur down to $\mathrm{pH} 4.2^{109}$. However, its inhibition is known to be amplified by high concentrations of undissociated acids ${ }^{59,110}$ and by sufficient mixing that prevents niche and biofilm formation ${ }^{111}$. As a result, total chain elongation selectivity increased from $84 \%$ to $96 \%$ (percentage of the summed formed $\mathrm{i}-\mathrm{C}_{4}, \mathrm{n}-\mathrm{C}_{4}$ and $\mathrm{n}-\mathrm{C}_{6}$ per total amount of formed products, based on carbon atoms). Overall the selection pressure in the reactor was 
sufficient to select for methanol based chain elongators and to prevent methanogenesis from becoming the dominant bioprocess.

\section{The $\mathrm{i}-\mathrm{C}_{4}$ and $\mathrm{n}-\mathrm{C}_{4}$ ratios meet thermodynamic equilibrium of isomerization}

In the continuous reactor experiment, the formation of $\mathrm{i}-\mathrm{C}_{4}$ and $\mathrm{n}-\mathrm{C}_{4}$ at $\mathrm{pH} 5.5$, as well as at $\mathrm{pH} 5.2$, seemed to occur towards concentrations that approached the thermodynamic equilibrium of isomerization (Figure S6A and S6B). The batch series A-M also showed i- $\mathrm{C}_{4}$ and $n-C_{4}$ formation progressing towards a similar ratio (Figure 4), as well as a previous study on methanol based chain elongation ${ }^{46}$. Moreover, an experiment that was performed with cell extracts and was designed to determine butyryl-CoA:isobutyryl-CoA mutase activity showed that in this setup isobutyryl-CoA to butyryl-CoA ratios approach a similar value $^{95}$. The genome of C. luticellarii (DSM No. 29923) has recently been sequenced and harbors genes encoding for enzymes within the isobutyryl-CoA mutase (ICM)-like subfamily (NCBI Reference Sequence: WP_106007777.1 and WP_106008210.1) ${ }^{112}$. In contrast, the sequences of two known Eubacterium limosum species (taxid: 1736 and 903814) do not contain any similarities to genes of the ICM-like subfamily (NCBI blastp queries with various fused isobutyryl-CoA mutases, icmF, show no similarity). Based on the stoichiometric and microbial analysis of this research and the work on pure C. luticellarii (DSM No 29923) ${ }^{104}$, it is suggested that this species forms both $\mathrm{i}-\mathrm{C}_{4}$ and $\mathrm{n}-\mathrm{C}_{4}$ during methanol based chain elongation of acetate coupled to a bidirectional isomerization reaction dictated by thermodynamic conditions. For this fermentation, $\mathrm{i}-\mathrm{C}_{4}$ and $\mathrm{n}-\mathrm{C}_{4}$ are effectively the main end products (apart from the formation of small $n-\mathrm{C}_{6}$ amounts), which allows their ratios to approach thermodynamic equilibrium. In contrast, during other isobutyrate-forming fermentations, e.g. methanogenesis systems ${ }^{91},{ }^{113}$ and bioelectrochemical systems ${ }^{80}, 81$, the metabolic fluxes are also geared towards the formation of alternative products from butyrate as intermediate, which could prevent equilibrium of isomerization from being established.

\section{Isobutyrate formation was observed to be coupled to catabolic activity of methanol chain elongation at low pH}

Isomerization of $\mathrm{n}-\mathrm{C}_{4}$ and $\mathrm{i}-\mathrm{C}_{4}$ can occur in both directions and is regularly observed within methanogenic cultures ${ }^{95,114}$. Within methanogenic cultures (de-)isomerization of butyrate species is coupled to beta-oxidation and consecutive hydrogenotrophic and acetotrophic methanogenesis ${ }^{91,94}$. In this research, however, the microbiome performed methanol based chain elongation, and methanogenesis was mainly methylotrophic. It took the microbiome three weeks in a batch (VI, at pH 6.5, Figure S9) to develop observable betaoxidation activity, which shows this activity was negligible during continuous reactor operation. The increase in relative abundance of Syntrophomonas and Methanoculleus ${ }^{105}$, 
115 (Table 4, batch VI) supports this observation. Moreover, deisomerization of $\mathrm{i}-\mathrm{C}_{4}$ occurred slowly, if not at all (batch VII and VIII).

In the first batch series (I-VIII), which was performed at low substrate concentrations, equilibrium of isomerization could not be achieved before substrate was depleted; in the absence of methanol, catabolic activity (chain elongation) was halted and so was (de-)isomerization activity. It shows that the $\mathrm{i}-\mathrm{C}_{4}$ formation is driven by the active formation of the butyrate species via methanol based chain elongation. The enzyme butyryl-CoA:isobutyryl-CoA mutase that performes reversible isomerization requires activation of the substrates via a Coenzyme $A$ bond ${ }^{116}$. It is known that transition from exponential phase (during substrate abundance) to stationary phase (when energy sources become limited) can lead to drastic changes of the metabolic fluxes ${ }^{117}$, accompanied by large changes in intracellular CoA-derivative concentrations ${ }^{118,119}$. The metabolic changes during substrate limitation, combined with other regulatory mechanisms ${ }^{120,121}$, could potentially have reduced the flux towards (de-)isomerization and explain the lack of passive (de-)isomerization. However, to experimentally verify this hypothesis, pure culture experiments with $C$. luticellarii to determine intracellular concentrations and enzyme activity assays to determine $\mathrm{K}_{\mathrm{m}}$ and $\mathrm{k}_{\mathrm{cat}}$ values would need to be performed.

\section{Isomerization of $\mathrm{n}-\mathrm{C}_{4}$ to $\mathrm{i}-\mathrm{C}_{4}$ may provide a competitive advantage to chain elongation microbes at low pH and a high undissociated acid concentration}

A physiological reason for $\mathrm{i}-\mathrm{C}_{4}$ formation can be found in exploring what selection advantage is gained under the supplied conditions. Isobutyrate formation was stimulated in an environment with a high concentration of fatty acids and a $\mathrm{pH}$ that approaches the pKa values of these acids (See Figure S4 for the undissociated acids concentration in the reactor). It is known that branched fatty acids have a lower toxic effect on microorganisms than unbranched forms ${ }^{122}$. Partly this can be explained because $\mathrm{i}-\mathrm{C}_{4}$ is predicted to have a lower diffusivity through cellular membranes compared to $n-C_{4}{ }^{123}$, thereby lessening the strain on maintenance due to futile cycling ${ }^{59}$. Thus, hypothetically, switching towards an i$\mathrm{C}_{4}$ forming catabolism could give a competitive advantage to the chain elongation microbe that is growing at low pH and at high undissociated acid concentrations. This would explain the dominance of $\mathrm{i}-\mathrm{C}_{4}$ formation at the lower applied $\mathrm{pH}$, and its absence at a higher $\mathrm{pH}$ where an anabolic investment of making an additional enzyme by organisms to perform the isomerization is not energetically rewarded. 


\section{Future outlook}

During the last phase (VII, at $\mathrm{pH} 5.2$ ) of the reactor the highest $\mathrm{i}-\mathrm{C}_{4}$ volumetric productivity was $111 \pm 15 \mathrm{mmol} \mathrm{C} \mathrm{L}^{-1}$ day $^{-1}$ or $2.4 \pm 0.3 \mathrm{~g} \mathrm{~L}^{-1}$ day ${ }^{-1}$ with an average broth concentration of $191 \pm 28 \mathrm{mM} \mathrm{C}$ or $4.2 \pm 0.6 \mathrm{~g} \mathrm{~L}^{-1}$ and a selectivity towards i- $\mathrm{C}_{4}$ of 0.65 carbon per carbon of total products. The achieved volumetric productivity was $20 \%$ higher (continuous reactor) and the achieved concentration was $80 \%$ higher (batch) than in an earlier study on $\mathrm{i}-\mathrm{C}_{4}$ formation via methanol based chain elongation ${ }^{35}$. The batch experiments showed $\mathrm{i}-\mathrm{C}_{4}$ production up to a concentration of around $400 \mathrm{mM} \mathrm{C}, 8.8 \mathrm{~g} \mathrm{~L}^{-1}$ (and $\mathrm{n}-\mathrm{C}_{4}$ up to a concentration of around $150 \mathrm{mM} \mathrm{C}, 3.3 \mathrm{~g} \mathrm{~L}^{-1}$ ), where chain elongation became constrained despite acetate and methanol still being available. It shows the limitations of methanol based chain elongation in the batch set up, but also that a higher broth concentration within the continuous system can likely be achieved. The necessary increased productivity may be reached by an increase of the amount of active biomass via biomass retention with e.g. techniques ${ }^{124,125}$. Further exploring the metabolic pathway(s) and investigating the potential physiological reasons for $\mathrm{i}-\mathrm{C}_{4}$ formation may also give more insight in the fundamental principles that drive the selection of the developed reactor microbiome. Finally, more researches on effective product recovery methods and specific case-tailored solutions are necessary to determine how valorization of organic residues by chain elongation can become economically feasible $e^{126}$.

\section{Supporting Information}

The Supporting Information is available in the Supporting Information Section of this thesis. Additional information regarding Materials and Methods and additional figures and graphs for the Results and Discussion, 22 figures, and 13 tables (PDF). 


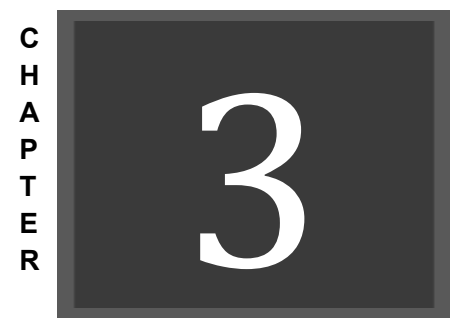

\section{Continuous n-Valerate Formation from Propionate and Methanol in an Anaerobic Chain Elongation Open-Culture Bioreactor}

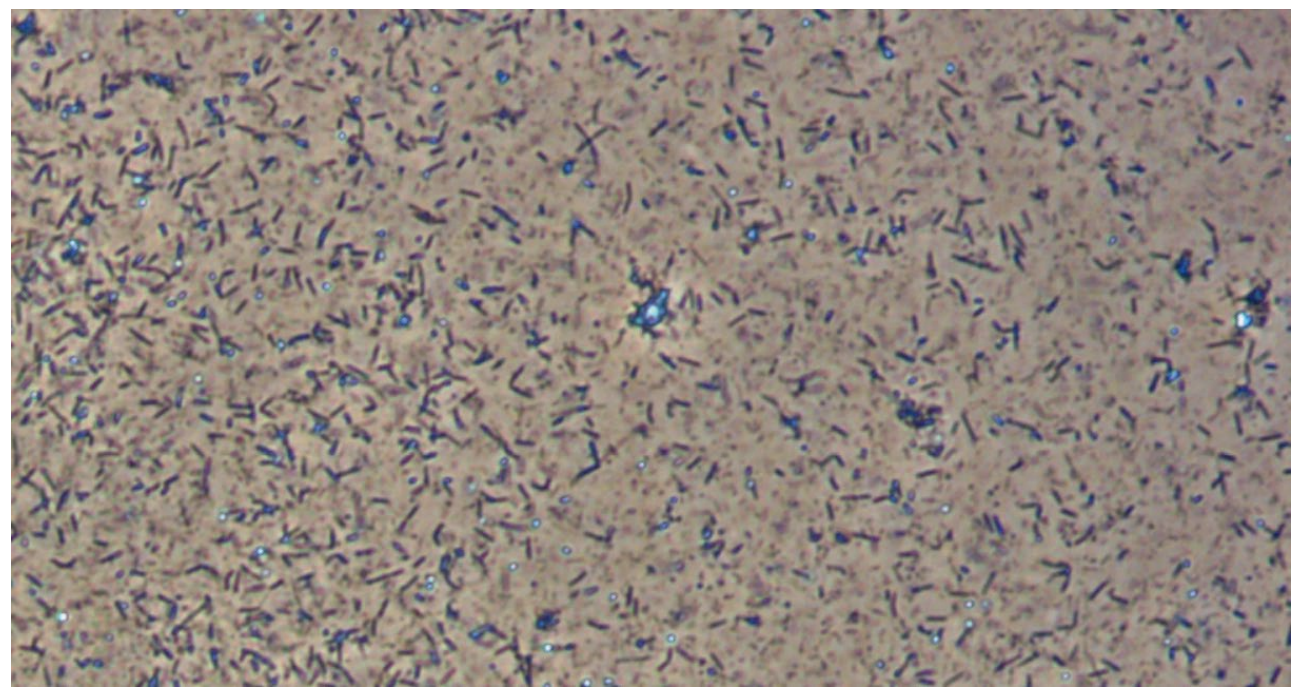

This chapter is published as:

Sanne M. de Smit*; Kasper D. de Leeuw*; Cees J.N. Buisman \& David P.B.T.B. Strik, 2020, Continuous $n$-valerate formation from propionate and methanol in an anaerobic chain elongation open-culture bioreactor

* Shared first author

Biotechnology for Biofuels volume 12, Article number: 132 (2019)

https://doi.org/10.1186/s13068-019-1468-x 


\section{Abstract}

Chain elongation forms a new platform technology for the circular production of biobased chemicals from renewable carbon and energy sources. This study aimed to develop a continuous methanol based chain elongation process for the open-culture production of a new generation biofuel precursor and potential platform chemical: $n$-valerate. Propionate was used as substrate for chain elongation to $n$-valerate in an anaerobic open culture bioreactor. Additionally, the co-production of $n$ - and iso-butyrate in addition to $n$-valerate via respectively acetate and propionate elongation was investigated. $N$-valerate was produced during batch and continuous experiments with a $\mathrm{pH}$ in the range $5.5-5.8$ and a hydraulic retention time of 95 hour. Decreasing the $\mathrm{pH}$ from 5.8 to 5.5 caused an increase of the selectivity for $n$-valerate formation (from 58 up to 70 weight\%) during methanol based propionate elongation. $n$-Valerate and both $n$ - and iso-butyrate were produced during simultaneous methanol based elongation of propionate and acetate. Propionate was within the open-culture preferred over acetate as substrate with $10-30 \%$ more consumption. Increasing the methanol concentration in the influent (from 250 to $400 \mathrm{mM}$ ) resulted in a higher productivity (from 45 to $58 \mathrm{mmol} \mathrm{C} / \mathrm{L} /$ day), but a lower relative product selectivity (from 49 to 43 weight\%) of $n$-valerate. The addition of acetate as substrate did not change the average $n$-valerate productivities. Within the continuous bioreactor experiments 6 to 17 weight\% of formed products was methane. The microbial community during all steady states in both methanol based elongation bioreactors was dominated by species related to Clostridium luticellarii and Candidatus Methanogranum. C. Iuticellarii is the main candidate for $n$-valerate formation from methanol and propionate. $n$-Valerate was for the first time proven to be produced from propionate and methanol by an open culture bioreactor. Methanogenic activity can be inhibited by decreasing the $\mathrm{pH}$, the $n$-valerate productivity can be improved by increasing the methanol concentration. The developed process can be integrated with various biorefinery processes from thermochemical, (bio)electrochemical, photovoltaic and microbial technologies. The findings from this study form a useful tool to steer the process of biological production of chemicals from biomass and other carbon and energy sources.

Keywords: Chain elongation, Selective pressure, Open-culture fermentation, Mixedculture fermentation, Biobased chemicals, Methanol, Butyrate, n-Valerate 


\section{Introduction}

The growing world population causes arable land to become more scarce, waste to be produced in larger quantities and carbon emissions to rise due to fossil fuel usage 127,128 . These developments emphasize the need for more sustainable and efficient production of chemicals. Microbial chain elongation processes can contribute to the realisation of a more circular economy by providing a versatile approach to convert complex organic waste streams to fatty acids (short (C1-C5) and medium (C6-C10) chain length). Currently the company ChainCraft is starting a commercial demonstration factory using ethanol based chain elongation to produce mixture of carboxylate salts for use as feed additive in the agro-food industry ${ }^{129}$.

Several chain elongation microbial pathways are known including homoacetogenesis (Wood-Ljungdahl pathway), the Arnon-Buchanan cycle and reverse beta-oxidation ${ }^{130}$. Chain elongation processes utilize short carbon chains as electron acceptor and by elongating the fatty acids the carbon atoms are getting increasingly reduced (with a limit to 6 electrons per carbon). The products of the chain elongation conversions are largely dependent on the supplied substrates. Various electron donors can be used for chain elongation such as ethanol, methanol, lactate, sugars or electrons provided via microbial electrosynthesis ${ }^{130-133}$.Currently, the usage of methanol as electron donor is not sufficiently investigated and therefore is the focus of this study.

Methanol is an available electron donor ${ }^{134}$, which could be further mass produced via various thermochemical and electrochemical methods. Substrates for methanol production include lignocellulosic biomass, waste streams (e.g. via syngas) or $\mathrm{CO}_{2}$ sources including air ${ }^{135-137}$. Hypothetically, when methanol is used as an electron donor for chain elongation, a cobalamin-dependent methyltransferase system coupled to the Wood-Ljungdahl pathway, could allow for its oxidation towards acetyl-CoA, which can then be utilized in a reverse beta oxidation to reduce and elongate short chain fatty acids to longer carbon chains ${ }^{138}$. The short chain fatty acids acetate and propionate, which are substrates for the currently studied chain elongation process, can be produced via a hydrolysis or acidogenesis fermentation process. Additionally, the acids can be produced from $\mathrm{CO}_{2}$ with pure or openculture microbial electrosynthesis, from acetyl-CoA by biosynthesis and from amino acids 139-141.

The known products that can be formed within an open-culture methanol based chain elongation of acidified supermarket waste are $n$-butyrate, iso-butyrate, $n$-valerate and $n$ caproate ${ }^{45}$. $\mathrm{N}$-valerate is a new generation biofuel precursor and potential platform chemical. The esterification product of protonated $n$-valerate (valeric acid) can be used as an additive to diesel fuels ${ }^{142,143}$, butyrate and n-valerate are also suitable precursors for 
bioplastic (Polyhydroxyalkanoates) production ${ }^{144}$ and Kolbe electrolysis of pure $n$-valerate would lead to gasoline (octane) formation ${ }^{145}$.

$\mathrm{N}$-valerate production from waste streams is a rarely investigated environmental biorefinery process ${ }^{146} . \mathrm{N}$-valerate formation during chain elongation processes occurred in previous research during methanol based chain elongation from acidified supermarket waste. However, due to presence of various electron donors (e.g. methanol and endogenous produced ethanol) it could not be shown whether methanol based propionate elongation occurred ${ }^{147}$. A pure culture experiment with Eubacterium Limosum also successfully showed propionate elongation with methanol to $n$-valerate ${ }^{148}{ }^{149}$. So far no study focussed on utilizing an open culture to elongate propionate with methanol to $n$ valerate. Such open-culture microbiome could be advantageous for application since no sterilization is needed and a waste stream (i.e. organic waste) could be used as feedstock during open-culture operation.

Fermentation of complex/mixed substrates using open-culture microbiomes typically results in a plethora of microbial processes that are either desired for chain elongation or are a competing process ${ }^{25}$. Reactor conditions such as temperature, $\mathrm{pH}$, gas composition and hydraulic retention time (HRT) become important selective pressure tools that will determine the product spectrum $25,133,147$. In this study $\mathrm{pH}$ and hydraulic retention time were varied to selectively inhibit competitive methanol consumption processes. Slow growing methylotrophic methanogens and acetogens 150 are competitive methanol consuming microbes and should be kept low in numbers by maintaining a suitably low HRT. This effect is enforced by operating at a low pH that causes more maintenance stress and overall lowers the growth rate of all microbes ${ }^{151}$. Moreover, the presence of undissociated acids at a low $\mathrm{pH}$ hinders bacteria due to futile cycling caused by diffusion of undissociated acids through the cell membranes ${ }^{152-154}$. Therefore in combination with a low $\mathrm{pH}$ high volatile fatty acids concentrations could provide additional selection pressure, assuming that the microorganisms performing the desired chain elongation reactions are better suited to withstand this compared to undesired methanogenic and acetogenic microorganisms ${ }^{155}$.

The aim of this study was to develop a continuous $n$-valerate production process using methanol based chain elongation in an anaerobic open-culture reactor. Also, the coproduction of $n$ - and iso-butyrate in addition to $n$-valerate from methanol based elongation using respectively acetate and propionate was investigated. Two continuous reactors were developed that successfully performed methanol based chain elongation where propionate was elongated to $n$-valerate and acetate was elongated to $n$-butyrate and iso-butyrate. 


\section{Materials and Methods}

Batch tests and continuous experiments were carried out during this study. A series of batch experiments was carried out in duplo with an initial pH ranging from 5 to 7.5 (with steps of 0.5 ) to study which $\mathrm{pH}$ range allowed propionate elongation to $n$-valerate. Serum bottles $(250 \mathrm{ml})$ were filled with $150 \mathrm{ml}$ medium with $250 \mathrm{mM}$ methanol and $150 \mathrm{mM}$ propionate and $10 \mathrm{ml}$ inoculum from a continuous methanol based acetate elongation reactor $^{156}$ and placed in a shaking cabinet at $308 \mathrm{~K}$. The initial headspace consisted of $20 \%$ $\mathrm{CO}_{2}$ and $80 \% \mathrm{~N}_{2}$. An elaborate overview of the medium composition can be found in the SI.

\section{Continuous methanol based chain elongation}

An 1-L upflow anaerobic bioreactor (UAB) with 0.2-L headspace, as used by Chen et al. ${ }^{45}$, was used for continuous methanol based propionate elongation (Figure 1). A gas counter was used to measure the amount of produced gas. The reactor content was recirculated with a peristaltic pump (Watson Marlow S CIQ 323, UK) with a velocity of $400 \mathrm{ml} / \mathrm{min}$. In the recirculation loop, the turbidity and the $\mathrm{pH}$ (Endress Hauser M, Netherlands) were measured. The $\mathrm{pH}$ was maintained constant by automatic addition of potassium hydroxide.
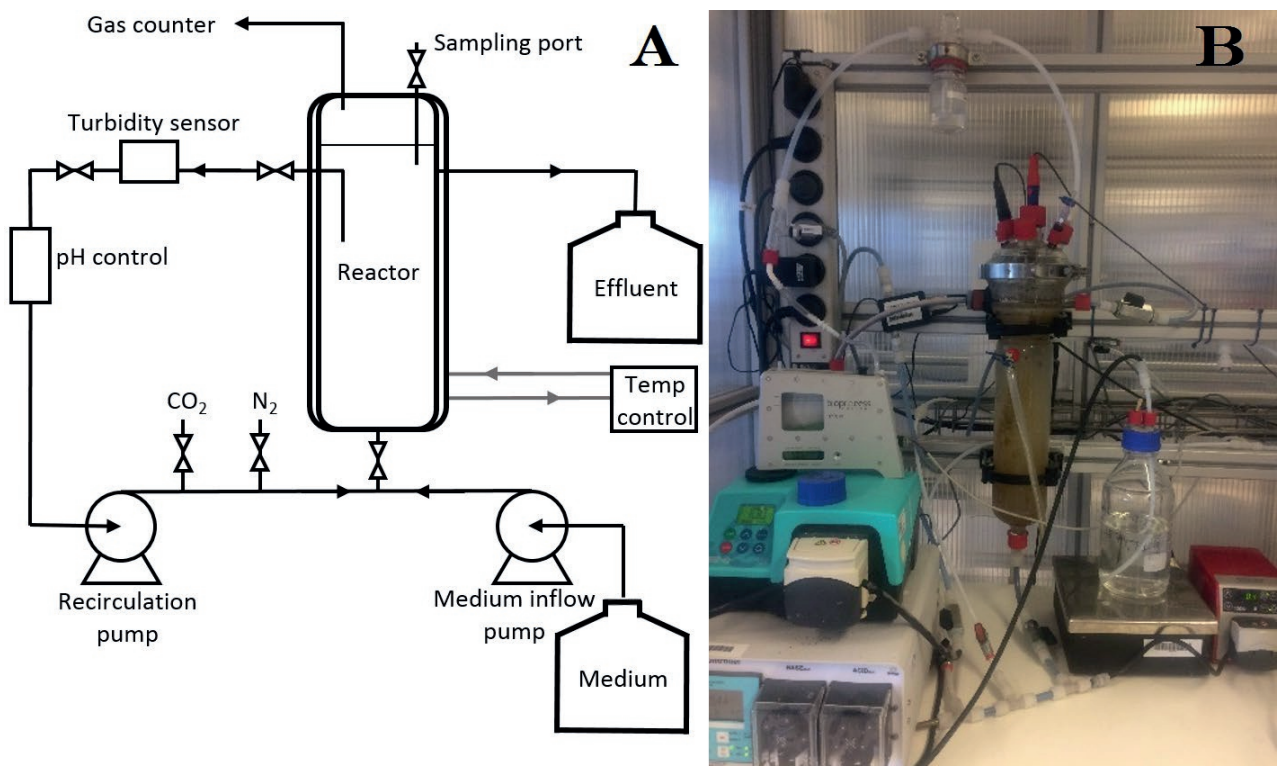

Figure 1. Schematic (A) and actual (B) setup of upflow anaerobic bioreactor used for continuous methanol based chain elongation.

The medium was pumped into the reactor by a peristaltic pump (Watson Marlow IP31, UK), the hydraulic retention time (HRT) could be adjusted by adjusting the pump rate. Carbon 
dioxide was supplied during the continuous methanol elongation process for acetate formation from methanol and bicarbonate (Table 3: R2). The temperature of the reactor was kept at $309 \mathrm{~K}$ using a water bath (Julabo 4, Germany). A medium with $250 \mathrm{mM}$ methanol, $150 \mathrm{mM}$ sodium propionate and $1 \mathrm{~g} / \mathrm{L}$ yeast extract was used. The exact composition of the medium is shown in the SI. The medium was stored in a camelbag (MSR Dromedary, $6 \mathrm{~L}$ ) in a fridge at $279 \mathrm{~K}$ that was continuously flushed with nitrogen.

A second reactor was used to study the simultaneous use of acetate and propionate as substrate for methanol based chain elongation. A 3-L upflow anaerobic bioreactor (UAB) with 0.6-L headspace was used for continuous methanol based acetate and propionate elongation (Figure 1A). The recirculation velocity of the reactor content was $300 \mathrm{ml} / \mathrm{min}$ (Watson Marlow $505 \mathrm{~S}, \mathrm{UK}$ ), the turbidity and pH were measured in the recirculation loop (Endress Hauser $\mathrm{M}$, Netherlands). The $\mathrm{pH}$ was maintained constant by automatic addition of potassium hydroxide.

The medium ( $250 \mathrm{mM}$ methanol, $75 \mathrm{mM}$ sodium propionate, $75 \mathrm{mM}$ sodium acetate and 1 $\mathrm{g} / \mathrm{L}$ yeast extract) was pumped into the reactor by a peristaltic pump (Watson Marlow IP31, UK). To compare the substrate use, propionate and acetate were added on a 1:1 molar base. The exact composition of the medium is shown in the SI. The medium was stored in a camelbag in a fridge at $277 \mathrm{~K}$ that was continuously flushed with nitrogen. Carbon dioxide gas was let into the reactor continuously and the temperature of the reactor was kept at $309 \mathrm{~K}$ using a water bath (Julabo F25, Germany).

\section{Analysis}

Three times a week, a gas sample was measured using gas chromatography to analyse the fractions of oxygen, carbon dioxide, methane, nitrogen and hydrogen in the gas phase (Shimadzu GC-2010, Japan). Additionally, a liquid sample was taken, from which the concentrations of volatile fatty acids and alcohols (methanol, ethanol, propanol, butanol, pentanol, hexanol, acetate, propionate, $n$-butyrate, iso-butyrate, $n$-valerate, iso-valerate, $n$-caproate, iso-caproate, heptylate and caprylate) were measured using gas chromatography. A liquid sample was also taken from the fresh and old medium when the medium was replaced. The concentration of volatile fatty acids and alcohols of the medium samples were measured as well using gas chromatography (HP5890, USA). The difference between 2-methylbutyrate and 3-methylbutyrate could not be measured using the available equipment. Therefore, no distinction could be made between the formation of 2and 3-methylbutyrate during this experiment and both compounds were measured as isovalerate. 


\section{Reactor setting changes}

The setup changes during different phases of the continuous methanol based propionate elongation process are shown in Table $\mathbf{1}$. The reactor was inoculated with biomass from the previously mentioned $n$-valerate formation batch experiments at day 0 and day 27 . During phase I, decrease of the $n$-valerate formation was observed, the HRT was doubled for phase II. The $\mathrm{pH}$ during the $n$-valerate formation phase of the batch experiments was 5.8 , so the $\mathrm{pH}$ was lowered to 5.8 to stimulate $n$-valerate formation even further in phase III. Steady state was reached at day 78 , and after 12 days of steady state $n$-valerate formation, the $\mathrm{pH}$ of the medium was decreased to 5.5 to inhibit methanogenesis (phase IV). The carbon dioxide supply was changed with changing $\mathrm{pH}$ to maintain a constant bicarbonate concentration during the formation process. The change of the concentration of dissolved $\mathrm{CO}_{2}$ and the $\mathrm{pH}$ in time (Figure $\mathrm{S} 1$ ) and the calculation of the required $\mathrm{CO}_{2}$ gasflow are shown in the SI.

Table 1. Setup for the different phases of the continuous formation of valerate from propionate and methanol to study the effect of $\mathrm{pH}$ lowering on $\mathrm{n}$-valerate formation. The $\mathrm{pH}$ in the reactor was controlled during phase III and phase IV.

\begin{tabular}{lllll}
\hline Phase conditions & Phase I & $\begin{array}{l}\text { Phase } \\
\text { II }\end{array}$ & $\begin{array}{l}\text { Phase } \\
\text { III }\end{array}$ & Phase IV \\
\hline \hline $\begin{array}{l}\text { Hydraulic retention time } \\
\text { (h) }\end{array}$ & 42.3 & $\mathbf{9 5 . 2}$ & 95.2 & 95.2 \\
Phase duration (days) & $0-27$ & $27-43$ & $43-91$ & $91-120$ \\
pH influent & 7.0 & 7.0 & $\mathbf{5 . 8}$ & $\mathbf{5 . 5}$ \\
& 6.4 & 6.3 & & \\
pH reactor & \pm 0.3 & \pm 0.2 & $\mathbf{5 . 8}$ & $\mathbf{5 . 5}$ \\
$\mathrm{CO}_{2}$ supply (ml/min) & 0.18 & 0.18 & $\mathbf{0 . 3 6}$ & $\mathbf{0 . 4 0}$ \\
\hline \hline
\end{tabular}

The setup changes during different phases of the continuous methanol based acetate and propionate elongation process are shown in Table $\mathbf{2}$. The reactor was inoculated with biomass from the $n$-valerate formation batch experiments at day 0 . No conversions of methanol and acetate were observed during phase $\mathrm{I}$, so the $\mathrm{pH}$ was lowered from 7.0 to 5.8. Carbon dioxide was supplied to the reactor from phase III and onwards. During phase III, the high methanol concentration in the reactor was presumed to be prohibiting microbial activity. Therefore, the reactor was set in batch mode during phase IV, so that the methanol could be consumed and biomass could be accumulated. The hydraulic retention time was increased after the batch phase (during phase $V$ ) to prevent washout of $n$-valerate producing organisms. A steady state was reached from day 64 to day 71 . In phase VI, the methanol concentration was increased to study whether increase of the concentration of methanol would stimulate the formation of $n$ - and iso-butyrate and $n$ valerate at the same biomass retention rate. 
At day 29 (during phase III) and 38 (start of phase IV), the reactor was inoculated with a mix of biomass from the methanol elongation reactor with propionate and a continuous methanol elongation reactor with acetate. The carbon dioxide supply was changed with changing $\mathrm{pH}$ to maintain a similar theoretically supplied the bicarbonate concentration constant during the formation process from phase IV (SI Figure S1).

Table 2. Setup for the different phases of the continuous formation of valerate and $n$ - and isobutyrate from propionate, acetate and methanol to study the effect of methanol increase on $\mathrm{n}$ and iso-butyrate and $\mathrm{n}$-valerate formation. The $\mathrm{pH}$ in the reactor was controlled during phase II to phase VI. The methanol concentration of the influent was increased from $250 \mathrm{mM}$ to $400 \mathrm{mM}$ in phase VI. The reactor was in batch mode during phase IV.

\begin{tabular}{|c|c|c|c|c|c|c|}
\hline Phase conditions & Phase I & Phase II & Phase III & Phase IV & Phase V & Phase VI \\
\hline $\begin{array}{l}\text { Hydraulic retention time } \\
\text { (h) }\end{array}$ & 46.3 & 42.5 & 45.4 & $\infty$ & 90.5 & 87.8 \\
\hline Phase duration (days) & $0-17$ & $17-20$ & $20-38$ & $38-45$ & $45-71$ & 71-104 \\
\hline $\mathrm{pH}$ influent & $\begin{array}{l}7.0 \\
6.9\end{array}$ & 5.8 & 5.8 & 5.8 & 5.8 & 5.8 \\
\hline $\mathrm{pH}$ reactor & \pm 0.1 & $6.1 \pm 0.2$ & $5.8 \pm 0.1$ & $5.8 \pm 0.0$ & $5.7 \pm 0.1$ & $5.8 \pm 0.1$ \\
\hline $\begin{array}{l}\mathrm{CO}_{2} \text { supply }(\mathrm{ml} / \mathrm{min}) \\
\text { Methanol in influent } \\
(\mathrm{mM})\end{array}$ & 250 & 250 & 0.18 & 0.36 & 0.36 & $\begin{array}{l}0.36 \\
\mathbf{4 0 0}\end{array}$ \\
\hline
\end{tabular}

\section{Stoichiometric analysis}

A stoichiometric analysis of the occurred conversions was performed based on the main possible conversions shown in Table 3 combined with the observed changes in the compound composition of the reactors and the obtained carbon and electron balances. The carbon balances per data point are shown in SI Figure S3. Further indications of the conversions were given by the measured production of protons and gases (methane, carbon dioxide, hydrogen). 


\section{Microbial community analysis}

In order to characterize the enrichment of the biomass during propionate methanol chain elongation samples of both inoculum and steady state biomass were used for 16S rRNA gene amplicon analyses. Inoculum biomass was taken three months after taking the last sample from one of the batches that was started at $\mathrm{pH}$ 6.5. Reactor biomass was taken from the propionate methanol chain elongation reactor on day 90 and day 125 (at the ends of the steady states). For the propionate/acetate reactor biomass samples were taken on day 69 and day 106 (also at the end of the steady states). All biomass samples were taken, in duplo, by spinning down $20 \mathrm{ml}$ in a centrifuge tube and snap freezing the pellet using liquid hydrogen. From here on the in duplo taken samples were analysed separately.

DNA was extracted from the pellets using the Powersoil DNA isolation kit according to their instruction manual. The isolated DNA was then used as template to amplify the V3-V4 regions of $16 \mathrm{~S}$ rRNA gene via PCR using the primer sets provided by Takahashi et al. ${ }^{98}$. This allowed simultaneous amplification of bacterial and archaean 16S rRNA genes. The illumina library generation ${ }^{98}$ methods were subsequently used to generate DNA sequence data.

After acquiring rDNA sequence data a statistical analysis allowed OTU picking, using the SILVA version 12816 S reference database and uclust 157, 158. The RDP classifier (version 2.2) ${ }^{159}$ was trained with the same SILVA reference database and subsequently used to classify the OTUs. Taxonomic analysis was performed using QIIME software version 1.9.1 160. This bioinformatics process was performed on the $21^{\text {st }}$ of August 2018. From the acquired data a heat map such as shown in SI Table S10 could be made using Microsoft Excel. Open source software Rstudio v3.5.0 was used to sort the data and create quantitative OTU tables (as in SI Table S10 to S13) that belonged to a chosen taxonomic group. This allowed counting the most abundant OTU's that were classified within a single genus. The rDNA sequences of selected abundant OTUs (as in SI Table ) were then used for Megablast to search within the NCBI nucleotide database on the 18th of April 2019. 


\section{Results}

\section{$\mathrm{N}$-valerate formation during batch experiments with $\mathrm{pH}$ ranging from 5 to 7.5}

The open-culture batch experiments showed that indeed methanol based chain elongation of propionate is feasible. The series of batch experiments started at an initial $\mathrm{pH}$ ranging from 5 to 7.5 (with steps of 0.5 ) and showed propionate elongation with methanol to form $n$-valerate $(n-\mathrm{C} 5)$. Figure 2 shows the results of a batch that started at $\mathrm{pH} 7$. Figure S2 in the SI shows the results of the methanol based propionate elongation batches with initial $\mathrm{pH}$ values ranging from 5.5 to 7.5. Table 3 shows the main conversions that could occur during continuous methanol based elongation of propionate and acetate.
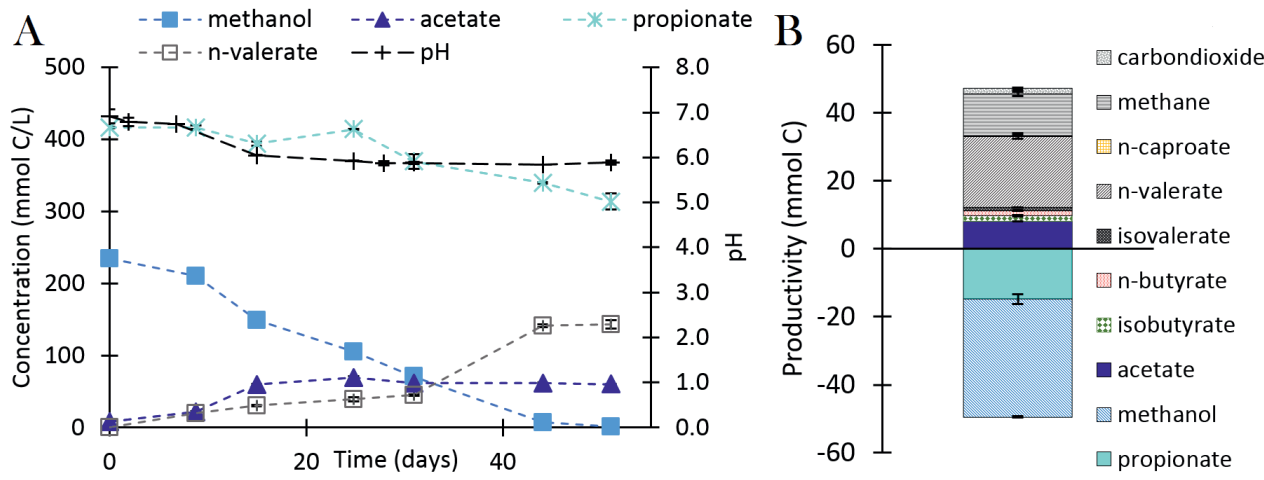

Figure 2. Concentration profile $(A)$ during a batch experiment with methanol and propionate with initial $\mathrm{pH} 7$ at $308 \mathrm{~K}$. Initially (day 0 - 25) acetate is formed via $\mathrm{CO}_{2}$ elongation with methanol concurrently with a $\mathrm{pH}$ drop. Propionate elongation to valerate starts slowly in the beginning, however after 40 days when the $\mathrm{pH}$ is $\mathbf{5 . 8}$ it is the most prevalent metabolic activity. The total conversions at the end of the batch experiment are shown as well (B). The error bars represent the minimum and maximum values measured in the duplo experiments. 
Table 3. Overview of main catabolic conversions shown occurring during continuous methanol based propionate and acetate elongation under anaerobic conditions at $308 \mathrm{~K}$ and pH 5.8 with the Gibbs free energy of the reactions. The calculation of the Gibbs free energy is shown in the SI.

\begin{tabular}{|c|c|c|c|}
\hline $\mathbf{N r}$ & Description & Catabolic reaction & $\begin{array}{l}\Delta \mathbf{G}_{\mathbf{r}}{ }^{1} \\
(k J / \text { reaction })\end{array}$ \\
\hline 1 & $\begin{array}{l}\text { Methylotrophic } \\
\text { methanogenesis } 161\end{array}$ & $4 \mathrm{CHH}_{3} \mathrm{OH} \rightarrow 3 \mathrm{CH}_{4}+\mathrm{HCO}_{3}^{-}+\mathrm{H}_{2} \mathrm{O}+\mathrm{H}^{+}$ & -310.8 \\
\hline 2 & $\begin{array}{l}\text { Methylotrophic } \\
\text { acetogenesis } 44\end{array}$ & $4 \mathrm{CH}_{3} \mathrm{OH}+2 \mathrm{HCO}_{3}^{-} \rightarrow 3 \mathrm{CH}_{3} \mathrm{COO}^{-}+\mathrm{H}^{+}+4 \mathrm{H}_{2} \mathrm{O}$ & -178.7 \\
\hline 3 & $\begin{array}{l}N \text {-valerate formation } \\
\text { from methanol and } \\
\text { propionate }\end{array}$ & $2 \mathrm{CH}_{3} \mathrm{OH}+\mathrm{C}_{3} \mathrm{H}_{5} \mathrm{O}_{2}^{-} \rightarrow \mathrm{C}_{5} \mathrm{H}_{9} \mathrm{O}_{2}^{-}+2 \mathrm{H}_{2} \mathrm{O}$ & -106.1 \\
\hline 4 & $\begin{array}{l}N \text {-butyrate formation } \\
\text { from methanol and } \\
\text { acetate } 149\end{array}$ & $2 \mathrm{CH}_{3} \mathrm{OH}+\mathrm{CH}_{3} \mathrm{COO}^{-} \rightarrow \mathrm{CH}_{3}\left(\mathrm{CH}_{2}\right)_{2} \mathrm{COO}^{-}+2 \mathrm{H}_{2} \mathrm{O}$ & -106.1 \\
\hline 5 & $\begin{array}{l}\text { Iso-butyrate formation } \\
\text { from methanol and } \\
\text { acetate }\end{array}$ & $2 \mathrm{CH}_{3} \mathrm{OH}+\mathrm{CH}_{3} \mathrm{COO}^{-} \rightarrow\left(\mathrm{CH}_{3}\right)_{2} \mathrm{CHCOO}^{-}+2 \mathrm{H}_{2} \mathrm{O}$ & -106.1 \\
\hline 6 & $\begin{array}{l}\text { Acetotrophic } \\
\text { methanogenesis } 162\end{array}$ & $\mathrm{CH}_{3} \mathrm{COO}^{-}+\mathrm{H}_{2} \mathrm{O} \rightarrow \mathrm{CH}_{4}+\mathrm{HCO}_{3}^{-}$ & -49.9 \\
\hline 7 & $\begin{array}{l}\text { Hydrogenogenic } \\
\text { propionate degradation } \\
163,164\end{array}$ & $\mathrm{CH}_{3} \mathrm{CH}_{2} \mathrm{COO}^{-}+3 \mathrm{H}_{2} \mathrm{O} \rightarrow \mathrm{CH}_{3} \mathrm{COO}^{-}+\mathrm{HCO}_{3}^{-}+\mathrm{H}^{+}+3 \mathrm{H}_{2}$ & $\begin{array}{l}26.7 \text { with } \mathrm{pH}_{2} \\
100 \mathrm{~Pa}\end{array}$ \\
\hline 8 & $\begin{array}{l}\text { Hydrogenotrophic } \\
\text { methanogenesis }{ }^{165}\end{array}$ & $4 \mathrm{H}_{2}+\mathrm{HCO}_{3}^{-}+\mathrm{H}^{+} \rightarrow \mathrm{CH}_{4}+3 \mathrm{H}_{2} \mathrm{O}$ & $\begin{array}{l}-68.4 \text { with } \mathrm{pH}_{2} \\
100 \mathrm{~Pa}\end{array}$ \\
\hline 9 & $\begin{array}{l}\text { Hydrogenotrophic } \\
\text { acetogenesis } 166\end{array}$ & $4 \mathrm{H}_{2}+2 \mathrm{HCO}_{3}^{-}+\mathrm{H}^{+} \rightarrow \mathrm{CH}_{3} \mathrm{COO}^{-}+4 \mathrm{H}_{2} \mathrm{O}$ & $\begin{array}{l}-36.1 \text { with } \mathrm{pH}_{2} \\
100 \mathrm{~Pa}\end{array}$ \\
\hline
\end{tabular}

\section{Continuous n-valerate formation with HRT 95h and pH 5.5-5.8}

$N$-valerate was produced continuously during methanol based propionate elongation by an anaerobic open-culture in a continuous reactor (Figure $\mathbf{3}$ ). No methanol was consumed until the HRT was changed from 42 to $95 \mathrm{~h}$ (day 27, start of phase II). At the start of phase III ( $\mathrm{CO}_{2}$ supply, day 43$)$, the methanol concentration decreased rapidly and increased again after the $\mathrm{pH}$ was lowered to 5.5 (SI Figure S3, day 90). The propionate consumption increased from the moment the $\mathrm{pH}$ was decreased to 5.8 (day 43 ). The $n$-valerate production was low ( $\pm 2.4 \mathrm{mmol} / \mathrm{L} /$ day) during phase II with $\mathrm{pH} 6.3$ (day 27-43) and increased after the $\mathrm{pH}$ was decreased to 5.8 (day 43 ) to a value of $9.3 \mathrm{mmol} / \mathrm{L} / \mathrm{day}$. A slight increase in the $n$-valerate productivity (to $9.7 \mathrm{mmol} / \mathrm{L} / \mathrm{day}$ ) and concentration (40 $\mathrm{mM}$ ) followed in phase IV, when the $\mathrm{pH}$ was 5.5 (day 111-120) (Figure 4, Table 4). The concentration profiles of the most important compounds with the carbon and electron balances are shown in the SI (Figure S3A). 


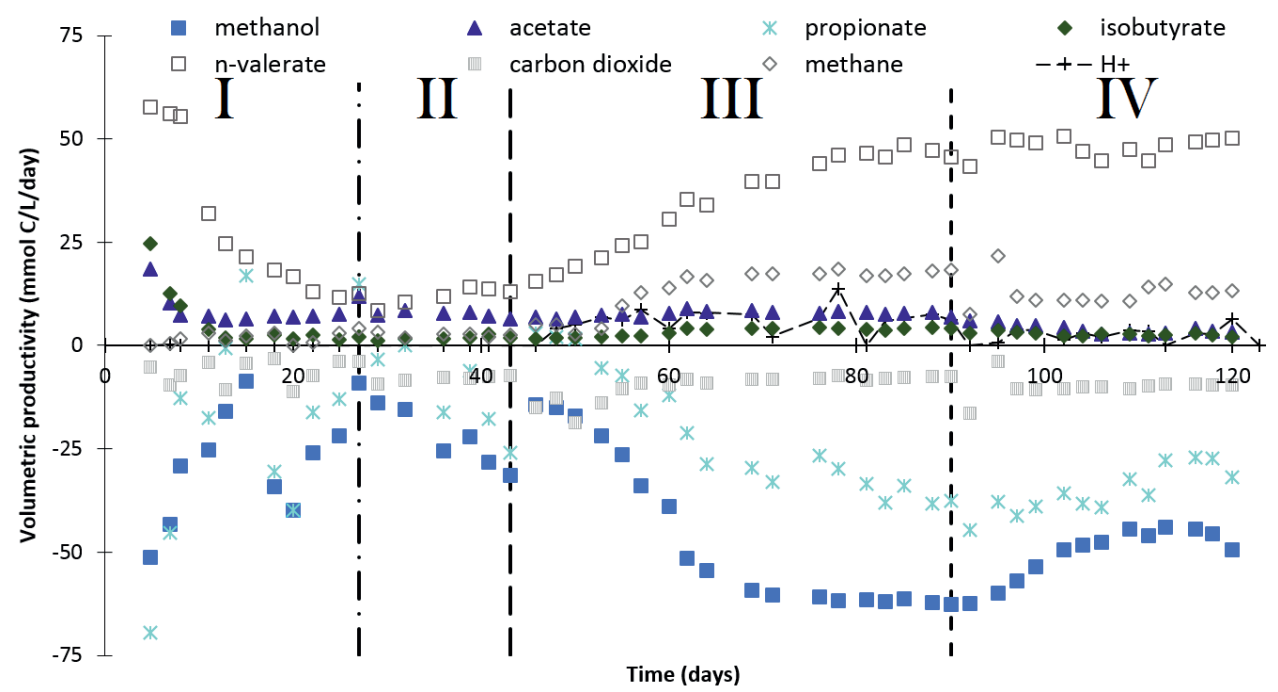

Figure 3. Volumetric productivities in time during continuous methanol based propionate elongation in an anaerobic open-culture reactor at $309 \mathrm{~K}$. The production of protons in $\mathrm{mmol} /$ day is also shown (-+--). The vertical lines indicate the major changes in the setup: change of the hydraulic retention time (HRT) from 42 to $95 \mathrm{~h}(-.-)$, pH change from 6.3 to 5.8 ( - - ) and pH change from 5.8 to 5.5 (- - - ).

\section{Formation of $n$ - and iso-butyrate in addition to $n$-valerate during simultaneous elongation of acetate and propionate}

The continuous experiment with simultaneous propionate and acetate elongation with methanol showed formation of both $n$ - and iso-butyrate and $n$-valerate. The concentration profile of the most important compounds is shown in the SI (Figure S3B). A steady state was reached after 64 days, subsequently the methanol concentration in the influent was increased (from 250 to $400 \mathrm{mM}$ ) to study whether a higher methanol concentration would lead to a higher chain elongation productivity. The concentrations of methanol in the reactor were $27 \pm 4 \mathrm{mM}$ and $76 \pm 14 \mathrm{mM}$ respectively during the steady states with 250 $\mathrm{mM}$ methanol and $400 \mathrm{mM}$ methanol in the reactor influent. After the increase of the methanol concentration in the influent from 250 to $400 \mathrm{mM}$, the $n$-valerate formation increased, while the productivities of iso-butyrate and $n$-butyrate stayed constant compared to the phase with $250 \mathrm{mM}$ methanol in the influent (Figure 4 and SI Figure S4). More iso-butyrate was formed compared to $n$-butyrate, the iso-butyrate/ $n$-butyrate ratios were $2.5 \pm 0.3$ and $2.1 \pm 0.1$ respectively in the steady states with 250 and $400 \mathrm{mM}$ methanol in the influent of the reactor with continuous methanol based propionate and acetate elongation (Figure 4: right two bars). Small amounts (1 $\mathrm{mM})$ of iso-valerate were observed during both continuous methanol based chain elongation processes. This isovalerate is likely produced from the amino acids in the yeast extract present in the medium 167,168 . 
Table 4. Average concentrations, volumetric productivities and relative product selectivities of $n$ valerate during the steady states of phase III (day 78-90) (pH 5.8) and phase IV (day 111-120) (pH 5.5) of continuous methanol based propionate elongation and during the steady state of phase $\mathrm{V}$ (day 64-71) (methanol in influent $250 \mathrm{mM}$ ) and during the last days of phase VI (day 97-104) (methanol in influent $400 \mathrm{mM}$ ) of continuous methanol based propionate and acetate elongation in an anaerobic open-culture reactor at $309 \mathrm{~K}$.

\begin{tabular}{|c|c|c|c|c|}
\hline & $\begin{array}{l}\text { Propionate } \\
\text {; pH 5.8; } \\
250 \mathrm{mM} \\
\text { methanol }\end{array}$ & $\begin{array}{l}\text { Propionate } \\
\text {; pH 5.5; } \\
250 \mathrm{mM} \\
\text { methanol }\end{array}$ & $\begin{array}{l}\text { Propionate } \\
\text { \& acetate; } \\
\text { pH 5.8; 250 } \\
\text { mM } \\
\text { methanol }\end{array}$ & $\begin{array}{l}\text { Propionate \& } \\
\text { acetate; pH } \\
5.8 ; 400 \mathrm{mM} \\
\text { methanol }\end{array}$ \\
\hline $\begin{array}{l}\text { Average } n \text {-valerate } \\
\text { concentration (mM) } \\
\text { Average } n \text {-valerate }\end{array}$ & $37.1 \pm 0.9$ & $38.4 \pm 1.4$ & $33.9 \pm 2.3$ & $42.8 \pm 0.7$ \\
\hline & $9.3 \pm 0.2$ & $9.7 \pm 0.4$ & $9.0 \pm 1.0$ & $11.5 \pm 0.2$ \\
\hline $\begin{array}{l}\text { Average } n \text {-valerate } \\
\text { selectivity (wt\%) }\end{array}$ & 58 & 70 & 49 & 43 \\
\hline
\end{tabular}

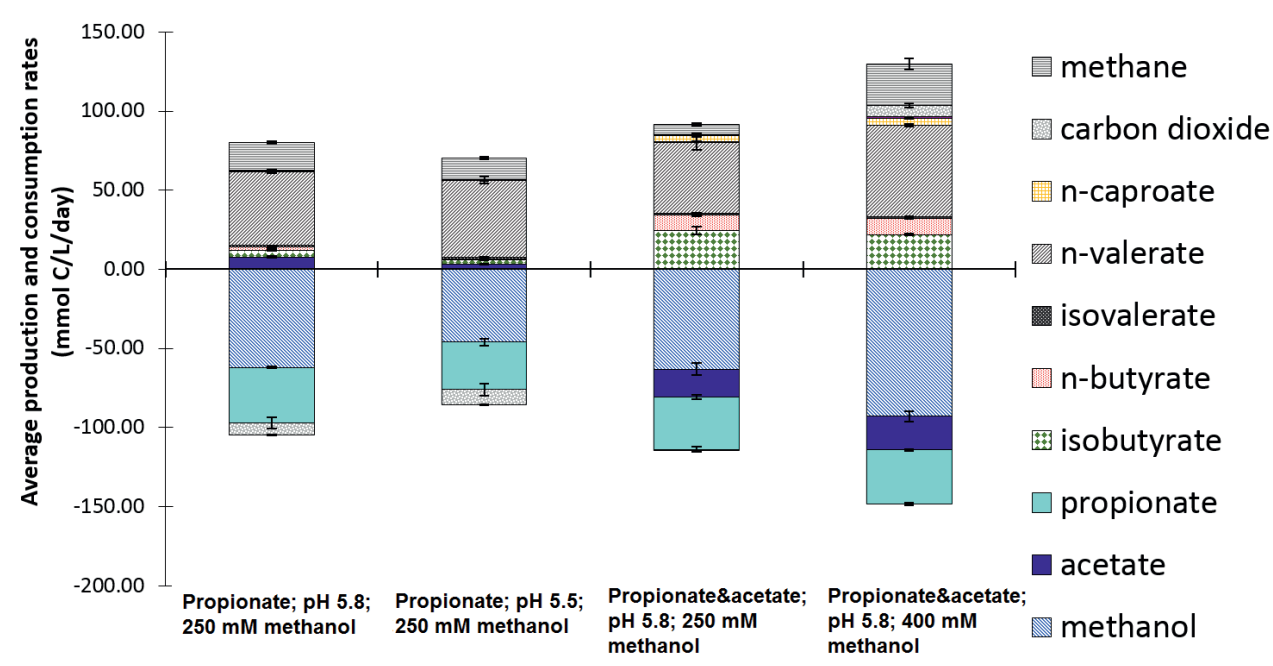

Figure 4. Average volumetric production and consumption rates during the steady states of phase III (day 78-90) (pH 5.8) and phase IV (day 111-120) (pH 5.5) of continuous methanol based propionate elongation in an anaerobic open-culture reactor and during the steady state of phase $\mathrm{V}$ (day 64-71) (methanol in influent $250 \mathrm{mM}$ ) and during the last days of phase VI (day 97-104) (methanol in influent $400 \mathrm{mM}$ ) of continuous methanol based propionate and acetate elongation in an anaerobic open-culture reactor at $309 \mathrm{~K}$. The error bars represent the minimum and maximum values measured during the phase. Table 4 shows an overview of the average concentrations, productivities and relative product selectivities of $n$-valerate during the same four phases. The carbon balances for the four steady states were $88 \pm 2,93 \pm 4,88 \pm 4$ and $92 \pm 3 \%$ from left to right, the electron balances were $92 \pm 2,98 \pm 4,87 \pm 4$ and $91 \pm 3 \%$, respectively. The balances can be found in SI Figure S3. 


\section{Increase of relative methane selectivity after increase of methanol concentration}

The increase of the methanol concentration in the reactor increased the $n$-valerate productivity, but decreased the relative selectivity for $n$-valerate production from $49 \mathrm{wt} \%$ to 43 wt\% of the total carbon containing products (Figure 5, Table 4 ). The main reason for the decreased selectivity is the net carbon dioxide formation and the increased methane production that occurred with a higher methanol concentration in the influent.
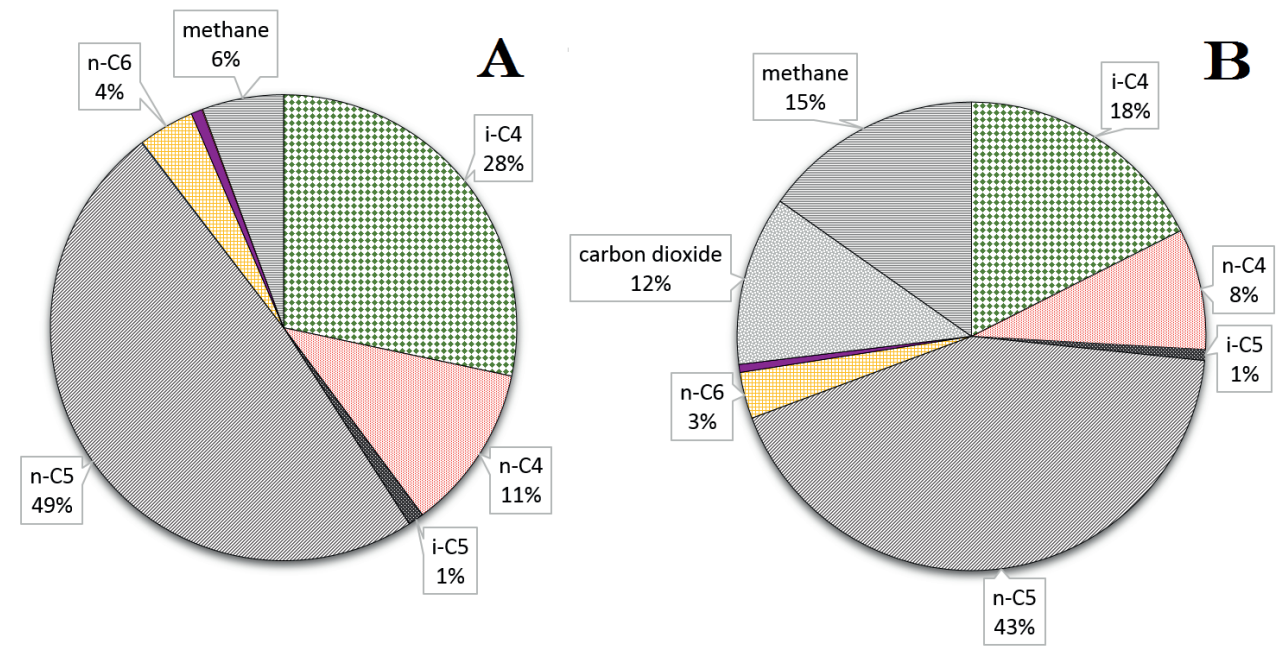

Figure 5. Relative selectivity of the formed n-butyrate (n-C4), iso-butyrate (i-C4), n-valerate ( $n$ $\mathrm{C5})$, iso-valerate (i-C5), n-caproate $(\mathrm{n}-\mathrm{C} 6)$, carbon dioxide $\left(\mathrm{CO}_{2}\right)$ and methane $\left(\mathrm{CH}_{4}\right)$ of continuous methanol based propionate and acetate elongation in an anaerobic open-culture reactor at $36^{\circ} \mathrm{C}$ at the steady state with 250 mM methanol in the influent (A) (day 64-71) and at the last days with $400 \mathrm{mM}$ methanol in the influent $(B)$ (day 111-120). The values are calculated based on the production rates in $\mathrm{g} / \mathrm{L} / \mathrm{day}$, the total production rates were $1.37 \pm 0.26$ (A) and $2.12 \pm 0.18$ (B) g/L/day.

\section{Clostridium Iuticellarii (species) dominant during both acetate and propionate elongation}

Within the methanol chain elongation reactor two orders predominantly were present: Clostridiales and Thermoplasmatales. Table $\mathbf{5}$ shows the composition of the microbial communities at the end of the two last phases in both the continuous methanol based propionate elongation reactor and the continuous methanol based acetate and propionate elongation reactor. The most abundant OTU from the Clostridiales bacteria ( $20 \%, \sim 17 \%$, $\sim 43 \%$ and $\sim 17 \%$ of the total OTU count, respectively for the phases from left to right in Table 5) appeared to be highly similar to Clostridium luticellarii ( $99.72 \%$ similarity, SI Table S12). In Table S10 to S13 (Supporting Information) more extensive information on genus level relative abundances, OTU counts and ncbi BLAST results can be found. 
Table 5. Overview of the relative abundances within the microbiomes, given in percentage (\%) at order level. The samples were taken at the end of the steady states of phase III (day 90) (pH 5.8) and phase IV (day 125) (pH 5.5) of continuous methanol-based propionate elongation in an anaerobic open-culture reactor and at the end of the steady state of phase $\mathrm{V}$ (day 69) (methanol in influent $250 \mathrm{mM}$ ) and during the last day of phase VI (day 106) (methanol in influent $400 \mathrm{mM}$ ) of continuous methanol-based propionate and acetate elongation in an anaerobic open-culture reactor at $309 \mathrm{~K}$. For all biomass samples the duplo results are shown indicated by $1 \& 2$. More detailed information and the relative abundances found for the inoculum samples can be found in the SI: Tables S10 to S13.

\begin{tabular}{|c|c|c|c|c|c|c|c|c|}
\hline \multirow[t]{2}{*}{ Order } & \multicolumn{2}{|c|}{$\begin{array}{l}\text { Propionate; pH 5.8; } \\
250 \text { mM methanol }\end{array}$} & \multicolumn{2}{|c|}{$\begin{array}{l}\text { Propionate; pH 5.5; } \\
250 \text { mM methanol }\end{array}$} & \multicolumn{2}{|c|}{$\begin{array}{c}\text { Propionate\&acetate; } \\
\text { pH } 5.8 ; 250 \mathrm{mM} \\
\text { methanol }\end{array}$} & \multicolumn{2}{|c|}{$\begin{array}{c}\text { Propionate\&acetate; } \\
\text { pH 5.8; } 400 \mathrm{mM} \\
\text { methanol }\end{array}$} \\
\hline & 1 & 2 & 1 & 2 & 1 & 2 & 1 & 2 \\
\hline Clostridiales & $42.3 \%$ & $43.2 \%$ & $39.1 \%$ & $46.7 \%$ & $58.1 \%$ & $58.4 \%$ & $38.0 \%$ & $34.6 \%$ \\
\hline Thermoplasmatales (Archaea) & $29.3 \%$ & $32.7 \%$ & $25.0 \%$ & $21.6 \%$ & $18.1 \%$ & $19.2 \%$ & $33.9 \%$ & $38.2 \%$ \\
\hline Pseudomonadales & $6.3 \%$ & $3.2 \%$ & $0.0 \%$ & $0.0 \%$ & $0.1 \%$ & $0.1 \%$ & $0.0 \%$ & $0.1 \%$ \\
\hline Rhodocyclales & $4.9 \%$ & $3.3 \%$ & $0.0 \%$ & $0.0 \%$ & $0.0 \%$ & $0.0 \%$ & $0.0 \%$ & $0.0 \%$ \\
\hline Bacteroidales & $3.4 \%$ & $4.0 \%$ & $12.1 \%$ & $11.5 \%$ & $8.5 \%$ & $9.0 \%$ & $10.3 \%$ & $10.3 \%$ \\
\hline Methanomicrobiales (Archaea) & $2.2 \%$ & $2.8 \%$ & $5.1 \%$ & $3.3 \%$ & $0.2 \%$ & $0.3 \%$ & $4.5 \%$ & $4.0 \%$ \\
\hline Coriobacteriales & $2.0 \%$ & $2.2 \%$ & $4.3 \%$ & $3.9 \%$ & $0.4 \%$ & $0.4 \%$ & $1.1 \%$ & $1.0 \%$ \\
\hline Burkholderiales & $1.3 \%$ & $0.7 \%$ & $2.1 \%$ & $1.9 \%$ & $1.0 \%$ & $0.9 \%$ & $1.2 \%$ & $1.3 \%$ \\
\hline Campylobacterales & $1.3 \%$ & $1.4 \%$ & $0.0 \%$ & $0.0 \%$ & $0.1 \%$ & $0.1 \%$ & $0.0 \%$ & $0.1 \%$ \\
\hline Synergistales & $0.8 \%$ & $1.0 \%$ & $0.4 \%$ & $0.4 \%$ & $0.3 \%$ & $0.2 \%$ & $0.3 \%$ & $0.3 \%$ \\
\hline Spirochaetales & $0.5 \%$ & $0.5 \%$ & $0.5 \%$ & $0.4 \%$ & $0.4 \%$ & $0.5 \%$ & $0.5 \%$ & $0.4 \%$ \\
\hline Erysipelotrichales & $0.5 \%$ & $0.5 \%$ & $7.6 \%$ & $6.6 \%$ & $9.4 \%$ & $8.2 \%$ & $8.3 \%$ & $8.0 \%$ \\
\hline Selenomonadales & $0.3 \%$ & $0.3 \%$ & $1.7 \%$ & $1.7 \%$ & $0.6 \%$ & $0.4 \%$ & $0.3 \%$ & $0.3 \%$ \\
\hline Methanobacteriales (Archaea) & $0.1 \%$ & $0.1 \%$ & $0.1 \%$ & $0.1 \%$ & $0.0 \%$ & $0.0 \%$ & $0.0 \%$ & $0.0 \%$ \\
\hline Sum of all order & $95.2 \%$ & $95.7 \%$ & $97.9 \%$ & $98.1 \%$ & $97.4 \%$ & $97.7 \%$ & $98.5 \%$ & $98.5 \%$ \\
\hline Other orders & $4.8 \%$ & $4.3 \%$ & $2.1 \%$ & $1.9 \%$ & $2.6 \%$ & $2.3 \%$ & $1.5 \%$ & $1.5 \%$ \\
\hline Total OTU count & 132846 & 221728 & 197444 & 208429 & 189896 & 194669 & 183205 & 200691 \\
\hline
\end{tabular}




\section{Discussion}

\section{$\mathrm{N}$-valerate formation during batch experiments with $\mathrm{pH}$ ranging from 5 to 7.5}

During the batches three predominant metabolic groups were deducted from the consumed substrates and produced biochemicals: (1) methylotrophic methanogens, methylotrophic acetogens and (3) methanol based propionate elongation (Table 3). Also traces of $n$-butyrate and iso-butyrate were found (Figure 2B) which indicates methanol based acetate elongation activity.

The degree in which the main metabolic activities occurred depended heavily on initial $\mathrm{pH}$ and final concentrations. An initial pH of 7 and 7.5 caused some methanogenic activity and acetogenesis to occur in the beginning of the batch, whereas methanol based chain elongation of propionate to $n$-valerate caught on after approximately 30 days when $\mathrm{pH}$ had already dropped to $5.8 \pm 0.1$. In the batch that started at $\mathrm{pH} 6.5$ this pattern was similar but showed a longer lag phase and more extended/slowed production profile. The batches performed at pH 6 and lower showed no significant production after 60 days (SI Figure S2).

\section{Continuous n-valerate formation with HRT 95h and pH 5.5-5.8}

The hydraulic retention time (HRT) and the $\mathrm{pH}$ appeared to be critical for continuous $n$ valerate production. The HRT of $42 \mathrm{~h}$ appeared to be too short for the valerate producing organisms, since the concentration of $n$-valerate and the $n$-valerate productivity decreased exponentially after the startup of the experiment (Figure 3: day 0 to 27). The $n$-valerate producing culture probably washed out during the first phase, since a decrease in optical density was by eye observed. For this study, a HRT of $95 \mathrm{~h}$ was used, so most microorganisms that carry out competitive methanol consuming processes (Table 3: R1 and R2) could now theoretically grow in the continuous reactor, based on their growth rates found in literature ${ }^{150}$. The minimum HRT will be between 42 and $95 \mathrm{~h}$.

The chain elongation reactions occurred at $\mathrm{pH}$ values between 5.5 and 5.8 during both batch and continuous experiments. A pH of 5.5 is optimal for $n$-valerate formation during continuous methanol based propionate elongation (Figure 3). The $\mathrm{pH}$ decrease from 5.8 to 5.5 led to selectivity increase for $n$-valerate formation from methanol and propionate (from $58 \%$ to $70 \%$, Table 4 ), which was attributed to two causes.

Firstly, the acetate formation (Table 3: R2) decreased after the $\mathrm{pH}$ was lowered from 5.8 to 5.5 (day 90) (Figure 3). The theoretical available amount of dissolved carbon dioxide was maintained equal at $\mathrm{pH} 5.8$ and 5.5 , so substrate availability was not the reason for the decreased acetogenic activity (SI Figure S1). The acetogenic activity could decrease at 
lower $\mathrm{pH}^{169}$ or at higher concentration of undissociated acids present at the lower $\mathrm{pH}^{152}$. Less acetate was apparently available for $n$ - and iso-butyrate formation at pH 5.5 (Table 3: R5), so the $n$ - and iso-butyrate formation decreased as well.

Secondly, the methanogenic productivity decreased after the $\mathrm{pH}$ was lowered from 5.8 to 5.5 (day 90) (Figure 4). This finding is supported by the decreased relative abundance of the Thermplasmatales family at pH 5.5 compared to $\mathrm{pH} 5.8$ in the continuous methanol based propionate elongation reactor (Table 5). The Thermoplasmatales family contains methane producing archaea; they were identified to fall within uncultured species of the methylotrophic methanogen genus Candidatus methanogranum ${ }^{170}$ (Table 3: R1). These archaea are known to utilize methanol as a substrate for methane formation, which well fits the observed methanogenesis in our system (Figure 4) ${ }^{171}$. The methanogenesis inhibition with the $\mathrm{pH}$ decrease could e.g. be caused by either the higher extracellular proton concentration or by the higher concentration of undissociated volatile fatty acids 152-154.

\section{Increase of methanol concentration caused increase of both $n$-valerate productivity and methanogenesis}

The $n$-valerate production improved after the increase of the methanol concentration in the influent, while the prior methanol concentration did not limit the $n$ - and iso-butyrate production (Figure 4). Methylotrophic methanogenesis (Table 3: R1), one of the competing methanol consuming processes, increased as result of the higher methanol influent concentration in phase VI. The relative abundance of the Candidatus Methanogranum genus also increased with the increasing methanol concentration (Table 5), indicating a growth of the methanogenic bacteria community. The methanol concentration in the influent and reactor was the only parameter that changed between the two steady states shown in Figure 5, indicating that the methylotrophic methanogenesis was limited by the methanol concentration ( $27 \pm 4 \mathrm{mM}$ ) during the first steady state. Further examination of the $\mathrm{K}_{\mathrm{s}}$ value for methylotrophic methanogenesis under the described conditions can verify whether kinetics were indeed limiting at lower methanol concentrations. The yield of $n$-valerate over propionate was $1.01 \mathrm{~mol} / \mathrm{mol}$, while the yield of $n$ - and iso-butyrate over acetate was $0.76 \mathrm{~mol} / \mathrm{mol}$ during phase VI. The lower yield of butyrate over acetate indicates that acetate was consumed for other processes than butyrate formation; likely acetate was utilized for biomass formation.

\section{Clostridium Iuticellarii (species) dominant candidate for continuous methanol based propionate elongation}

Clostridium luticellarii is a known butyrate producing strain (SI Table S11) within the Clostridium sensu stricto 12 genus. It shares highest similarities with Clostridium 
Ijungdahlii and Clostridium kluyverii ${ }^{172}$. C. luticellarii is the prime candidate for performing the methanol based chain elongation, which well fits its similarities to a Wood-Ljungdahl harbouring ( $C$. ljungdahlii) microorganism and a reverse $\beta$-oxidation harbouring ( $C$. kluyverii) microorganism. The OTU with a high similarity (100\% cover, $99.72 \%$ identity, Table S12) to $C$. Iuticellarii has the highest relative abundance among the Clostridium sensu stricto 12 for both the methanol based propionate elongation reactor and the methanol based propionate and acetate elongation reactor. Based on this finding can be suggested that $C$. luticellarii is responsible for both the elongation reactions from acetate to $n$ - and isobutyrate (Table 3: R4 and R5) and the elongation from propionate to $n$-valerate (Table 3: R3). The proposed mechanism by which C. luticallarii performs the methanol based chain elongation reaction (for propionate elongation to $n$-valerate) is shown in Figure 6. Methanol as electron donor is known to be metabolized within the Wood-Ljungdahl pathway where some methanol is oxidized to $\mathrm{CO}$, while the rest of the methanol is used for elongation with this $\mathrm{CO}$ to form acetyl-COA 30,173 . It was observed in this study that the electron acceptor (acetate and propionate) was always elongated with two-carbon units. This stoichiometry suggests that the elongation is executed via an acetyl-CoA thiolasedriven reaction similar to reverse beta-oxidation 33,34 . The proposed route should be verified by isolating the responsible strain and by performing a genome analysis to identify the corresponding enzymes. 


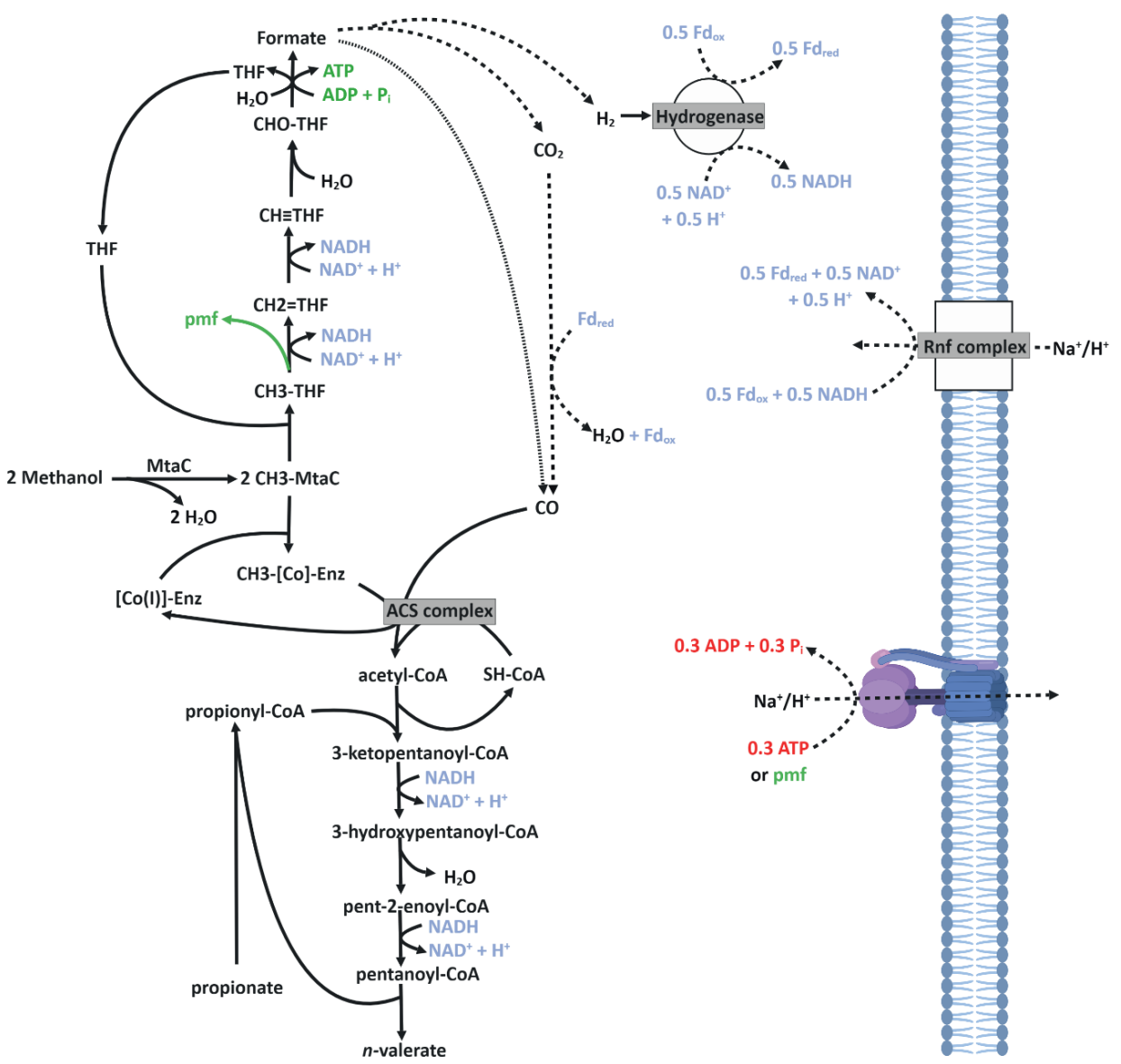

Figure 6. The figure shows the hypothetical proposed mechanism for methanol based propionate elongation to $\mathrm{n}$-valerate. Within the Wood-Ljungdahl pathway one methanol is oxidized via the THFroute to formate/CO, while another methanol is supplied to the ACS complex via a $\mathrm{CH}_{3}-[\mathrm{Co}]-E n z y m e$ intermediate ${ }^{30,173}$. The ACS complex then catalyses the formation of acetyl-CoA. Depending on the intracellular potential formate could either be directly utilized for the formation of CO (dotted line) 173 , or alternatively $\mathrm{CO}$ formation would require the bifurcating hydrogenase as well as a Rnf complex to balance the redox compounds (dashed line) ${ }^{30}$. The formed acetyl-CoA is then likely used in a thiolase-driven condensation step with propionyl-CoA to form 3-ketopentanoyl-CoA, similar to the reverse beta-oxidation mechanism in $C$. kluyveri ${ }^{132}$. The two NADH generated during the oxidation of methanol are subsequently used to reduce 3-ketopentanoyl-CoA to 3-hydroxypentanoyl-CoA and to reduce pent-2-enoyl-CoA to pentanoyl-CoA. Because the methanol based chain elongation of propionate to $\mathrm{n}$-valerate (Table 3:R3) has a $\triangle \mathrm{G}$ of $-106.1 \mathrm{~kJ} /$ reaction an ATP yield of 1.5 ATP would be expected $\left(106.1 \mathrm{~kJ} / \sim 70 \mathrm{~kJ} /\right.$ ATP $\left.{ }^{174}=1.5 \mathrm{ATP}\right)$. This suggests that additional energy would be gained via a proton/ $\mathrm{Na}^{+}$motive force (pmf) that is likely generated at the oxidation of $\mathrm{CH}_{3}-\mathrm{THF}^{174}$. Potentially additional bifurcation steps within the reverse beta-oxidation part might be necessary, depending on the intracellular redox potentials of the redox cofactors ${ }^{174,175}$. 


\section{Shift in catabolic conversions as a result of the pH change and the methanol increase}

During continuous methanol based propionate elongation, the $\mathrm{pH}$ decrease from 5.8 to 5.5 caused a decrease in both acetate formation and $n$-valerate degradation (Figure 7: $\mathrm{A}$ and $B)$. The microbial analysis that was performed during the steady state at $\mathrm{pH} 5.8$ was combined with the stoichiometric analysis to obtain an overview of the main catabolic conversions (Figure 7A). The proton production decreased after the $\mathrm{pH}$ decrease (SI Figure S3), suggesting methylotrophic acetogenesis (Table 3: R2) is less likely to occur at pH 5.5.

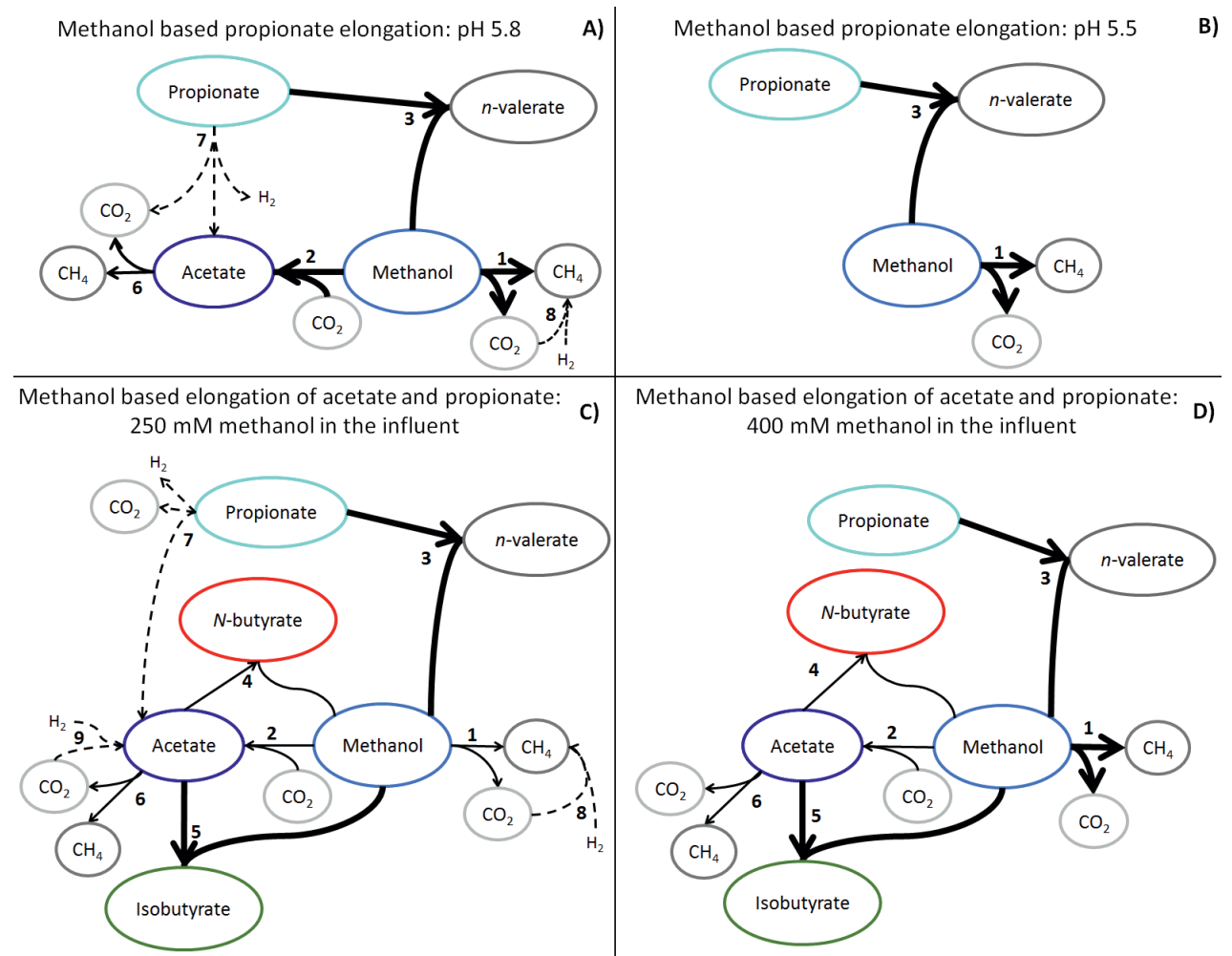

Figure 7. Schematic overview of the proposed main conversions that occurred during continuous methanol based elongation of propionate (Pro) and simultaneous propionate and acetate (Pro\&Ac) elongation at $36^{\circ} \mathrm{C}$. The four scenarios represent the steady states in the propionate elongation reactor at $\mathrm{pH} 5.8(\mathrm{~A})$ and $\mathrm{pH} 5.5$ (B) and in the simultaneous propionate and acetate elongation reactor with 250 (C) and 400 (D) $\mathrm{mM}$ methanol in the reactor influent. The conversions with dashed arrows are proposed to maintain the electron balance. The main reactions are indicated with thicker arrows. For simplicity, only the productivities of the compounds with a value higher than 5 $\mathrm{mmol} / \mathrm{L} /$ day are shown. The reaction equations and Gibbs free energy of the numbered conversions can be found in the SI. 
The change in the molar yield of $n$-valerate over propionate (from 0.80 at pH 5.8 to 0.97 at $\mathrm{pH}$ 5.5) indicated that the degradation reaction of propionate (Table 3: R7) did not occur during the steady state at pH 5.5 (Figure 7B). Only very small traces of $\mathrm{H}_{2}$ were observed during the continuous methanol based elongation of acetate ( $\max 0.02$ $\mathrm{mmol} / \mathrm{L} /$ day) and during the simultaneous elongation of acetate and propionate ( $\max 0.03$ $\mathrm{mmol} / \mathrm{L} /$ day). Some blasted OTUs from the Clostridium sensu stricto 21 genus found during this study (SI Table S11) were also found in mixed cultures where hydrogen production was observed 176,177 , so possibly propionate degradation by $\mathrm{H}_{2}$-producing bacteria took place at $\mathrm{pH}$ 5.8. However, the Gibbs free energy of the propionate degradation is positive at $100 \mathrm{~Pa} \mathrm{H}_{2}$ (becomes <-20 kJ/reaction at $0.1 \mathrm{~Pa}$ ) making this reaction unlikely to have occurred. The conversion is dashed since it is unknown whether hydrogen produced during the propionate degradation. The competing methanol and propionate consumption processes ( 2 and 7 ) were almost completely inhibited at pH 5.5 (Figure 7).

The increase of the methylotrophic methanogenesis conversion (Table 3: R1) is the main change observed from the stoichiometric analysis of the steady states in the reactor with simultaneous acetate and propionate elongation (Figure 7: $\mathrm{C}$ and $\mathrm{D}$ ). The molar ratio between the productivity of methane and $\mathrm{CO}_{2}$ is $3: 1$ (Figure 4), supporting the proposed methylotrophic methanogenesis (Table 3: R1). The molar yield of $n$-valerate per propionate increased (from 0.81 to 1.01 ) after the increased methanol concentration in the influent. This indicates that the propionate degradation reaction (Table 3: R7) mainly occurred during the phase with $250 \mathrm{mM}$ methanol in the influent. Since no significant net hydrogen production was measured in the reactor during the continuous acetate and propionate elongation process, a hydrogen-consuming process must have taken place if propionate was degraded to bicarbonate and hydrogen. Hydrogenotrophic methanogenesis (Table 3: R8) and hydrogenotrophic acetogenesis (Table 3: R9) are suggested to have occurred as hydrogen-consuming process during the steady state with $250 \mathrm{mM}$ methanol in the influent (Figure 7C).

The molar yield of $n$ - and iso-butyrate over acetate was 0.99 during the steady state with $250 \mathrm{mM}$ methanol in the influent, so when propionate degradation occurred, the hereby produced acetate (Table 3: R7) has been consumed in another process. Acetotrophic methanogenesis (Table 3: R6) is suggested to have occurred during the steady state with $250 \mathrm{mM}$ methanol in the influent (Figure 7C). The molar yield of $n$ - and iso-butyrate over acetate decreased (to 0.76) after the methanol concentration in the influent was increased, so presumably acetate was consumed in other processes than butyrate formation during the phase with $400 \mathrm{mM}$ methanol in the influent (Figure 7D). 


\section{Outlook on application and process improvements}

Microbially formed $n$-valerate can become an additional platform chemical for various applications. Instead of sugarcane molasses ${ }^{178}$, more and possible cheaper substrates such as organic waste could be used as a substrate for $n$-valerate production. A recent study from Gonzalez-Garcia et al. ${ }^{140}$ showed that propionate production can be achieved via various metabolic pathways. Microbial produced propionate will enter the market in the near future ${ }^{140}$, which will make $n$-valerate production easier and more attractive. Mixtures of acetate and propionate can also be produced from the earlier used supermarket/food waste ${ }^{35}$ but are also reported for microbial electrosynthesis from $\mathrm{CO}_{2}{ }^{141,179}$. Methanol can be produced from syngas from lignocellulose or waste, but can also be produced by thermo-chemical $\mathrm{CO}_{2}$ conversions 135, 137, 180. The electrosynthesis and thermochemical processes can be driven by electricity obtained from photovoltaics. In summary, with substrates from various waste sources and renewable energy, the developed process can be applied in an integrated and sustainable $n$-valerate biorefinery processes.

The developed $n$-valerate formation process can be further improved. In the experiments, the addition of acetate as substrate did not lower the effectiveness of propionate elongation with methanol. Using a mixture of acetate and propionate for $n$-valerate production gave similar average $n$-valerate productivities $(45.1 \pm 4.8 \mathrm{mmol} \mathrm{C} / \mathrm{L} /$ day with propionate and acetate, and $46.6 \pm 1.1 \mathrm{mmol} \mathrm{C} / \mathrm{L} /$ day with only propionate, at pH 5.8 and $250 \mathrm{mM}$ methanol in the influent). Depending on the desired product spectrum the decreased selectivity of the $n$-valerate production as result of the addition of acetate as substrate (from 58 to $43 \%$ ), could form a disadvantage during $n$-valerate production with multiple substrates.

Two main findings can be used to increase the selectivity and productivity of methanol based propionate elongation to n-valerate. Firstly, the selectivity was improved by lowering the $\mathrm{pH}$ from 5.8 to 5.5 (within the propionate methanol elongation reactor) (Table 4). This increase in selectivity is mainly caused by the decrease of methylotrophic methanogenic activity. Secondly, the productivity was improved by increasing the methanol concentration in the influent from $250 \mathrm{mM}$ to $400 \mathrm{mM}$ (Table 4). The methanol concentration prior to increase was a limiting factor for the propionate chain elongation.

When by combining these findings that allow for increased selectivity and productivity the next step in optimizing process performance is to increase the biomass concentration. The use of carrier materials or biomass granulation are efficient strategies to improve the biomass concentration and retention, as shown during other studies ${ }^{124,181,182}$. Challenges then lie in maintaining the correct selective pressure, as decoupling solid retention time (SRT) from HRT might introduce new problems with methanogen retention in the system. 
Further optimizing additional factors like $\mathrm{pH}$, volatile fatty acid concentrations, $\mathrm{CO}_{2}$ availability in the bioreactor could provide methods to increase the selective pressure towards methanol chain elongation, by inhibiting methanogenic activity.

Chen et al. ${ }^{147}$ used acidified supermarket waste as a substrate for methanol based chain elongation at approximately pH 6.2 and a hydraulic retention time of $40 \mathrm{~h}$. Based on the results from the current study it can be suggested to decrease the $\mathrm{pH}(5.5-5.8)$ and increase the hydraulic retention time (to $95 \mathrm{~h}$ ) to stimulate $n$-valerate from propionate containing supermarket waste. Additionally, the methane production could be decreased by decreasing the $\mathrm{pH}$ to 5.5 . Implementing this knowledge about selective pressure can reduce the costs for $n$-valerate production and create a new open-culture process of biological production of chemicals from biomass and other carbon sources.

\section{Conclusions}

$\mathrm{N}$-valerate was for the first time proven to be produced from propionate and methanol during a continuous methanol based chain elongation process in an anaerobic open culture reactor. Acetate formation from methanol occurred at pH 5.8 and decreased at pH 5.5 due to the higher proton concentration and/or the accompanying increased undissociated acids concentration. Methanol consumption did not occur at a HRT of $42 \mathrm{~h}$, while a HRT of $95 \mathrm{~h}$ showed to be long enough for methanol consuming chain elongation processes. The product selectivity for $n$-valerate was increased with $\mathrm{pH}$ lowering from 5.8 to 5.5 during continuous methanol based propionate elongation. Propionate elongation to $n$-valerate and acetate elongation to iso-butyrate and $n$-butyrate occurred simultaneously in a continuous methanol based chain elongation reactor with both propionate and acetate present as chain elongation substrate. The addition of acetate as substrate did not cause a decrease of the $n$-valerate productivity. The productivity of $n$-valerate was improved by increasing the methanol concentration in the influent from 250 to $400 \mathrm{mM}$ during methanol based elongation of propionate and acetate. Clostridium luticellarii was suggested to be most abundant during all steady states of methanol based elongation of both propionate and simultaneous elongation of acetate and propionate and is therefore proposed as main candidate for methanol based chain elongation.

\section{Supporting Information}

The Supporting Information is available in the Supporting Information Section of this thesis. Additional information regarding Materials and Methods and additional figures and graphs for the Results and Discussion, 4 figures, and 13 tables (PDF). 


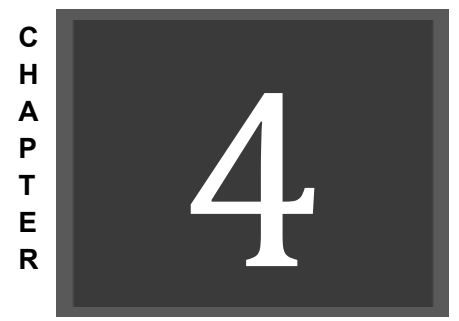

\section{Branched Medium Chain Fatty Acids: Isocaproate Formation from Isobutyrate Broadens the Product Spectrum for Microbial Chain Elongation}

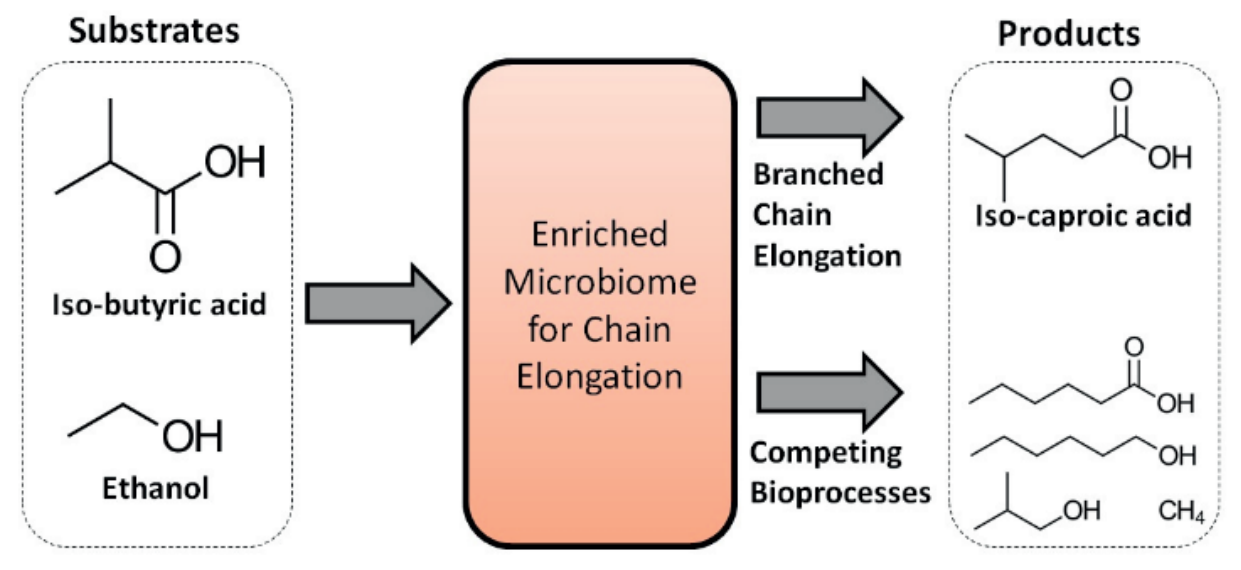

This chapter is published as

Kasper D. de Leeuw, Cees J.N. Buisman, David P.B.T.B. Strik, 2019, Branched Medium Chain Fatty Acids: iso-caproate formation from iso-butyrate broadens the product spectrum for microbial chain elongation

Environ. Sci. Technol. 2019, 53, 13, 7704-7713

https://doi.org/10.1021/acs.est.8b07256 


\section{Abstract}

Chain elongation fermentation can be used to convert organic residues into biobased chemicals. This research aimed to develop a bioprocess for branched medium chain fatty acids (MCFAs) production. A long-term continuous reactor experiment showed that isocaproate (4-methyl pentanoate, $\mathrm{i}-\mathrm{C}_{6}$ ) can be produced via ethanol based chain elongation. The enriched microbiome formed iso-caproate from iso-butyrate at a rate of $44 \pm 6 \mathrm{mmol}$ $\mathrm{Cl}^{-1}$ day $^{-1}$ during the last phase. This amounted to $20 \%$ of all formed compounds based on carbon atoms. The main fermentation product was $n$-caproate ( $55 \%$ of all carbon), as a result of acetate and subsequent n-butyrate elongation. The microbiome preferred straight-chain elongation over branched-chain elongation. Lowering the acetate concentration in the influent led to an increase of excessive ethanol oxidation (EEO) into electron equivalents (e.g. $\mathrm{H}_{2}$ ) and acetate. The formed acetate in turn stimulated straight chain elongation, but the resulting lower nett acetate supply rate towards straight chain elongation led to an increased selectivity towards and productivity of $\mathrm{i}-\mathrm{C}_{6}$. The electrons produced via oxidation routes and chain elongation were apparently utilized by hydrogenotrophic methanogens, homoacetogens and carboxylate-to-alcohol reducing bacteria. Further improvements could be achieved if the acetate-producing EEO was minimized and limitations of ethanol and $\mathrm{CO}_{2}$ were prevented.

Keywords: Chain elongation, Open-culture, Fermentation, isobutyrate, isocaproate, ethanol, n-caproate 


\section{Introduction}

Sustainable alternative technologies need to be developed to transform the linear fossilbased economy into a circular economy ${ }^{183}$. Biomass residues, organic waste streams, and gaseous $\mathrm{CO} 2$ streams offer microbial convertible feedstocks for procuring new biochemicals. The carboxylate platform is regarded as a potent tool that can supply alternatives to some of the still widely used fossil-derived products ${ }^{25}, 184$. One emerging bioprocess is the elongation of short chain fatty acids (SCFA, carbon length $C_{1}-C_{5}$ ) into medium chain fatty acids (MCFA, carbon length $\mathrm{C}_{6}-\mathrm{C}_{12}$ ) via microbial chain elongation ${ }^{185}$. It provides the means to catalyze the conversion of complex organic residues into a broad range of useful chemicals ${ }^{23}, 186,187$. MCFAs as potential new platform chemicals can be used as feedstocks for the fuel industry, chemicals for antimicrobial agents, additives in animal feed ${ }^{188}$, flavor additives, lubricants and plasticizers ${ }^{22}$. The global market size for MCFAs shows a growing trend and was predicted to grow from 5.32 billion USD in 2014 to 8 billion USD by the end of $2023^{189}$. Of all MCFAs, $\mathrm{C}_{6}$ has by far the lowest availability in traditional resources ( $C_{6}$ constitutes around $2 \%$ of the total fatty acids in palm and kernel oil ${ }^{190}$.) and thus its scarcity provides a window of opportunity for alternative production by microbial chain elongation. A recent research effort has led to the implementation of chain elongation technology on a large scale in the Netherlands. ChainCraft B.V. is building the first largescale demonstration plant in Amsterdam that will produce MCFAs from organic waste streams via a multi-step fermentation process at a few metric tons per year ${ }^{26}$.

During chain elongation microbes that employ reverse beta-oxidation use an electron donor such as ethanol, to elongate SCFAs to MCFAs ${ }^{22,191}$. The elongation is always executed with two carbon atoms via an acetyl-CoA condensation step. Besides for the production of $n-$ caproate $\left(n-C_{6}\right)$, the microbes can also be used to produce n-valerate ( $\left.n-C_{5}\right)$, n-heptanoate $\left(n-C_{7}\right)$ and $n$-caprylate $\left(n-C_{8}\right)^{192}, 193$. To date, reports have mainly mentioned straightchained ( $n$-forms) MCFAs production via open culture chain elongation with the focus on $\mathrm{n}-\mathrm{C}_{6}$ as dominant product, whereas branched chained (iso-forms) MCFAs have been reported to be produced in low amounts only ${ }^{40}$. Moreover, in the processes studied it was not clear if $\mathrm{i}-\mathrm{C}_{6}$ had been produced via either chain elongation or protein degradation. So far the only known iso- $\mathrm{C}_{6}$ bioprocess has been created while using a pure culture of genetically-modified $E$. coli, which converted glucose to $\mathrm{i}-\mathrm{C}_{6}$ at a high titer of 13 grams per liter $^{194}$.

Branched fatty acids have various interesting applications because of their different physical properties compared to straight forms. These properties include a lower boiling point, higher viscosity, a reducing effect on crystallization ${ }^{36}$ and higher oxidative stability ${ }^{37}$, 38. For example, branched MCFAs can be added in the substrate mix for Kolbe electrolysis ${ }^{24,}$ 195 to acquire fuels that have an increased octane number ${ }^{196}$. Successful microbial 
production of branched MCFAs would greatly expand the application spectrum of chain elongation technologies.

Protein-rich waste streams are an attractive substrate for potential branched chain elongation. Anaerobic degradation of protein-rich waste leads to formation of branched fatty acids, such as iso-butyrate, iso-valerate and iso-caproate ${ }^{168,197}$. Valine, leucine and iso-leucine are substrates for branched carboxylic acids formation in this fashion, and represent a small but significant fraction of protein mass in organic residues ${ }^{198}$. Moreover, (de-)isomerization - of $n /$ iso-butyrate in particular - is stimulated in some acidifications ${ }^{199}$ that are associated with methanogenesis 20091,94 , which leads to temporarily increased isobutyrate titers. Alternatively, as recently shown $\mathrm{i}-\mathrm{C}_{4}$ feedstock can be obtained from organic residues via a mixed culture methanol chain elongation bioprocess that simultaneously stimulates isomerization ${ }^{35}$. Such a precursor could eventually be used to produce $\mathrm{i}-\mathrm{C}_{6}$ from any type of fermentable organic residue.

Chain elongation of iso-butyrate ( $\left.\mathrm{i}-\mathrm{C}_{4}\right)$ with various electron donors including ethanol was attempted via batch experiments, but did not show iso-caproate (i- $C_{6}$ ) formation ${ }^{133}$. Theoretically, though, it should be possible to form i- $\mathrm{C}_{6}$ via $\mathrm{i}-\mathrm{C}_{4}$ elongation with ethanol, assuming the enzymes within the microbiome have affinity for branched carboxylic acids. The objective of this study was to develop a chain elongation bioreactor system capable of producing branched MCFAs. A continuously fed bioreactor was used to enrich an i- $\mathrm{C}_{6}$ producing community. As starting microbiome, biomass from an ethanol chain elongation reactor that produced $\mathrm{n}_{-} \mathrm{C}_{6}{ }^{201}$ was used, as well as biomass from a methanol chain elongation reactor that produced $\mathrm{i}-\mathrm{C}_{4}{ }^{35}$. Within the enriched microbiome $\mathrm{i}-\mathrm{C}_{4}$ was successfully elongated to i- $\mathrm{C}_{6}$ (4-methyl pentanoate). 


\section{Material and Methods}

This study aimed to develop a chain elongation bioreactor system capable to produce branched MCFAs. The experiment was divided into six phases to investigate the effect on iso-caproate production of iso-butyrate, methanol, acetate and vitamin $\mathrm{B}_{12}$ Cobalamin. During each of these six phases the medium composition was changed, as shown in Table 1. The reactor system was characterized by analysis of the steady state mass balances for the total amount of carbon and electrons in the liquid and gas phase.

\section{Reactor set-up and operation}

The experiment was performed in a lab-scale continuous up-flow anaerobic reactor (schematic depiction shown in Figure S1) with a height of $35 \mathrm{~cm}$ and an internal column diameter of $6.5 \mathrm{~cm}$ (working liquid volume of $1 \mathrm{I}$ with a headspace of $0.15 \mathrm{I}$ ). The medium inflow was set at $25 \mathrm{ml} \mathrm{hour}^{-1}$. This flow led to a hydraulic retention time (HRT) of approximately $40 \pm 2$ hours, which, at the start of the experiment when no biofilms were observed, was sufficient for growth and accumulation of chain elongating bacteria192, 202, while low enough to provide a selective pressure against dominant growth of suspended methanogens ${ }^{90}$. The liquid phase was partially mixed by internal recirculation $(150 \mathrm{ml}$ minute $^{-1}$ ) with a pump (Watson-Marlow $® 323 \mathrm{Dz}$, UK). Biofilms were allowed to grow in the reactor, which then decoupled the HRT from the SRT. In the first five phases biomass growth was mainly suspended, while a thin biofilm grew on the wall of the reactor. In phase VI black granular biofilms developed and started to accumulate in the recirculation tubes and at the bottom of the reactor. The temperature was kept constant at $35^{\circ} \mathrm{C}$, using a water jacket and water bath (Fisher Scientific Polystat 37, USA). The pH was controlled ( $\mathrm{pH} 6.5 \pm 0.1$ ) to maintain a suitable $\mathrm{pH}$ for chain elongation ${ }^{192,203}$ by automatic titration using $1 \mathrm{M} \mathrm{KOH}$ as base. $\mathrm{CO}_{2}$ was continuously added (at a rate of $240 \mathrm{mln}^{-1}$ day ${ }^{-1}$ with the aim of maintaining a sufficient ( $>1 \mathrm{kPa}$ ) $\mathrm{CO}_{2}$ partial pressure needed for growth of ethanol chain elongating bacteria 204,205 .

\section{Inoculum}

The reactor was inoculated with a mixture of two undefined anaerobic cultures that originated $(1)^{201}$ from an ethanol based chain elongation reactor and $(2)^{35}$ from a reactor performing methanol based chain elongation to produce iso-butyrate.

\section{Medium}

The synthetic growth medium contained the following macro nutrients $\left(\mathrm{g} \mathrm{l}^{-1}\right): \mathrm{NH}_{4} \mathrm{H}_{2} \mathrm{PO}_{4}$ 3.60; $\mathrm{MgCl}_{2} \cdot 6 \mathrm{H}_{2} \mathrm{O} 0.33 ; \mathrm{MgSO}_{4} \cdot 7 \mathrm{H}_{2} \mathrm{O} 0.20 ; \mathrm{CaCl}_{2} \cdot 2 \mathrm{H}_{2} \mathrm{O} 0.20 ; \mathrm{KCl} 0.20$. In addition, the micro nutrients (Pfennig trace metals and B-vitamins) of the designed basal medium described in Phillips et al. (1993) ${ }^{206}$ were used. The B-vitamin composition of this medium 
differed in phases I, II, IV, V and VI. The vitamin $\mathrm{B}_{12}$ cobalamin concentration in these phases was 30 times higher than in phase III ( $0.375 \mathrm{mg} \mathrm{l}^{-1}$ compared to $\left.0.0125 \mathrm{mg} \mathrm{l}^{-1}\right)$. The carbon sources were acetate, methanol, ethanol, n-butyrate and i-butyrate. The composition of the carbon sources during each of the six phases changed as shown in Table 1. The reason for the shifts in vitamin $B_{12}$ concentration and the addition/removal of methanol are given in the Supplementary Information section.

Table 1. The overview shows changes of the influent carbon source compositions (mM C), the steady state period per phase and the acquired values for $\mathrm{pH}, \mathrm{HRT}$ and $\mathrm{C} / \mathrm{e}$-balances. During phases III and IV there was also a change in vitamin $B_{12}$ concentration.

\begin{tabular}{|c|c|c|c|c|c|c|c|}
\hline & & Phase I & Phase II & Phase III & Phase IV & Phase V & Phase VI \\
\hline \multirow{5}{*}{ Influent } & acetate & 150 & 150 & 150 & 150 & 150 & 14 \\
\hline & $\mathrm{n}-\mathrm{C}_{4}$ & 136 & 272 & $\mathbf{0}$ & - & - & - \\
\hline & $\mathrm{i}-\mathrm{C}_{4}$ & 136 & $\mathbf{0}$ & 272 & 272 & 272 & 272 \\
\hline & $\mathrm{MeOH}$ & 94 & 94 & 94 & 94 & $\mathbf{0}$ & 0 \\
\hline & $\mathrm{EtOH}$ & 369 & 369 & 369 & 369 & 369 & 369 \\
\hline Description & & $\begin{array}{l}\mathrm{i}-\mathrm{C}_{4} / \mathrm{n}-\mathrm{C}_{4} \\
\text { ratio: } 1 / 1\end{array}$ & $\begin{array}{c}\mathrm{i}-\mathrm{C}_{4} \text { was } \\
\text { replaced by } \\
\mathrm{n}-\mathrm{C}_{4}\end{array}$ & $\begin{array}{l}\mathrm{n}-\mathrm{C}_{4} \text { was } \\
\text { replaced by } \\
\mathrm{i}-\mathrm{C}_{4} \& \text { vit } \\
\mathrm{B}_{12} \text { from } \\
375 \mu \mathrm{g} \mathrm{I}^{-1} \text { to } \\
12.5 \mathrm{~g} \mathrm{I}^{-1}\end{array}$ & $\begin{array}{c}\text { vit } B_{12} \text { from } \\
12.5 \mu \mathrm{g} \mathrm{I}^{-1} \\
\text { to } 375 \mu \mathrm{g} \mathrm{I}_{1}^{-}\end{array}$ & $\begin{array}{l}\text { removed } \\
\text { methanol } \\
\text { from feed }\end{array}$ & $\begin{array}{c}\text { lowered } \\
\text { acetate } \\
\text { concentrati } \\
\text { on }\end{array}$ \\
\hline $\begin{array}{l}\text { Phase } \\
\text { period } \\
\text { Steady } \\
\text { state } \\
\text { period } \\
\end{array}$ & & $\begin{array}{c}\text { day } 1 \text { to } 38 \\
\text { day } 16 \text { to } \\
36\end{array}$ & $\begin{array}{c}\text { day } 38 \text { to } \\
112 \\
\text { day } 52 \text { to } \\
65\end{array}$ & $\begin{array}{c}\text { day } 112 \text { to } \\
190 \\
\text { day } 157 \text { to } \\
181\end{array}$ & $\begin{array}{c}\text { day } 190 \text { to } \\
262 \\
\text { day } 225 \text { to } \\
254\end{array}$ & $\begin{array}{c}\text { day } 262 \text { to } \\
297 \\
\text { day } 269 \text { to } \\
290\end{array}$ & $\begin{array}{c}\text { day } 297 \text { to } \\
388 \\
\text { day } 330 \text { to } \\
358\end{array}$ \\
\hline $\mathrm{pH}$ & & $6.40 \pm 0.09$ & $6.60 \pm 0.09$ & $6.50 \pm 0.06$ & $6.47 \pm 0.06$ & $6.46 \pm 0.04$ & $6.50 \pm 0.05$ \\
\hline $\begin{array}{l}\text { HRT } \\
\text { (hours) }\end{array}$ & & $42.6 \pm 2.2$ & $42.1 \pm 2.8$ & $40.2 \pm 1.2$ & $41.59 \pm 0.6$ & $43.1 \pm 0.8$ & $39.1 \pm 0.6$ \\
\hline C-balance & & $97 \pm 2 \%$ & $90 \pm 4 \%$ & $100 \pm 2 \%$ & $103 \pm 1 \%$ & $105 \pm 2 \%$ & $100 \pm 2 \%$ \\
\hline e-balance & & $93 \pm 2 \%$ & $87 \pm 4 \%$ & $97 \pm 2 \%$ & $100 \pm 1 \%$ & $102 \pm 2 \%$ & $96 \pm 1 \%$ \\
\hline
\end{tabular}

\section{Sampling and measurement}

The reactor was sampled approximately three times per week. Liquid samples were stored at $-20^{\circ}$ and analyzed once every two weeks using gas chromatography according to the procedure described by Jourdin $(2018)^{207}$. This method allowed the quantification of volatile fatty acids from $n-C_{2}$ to $n-C_{8}, i-C_{4}, b-C_{5}$ (both 2- and 3-methylbutanoic acid have the same retention time) and $\mathrm{i}-\mathrm{C}_{6}$ (4-methyl-pentanoic acid). The $\mathrm{i}-\mathrm{C}_{6}$ isoform, 4-methyl-pentanoic acid, has a different retention time than 2-methyl-pentanoic acid, as is shown in Figure S2. In addition to the volatile fatty acids, the alcohols methanol to hexanol, including isobutanol and iso-hexanol could be quantified. Prior to injection, samples were acidified in a final concentration of $1.5 \%$ wt formic acid. 
The headspace gas was analyzed every time a liquid sample was taken. The concentrations of $\mathrm{O}_{2}, \mathrm{~N}_{2}, \mathrm{CH}_{4}, \mathrm{H}_{2}$, and $\mathrm{CO}_{2}$ were determined via gas chromatography using $2 \mathrm{GC}$ systems with a standardized method 45,185 . The headspace was connected to a gas meter ( $\mu$ Flow Bioprocess Control, Sweden) to measure gas production. In this internal recirculation loop the turbidity was continuously measured to indicate the suspended biomass concentration.

\section{Steady state characterization}

During all six phases the reactor was assumed to be in a steady state when it met the following criteria: (i) the reactor had been operating at the same conditions for at least 5 times the HRT value and (ii) the main metabolite concentrations and corresponding conversion rates were relatively constant. The second criteria was defined as when the confidence interval was less than $20 \%$ of the steady state average values. Confidence intervals were determined with an a of 0.01 and are indicated with $a \pm$.

\section{Calculation for excessive ethanol oxidation}

There are various competing excessive ethanol oxidation (EEO) routes that lower the efficiency of ethanol usage in chain elongation processes (see Table S1). Moreover, ethanol oxidation yields acetate that directly causes a favorable selective pressure towards straight chain elongation, which competes with branched chain elongation. The percentage of ethanol that was not used for chain elongation could be quantified via stoichiometric analysis of chain elongation activity (using the measured formation of $n-C_{4}, n-C_{4} O H, n / i-C_{6}$, $\mathrm{n} / \mathrm{i}-\mathrm{C}_{6} \mathrm{OH}$ and $\mathrm{n}-\mathrm{C}_{8}$ ) and the measured ethanol consumption. The stoichiometry of chain elongation was generalized as reverse beta-oxidation combined with ethanol oxidation coupled in a $5(n=5)$ to $1(p=1)$, or $4(n=4)$ to $1(p=1)$ ratio (reaction 1 for acetate and 2 for longer carboxylates) $)^{22}$ :

$$
\begin{aligned}
& \left(n_{\mathrm{C} 2}+p\right) \mathrm{CH}_{3} \mathrm{CH}_{2} \mathrm{OH}+\left(n_{\mathrm{C} 2}-p\right) \mathrm{CH}_{3} \mathrm{COO}^{-} \rightarrow n_{\mathrm{C} 2} \mathrm{C}_{3} \mathrm{H}_{7} \mathrm{COO}^{-}+2 \mathrm{pH}_{2}+\left(n_{\mathrm{C} 2}-p\right) \mathrm{H}_{2} \mathrm{O}+p \mathrm{H}^{+} \\
& \left(n_{\mathrm{Ci}}+p\right) \mathrm{CH}_{3} \mathrm{CH}_{2} \mathrm{OH}+n_{\mathrm{Ci}} \mathrm{C}_{x} \mathrm{H}_{2 x+1} \mathrm{COO}^{-} \rightarrow n_{\mathrm{Ci}} \mathrm{C}_{x+2} \mathrm{H}_{2(x+2)+1} \mathrm{COO}^{-}+2 p \mathrm{H}_{2}+\left(n_{\mathrm{Ci}}-p\right) \mathrm{H}_{2} \mathrm{O}+p \mathrm{H}^{+}+p \mathrm{CH}_{3} \mathrm{COO}^{-}
\end{aligned}
$$

EEO as a percentage of total ethanol consumption was calculated as follows :

$$
E E O(\%)=\left(1-\frac{\sum \text { Chain_elongation_activity }}{\text { Observed_ethanol_consumption }}\right) \bullet 100
$$

where $\Sigma$ Chain_elongation_activity is the amount of ethanol necessary to perform all

observed chain elongation and as such

$$
n=\sum_{C i=C 2, n-C 4, i-C 4, e t c} n_{C i} \text {. }
$$


Furthermore, for the calculation the following assumptions were made:

- Consumed methanol was used for methanol based chain elongation of acetate to butyrate (reaction 9 in Table S1).

- EEO was calculated using two different stoichiometries for chain elongation (i) where $n=5$ and $p=1$ and (ii) where $n=4$ and $p=1$. For simplicity it was assumed that elongation of externally added carboxylates, besides acetate, occurs with similar stoichiometry. The letter $n$ stands for the amount of ethanol used for the summed chain elongation activity and $p$ stand for the amount of ethanol used for substrate level phosphorylation and acetate $+\mathrm{H}_{2}$ formation. Both ratios were used because thermodynamic calculations of the chain elongation reaction (Figure S5) showed that the reaction Gibbs free energy was not always sufficient for generation of 2.5 ATP22.

- If $\beta$-oxidation was occurring, then this will cause a net increase of $\operatorname{EEO}(\%)$. Chain elongation (reaction 1 ) followed by five times $\beta$-oxidation (reaction 5 in Table S1) yields the same stoichiometric outcome as six times direct ethanol oxidation (reaction 2 in Table S1). Thereby potential $\beta$-oxidation activity is incorporated within this calculation.

\section{Microbial community analysis}

Biomass samples during the steady states in phase I, II, IV and VI ( for VI both suspended biomass and granules) were used for 16S rRNA gene amplicon analysis to determine the microbial compositions. The biomass samples were taken, in duplicate, by spinning down $\sim 20 \mathrm{ml}$ (for suspended growth) and $\sim 1 \mathrm{ml}$ (wet granular biomass) in $2 \mathrm{ml}$ Eppendorf centrifuge tubes for 5 minutes at $9300 \mathrm{RCF}$, snap freezing the pellets using liquid nitrogen and storing the frozen pellets at $-80^{\circ} \mathrm{C}$ until DNA extraction. The samples taken in duplicate were then analyzed separately.

DNA was extracted from the pellets applying a Powersoil DNA isolation kit, according to the instruction manual. The isolated DNA was used as template for amplifying the V3-V4 region of 16S rRNA gene via Illumia sequencing using the primer sets described by Takahashi et $\mathrm{a}^{98}$. This allowed simultaneous amplification of the both bacterial and archaean 16S rRNA gene regions. The 16S rRNA gene amplicon analysis and subsequent taxonomic analysis was performed using QIIME software version 1.9.99, 101, 208, 209. (For more details see supplementary information.) This bioinformatics process was performed on the December 31, 2017.

From the acquired data a heat map was made using Microsoft Excel. Open source software Rstudio v3.5.0 was used to sort the data and create quantitative OTU tables that belonged 
to the chosen taxonomic group. This allowed counting the most abundant OTU's that were classified within a single genus. The rDNA sequences of selected abundant OTUs were then used for Megablast to search within the NCBI nucleotide database on September 27, 2018.

It is important to emphasize that the performed 16s rRNA gene amplicon analysis limits the microbial community analysis to taxonomic and phylogenetic determination. Functional roles within the microbiome cannot be attributed to the observed taxa using this data. Instead the microbial community analysis was used to link the stoichiometric analysis of the experiment to existing literature. Moreover, the relative abundances estimated by this NGS method are an indication but should not be used for quantitative analyses.

\section{Results and Discussion}

\section{Elongation of iso-butyrate with ethanol to iso-caproate}

A reactor system with an ethanol based chain elongation microbiome was developed that formed iso-caproate ( $\mathrm{i}-\mathrm{C}_{6}$ ) continuously for 250 days (Figure 1). The highest volumetric production rate was $44 \pm 6 \mathrm{mmol} \mathrm{Cl}^{-1}$ day $^{-1}\left(0.86 \pm 0.1 \mathrm{~g} \mathrm{l}^{-1}\right.$ day $\left.^{-1}\right)$ during the last phase of operation with a broth concentration of $73 \pm 7 \mathrm{mM} \mathrm{C}$ or $1.4 \pm 0.1 \mathrm{~g} \mathrm{l}^{-1}$. This amounted to $20 \%$ of all the formed compounds based on carbon atoms. The complete concentration profiles of all measured carbon compounds, as well as the headspace gas pressures, are shown in Figure 1. From these profiles it can be seen that the presence of $\mathrm{i}-\mathrm{C}_{4}$ steers towards $\mathrm{i}-\mathrm{C}_{6}$ formation.

The measured $\mathrm{C}_{6}$ isoform, 4-methyl pentanoate, matches the predicted compound for $\mathrm{i}-\mathrm{C}_{4}$ elongation. According to the described mechanism for ethanol based chain elongation ${ }^{22}$, the carboxylates are always elongated with two carbon chain units via acetyl-CoA condensation. In the case of ethanol based chain elongation this suggests that the first step is a thiolase driven catalysis in which the alkyl-transfer reaction allows for $\mathrm{i}-\mathrm{C}_{4}$ to covalently bind one of the cysteines in the catalytic site. Next, a Claisen-type condensation reaction adds the subsequent acetyl- $\mathrm{CoA}^{33,34}$. For $\mathrm{i}-\mathrm{C}_{4}$ elongation, reduction of the acquired 3-keto-4-methyl-pentanoyl-CoA should then occur via 3-hydroxy-4-methyl-pentanoyl-CoA, 4-methyl-2-pentenoyl-CoA to 4-methyl-pentanoate, as shown in Figures S3 and S4. 


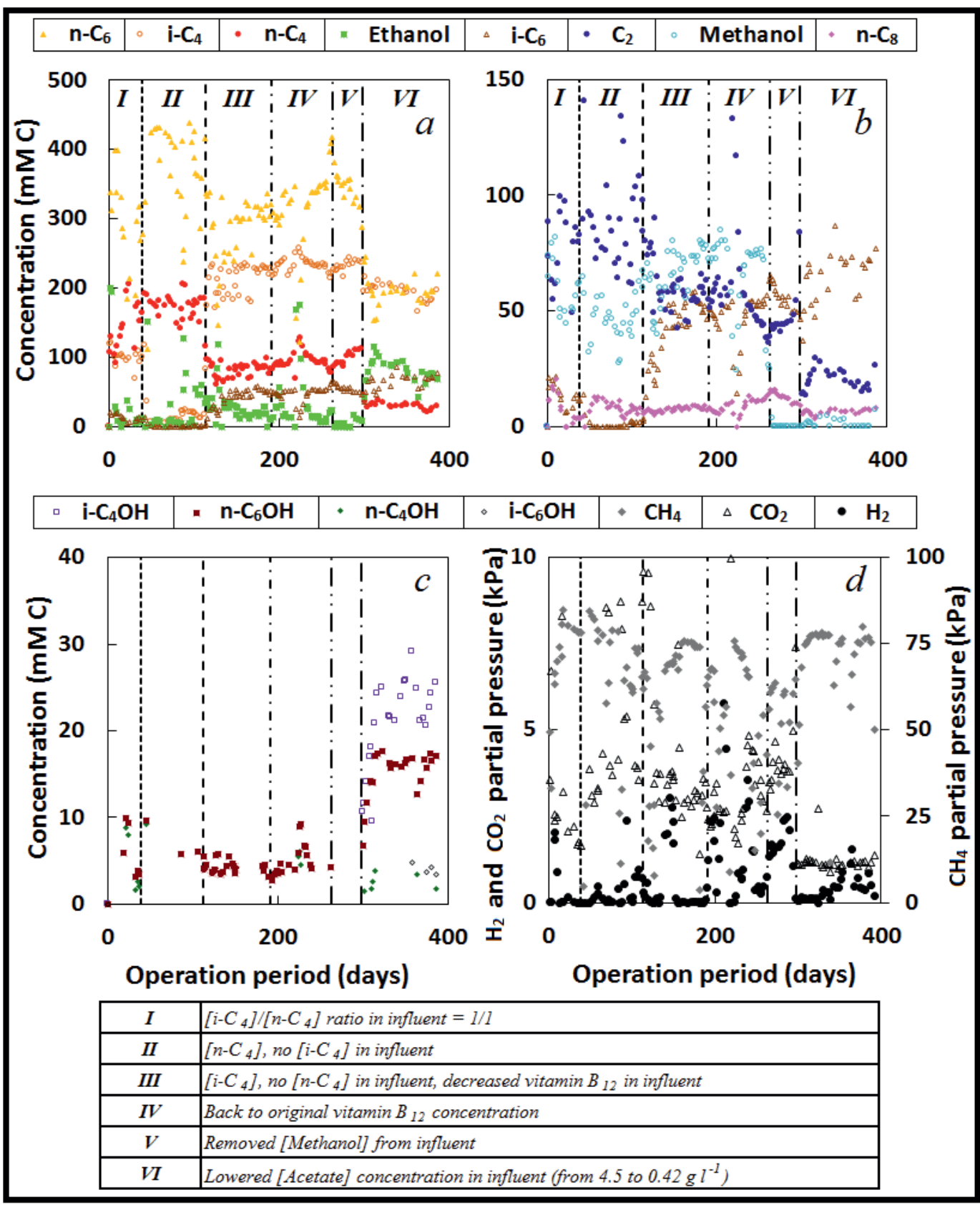

Figure 1. Graph $a, b$ and $c$ show the reactor broth concentrations ( $\mathrm{mM} \mathrm{C}$ ) of the soluble compounds throughout the six operation phases. Carboxylic acids are indicated according to their carbon number and straight ( $\mathrm{n}-)$ or branched ( $\mathrm{i}-)$ form. Alcohols are indicated by name or the $\mathrm{OH}$ suffix. Graph $d$ shows the headspace gas partial pressures throughout the phases, excluding nitrogen gas and oxygen (which was kept under $1 \%$ ). 
During start up, in phase I when both $\mathrm{n}-\mathrm{C}_{4}$ and $\mathrm{i}-\mathrm{C}_{4}$ were fed into the system, only small amounts of $\mathrm{i}-\mathrm{C}_{6}$ were formed $\left(6 \pm 2 \mathrm{mmol} \mathrm{Cl}^{-1}\right.$ day $\left.^{-1}\right)$. Mainly straight $(\mathrm{n}-) \mathrm{C}_{6}$ was formed. The $\mathrm{i}-\mathrm{C}_{6}$ formation stopped completely in the following phase (II), when $\mathrm{i}-\mathrm{C}_{4}$ was removed from the influent and replaced by the same amount of $n-\mathrm{C}_{4}$. In the phase thereafter (III) the $\mathrm{n}-\mathrm{C}_{4}$ was replaced by $\mathrm{i}-\mathrm{C}_{4}$ and branched $(\mathrm{i}-) \mathrm{C}_{6}$ was formed again. In Table $\mathrm{S} 2$ the molar ratios of $\mathrm{i}-\mathrm{C}_{4}$-derived production (e.g. the formation of $\mathrm{i}-\mathrm{C}_{4} \mathrm{OH}, \mathrm{i}-\mathrm{C}_{6}, \mathrm{i}-\mathrm{C}_{6} \mathrm{OH}$ ) and $\mathrm{i}-\mathrm{C}_{4}$ consumption are given for each steady state during the phases. In phases III, IV, $\mathrm{V}$ and VI these ratio were between $98 \%$ and $112 \%$. The fact that $\mathrm{i}-\mathrm{C}_{6}$ formation was equivalent to $\mathrm{i}-\mathrm{C}_{4}$ consumption further supports the proposed mechanism for ethanol based $\mathrm{i}-\mathrm{C}_{4}$ elongation and suggests that in these phases no de-isomerization took place.

\section{Iso-caproate formation without elevated $B_{12}$ or methanol}

As earlier work on $\mathrm{i}-\mathrm{C}_{4}$ formation indicated the necessity for methanol and high vitamin $\mathrm{B}_{12}$ concentrations ${ }^{35}$, the reactor system was started with similar amounts in the influent. The vitamin $B_{12}$ concentration was lowered (phase III) and methanol was removed from the influent (phase $V$ ). Figure $\mathbf{1 b}$ shows that lowering the $B_{12}$ concentration and removing methanol from the influent did not affect $\mathrm{i}-\mathrm{C}_{6}$ formation.

\section{Straight chained MCFA $n-C_{6}$ formation was preferred over i-C 6 formation}

In Figure 2 the volumetric conversion rates show that throughout the six phases $n-C_{6}$ was the most abundantly formed compound. When $\mathrm{n}-\mathrm{C}_{4}$ and $\mathrm{i}-\mathrm{C}_{4}$ were present in equal ratios (phase I), there was 26 times as much $n-\mathrm{C}_{6}$ formation than $\mathrm{i}-\mathrm{C}_{6}$ formation $\left(0.038 \mathrm{i}-\mathrm{C}_{6}\right.$ per $\mathrm{n}-\mathrm{C}_{6}$ ). Even when no $\mathrm{n}-\mathrm{C}_{4}$ was fed (from phase III onwards) the major part of the formed MCFAs was $n-C_{6}$. Formation of this $n-C_{6}$ could be attributed to regular chain elongation of $C_{2}$ via $n-C_{4}$ to $n-C_{6}$. There is a preference for $C_{2}$ and straight- $C_{4}$ over branched- $C_{4}$ during ethanol based chain elongation. Straight chain elongation should thus be prevented to develop a more selective branched MCFA bioprocess.

In the final phase (VI) the effect of a lowered acetate concentration in the influent (reduction from $4.5 \mathrm{~g} \mathrm{l}^{-1}$ to $0.42 \mathrm{~g} \mathrm{l}^{-1}$ ) was studied with the aim to prevent $\mathrm{n}-\mathrm{C}_{6}$ formation via $\mathrm{C}_{2}$ and to increase $\mathrm{i}-\mathrm{C}_{6}$ formation. However, it was observed that direct (excessive) ethanol oxidation (EEO) was stimulated which resulted into the formation of acetate and subsequent elongation to $n-C_{4}$ and $n-C_{6}$. Yet, less $n-C_{6}$ was formed than at the high acetate load during preceding phases. Still, the ratio of $i-C_{6}$ per $n-C_{6}$ in the final phase turned out the be the highest ever achieved in this system ( $0.4 \mathrm{i}-\mathrm{C}_{6}$ per $\left.1 \mathrm{n}-\mathrm{C}_{6}\right)$. 


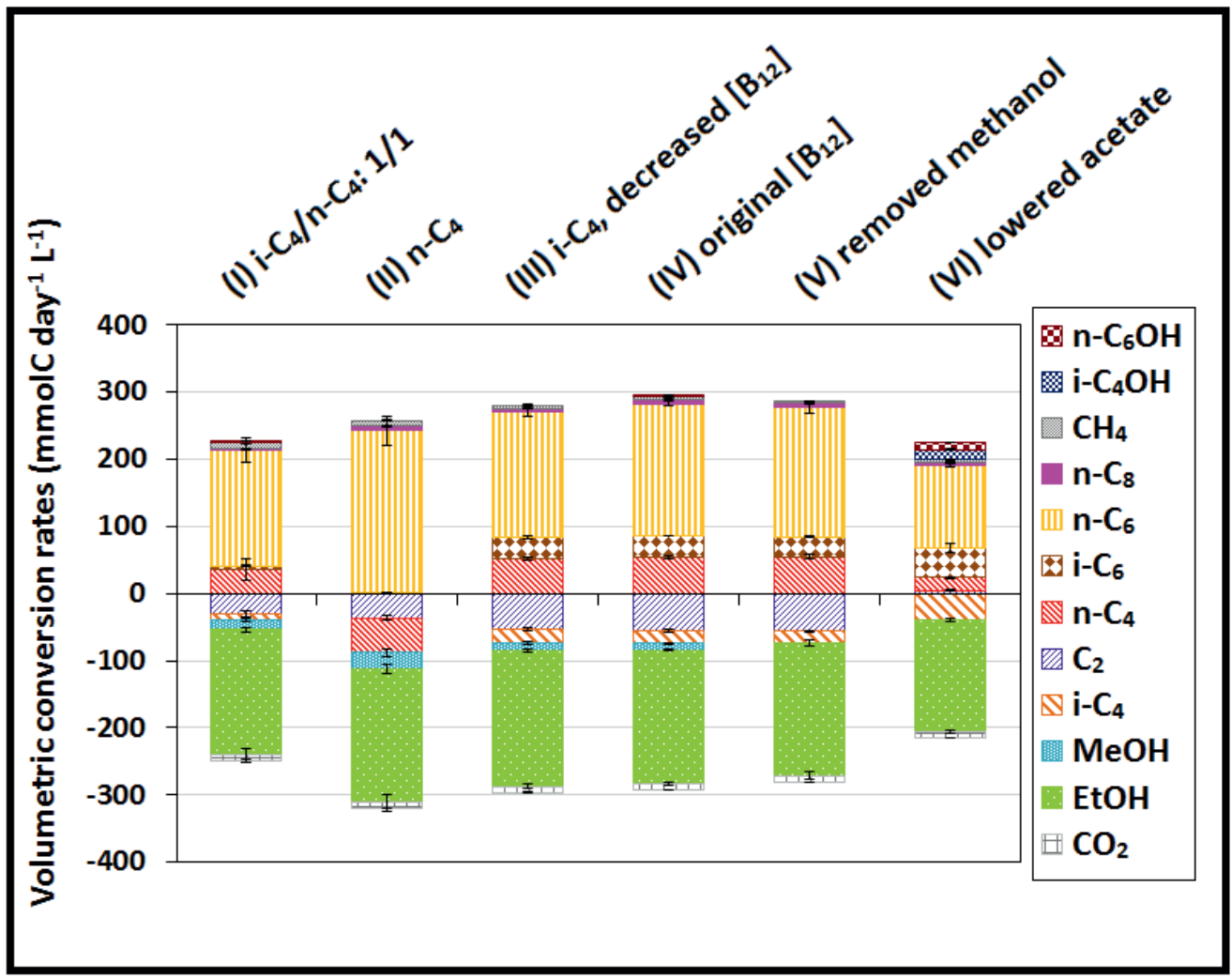

Figure 2. The figure shows an overview of the net volumetric conversion rates, averaged over the steady state periods with corresponding confidence intervals. The phases are indicated by a roman numeral and a designation at the top. Consumption of compounds is indicated as negative volumetric conversion, while formation is indicated with a positive number. Values of conversion rates and steady state concentrations are shown in Table S3 in the supplementary information.

\section{Excessive ethanol oxidation and acetate limitations}

The excessive ethanol oxidation in Figure $\mathbf{3}$ shows a decreasing trend during the first five phases. This coincides with a low ethanol broth concentration $\left(0\right.$ to $\left.0.5 \mathrm{~g} \mathrm{l}^{-1}\right)$ and a $\mathrm{C}_{2}$ broth concentration of 1.0 to $2.5 \mathrm{~g} \mathrm{l}^{-1}$. Moreover, when the EEO was near zero during phases IV and $\mathrm{V}$, the headspace hydrogen partial pressure $\left(\mathrm{p}_{\mathrm{H} 2}\right)$ was regularly above $1 \mathrm{kPa}$ (Figure 1d), during peaks around day 196, 210, 240 in phase IV and constantly above 1 kPa during phase $\mathrm{V}$. A pH2 higher than approximately $1 \mathrm{kPa}$ thermodynamically inhibits direct ethanol oxidation to acetate and $\mathrm{H}_{2}$, as is shown in Table $\mathrm{S} 1$. 


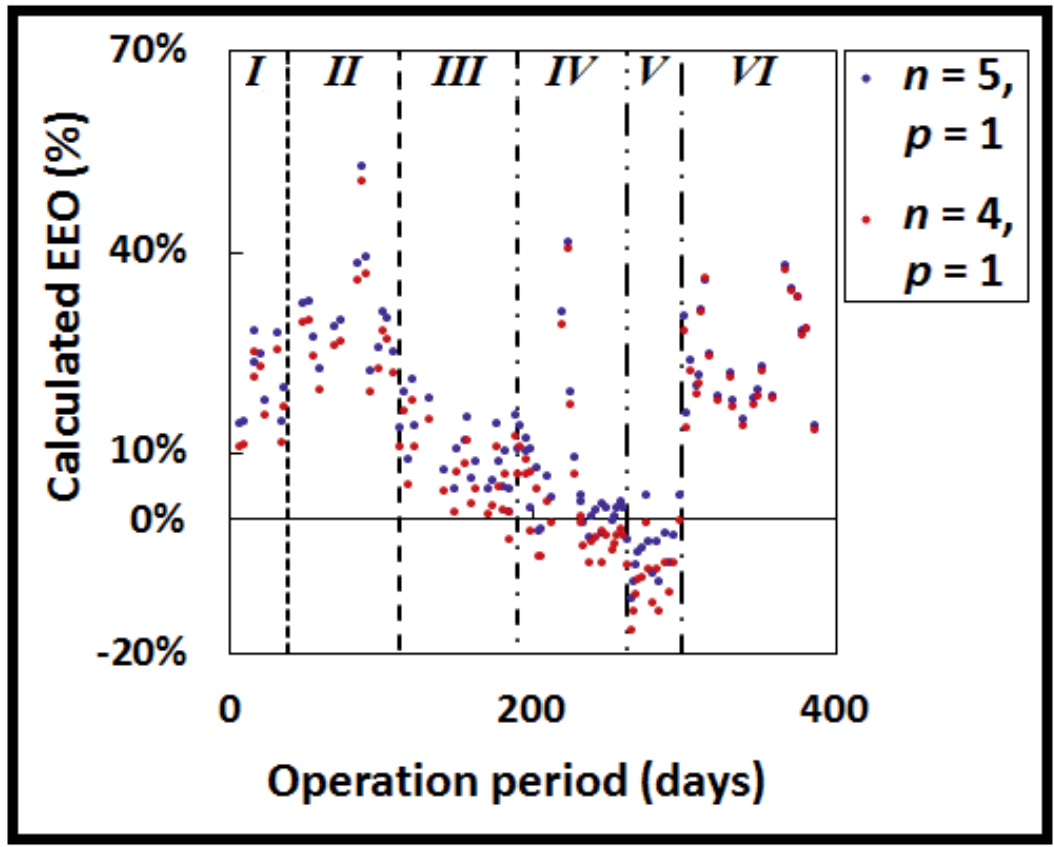

Figure 3. The percentage of ethanol consumption that is not used for chain elongation (EEO) is shown. Data points from measurements that did not fall in-between a $90 \%-110 \%$ carbon balance were omitted. The sudden increase of EEO during phase IV around day 217 occurred during a pump malfunction which resulted in a temporary high HRT. The graph shows the EEO in case of NADH driven reduction (Figure S3, $n=5$ and $p=1$ ) and NADPH driven reduction (Figure S4, $n=4$ and $p$ $=1$ ) of the 3-ketoalkanoyl-CoA intermediate. Note that the ratio $(n=5$ and $p=1)$ was derived for simplified chain elongation at high substrate concentrations ${ }^{22}$ and only holds in the scenario where solely acetate is supplied for elongation with ethanol. Adding extra - not internally produced carboxylates as substrate breaks the constraint put to this stoichiometric model and could result in $\Sigma n_{i}>5$.

The negative values in Figure 3 during phase IV and $V$ can be explained by: (i) chain elongation with a higher than 5 to 1 reverse beta-oxidation to ethanol oxidation ratio; (ii) utilization of electrons (i.e. hydrogen gas) to reduce acetate to ethanol leading to additional chain elongation ${ }^{210}$; (iii) additional SCFAs production due to biomass decay that had accumulated during the preceding days. (The averaged carbon balance in phase $\mathrm{V}$ was 105\%. See Table 1 in the Material and Methods section.)

In contrast, during the last phase (VI), when the acetate influent concentration was lowered to $0.42 \mathrm{~g} \mathrm{l}^{-1}$ the EEO increased significantly to $20-40 \%$ of the total ethanol consumption. Chain elongation became less efficient, as is shown by the higher amount of unconverted ethanol in the effluent $\left(2 \mathrm{~g} \mathrm{l}^{-1}\right)$ and lower $C_{6}$ production. Instead of $C_{2}$ being consumed, there was a net $C_{2}$ production which led to a $C_{2}$ broth concentration of 0.63 $\pm 0.06 \mathrm{~g} \mathrm{l}^{-1}$. The acetate for straight chain elongation had to come from ethanol oxidation, (niche-driven) $\beta$-oxidation or homoacetogenesis, the rates of which would determine 
acetate supply to straight chain elongating microbes. Although the $\mathrm{i}-\mathrm{C}_{4} / \mathrm{i}-\mathrm{C}_{6}$ elongation rate increased by a factor of 1.5 , this was insufficient to keep the ethanol in the previous 0 $0.5 \mathrm{~g} \mathrm{l}^{-1}$ concentration range. During this period the total $i-C_{6}$ formation was highest.

\section{From ethanol limited conditions to carbon dioxide (and acetate) limited conditions}

During the last phase (VI) the carbon dioxide partial pressure in the headspace was consistently around $1 \mathrm{kPa}$ (Figure 1d), whereas in previous phases it was in a higher range $(2-10 \mathrm{kPa})$. It is known that an increased $\mathrm{CO}_{2}$ supply (i.e. greater availability) does enhance the activity of hydrogenotrophic methanogens and EEO in chain elongation microbiomes ${ }^{211}$. In this case, however, it seemed that increased EEO led to more reducing equivalents that could be harvested with $\mathrm{CO}_{2}$ as electron acceptor, leading to a lower $\mathrm{CO}_{2}$ concentration.

The increased activity of methanogens (from $3.0 \pm 0.5$ to $4.8 \pm 1.2 \mathrm{mmol} \mathrm{Cl}^{-1}$ day $^{-1}$, Table S3) and possibly acetogens caused $\mathrm{CO}_{2}$ to reach limiting concentrations for chain elongation (Figure S6 shows the total dissolved carbon dioxide species) ${ }^{53}$. Moreover, the fact that in the last phase ethanol is no longer completely consumed suggests that the chain elongation microbes were inhibited by the low carbon dioxide concentrations, and possibly also by the low acetate concentrations. Additionally, a shift away from the confurcating metabolism of chain elongation bacteria (Figure S5) would lower the amount of internally produced acetate by stoichiometry. In this final phase branched chain elongation had - relative to straight chain elongation - a higher probability to occur than in the previous phase because more ethanol was present in conjunction with the high $\mathrm{i}-\mathrm{C}_{4}$ concentration, while there was less acetate supply, both by influent and by production of the microbiome itself.

\section{Concurrent methanogenesis, acetogenesis and carboxylate-to alcohol reduction}

In the scenario of $\mathrm{CO}_{2}$ limitation, excess reducing equivalent (e.g. hydrogen released during ethanol oxidation, $\beta$-oxidation and chain elongation) is present without sufficient bicarbonate for hydrogenotrophic methano- and/or acetogenesis. Therefore an alternative electron acceptor must be reduced ${ }^{212}$. The remaining electron equivalents were utilized by carboxylate reducers that performed biohydrogenation of fatty acids to alcohols ${ }^{213}$, or ethanol was consumed via direct carboxyl-hydroxyl exchange (reaction 14 in Table S1). By these processes, iso-butanol could be formed up to a concentration of $0.5 \mathrm{~g} \mathrm{l}^{-1}$ and $\mathrm{n}$ hexanol up to $0.3 \mathrm{~g} \mathrm{l}^{-1}$; also trace amounts of $\mathrm{n}$ - butanol and iso-hexanol were observed. This is the first prospective observation of branched carboxylic acid and n-caproate reduction at these levels during mixed culture chain elongation, without other electron donors being supplied besides ethanol (e.g. carbon monoxide or via biocathodes). 
Production rates of higher alcohols were similar to those of a chain elongation co-culture growing on hydrogen, carbon monoxide and carbon dioxide ${ }^{214}$. Besides the carboxylate-toalcohol reduction mechanism as energy yielding pathway, chain elongating bacteria could also be responsible for the observed n-butanol and n-hexanol formation throughout the operation period, where it acts as an additional electron sink $\mathrm{k}^{203,215}$.

Thermodynamic calculations of the carboxylate-to-alcohol reduction suggest that a high reactant to product ratio for hydroxyl-carboxyl exchange is necessary to maintain a $\Delta G$ lower than $-20 \mathrm{~kJ}$ reaction ${ }^{-1}$ (Table S1) when the hydrogen pressure is insufficient and $\mathrm{pH}$ too high to drive the reduction. During earlier phases (I and II) where EEO was also around $20 \%$ to $40 \%$, less carboxylate-to-alcohol reduction occurred. This could be explained by the too low reactant-to-product ratio in these phases and abundance of $\mathrm{CO}_{2}$ as external electron acceptor (Figure S6). Moreover, during phase VI small black granules were observed. The emergence of these granules, together with the microbiota data, suggests a syntrophic culture that created its own niche to facilitate otherwise thermodynamically unfeasible reactions (see Table S1). Close proximity of various microbes within a biofilm facilitates syntrophic interactions ${ }^{216}$. It is known that effective electron transfer (via hydrogen or Direct Interspecies Electron Transfer (DIET)) between ethanol (or $\beta$-) oxidizers and syntrophic partner organisms, such as methanogens, is feasible $217-219$. Therefore we can hypothesize that here the ethanol oxidation, or possibly beta-oxidation, was linked via reducing equivalent transfer (hydrogen, formate, or other) or DIET to methanogenesis, acetogenesis and/or carboxylate-to-alcohol reduction. 


\section{Microbial community analysis}

In Table 2 a heat map shows the relative abundances of orders within the microbiome for phases I, II, IV and VI. In Table S4 the heatmap is extended towards genus level. OTU counts and BLAST results are shown in Table S5 to S28. The black granules $(0.5$ to $1 \mathrm{~mm}$ diameter) that were formed during phase VI were also analyzed and are referred to as 'VI granule'.

Table 2. The heat map shows the relative abundance in percentage of the different orders within the microbial community. Data was acquired via Illumina sequencing the V3-V4 regions of the $16 \mathrm{~S}$ rRNA gene and OTU taxonomic classification. For each phase two rows are shown which represent the values for the duplicates.

$\begin{array}{llllllllll}\text { Order } & \text { I } & \text { I } & \text { II } & \text { II } & \text { IV } & \text { IV } & \text { VI } & \text { VI } & \begin{array}{l}\text { VI } \\ \text { granule }\end{array} \\ & & & & & & & & & \\ \text { granule }\end{array}$

The heat map shows that in all analyzed samples Clostridiales is the most abundant order, in which the most abundant OTU belonged to Clostridium kluyveri (100\% coverage and $98 \%$ similarity, Table S5 \& S6). C. kluyveri is a well described bacterium that performs the chain elongation. It is likely that in this case a strain of $C$. kluyveri is present that can perform the branched chain elongation. Hypothetically the elongation is executed using the same enzymes that are used for straight-chain elongation, albeit that these enzymes have different affinities for the metabolites involved in the branched-chain elongation. Alternatively a different microbe could be responsible for $\mathrm{iC}_{6}$ formation. By isolating and characterizing the responsible bacteria a definitive explanation could be given.

As mentioned earlier, the microbiome contains several other functional groups apart from chain elongation. Figure S7 shows a putative overview of possible metabolic activities of the presented microbiome. Some conversions were attributed to microorganisms that were 
identified within the community. Among these are bioprocesses such as beta-oxidation and direct ethanol oxidation ${ }^{220}$. These bioconversions form reducing equivalents, such as hydrogen gas that can be utilized via hydrogenotrophic methanogenesis, homoacetogenesis and carboxylate-to-alcohol reduction.

It should be noted that the reactor was not a sole ideally mixed system because some biofilms (in the form of wall growth and granules) were present. Niche formation, which is underlined by the granule formation in phase VI, increases the possibility for these syntrophic processes to occur. Biofilm growth decouples solids retention time from hydraulic retention time and allows microbes that grow slower than the reactor dilution rate to be maintained in the reactor. The $\beta$-oxidizers Syntrophomonadaceae in phase I had some similarity (100\% coverage, $96 \%$ similarity, Table S8) with Syntrophomonas zehnderi $O L-4$. These microbes are thermodynamically only able to oxidize fatty acids at a partial $\mathrm{H}_{2}$ pressure below $1 \mathrm{~Pa}$ (Table $\left.\mathrm{S} 1\right)^{115,217}$, although higher hydrogen partial pressures were measured. Due to local differences and fluctuations in the micro environment around the bacteria, thermodynamic calculations performed with macroscopic data (e.g. headspace partial pressures) should therefore be interpreted with a wider range of uncertainty. In phase VI the granules contained a syntrophic culture of $\beta$-oxidizing microbes within the Anaerolineaceae UCG-001 order and methane forming Methanoculleus palmolei (99\% coverage, 99\% similarity, Table S28, Methanomicrobiales order) ${ }^{105}, 221$.

The Methanoculleus genus has been reported to perform hydrogenotrophic methanogenesis in other mixed culture chain elongation microbiomes ${ }^{191,211}$. In contrast to Methanoculleus palmolei, that had a high presence within the granular biofilm, the Thermoplasmatales had a relatively low abundance in the granular biofilm compared to their abundance within the suspended biomass. The OTU classification of the Thermoplasmatales showed very low similarity to Methanomassiliicoccus luminyensis B10 (99\% coverage, $88 \%$ similarity, Candidatus methanogranum genus). On account of its low abundance in the granular biofilm compared to the abundance in suspension this unknown C. methanogranum is hypothesized to not be a syntrophic reduction partner. Instead, assuming it is a methane producing organism, it could be involved in either alcoholotrophic ${ }^{64}$ or acetoclastic methanogenesis (reaction 4 and 13 respectively in Table S1), which both are thermodynamically feasible independent on hydrogen partial pressure.

The observed Desufovibrio most likely work in conjunction with the Anaerolineaceaea ICG001 and Methanoculleus palmolei. Desulfovibrio are known to be able to perform $\beta$ oxidation in the absence of an extracellular electron acceptor 222,223 . The most abundant Desulfovibrio OTU was identified to resemble Desulfovibrio legallii H1 (95\% coverage and $99 \%$ similarity, Table S20), which is also known to be able to perform direct ethanol oxidation ${ }^{223}$. These type of sulfate reducing bacteria are versatile in bioelectrochemical 
processes and can donate or take up electrons from electrodes while converting various organics or $\mathrm{CO}_{2}$. Possibly the Desulfovibrionales were (partly) responsible for the usage of electrons or $\mathrm{H}_{2}$ while they were reducing $\mathrm{CO}_{2}$ to acetate and/or forming alcohols ${ }^{224}, 225$. In addition to these fermentation processes, the Desulfovibrio could also have been responsible for the black coloration of the granules by causing black precipitation of FeS after reducing the little amounts of present sulfate and iron(III) 226,227 . Some types of FeS compounds have been shown to enhance direct interspecies electron transfer ${ }^{219}$.

\section{Future outlook}

Here we report on iso-caproate bioformation via chain elongation. We also observed that higher alcohols such as iso-butanol, hexanol and iso-hexanol were formed. Branched alcohol formation has not been reported in earlier chain elongation research work. Higher branched alcohols could be interesting fermentation products because of their advantages over bioethanol such as higher energy density, lower hygroscopicity, lower vapor pressure, and compatibility with existing transportation infrastructures ${ }^{228}$. The developed mixed culture microbiome represents a complex mix of microbial processes with several possible competing and syntrophic interactions. The reactor system was not operated with conditions for maximal production rate and product titers. By increasing ethanol loads and preventing $\mathrm{CO}_{2}$ to become limiting for chain elongation higher MCFA production rates and concentrations can likely be achieved. In addition, a more stringent selective pressure could be achieved by lowering the hydraulic retention time ${ }^{192,202}$ and by increasing the mixing to prevent niche-formation.

In order to improve the selectivity of $\mathrm{i}-\mathrm{C}_{6}$ production via $\mathrm{i}-\mathrm{C}_{4}$ elongation, the microbiome must be enriched to the point where $C_{2} / n-C_{4} / n-C_{6}$ elongation is no longer the dominant chain elongation process. Excessive ethanol oxidation and beta-oxidation should be prevented to the point where the resulting acetate production in turn does not allow for dominant $\mathrm{C}_{2} / \mathrm{n}-\mathrm{C}_{4}$ elongation. A $100 \%$ selective production process of $\mathrm{i}-\mathrm{C}_{6}$ is impossible in principle, due to the fact that some $\mathrm{C}_{2}$ is always present. There will always be acetate production during the reverse beta-oxidation process, due to substrate level phosphorylation coupled to ethanol oxidation ${ }^{22}$.

The feedstock to produce $\mathrm{i}-\mathrm{C}_{6}$ could be derived from an $\mathrm{i}-\mathrm{C}_{4}$ bioproduction process that uses methanol and organic waste (e.g. outdated food waste) ${ }^{35}$. The presence of methanol remaining from such a first stage will likely not hamper the $\mathrm{i}-\mathrm{C}_{6}$ formation, as in this study methanol did not have an effect on $\mathrm{i}-\mathrm{C}_{6}$ formation. Hypothetically, earlier formed isovalerate too could be elongated to $\mathrm{i}-\mathrm{C}_{7}$ within the developed microbiome.

Further studies will be required to exploit the formation of the branched MCFAs and alcohols. The $\mathrm{i}-\mathrm{C}_{6}$ production rate of $0.86 \mathrm{~g} \mathrm{l}^{-1}$ day $^{-1}$ is still 65 times lower than optimized $\mathrm{n}-\mathrm{C}_{6}(55.8$ 
g n-caproate $\mathrm{l}^{-1}$ day $^{-1}$ ) chain elongation systems $\mathrm{s}^{229}$ and thus needs to be increased. Moreover, the $\mathrm{i}-\mathrm{C}_{6}$ broth concentration $\left(1.4 \mathrm{~g} \mathrm{l}^{-1}\right)$ in this study was 10 times lower than the maximum solubility of the protonated acid-form. Concentrations in this higher range are necessary for effective extraction ${ }^{230}$. Once these criteria are met, efficient downstream processing methods can be developed that will allow for branched chain elongation to become viable in industry.

\section{Supporting Information}

The Supporting Information is available in the Supporting Information Section of this thesis. Additional information regarding Materials \& Methods, a summary table with bioprocesses and thermodynamic calculations, additional depictions for Results \& Discussion, 13 figures and 29 tables. 


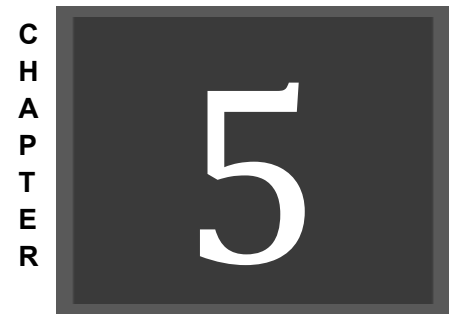

\section{Expanding the Usage of Branched Electron Acceptors for the Formation of Iso-caproate and Iso-heptanoate by Ethanol-based Chain Elongation Reactor Microbiomes}

This chapter is submitted as

Kasper D. de Leeuw, Theresa Ahrens, Cees J.N. Buisman, David P.B.T.B. Strik, 2020, Expanding the Usage of Branched Electron Acceptors for the Formation of Iso-caproate and Iso-heptanoate by Ethanol-based Chain Elongation Reactor Microbiomes. 


\section{Abstract}

Chain elongation fermentation technologies allow for the synthesis of useful biobased chemicals from complex organic residue streams. In order to expand the product spectrum of chain elongation technology and its application range we investigated (i) how to increase selectivity towards branched chain elongation and (ii) whether alternative branched carboxylates such as branched valerates can be used as electron acceptors. Elongation of isobutyrate $\left(\mathrm{i}-\mathrm{C}_{4}\right)$ elongation towards 4-methyl-pentanoate $\left(\mathrm{i}-\mathrm{C}_{6}\right)$ was achieved with a selectivity of $27 \%$ (of total products, based on carbon atoms) in a continuous system that operated under $\mathrm{CO}_{2}$ and acetate limited conditions. Increasing the $\mathrm{CO}_{2}$ load led to more in situ acetate formation that increased overall chain elongation rate, but decreased the selectivity of branched chain elongation. A part of the in situ acetate formation was related to alcohol formation that seemed to occur via hydrogenogenic ethanol oxidation thermodynamically coupled to hydrogentrophic carboxylate reduction. Several alcohols including isobutanol and $n$-hexanol were formed. The microbiome from the continuous reactor was also able to form small amounts of 5-methyl-hexanoate (i- $\mathrm{C}_{7}$ ) likely from 3methyl-butanoate $\left(\mathrm{i}-\mathrm{C}_{5}\right)$ and ethanol as substrate in batch experiments. The highest achieved concentration of $i-C_{7}$ was $6.4 \pm 0.9 \mathrm{mM} \mathrm{C}$, or $118 \pm 17 \mathrm{mg} / \mathrm{L}$, which contributed for $7 \%$ to the total amount of products (in carbon). The formation of $\mathrm{i}-\mathrm{C}_{7}$ was dependent on the isoform of branched-C5. While 3-methyl-butanoate (i- $\left.\mathrm{C}_{5}\right)$ was likely elongated to 5methylhexanoate (i-C7), a racemic mixture of L/D 2-methyl-butanoate was not. When i$\mathrm{C}_{4}$ and $\mathrm{i}-\mathrm{C}_{5}$ were added simultaneously as substrates there was a large preference for elongation of $\mathrm{i}-\mathrm{C}_{4}$ over $\mathrm{i}-\mathrm{C}_{5}$. Overall, this work show that chain elongation microbiomes can be further engineered with supplement of branched-electron acceptors towards the formation of iso-caproate and iso-heptanoate as well as that longer alcohol formation can be stimulated. 


\section{Introduction}

Chain elongation is an emerging bioprocess by which medium chain carboxylates (MCCs) can be produced in an alternative way. Currently, MCCs are mainly produced by either non-renewable fossil resources or palm and kernel oil-crops, both of which aggravate environmental problems ${ }^{231}, 232$. Chain elongation fermentations provide a sustainable alternative by utilizing organic residue streams as substrate ${ }^{25,191}$. The formation of MCCs from organic residues provide an economically attractive alternative to biogas formation ${ }^{23}$. The amount of research groups that study the formation of straight MCCs such as ncaproate $\left(n-C_{6}\right)$ and $n$-caprylate $\left(n-C_{8}\right)$ via microbial chain elongation is quickly expanding ${ }^{22}$ 185, 233-238. Commercial application by ChainCraft B.V. in Amsterdam will demonstrate large scale production of $\mathrm{n}-\mathrm{C}_{6}$ from organic residues ${ }^{239}$. Chain elongation fermentation technologies could become an impactful recycling method that can aid in the development of a circular economy ${ }^{32}$.

Recently it was also shown that branched MCFA iso-caproate ( $\mathrm{i}-\mathrm{C}_{6}$ ) can be formed in significant amounts when iso-butyrate ( $\left.\mathrm{i}-\mathrm{C}_{4}\right)$ is used as electron acceptor in an open-culture ethanol based chain elongation fermentation ${ }^{240}$. This research suggests that formation of other branched MCFAs such as iso-heptanoate $\left(i-C_{7}\right)$ should be possible. The necessary iso-valerate $\left(i-C_{5}\right)$ substrate could be formed via protein degradation steps ${ }^{168,241}$ or genetically engineering organisms ${ }^{194}$. While iso-caproate was sometimes in trace amounts detected in chain elongation microbiomes, to our best knowledge, iso-heptanoate was not ${ }^{22}$ 40. Branched MCFAs are interesting compounds because their physical properties differ from those of straight MCFAs. Branched MCFAs have a higher viscosity, they reduce crystallization $^{36}$ and have an oxidative stability 38,242 that can improve fuel combustion ${ }^{243}$. MCFAs in general can be used in the production of e.g. solvents, feed additives ${ }^{188}$, lubricants, surfactants, emulsifiers, pharmaceuticals ${ }^{22}$ and as precursors for plastics and fuels ${ }^{38,195}$. In addition to MCFAs, higher alcohols are also interesting fermentation products and can be coproduced within a chain elongation microbiome $214,240,244$. A better understanding on their production mechanism could facilitate the development of higher alcohol formation from organic residues, rather than from more expensive glucose based fermentations ${ }^{245,246 .}$

A challenge with ethanol based chain elongation fermentations is minimizing the (excessive) direct ethanol oxidation. Excessive ethanol oxidation is thermodynamically feasible at a hydrogen partial pressure below approximately $1 \mathrm{kPa}$ and can be stimulated when syntrophic partners in biofilms utilize the produced hydrogen 247.248 The usage of $1^{\text {st }}$ generation ethanol has a big impact on the sustainability and costs of the ethanol-based chain elongation process ${ }^{230}$. Additionally, the in situ acetate formation reduces the selectivity of branched and odd-chain carboxylate elongation ${ }^{192,240}$. Earlier research has 
shown that reducing the $\mathrm{CO}_{2}$ dosage could reduce excessive ethanol oxidation and increase carboxylate elongation selectivity ${ }^{211}$.

The objective of this study was to expand the usage of branched electron acceptors for the formation of isocaproate and isoheptanoate by ethanol-based chain elongation reactor microbiomes. A continuous anaerobic filter bioreactor that was fed with ethanol and isobutyrate was operated under two different $\mathrm{CO}_{2}$ loads was used to grow a chain elongation dominant microbiome. The results indicated that branched chain elongation selectivity was indeed higher at low $\mathrm{CO}_{2}$ loads. This effect, however, could be transient due to microbiome adaptation that gain acquired increased functionality towards alcohol formation that seemed to be coupled to excessive ethanol oxidation.

The same microbiome was used in batch bioreactors to evaluate the feasibility of using branched five-carbon fatty acids (3-methyl butanoate i.e. iso-valerate and L/D 2-methyl butanoate) as substrate and electron acceptor within an ethanol based chain elongation microbiome. We show the first appearance of $\mathrm{i}-\mathrm{C}_{7}$ (5-methylhexanoate) which was likely formed from microbial iso-valerate (3-methylbutanoate) elongation using ethanol as electron donor. After this observation another batch series was performed to evaluate the effect of initial higher hydrogen partial pressure and acetate concentrations that are known to influence the chain elongation activity $22,185,187,240$. In addition substrate specificity of iso-valerate was compared to that of iso-butyrate as an alternative substrate and electron acceptor. The results led to a discussion which highlighted that branched chain elongation occurs as cometabolism during straight chain elongation, meaning that a minimum amount of acetate is required for chain elongation to occur at all.

\section{Materials and Methods}

\section{Continuous reactor setup}

A continuous anaerobic filter bioreactor was set up to investigate the effect of $\mathrm{CO}_{2}$ supply microbial chain elongation reactor that was fed with isobutyrate $(650 \mathrm{mM} \mathrm{C})$, ethanol (540 $\mathrm{mM} \mathrm{C}$ ) and yeast extract ( $\left.1 \mathrm{~g} \mathrm{~L}^{-1}\right)$ as carbon sources (acetate was omitted from the influent). The reactor system was similar to the previous research on isocaproate formation via ethanol based chain elongation ${ }^{240}$. Different was the addition of a carrier material to retain microbial biomass increase the rate of chain elongation activity ${ }^{249}$.

After startup the reactor was completely filled with sponge carrier material $(0.15 \mathrm{~L}$ liquid exclusion volume of 15 by $15 \mathrm{~mm}$ polyurethane cubes; Recticel, Belgium) to support additional growth of biomass (phase II). To maintain anaerobic conditions during this procedure the reactor was flushed with $\mathrm{N}_{2}$ gas. The addition of cubes changed the active 
liquid volume of the reactor from $1 \mathrm{~L}$ to $0.85 \mathrm{~L}$. The influent rate was adjusted accordingly (from $22.2 \mathrm{~mL}$ hour ${ }^{-1}$ to $18.9 \mathrm{~mL}$ hour ${ }^{-1}$ ) to maintain an HRT of around 45 hours. The $\mathrm{CO}_{2}$ supply was doubled in phase III and halved again in phase IV. An overview of the influent carbon sources, the steady state duration for each phase, the HRT, pH and the CO2 supply are listed over the different phases in Table 1.

Table 1. Overview over the different phases in the reactor. The influent carbon sources, the steady state duration for each phase, the HRT, $\mathrm{pH}$ and $\mathrm{CO} 2$ supply are listed.

\begin{tabular}{l|cccc} 
Phase & $\boldsymbol{I}$ & $\boldsymbol{I I}$ & $\boldsymbol{I I I}$ & $\boldsymbol{I V}$ \\
\hline Condition & Start-up & $\begin{array}{c}\text { Add carrier } \\
\text { material }\end{array}$ & $\mathrm{CO}_{2}$ increase & $\mathrm{CO}_{2}$ decrease \\
$\begin{array}{l}\text { Phase period } \\
\text { (days) }\end{array}$ & $1-45$ & $45-78$ & $78-94$ & $94-129$ \\
HRT (h) & $44 \pm 7$ & $46 \pm 8$ & $44 \pm 2$ & $47 \pm 6$ \\
Isobutyrate (mM C) & 650 & 650 & 650 & 650 \\
Ethanol (mM C) & 540 & 540 & 540 & 540 \\
pH & $6.65 \pm 0.07$ & $6.6 \pm 0.03$ & $6.6 \pm 0.02$ & 6.62 \\
CO supply & 0.18 & 0.18 & 0.36 & 0.18 \\
(mLn/min) & & & &
\end{tabular}

\section{Batch experimental setup}

The batch experiments were performed in $250 \mathrm{~mL}$ serum bottles ( $150 \mathrm{~mL}$ liquid medium). The remaining $100 \mathrm{~mL}$ gas headspace was replaced at the start of each batch up to a pressure of $150 \mathrm{kPa}$. The macronutrients and micronutrients medium composition for the batches is given in Tables S1-S3 in the Supplementary Information. The batch bottles were kept in a shaker at $35^{\circ} \mathrm{C}$ and $150 \mathrm{rpm}$ throughout the whole experiment. The exact stepby-step protocol for the batches is given in the Supplementary Information. All batches were carried out in duplicates.

\section{Investigating i-C5 elongation proof of concept - first batch series}

The first experimental series consisted of eight batches (1.A to $1 . \mathrm{H}$ ) that aimed to investigate if an enriched microbiome that produced $\mathrm{i}-\mathrm{C}_{6}$ could also elongate branched valerates to branched heptanoates. Ethanol and a small amount of acetate were always added as substrate, whereas the types of branched valerates were varied throughout the series. In batch 1.A and 1.B a racemic mixture of L/D 2-methylbutanoate was added. Isovalerate (i.e. 3-methylbutanoate, $\mathrm{i}-\mathrm{C}_{5}$ ) was added in batch 1.C and 1.D. In batch 1.E and 1.F a 50:50 mixture of the L/D 2-methylbutanoate racemate and iso-valerate was added to investigate their combined effect on chain elongation. All these batches were performed at two different substrate concentrations. BES (2-bromoethanesulfanoate) was added at $10 \mathrm{~g} / \mathrm{L}$ to inhibit methanogenesis ${ }^{250}$, except in the control batch 1.G. Additionally a control 
batch $(1 . \mathrm{H})$ was performed without yeast extract to be able to exclude the possible formation of $\mathrm{i}-\mathrm{C}_{7}$ from yeast extract. Table 2 shows the different carbon source concentrations that were used as well as other parameters that were varied throughout the two batch series.

Table 2. Overview of the different starting parameters for the first batch series.

\begin{tabular}{l|llllllll}
\multicolumn{1}{l}{} & $1 . A$ & $1 . B$ & $1 . C$ & $1 . D$ & $1 . E$ & $1 . F$ & $1 . G$ & $1 . H$ \\
\hline $\begin{array}{l}\text { EtOH }(m M C) \\
\text { Acetate }(m M C)\end{array}$ & 320 & 160 & 320 & 160 & 320 & 160 & 160 & 160 \\
$\begin{array}{l}\text { L/D 2- } \\
\text { methylbutanoate } \\
(m M C)\end{array}$ & 13 & 6.5 & 13 & 6.5 & 13 & 6.5 & 6.5 & 6.5 \\
$(3-) i-C_{5}(m M C)$ & - & - & 325 & 162.5 & 162.5 & 81.3 & 162.5 & 162.5 \\
BES $(g / L)$ & 10 & 10 & 10 & 10 & 10 & 10 & - & 10 \\
Yeast $(g / L)$ & 0.5 & 0.5 & 0.5 & 0.5 & 0.5 & 0.5 & 0.5 & - \\
$p H$ & 6.5 & 6.5 & 6.5 & 6.5 & 6.5 & 6.5 & 6.5 & 6.5 \\
$\mathrm{~N}_{2} \%$ & 90 & 90 & 90 & 90 & 90 & 90 & 90 & 90 \\
$\mathrm{CO}_{2} \%$ & 10 & 10 & 10 & 10 & 10 & 10 & 10 & 10 \\
$\mathrm{H}_{2} \%$ & 0 & 0 & 0 & 0 & 0 & 0 & 0 & 0
\end{tabular}

\section{Investigating the substrate specificity and limiting factors for chain elongation activity - secondary and tertiary batch series}

In the first batch series ethanol was not completely converted and it remained unclear if this was caused by the drop in $\mathrm{pH}$, a limiting acetate concentration, the increased hydrogen partial pressure or something else (such as product inhibition on the microbiome ${ }^{57}$ ). Therefore a second and third series was performed to further investigate the effect of increased hydrogen partial in combination with different starting acetate concentrations. In contrast to the first series that contained no hydrogen at the start of the experiment, the second series was performed with hydrogen already present in the headspace at the start of the batch ( $20 \%$ for all batches, except 2.C which contained $80 \% \mathrm{H}_{2}$ at the start). This was done to minimize acetate formation via potential excessive ethanol oxidation which is thermodynamically inhibited at higher $\mathrm{H}_{2}$ pressures ${ }^{211}$ and to investigate the effect of an even higher $\mathrm{H}_{2}$ pressure on the chain elongation itself ${ }^{22}, 251$. These batches were all started at $\mathrm{pH} 7$ instead of 6.5 to allow for more potential proton formation due to ethanol oxidation before $\mathrm{pH}$ becomes a limiting factor and prevent a subsequent limiting bicarbonate concentration ${ }^{204,252}$. One batch (2.B) was started with an initial acetate concentration ten times higher than the other batches (2.A as control). Additionally to investigate the necessity of acetate during chain elongation, batch 3.B was started with zero added acetate (3.A as control, in the third batch series). 
To batch 2. $\mathrm{D}$ i- $\mathrm{C}_{4}$ was added in addition to $\mathrm{i}-\mathrm{C}_{5}$ to get insight into substrate preferences for branched chain elongation. In the third batch series $n$-valerate was added (batch 1.D) to compared its utilization as electron acceptor with $\mathrm{i}-\mathrm{C}_{5}$ and exclude possible $\mathrm{i}-\mathrm{C}_{7}$ formation via $n-C_{5}$. Table 3 and 4 show overviews of the second and third batch series respectively. The medium was the same as the medium from the first series, except for the indicated changes in the tables.

Table 3. Overview of the different starting parameters for the second batch series.

\begin{tabular}{l|llll}
\multicolumn{2}{c}{$\begin{array}{l}2 . A \\
\text { (low acetate) }\end{array}$} & $\begin{array}{l}\text { 2.B } \\
\text { (high acetate) }\end{array}$ & $\begin{array}{l}\text { 2.C } \\
\text { (high hydrogen) }\end{array}$ & $\begin{array}{l}\text { 2.D } \\
\text { (including i-C4) }\end{array}$ \\
\hline Inoculum & Batch 1.D & Batch 1.D & Batch 1.D & Batch 1.D \\
EtOH $(m M ~ C)$ & 160 & 160 & 160 & 160 \\
$(3-) i-C_{5}(m M C)$ & 162.5 & 162.5 & 162.5 & 162.5 \\
$i-C_{4}(m M C)$ & - & - & - & 64.5 \\
Acetate $(m M C)$ & 6.5 & 65 & 6.5 & 6.5 \\
BES $(g / L)$ & 10 & 10 & 10 & 10 \\
Yeast $(g / L)$ & 0.5 & 0.5 & 0.5 & 0.5 \\
$p H$ & 7 & 7 & 7 & 7 \\
$\mathrm{~N}_{2}(\%)$ & 70 & 70 & 10 & 70 \\
$\mathrm{CO}_{2} \%$ & 10 & 10 & 10 & 10 \\
$\mathrm{H}_{2}(\%)$ & 20 & 20 & 80 & 20
\end{tabular}

Table 4. Overview of the different starting parameters for the third batch series.

\begin{tabular}{|c|c|c|c|c|}
\hline & $\begin{array}{l}\text { 3.A } \\
\text { (low acetate) }\end{array}$ & $\begin{array}{l}3 . B \\
\text { (no acetate) }\end{array}$ & $\begin{array}{l}\text { 3.C (no extra } \\
\text { carboxylate) }\end{array}$ & $\begin{array}{l}3 . D \\
\left(n-C_{5}\right)\end{array}$ \\
\hline Inoculum & regrown 2.D & regrown 2.D & regrown 2.D & regrown 2.D \\
\hline EtOH (mM C) & 320 & 320 & 320 & 320 \\
\hline$\left(3^{-}\right) i-C_{5}(m M C)$ & 162.5 & 162.5 & & \\
\hline$n-C_{5}(m M C)$ & & & & 162.5 \\
\hline Acetate (mM C) & 13 & 0 & 13 & 13 \\
\hline$B E S(g / L)$ & 10 & 10 & 10 & 10 \\
\hline Yeast $(g / L)$ & 0.5 & 0.5 & 0.5 & 0.5 \\
\hline$p H$ & 7 & 7 & 7 & 7 \\
\hline$N_{2}(\%)$ & 60 & 60 & 60 & 60 \\
\hline $\mathrm{CO}_{2} \%$ & 20 & 20 & 20 & 20 \\
\hline$H_{2}(\%)$ & 20 & 20 & 20 & 20 \\
\hline
\end{tabular}




\section{Inoculum}

The continuous reactor as well as the first batch series was inoculated with a mixture of two undefined anaerobic cultures. One volume part was taken from the continuous reactor that elongated $\mathrm{i}-\mathrm{C}_{4}$ to $\mathrm{i}-\mathrm{C}_{6}{ }^{240}$ and a second equal volume part came from a mixed bovine rumen sample. The bovine rumen liquid from three cows was provided by the Animal Science Department of Wageningen University and Research. Biomass concentration was not measured within the inocula. The inoculum mixture was put in $50 \mathrm{~mL}$ centrifuge tubes and spinned down at 4500 rcf for 10 minutes. The cell pellets were resuspended in carbon source free medium prior to inoculation as described within the step-by-step protocol in the Supplementary Information section.

The inoculum for the second batch series was taken from batch 1.D of the first series. Its contents were centrifuged in $50 \mathrm{~mL}$ tubes at $4500 \mathrm{rfc}$ and the pellets were subsequently combined and re-suspended with $50 \mathrm{~mL}$ carbon source free medium. These re-suspended cells were then used as inoculum for the second batch series as described within the stepby-step protocol. Similarly, the third batch series was inoculated with biomass that originated from batch 2.D. However, before inoculating, batch 2.D was stored for one and a half year at room temperature. Sporulation of bacteria was observed under the microscope, prior to activation. Before starting the third batch series an activation batch was performed using the same conditions as in batch 2.D. The third batch series was then inoculated with this freshly activated biomass.

\section{Sampling and measurement}

Samples of the gas phase were taken once per week and analyzed using an established protocol for gas chromatography to determine the fractions of $\mathrm{O}_{2}, \mathrm{~N}_{2}, \mathrm{CH}_{4}, \mathrm{H}_{2}$, and $\mathrm{CO}_{2}$. ${ }^{185}$, 253 Before sampling the pressure was measured using a pressure meter (GMH 3151). At the same time liquid samples $(3.5 \mathrm{~mL})$ were taken, centrifuged at $10000 \mathrm{rfc}$ and stored in a freezer at $-20^{\circ} \mathrm{C}$. Every two weeks these samples were analyzed according an earlier described method ${ }^{97}$ to determine the concentrations of primary alcohols and volatile carboxylic acids (ethanol, propanol, butanol, iso-butanol, pentanol, b-pentanol, n-hexanol, iso-hexanol and acetic, n-butyric, iso-butyric, n-valeric, b-valeric, n-caproic, iso-caproic, $\mathrm{n}$-heptanoic, iso-heptanoic and n-caprylic acid). The isomers 3-methylbutyric acid (i-C5) and L/D 2-methylbutyric acid (L/D 2-MB) indicated as b-valeric acid (prefix b- refers to branched compounds, regardless of which of the isomers), as well as the isomers 3methylbutanol and L/D 2-methylbutanol, indicated as b-pentanol, could not be distinguished with the available equipment because the isomers exhibited the same retention time. Therefore the batches were designed to investigate their effect on chain elongation separately to analyze which isomers of branched $C_{5}$ were used for the formation of which branched $C_{7}$. The expected forms of branched heptanoic acids, (4-) and (5-) 
methylhexanoic acid (MHA), could be distinguished as is shown in Figure $\mathrm{S} 1$ in the Supplementary Information. Hypothetically a L/D 4-MHA racemate is the elongation product of $L / D$ 2-MB, whereas $5-M H A\left(i-C_{7}\right)$ is the elongation product of $i-C_{5}$, assuming the elongation occurs in a similar fashion as during earlier observed $\mathrm{i}-\mathrm{C}_{4}$ elongation to $\mathrm{i}-\mathrm{C}_{6}$ and other chain elongation mechanisms ${ }^{254}$. For the continuous experiment the data is presented using the averaged values during each phase and a confidence interval ( \pm ) using an a of 0.1 . For the batches the duplicate results are averaged and the error bars $( \pm)$ indicate the differences between each measurement. 


\section{Results}

\section{CONTINUOUS REACTOR}

\section{Non-reversible reactor performance behavior after $\mathrm{CO2}$ increase and decrease}

During the first three weeks after starting the isobutyrate and ethanol fed continuous reactor, acetate accumulated in the broth, followed by n-butyrate, n-butanol and isobutanol. Around day 30 the broth concentration of these compounds, except for isobutanol, lowered while n-caproate and isocaproate formation started to occur. When caproate concentrations no longer increased at the end of the start-up (phase I) carrier material was added on day 45 . The reactor then reached a steady state from day 62 to day 78 in phase II. The $\mathrm{CO}_{2}$ headspace partial pressure was consistently below $1 \mathrm{kPa}$ as soon as chain elongation activity was observed, even after doubling the $\mathrm{CO}_{2}$ in phase III. The highest volumetric productivities and concentrations of $\mathrm{i}-\mathrm{C}_{6}$ were obtained during phase III at increased $\mathrm{CO}_{2}$ supply, reaching a rate of $57 \pm 4 \mathrm{mM} \mathrm{C} /$ day, or $1.1 \pm 0.07$ $\mathrm{g} / \mathrm{L} /$ day and a concentration of $125 \pm 6.6 \mathrm{mCM}$, or $2.43 \pm 0.13 \mathrm{~g} / \mathrm{L}$. However, selectivity towards $\mathrm{iC}_{6}$ (carbon per tot carbon in products) was highest in in phase II (27\%) and dropped to $20 \%$ in phase III. The metabolite broth concentrations are shown in Figure 1 and the product yields per phase are shown in Table 5. Excessive ethanol oxidation (EEO) as shown in the table is calculated from the observed chain elongation activity and ethanol consumption using an earlier described method ${ }^{240}$.
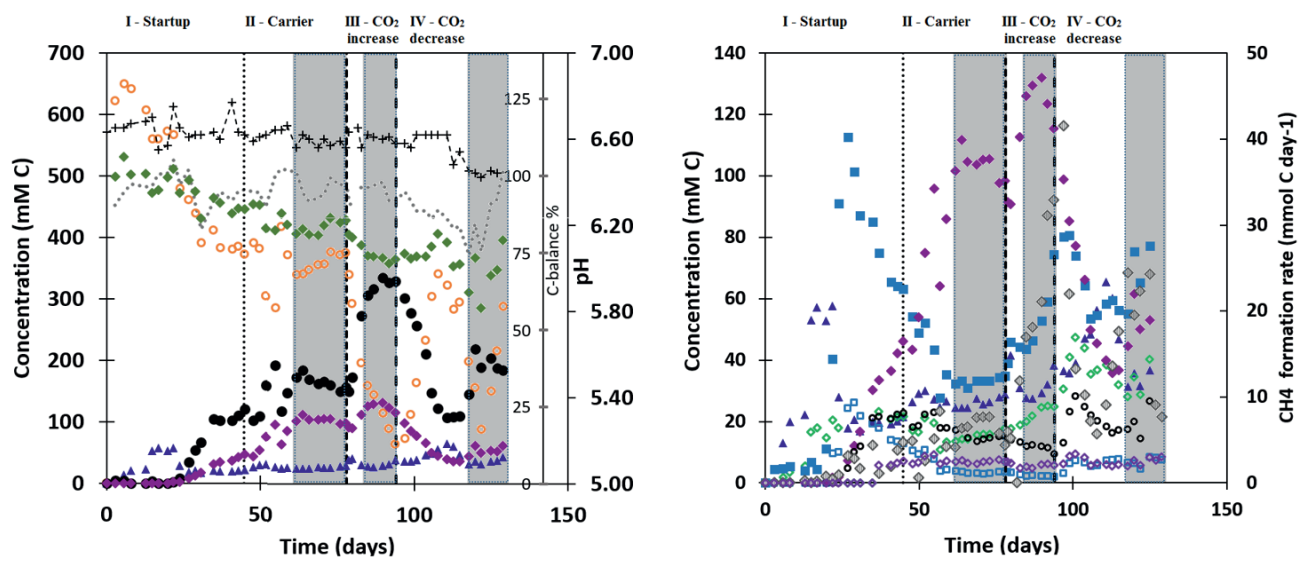

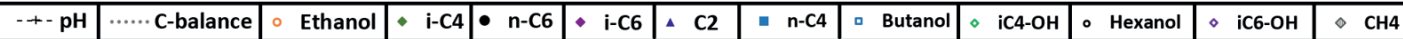

Figure 1. Broth concentrations of metabolites within the continuous reactor system. Additionally the carbon balance and $\mathrm{pH}$ are shown in (A) and methane formation is rate shown in (B). The grey boxes at the end of each phase show the range where the averages were taken for the values in Table 5. 
The reactor was not allowed to develop a steady state in phase III because methane formation kept increasing, which was deemed unfavorable for chain elongation activity in the long term. Instead, the $\mathrm{CO}_{2}$ load was lowered from $0.36 \mathrm{mLn} / \mathrm{min}$ (phase III) to 0.18 $\mathrm{mLn} / \mathrm{min}$ (phase IV) with two aims: (i) lower methanogenic activity and (ii) investigate if the $\mathrm{i}-\mathrm{C}_{6}$ selectivity could be increased again. However, the reactor had also developed an increased alcohol (in particular $\mathrm{i}-\mathrm{C}_{4} \mathrm{OH}$ ) productivity. As a consequence different conversion rates were observed in phase VI compared to phase II, although reactor operating conditions were the same. Alcohol $\left(n-\mathrm{C}_{4} \mathrm{OH}, \mathrm{i}-\mathrm{C}_{4} \mathrm{OH}, \mathrm{n}-\mathrm{C}_{6} \mathrm{OH}\right.$ and $\mathrm{i}-\mathrm{C}_{6} \mathrm{OH}$ ) formation had increased from a combined selectivity of $10 \%$ in phase II to $16 \%$ in phase IV. Also an increase in straight carboxylates was observed relative to phase II, while i-C $\mathrm{C}_{6}$ selectivity had dropped down to $12 \%$ in Phase IV. Additionally the hydrogen partial pressure had dropped below $1 \mathrm{kPa}$ from phase III onwards and did not recover to the levels observed in phase II (up to $10 \mathrm{kPa}$ ). The gas partial pressures in the reactor headspace are shown in Figure S2 in the SI. Evidently, the average conversion rates in the reactor show a nonreversible behavior after the $\mathrm{CO}_{2}$ increase and decrease. 
Table 5. Overview of averaged operating parameters and conversion rates including confidence intervals. Averages from phase IV are taken when caproate formation had stabilized (day 106 to 113).

\begin{tabular}{|c|c|c|c|c|c|c|}
\hline & \multicolumn{2}{|c|}{ Phase II } & \multicolumn{2}{|c|}{ Phase III } & \multicolumn{2}{|c|}{ Phase IV } \\
\hline Discription & \multicolumn{2}{|c|}{$\begin{array}{l}\text { Add carrier } \\
\text { material }\end{array}$} & \multicolumn{2}{|c|}{ CO2 increase } & \multicolumn{2}{|c|}{$\mathrm{CO} 2$ decrease } \\
\hline Phase period & \multicolumn{2}{|c|}{ day $45-78$} & \multicolumn{2}{|c|}{ day $78-94$} & \multicolumn{2}{|c|}{ day $94-129$} \\
\hline Averages taken from & \multicolumn{2}{|c|}{ day $62-78$} & \multicolumn{2}{|c|}{ day $85-94$} & \multicolumn{2}{|c|}{ day 117-129 } \\
\hline Calculated EEO (\%) & 21 & \pm 7 & 25 & \pm 6 & 44 & \pm 11 \\
\hline HRT (hours) & 46 & \pm 8 & 44 & \pm 2 & 43 & \pm 1 \\
\hline $\mathrm{pH}$ & 6.6 & \pm 0.03 & 6.6 & \pm 0.02 & 6.5 & \pm 0.04 \\
\hline $\mathrm{CO}_{2}$ loading ( $\mathrm{mLn} \mathrm{min}^{-1}$ ) & 0.18 & & 0.36 & & 0.18 & \\
\hline C-balance (\%) & 96 & \pm 3 & 95 & \pm 3 & 87 & \pm 10 \\
\hline e-balance (\%) & 96 & \pm 3 & 97 & \pm 3 & 84 & \pm 9 \\
\hline \multicolumn{7}{|c|}{ Volumetric C molar conversion rates (mmol C L-1 day-1) } \\
\hline $\mathrm{C2}$ & 13 & \pm 1 & 14 & \pm 2 & 17 & \pm 2 \\
\hline $\mathrm{i}-\mathrm{C} 4$ & -49 & \pm 8 & -58 & \pm 6 & -67 & \pm 20 \\
\hline$n-C 4$ & 17 & \pm 0 & 25 & \pm 5 & 31 & \pm 4 \\
\hline i-C6 & 50 & \pm 3 & 57 & \pm 4 & 25 & \pm 3 \\
\hline $\mathrm{n}-\mathrm{C} 6$ & 82 & \pm 5 & 146 & \pm 4 & 88 & \pm 11 \\
\hline$n-C 8$ & 2 & \pm 0 & 5 & \pm 0 & 2 & \pm 0 \\
\hline EtOH & -134 & \pm 13 & -219 & \pm 20 & -203 & \pm 32 \\
\hline $\mathrm{n}-\mathrm{C} 4 \mathrm{OH}$ & 2 & \pm 0 & 1 & \pm 0 & 3 & \pm 1 \\
\hline $\mathrm{i}-\mathrm{C} 4 \mathrm{OH}$ & 8 & \pm 1 & 11 & \pm 1 & 16 & \pm 2 \\
\hline Hexanol & 8 & \pm 1 & 5 & \pm 1 & 10 & \pm 2 \\
\hline $\mathrm{i}-\mathrm{C} 6 \mathrm{OH}$ & 1 & \pm 0 & 1 & \pm 0 & 1 & \pm 0 \\
\hline $\mathrm{CH}_{4}$ (gas) & 6 & \pm 1 & 24 & \pm 8 & 18 & \pm 7 \\
\hline $\mathrm{CO}_{2}$ (gas) & -11 & \pm 0 & -23 & \pm 0 & -11 & \pm 0 \\
\hline
\end{tabular}




\section{BATCHES}

\section{Chain elongation using different carboxylates as additional electron acceptors at acetate limitation}

The dominant bioprocess throughout all batch series was straight chain elongation towards $n-C_{6}$. Depending on the added carboxylate $\left(i-C_{4}, n-C_{5}, i-C_{5}\right)$ besides acetate, varying amounts of alternative elongation products $\left(i-C_{6}, n-C_{7}\right.$ and $\left.i-C_{7}\right)$ were formed. The amount of acetate that was present in the beginning of the batch significantly affected the time it took for chain elongation to be observed. Concentration profiles of the second and third batch series are shown in Figure $\mathbf{2}$ and Figure $\mathbf{3}$ respectively.

It is relevant to emphasize that during all batches, except batch 2.B (where $65 \mathrm{mM} \mathrm{C}$ acetate was added), a large fraction of ethanol was not consumed and therefore still available as electron donor. Increasing the initial acetate concentration (2.B, Table 4 and Figure 2) caused a substantial higher chain elongation activity towards $n-C_{6}$ (2.6 times compared to 2.A); significantly more ethanol was consumed in this batch up to a higher $\mathrm{P}_{2}$ and higher final acetate concentration. However, only $3.1 \mathrm{mM} \mathrm{Ci-C_{7 }}$ was formed versus

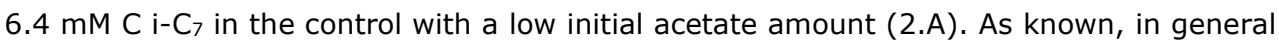
the low acetate concentration in combination with a high hydrogen partial pressure 211,255 was identified as the plausible cause that hampered chain elongation activity in the first two series, excluding control batch 1.G. Here, the absence of BES allowed methanogenesis to consume $\mathrm{CO}_{2}$ down to a partial pressure $<1 \mathrm{kPa}$, concomitant with more excessive ethanol oxidation to acetic acid that led to a $\mathrm{pH}$ drop to $<5.7$. These low $\mathrm{CO}_{2}$ partial pressure and $\mathrm{pH}$ values are limiting conditions for (ethanol-based) chain elongation bacteria 53,256 . 

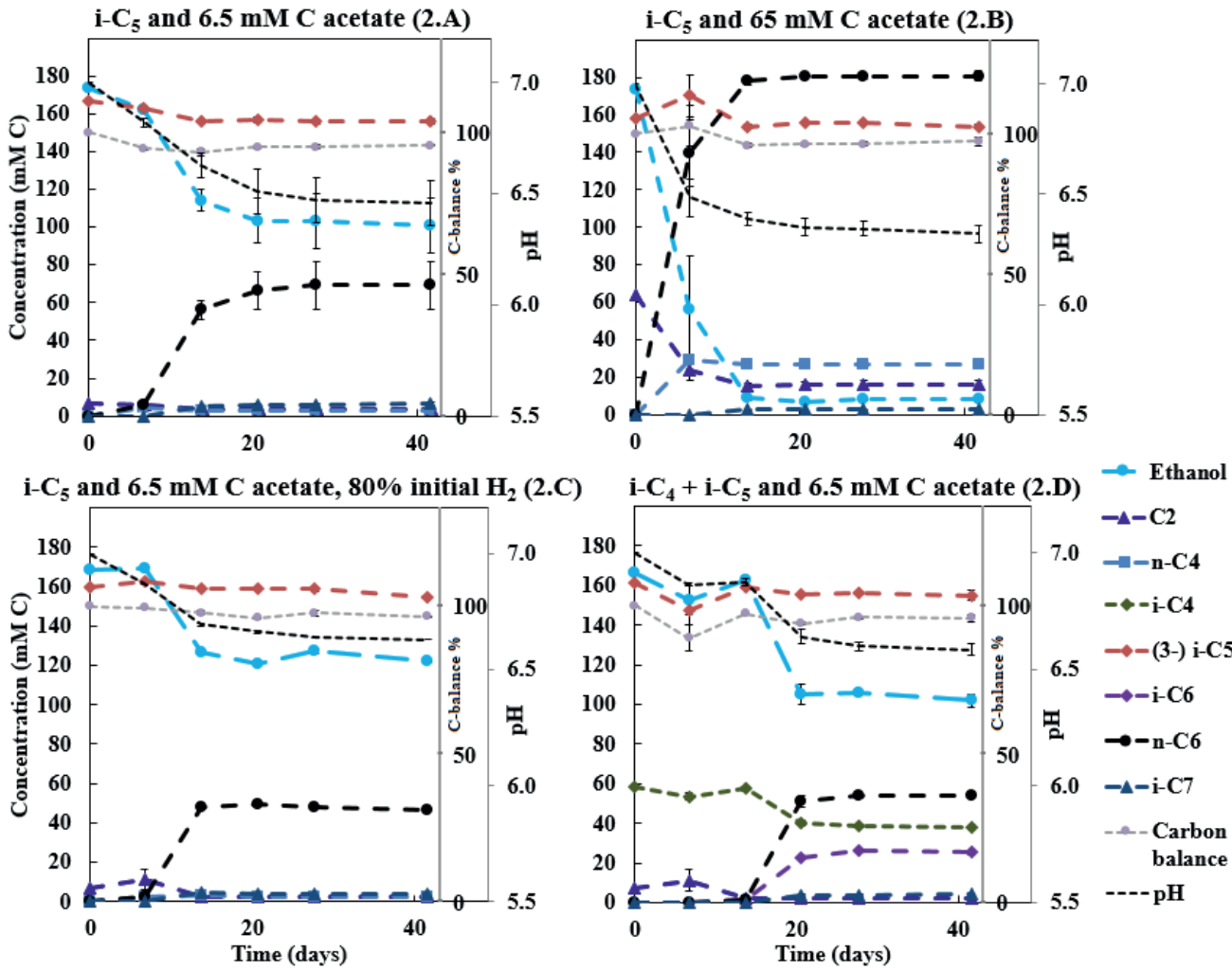

Figure 2. Concentration profiles of batches performed with $\mathrm{i}-\mathrm{C}_{5}$ as additional electron donor and varying levels of starting acetate amounts and hydrogen partial pressures (2.A-2C). Batch 2.D shows the preference for $\mathrm{i}-\mathrm{C}_{4}$ over $\mathrm{i}-\mathrm{C}_{5}$ as alternative electron acceptor leading to branched MCFA formation concomitant with straight chain elongation. The $\mathrm{i}-\mathrm{C}_{5}$ addition results only in marginal $\mathrm{i}-\mathrm{C}_{7}$ formation. 

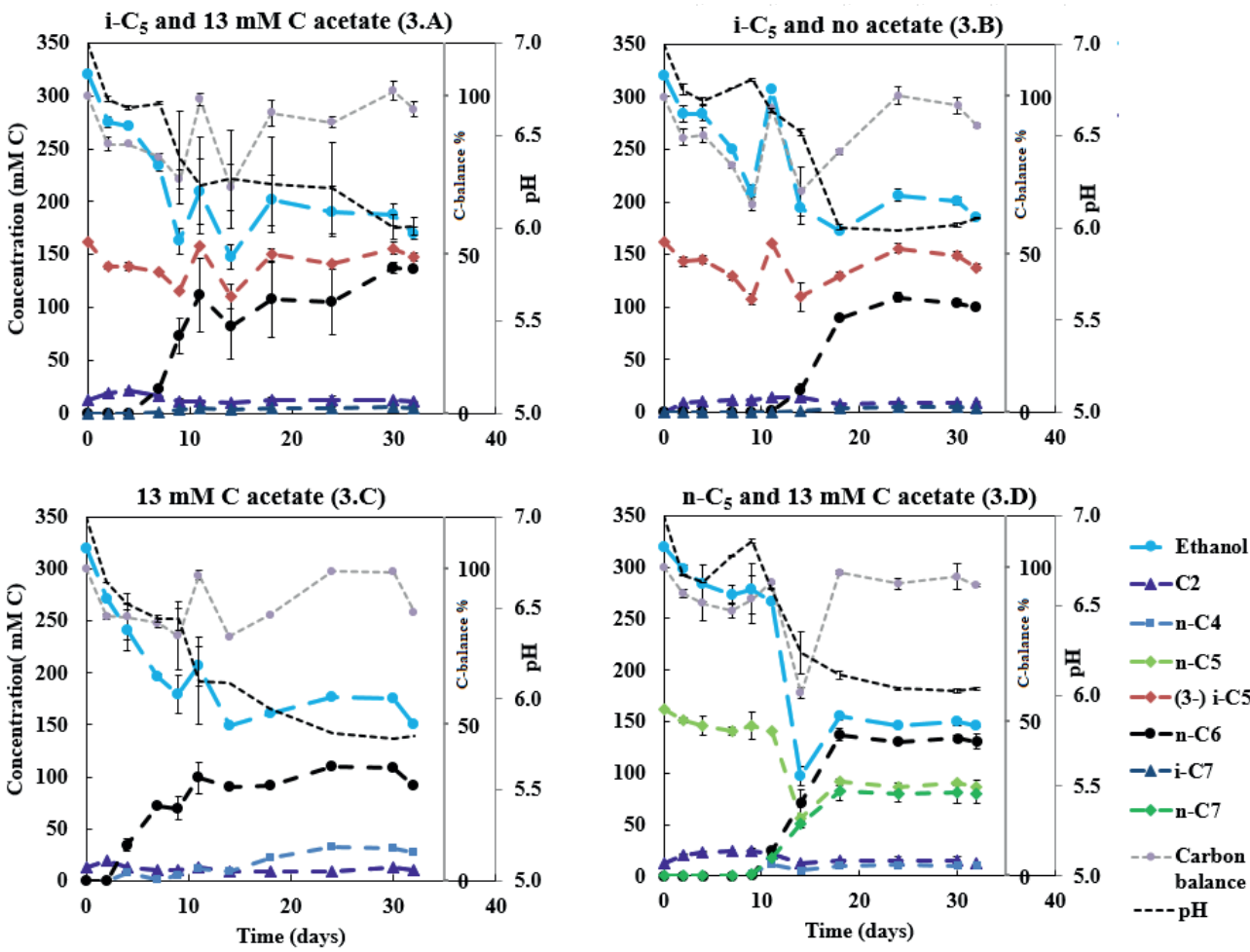

Figure 3. The four batches illustrate the effect of different initial acetate amounts and added carboxylates beside acetate on chain elongation activity. Complete absence of initial acetate (3.B) causes a prolonged lag phase, whereas the addition of a small amount (3.A) sped up the onset of chain elongation by approximately 7 days. Additionally, the batch without any additional carboxylate besides acetate shows an even faster onset of chain elongation activity (3.C). The batch with added $n-C_{5}$ (3.D) shows $n-C_{6}$ and $n-C_{7}$ formation and no $i-C_{7}$ formation.

Alcohol formation was observed in all batches and showed a general trend that whenever a carboxylate is present, the corresponding alcohol is formed albeit up to a significantly lower concentration (i.e. i- $\mathrm{C}_{4}$ led to $\mathrm{i}-\mathrm{C}_{4} \mathrm{OH}, \mathrm{n}-\mathrm{C}_{6}$ led to $\mathrm{n}-\mathrm{C}_{6} \mathrm{OH}$, etc.). The alcohol formation occurred in all batches up to a final concentration range between 1 and $5 \mathrm{mM} \mathrm{C}$ for each produced alcohols species. The concentration profiles of metabolites in this lower concentration range are shown for all batches in Figure S4, S6, S7 and S9. Gas headspace partial pressure profiles of all batches are shown in Figures S5, S6, S8 and S10. 


\section{Formation of small $\mathrm{i}-\mathrm{C}_{7}$ amounts is observed during presence of $\mathbf{i}-\mathbf{C}_{\mathbf{5}}$.}

The first batch series showed 5-methylhexanoate $\left(\mathrm{i}-\mathrm{C}_{7}\right)$ formation in the batches fed with 3-methylbutanoate (i-C5), albeit in very low amounts (Figures S3 and S4). The L/D 2methylbutanoate racemate (L/D 2-MB) was seemingly not utilized at all as substrate for chain elongation, as no hypothetical elongation product (4-MHA) was observed. Small amounts of branched pentanol formation were observed in all batches regardless which form of branched pentanoate was available. Due to a relative high standard error of the branched $\mathrm{C}_{5}$ analysis compared to the low $\mathrm{i}-\mathrm{C}_{7}$ and b-pentanol concentrations, it could not be determined whether i-C5 was molar-equally consumed. However, in all batches that did not contain isovalerate no isoheptanoate formation occurred. Additionally, the positive control batch without added yeast extract also showed $\mathrm{i}-\mathrm{C}_{7}$ formation, excluding yeast extract conversion, as potential cause for $\mathrm{i}_{-} \mathrm{C}_{7}$ formation. The likely chain elongation of $\mathrm{i}$ $\mathrm{C}_{5}$ towards $\mathrm{i}-\mathrm{C}_{7}$ accounted to $7.2 \%$ (based on carbon atoms) of the formed compounds in the best performing batch in regards to $\mathrm{i}_{-} \mathrm{C}_{7}$ formation (2.A). However, in this batch $98 \%$ of the supplied $\mathrm{i}-\mathrm{C}_{5}$ still remained unconverted. The $\mathrm{n}-\mathrm{C}_{6}$ accounted to $79.6 \%$ (based on carbon atoms) of formed compounds. Hexanol and iso-pentanol constituted to $4.7 \%$ and $0.6 \%$ respectively of the formed compounds. 


\section{Discussion}

\section{Tradeoff between branched carboxylate selectivity and productivity dependent on acetate availability}

The continuous reactor experiment was operated without any acetate in the influent to maximize isobutyrate utilization during chain elongation and thereby maximize selectivity towards isocaproate formation. Compared to a previous study on isocaproate formation 240 this system achieved a $30 \%$ higher volumetric $\mathrm{i}-\mathrm{C}_{6}(57 \pm 4 \mathrm{mM} \mathrm{C} / \mathrm{day}$, or $1.1 \pm 0.07$ $\mathrm{g} / \mathrm{L} /$ day) formation rate and a $70 \%$ higher average $\mathrm{i}-\mathrm{C}_{6}$ broth concentration (125 \pm 6.6 $\mathrm{mCM}$, or $2.43 \pm 0.13 \mathrm{~g} / \mathrm{L}$ ) in phase III. During the whole operation period the reactor was operating under apparent $\mathrm{CO}_{2}$ limited conditions $(<1 \mathrm{kPa})$, meaning that the low availability limits chain elongation activity of well-known chain elongators such as C. kluyveri ${ }^{53}$. When the $\mathrm{CO}_{2}$ load in phase III was increased, overall chain elongation activity increased. There was a higher (branched) $\mathrm{i}_{-} \mathrm{C}_{6}$ productivity, although selectivity towards $\mathrm{i}-\mathrm{C}_{6}$ had dropped (from $27 \%$ in phase II to $20 \%$ in phase III). Higher in situ acetate formation, both directly via the chain elongation metabolism and via increased excessive ethanol oxidation led to increased straight chain elongation (see Table S5). It shows there is a tradeoff to be made when designing the system: (i) selectivity towards isobutyrate elongation is high during acetate and $\mathrm{CO}_{2}$ limitation (which reduces overall chain elongation activity), or (ii) straight chain elongation is stimulated by lifting the $\mathrm{CO}_{2}$ limitation leading to a decreased selectivity towards alternative carboxylate elongation. In all phases $\mathrm{i}-\mathrm{C}_{4}$ was abundantly available, while acetate was only available via in situ formation. The sensitivity to increases in acetate show that there is a preference towards acetate as electron acceptor over $\mathrm{i}-\mathrm{C}_{4}$ (and $\mathrm{i}-\mathrm{C}_{5}$, in the batches) within the established chain elongation microbiome.

\section{The affinity for $\mathrm{i}-\mathrm{C}_{5}$ elongation is limited, a co-metabolism with straight chain elongation is suggested}

The degree by which b-valerates and iso-butyrate are elongated in a batch system varied. Formation of $\mathrm{i}-\mathrm{C}_{7}$ contributed only $4 \%$ (based on carbon atoms) to the total produced compounds in the batch with both $\mathrm{i}-\mathrm{C}_{5}$ and $\mathrm{i}-\mathrm{C}_{4}$ (2.D). In contrast, $\mathrm{i}-\mathrm{C}_{6}$ formation contributed for $27 \%$ to the total product spectrum, even though the molar concentration of $\mathrm{i}-\mathrm{C}_{5}$ was higher than $\mathrm{i}-\mathrm{C}_{4}$. With the $\mathrm{L} / \mathrm{D}$ 2-methylbutanoate racemate batches no elongation product was observed at all and overall the chain elongation rate diminished. Moreover, in the batch reactors a higher acetate availability negatively influences the selectivity towards branched chains, similar to what was observed in the continuous reactor. This is emphasized by the batches performed at $65 \mathrm{mM} \mathrm{C}$ and $6.5 \mathrm{mM} \mathrm{C}$ initial acetate. A higher initial acetate concentration (batch 2.B) increased total chain elongation activity, but significantly lowered the selectivity towards $\mathrm{i}_{-} \mathrm{C}_{7}(1.4 \%)$ compared to the control $(7.3 \%)$ at low initial acetate amounts (batch 2.A). The results suggest the microbiome harbors 
affinities for branched carboxylate elongation. Hypothetically, the higher $\mathrm{i}-\mathrm{C}_{7}$ selectivity during acetate limitation (2.A) can arise from kinetic impairment of acetate elongation at low acetate concentrations. Consequently at higher acetate concentrations (2.B) the alleviated kinetic impairment leads to more acetate elongation compared to branched chain elongation. These two scenarios where varying kinetics lead to different selectivities are illustrated in Figure S12.

The initially available acetate $(6.5 \mathrm{mM} \mathrm{C}$ in $2 . A$ versus $65 \mathrm{mM} \mathrm{C}$ in $2 . B$ and $13 \mathrm{mM} \mathrm{C}$ in $3 . \mathrm{A}$ versus $0 \mathrm{mM} \mathrm{C}$ in $3 . B$ ) in the batch series greatly affected the lag time of chain elongation. These results are in line with earlier studies that show a reduced chain elongation activity during acetate limitation ${ }^{255}$. Despite presence of sufficient alternative electron acceptors, a minimum amount of acetate seems to be required for chain elongation to occur. The requirement of acetate hints towards a cometabolism for the branched electron acceptors within chain elongation; i.e. branched carboxylates are only elongated during straight chain elongation.

\section{Alcohol formation driven by high substrate to product ratios as alternative source for in situ acetate production}

The observed longer alcohol formation during the continuous reactor experiment followed a dependency on the concentrations of ethanol and acetate as well as on the concentration of the longer carboxylates and their corresponding alcohols species. This finding was in line with the earlier study where $\mathrm{i}-\mathrm{C}_{6}$ and alcohol $\left(\mathrm{i}-\mathrm{C}_{4} \mathrm{OH}, \mathrm{n}-\mathrm{C}_{6} \mathrm{OH}\right.$ and $\left.\mathrm{i}-\mathrm{C}_{6} \mathrm{OH}\right)$ formation were found ${ }^{240}$. It suggests that (hydrogenogenic) ethanol oxidation and (hydrogenotrophic) carboxylate reduction are coupled within the microbiome as shown in Table 6, resulting in a net carboxyl-hydroxyl exchange reaction. A coupling of reactions would imply that the thermodynamics driving force is no longer affected by $\mathrm{pH}$ and hydrogen partial pressure $\left(\mathrm{p}_{\mathrm{H} 2}\right)$, in contrast to hydrogenotrophic carboxylate reduction to alcohols that is favored at a lowered $\mathrm{pH}$ and an elevated $\mathrm{p}_{\mathrm{H} 2}$ (See Figure $\mathrm{S} 2$ for the $\mathrm{p}_{\mathrm{H} 2}$ in the continuous reactor) ${ }^{213}$. Figure 5 shows that after startup the $\Delta \mathrm{rG}^{1}$ of the combined reactions for each carboxylate - alcohol pair (when correcting for the broth concentrations of the reactants and products) remained between $15-25 \mathrm{~kJ}$ reaction ${ }^{-1}$. This value is close to the currently known minimum required energy gain for a catabolic reaction to sustain microbial growth ${ }^{257}$, and suggests that this bioconversion could be utilized as energy-providing route by organisms growing in a specific niche. It still needs to be revealed which organism(s) play(s) a role in this alcohol formation.

Possibly chain elongation microorganisms themselves are solely responsible for the formation of the longer alcohols. It is reported that Clostrdium kluyveri, a well-known chain elongator, is able to produce small amounts of higher alcohols ${ }^{244}$. A batch series performed 
using pure Clostridium kluyveri with propionate and ethanol under different hydrogen pressures showed that propanol formation increased with an increasing $\mathrm{PH}_{2}{ }^{258}$. The alcohol formation during acetate limitation in combination with a high $\mathrm{pH}_{2}$ could hypothetically be method to get rid of excess electrons when chain elongation-coupled ethanol oxidation is thwarted due to high hydrogen partial pressures. Carboxylate reduction then replaces hydrogen formation as electron sink.

Alternatively, another specialized organism performing the hydroxyl-carboxyl exchange is present. It would require a $C$. autoethanogenum-like species ${ }^{214}$ that can harvest the energy from ethanol-derived electrons via an energy-coupled transhydrogenase (Rnf complex) ${ }^{47}$ before reducing the larger carboxylates. A second alternative would be syntrophic interaction between ethanol oxidizers and "hydrogenotrophic" carboxylate reducers (via $\mathrm{H}_{2}$ exchange and/or Direct Interspecies Electron Transfer (DIET) ${ }^{248}$ ). Although the thermodynamic calculations performed with macroscopic data show that the hydrogenotrophic carboxylate reduction is often unfeasible (Figure 5B), a syntrophic coupling of ethanol oxidation and carboxylate reduction would imply that the actual microscopic conditions are such that both (in syntophy-growing) microorganisms are able to proliferate 259 .

Table 6. Thermodynamic calculations for ethanol oxidation and carboxylate ( $n$-butyrate as example) reduction towards the corresponding alcohol (n-butanol). $\Delta_{r} G^{01}$ indicates the reaction Gibbs free energy change at standard biological conditions $\left(298^{\circ} \mathrm{C}, \mathrm{pH} 7\right) . \Delta_{\mathrm{r}} \mathrm{G}^{2}$ and $\Delta_{\mathrm{r}} \mathrm{G}^{3}$ are corrected for reactants to products ratios (carboxylates and alcohols only) of 100 and 0.01 respectively.

\begin{tabular}{|c|c|c|c|c|}
\hline Bioprocesses & Reaction & $\Delta_{\mathrm{r}} \mathbf{G}^{\mathbf{0 1}}$ & $\mathbf{\Delta}_{\mathbf{r}} \mathbf{G}^{2}$ & $\Delta \mathbf{r} \mathbf{G}^{3}$ \\
\hline Hydrogenogenic ethanol oxidation & $\mathrm{CH}_{3} \mathrm{CH}_{2} \mathrm{OH}+\mathrm{H}_{2} \mathrm{O} \rightarrow \mathrm{CH}_{3} \mathrm{COO}^{-}+2 \mathrm{H}_{2}+\mathrm{H}^{+}$ & 9.6 & -1.8 & 21.1 \\
\hline $\begin{array}{l}\text { Hydrogenotrophic carboxylate } \\
\text { reduction (butyrate) }\end{array}$ & $\mathrm{C}_{3} \mathrm{H}_{7} \mathrm{COO}^{-}+2 \mathrm{H}_{2}+\mathrm{H}^{+} \rightarrow \mathrm{C}_{3} \mathrm{H}_{7} \mathrm{CH}_{2} \mathrm{OH}+\mathrm{H}_{2} \mathrm{O}$ & -16.4 & -27.9 & -5.0 \\
\hline $\begin{array}{l}\text { Combined: Hydroxyl-carboxyl } \\
\text { exchange }\end{array}$ & $\mathrm{C}_{3} \mathrm{H}_{7} \mathrm{COO}^{-}+\mathrm{CH}_{3} \mathrm{CH}_{2} \mathrm{OH} \rightarrow \mathrm{C}_{3} \mathrm{H}_{7} \mathrm{CH}_{2} \mathrm{OH}+\mathrm{CH}_{3} \mathrm{COO}^{-}$ & -6.8 & -29.6 & 16.0 \\
\hline
\end{tabular}

The gradual increase of $\mathrm{i}-\mathrm{C}_{4} \mathrm{OH}$ formation during phase III and the increased alcohol formation in phase IV compared to phase II indicate that this additional bioconversion capability had slowly become more prominent within microbiome. Consequently, the $\mathrm{p}_{\mathrm{H} 2}$ did not recover in phase IV to the earlier values in phase II ( $9.2 \pm 1.3 \mathrm{kPa})$ after reducing the $\mathrm{CO}_{2}$ load; it was kept in a lower range $(0.6 \pm 0.4 \mathrm{kPa}$ in phase IV) by the microbiome, while alcohol formation spiked. The alcohol formation likely acted as an alternative electron sink when methane formation had dropped due to the sudden lower availability of $\mathrm{CO}_{2}$, as was also observed previously 240 . 
The onset of the alcohol formation implies that the earlier achieved high selectivity towards $\mathrm{i}_{-} \mathrm{C}_{6}$ in phase II could be transient. A low acetate concentration is used as steering parameter in this research to increase the selectivity towards $\mathrm{i}-\mathrm{C}_{4}$ elongation. However, in combination with high ethanol and high other carboxylate amounts, a low acetate concentration leads to a thermodynamic potential that allows an alternative source of in situ acetate formation via hydroxyl-carboxyl exchange.
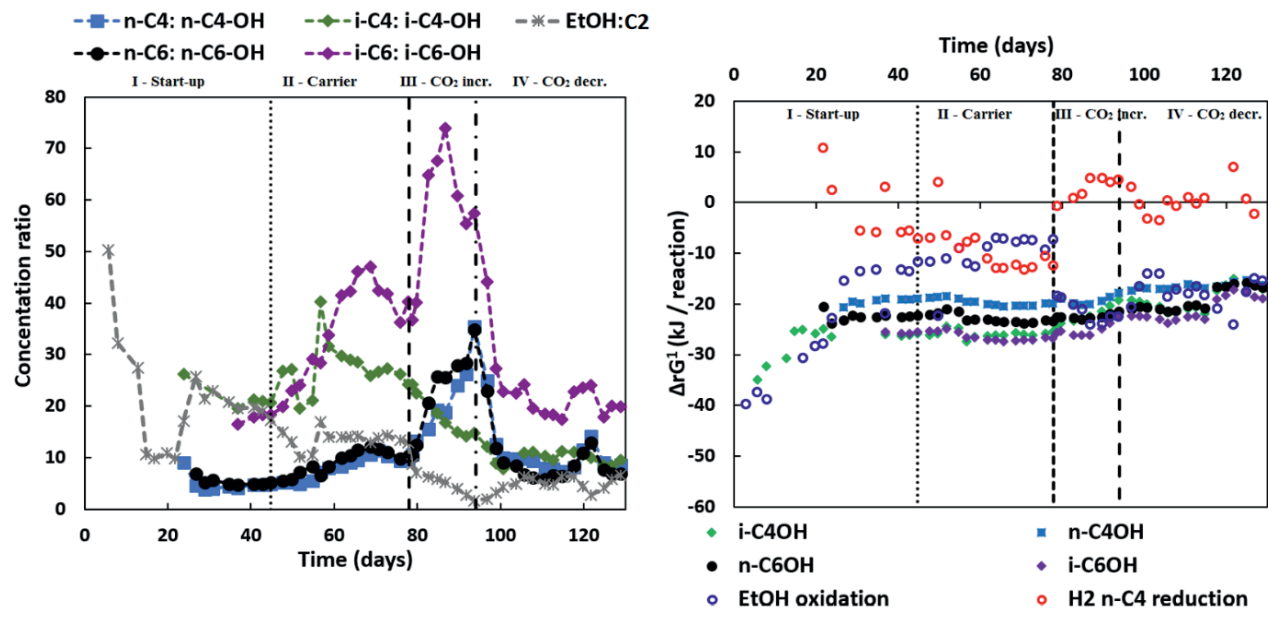

Figure 5. A) The concentration ratios of carboxylates to corresponding alcohols (and ethanol to acetate) are shown. These ratios seem to be inversely correlated to the ethanol to acetate ratio, especially in the later phases (III and IV), except for the $\mathrm{i}-\mathrm{C}_{4}$ to $\mathrm{i}-\mathrm{C}_{4} \mathrm{OH}$ ratio. Isobutyrate was continuously fed into the reactor and present at high concentrations in the broth. This would contribute to the driving force of isobutanol formation, while lessening the inverse correlation of the $\mathrm{i}-\mathrm{C}_{4}$ to $\mathrm{i}-\mathrm{C}_{4} \mathrm{OH}$ ratio to the ethanol to acetate ratio. $\left.\mathrm{B}\right)$ The Gibbs free energy change $\left(\Delta \mathrm{rG}^{1}\right)$ is shown for the reactions: Ethanol + Carboxylate $\rightarrow$ Acetate + Corresponding Alcohol (for $\mathrm{iC}_{4} \mathrm{OH}, \mathrm{n}-\mathrm{C}_{4} \mathrm{OH}, \mathrm{n}-\mathrm{C}_{6} \mathrm{OH}$ and $\mathrm{i}-\mathrm{C}_{6} \mathrm{OH}$ ), direct ethanol oxidation and hydrogenotrophic butyrate reduction. The Gibbs free energy change was adjusted for the conditions (including $\mathrm{pH}$ and hydrogen partial pressure for ethanol oxidation and hydrogenotrophic reduction) in the reactor. The combined hydroxyl-carboxyl exchange reaction seems to remain stable in the range of -15 to $25 \mathrm{~kJ}$ reaction ${ }^{-1}$. The used Gibbs formation energies and assumption for the calculations are given in Table S6. 


\section{Outlook for further bioprocess development}

Chain elongation microbiomes can be engineered to produce various chemicals depending on the supplied feedstock and steered reactor conditions. The higher branched and straight alcohol formations described in this research are thermodynamically dependent on the reactant to product ratio. If the products could be removed in situ this would drive the reaction towards more straight and branched alcohol formation. Increasing the alcohol formation in this way could lead to an interesting biochemical production process in itself; the observed alcohol titers are in a suitable range for in situ extraction via gas stripping 244. This process may be used to develop processes that upgrade the ethanol in dilute ethanol-containing residue streams to higher alcohols.

Branched carboxylates such as $\mathrm{i}-\mathrm{C}_{4}$ and apparently also $\mathrm{i}-\mathrm{C}_{5}$ can be used as electron acceptor during chain elongation fermentations with a varying degree of efficiency. Operating the reactor under acetate and $\mathrm{CO}_{2}$ limited conditions increases the selectivity towards branched carboxylate elongation, but as a tradeoff overall chain elongation activity is reduced. The conversions of branched carboxylates to longer chains seem only to occur as a form of co-metabolism during straight chain elongation. It remains to be seen if the co-metabolism, that is expressed as a dependency on straight chain elongation activity, can be lifted. Acetate plays a pivotal role within the chain elongation metabolism as it can both serve as a primer and elongation (acetyl-CoA) unit ${ }^{260}$. However, research has already shown that it is possible to increase the affinity of butyrate relative to acetate for an engineered thiolase ${ }^{261}$, as well as first efforts to modify thiolases to use branched carboxylates as primers ${ }^{262}$. Further efforts to tailor the thiolase and other involved enzymes via metabolic engineering could offer perspectives where the $k_{c a t}$ and $K_{m}$ values for branched carboxylates and their conversion intermediates are increased.

Production of $\mathrm{i}-\mathrm{C}_{7}$ in the observed amounts in this study at this stage are unattractive for direct industrial applications compared to the formation of $n-C_{6}$. Still with fractional distillation of the produced broth significant amounts of $\mathrm{i}-\mathrm{C}_{7}$ may be obtained. In the bioreactors $\mathrm{i}-\mathrm{C}_{5}$ is hardly elongated ( $98 \%$ remains unconverted) in the case where it is supplied in excess and acetate is only present in low amounts. So far, it is remarkable that $\mathrm{i}-\mathrm{C}_{4}$ elongation has different kinetics compared to $\mathrm{i}-\mathrm{C}_{5}$ elongation. It potentially shows that the involved microbiome has not developed fully optimized enzymes for the artificially imposed selective pressure with low amounts of acetate and large amounts of alternative electron acceptors. In addition to metabolic engineering approaches further research on selection pressure and natural adaptation in open culture microbiomes can provide a potential $\mathrm{i}-\mathrm{C}_{7}$ bioprocess development utilizing organic residual streams. 


\section{Supporting Information}

The Supporting Information is available in the Supporting Information Section of this thesis. Additional information regarding Materials \& Methods and Results \& Discussion, 12 figures and 6 tables. 


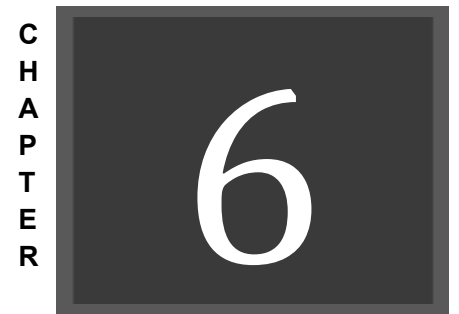

\section{General Discussion}




\section{Introduction}

In this thesis we explored how open-culture microbiomes can be enriched for the production of branched small and medium chain carboxylates and alcohols. The research chapters can be categorized by the two main metabolic functionalities that were the focus of enrichment: (i) methanol-based chain elongation and (ii) ethanol-based chain elongation. In this chapter the most important findings of each study are highlighted. Then the studies are reevaluated in the context of the microbial activity and reactor performances. Finally biotechnological implementations of both types of fermentations are discussed and how these can contribute to circular economy principles. All researches were performed using synthetic defined media that contained fixed amounts of carboxylates (used as electron acceptor) and alcohols (used as electron donor). Further development of the discovered processes into useful technologies would require applying the knowledge from this thesis to engineer case-tailored solutions for recycling appropriate organic residue streams to useful and valuable products.

\section{Methanol based chain elongation of acetate and propionate to iso/n-butyrate and n-valerate}

The first two research chapters entailed the enrichment of methanol-based chain elongation microbiomes. In Chapter 2 formation of $n$-butyrate and iso-butyrate via the elongation of acetate with methanol was elucidated. We showed that the microbiomes could be enriched in multiple ways to control the product spectrum using $\mathrm{pH}$ as a selection pressure tool. At $\mathrm{pH} 6.75$ most of the fermentation product was $\mathrm{n}$-butyrate, whereas at a $\mathrm{pH} 5.2$ the formation of the butyrate species approached a ratio of $0,69 \mathrm{i}-\mathrm{C}_{4}$ to $0.31 \mathrm{n}-\mathrm{C}_{4}$, determined by the thermodynamic equilibrium of isomerization. Chapter 3 showed that methanol-based propionate elongation led to mainly $n$-valerate formation and no significant amounts of branched valerates. Using an influent with both acetate and propionate led to simultaneous isobutyrate, $n$-butyrate, $n$-valerate and $n$-caproate formation. The results suggested that Clostridium luticellarii is responsible for methanolbased chain elongation of both acetate and propionate in the investigated reactor.

\section{A revised hypothesized metabolic pathway for Clostridium Iuticellarii}

A hypothetical metabolic pathway for methanol-based chain elongation was proposed in Figure 5 of Chapter 3. Although it was suggested that the ATP yield of overall energyproviding metabolism should be around 1.5 ATP reaction ${ }^{-1}$, the proposed pathway did not show this. Moreover, the mechanism for $\mathrm{CO}$ formation was ambiguous and possible electron bifurcation steps during the pent-2-enoyl-CoA reduction were proposed but 
excluded from the figure. In Figure $\mathbf{1}$ of this section a revised metabolic pathway is proposed. The figure is adapted to generalize the methanol based chain elongation mechanism. Additionally the isomerization step is added; for now this has only been verified for $\mathrm{n}$-butyrate/isobutyrate isomerization. The proteome of $C$. luticellarii was checked for enzymes that are required for performing the proposed conversions. Indeed, NCBI protein-protein BLAST results verify that $C$. luticellarii harbours the genes necessary for conducting conversions of both the Wood-Ljungdahl pathway and the reverse betaoxidation pathway (NCBI protein-protein BLAST results are shown in Table S1 and S2 in the Appendix of this chapter).

It is important to emphasize that the hypothetically proposed pathway is by no means proven. To verify the pathway, extensive experimentation is necessary. Pure culture experiments with the $C$. Iuticellarii strain using $C_{13}$ labeled substrate could help track intermediate formations and thereby quantize the metabolic fluxes ${ }^{263}$. Additionally, the identified genes should be tested for their actual functionality; extraction of enzymes and performing activity assays could be used to experimentally reconstruct and verify individual elements of the proposed pathway 264,265 . 


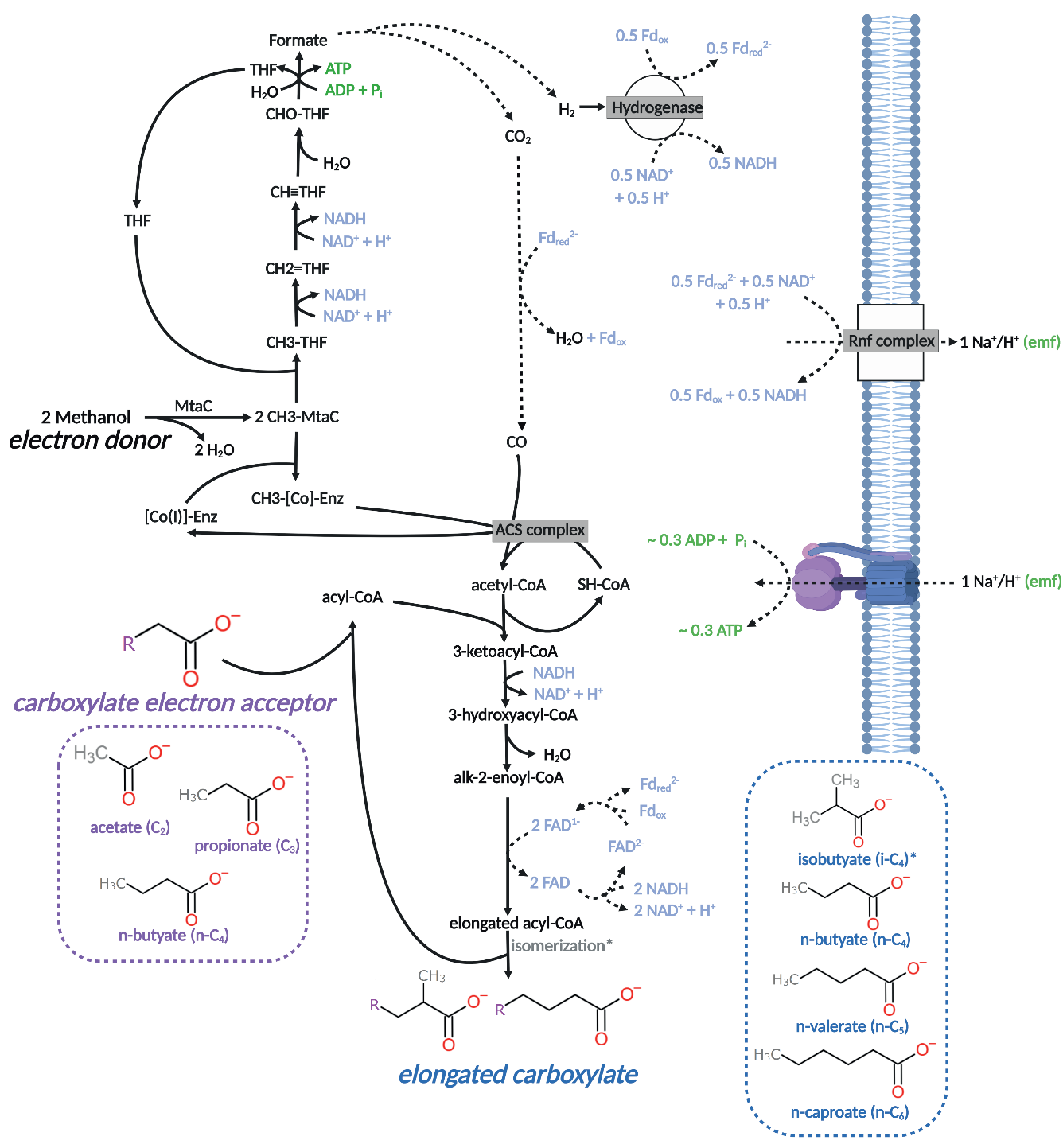

Figure 1. Revised hypothesized methanol-based chain short-chain carboxylate elongation pathway. The figure shows how the Wood-Ljungdahl pathway could be coupled to the reverse beta-oxidation pathway, while conserving electron mediator homeostasis and providing a source for ATP formation. It shows an extension to Figure 5 in Chapter 3 by incorporating electron bifurcation at the alk-2enoyl-CoA reduction step, similar to how it is suggested to occur during reverse-beta oxidation in Clostridium kluyveri ${ }^{31}$. By introducing this electron bifurcation step, a small electromotive force can be established. Consequently the overall ATP yield approaches 1.3 ATP reaction $^{-1}$, using the same efficieny for the Rnf complex and ATPase as proposed for Acetobacter woodii ${ }^{30}$. The ATP yield is approximately in line with the expected yield when considering the $\Delta_{r} G$ of the reaction $(-106.1 \mathrm{~kJ}$ reaction ${ }^{-1} / 70 \mathrm{~kJ} \mathrm{ATP}^{-1} \approx 1.5$ ATP reaction $^{-1}$ ). 


\section{Outlook for methanol based chain elongation: Improving reactor performance and conceiving potential applications}

The developed methanol-based chain elongation processes showed high product selectivities for isobutyrate, n-butyrate and n-valerate formation. However, there are many more factors that determine if and how methanol-based chain elongation can become an effective, economically viable, recycling technology. These factors include and are not limited to: (i) the extend of substrate conversion, (ii) volumetric conversion rates, (iii) product titers and, (iv) not unrelated to the previous point, the difficulty of downstream processing (DSP). The process performance for points i to iii for the methanol-based chain elongation reactors is shown in Table $\mathbf{1}$.

Table 1. Reactor performance parameters for the investigated methanol-based chain elongation reactors.

$\begin{array}{llll}\text { Phase V, pH } & \text { Phase VII, } & \text { Phase IV } & \text { Phase VI } \\ 6.75 & \text { pH } 5.2 & \text { (pro), pH } & \text { (pro+ac), } \\ \text { (Chpt. 2) } & \text { (Chpt. 2) } & 5.5 \text { (Chpt. 3) } & \text { pH } 5.8\end{array}$

(Chpt. 3)

\begin{tabular}{|c|c|c|c|c|}
\hline Unconverted acetate & $61 \%$ & $60 \%$ & - & $49 \%$ \\
\hline Unconverted propionate & - & - & $74 \%$ & $46 \%$ \\
\hline Unconverted methanol & $24 \%$ & $46 \%$ & $29 \%$ & $18 \%$ \\
\hline $\begin{array}{l}\text { Volumetric n-butyrate } \\
\left.\text { productivity (g L } L^{-1} \text { day }^{-1}\right)\end{array}$ & $3.1 \pm 0.3$ & $0.9 \pm 0.3$ & $0.020 \pm 0.003$ & $0.23 \pm 0.008$ \\
\hline $\begin{array}{l}\text { Vvolumetric i-butyrate } \\
\left.\text { productivity (g L } L^{-1} \text { day }^{-1}\right)\end{array}$ & $0.10 \pm 0.04$ & $2.4 \pm 0.3$ & $0.055 \pm 0.007$ & $0.48 \pm 0.015$ \\
\hline $\begin{array}{l}\text { Volumetric n-valerate } \\
\left.\text { productivity (g L }{ }^{-1} \text { day }^{-1}\right)\end{array}$ & - & - & $0.99 \pm 0.05$ & $1.18 \pm 0.02$ \\
\hline $\begin{array}{l}\text { n-butyrate broth } \\
\text { concentration }\left(g L^{-1}\right)\end{array}$ & $4.5 \pm 1.0$ & $1.6 \pm 0.2$ & $0.09 \pm 0.01$ & $0.86 \pm 0.03$ \\
\hline $\begin{array}{l}\text { i-butyrate broth } \\
\text { concentration }\left(g L^{-1}\right)\end{array}$ & $0.5 \pm 0.1$ & $4.2 \pm 0.6$ & $0.22 \pm 0.01$ & $1.80 \pm 0.05$ \\
\hline $\begin{array}{l}\text { n-valerate broth } \\
\text { concentration }\left(g L^{-1}\right)\end{array}$ & - & - & $3.9 \pm 0.1$ & $1.37 \pm 0.08$ \\
\hline
\end{tabular}




\section{Biomass retention could aid in increasing conversion rates, but creates additional challenges of maintaining selection pressure}

Significant improvements can still be made regarding the extend of substrate conversion. In most phases, sufficient substrate was still available in the reactors for additional conversion. Likely, the reactor performance was limited by the amount of active biomass in the system. Volumetric conversion rates were relatively low in comparison to what was achieved for ethanol-based chain elongation to $\mathrm{n}$-caproate $\left(46.5 \mathrm{~g} \mathrm{n}-\mathrm{C}_{6} \mathrm{~L}^{-1} \text { day }^{-1}\right)^{229}$. As was already shortly discussed in chapter 2 and 3, the conversion rates could be improved via accumulation of the biocatalyst via biofilm retention techniques. Biomass retention could create additional challenges in maintaining the right selection pressure. The openculture fermentations with methanol-based chain elongation always showed a competition for methanol as substrate between the chain elongators and methylotrophic methanogens. Chain elongation activity was kept dominant by a combination of factors: (i) high substrate concentrations, (ii) high volatile fatty acids concentrations, (iii) a low $\mathrm{pH}$ and (iv) a relatively low HRT. By allowing growth of biofilms, however, microbes are better able to protect themselves from adverse effects of the environment ${ }^{266}$. Moreover, they are able to proliferate in the reactor at low growth rates with less risk of washout ${ }^{267}$. Allowing biofilm retention techniques could assist in acquiring a higher volumetric productivity and possibly also higher product titers. But, further experimentation will need to explore whether methanol-based chain elongation dominance can then still be maintained. A positive sign that this should be possible are the results from Chapter 2 of phase VI and VII - reduction of operating $\mathrm{pH}$ from 5.5 to 5.2. Here, methane formation had dropped strongly by $90 \%$, whereas chain elongation was only slight affected, suggesting that methanol-based chain elongators are more suited than the methylotrophic methanogens to operate at that respective $\mathrm{pH}$ and undissociated acid concentration.

\section{Operation at low pH invites in situ extraction for n- valerate}

The product titers were relatively low compared to what is needed for economically viable DSP. In general high product titers in the range of $>50 \mathrm{~g} \mathrm{~L}^{-1}$ are necessary to facilitate efficient DSP, especially for short-chain fatty acids ${ }^{268}$. Depending on the chain length, as well as on the desired final product, lower titers might also suffice ${ }^{269}$. For the investigated methanol-based chain elongation process, $n$-valerate was the longest observed main chain elongation product. Recent advances on in situ extraction methods suggest that $n$-valerate (and longer chains) could be efficiently extracted in situ at $\mathrm{pH}$ values ( $\sim 5.5)$ that still allow methanol based chain elongation ${ }^{270}$. Experiments performed with ethanol-based chain elongation of glycerol-derived propionate showed successful n-valerate production and 
simultaneous extraction 271,272 . The achieved $n$-valerate titers and conversion rates within these fermentations were comparable to the rates and titers achieved in this thesis for methanol-based chain elongation. Methanol-based $n$-valerate formation could provide an interesting alternative to ethanol-based chain elongation, depending on the cost differences for methanol and ethanol which can strongly vary depending on the geographical location 273 . The fact that methanol is used during biodiesel (and glycerol residue) production 274 presents an opportunity that could favor valorization methanolbased chain elongation, provided that its cost-effectiveness outweighs alternative recycling methods.

\section{Methanol-based chain elongation in multi-step production schemes}

Performing direct cost-effective DSP for n-butyrate and iso-butyrate production from these fermentations will likely be challenging. Concentration steps via reverse osmosis or evaporation (after centrifugation and filtrations) might be necessary before purification of the compounds can be efficiently performed ${ }^{126,275}$. An alternative to purification of $n$ - and isobutyrate would be to utilize the fermentation broth for subsequent fermentations and further upgrade the compounds. The short chain carboxylates could be converted towards their corresponding alcohols or towards medium chain fatty acids via ethanol-based chain elongation methods presented in Chapter 4 and 5 . The presence of both n-butyrate and isobutyrate in the broth, however, will affect the selectivity of ethanol-based isobutyrate elongation. Likely, first all n-butyrate will be converted before significant amounts of isobutyrate are elongated to isocaproate, due to preferential/kinetic differences within the chain elongation metabolism (Chapter 4).

An alternative method to concentrate the products of methanol-based chain elongation would be to utilize the fermentation effluent for polyhydroxyalkanoate (PHA) production. In this scheme the carboxylates in the effluent are transferred to an accumulation reactor where Plasticicumulans acidivorans-like bacteria can concentrate the compounds as PHA in their cell mass ${ }^{276}$. This will introduce complexities such as that the effluent needs to be nitrogen-limited to maintain the feed-famine regimes that are used to enrich the accumulation reactor ${ }^{277}$. The methanol-based chain elongation experiments in this thesis were performed with an overabundance of nitrogen in the broth, so it will need to be researched if these fermentation can also be performed using less ammonium. PHA production is attractive concept to valorize very low cost and low quality organic residue streams. It remains to be seen if methanol-based chain elongation can compete on an economic and sustainability level with such anaerobic digestion methods. The DSP of PHA, however, is also challenging in itself and currently subject of many research attempts to increase efficacy ${ }^{278}$. 
An interesting property of methanol based chain elongation, is that it provides a method to form n-butyrate from acetate and methanol when fermenting at $\mathrm{pH} 6.75$ (Chapter 2). A next research step would be to investigate whether an enriched microbiome could also achieve similar $\mathrm{n}$-butyrate productivities and titers from methanol and $\mathrm{CO}_{2}$ alone. Methanol, in turn could be produced via aqueous electrochemical reduction of $\mathrm{CO}_{2}$ using sustainable electricity sources 279, 280. An advantage would be that the electrochemically derived aqueous methanol solution would not need DSP before entering the fermentation. Combining the electrochemical reduction of $\mathrm{CO}_{2}$ to methanol with methanol based chain elongation in that way could provide an effective carbon sequestration process that allows the formation of longer-chain reduced carbon compounds.

\section{Ethanol-based branched carboxylate elongation versus ethanol oxidation and straight-chain elongation}

The third and fourth research chapters focused on ethanol based chain elongation using branched short chain ( $\mathrm{i}-\mathrm{C}_{4}$ and $\mathrm{i}-\mathrm{C}_{5}$ ) carboxylate as electron acceptors. In chapter 4 isobutyate elongation towards isocaproate was achieved. Microbiome analysis showed that a Clostridium kluyveri species is likely responsible for the observed chain elongation activity. The elongation of isobutyrate occurred concomitant with the formation of straight chained carboxylates due to in situ produced acetate; the main product in all researched ethanol based fermentation remained straight-chained $n$-caproate. In Chapter 5 the selectivity of isobutyrate was increased by introducing acetate and $\mathrm{CO}_{2}$ limitations. However, a drawback of this strategy was reduction of the overall chain elongation rate. This research indicated that small amounts of $\mathrm{i}-\mathrm{C}_{5}$ could also be elongated, albeit at significantly lower rates than $\mathrm{i}-\mathrm{C}_{4}$ elongation. It was proposed that branched chain elongation occurs only as cometabolism to straight chain elongation and is reliant on sufficient ethanol oxidation towards acetate. Coupled to ethanol oxidation, in some cases carboxylate reduction towards the corresponding alcohols was observed. This carboxylate reduction seemed to be driven by a high substrate to products ratio of two coupled reactions: ethanol oxidation and hydrogenotrophic carboxylate reduction ${ }^{259}$.

\section{Reevaluation of ethanol oxidation during ethanol-based chain elongation in view of its versatile stoichiometry}

The degree of ethanol oxidation within chain elongation, while it is coupled to reverse-beta oxidation is suggested to be versatile ${ }^{22}$. Depending on the substrate and product concentrations the chain elongators can apply different strategies to maximize the energy yield of the conversions, to keep its metabolism going and to maintain competitive conversion rates. As presented in the introduction, the stoichiometry of chain elongation 
can shift depending on (i) possibility to use electron bifurcation for electromotive force buildup and (ii) the need for electron confurcation towards NADP ${ }^{+} /$NADPH reduction (Refer back to Box 1 in the introduction). In the first case $(n=5, p=1)$ ATP yield for the entire reaction is predicted to be around 2.5 ATP reaction ${ }^{-1}, 1$ ATP coming from substrate level phosphorylation (SLP) and 1.5 ATP coming from an electromotive force-driven ATPase 22 . In the second case $(n=4, p=1)$ the ATP yield is suggested to be 1 ATP reaction ${ }^{-1}$, its only source for production being SLP as no electromotive force is generated. The overall stoichiometry for ethanol-based chain elongation is given by equation (1) and (2).

$\left(n_{c 2}+p\right) \mathrm{CH}_{3} \mathrm{CH}_{2} \mathrm{OH}+\left(n_{c 2}-p\right) \mathrm{CH}_{3} \mathrm{COO}^{-} \rightarrow n_{c 2} \mathrm{C}_{3} \mathrm{H}_{7} \mathrm{COO}^{-}+2 p \mathrm{H}_{2}+\left(n_{c 2}-p\right) \mathrm{H}_{2} \mathrm{O}+p \mathrm{H}^{+}$

$\left(n_{c i}+p\right) \mathrm{CH}_{3} \mathrm{CH}_{2} \mathrm{OH}+n_{c i} \mathrm{C}_{x} \mathrm{H}_{2 x+1} \mathrm{COO}^{-} \rightarrow n_{c i} \mathrm{C}_{x+2} \mathrm{H}_{2(x+2)+1} \mathrm{COO}^{-}+2 p \mathrm{H}_{2}+\left(n_{c i}-p\right) \mathrm{H}_{2} \mathrm{O}+p \mathrm{H}^{+}+$ $p \mathrm{CH}_{3} \mathrm{COO}^{-}$

However, besides these two scenarios, another stoichiometry was proposed where additional ethanol oxidation could provide an increased ATP yield during a high ethanol to acetate ratio ${ }^{22}$. In this case $p=3.33$ and $n=3.33$, and during acetate elongation all acetate is formed in situ by the ethanol oxidation step and elongated to $n$-caproate. This results in an overall stoichiometry shown in equation (3).

10 Ethanol $\rightarrow 3.33$ n-Caproate- $+3.33 \mathrm{H}++6.67 \mathrm{H} 2+3.33 \mathrm{H} 2 \mathrm{O}(+3.33 \mathrm{ATP})$

For each reverse beta-oxidation cycle, the chain elongator then metabolizes a higher amount of ethanol. This change in stoichiometry has partly been experimentally verified (and extended to $n$-caprylate formation) and is thought to occur at very high ethanol to carboxylate ratios 255 . Consequently this stoichiometric versatility necessitates a reevaluation of the calculated amounts of excessive ethanol in this thesis. In chapter 5 the ethanol to acetate ratios were especially high and could have been triggered such a stoichiometric shift. The introduction of an externally provided carboxylate creates adds complexity in evaluating the stoichiometry of chain elongation, as an extra driving force is competing with acetate elongation. How the stoichiometry is affected then relies on kinetic differences of the different conversions. Potentially, an overestimation of the calculated "excessive ethanol oxidation" is the result of the chain elongators themselves adjusting the stoichiometry of conversion. A way to establish the true overall stoichiometry of chain elongation would require tracking all electron equivalents, including correcting for biomass yield. Such investigation would need further experimentation with high measurement resolutions, because the required stochiometric analysis is sensitive to measurement errors. 


\section{Exploring branched chain elongation co-metabolism in view of thermodynamics and dependency on acetate availability}

The availability of acetate plays a pivotal role in the progression of ethanol-based chain elongation activity 22,255 . Acetate can be formed during chain elongation of other electron acceptors besides acetate, however, its formation was not always sufficient to keep branched carboxylate chain elongation ongoing without continuous in situ formation of acetate. This is illustrated by the batches performed with isovalerate as electron donor in Chapter 5 . Figure 2 shows the calculated $\Delta_{r} G$ for straight chain elongation and isovalerate elongation within two batches that were discussed in chapter 5: batch 2.A, with low initial acetate and batch 2.B, with high initial acetate. A driving force is present for isovalerate elongation in all cases. However, the batch with low initial acetate amounts stagnates nonetheless. In contrast, the batch with high initial acetate amounts proceeds to continue chain elongation (mainly straight chains $n-C_{4}$ and $n-C_{6}$ ) until the $\Delta_{r} G$ of the reaction approaches the threshold for 1 ATP. At that point SLP is suggested to be the only source of ATP generation, because the confurcating mechanism prevents the formation of an electromotive force due to the necessary NADPH formation ${ }^{22}$; $-72 \mathrm{~kJ}$ reaction ${ }^{-1}$ becomes the minimum threshold for ethanol-based chain elongation to conserve energy. Chain elongation in the non-acetate limited batch is able to proceed completely towards eventual mechanistic limitation for energy conservation, while ethanol was still available.

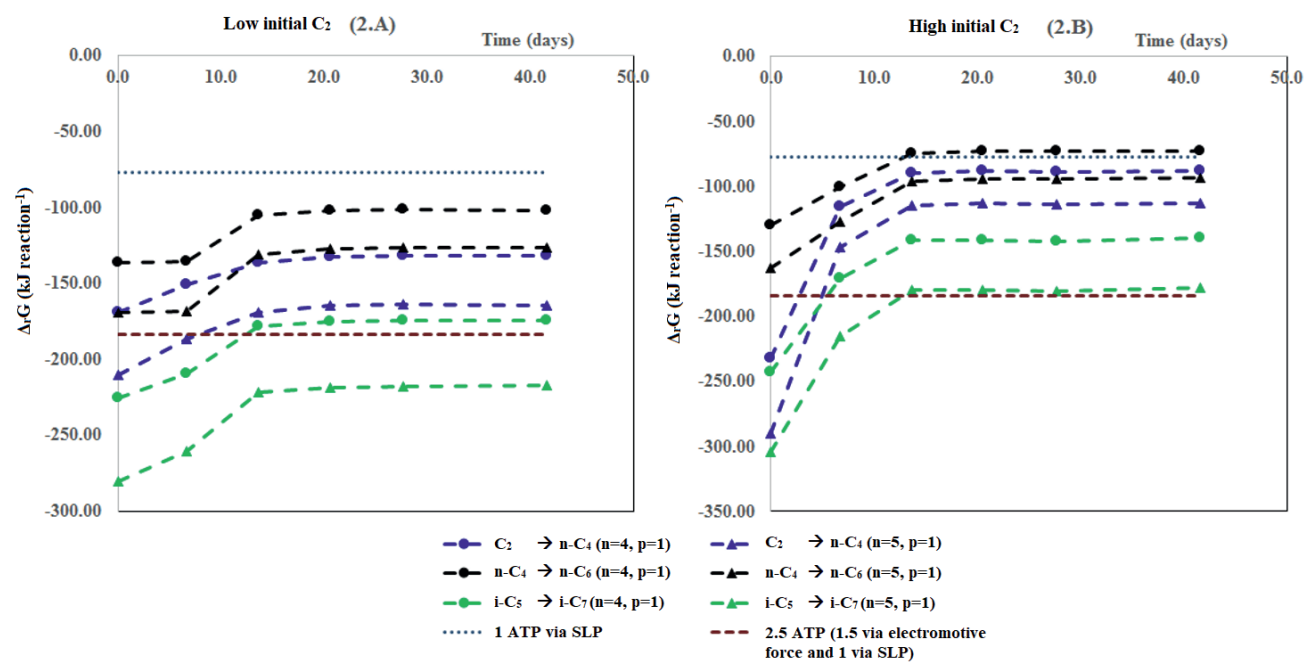

Figure 2. Thermodynamic calculations for observed ethanol-based chain elongation in Batch 2.A and 2.B, Chapter 5, corrected for the measured reactor conditions (e.g. extracellular metabolite concentrations, headspace partial pressure of $\mathrm{H}_{2}$ ). For the calculations the stoichiometries for a 2.5 
ATP yield $(n=5, p=1)$, as well as for a 1 ATP yield were used. It was assumed that chain elongation of branched compounds ( $\mathrm{i}-\mathrm{C}_{5}$ in this case) occurs via the same stoichiometry. Due to the lack of thermodynamic data on isoheptanoate, the $\Delta_{r} \mathrm{G}^{0}$ for $\mathrm{i}-\mathrm{C}_{5}$ elongation to $\mathrm{i}-\mathrm{C}_{7}$ was assumed to be the same as that of $n-C_{4}$ elongation to $n-C_{6}$. Although as result there could be an error of a few $k J$, the effects of the reactor conditions on the corrected $\Delta_{r} G^{1}$ will be sufficiently representative.

In contrast, the catabolic reaction energies for branched chain elongation were far more exergonic $\left(<<-72 \mathrm{~kJ}\right.$ reaction $\left.{ }^{-1}\right)$, and must have been inhibited by other factors. In chapter 5 it was discussed that the inhibition in this case could not have been caused by limited $\mathrm{CO}_{2}$ availability or $\mathrm{pH}$. The fact that the thermodynamic calculations for branched carboxylate chain elongation in the batches show feasible $\Delta_{\mathrm{r}} \mathrm{G}$ values but nonetheless no branched chain elongation occurs without concomitant straight chain elongation, suggests that there are physiological limitations that may arise from the differences in kinetic parameters.

\section{Exploring branched chain elongation co-metabolism in view of kinetics and intracellular intermediate concentrations - hypothesizing a negative feedback loop}

Acetate consumption due to straight chain elongation hypothetically outpaces acetate formation from branched chain elongation and can lead to limiting intracellular concentrations of chain elongation intermediates (e.g. acetyl-CoA), despite the presence of abundant alternative electron acceptors such a branched carboxylates and sufficient thermodynamic driving force. For simplicity only the effect of intracellular acetyl-CoA is considered with $\mathrm{K}_{\mathrm{m}, \mathrm{COA}}$ being the affinity constant during the Claisen condensation step. $\mathrm{A}$ negative feedback loop could occur when kinetic parameters for straight chain elongation

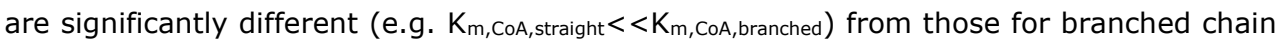
elongation. Straight chain elongation would cause acetate to be consumed down to levels where branched chain elongation is significantly inhibited; the resulting intracellular concentrations of acetyl-CoA ( $<<\mathrm{K}_{\mathrm{m}}, \mathrm{CoA}$, branched) could become too low for reactions with the branched carboxylates as electron acceptor to proceed, causing a hampering of the whole catalytic machinery that is the primary source for acetate/acetyl-CoA formation. Consequently, although there might be a high driving force for branched chain elongation, it can only occur as cometabolism during straight chain elongation; branched chain elongation does occur, but only during periods of straight chain elongation, when intermediate concentrations are temporarily higher than during stationary phase. A premise of this hypothesis is that acetyl-CoA levels are elevated during chain elongation activity and slow down during stagnation of the energy metabolism. This explained cometabolism can evidently be observed during acetate limitation and high hydrogen partial pressures that inhibit direct ethanol oxidation towards acetate. A contrasting case, with an elevated hydrogen pressure where solely propionate and ethanol were provided as respective electron acceptor and donor, has shown continued chain elongation to $n$ - 
valerate and $\mathrm{n}$-heptanoate, despite the absence of initial acetate ${ }^{258}$. It indicates that differences for kinetic parameters between propionate and acetate elongation are smaller than the proposed kinetic differences between branched (i- $C_{5}$ in particular) and straight chain elongation in this research.

\section{Outlook for ethanol-based branched carboxylate chain elongation in industry}

The developed ethanol-based branched carboxylate elongation process provides the first steps towards a biorefinery capable of producing isocaproate. With volumetric productions rates of $0.86 \mathrm{~g} \mathrm{i}^{-} \mathrm{C}_{6} \mathrm{~L}^{-1}$ day $^{-1}$ (Chapter 4 ) and $1.2{\mathrm{~g} \mathrm{i}-\mathrm{C}_{6} \mathrm{~L}^{-1} \text { day }}^{-1}$ (Chapter 5) the performance of the reactor was a few times lower than that of an earlier researched oddchain elongation reactor system that was designed to produce heptanoate from propionate (4.5 $\mathrm{g} \mathrm{n}^{-} \mathrm{C}_{7} \mathrm{~L}^{-1} \mathrm{day}^{-1}$ ) ${ }^{192}$. Possibly, by following similar strategies employed to increase volumetric productivity - e.g. facilitate biomass retention and decrease the HRT 229, 249isocaproate productivity can also be increased. Evidently the introduction of biofilms results in a selection pressure that can lead to more ethanol oxidation (and other associated conversions). Consequently this will likely affect selectivity of branched-chain elongation, but it is a tradeoff that needs to be evaluated when designing a commercial industrial process.

A recent insight is that the ethanol to carboxylate ratio is an important factor that affects selectivity of odd-chain elongation selectivity ${ }^{258}$. That research shows that as the ethanol to carboxylate (propionate in their case) ratio approaches 0.5 , chain elongation selectivity of the odd-chain carboxylates increases significantly. Their results seem to show that propionate elongation towards $n$-valerate can approach the theoretical maximum amount where $\sim 83.3 \%$ of the ethanol $\left(5 / 6^{\text {th }}\right)$ is used for elongation of propionate alone (assuming that the remaining $1 / 6^{\text {th }}$ ethanol is being used for SLP-driven acetate formation and excluding biomass formation). Usage of this strategy for branched chain elongation could similarly lead to higher branched carboxylate conversion selectivities. Consequently rates may be negatively affected, and a lower ethanol to carboxylate ratio would lead to less alcohol formation coupled to ethanol oxidation. Again, deciding upon how to utilize these sensitive process-determining operating conditions for industrial use will depend on the desired end-products and accompanying DSP methods.

\section{Concluding remarks}

Open culture fermentation offer potential to discover new metabolic functionalities that could aid in the development of new processes. At the start of this thesis it was largely unknown if and how branched carboxylates could be formed via chain elongation fermentations. Isobutyrate formation was considerably uncovered: (i) the sole substrates 
were identified, (ii) the key microbial player (C. luticellarii) was identified and (iii) a successful selection pressure was applied to enrich the culture to the point that isobutyrate was formed according to thermodynamic equilibrium of isomerization from n-butyrate. Furthermore an efficient $n$-valerate production method via methanol-based chain elongation was developed. In the realm of ethanol-based chain elongation isocaproate formation was discovered as well formation of isoheptanoate. This has led to more insights into the versatility of ethanol-based chain elongation microbiomes and of the associated metabolic functionalities.

Very recently branched-chain carboxylate production (i- $\mathrm{C}_{6}, \mathrm{i}-\mathrm{C}_{7}$ and $\mathrm{i}-\mathrm{C}_{8}$ ) was for the first time achieved using waste biomass as substrate supplemented with ethanol ${ }^{281}$. Interestingly, this study shows that also i-C8 was formed during the fermentation, which further expands the ethanol-based chain elongation product spectrum beyond what was discovered in this thesis. Due to the nature of chain elongation leading to a mixture of compounds in the broth, especially in the case when branched electron acceptors are introduced, an industrial process would likely need to incorporate DSP schemes that lead to (i) either a commercial product wherein the carboxylates are present in a mix or (ii) will require separating the compounds via e.g. distillation or chromatography techniques ${ }^{126}$, 269,282 . This thesis focused on the fermentation aspect of chain elongation biorefineries. However, the cost-effectiveness of DSP will also largely determine if and how branched medium chain carboxylate production can be realized on an industrial scale. Moreover, the introduction of new biochemicals require industrial purchasers that design commercial products and require the usage of these newly introduced biochemicals as resource.

By expanding the available knowledge on energy efficient resource recovery methods, chain elongation fermentation technology can act as a new catalyst to more efficiently recycle resources and to create value. The knowledge presented in this thesis contributes to the field of biological resource recovery methods to enable a more sustainable environment on this planet. Further research and applications of the developed processes should align with the Sustainable Development goals set by the United Nations to facilitate a smooth transition towards a circular economy. 
To explore the potential of nature to improve the quality of life. ${ }^{283}$ 


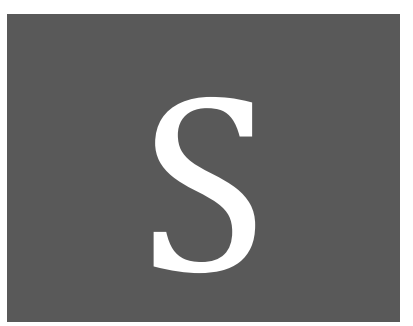

\section{Summary}




\section{Summary}

In order to achieve a sustainable society it is paramount to develop technologies that can aid in recycling waste streams and in reducing the environmental footprint of human activity on this planet. The introduction of this thesis underlines the necessity of transitioning towards a circular economy and presents a technology that can help with recycling and valorising organic residues. Chain elongation fermentation allows the production of medium chain carboxylates (MCC) from complex organic waste. The fermentation products can be used for a wide range of applications within agriculture and the chemical industry. In this thesis new methods for chain elongation are discovered and researched that broaden the product spectrum of the technology. Besides straight molecular chains, branched chained MCCs have been shown as dominant products in these new fermentation types. The products with a different molecular structure inherently have different physical properties that might make them better suited for certain applications within society.

The research chapters elaborated on how specific selection pressures in open culture fermentations can be used to enrich microbiomes to harbour desired biocatalytic capabilities. Two different types of chain elongation fermentation are the subject of these investigations: methanol-based and ethanol-based chain elongation. These two alcohols are used by the microbiomes as electron donors within the fermentation. In order to harvest energy and grow, the organism use a metabolism where they process the energyrich electrons from the alcohols. The electrons are subsequently used to reduce a carboxylate electron acceptor, which is simultaneously elongated in the process.

Within methanol-based chain elongation microbiomes, the elongation of acetate leads to mainly butyrate formation. Depending on the $\mathrm{pH}$, the microbiome could be enriched to the point that isomerization of $n$-butyrate to isobutyrate occurred (Chapter 2 ). At a pH around 6.75 no isomerization happened, but at a pH around 5.5 it did. The ratio of the n-butyrate and isobutyrate concentrations were found to be coupled to the thermodynamic equilibrium of isomerization. The responsible microorganism for isobutyrate formation was found to be closely related to an earlier described Clostridium luticellarii.

When propionate was used as electron acceptor, elongation to n-valerate occurred (Chapter 3). The enriched microbiome also contained $C$. luticellari as dominant microorganism. The microbiome was capable to simultaneously elongate both acetate and propionate to n-butyrate, isobutyrate, n-valerate as dominant products. Also small amounts of $n$-caproate were formed whenever $n$-butyrate was present within methanolbased chain elongation microbiomes. Based on literature a metabolic pathway for 
methanol-based chain elongation was proposed that could describe the experimentally observed stoichiometry.

Microbiomes were also enriched to perform ethanol-based chain elongation, in particular for the elongation of branched electron acceptors. When isobutyrate was fed to the microbiome together with ethanol, elongation towards isocaproate was stimulated (Chapter 4). However, due to the nature of ethanol-based chain elongation in situ acetate formation always occurs. Additionally ethanol can in some situations be directly converted towards acetate and hydrogen by other microbes that compete for substrate. This leads to a situation where the chain elongators can use an increasing amount of acetate as electron acceptor, which seemed to be preferred over isobutyrate. Limiting acetate supply led to isocaproate production up to $20 \%$ of the total products.

In an attempt to control the excessive ethanol oxidation in the reactor, conditions were adjusted to limited $\mathrm{CO} 2$ supply (Chapter 5). Limitation of $\mathrm{CO} 2$ leads to a deficiency for hydrogenotrophic methanogens; they need $\mathrm{CO} 2$ as electron acceptor for their energyproviding, methane-producing metabolism. However, the conditions of the reactor were such that an alternative route for ethanol oxidation was stimulated. High ethanol to acetate ratios, and high (other) carboxylate to corresponding alcohol ratios created the potential for carboxylate reduction coupled to ethanol oxidation. In turn in situ acetate formation persisted, whereby straight chain elongation remained the most dominant metabolic functionality.

In the general discussion hypotheses are presented that could further mechanistically explain the observed metabolic functionalities. For methanol-based chain elongation the metabolic pathway is revised, using supporting evidence from the genome of $C$. luticellarii. Improvements on reactor operation are suggested to increase the performances. Additionally recommendations are given on how integrated bioprocess designs could circumvent downstream processing difficulties. Finally an outlook on ethanol-based chain elongation fermentation for branched carboxylate production is presented. 


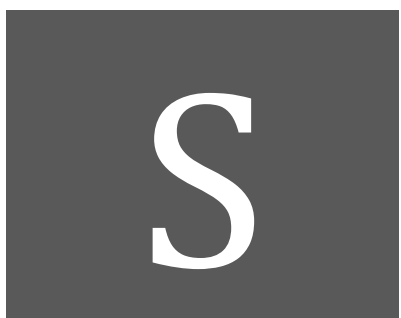

\section{Supporting Information}




\section{Supporting Information - Chapter 2}

Methanol based chain elongation with acetate to $n$-butyrate and isobutyrate at varying selectivities dependent on $\mathrm{pH}$

Kasper D. de Leeuw, Sanne M. de Smit, Sabine van Oossanen, Marinus J. Moerland, Cees J.N. Buisman, David P.B.T.B. Strik 


\section{Material and Methods}

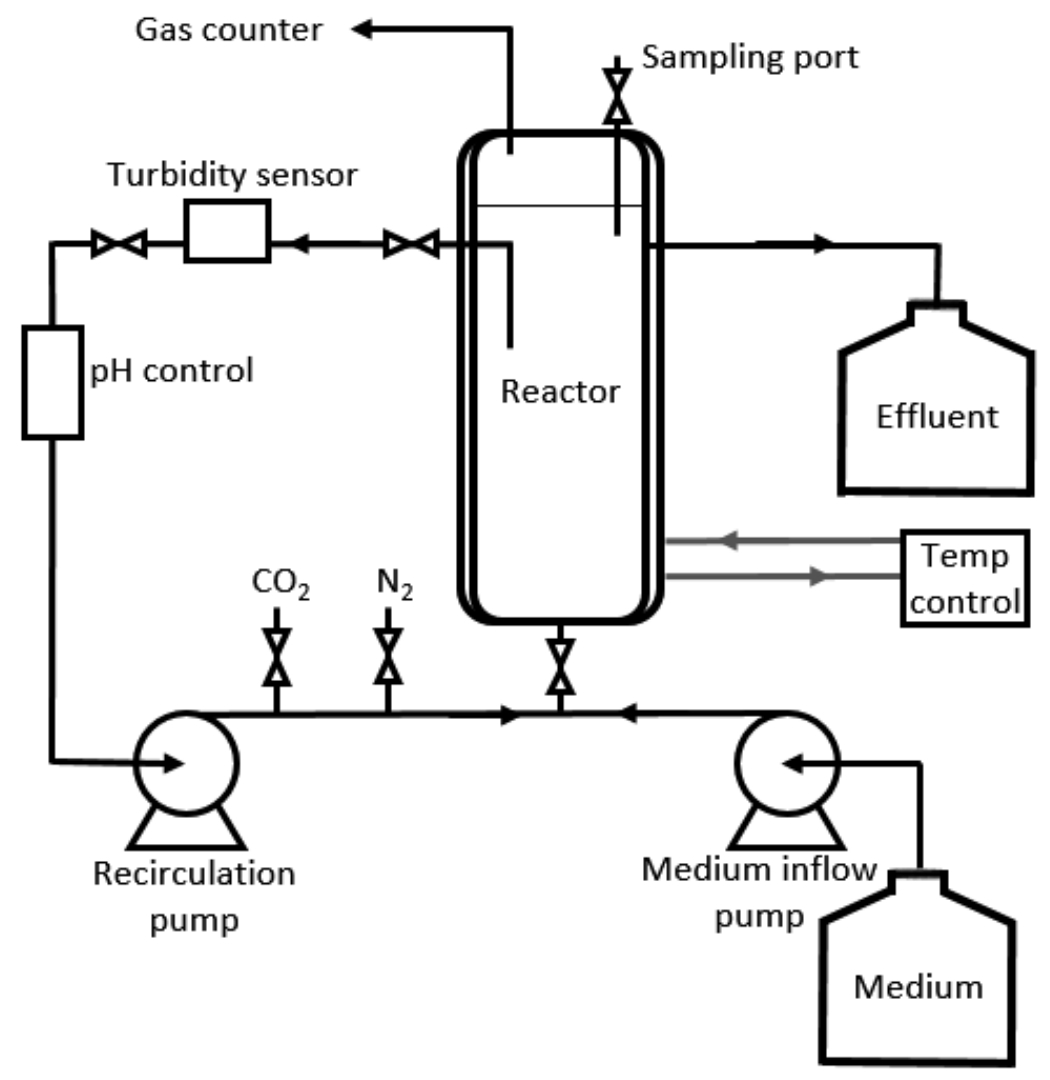

Figure S1. Schematic overview of the continuous reactor setup. 


\section{Protocol batch experiments}

\subsection{Inoculum preparation}

Inoculum for batch I to VIII was taken directly from the reactor during phase VII. Ten percent of the total batch volume in this first series was inoculum.

The second series (A-M) was performed after the first and used an inoculation method that prevented carbon source from the inoculum affecting the batch experiment. The culture containing $\mathrm{iC}_{4}$ producing chain elongating bacteria was taken from a batch III. The inoculum was was kept active in repeated batches with conditions that were that same as batch $A$. The medium contained $150 \mathrm{mM}$ acetate and $300 \mathrm{mM}$ methanol as substrate. A 21 day old culture of $300 \mathrm{~mL}$ was used to inoculate series $A$ to $M$. To prepare the inoculum for batch series $A-M$, the following protocol was followed:

1. Distribute the $300 \mathrm{~mL}$ culture over $25 \mathrm{~mL}$ tubes

2. Centrifugate at $10000 \mathrm{rpm}$ for 15 minutes

3. Discard the supernatant

4. Resuspend each pellet in $20 \mathrm{~mL}$ medium without carbon source

5. Repeat step 1-2 to wash.

6. Resuspend all pellets and combine in one flask using $150 \mathrm{~mL}$ medium without carbon source. This is the prepared inoculum, from which $12 \mathrm{~mL}$ is used during preparation for each duplicate batch.

For the pure Eubacterium limosum the protocol provided by dsmz was used to activate the culture. The batches performed with this inoculum (batch EL) were treated in a laminar flow cabinet with extra sterilization measures (by continually burning the stopper and needles with flame and $70 \%$ ethanol) to ensure the culture was kept pure. 


\subsection{Batch series preparation}

The following protocol was repeated for each batch, except the pure Eubacterium limosum batch. The protocol provides enough medium for two batch bottles (duplicate batches were performed for each different set of parameters). The compositions of the Stock, Vitamin and Trace element solutions are given in Table S1 to S6. As shown in Table 2 in the main manuscript, substrate concentrations, $\mathrm{iC}_{4}$ concentrations and $\mathrm{pH}$ vary per experiment.

1. Mark the $150 \mathrm{~mL}$ level of two clean serum bottles ( $250 \mathrm{~mL}$ total volume) using demi water.

2. Add in the following order to a $500 \mathrm{~mL}$ beaker

- 300 mL water

- $0.50 \mathrm{~g}$ yeast extract

- Acetate ( $\mathrm{NaCH} 3 \mathrm{COO} \cdot 3 \mathrm{H} 2 \mathrm{O})$ (amount varies per series)

- Methanol (amount varies per series)

- $\mathrm{i}-\mathrm{C}_{4}$ or $\mathrm{n}-\mathrm{C}_{4}$ (amount varies per series)

- $0.50 \mathrm{~mL}$ Vitamin solution

- $0.25 \mathrm{~mL}$ Trace element solution I

- $0.25 \mathrm{~mL}$ Trace element solution II

- $10 \mathrm{~mL}$ Stock solution I

- $10 \mathrm{~mL}$ Stock solution II

3. Fill to a volume of $400 \mathrm{~mL}$ with demi water.

4. Set the $\mathrm{pH}$ according to either $5.2,5.5$ or 6.5 . using $1 \mathrm{M} \mathrm{HCl}$ or $1 \mathrm{M} \mathrm{KOH}$.

5. Transfer the medium solution to a $500 \mathrm{~mL}$ flask.

6.

For series I to VIII, $50 \mathrm{ml}$ inoculum taken directly from the reactor during phase VII was added, after which the volume was adjusted up to $500 \mathrm{~mL}$ using demi water.

For series $A-M$, the volume was adjusted up to $500 \mathrm{~mL}$ using demi water and the inoculation was performed at step 8 . 
7. Transfer the medium solution to a $600 \mathrm{~mL}$ beaker.

8a. (for batch I to VIII) Inoculation was performed at step 6.

8b. (for batch A-M)

Centrifugate $12 \mathrm{~mL}$ of previously prepared inoculum at $10000 \mathrm{rpm}$ for 15 minutes.

- Prepared inoculum: suspended cells originating from batch III that were kept active in a repeated batch at the same conditions as batch $A$.

Discard the supernatant.

- $\quad$ Resuspend the pellet in the prepared medium.

- $\quad$ Add to the beaker, mix shortly.

9. Fill up the batch bottles with solution to the $150 \mathrm{~mL}$ mark.

10. Take $2 \times 5 \mathrm{~mL}$ sample, spin down at $10000 \mathrm{rpm}$ for 15 minutes and store for further analysis at $-20^{\circ} \mathrm{C}$.

11. Cap the batch bottles.

12. Replace the headspace with $90 \%$ (or $80 \%$ for the EL batch) $\mathrm{N}_{2}$ and $10 \%$ (or $20 \%$ for the $\mathrm{EL}$ batch) $\mathrm{CO}_{2}$ at 1.5 bar using the gas exchanger.

14. Place the bottles in a $35^{\circ} \mathrm{C}$ incubator mixing at $120 \mathrm{rpm}$ (Series A-F) or $130 \mathrm{rpm}$ (Series G-M). Mixing was increased in the second series, to reduce biofilm formation.

N.B. For the batches (EL batch) performed with pure Eubacterium limosum the medium (excluding the vitamins) was added to the batch prior to an additional autoclave step. Vitamins were subsequently added through a styrile $0.2 \mu \mathrm{m}$ filter. Every sampling step during this batch was performed in a sterile environment within a laminar flow cabinet.

\subsection{Biomass optical density and dry weight determination (Protocol OD/dry weight correlation curves)}

The biomass concentrations in batch experiments $A-M$ were correlated to optical density using dry weight measurements and spectrometry. Two correlation curves were made for the $\mathrm{i}-\mathrm{C}_{4}$ producing microbiome. Biomass was spinned down initially in order to concentrate the suspended cells and acquire a complete calibration curve and determine the linear range.

Protocol for spectrometry measurements 
1. Spin down $20 \mathrm{~mL}$ of $\mathrm{i}-\mathrm{C}_{4}$ producing culture for 10 minutes at $15000 \mathrm{rpm}$

2. Take off supernatant

3. Resuspend the pellets in medium without added carbon. use $5 \mathrm{ml}$ of medium without carbon source ( $4 x$ concentrated. or $0.25 x$ diluted)

4. Add $2 \mathrm{~mL}$ of resuspended pellet to $2 \mathrm{~mL}$ of medium without carbon source ( $0.5 \mathrm{x}$ diluted).

5. Add $2 \mathrm{~mL}$ of the suspension from step 4 to $2 \mathrm{~mL}$ medium without carbon. Repeat so a dilution series is made until a $64 x$ dilution.

6. Calibrate a spectrometer at $\lambda=660 \mathrm{~nm}$ using medium without carbon as blanc

7. Measure the optical density of each dilution

Protocol for dry weight measurements

1. Dry a $2 \mu \mathrm{m}$ filter (Whatman. $47 \mathrm{~mm}$. grade GMF) in an aluminium cup for 1 hour at $105^{\circ} \mathrm{C}$

2. Weigh the cup and filter (m0)

3. Weigh a $30 \mathrm{~mL}$ tube (mB1)

4. Add $20 \mathrm{~mL}$ of iC4 producing culture to the tube. weigh the tube again (mB2)

5. Put the filter on a Büchner funnel with under pressure. wash the filter three times with demi water

6. Filter the tube content

7. Fill the tube with demi water. filter the tube content. Repeat three times

8. Turn off under pressure. take off the filter with tweezers and return to the aluminium cup

9. Dry the filter+cup overnight at $105^{\circ} \mathrm{C}$

10. Weigh the filter+cup (m1)

12. Dry the filter+cup for at least 2 hours at $550^{\circ} \mathrm{C}$

13. Weigh the filter+cup (m2)

The total suspended solids (TSS) and volatile suspended solids (VSS) are defined as follows: 
$\mathrm{TSS}=\frac{\mathrm{m} 1-\mathrm{m} 0}{\mathrm{mB} 2-\mathrm{mB} 1} \mathrm{ASH}=\frac{\mathrm{m} 2-\mathrm{m} 0}{\mathrm{mB} 2-\mathrm{mB} 1} \quad$ VSS $=T S S-A S H$

Conversion of dry weight to moles carbon

The filter was assumed to only contain biomass as carbon source. other carbons were assumed to be smaller than $2 \mu \mathrm{m}$ and to be washed away. The molar biomass concentration was calculated by dividing the average TSS of triplicates over the molar weight of biomass. When assuming a biomass composition of $\mathrm{CH}_{1.8} \mathrm{O}_{0.5} \mathrm{~N}_{0.2}{ }^{284}$, the molar weight of biomass is $24.6 \mathrm{~g} / \mathrm{Cmol}$.

\section{Calibration curves}

For the biomass calibration (See Figure S2) of the iC4 producing inoculum of the reactor. the OD660 measurements were performed in duplicate and the dry weight measurements in triplicate. To find the correlation between the optical density and the VSS content. the average VSS content of triplicates was multiplied with the dilution factors used for the optical density samples.

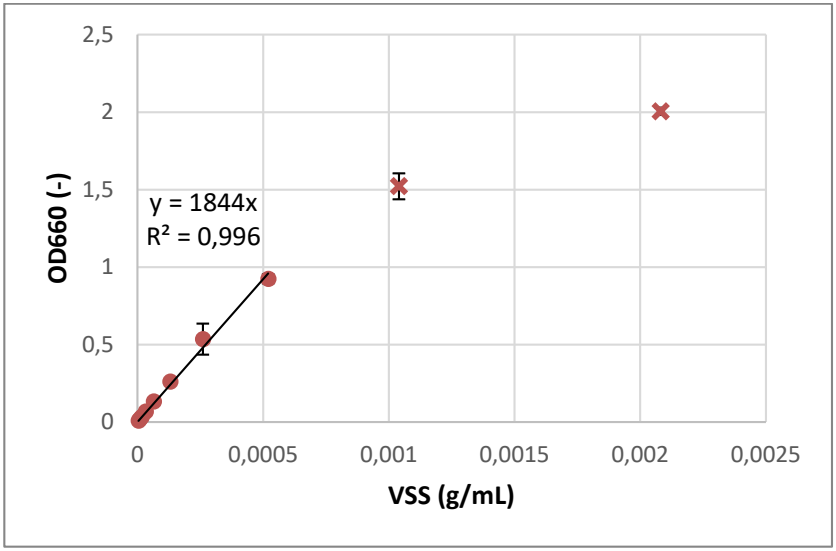

Figure S2. Biomass calibration curve of the $\mathrm{i}-\mathrm{C}_{4}$ producing microbiome used as inoculum for batch series G-M. correlating biomass optical density and VSS content. Error bars mark the confidence interval of triplicate optical density measurements. Optical density measurements are linear up to an OD660 absorption of 0.92 .

N.B.: Biomass determinations were only completely performed for batches $G$ to $M$ (only halfway A-F was biomass determination introduced, after the exponential phase). However, some solid biofilm-like particles were observed that greatly affected the measurements. Therefore these results are not discussed in the main article.

Additionally the method used to make the calibration line - concentrating before making the dilution series - could have introduced errors by the extra sample handling steps. This 
needs to be checked in the future when the results are to be used for precise calculations of biomass specific rates and such.

\section{Medium compositions}

I.1 Medium composition for batch experiments- methanol based chain elongation of acetate

Table S1. Medium composition for methanol chain elongation experiments with acetate as electron acceptor. The compositions of the stock solutions, vitamin solution and trace element solutions are shown in Table S2 to Table S6.

\begin{tabular}{|l|l|l|}
\hline Compound & $\begin{array}{l}\text { Amount per } \\
\text { L }\end{array}$ & Unit \\
\hline NaCH3COO-3H2O & Variable & $\mathrm{g}$ \\
\hline Yeast Extract & 1 & $\mathrm{~g}$ \\
\hline Stock I & 20 & $\mathrm{~mL}$ \\
\hline Stock II & 20 & $\mathrm{~mL}$ \\
\hline Methanol (liquid) & Variable & $\mathrm{g}$ \\
\hline n-butyric acid & Variable & $\mathrm{g}$ \\
\hline i-butyric acid & Variable & $\mathrm{g}$ \\
\hline Vitamin solution & 1 & $\mathrm{~mL}$ \\
\hline Trace elements I & 0.5 & $\mathrm{~mL}$ \\
\hline Trace elements II & 0.5 & $\mathrm{~mL}$ \\
\hline
\end{tabular}

Table S2. Composition of stock solution I

\begin{tabular}{|c|c|}
\hline Compound & $\begin{array}{l}\text { Concentration }(\mathrm{g} \\
\left.\mathrm{L}^{-1}\right)\end{array}$ \\
\hline $\mathrm{NH}_{4} \mathrm{H}_{2} \mathrm{PO}_{4}$ & 180 \\
\hline $\mathrm{MgCl}_{2} \cdot 6 \mathrm{H}_{2} \mathrm{O}$ & 16.5 \\
\hline $\mathrm{MgSO}_{4} \cdot 7 \mathrm{H}_{2} \mathrm{O}$ & 10 \\
\hline
\end{tabular}


Table S3. Composition of stock solution II

\begin{tabular}{|l|l|}
\hline Compound & $\begin{array}{l}\text { Concentration (g L- } \\
{ }^{1} \text { ) }\end{array}$ \\
\hline $\mathbf{C a C l}_{\mathbf{2}} \cdot \mathbf{2} \mathbf{H}_{2} \mathbf{O}$ & 10 \\
\hline $\mathbf{K C l}$ & 7.5 \\
\hline
\end{tabular}

Table S4. Composition of the vitamin solution, 1000x concentrated.

\begin{tabular}{|l|l|}
\hline Compound & $\begin{array}{l}\text { Concentration (g L- } \\
\mathbf{1})\end{array}$ \\
\hline Biotin & 0.106 \\
\hline Folic acid & 0.005 \\
\hline Pyridoxal-HCL & 0.0025 \\
\hline Lipoic acid & 0.015 \\
\hline Riboflavin & 0.0125 \\
\hline Thiamine HCl & 0.266 \\
\hline Ca-D-Pantothenate & 0.413 \\
\hline Cyanocobalamin & 0.0125 \\
\hline P-aminobenzoic acid & 0.0125 \\
\hline Nicotinic acid & 0.0125 \\
\hline
\end{tabular}

Table S5. Composition of trace element solution I, 2000x concentrated

\begin{tabular}{|c|c|}
\hline Compound & Concentration $\left(\mathrm{g} \mathrm{L}^{-1}\right)$ \\
\hline $\mathrm{FeCl}_{2} \cdot 4 \mathrm{H} 2 \mathrm{O}$ & 30 \\
\hline $\mathrm{HCl}(1 \mathrm{M})$ & 77 \\
\hline $\mathrm{MnCl}_{2} \cdot \mathbf{4 \mathrm { H } _ { 2 } \mathrm { O }}$ & 0.6 \\
\hline $\mathrm{H}_{3} \mathrm{BO}_{3}$ & 6.0 \\
\hline $\mathrm{COCl}_{2} \cdot 6 \mathrm{H}_{2} \mathrm{O}$ & 4.0 \\
\hline $\mathrm{CuCl}_{2} \cdot 4 \mathrm{H}_{2} \mathrm{O}$ & 0.2 \\
\hline $\mathrm{NiCl}_{2} \cdot \mathbf{6} \mathrm{H}_{2} \mathrm{O}$ & 0.4 \\
\hline $\mathrm{ZnSO}_{4} \cdot 7 \mathrm{H}_{2} \mathrm{O}$ & 2.0 \\
\hline
\end{tabular}


Table S6. Composition of trace element soution II, 2000x concentrated

\begin{tabular}{|l|l|}
\hline Compound & Concentration $\left(\mathrm{g} \mathrm{L}^{1}\right)$ \\
\hline $\mathrm{Na}_{2} \mathrm{MOO}_{4} * 2 \mathrm{H}_{2} \mathrm{O}$ & 0.6 \\
\hline $\mathrm{Na}_{2} \mathrm{SeO}_{3}$ & 0.2 \\
\hline $\mathrm{KOH}(4 \mathrm{M})$ & 3.1 \\
\hline
\end{tabular}

\section{Calculations}

Total inorganic carbon

The total dissolved inorganic carbon was calculated rewriting the Herderson-Hasselbach equation (1):

$$
[\text { total inorganic carbon }(a q)]=\frac{\left[H_{2} C O_{3}\right] *\left(\left[H^{+}\right]^{2}+K_{A_{1}} *\left[H^{+}\right]+K_{A_{2}} * K_{A_{1}}\right)}{\left[H^{+}\right]^{2}}
$$

With $\left(\left[\mathrm{H}^{+}\right]\right.$in $\left.\mathrm{M}\right)$ using (2):

$$
\left[H^{+}\right]=10^{-p H}
$$

Carbonic acid dissociates into $\mathrm{HCO}_{3}^{-}$(3) and $\mathrm{CO}_{3}{ }^{2-}$ (4):

$$
\begin{gathered}
\mathrm{H}_{2} \mathrm{CO}_{3} \rightarrow \mathrm{HCO}_{3}^{-}+H^{+} \text {with } p K_{A_{1}}=6.35 \\
\mathrm{HCO}_{3}^{-} \rightarrow \mathrm{CO}_{3}^{2-}+H^{+} \text {with } p K_{A_{2}}=10.33
\end{gathered}
$$

And (5):

$$
\left[\mathrm{H}_{2} \mathrm{CO}_{3}(\mathrm{M})\right]=\frac{\gamma_{\mathrm{CO} 2}(-) * P_{\text {tot }}(\mathrm{atm})}{K_{H}}
$$

$\mathrm{YcO2}=$ fraction $\mathrm{CO}_{2}$ in the headspace

$P_{\text {tot }}=$ total pressure in the batch bottle, or atmospheric pressure for the continuous reactor system

$\mathrm{K}_{\mathrm{H}}=$ Henry constant $=29.41 \mathrm{~atm} / \mathrm{M}^{285}$

Equilibrium of isomerization concentrations $-i-C_{4}$ and $n-C_{4}$ to total butyrate species-ratios In figure $\mathrm{S} 3$ a graph is given showing the $\Delta \mathrm{Gr}^{1}$ of de-isomerization and isomerization, deisomerization being Isobutyrate $\rightarrow \mathrm{n}$-butyrate $\left(\Delta \mathrm{Gr}^{0, a}=+1.98 \mathrm{~kJ}\right)(6)$

$$
\Delta G_{r}^{1}=\Delta G_{r}^{0}+R T \ln \left(\frac{\left[n C_{4}\right]}{\left[i C_{4}\right]}\right)
$$


and isomerization being $\mathrm{n}$-butyrate $\rightarrow$ isobutyrate $\left(\Delta \mathrm{Gr}^{0, \mathrm{~b}}=-1.98 \mathrm{~kJ}=-\Delta \mathrm{Gr}^{0, \mathrm{a}}\right)^{3}(7)$.

$$
\Delta G_{r}^{1}=\Delta G_{r}^{0, b}+R T \ln \left(\frac{\left[i C_{4]}\right]}{\left[n C_{4}\right]}\right)
$$

Using $\left[\mathrm{C}_{4, \text { total }}\right]=\left[\mathrm{iC}_{4}\right]+\left[\mathrm{nC}_{4}\right]$ for substitution and rewriting yields $(8)$

$$
\frac{\left[i \text { or } n C_{4}\right]}{\left[C_{4} \text { total }\right]}=\frac{1}{\exp \left(\frac{\Delta G_{r}^{1}-\Delta G_{r}^{0, a \text { or b }}}{R T l n}\right)+1}
$$

Which with $\Delta \mathrm{Gr}^{1}{ }^{1}=0$, gives the equilibrium ratios for $\mathrm{i}-\mathrm{C}_{4}$ to total $\mathrm{C}_{4}$ and $\mathrm{n}-\mathrm{C}_{4}$ to total $\mathrm{C}_{4}$.

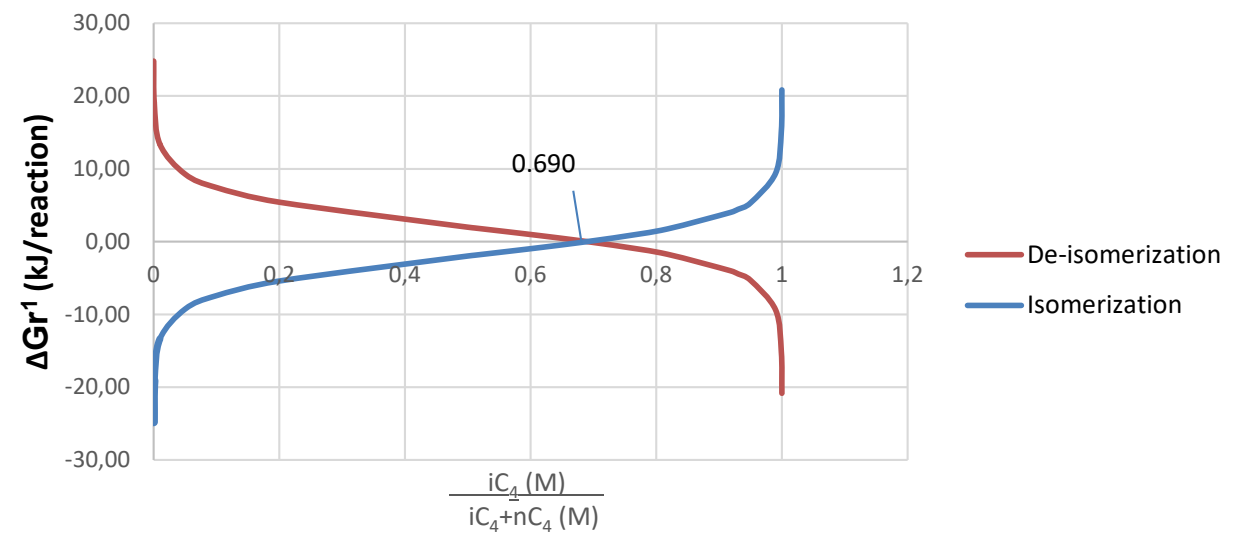

Figure S3. The figure shows where the $\Delta \mathrm{Gr}_{\mathrm{r}}{ }^{1}$ for de-isomerization and isomerization intersect and at what ratio equilibrium of isomerization would set in.

\section{Volumetric conversion rate}

Volumetric conversion rate (10) is calculated for the continuous reactor between each two measurement points using the following parameters

- $V_{r}$ : Reactor volume was $1 \mathrm{~L}$.

- Fin: Inflow was determined by using a scale and weighing the amount of influent that had flowed into the reactor. Unit: $\mathrm{kg} \mathrm{day}^{-1}$, converted to $\mathrm{L}$ day ${ }^{-1}$ using an experimentally determined density of the medium.

- Fout: Outflow was determined by taking the inflow and adding the volume amount of dosed $1 \mathrm{M} \mathrm{KOH}$. Unit $\mathrm{kg} \mathrm{day}^{-1}$, converted to $\mathrm{L}_{\text {day }}{ }^{-1}$ using an experimentally determined density of the broth. 
- (Cinfleunt) Concentrations of compounds of the influent were known

- (Cbroth) Concentrations of compounds in the effluent were measured via GC.

$$
\begin{gathered}
\text { Total Conversion Rate }=F_{\text {out }} \cdot C_{\text {broth }}-F_{\text {in }} \cdot C_{\text {influent }}\left[\frac{\mathrm{mmolC}}{\text { day }}\right] \\
\text { Volumetric Conversion Rate }=\frac{\text { Total Conversion Rate }}{\boldsymbol{V}_{\boldsymbol{r}}}\left[\frac{\mathrm{mmol} \mathrm{C}}{\text { Lday }}\right]
\end{gathered}
$$




\section{Results and Discussion}

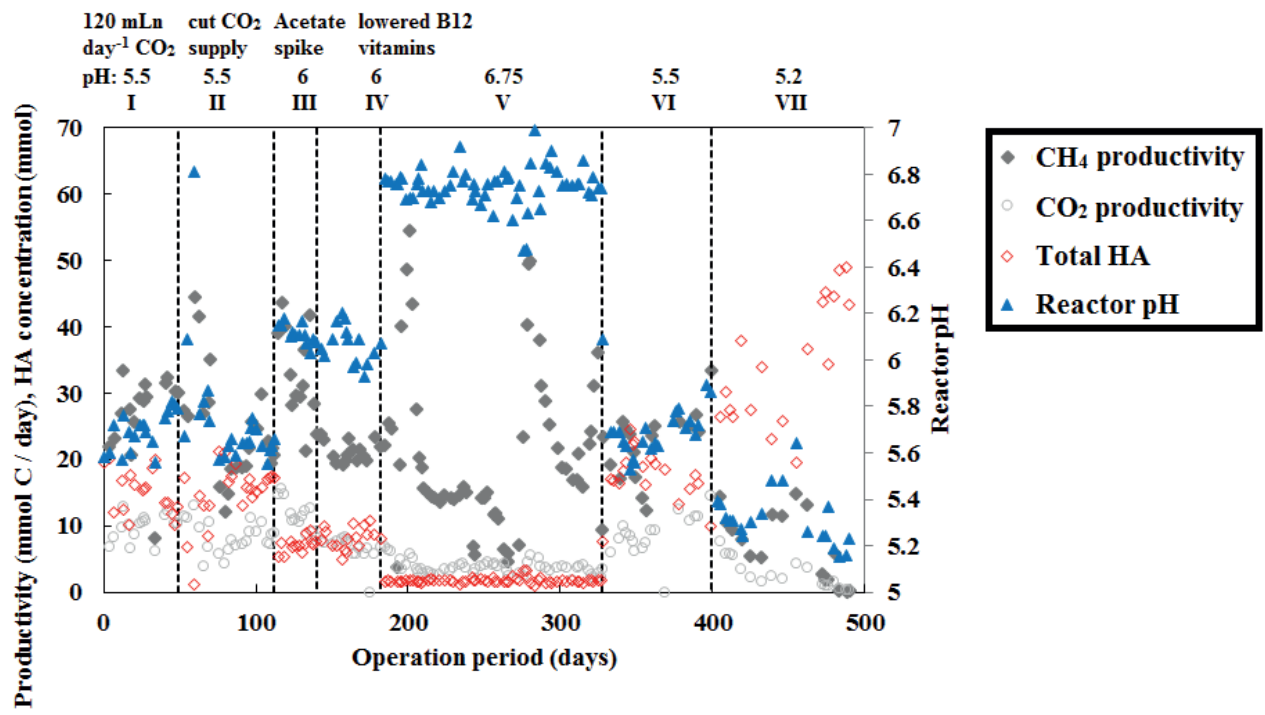

Figure S4. Graph showing the measured $\mathrm{pH}$ values in the continuous reactor and the calculated undissociated acids concentrations using the measured values for the broth fatty acids concentrations and their associated $\mathrm{pK}_{\mathrm{a}}$ values.

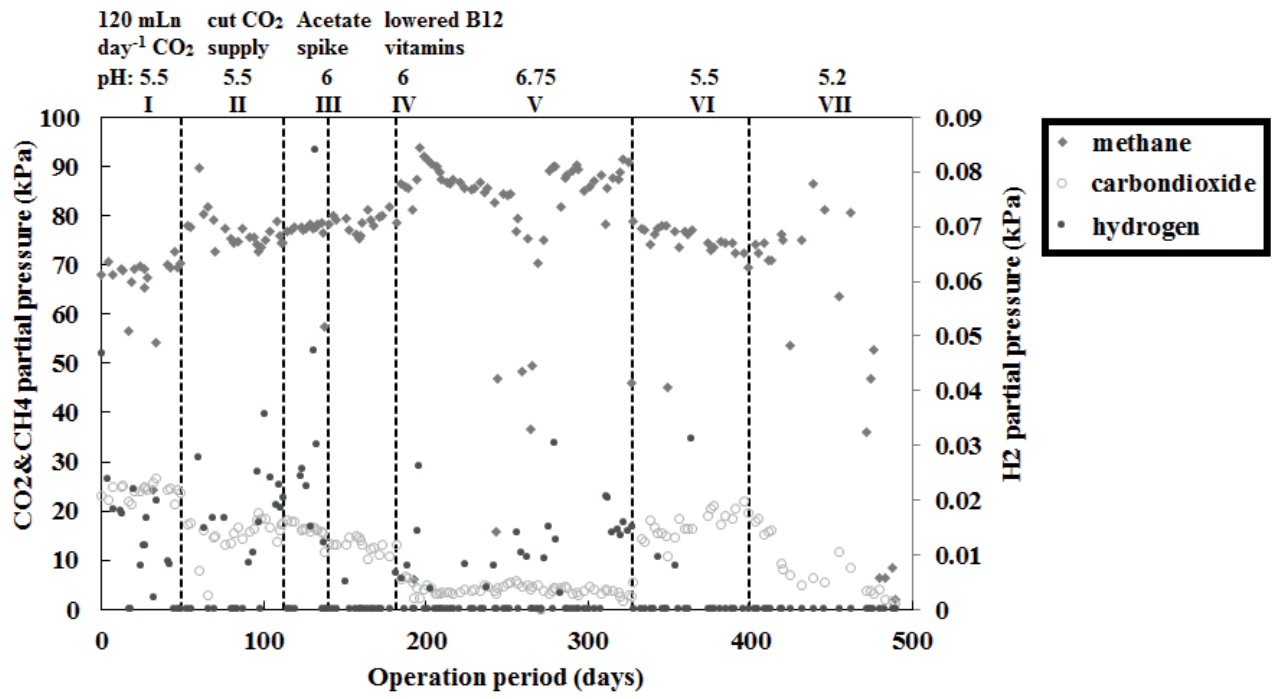

Figure S5. Measured gas headspace partial pressure through the operation period of the continuous reactor. 

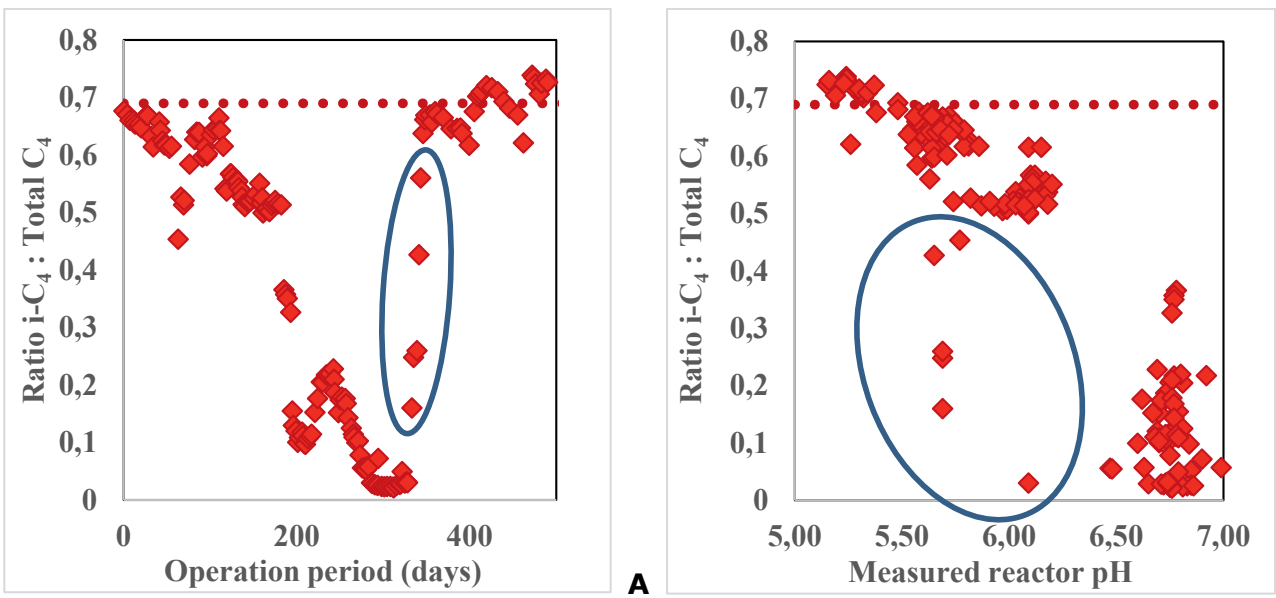

B

Figure S6. The graph shows the $\mathrm{i}-\mathrm{C}_{4} /\left(\mathrm{i}-\mathrm{C}_{4}+\mathrm{n}-\mathrm{C}_{4}\right)$ ratio calculated from the measured concentrations on the $y$-axis, with the operation period $(A)$ and the measured reactor $\mathrm{pH}(\mathrm{B})$ on the $\mathrm{x}$-axis. The dotted lines represent the calculated equilibrium ratio using an isomerization $\Delta \mathrm{G}_{\mathrm{r}}{ }^{01}$ of $-1.98 \mathrm{~kJ} 28,103$. The dots within the encircled part are from measurements during the first 2 weeks of operation at pH 5.5 (phase VI) after a long period at pH 6.75 (phase $\mathrm{V}$ ) when the microbiome was adapting to the change in reactor conditions. 


\section{Batches I - VIII}

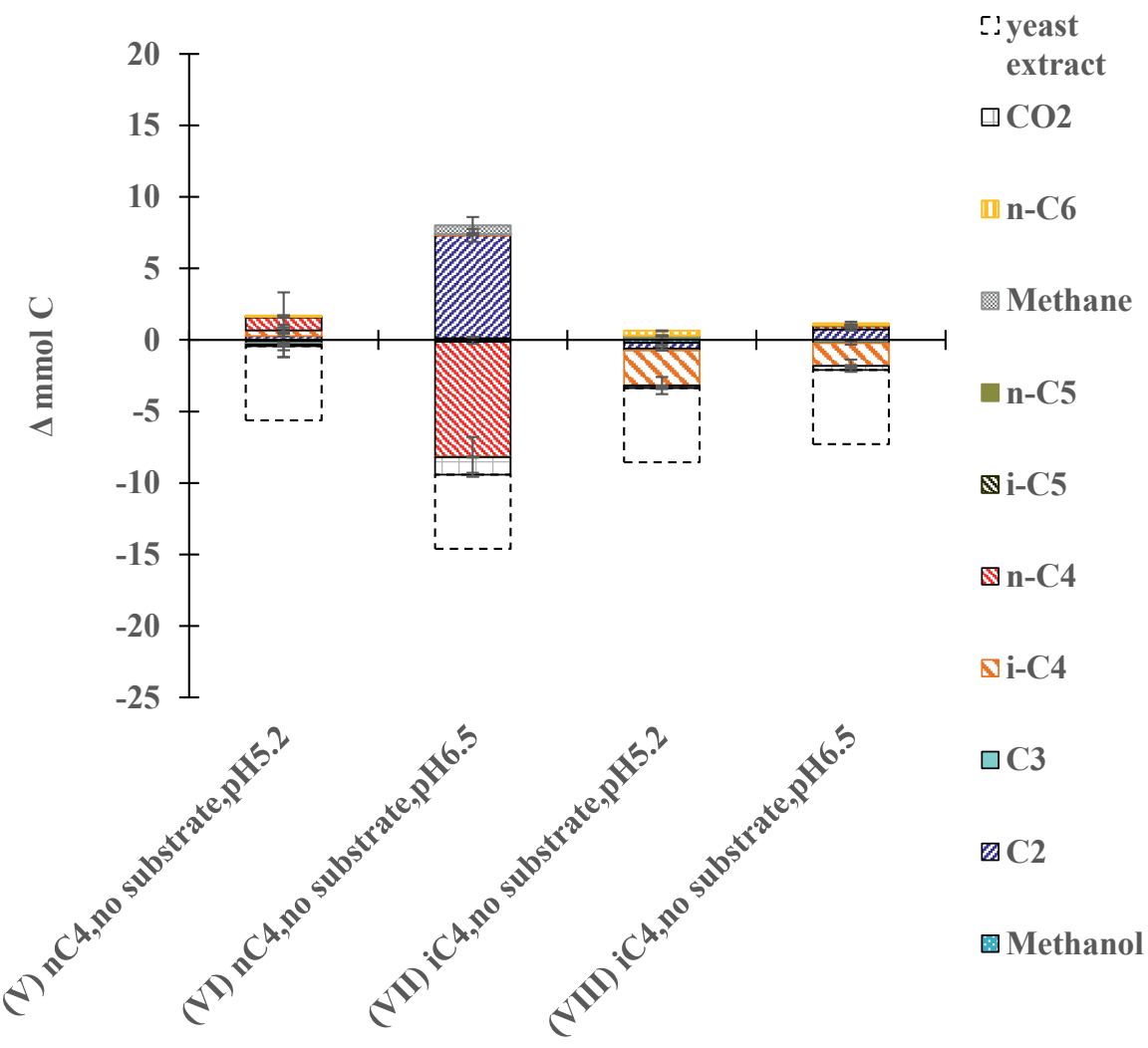

Figure S7. Formation and consumption of carbon containing components calculated for the period after methanol was consumed for batches $V$ to VIII. Error bars show difference between the duplicate experiments. The yeast extract that was added to the medium is shown as well and is shown to be fully consumed, however, likely it was consumed during the active period when methanol was available. The shown conversions are calculated from day 14 and onwards, when the small amount of methanol that came from the inoculum was depleted, except for batch VII. During batch VII the small amount of methanol was only slowly consumed and the shown conversions are calculated from day 63 and onwards. 

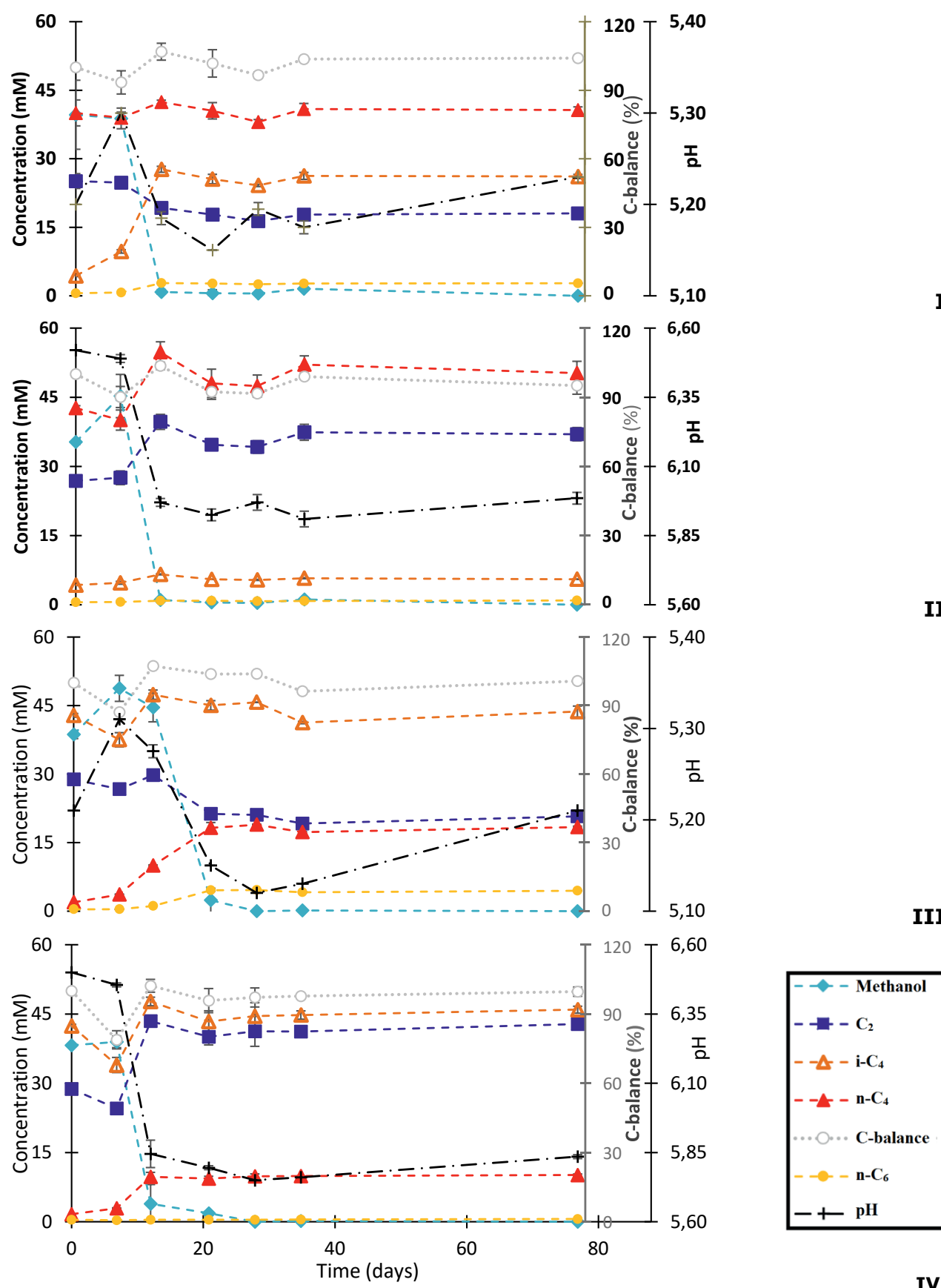

IV

Figure S8. Concentrations of methanol, acetate, n-butyrate and iso-butyrate during the batch experiments I to IV. 

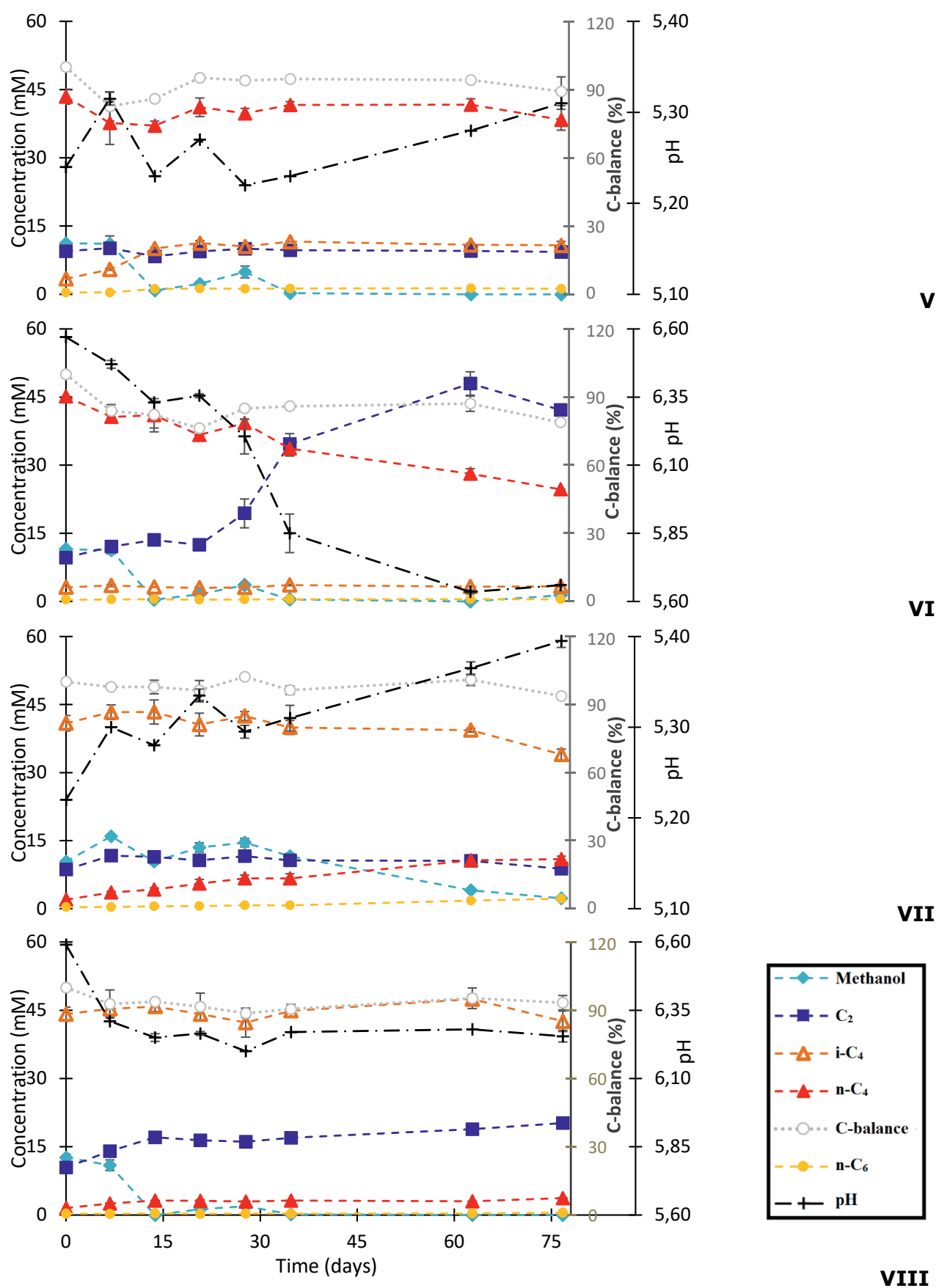

Figure 59. Concentrations of methanol, acetate, n-butyrate and iso-butyrate during the batch experiments $\mathrm{V}$ to VII. 

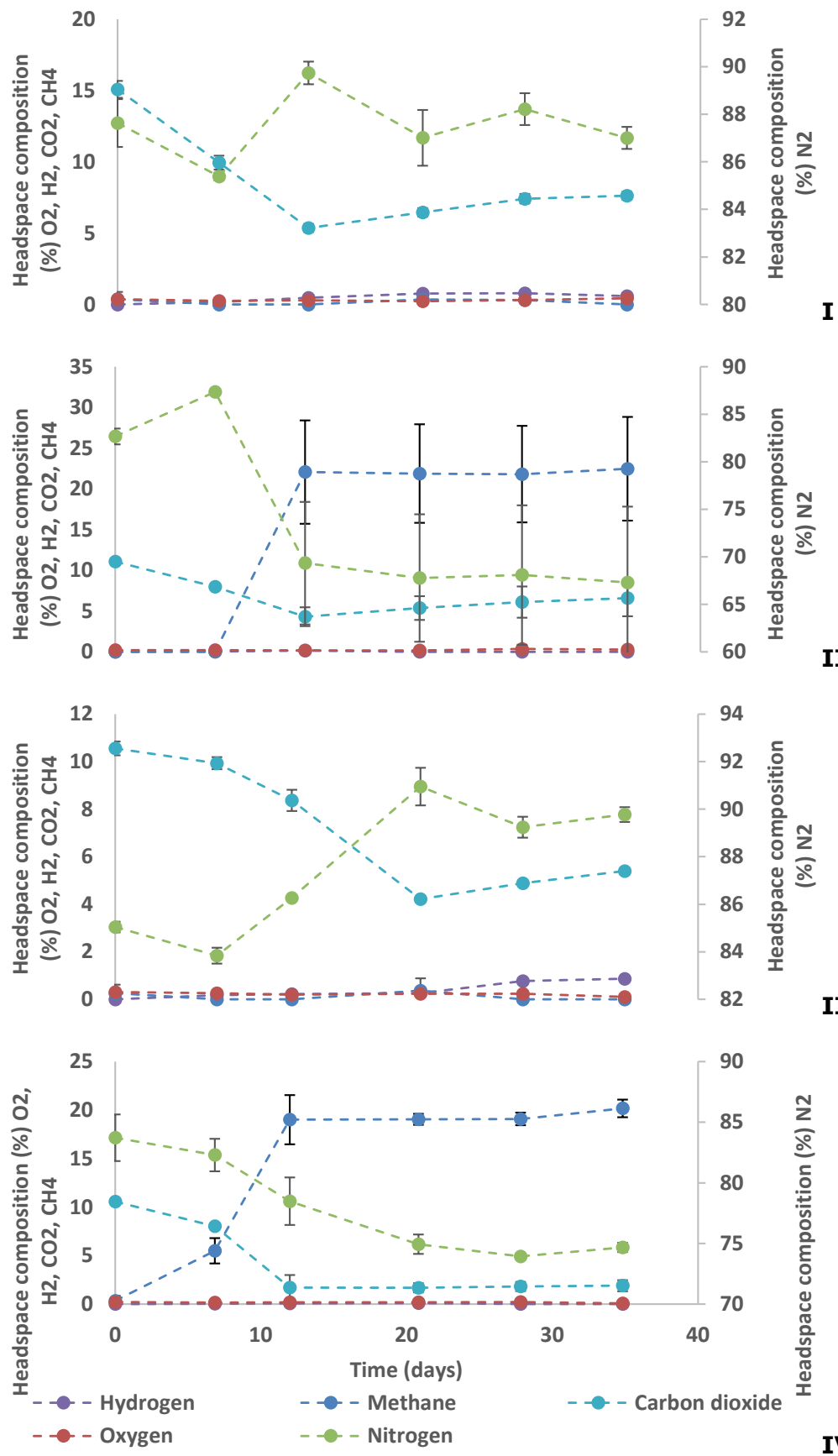

III

Figure S10. Headspace percentage of hydrogen, methane, carbon dioxide, oxygen and nitrogen during the batch experiments I to IV. Pressures in the headspace were always in between 1 and 1.5 bar. 

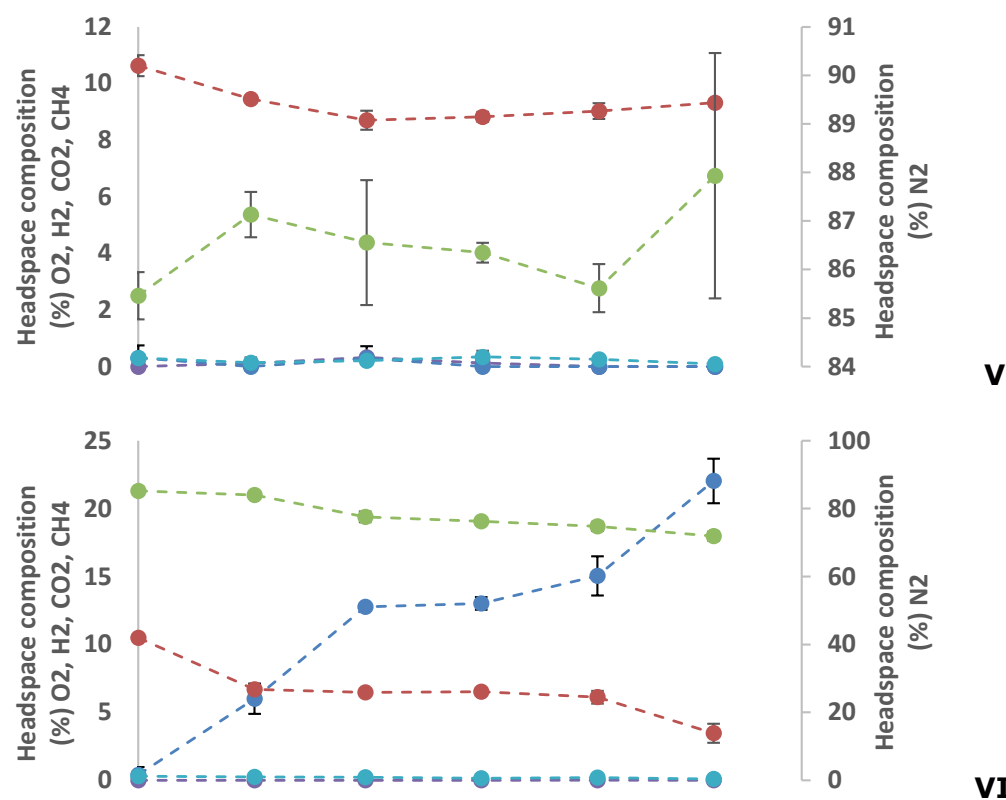

VI
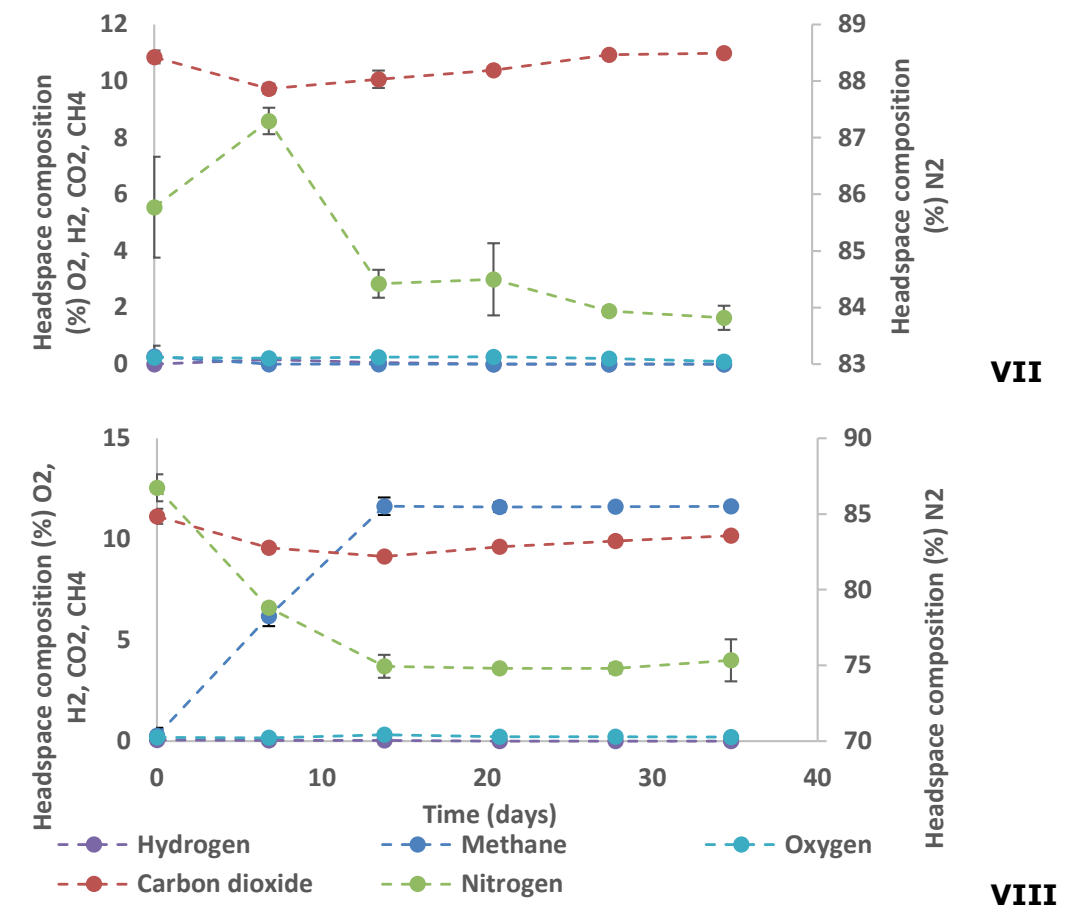

VII

Figure S11. Headspace percentage of hydrogen, methane, carbon dioxide, oxygen and nitrogen during the batch experiments V to VIII. Pressures in the headspace were always in between 1 and 1.5 bar. 


\section{Batches A - F}

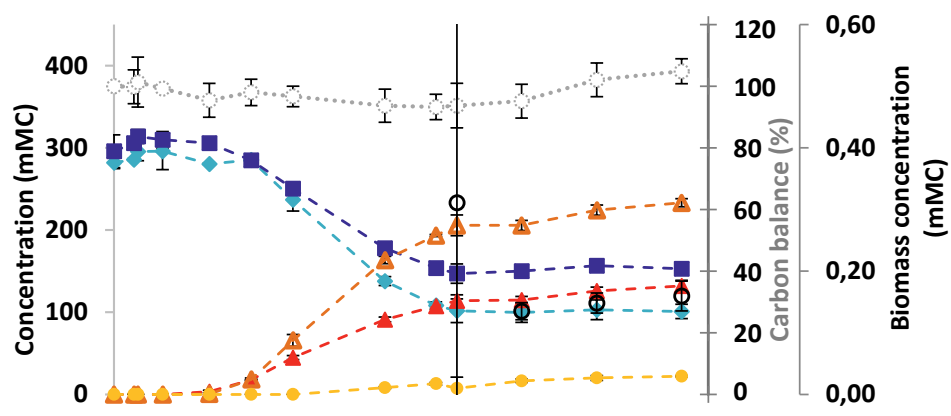

A

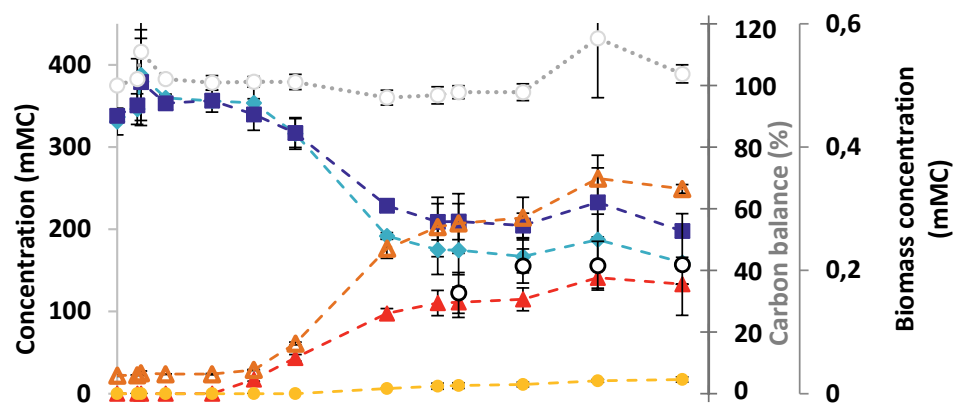

B
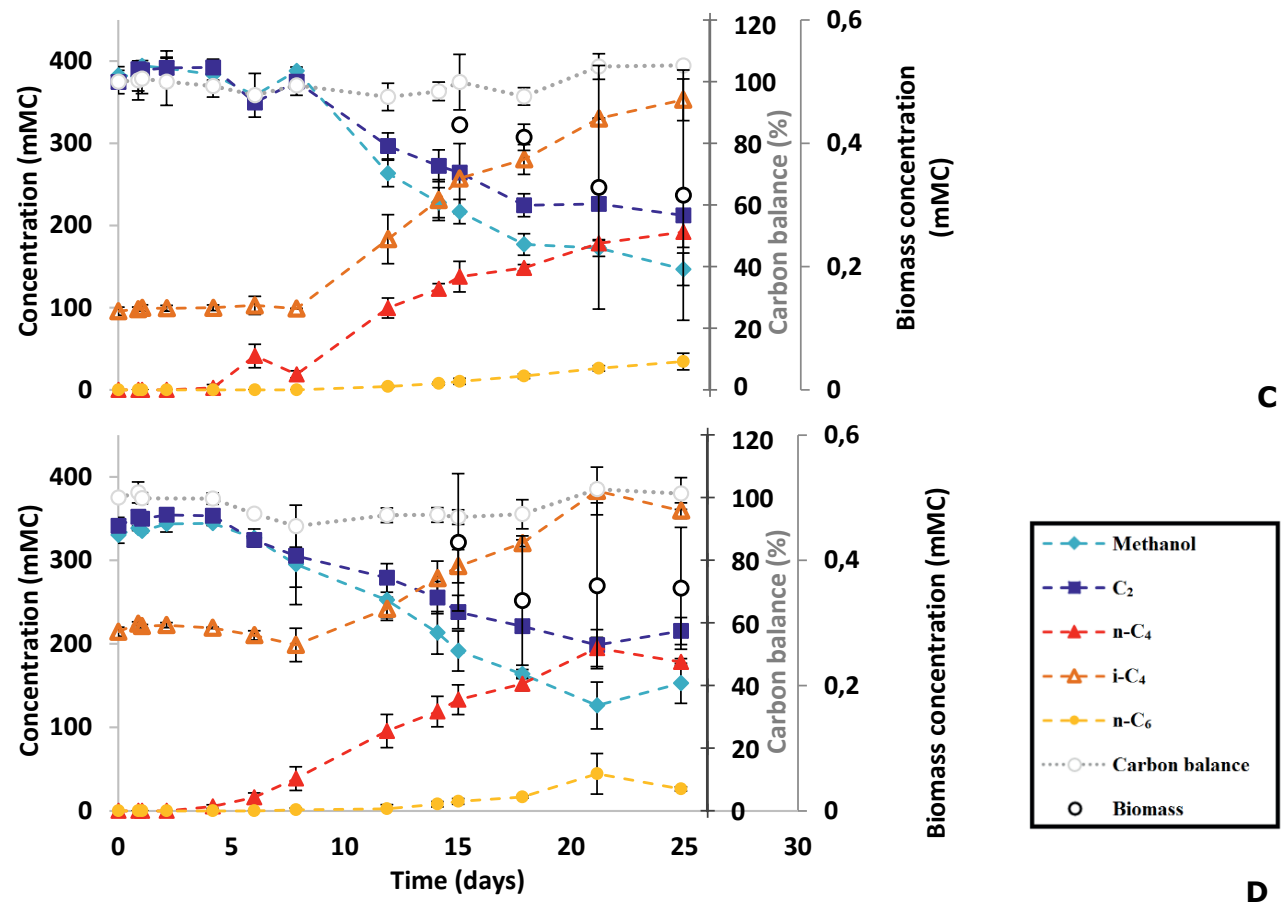

Figure S12. Concentrations of methanol, acetate, $n$-butyrate, iso-butyrate and the determined biomass concentration during the batch experiments $A$ to $D$. 

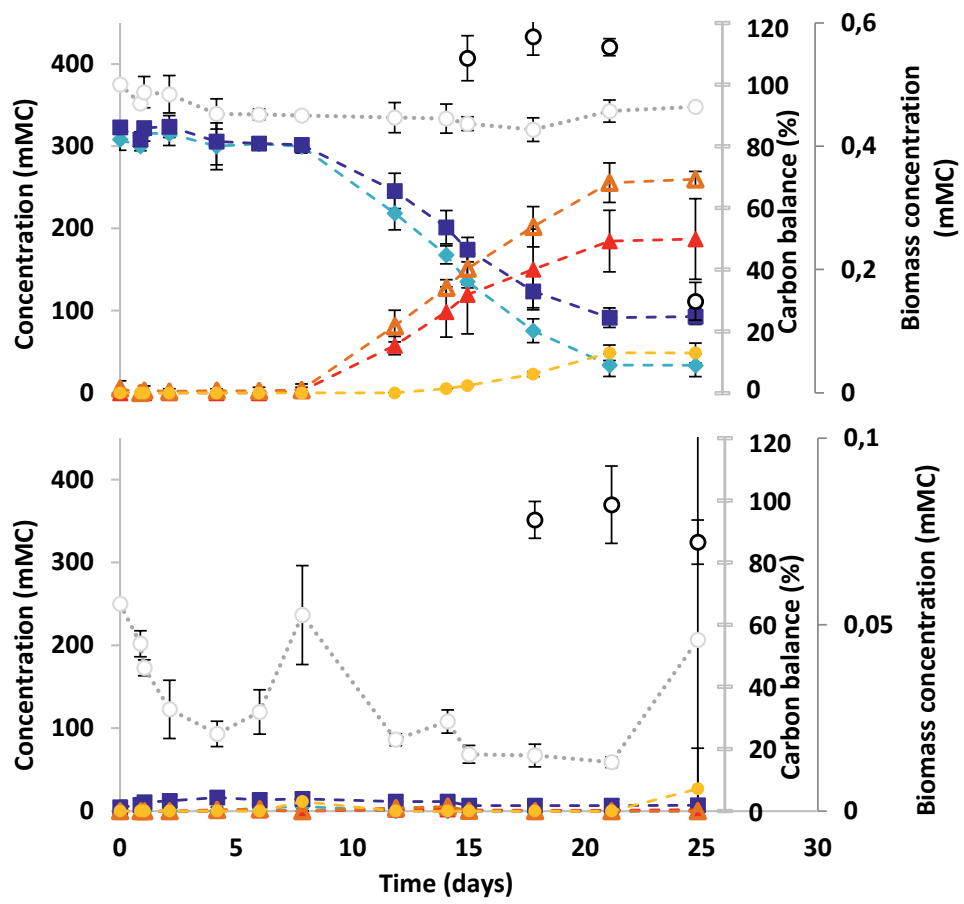

Figure S13. Concentrations of methanol, acetate, n-butyrate, iso-butyrate and the determined biomass concentration during the batch experiments $\mathrm{E}$ and $\mathrm{F}$. 

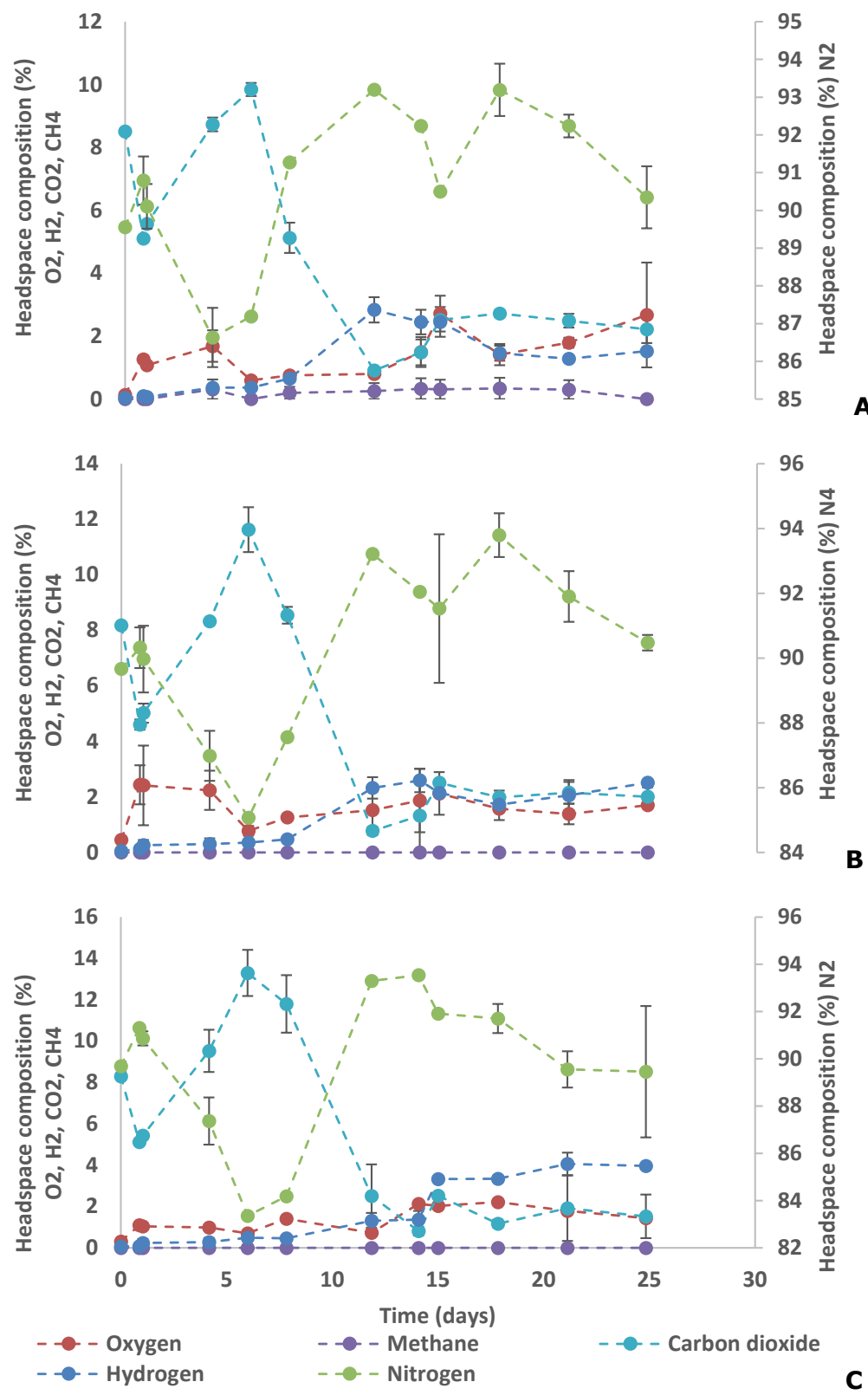

Figure S14. Headspace percentage of hydrogen, methane, carbon dioxide, oxygen and nitrogen during the batch experiments A to C. Pressures in the headspace were always In between 1 and 1.5 bar. 

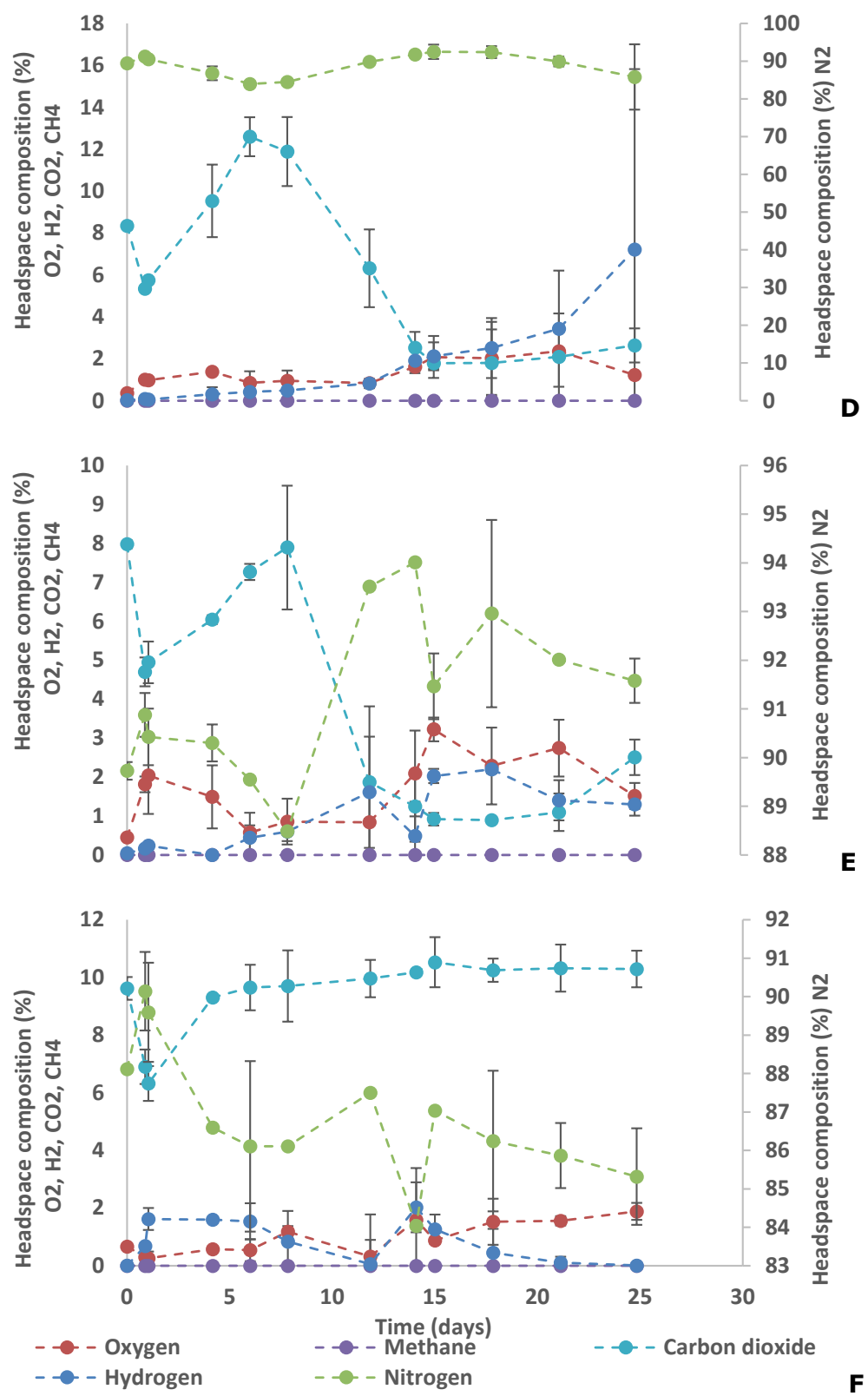

Figure S15. Headspace percentage of hydrogen, methane, carbon dioxide, oxygen and nitrogen during the batch experiments $D$ to $F$. Pressures in the headspace were always In between 1 and 1.5 bar. 


\section{Batches G-M}
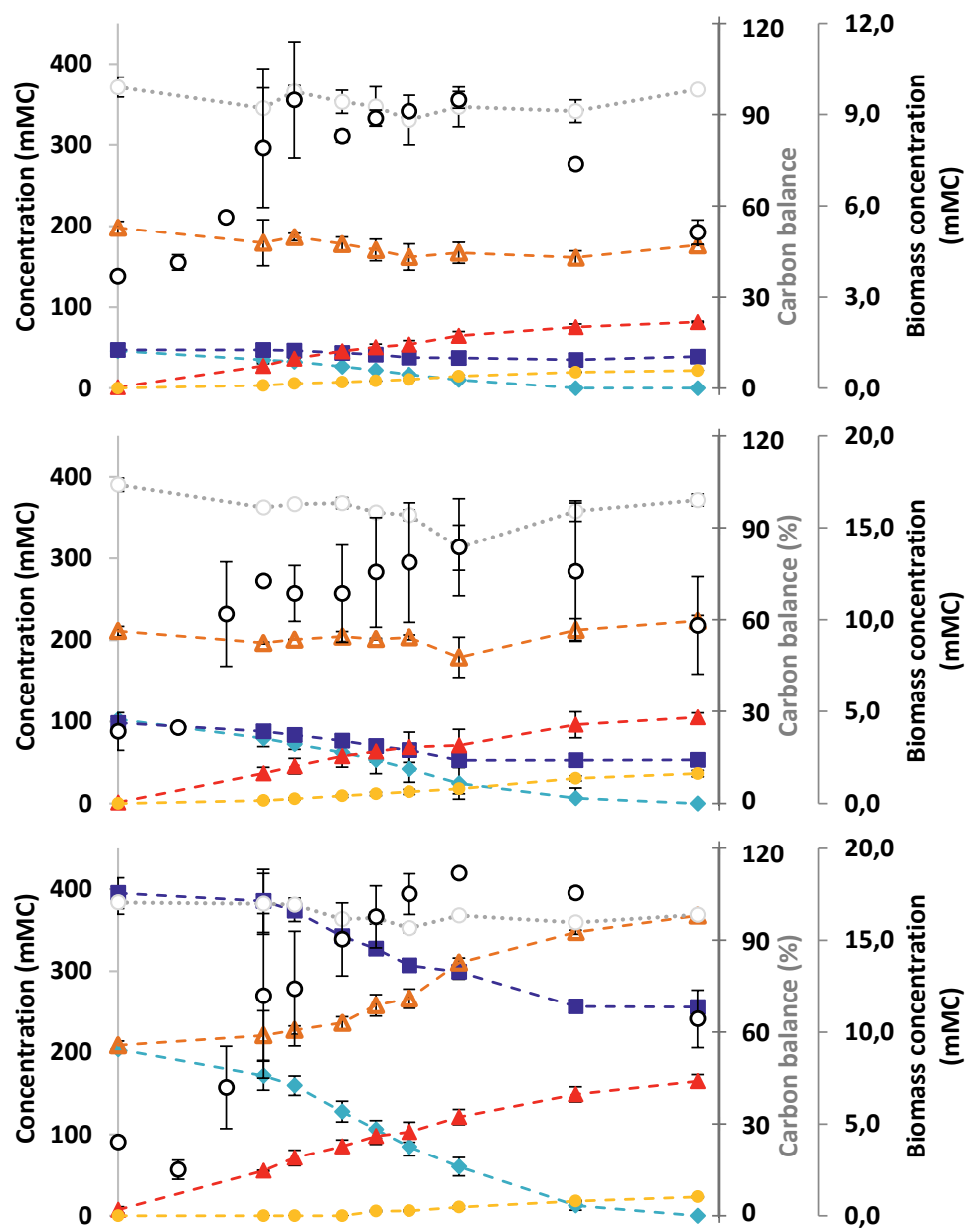

H
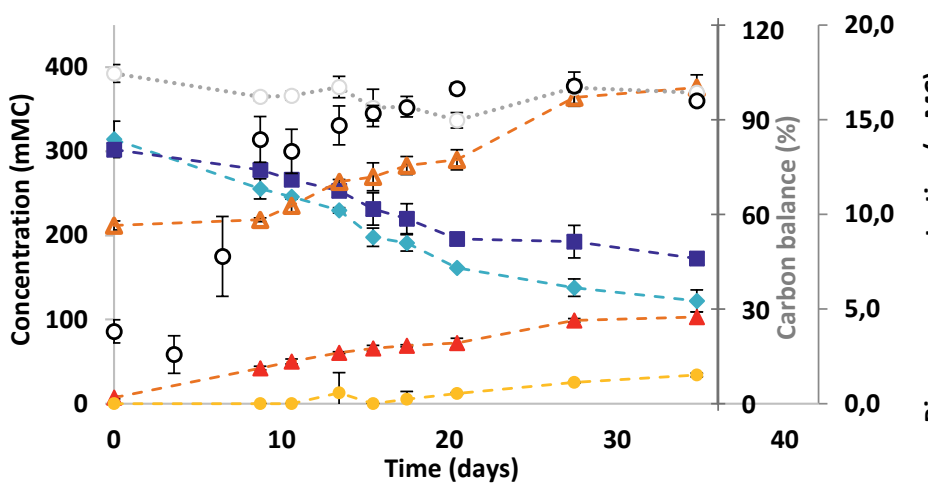

Figure S16. Concentrations of methanol, acetate, $n$-butyrate, iso-butyrate and the determined biomass concentration during the batch experiments $\mathrm{G}, \mathrm{H}, \mathrm{J}$ and $\mathrm{K}$. 

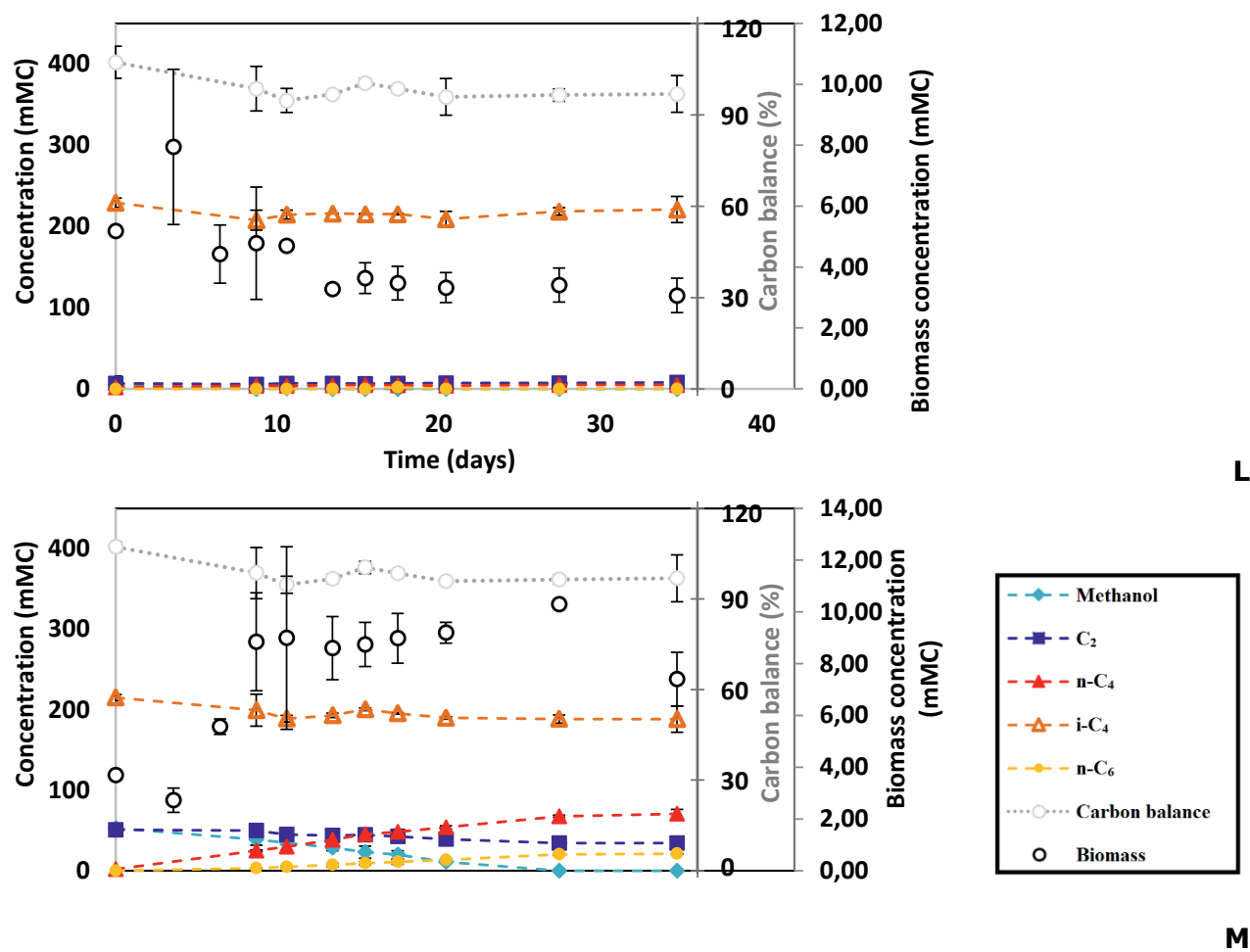

Figure S17. Concentrations of methanol, acetate, n-butyrate, iso-butyrate and the determined biomass concentration during the batch experiments $L$ and $M$. 

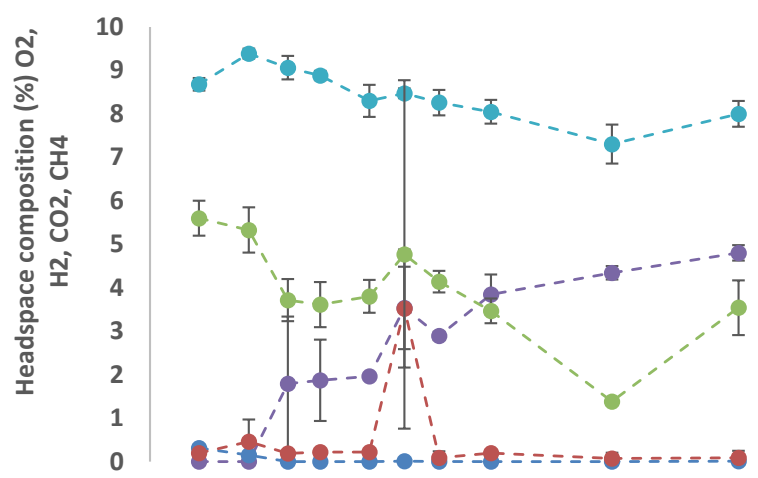

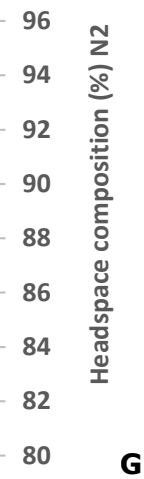
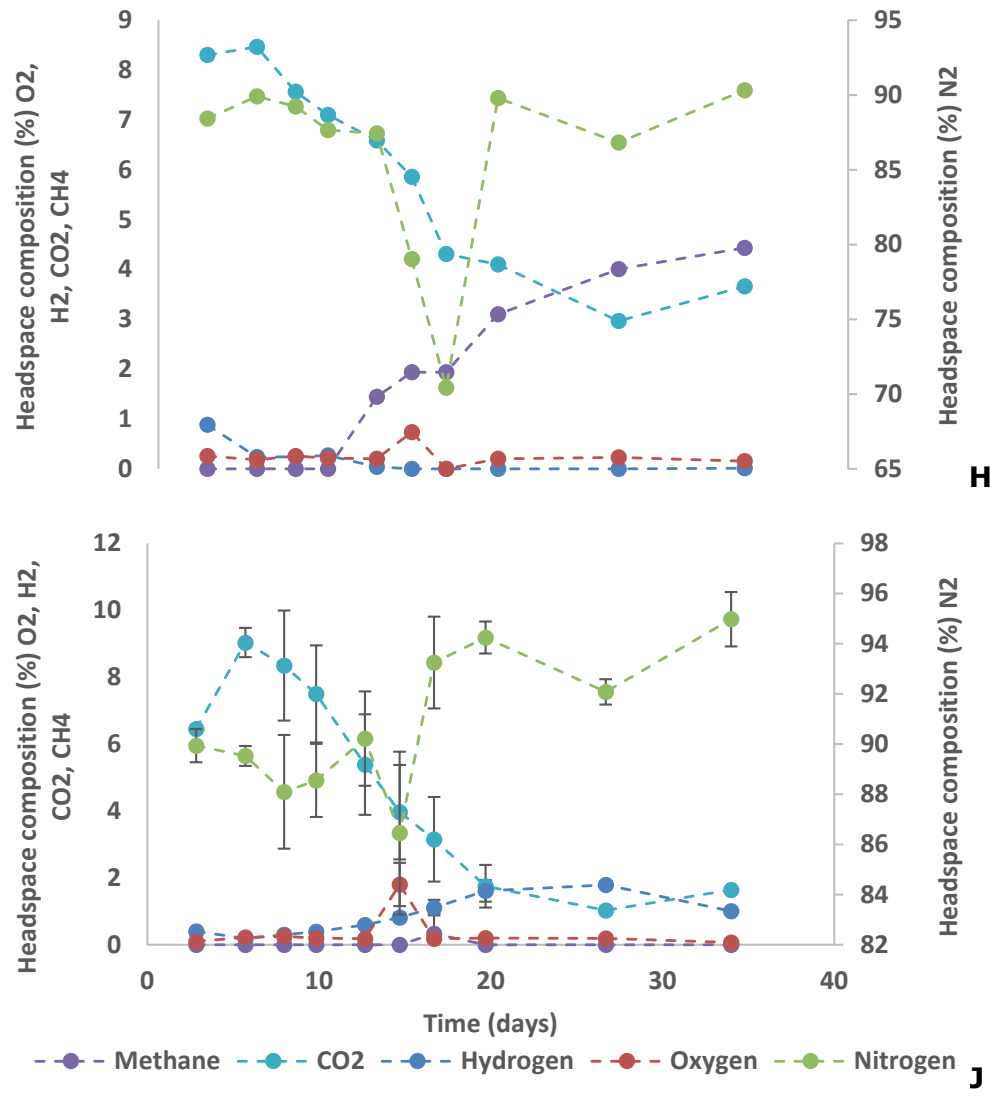

Figure S18. Headspace percentage of hydrogen, methane, carbon dioxide, oxygen and nitrogen during the batch experiments $\mathrm{G}, \mathrm{H}$ and J. Pressures in the headspace were always In between 1 and 1.5 bar. 

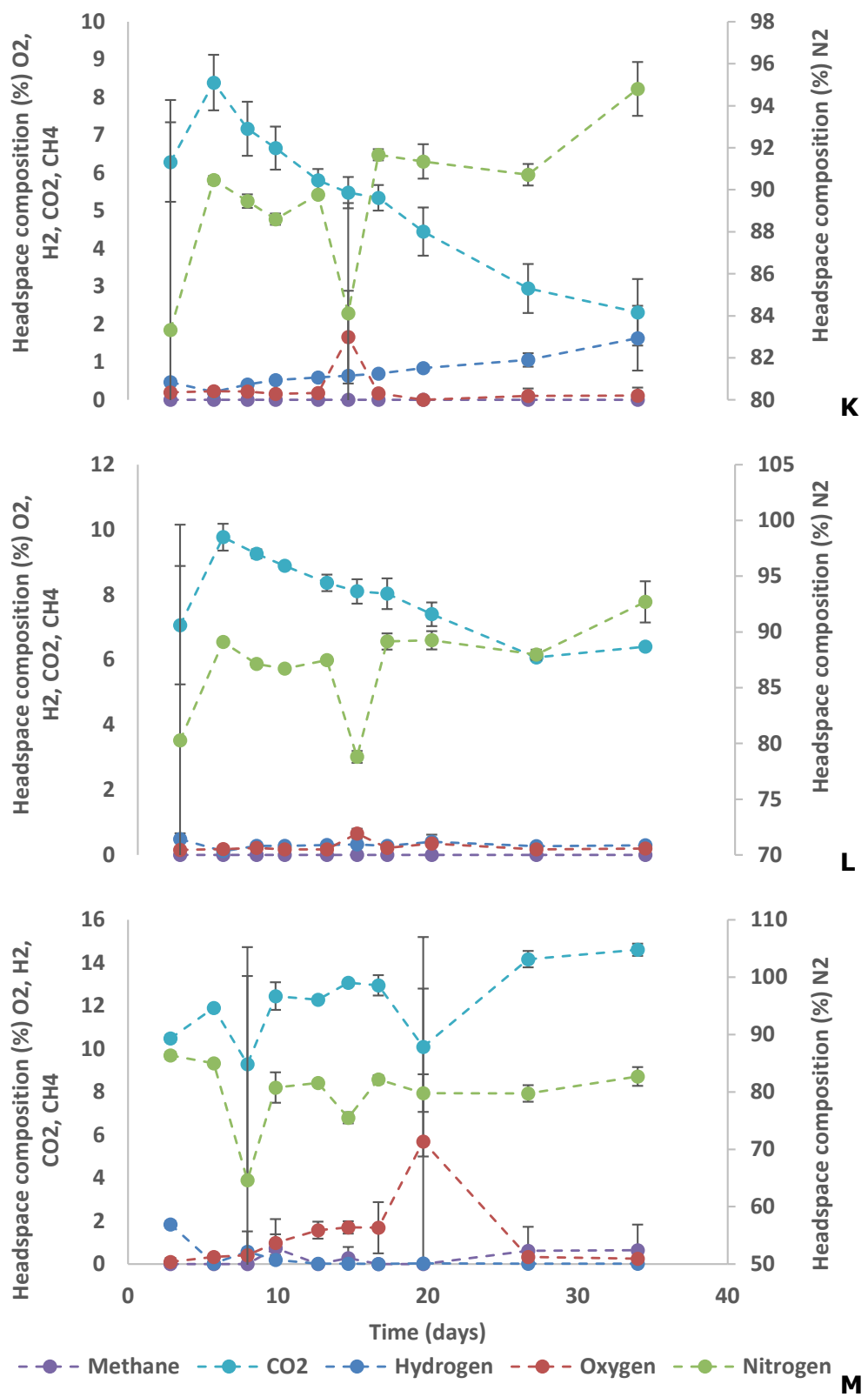

Figure S19. Headspace percentage of hydrogen, methane, carbon dioxide, oxygen and nitrogen during the batch experiments $\mathrm{K}, \mathrm{L}$ and $\mathrm{M}$. Pressures in the headspace were always In between 1 and 1.5 bar. 


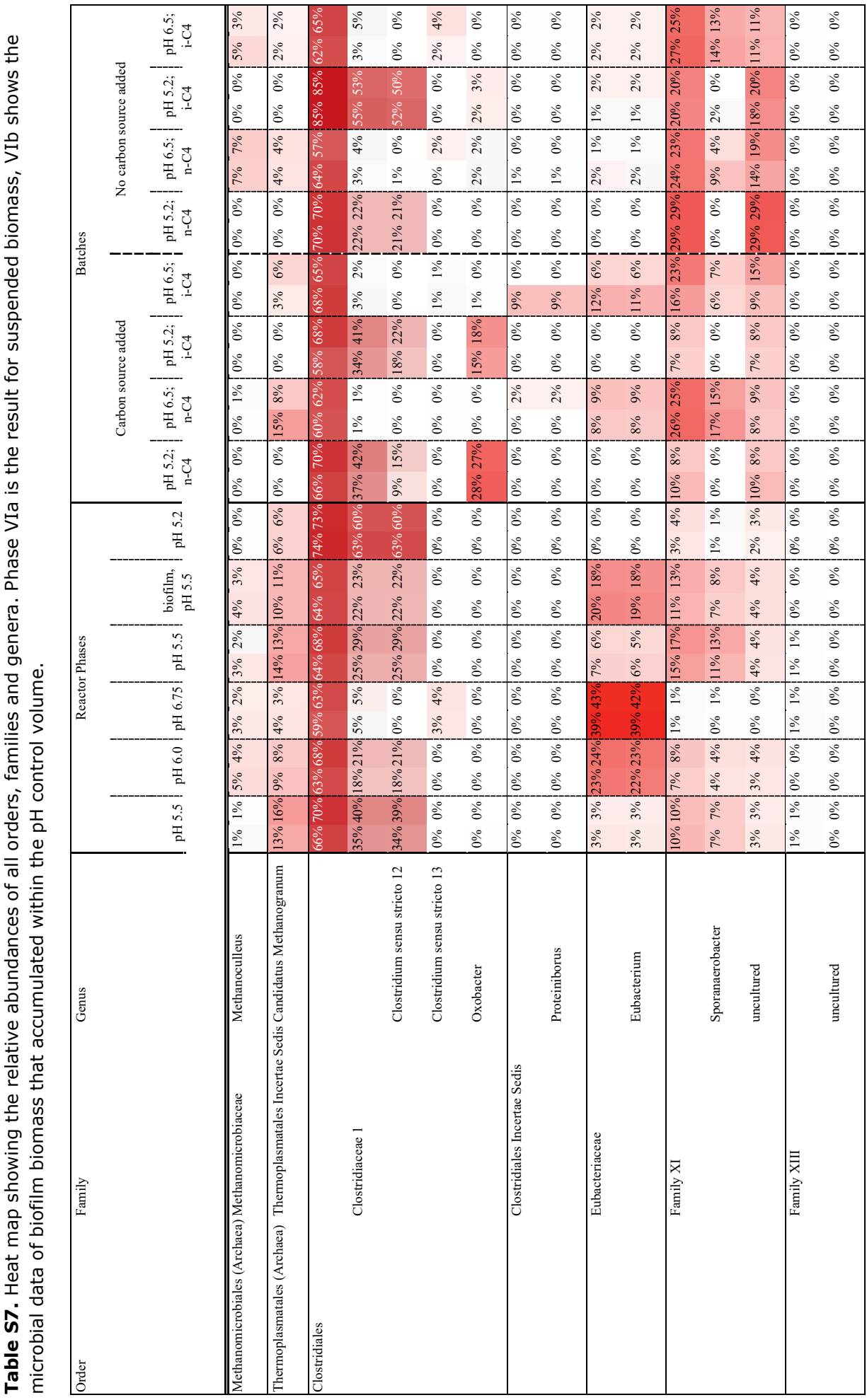




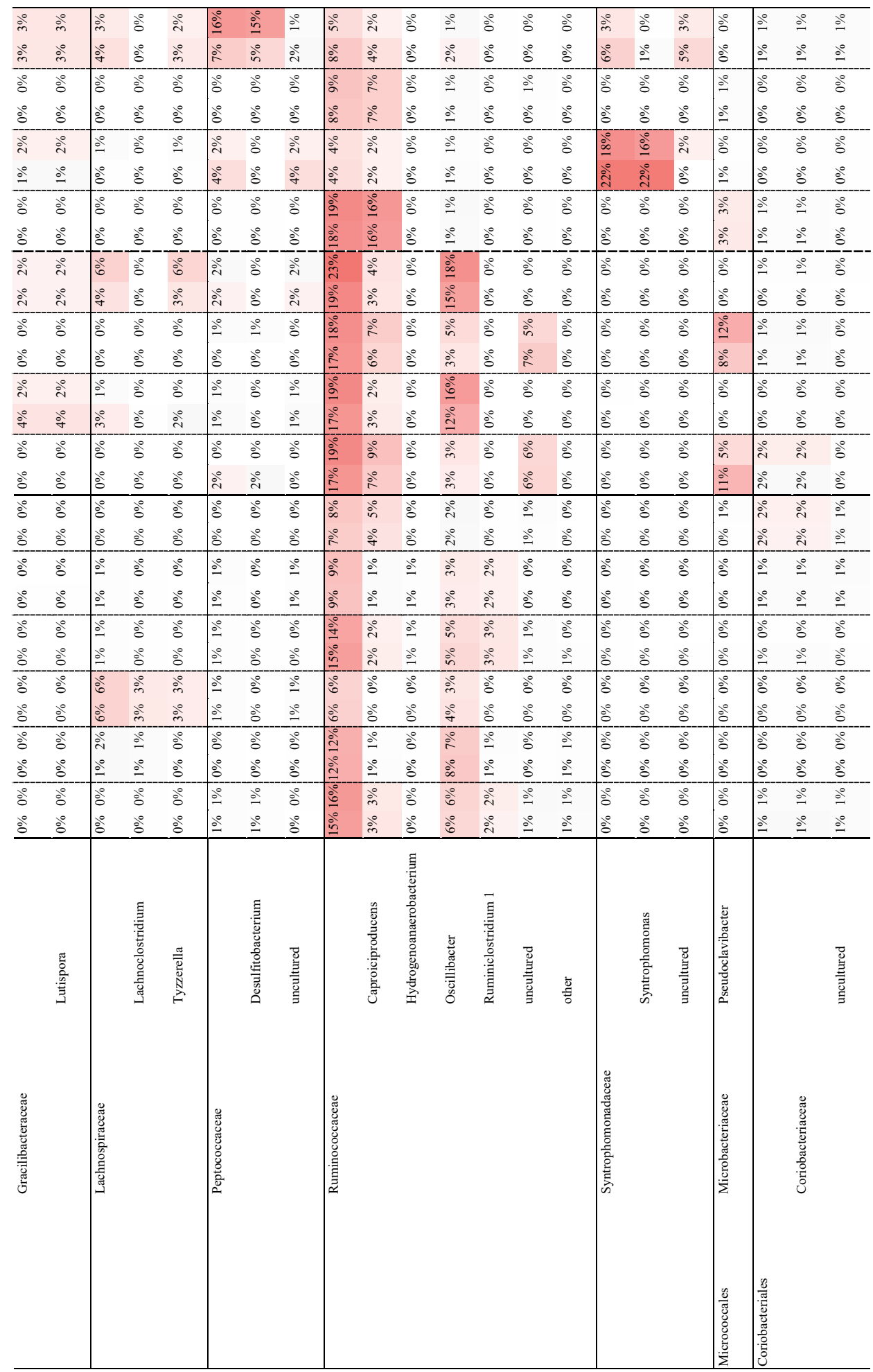




\begin{tabular}{|c|c|c|c|c|c|c|c|c|c|c|c|c|c|c|c|c|c|c|c|c|c|}
\hline aे & 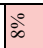 & $\stackrel{\circ}{\circ}$ & $\because$ & gे & ळें & ふें & $\mathrm{a}^{\circ}$ & $\cong$ & $\stackrel{\circ}{\circ}$ & 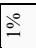 & $\therefore$ & 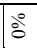 & 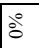 & 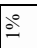 & $\mathrm{a}$ & ò & aे & & & å & @̊ \\
\hline $8^{\circ}$ & $i^{\circ}$ & $8^{\circ}$ & $3^{\circ}$ & $\mathrm{s}^{\circ}$ & ळे & iे & $8^{\circ}$ & $\therefore$ & $8^{\circ}$ & $\stackrel{\circ}{\circ}$ & $\stackrel{\circ}{\circ}$ & $8^{\circ}$ & $8^{\circ}$ & $\stackrel{\circ}{\circ}$ & $\mathrm{s}^{\circ}$ & $8^{\circ}$ & $8^{\circ}$ & $8^{\circ}$ & $\therefore$ & $8^{\circ}$ & 8 \\
\hline 8 & in & $8^{\circ}$ & $\therefore$ & $\mathrm{g}^{\circ}$ & $\mathrm{g}^{\circ}$ & 80 & $8^{\circ}$ & $\because$ & $8^{\circ}$ & $\stackrel{\circ}{\circ}$ & के & $8^{\circ}$ & $\because$ & $8^{\circ}$ & 8 & $\therefore$ & 8 & กั & 8 & خे & $\vdots$ \\
\hline $8^{\circ}$ & $\stackrel{\circ}{\circ}$ & 8 & 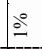 & $8^{\circ}$ & $8^{\circ}$ & $8^{\circ}$ & 8 & $\cong$ & $8^{\circ}$ & $\stackrel{\circ}{\circ}$ & ळे & $3^{\circ}$ & $8^{\circ}$ & $8^{\circ}$ & 8 & 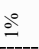 & 8 & సิ & 8 & ふे & $\stackrel{\circ}{\circ}$ \\
\hline 8 & $\ddot{\circ}$ & $8^{\circ}$ & iे & 8 & $\therefore$ & 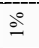 & $8^{\circ}$ & $\therefore$ & $8^{\circ}$ & ลे & ลे & $8^{\circ}$ & $8^{\circ}$ & $8^{\circ}$ & $8^{\circ}$ & 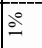 & 8 & $8^{\circ}$ & 8 & $8^{\circ}$ & $\because$ \\
\hline $8^{\circ}$ & in & $8^{\circ}$ & $\stackrel{\circ}{\circ}$ & $8^{\circ}$ & $\therefore$ & $8^{\circ}$ & $8^{\circ}$ & $\because$ & $8^{\circ}$ & 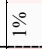 & $\stackrel{\circ}{\circ}$ & $\Xi^{\circ}$ & $8^{\circ}$ & $8^{\circ}$ & $8^{\circ}$ & $\AA^{\circ}$ & $8^{\circ}$ & $8^{\circ}$ & 8 & $8^{\circ}$ & $\stackrel{\circ}{\circ}$ \\
\hline $8^{\circ}$ & $\stackrel{\circ}{\circ}$ & $\mathrm{g}^{\circ}$ & $\therefore$ & $\therefore$ & $\therefore$ & $\therefore$ & $\mathrm{a}^{\circ}$ & $\stackrel{\circ}{\circ}$ & $8^{\circ}$ & $\because 0$ & $8^{\circ}$ & $\mathrm{g}^{\circ}$ & $\therefore$ & $\therefore$ & $\therefore$ & $\stackrel{\circ}{\circ}$ & $\therefore$ & $2^{\circ}$ & $\mathrm{B}^{\circ}$ & $\stackrel{\circ}{\circ}$ & $\stackrel{\circ}{\circ}$ \\
\hline $8^{\circ}$ & 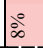 & aे & $\cong$ & aे & aे & aे & $\stackrel{\circ}{\circ}$ & ڤे & $\mathrm{a}^{\circ}$ & in & in & 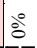 & $\cong$ & $\mathrm{a}^{\circ}$ & aे & $\cong$ & aे & 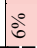 & aे & ذे & in \\
\hline
\end{tabular}

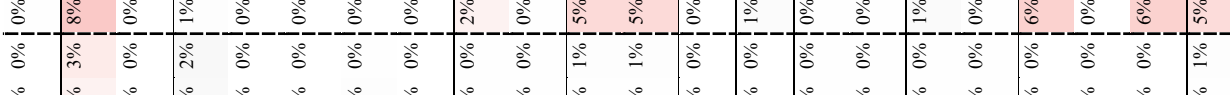

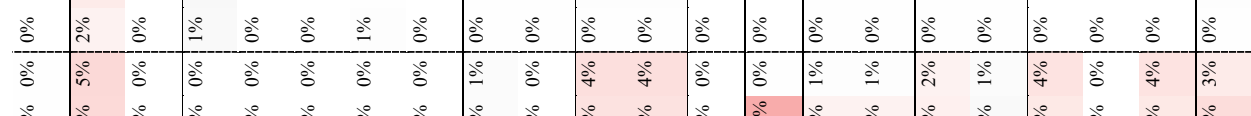

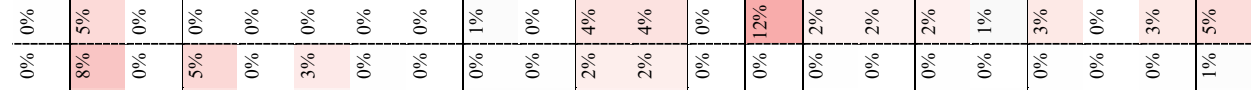

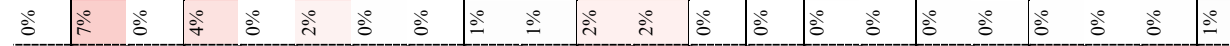

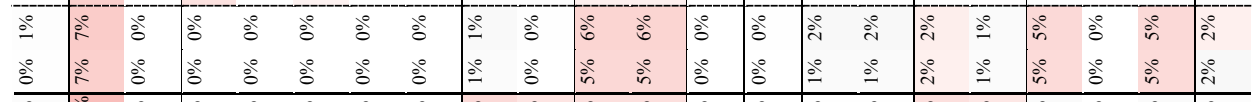

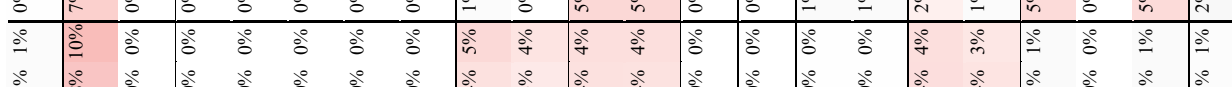

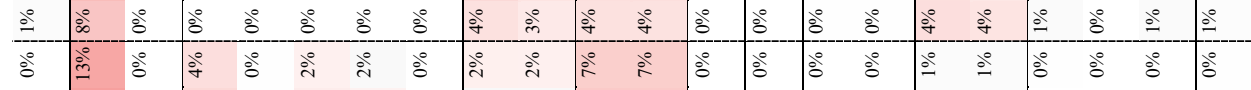

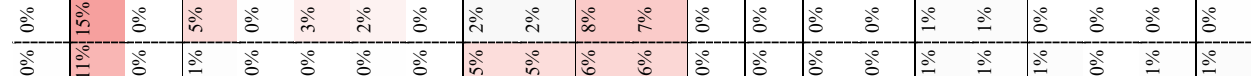

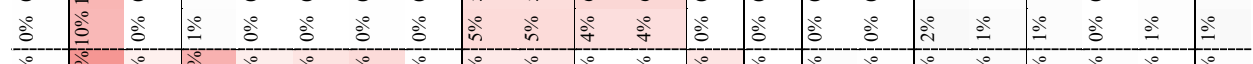

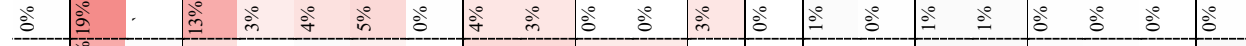

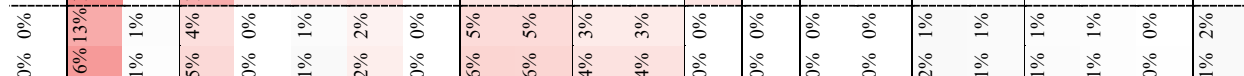

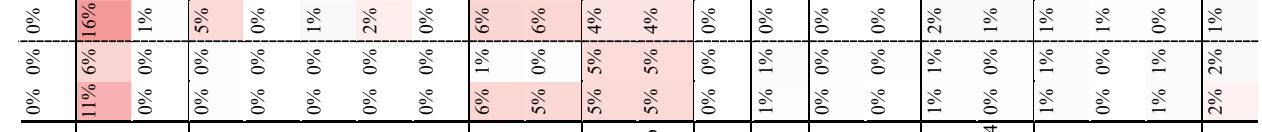

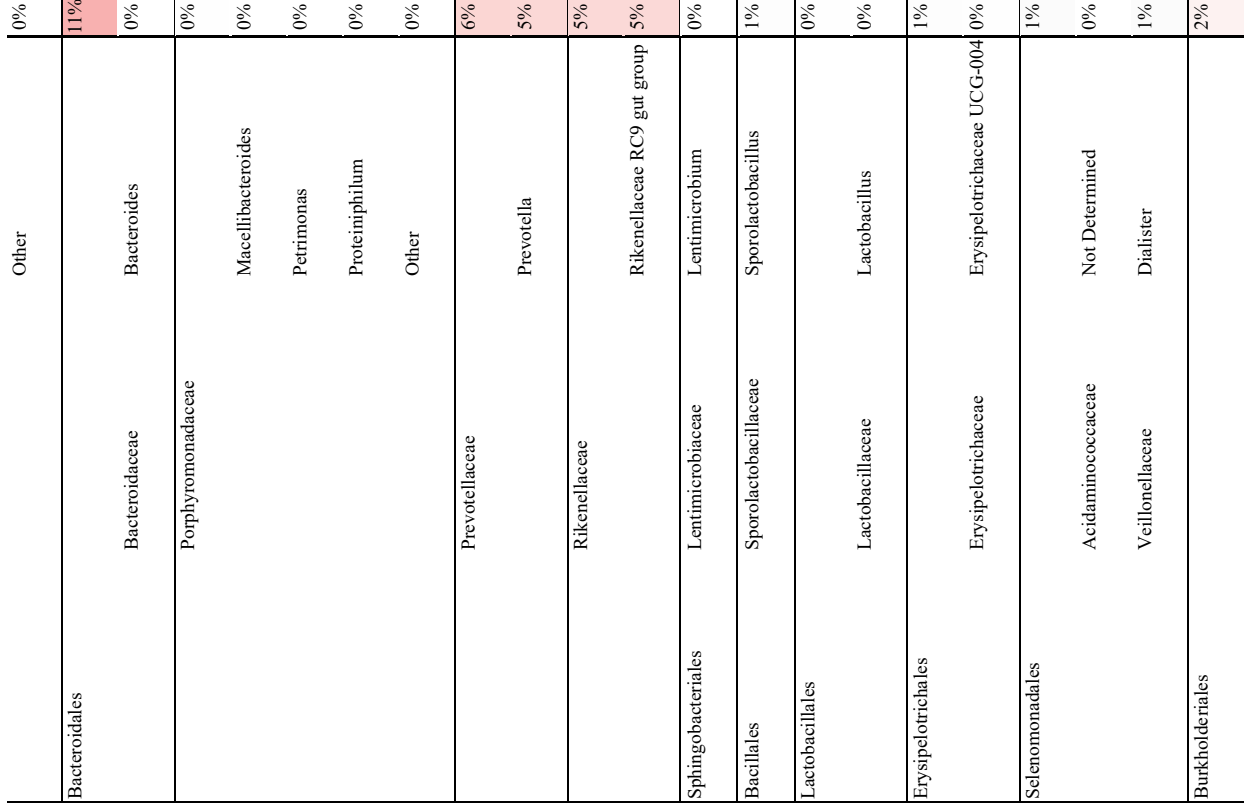




\begin{tabular}{|c|c|c|c|c|c|c|c|c|c|c|c|c|c|c|c|}
\hline$\overline{8}$ & $\lesssim$ & $\stackrel{\circ}{\circ}$ & 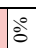 & вे & 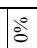 & $\stackrel{8}{\circ}$ & $2^{\circ}$ & ฉ̊ & $8^{\circ}$ & $\dot{8}^{\circ}$ & & $\mathrm{a}^{\circ}$ & 怒 & ळे & \\
\hline $\mathrm{a}^{\circ}$ & 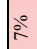 & $\stackrel{2}{2}$ & $\stackrel{8}{\circ}$ & $\stackrel{8}{\circ}$ & $\therefore$ & s̊ & $\fallingdotseq$ & $\stackrel{\varrho}{ٍ}$ & $\mathrm{~g}^{\circ}$ & $\mathrm{s}^{\circ}$ & 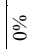 & 8 & $亠_{\infty}^{\circ}$ & ふे & \\
\hline خे & ลे & ৯े & $8^{\circ}$ & 80 & $\because$ & $\therefore$ & $8^{\circ}$ & 8 & $8^{\circ}$ & $8^{\circ}$ & $8^{\circ}$ & 80 & 高 & $\therefore$ & \\
\hline$\stackrel{\circ}{\cong}$ & ஓे & ஓे & $\therefore$ & s̊ & $\cong$ & $\therefore$ & 8 & $\mathrm{~s}$ & $\mathrm{~B}^{\circ}$ & $\mathrm{a}^{\circ}$ & 8 & $\mathrm{a}^{\circ}$ & 送 & $\stackrel{\circ}{\varrho}$ & \\
\hline$\therefore$ & $\because$ & $\therefore$ & 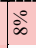 & $\infty$ & $\because$ & 8 & $2^{\circ}$ & ¿े & $8^{\circ}$ & 8 & 8 & $8^{\circ}$ & $\frac{8}{8}$ & $\therefore$ & \\
\hline 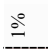 & $\therefore$ & $2^{\circ}$ & $\because$ & $\stackrel{\circ}{=}$ & 8 & $8^{\circ}$ & $\because$ & ذ̊ & $3^{\circ}$ & $8^{\circ}$ & 8 & 8 & 送 & 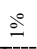 & \\
\hline ஓे & $\because$ & $\therefore$ & 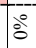 & $\ddot{\circ}$ & ๙े & 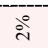 & 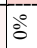 & वे & $\mathrm{a}^{\circ}$ & $\therefore$ & 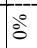 & $\mathrm{a}^{\circ}$ & के & 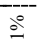 & \\
\hline in & $\cong$ & $\stackrel{\circ}{\circ}$ & $\vdots$ & aे & ळे & ळे & $a^{\circ}$ & $\stackrel{a}{a}$ & $\therefore$ & $\therefore$ & $a^{\circ}$ & $\mathrm{a}^{\circ}$ & $\mathrm{\sigma}^{\circ}$ & $\therefore$ & \\
\hline$\therefore$ & すo & 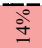 & $\because$ & aे & @ & aे & 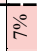 & ¿े & $\therefore$ & $\therefore$ & $\because$ & $8^{\circ}$ & 㐫 & ते & \\
\hline aे & 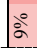 & ふे & 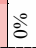 & oे & 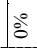 & aे & $\Xi^{\circ}$ & ذे & $\mathrm{a}^{\circ}$ & $\mathrm{a}^{\circ}$ & 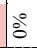 & $\mathrm{a}^{\circ}$ & $\overbrace{\infty}^{\circ}$ & ふें & \\
\hline iे & $\because$ & 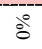 & $\because$ & $\therefore$ & $\stackrel{े}{\circ}$ & ৯े & $\because$ & $\therefore$ & $\therefore$ & $\therefore$ & $\because$ & वे & $\sigma^{\circ}$ & $\therefore$ & \\
\hline ஓे & $\because$ & oे & $\therefore$ & oे & ळे & ळे & 8 & sे & 3 & $\mathrm{~s}^{\circ}$ & 8 & 8 & 容 & 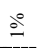 & \\
\hline$\stackrel{\circ}{\varrho}$ & $\cong$ & 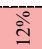 & 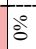 & $\ddot{\circ}$ & $\because$ & $\therefore$ & $\because$ & ठ̊ & $\because$ & $\therefore$ & $\because$ & $\therefore$ & के & $\therefore$ & \\
\hline$\stackrel{\circ}{\circ}$ & $\fallingdotseq$ & $\stackrel{\circ}{ٍ}$ & 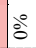 & $\stackrel{\circ}{\circ}$ & 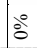 & $\stackrel{8}{\circ}$ & $\stackrel{\circ}{\circ}$ & $\stackrel{\circ}{\circ}$ & $\because$ & $\therefore$ & 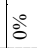 & $\mathrm{a}^{\circ}$ & 家 & $\stackrel{\circ}{\circ}$ & \\
\hline خे & $\because$ & 8 & $\because$ & $\ddot{\circ}$ & $\stackrel{\circ}{\circ}$ & ¿े & $8^{\circ}$ & 8 & $\because$ & $\mathrm{o}^{\circ}$ & $\because$ & $8^{\circ}$ & 家 & $\therefore$ & \\
\hline ふे & 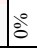 & oे & $\vdots$ & oे & ळे & ळे & 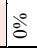 & oे & $\because$ & aे & 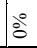 & $\mathrm{a}^{\circ}$ & 容 & 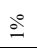 & \\
\hline$\stackrel{\circ}{\circ}$ & $\because$ & gे & 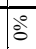 & o̊ & $\stackrel{\circ}{\circ}$ & aे & $\because$ & $\mathrm{g}^{\circ}$ & $\because$ & $\mathrm{a}^{\circ}$ & $a^{\circ}$ & $\mathrm{a}^{\circ}$ & 产 & 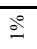 & \\
\hline 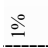 & $\because$ & $\stackrel{8}{0}$ & $\because$ & $\stackrel{8}{\circ}$ & $\therefore$ & $\therefore$ & $\therefore$ & aे & $\because$ & $\therefore$ & $\therefore$ & $\therefore$ & $\check{2}^{\circ}$ & $\therefore$ & \\
\hline $\mathrm{a}^{\circ}$ & $\because$ & $\therefore$ & $\because$ & $\ddot{\circ}$ & $\because$ & $\therefore$ & $\because$ & $\therefore$ & $\because$ & $\therefore$ & $\because$ & $\mathrm{a}^{\circ}$ & $\sigma^{\circ}$ & $\therefore$ & \\
\hline$\stackrel{\circ}{\circ}$ & $\cong$ & $\therefore$ & 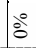 & aे & $\cong$ & $\therefore$ & $\cong$ & $\therefore$ & $\because$ & $\stackrel{\circ}{\circ}$ & 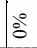 & $\stackrel{\circ}{\circ}$ & 送 & $\stackrel{\circ}{ }$ & \\
\hline$\stackrel{-}{\circ}$ & $\because$ & $\therefore$ & $\because$ & $\therefore$ & $\because$ & 8 & $\because$ & 8 & $\because$ & 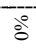 & $\because$ & $\therefore$ & कू & $\therefore$ & \\
\hline$\stackrel{\circ}{\varrho}$ & $\cong$ & $\stackrel{\circ}{\circ}$ & 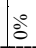 & $\stackrel{8}{\circ}$ & $\therefore$ & $\dot{8}$ & $8^{\circ}$ & $\mathrm{s}^{\circ}$ & $3^{\circ}$ & $\mathrm{s}^{\circ}$ & $\cong$ & $\stackrel{\circ}{\circ}$ & 送 & 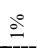 & \\
\hline oे & $\because$ & $\therefore$ & $\therefore$ & ळे & $\because$ & $\mathrm{a}$ & 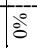 & वे & $\because$ & 8 & $\therefore$ & ลे & Sू & $\because$ & \\
\hline$\stackrel{\circ}{\circ}$ & $\because$ & $\stackrel{\circ}{\circ}$ & ळ & $\stackrel{\circ}{\circ}$ & $\because$ & $\therefore$ & 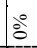 & $\stackrel{\circ}{\circ}$ & $\because$ & $\therefore$ & 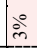 & $\dot{ }^{\circ}$ & $\approx$ & ふे & \\
\hline$\therefore$ & $\because$ & $\therefore$ & $\because$ & $\therefore$ & $\because$ & $\therefore$ & $\because$ & $\therefore$ & $\because$ & 8 & $\because$ & $\therefore$ & $\begin{array}{l}\frac{a}{2} \\
a^{2}\end{array}$ & $\therefore$ & \\
\hline$\stackrel{\circ}{\varrho}$ & $\because$ & $\stackrel{\circ}{\circ}$ & 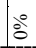 & $\stackrel{\circ}{\circ}$ & $\therefore$ & $\therefore$ & 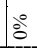 & $\mathrm{a}^{\circ}$ & $\Xi^{\circ}$ & $\therefore$ & $\cong$ & $\stackrel{\circ}{\circ}$ & : & 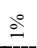 & \\
\hline ৯े & $\because$ & $\therefore$ & $\because$ & $\therefore$ & $\because$ & $\therefore$ & $\because$ & $\mathrm{o}^{\circ}$ & $\because$ & $\therefore$ & $\because$ & $a^{\circ}$ & क- & 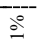 & \\
\hline خे & 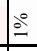 & $\stackrel{\circ}{-}$ & $3^{\circ}$ & 8 & $\because$ & $\dot{B}^{\circ}$ & $3^{\circ}$ & $\mathrm{o}^{\circ}$ & $\because$ & $\mathrm{a}^{\circ}$ & $\cong$ & $\stackrel{\circ}{\circ}$ & 今 & $\stackrel{\circ}{\circ}$ & \\
\hline 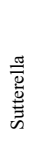 & & 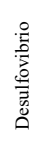 & & 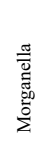 & & 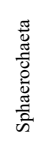 & & 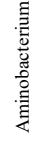 & & 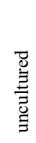 & & 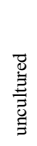 & & & \\
\hline 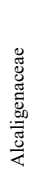 & & 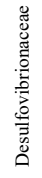 & & 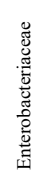 & & 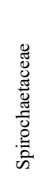 & & 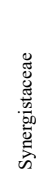 & & $\begin{array}{l}\text { 总 } \\
\text { 竧 } \\
\text { 音 }\end{array}$ & & 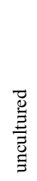 & & & \\
\hline & 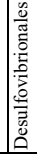 & & 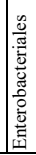 & & 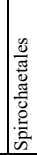 & & 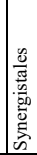 & & 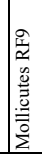 & & $\frac{\frac{7}{9}}{\frac{m}{2}}$ & & 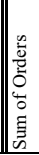 & 总 & 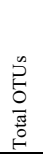 \\
\hline
\end{tabular}

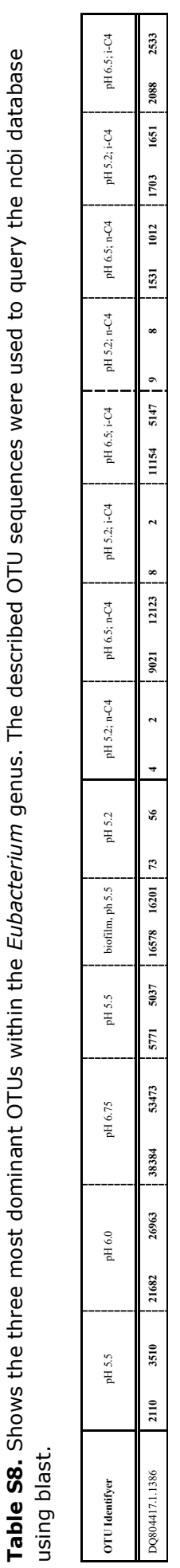




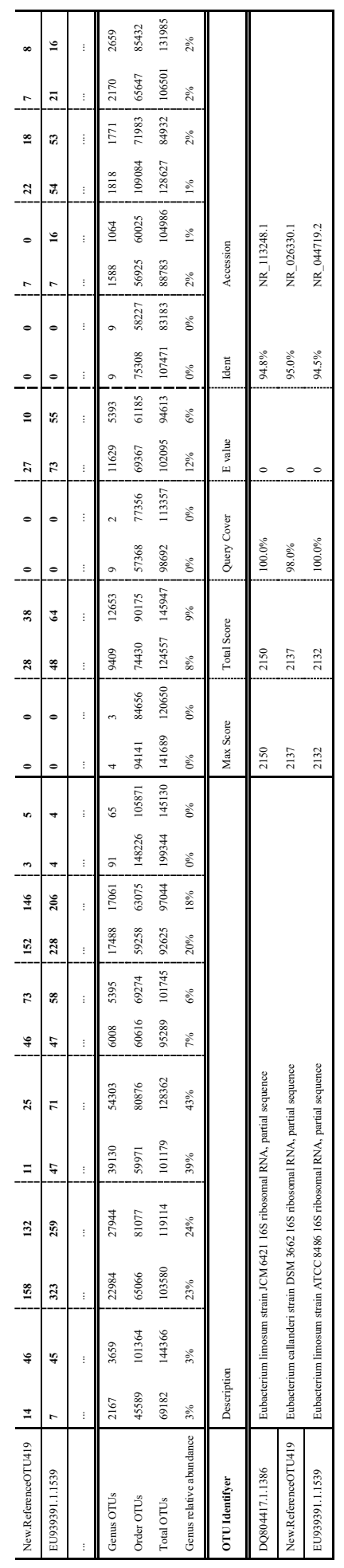

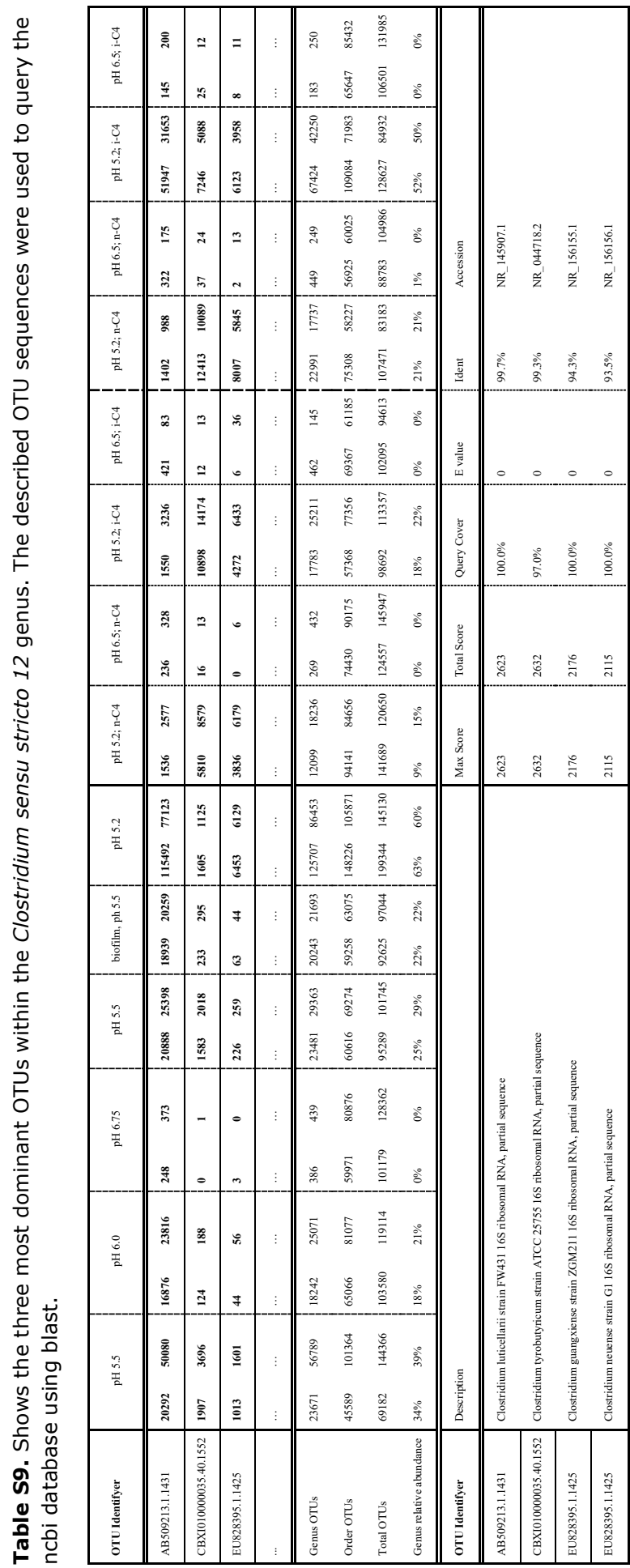



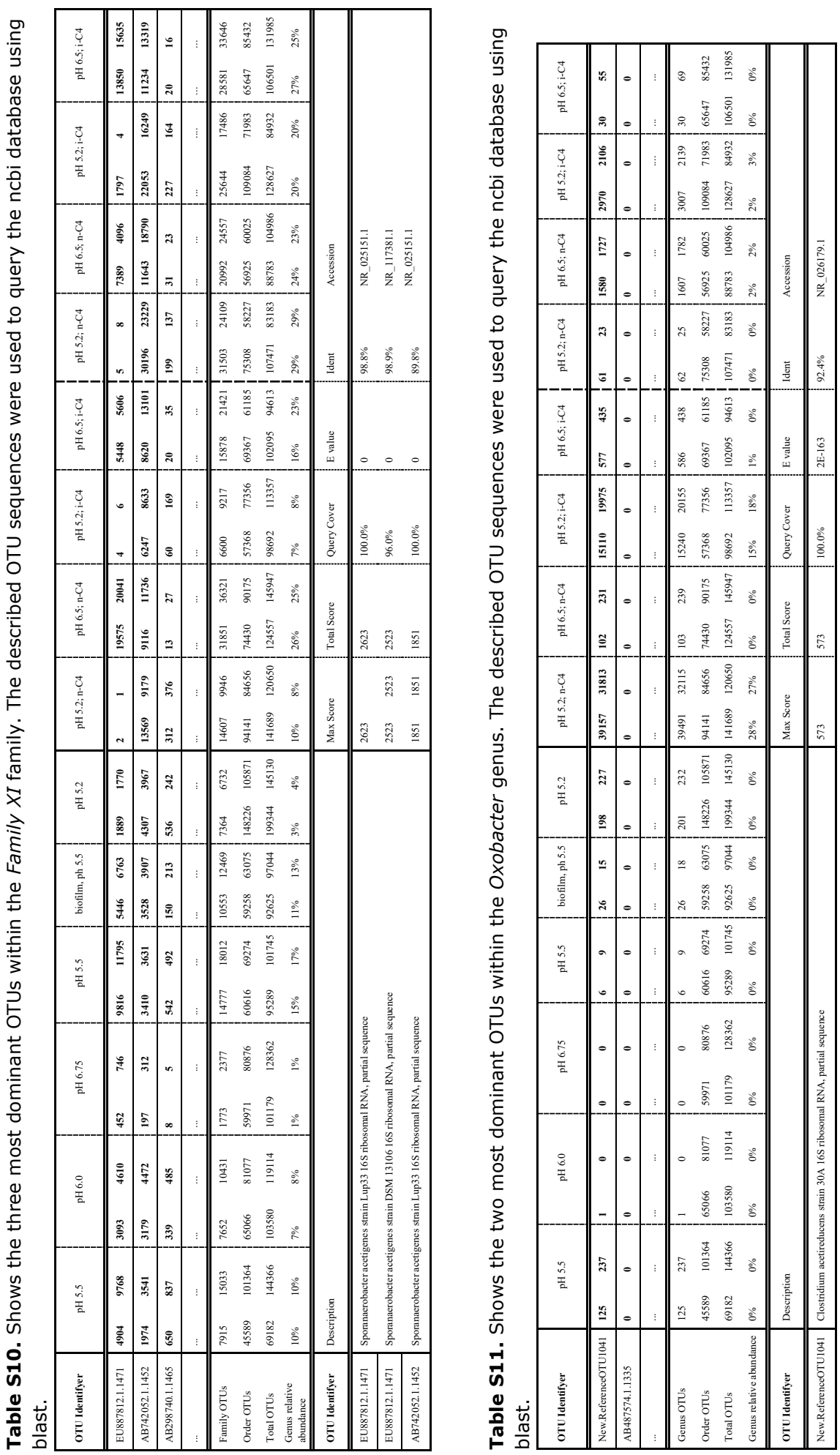

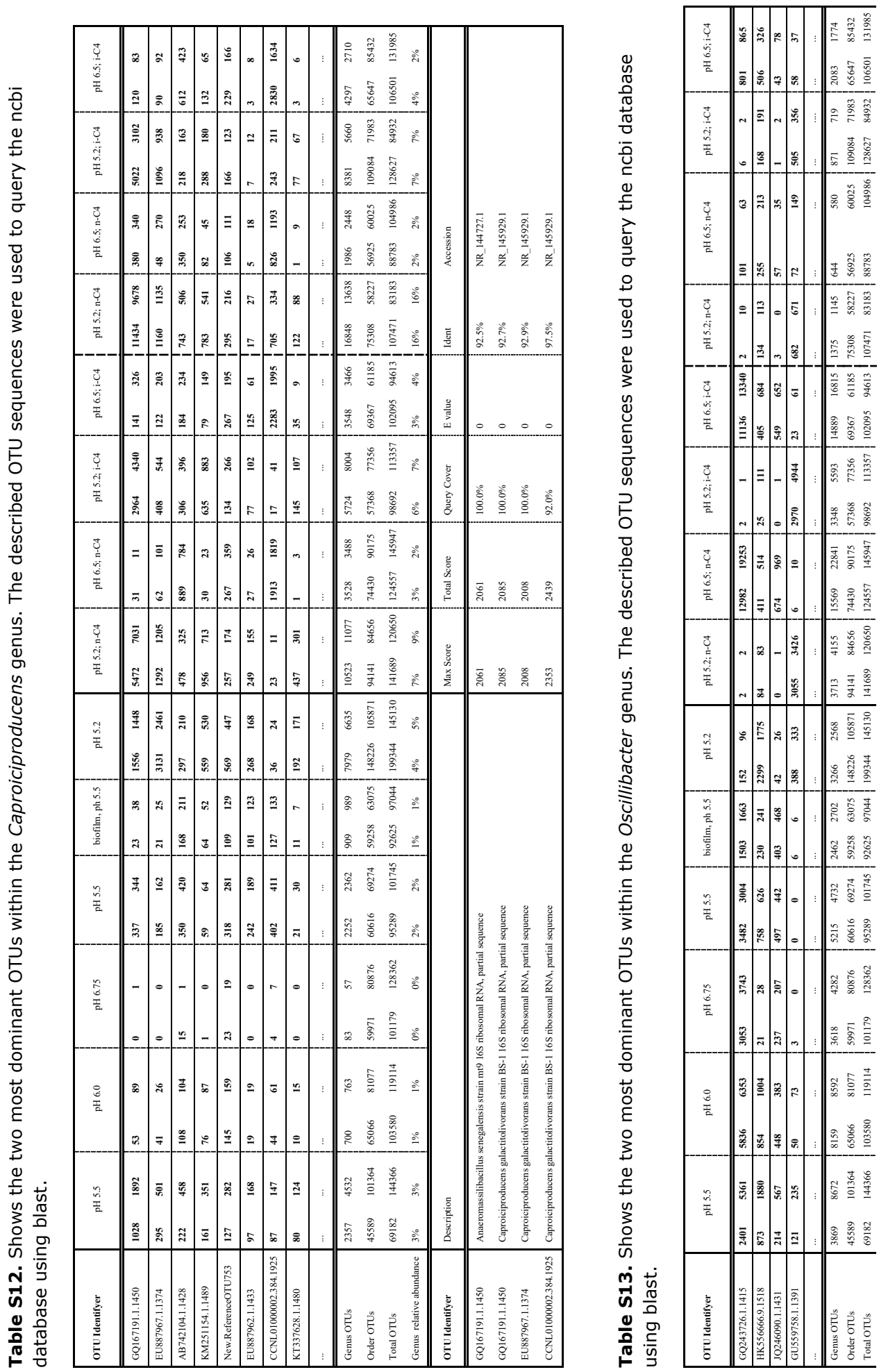


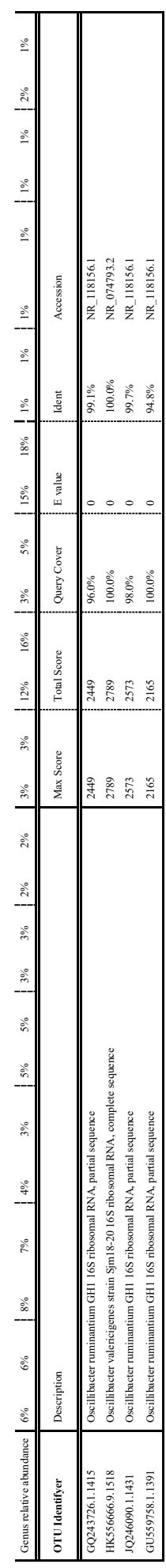



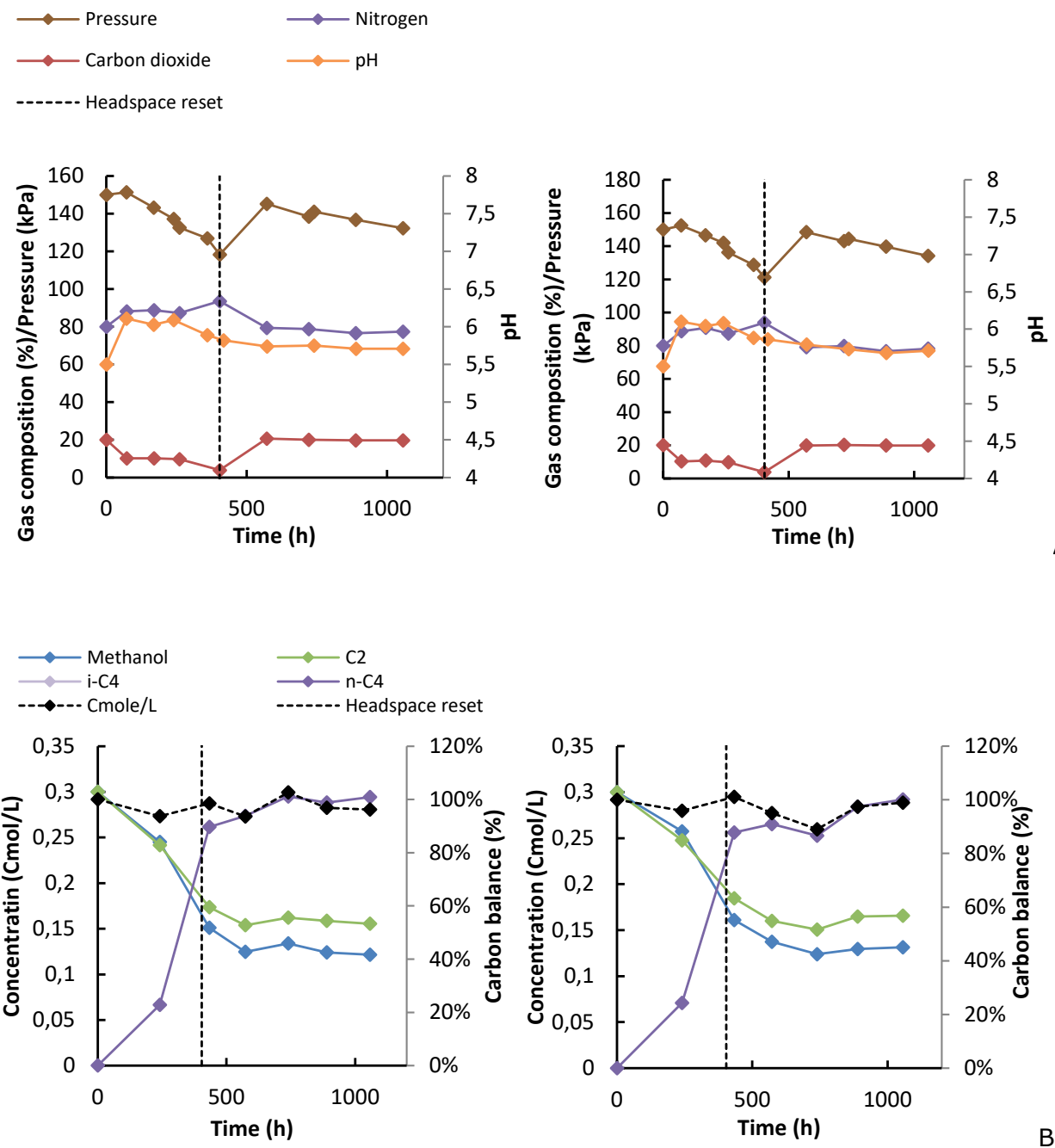

Figure S20. A) These two figures show the $\mathrm{pH}$ and headspace gas composition of two in duplo Eubacterium limosum pure culture batches. B) These two figures show the concentration profiles of the main metabolites (Methanol, acetate, n-butyrate) and the carbon balance of the two in duplo Eubacterium limosum pure culture batches. 


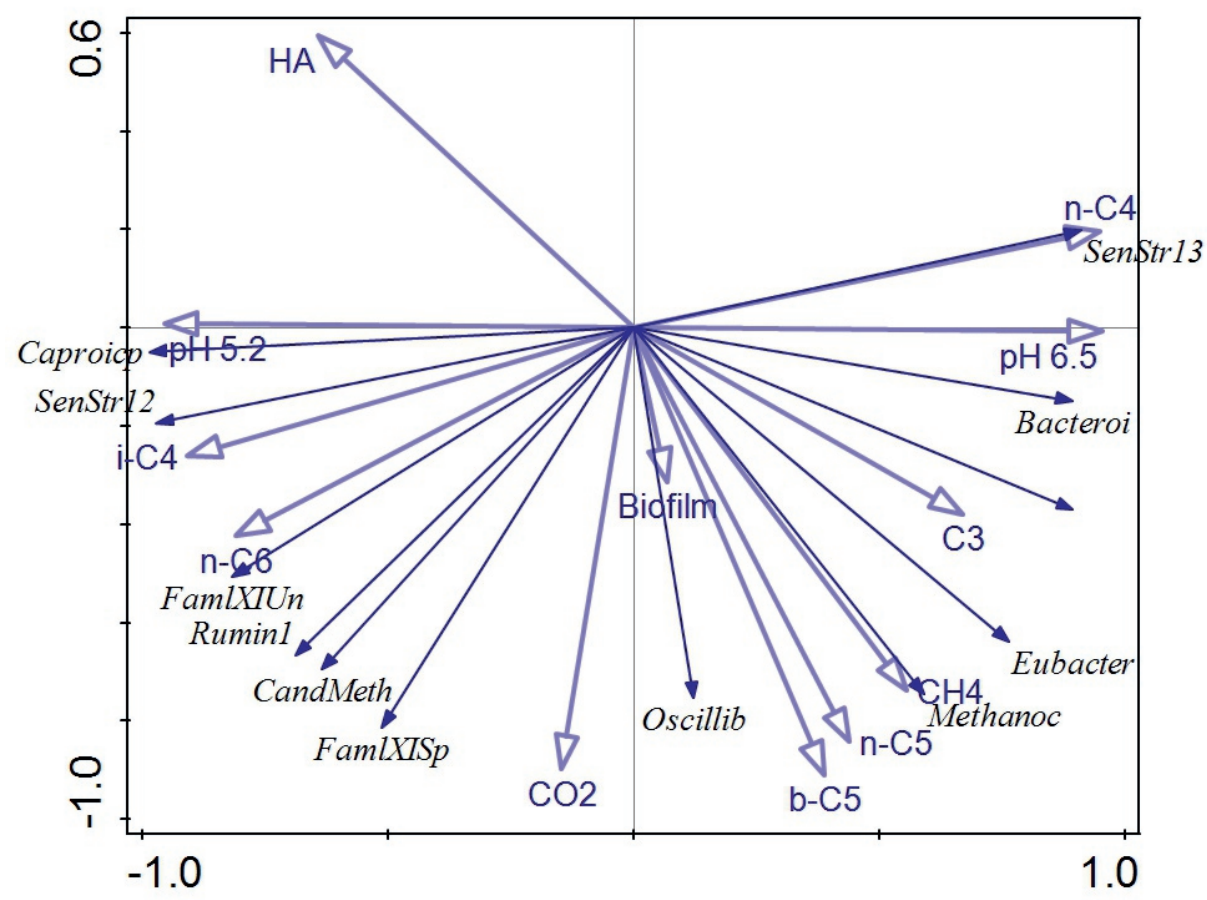

Figure S21. Unconstrained redundancy triplot using as parameters: the steady state average conversions for the phases in the reactor (positive=formation, negative=consumption), the difference between the $\mathrm{pH}$ levels (Using a positive difference for $\mathrm{pH} 5.2$ compared to 6.5 and vice versa) and the steady state average total undissociated fatty acids concentration. Microbioal data of the genera for phase II, IV, V, VI and VII was used creating this plot. 


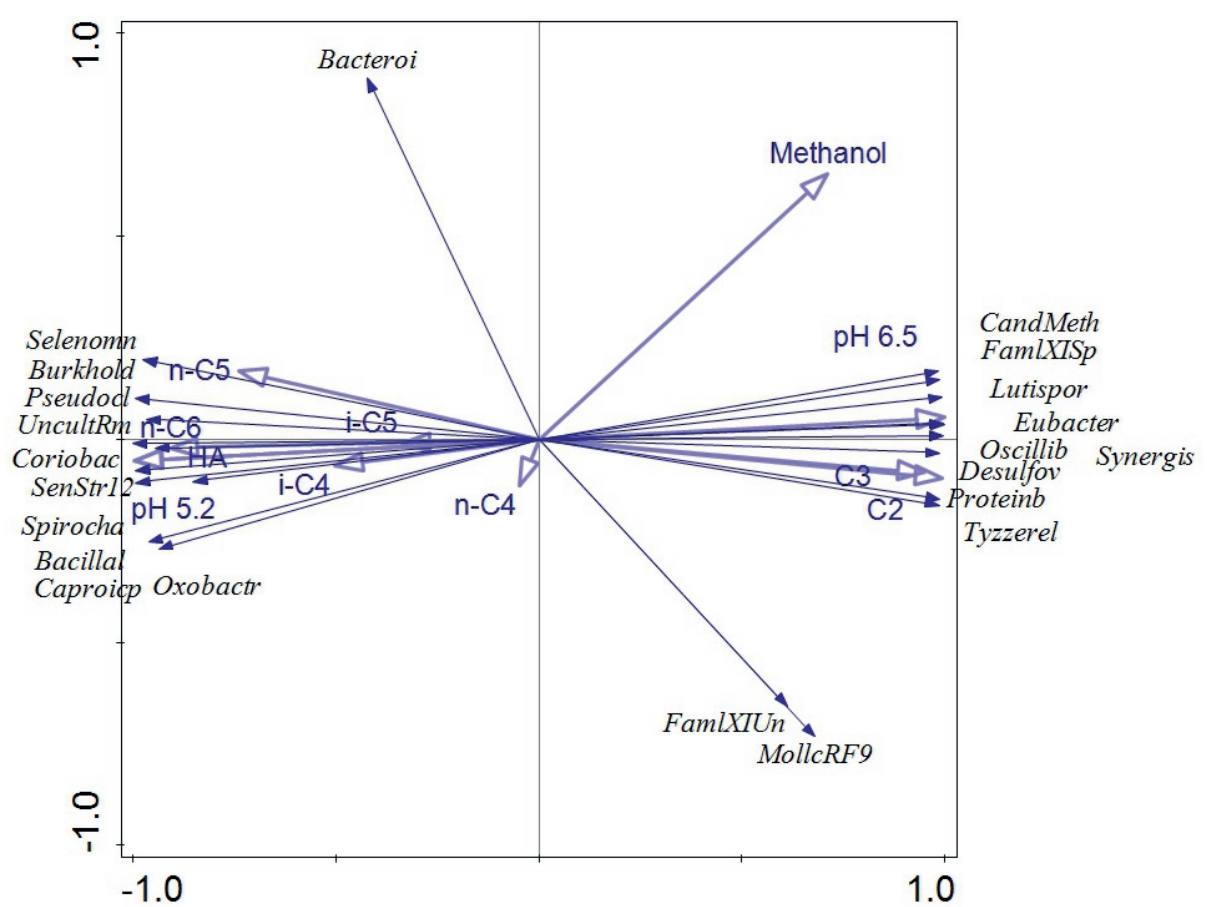

Figure S22. Unconstrained redundancy triplot using as parameters: the total conversions at the end of the batch (positive=formation, negative=consumption), the difference between the $\mathrm{pH}$ levels (Using a positive difference for $\mathrm{pH} 5.2$ compared to 6.5 and vice versa) and the final total undissociated fatty acids concentration. Microbioval data of the genera for batch I to IV was used creating this plot. 


\section{Supporting Information - Chapter 3}

Continuous n-Valerate Formation from Propionate and Methanol in an Anaerobic Chain Elongation Open-Culture Bioreactor

Sanne M. de Smit*, Kasper D. de Leeuw*, Cees J. N. Buisman and David P. B. T. B. Strik*

*Sanne M. de Smit and Kasper D. de Leeuw contributed equally to this work 


\section{Material and Methods}

Medium composition for batch methanol based elongation with propionate

Table S1. Medium composition for the batch methanol elongation reactor with propionate as chain elongation substrate. The compositions of stock I, stock II, vitamin B solution, trace element 1 and trace element 2 solution are shown in Table S4 to Table S8.

\begin{tabular}{lll}
\hline Compound & Amount per & Unit \\
\hline Sodium & 14.4 & $\mathrm{~g}$ \\
propionate & 1.00 & $\mathrm{~g}$ \\
Yeast Extract & 20 & $\mathrm{ml}$ \\
Stock I & 20 & $\mathrm{ml}$ \\
Stock II & 8.0 & $\mathrm{~g}$ \\
Methanol (liquid) & & $\mathrm{ml}$ \\
Vitamin B & 1 & $\mathrm{ml}$ \\
solution & & $\mathrm{ml}$ \\
Trace element 1 & 0.5 & \\
Trace element 2 & 0.5 & \\
\hline \hline
\end{tabular}

Medium composition for continuous methanol based elongation with propionate

Table S2. Medium composition for the continuous methanol elongation reactor with propionate as chain elongation substrate. The compositions of stock I, stock II, vitamin B solution, trace element 1 and trace element 2 solution are shown in Table S4 to Table S8.

\begin{tabular}{lll}
\hline Compound & $\begin{array}{l}\text { Amount per } \\
\text { L }\end{array}$ & Unit \\
\hline \hline Sodium & 14.4 & $\mathrm{~g}$ \\
propionate & 1.00 & $\mathrm{~g}$ \\
Yeast Extract & 20 & $\mathrm{ml}$ \\
Stock I & 20 & $\mathrm{ml}$ \\
Stock II & 8.0 & $\mathrm{~g}$ \\
Methanol (liquid) & & $\mathrm{ml}$ \\
Vitamin B & 1 & \\
solution & & $\mathrm{ml}$ \\
Trace element 1 & 0.5 & $\mathrm{ml}$ \\
Trace element 2 & 0.5 & \\
\hline \hline
\end{tabular}


Medium composition for continuous methanol based elongation with propionate and acetate

Table S3. Medium composition for the continuous methanol elongation reactor with propionate and acetate as chain elongation substrates. The compositions of stock I, stock II, vitamin B solution, trace element 1 and trace element 2 solution are shown in Table S4 to Table S8.

\begin{tabular}{lll}
\hline Compound & $\begin{array}{l}\text { Amount per } \\
\text { L }\end{array}$ & Unit \\
\hline Sodium propionate & 7.2 & $\mathrm{~g}$ \\
$\begin{array}{l}\text { Sodium acetate } \\
\text { trihydrate }\end{array}$ & 10.2 & $\mathrm{~g}$ \\
Yeast Extract & 1.00 & $\mathrm{~g}$ \\
Stock I & 20 & $\mathrm{ml}$ \\
Stock II & 20 & $\mathrm{ml}$ \\
Methanol (liquid) & 8.0 & $\mathrm{~g}$ \\
Vitamin B solution & 1 & $\mathrm{ml}$ \\
Trace element 1 & 0.5 & $\mathrm{ml}$ \\
Trace element 2 & 0.5 & $\mathrm{ml}$ \\
\hline \hline
\end{tabular}

\section{Composition of stock solutions}

The composition of the stock solutions is given in the tables below.

Table S4. Composition of "Stock I" solution.

\begin{tabular}{ll}
\hline Compound & $\begin{array}{l}\text { Concentration } \\
(\mathbf{g} / \mathbf{L})\end{array}$ \\
\hline \hline $\mathrm{NH}_{4} \mathrm{H}_{2} \mathrm{PO}_{4}$ & 180.0 \\
$\mathrm{MgCl}_{2} * 6 \mathrm{H}_{2} \mathrm{O}$ & 16.5 \\
$\mathrm{MgSO}_{4} * 7 \mathrm{H}_{2} \mathrm{O}$ & 10.0 \\
\hline \hline
\end{tabular}

Table S5. Composition of "Stock II" solution.

\begin{tabular}{ll}
\hline Compound & $\begin{array}{l}\text { Concentration } \\
(\mathbf{g} / \mathbf{L})\end{array}$ \\
\hline \hline $\mathrm{KCl}$ & 7.5 \\
$\mathrm{CaCl}_{2} * 2 \mathrm{H}_{2} \mathrm{O}$ & 10.0 \\
\hline \hline
\end{tabular}


Table S6. Composition of "Vitamin solution".

\begin{tabular}{ll}
\hline Compound & $\begin{array}{l}\text { Concentration } \\
(\mathbf{g} / \mathbf{L})\end{array}$ \\
\hline Biotin & 35.33 \\
Folic acid & 1.67 \\
Pyridoxal-HCl & 0.83 \\
Lipoic acid & 5.00 \\
Riboflavin & 4.17 \\
Thiamine HCl & 88.67 \\
Ca-D- & \\
Pantothenate & 137.67 \\
Cyanocobalamin & 4.17 \\
P-aminobenzoic & \\
acid & 4.17 \\
Nicotinic acid & 4.17 \\
\hline
\end{tabular}

Table S7. Composition of "Trace element I" solution.

\begin{tabular}{ll}
\hline Compound & $\begin{array}{l}\text { Concentration } \\
(\mathbf{g} / \mathbf{L})\end{array}$ \\
\hline \hline $\mathrm{FeCl} 2 * 4 \mathrm{H}_{2} \mathrm{O}$ & 10000 \\
$\mathrm{HCl}(1 \mathrm{M})$ & \\
$\mathrm{MnCl}_{2} * 4 \mathrm{H}_{2} \mathrm{O}$ & 200 \\
$\mathrm{H}_{3} \mathrm{BO}_{3}$ & 2000 \\
$\mathrm{CoCl}_{2} * 6 \mathrm{H}_{2} \mathrm{O}$ & 1333 \\
$\mathrm{CuCl}_{2} * \mathrm{H}_{2} \mathrm{O}$ & 67 \\
$\mathrm{NiCl}_{2} * 6 \mathrm{H}_{2} \mathrm{O}$ & 133 \\
$\mathrm{ZnSO} \mathrm{Zn}_{4} * 7 \mathrm{H}_{2} \mathrm{O}$ & 667 \\
\hline \hline
\end{tabular}

Table S8. Composition of "Trace element II" solution.

\begin{tabular}{ll}
\hline Compound & $\begin{array}{l}\text { Concentration } \\
(\mathbf{g} / \mathbf{L})\end{array}$ \\
\hline \hline $\mathrm{Na}_{2} \mathrm{MoO}_{4} * 2 \mathrm{H}_{2} \mathrm{O}$ & 200 \\
$\mathrm{Na}_{2} \mathrm{SeO}_{3}$ & 67 \\
$\mathrm{NaOH}(4 \mathrm{M})$ & 167 \\
\hline \hline
\end{tabular}




\section{Calculation of the concentration dissolved $\mathrm{CO}_{2}$}

When $\mathrm{CO}_{2}$ comes in contact with water, the following reaction takes place:

$$
\mathrm{CO}_{2}+\mathrm{H}_{2} \mathrm{O} \rightarrow \mathrm{H}_{2} \mathrm{CO}_{3}
$$

Carbonic acid dissociates into $\mathrm{HCO}_{3}^{-}$and $\mathrm{CO}_{3}{ }^{2-}$ :

$$
\mathrm{H}_{2} \mathrm{CO}_{3} \rightarrow \mathrm{HCO}_{3}^{-}+\mathrm{H}^{+} \text {with } p K_{A_{1}}=6.35
$$

$\mathrm{HCO}_{3}^{-} \rightarrow \mathrm{CO}_{3}^{2-}+\mathrm{H}^{+}$with $p K_{A_{2}}=10.33^{286}$

This gives the following equations (with all concentrations in $\mathrm{M}$ ):

$$
\begin{array}{cc}
K_{A_{1}}=\frac{\left[\mathrm{H}^{+}\right] *\left[\mathrm{HCO}_{3}^{-}\right]}{\left[\mathrm{H}_{2} \mathrm{CO}_{3}\right]} & \text { Equation 1 } \\
K_{A_{2}}=\frac{\left[\mathrm{H}^{+}\right] *\left[\mathrm{CO}_{3}^{2-}\right]}{\left[\mathrm{HCO}_{3}^{-}\right]} & \text {Equation 2 }
\end{array}
$$

The total dissolved inorganic carbon is given by:

$$
\begin{aligned}
& \text { [total inorganic carbon }(\mathrm{aq})] \\
& \qquad=\left[\mathrm{HCO}_{3}^{-}\right]+\left[\mathrm{CO}_{3}^{2-}\right]+\left[\mathrm{H}_{2} \mathrm{CO}_{3}\right]
\end{aligned}
$$

$\mathrm{H}_{2} \mathrm{CO}_{3}$ and $\mathrm{CO}_{3}{ }^{2-}$ can be expressed in $\mathrm{H}^{+}, \mathrm{HCO}_{3}{ }^{-}$and $\mathrm{K}_{\mathrm{A}}$ from Equation 1 and Equation 2:

$$
\begin{array}{cc}
{\left[\mathrm{H}_{2} \mathrm{CO}_{3}\right]=\frac{\left[\mathrm{H}^{+}\right] *\left[\mathrm{HCO}_{3}^{-}\right]}{K_{A_{1}}}} & \text { Equation } 4 \\
{\left[\mathrm{CO}_{3}^{2-}\right]} & =\frac{K_{A_{2}} *\left[\mathrm{HCO}_{3}^{-}\right]}{\left[\mathrm{H}^{+}\right]} \quad \text { Equation } 5
\end{array}
$$

Combining Equation 3, Equation 4 and Equation 5 gives:

$$
\begin{array}{ll}
\text { [total inorganic carbon }(\mathrm{aq})] & \text { Equation } 6 \\
\qquad=\left[\mathrm{HCO}_{3}^{-}\right]+\frac{K_{\mathrm{A}_{2}} *\left[\mathrm{HCO}_{3}^{-}\right]}{\left[\mathrm{H}^{+}\right]}+\frac{\left[\mathrm{H}^{+}\right] *\left[\mathrm{HCO}_{3}^{-}\right]}{K_{\mathrm{A}_{1}}}
\end{array}
$$

Dividing $\left[\mathrm{H}_{2} \mathrm{CO}_{3}\right]$ (Equation 4) by the total $\mathrm{CO}_{2}$ (Equation 6) gives: 


$$
\frac{\left[\mathrm{H}_{2} \mathrm{CO}_{3}\right]}{[\text { total inorganic carbon }(\mathrm{aq})]}=\frac{\frac{\left[\mathrm{H}^{+}\right] *\left[\mathrm{HCO}_{3}^{-}\right]}{K_{\mathrm{A}_{1}}}}{\left[\mathrm{HCO}_{3}^{-}\right]+\frac{K_{A_{2}} *\left[\mathrm{HCO}_{3}^{-}\right]}{\left[\mathrm{H}^{+}\right]}+\frac{\left[\mathrm{H}^{+}\right] *\left[\mathrm{HCO}_{3}^{-}\right]}{K_{A_{1}}}}
$$

Simplifying gives:

$$
\frac{\left[\mathrm{H}_{2} \mathrm{CO}_{3}\right]}{[\text { total inorganic carbon }(\mathrm{aq})]}=\frac{\left[\mathrm{H}^{+}\right]^{2}}{\left[\mathrm{H}^{+}\right]^{2}+K_{A_{1}} *\left[\mathrm{H}^{+}\right]+K_{A_{1}} * K_{A_{2}}}
$$

The total dissolved inorganic carbon can be calculated:

$$
[\text { total inorganic carbon }(\mathrm{aq})]=\frac{\left[\mathrm{H}_{2} \mathrm{CO}_{3}\right] *\left(\left[\mathrm{H}^{+}\right]^{2}+K_{A_{1}} *\left[\mathrm{H}^{+}\right]+K_{A_{2}} * K_{A_{1}}\right)}{\left[H^{+}\right]^{2}}
$$

With $\left(\left[\mathrm{H}^{+}\right]\right.$in $\left.\mathrm{M}\right)$ :

$$
\left[H^{+}\right]=10^{-p H}
$$

And:

$$
\left[\mathrm{H}_{2} \mathrm{CO}_{3}(\mathrm{M})\right]=\frac{\gamma_{\mathrm{CO} 2}(-) * P_{\text {tot }}(\mathrm{atm})}{K_{H}}
$$

$\mathrm{YCO}_{\mathrm{C}}=$ fraction $\mathrm{CO}_{2}$ in the headspace

$P_{\text {tot }}=$ total pressure in the batch bottle

$\mathrm{K}_{\mathrm{H}}=$ Henry constant $=29.41 \mathrm{~atm} / \mathrm{M}^{287}$ 


\section{Calculation of the required $\mathrm{CO}_{2}$ inflow}

The concentration of total dissolved $\mathrm{CO}_{2}$ at $\mathrm{t}=1\left(\left[\mathrm{CO}_{2}\right]_{1}\right.$ in $\left.\mathrm{M}\right)$ was calculated using the equations above and the $\mathrm{CO}_{2}$ concentration in the headspace of the bioreactor ( $\mathrm{F}_{\mathrm{CO} 2,1}$ in atm $\left.* \mathrm{M}^{2}\right)$ with a known $\mathrm{CO}_{2}$ inflow rate $\left(\mathrm{R}_{\mathrm{cO} 2,1} \mathrm{in} \mathrm{ml} / \mathrm{min}\right)$ and $\mathrm{pH}\left(\mathrm{pH}_{1}\right)$. For the total pressure $\left(\mathrm{P}_{\mathrm{tot}}\right), 1 \mathrm{~atm}$ was assumed. Changing the $\mathrm{pH}$ will change the amount of total dissolved $\mathrm{CO}_{2}$. To maintain the same concentration of total dissolved $\left[\mathrm{CO}_{2}\right]$, the $\mathrm{CO}_{2}$ supply needs to be changed. The fraction of $\mathrm{CO}_{2}\left(\mathrm{~F}_{\mathrm{CO} 2,2}\right)$ needed in the headspace at $\mathrm{pH}_{2}$ and $\mathrm{t}=2$ can be calculated from $\left[\mathrm{CO}_{2}\right]_{2}=\left[\mathrm{CO}_{2}\right]_{1}$ :

$$
F_{C O 2,2}=\frac{\left[\text { total } \mathrm{CO}_{2}(a q)\right]_{2} * K_{H} *\left[{\left.H^{+}\right]_{2}}^{2}\right.}{P_{\text {tot }} *\left(\left[H^{+}\right]_{2}^{2}+K_{A_{1}} *\left[H^{+}\right]_{2}+K_{A_{1}} * K_{A_{2}}\right)}
$$

The rate of $\mathrm{CO}_{2}$ supply needed to provide this concentration in the headspace was calculated by:

$$
R_{C O 2,2}=\frac{R_{C O 2,1} * F_{C O 2,2}}{F_{C O 2,1}}
$$




\section{Results and Discussion}

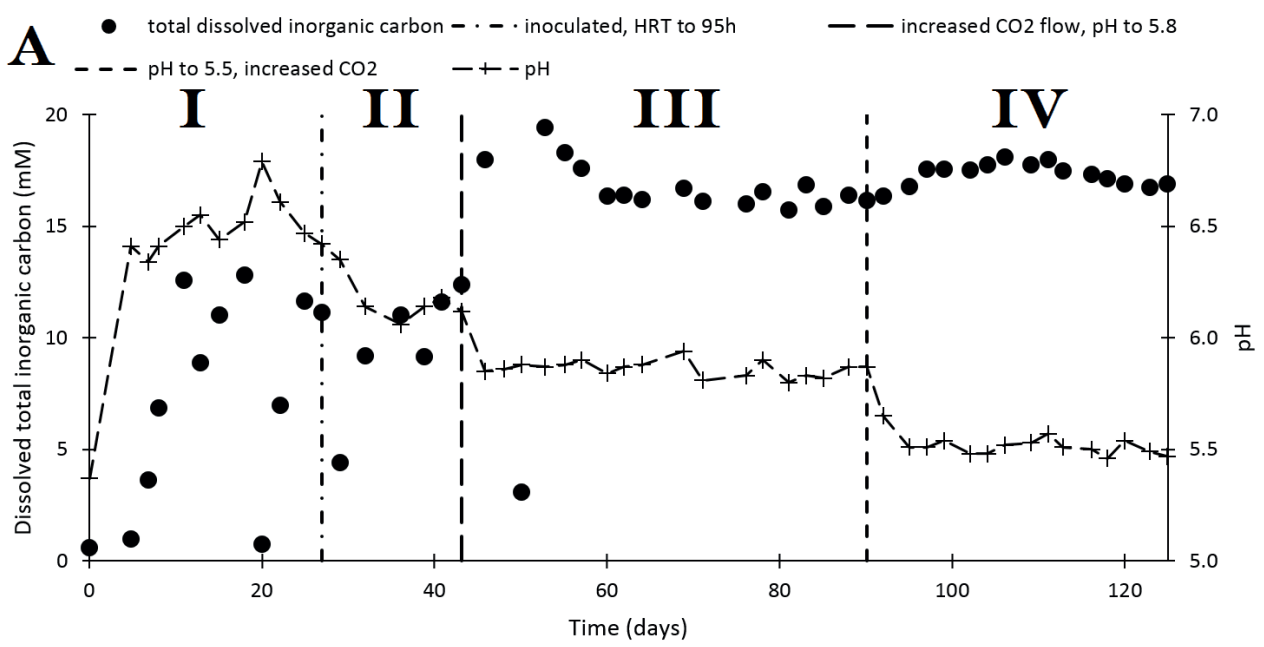

$\$ \begin{array}{ll}\text { total dissolved inorganic carbon } & -\cdots \mathrm{pH} \text { to } 5.8 \\ - & -\end{array}$

- CO2 supply
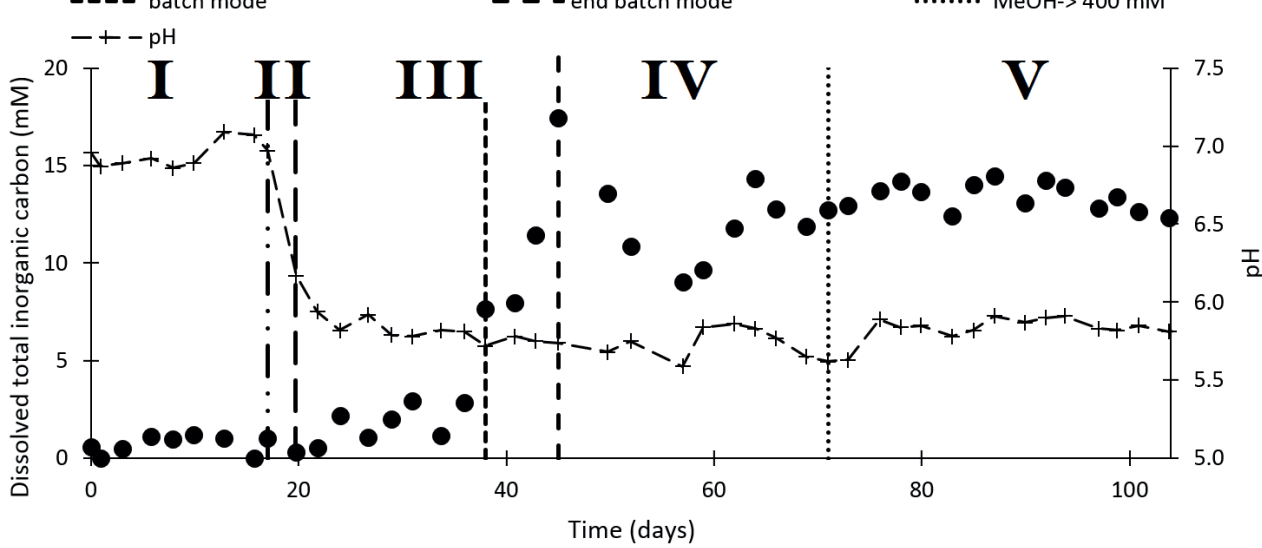

Figure S1. Change of the concentration of dissolved total inorganic carbon in the reactor and $\mathrm{pH}$ in time during the different phases in the continuous open culture reactors with methanol based propionate elongation $(A)$ and simultaneous methanol based elongation of propionate and acetate (B). The vertical lines indicate the major setup changes. The required $\mathrm{CO}_{2}$ inflow was calculated as described above. 


\section{Calculation of Gibbs free energy}

$$
\begin{array}{cc}
a[W]+b[X] \rightarrow c[Y]+d[Z] & \text { Equation 7 } \\
\Delta_{r} G^{0}=c \Delta_{f} G_{Y}^{0}+d \Delta_{f} G_{Z}^{0}-a \Delta_{f} G_{W}^{0}-d \Delta_{f} G_{X}^{0} & \text { Equation 8 } \\
\Delta_{r} G=\Delta_{r} G^{0}+R T \ln \frac{[Y]^{c}[Z]^{d}}{[W]^{a}[X]^{b}} & \text { Equation 9 }
\end{array}
$$

The Gibbs free energy from a reaction (Equation 7) can be calculated from the formation energy of the substrates and products from the reaction (Table S9), as shown in Equation 8. When the concentrations of the substrates and products are taken into account, the Gibbs free energy for a reaction can be calculated as shown in Equation 9, with $\mathrm{R}$ the gas constant $\left(\mathrm{kJ} /\left(\mathrm{K}^{*} \mathrm{~mol}\right)\right)$ and $\mathrm{T}$ the temperature $(\mathrm{K})$.

Table 59. Gibbs free energy values used for the calculation of the Gibbs reaction energy values given in Error! Reference source not found..

\begin{tabular}{llll}
\hline Compound & Name & Phase & $\left.\mathbf{G}_{\mathbf{f}} \mathbf{0}^{\mathbf{0}} \mathbf{k J} / \mathbf{m o l}\right)$ \\
\hline \hline $\mathrm{H}^{+}$ & Proton & $\mathrm{Aq}$ & $0.0^{61}$ \\
$\mathrm{H}_{2}$ & Hydrogen & $\mathrm{G}$ & $0.0^{61}$ \\
$\mathrm{H}_{2} \mathrm{O}$ & Water & $\mathrm{L}$ & $-237.2^{61}$ \\
$\mathrm{CHO}_{3}{ }^{-}$ & Bicarbonate & $\mathrm{Aq}$ & $-586.9^{288}$ \\
$\mathrm{CH}_{4}$ & Methane & $\mathrm{G}$ & $-50.8^{61}$ \\
$\mathrm{CH}_{4} \mathrm{O}$ & Methanol & $\mathrm{Aq}$ & $-175.4^{288}$ \\
$\mathrm{C}_{2} \mathrm{H}_{3} \mathrm{O}_{2}{ }^{-}$ & Acetate & $\mathrm{Aq}$ & $-369.4^{288}$ \\
$\mathrm{C}_{3} \mathrm{H}_{5} \mathrm{O}_{3}-$ & Propionate & $\mathrm{Aq}$ & $-361.1^{288}$ \\
$\mathrm{C}_{4} \mathrm{H}_{7} \mathrm{O}_{2}-$ & Butyrate & $\mathrm{Aq}$ & $-352.6^{288}$ \\
$\mathrm{C}_{5} \mathrm{H}_{9} \mathrm{O}_{2}^{-}$ & Valerate & $\mathrm{Aq}$ & $-344.3^{61}$ \\
\hline \hline
\end{tabular}




\section{Concentration and conversion profile of batch experiments with initial pH from 5 to $\mathbf{7 . 5}$}
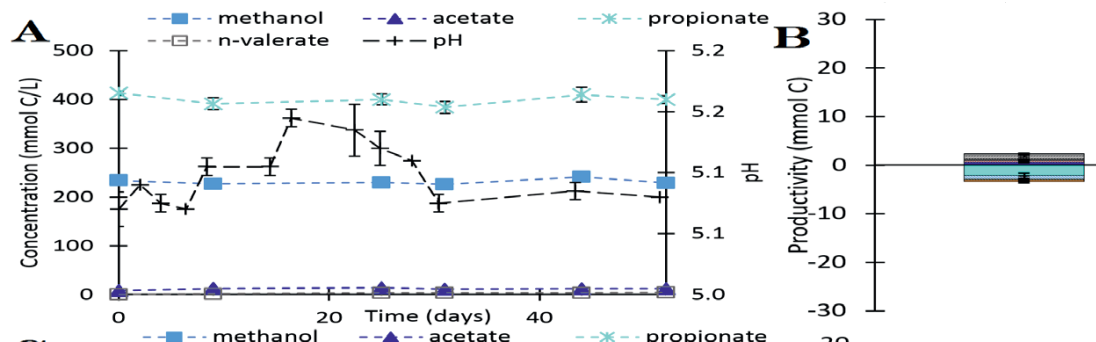

$\square$ carbondioxide

$\square$ methane

$\square$ n-caproate

n-valerate

isovalerate

$\square$ n-butyrate

ItI isobutyrate

acetate

$\square$ methanol

$\square$ propionate

$\mathrm{C}_{500}$ - -

-30
30
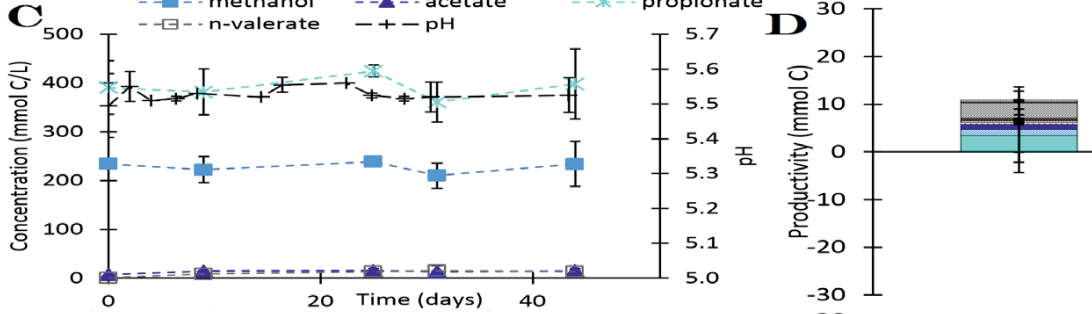

$\square$ carbondioxide

$\square$ methane

n-caproate

$\square$ n-valerate

isovalerate

$\square$ n-butyrate

m isobutyrate

acetate

$\square$ methanol

$\square$ propionate

H. - - methanol - - - acetate - - - - propionate
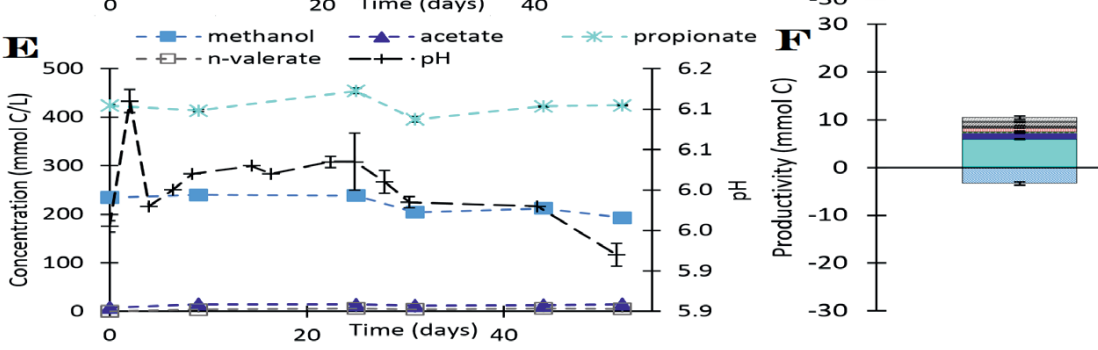

carbondioxide

$\square$ methane

$\square$ n-caproate

n-valerate

- isovalerate

$\square$ n-butyrate

it: isobutyrate

acetate

$\square$ methanol

$\square$ propionate
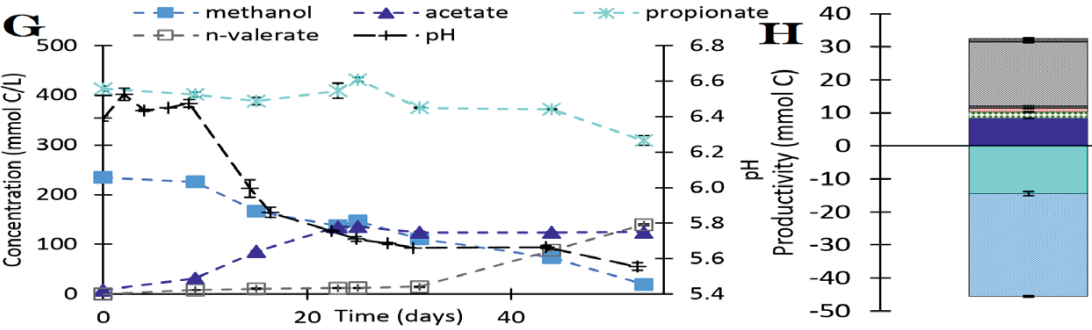

$\square$ carbondioxide

$\square$ methane

$\square$ n-caproate

n-valerate

isovalerate

$\square$ n-butyrate

min isobutyrate

- acetate

$\square$ methanol

$\square$ propionate
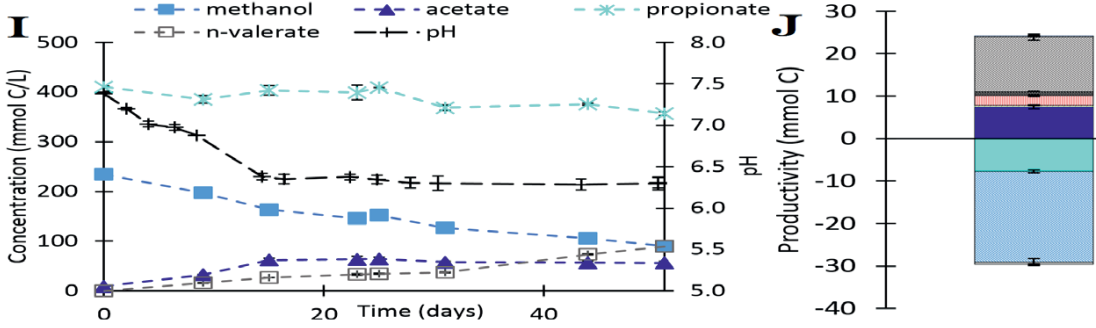

$\square$ carbondioxide

$\square$ methane

$\square$ n-caproate

$\square$ n-valerate

isovalerate

$\square$ n-butyrate

mititisobutyrate

acetate

$\square$ methanol

$\square$ propionate

Figure S2. Concentration profiles and total conversions during batch experiments with methanol and propionate with initial pH values of 5.0 ( $A$ and $B$ ), 5.5 ( $C$ and $D), 6.0$ (E and F), 6.5 ( $G$ and $H$ ) and 7.5 ( $\mathrm{I}$ and $\mathrm{J}$ ) at $308 \mathrm{~K}$. The error bars represent the minimum and maximum values measured in the duplo experiments. 


\section{Concentration profiles of continuous methanol based chain elongation reactors}
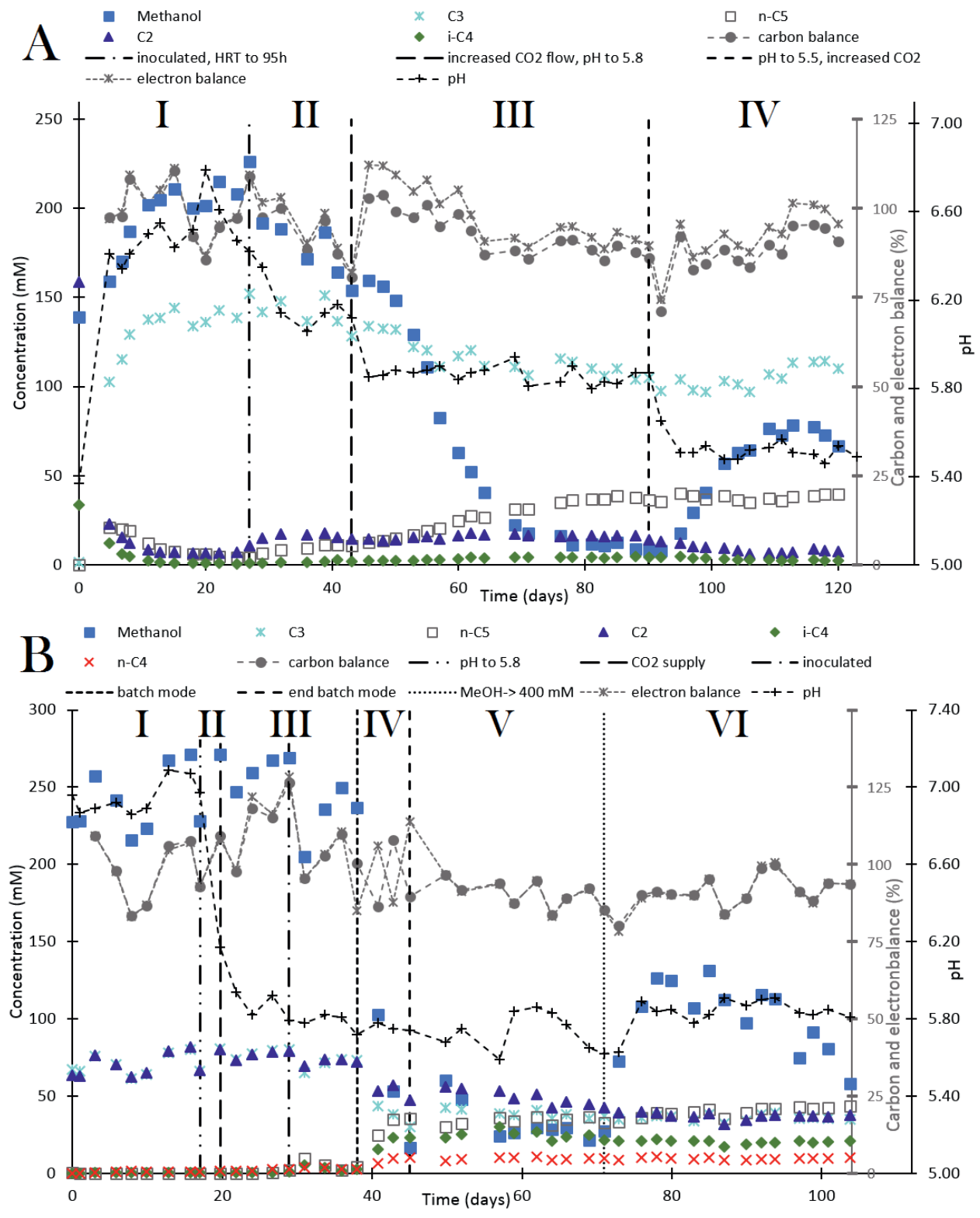

Figure S3. Change in concentrations of methanol, acetate (C2), propionate (C3), n-butyrate (n$\mathrm{C4})$, iso-butyrate (i-C4) and $n$-valerate $(n-C 5)$ in time during continuous methanol based propionate elongation (A) and simultaneous acetate and propionate elongation (B). The change of the $\mathrm{pH}$, the carbon balance and the electron balance are shown as well. The vertical lines indicate the major setup changes. The carbon balance was calculated by dividing the total amount 
of carbon in mmol $\mathrm{C} /$ day in the liquid and gas effluent streams over the total amount of carbon in $\mathrm{mmol} \mathrm{C} /$ day in the liquid and gas influent. The electron balance was calculated by dividing the total amount of electrons in mmol e/day in the outgoing liquid and gas stream by the total amount of electrons in $\mathrm{mmol}$ e/day in the ingoing liquid stream. The amount of electrons per compound is equal to the degree of reduction of that compound. The compounds taken into account for the carbon and electron balance are: methanol, ethanol, propanol, butanol, pentanol, hexanol, acetate, propionate, iso-butyrate, $n$-butyrate, isovalerate, $n$-valerate, isocaproate, caproate, heptylate, caprylate, methane and carbon dioxide.

\section{Volumetric productivities during continuous methanol based elongation of acetate and propionate}

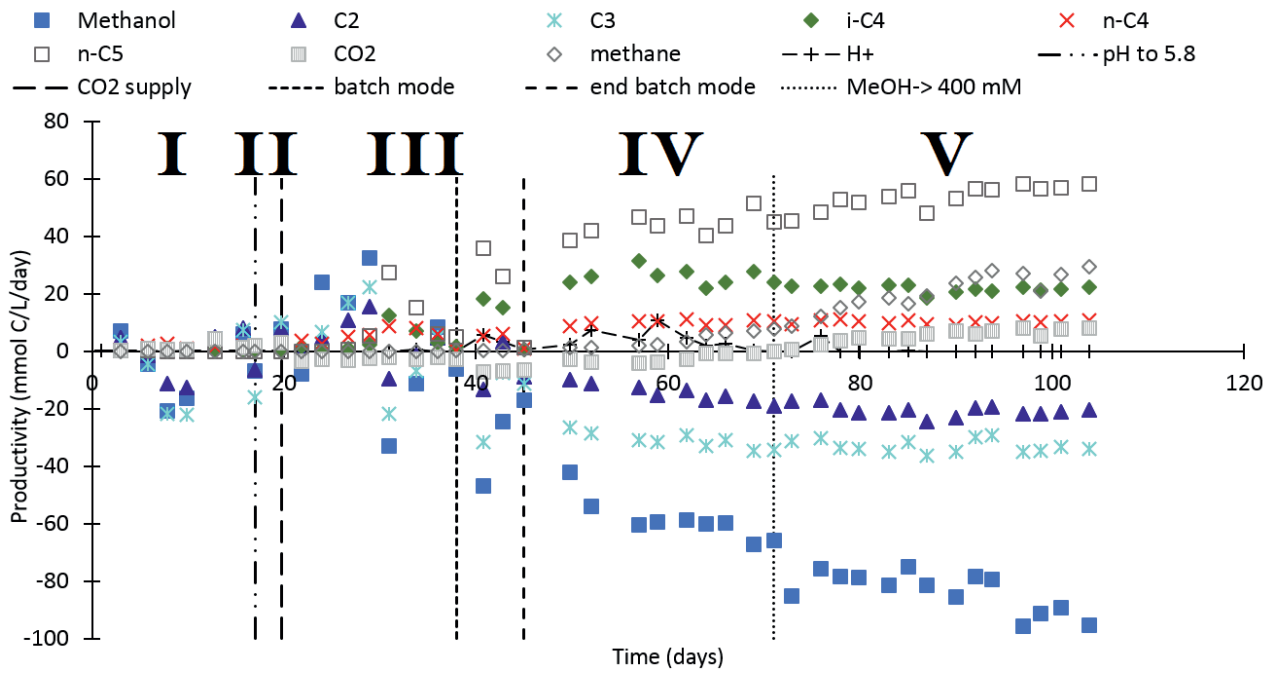

Figure S4. Volumetric productivities of methane, carbon dioxide ( $\mathrm{CO} 2)$, methanol, acetate ( $\mathrm{C} 2)$, propionate (C3), n-butyrate ( $n-C 4)$, iso-butyrate (i-C4) and n-valerate (n-C5) in time during continuous methanol based elongation of acetate and propionate in an anaerobic open-culture reactor at $309 \mathrm{~K}$. The production of protons in $\mathrm{mmol} / \mathrm{day}$ is also shown (-+--). 


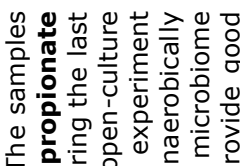

F 응

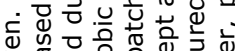

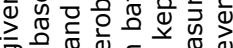

ब.

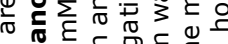

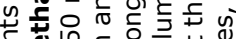

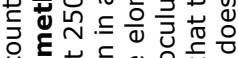

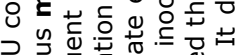

ว

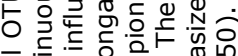

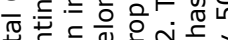

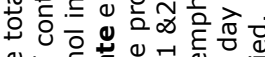

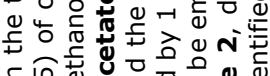

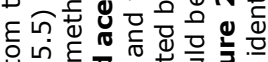

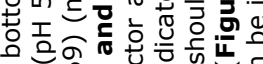

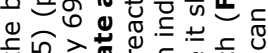

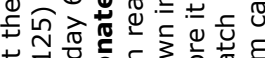

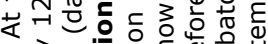
这> है

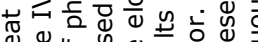

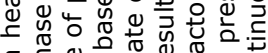

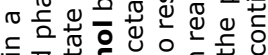

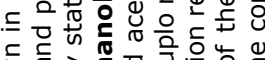

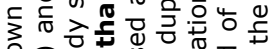
○ व

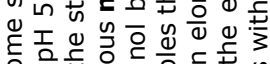

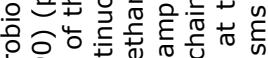
은 응 능

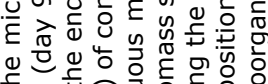

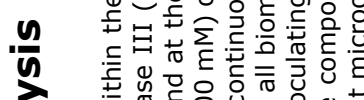

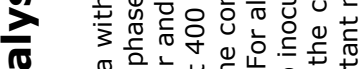

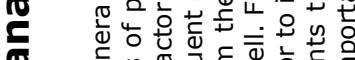

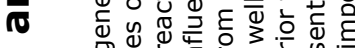

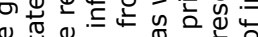

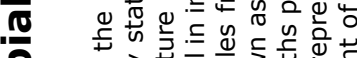

능

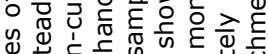
is

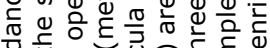

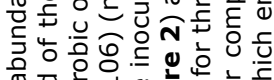

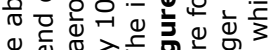

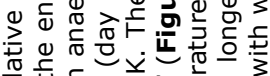

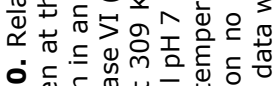

प)

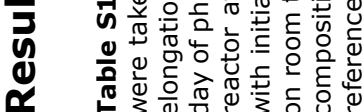

\section{矛字 \\ 这完}

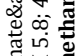

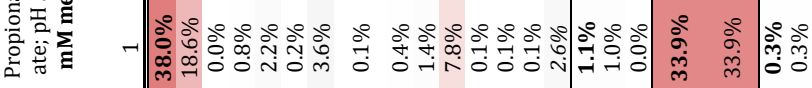

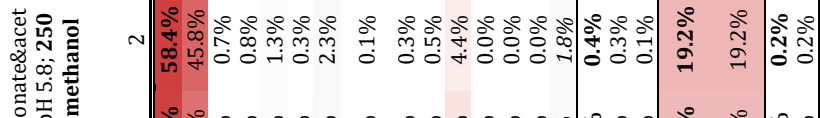

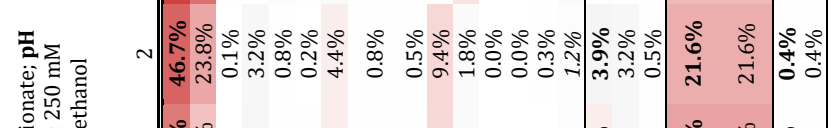

竞㫄

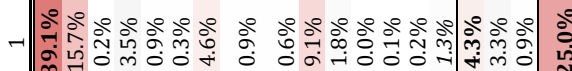

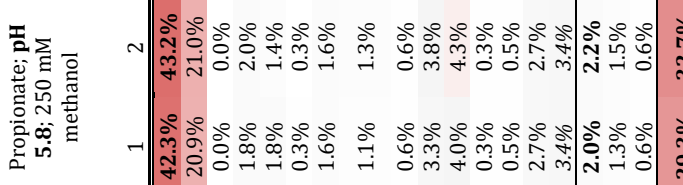

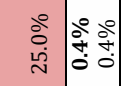

空- 월

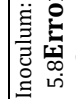

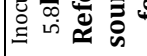

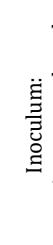

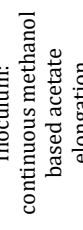

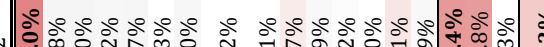

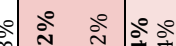

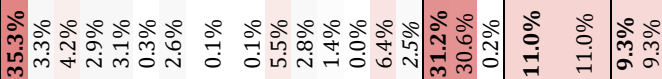
レ|

-1|

离

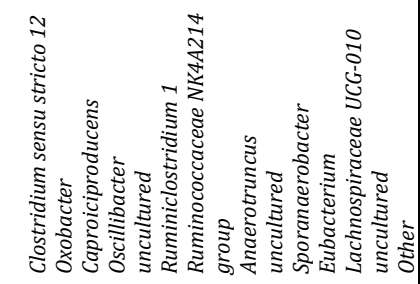

辛
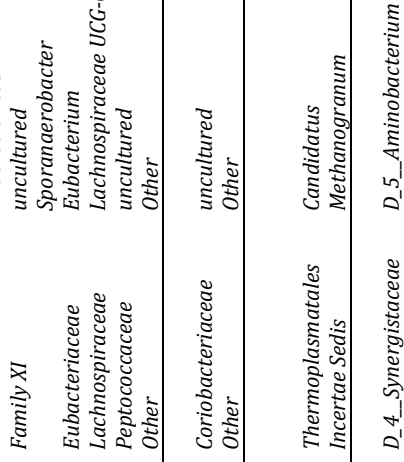

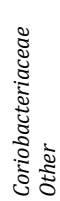
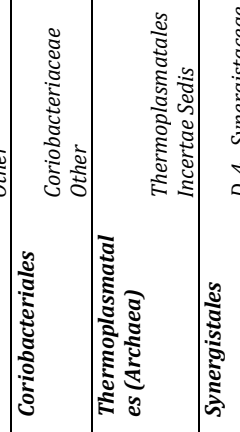


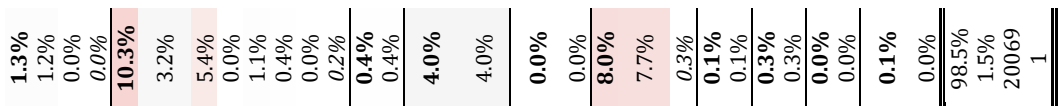

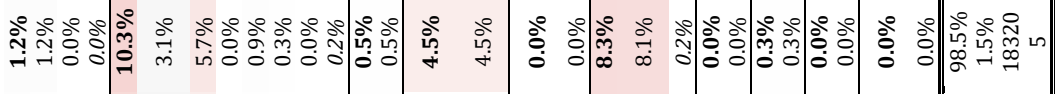

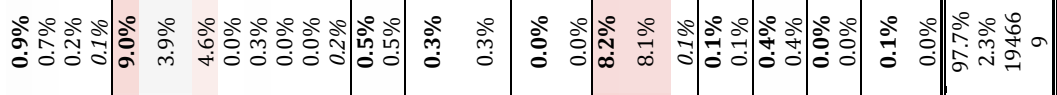

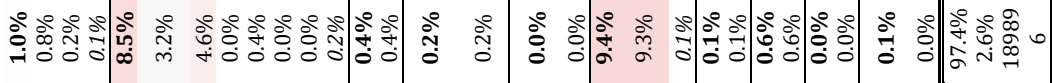

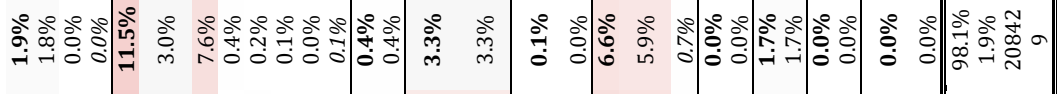

ลें

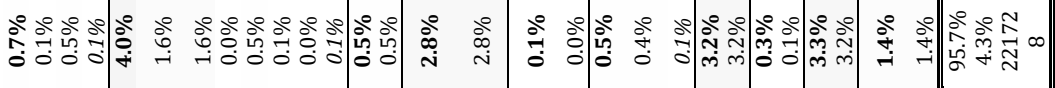

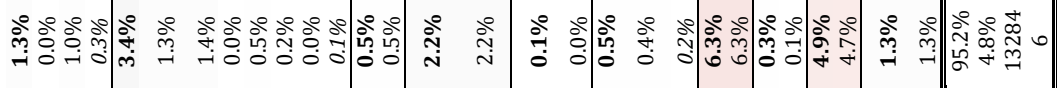

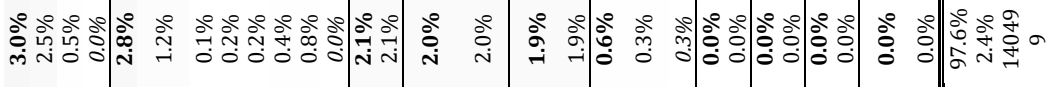

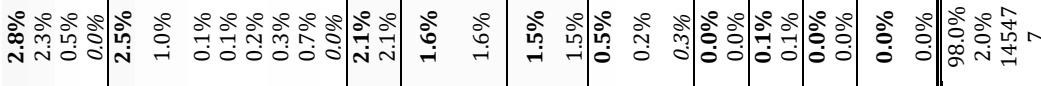

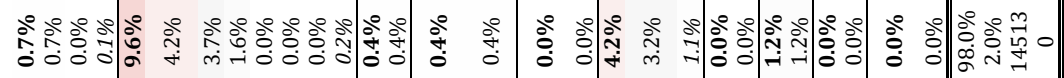

के

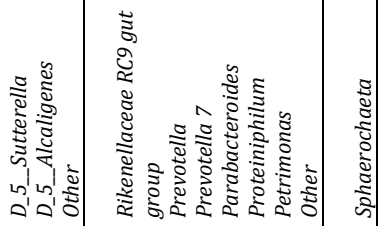

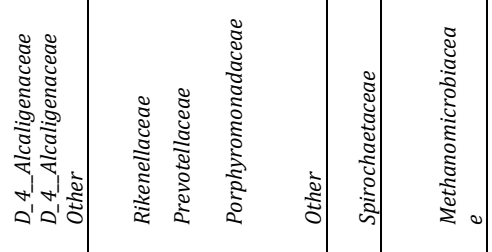

ปัँ

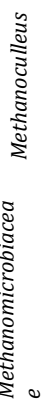

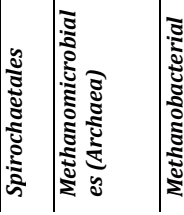

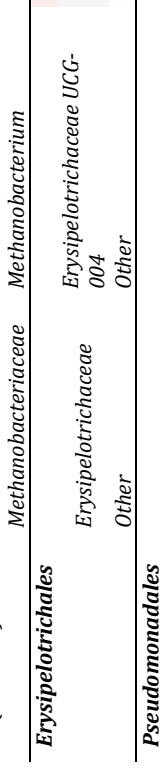

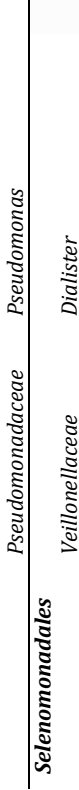

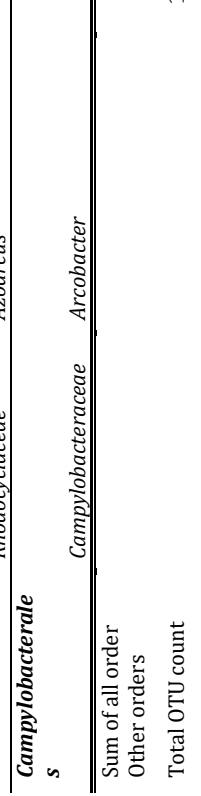


Table S11. The table shows the OTU counts as classified within the Clostridium sensu stricto 12 genus. The samples were taken at the end of the steady states of phase III (day 90) ( $\mathrm{pH} \mathrm{5.8)} \mathrm{and} \mathrm{phase} \mathrm{IV} \mathrm{(day}$ 125) ( $\mathrm{pH} \mathrm{5.5)} \mathrm{of} \mathrm{continuous} \mathrm{methanol} \mathrm{based} \mathrm{propionate} \mathrm{elongation} \mathrm{in} \mathrm{an} \mathrm{anaerobic} \mathrm{open-culture} \mathrm{reactor}$ and at the end of the steady state of phase $\mathrm{V}$ (day 69) (methanol in influent $250 \mathrm{mM}$ ) and during the last day of phase VI (day 106) (methanol in influent $400 \mathrm{mM}$ ) of continuous methanol based propionate and acetate elongation in an anaerobic open-culture reactor at $309 \mathrm{~K}$. The inocula samples from the continuous methanol based acetate elongation reactor and the propionate elongation batch experiment with initial pH 7 (Figure 2) are shown as well. For all biomass samples the duplo results are shown indicated by $1 \& 2$. OTUs where only one hit was found in total in all 12 samples were omitted due to the size of the table.

\begin{tabular}{|c|c|c|c|c|c|c|c|c|c|c|c|c|}
\hline \multirow[b]{3}{*}{ OTU identifiers } & \multicolumn{12}{|c|}{ OTU counts } \\
\hline & \multicolumn{2}{|c|}{$\begin{array}{c}\text { Inoculum: } \\
\text { continuous } \\
\text { methanol based } \\
\text { acetate } \\
\text { elongation }\end{array}$} & \multicolumn{2}{|c|}{$\begin{array}{c}\text { Inoculum: } \\
\text { Propionate; } \\
\text { pH } 5.8 \text { (Figure } \\
\text { 1) }\end{array}$} & \multicolumn{2}{|c|}{$\begin{array}{l}\text { Propionate; pH } \\
\mathbf{5 . 8} ; 250 \mathrm{mM} \\
\text { methanol }\end{array}$} & \multicolumn{2}{|c|}{$\begin{array}{c}\text { Propionate; } \mathbf{p H} \\
\text { 5.5; } 250 \mathrm{mM} \\
\text { methanol }\end{array}$} & \multicolumn{2}{|c|}{$\begin{array}{l}\text { Propionate\&ac } \\
\text { etate; pH 5.8; } \\
\mathbf{2 5 0} \mathbf{~ m M} \\
\text { methanol }\end{array}$} & \multicolumn{2}{|c|}{$\begin{array}{c}\text { Propionate\&ac } \\
\text { etate; pH 5.8; } \\
\mathbf{4 0 0} \mathbf{~ m M} \\
\text { methanol }\end{array}$} \\
\hline & 1 & 2 & 1 & 2 & 1 & 2 & 1 & 2 & 1 & 2 & 1 & 2 \\
\hline AB509213.1.1431 & $\begin{array}{c}11549 \\
2\end{array}$ & 77123 & 4606 & $\begin{array}{c}505 \\
4\end{array}$ & 26595 & 44552 & $\begin{array}{c}2497 \\
5\end{array}$ & $\begin{array}{c}4265 \\
8\end{array}$ & $\begin{array}{c}7772 \\
5\end{array}$ & $\begin{array}{c}8690 \\
4\end{array}$ & $\begin{array}{c}3259 \\
0\end{array}$ & $\begin{array}{c}3222 \\
4\end{array}$ \\
\hline $\begin{array}{l}\text { CBXI010000035.40.155 } \\
2\end{array}$ & 1605 & 1125 & 46 & 59 & 356 & 657 & 3908 & 4337 & 3 & 3 & 9 & 15 \\
\hline GU907811.1.1429 & 2 & 0 & 0 & 0 & 35 & 78 & 570 & 946 & 0 & 0 & 0 & 0 \\
\hline KM251144.1.1452 & 253 & 229 & 0 & 0 & 6 & 9 & 341 & 318 & 42 & 26 & 43 & 40 \\
\hline EU828395.1.1425 & 6453 & 6129 & 3 & 6 & 37 & 26 & 156 & 143 & 1159 & 800 & 495 & 406 \\
\hline New.Reference0TU249 & 105 & 153 & 15 & 15 & 101 & 235 & 119 & 185 & 293 & 185 & 243 & 148 \\
\hline EU307088.1.1475 & 265 & 153 & 8 & 6 & 63 & 88 & 67 & 100 & 116 & 115 & 66 & 44 \\
\hline Cropped... & $\ldots$ & $\ldots$ & $\ldots$ & $\ldots$ & $\ldots$ & $\ldots$ & $\ldots$ & $\ldots$ & $\ldots$ & $\ldots$ & $\ldots$ & ... \\
\hline $\begin{array}{l}\text { Sum of OTU counts } \\
\text { within genus }\end{array}$ & 125707 & 86453 & 4866 & 5396 & 27704 & 46595 & 30944 & 49631 & 80320 & 89248 & 34005 & 33518 \\
\hline $\begin{array}{l}\text { Total OTU counts in } \\
\text { sample }\end{array}$ & 199344 & 145130 & $\begin{array}{c}14547 \\
7 \\
\end{array}$ & $\begin{array}{c}1404 \\
99 \\
\end{array}$ & 132846 & 221728 & $\begin{array}{c}19744 \\
4 \\
\end{array}$ & $\begin{array}{c}20842 \\
9 \\
\end{array}$ & $\begin{array}{c}18989 \\
6 \\
\end{array}$ & $\begin{array}{c}19466 \\
9 \\
\end{array}$ & $\begin{array}{c}18320 \\
5 \\
\end{array}$ & $\begin{array}{c}20069 \\
1 \\
\end{array}$ \\
\hline
\end{tabular}

Table S12. Result of searching the NCBI 16S rRNA gene amplicon sequence (Baceria and Archaea) database using Megablast (executed in april 2019) using the most abundant Clostridium sensu stricto OTUs.

\begin{tabular}{|c|c|c|c|c|c|c|c|}
\hline OTU identifyers & Description & $\begin{array}{c}\text { Max } \\
\text { scor } \\
\text { e }\end{array}$ & $\begin{array}{l}\text { Total } \\
\text { score }\end{array}$ & $\begin{array}{l}\text { Query } \\
\text { cover }\end{array}$ & $\begin{array}{c}\mathbf{E} \\
\text { value }\end{array}$ & Ident & Accession \\
\hline AB509213.1.1431 & $\begin{array}{l}\text { Clostridium } \\
\text { luticellarii } \\
\text { strain FW431 } \\
\text { 16S ribosomal } \\
\text { RNA, partial } \\
\text { sequence }\end{array}$ & 2623 & 2623 & $100 \%$ & 0.0 & $99.72 \%$ & NR_145907.1 \\
\hline $\begin{array}{l}\text { CBXI010000035.40.1 } \\
552\end{array}$ & $\begin{array}{l}\text { Clostridium } \\
\text { tyrobutyricum } \\
\text { strain KCTC } \\
\text { 5387, complete } \\
\text { genome }\end{array}$ & 2750 & 16493 & $100 \%$ & 0.0 & $100.00 \%$ & СР014170.1 \\
\hline
\end{tabular}




\section{AB509213.1.1431 OTU sequence:}

GACGAACGCTGGCGGCGTGCCTAACACATGCAAGTCGAGCGAAGAAGCTCCTTCGGGAGGTTCT TAGCGGCGGACGGGTG

AGTAACACGTGGGTAACCTGCCTCAAAGAGGGGGATAGCCTCCCGAAAGGGAGATTAATACCGC ATAATAAGTGCAGTTC

GCATGAACCGCACTTTAAAGGAGAAATCCGCTTTGAGATGGACCCGCGGCGCATTAGCTAGTTG GTAAGGCAGCGGCTTA

CCAAGGCAACGATGCGTAGCCGACCTGAGAGGGTGAACGGCCACATTGGAACTGAGAGACGGT CCAGACTCCTACGGGAG

GCAGCAGTGGGGAATATTGCACAATGGGCGAAAGCCTGATGCAGCAACGCCGCGTGAGTGAAG AAGGTTITCGGATTGTA

AAGCTCTGTCATCTGGGACGATAATGACGGTACCAGATGAGGAAGCCACGGCTAACTACGTGCC AGCAGCCGCGGTAATA

CGTAGGTGGCAAGCGTTGTCCGGAATTACTGGGCGTAAAGGGTGCGCAGGCGGACATTTAAGTG AGATGTGAAAGACCCG

GGCTTAACTTGGGCAGTGCATTTCAAACTGGATGTCTGGAGTGCAGGAGAGGAGAACGGAATTC CTAGTGTAGCGGTGAA

ATGCGTAGAGATTAGGAAGAACACCAGTGGCGAAGGCGGTTCTCTGGACTGTAACTGACGCTGA GGCACGAAAGCGTGGG

TAGCAAACAGGATTAGATACCCTGGTAGTCCACGCCGTAAACGATGAGTACTAGGTGTAGGAGG TATCGACNCCTTCTGT

GCCGCAGTAAACACAATAAGTACTCCGCCTGGGAAGTACGATCGCAAGATTAAAACTCAAAGGAA TTGACGGGGCCCGCA

CAAGCAGCGGAGCATGTGGTTTAATTCGAAGCAACGCGAAGAACCTTACCTGGACTTGACATCCC CTGCATATCTTAGAG

ATAAGAGAAGCCCTTCGGGGCAGGGAGACAGGTGGTGCATGGTTGTCGTCAGCTCGTGTCGTGA GATGTTAGGTTAAGTC

CTGCAACGAGCGCAACCCCTATTGTTAGTTGCTAGCAGTAAGATGAGCACTCTAACGAGACAGCC GCGGTTAACGCGGAG

GAAGGTGGGGATGACGTCAAATCATCATGCCCCTTATGTCCAGGGCAACACACGTGCTACAATG GGCAGAACAGAGAGAA

GCAAGACCGCGAGGTGGAGCGAACCTTGAAAACTGCTCCCAGTTCGGATTGCAGGCTGAAACCC GCCTGCATGAAGCTGG

AGTTGCTAGTAATCGCGAATCAGCATGTCGCGGTGAATACGTTCCCGGGCCTTGTACACACCGCC CGTCACACCATGAGA

GCTGGCAACACCCGAAGTCCGTAGTCTAACGAAAGAGGACGCGGCCGAAGGTGGGGTTAGTGA TTGGGGTG

\section{CBXI010000035.40.1552 OTU sequence:}

AGAGTITGATCCTGGCTCAGGACGAACGCTGGCGGCGTGCCTAACACATGCAAGTCGAGCGATG AAACCCCTTCGGGGGT

GGATTAGCGGCGGACGGGTGAGTAACACGTGGGTAACCTGCCTCAAAGTGGGGGATAGCCTTCC GAAAGGAAGATTAATA

CCGCATAAAGCCAAGTTTCACATGGAATTTGGATGAAAGGAGTAATTCGCTTTGAGATGGACCCG CGGCGCATTAGTTAG

TTGGTGGGGTAATGGCCTACCAAGACAGCGATGCGTAGCCGACCTGAGAGGGTGATCGGCCACA TTGGAACTGAGATACG

GTCCAGACTCCTACGGGAGGCAGCAGTGGGGAATATTGCACAATGGGCGAAAGCCTGATGCAGC AACGCCGCGTGAGTGA 
TGAAGGTCTTCGGATTGTAAAGCTCTGTCTITTGGGACGATAATGACGGTACCAAAGGAGGAAGC CACGGCTAACTACGT GCCAGCAGCCGCGGTAATACGTAGGTGGCGAGCGTTGTCCGGATTTACTGGGCGTAAAGGGTG CGTAGGCGGATGTTTAA GTGAGATGTGAAATACCCGGGCTTAACTTGGGTGCTGCATTTCAAACTGGATATCTAGAGTGCAG GAGAGGAGAATGGAA

TTCCTAGTGTAGCGGTGAAATGCGTAGAGATTAGGAAGAACACCAGTGGCGAAGGCGATTCTCT GGACTGTAACTGACGC

TGAGGCACGAAAGCGTGGGTAGCAAACAGGATTAGATACCCTGGTAGTCCACGCCGTAAACGAT GAGTACTAGGTGTAGG AGGTATCGACCCCTTCTGTGCCGCAGTAAACACATTAAGTACTCCGCCTGGGAAGTACGATCGCA AGATTAAAACTCAAA GGAATTGACGGGGGCCCGCACAAGCAGCGGAGCATGTGGTTTAATTCGAAGCAACGCGAAGAA CCTTACCTGGACTTGAC

ATCCCCTGAATAACCTAGAGATAGGCGAAGCCCTTCGGGGCAGGGAGACAGGTGGTGCATGGTT GTCGTCAGCTCGTGTC

GTGAGATGTTAGGTTAAGTCCTGCAACGAGCGCAACCCTTATTGTTAGTTGCTAACATTCAGTTGA GCACTCTAACGAGA

CTGCCGCGGTTAACGCGGAGGAAGGTGGGGATGACGTCAAATCATCATGCCCCTTATGTCCAGG GCAACACACGTGCTAC

AATGGGCAGAACAAAGAGAAGCAATACCGCGAGGTGGAGCCAAACTCAAAAACTGCTCTCAGTT CGGATTGCAGGCTGAA

ACTCGCCTGCATGAAGCTGGAGTTGCTAGTAATCGCGAATCAGCATGTCGCGGTGAATACGTTCC CGGGCCTTGTACACA

CCGCCCGTCACACCATGAGAGCTGGCAACACCCGAAGTCCGTAGTCTAACGTAAGAGGACGCGG CCGAAGGTGGGGTTAG

TGATTGGGGTGAAGTCGTAACAAGGTAGCCGTAGGAGAACCTGCGGCTG 
Table S13. The table shows the OTU counts as classified within the Candidatus Methanogranum genus. The samples were taken at the end of the steady states of phase III (day 90) (pH 5.8) and phase IV (day 125) ( $\mathrm{pH} \mathrm{5.5)} \mathrm{of} \mathrm{continuous} \mathrm{methanol} \mathrm{based} \mathrm{propionate} \mathrm{elongation} \mathrm{in} \mathrm{an} \mathrm{anaerobic} \mathrm{open-culture} \mathrm{reactor}$ and at the end of the steady state of phase $V$ (day 69) (methanol in influent $250 \mathrm{mM}$ ) and during the last day of phase VI (day 106) (methanol in influent $400 \mathrm{mM}$ ) of continuous methanol based propionate and acetate elongation in an anaerobic open-culture reactor at $309 \mathrm{~K}$. The inocula samples from the continuous methanol based acetate elongation reactor and the propionate elongation batch experiment with initial $\mathrm{pH}$ 7 (Figure 2) are shown as well. For all biomass samples the duplo results are shown indicated by $1 \& 2$. OTUs where only one hit was found in total in all 12 samples were omitted due to the size of the table.

\begin{tabular}{|c|c|c|c|c|c|c|c|c|c|c|c|c|}
\hline \multirow[b]{3}{*}{$\begin{array}{c}\text { OTU } \\
\text { identifiers }\end{array}$} & \multicolumn{12}{|c|}{ OTU counts } \\
\hline & \multicolumn{2}{|c|}{$\begin{array}{c}\text { Inoculum: } \\
\text { continuous } \\
\text { methanol based } \\
\text { acetate } \\
\text { elongation }\end{array}$} & \multicolumn{2}{|c|}{$\begin{array}{c}\text { Inoculum: } \\
\text { Propionate; } \mathrm{pH} \\
5.8 \text { (Figure 1) }\end{array}$} & \multicolumn{2}{|c|}{$\begin{array}{c}\text { Propionate; } \mathbf{p H} \\
\mathbf{5 . 8} ; 250 \mathrm{mM} \\
\text { methanol }\end{array}$} & \multicolumn{2}{|c|}{$\begin{array}{l}\text { Propionate; pH } \\
\text { 5.5; } 250 \mathrm{mM} \\
\text { methanol }\end{array}$} & \multicolumn{2}{|c|}{$\begin{array}{c}\text { Propionate\&acet } \\
\text { ate; pH 5.8; } \mathbf{2 5 0} \\
\text { mM methanol }\end{array}$} & \multicolumn{2}{|c|}{$\begin{array}{c}\text { Propionate\&acet } \\
\text { ate; pH 5.8; } 400 \\
\text { mM methanol }\end{array}$} \\
\hline & 1 & 2 & 1 & 2 & 1 & 2 & 1 & 2 & 1 & 2 & 1 & 2 \\
\hline $\begin{array}{r}\text { JF980498.1.141 } \\
9\end{array}$ & 12109 & 8781 & 15769 & $\begin{array}{r}1834 \\
8\end{array}$ & 38560 & 71682 & 48792 & 44685 & 33959 & 36779 & 61411 & 75857 \\
\hline $\begin{array}{r}\text { New.CleanUp.Re } \\
\text { ferenceOTU147 } \\
471\end{array}$ & 17 & 28 & 58 & 40 & 107 & 203 & 110 & 99 & 43 & 78 & 129 & 179 \\
\hline $\begin{array}{r}\text { New.Reference } \\
\text { OTU188 }\end{array}$ & 83 & 64 & 7 & 16 & 70 & 98 & 45 & 59 & 135 & 173 & 55 & 73 \\
\hline $\begin{array}{r}\text { New.CleanUp.Re } \\
\text { ferenceOTU233 } \\
223\end{array}$ & 83 & 61 & 3 & 2 & 38 & 57 & 40 & 43 & 112 & 145 & 46 & 67 \\
\hline $\begin{array}{r}\text { New.CleanUp.Re } \\
\text { ferenceOTU119 } \\
462\end{array}$ & 0 & 0 & 5 & 7 & 32 & 63 & 73 & 32 & 0 & 3 & 97 & 85 \\
\hline Cropped... & $\ldots$ & $\ldots$ & $\ldots$ & $\ldots$ & $\ldots$ & $\ldots$ & ... & ... & $\ldots$ & ... & $\ldots$ & $\ldots$ \\
\hline $\begin{array}{l}\text { Sum of OTU } \\
\text { counts within } \\
\text { genus }\end{array}$ & 12359 & 8979 & 15956 & $\begin{array}{c}1856 \\
8\end{array}$ & 38965 & 72400 & 49309 & 45119 & 34425 & 37357 & 62043 & 76629 \\
\hline $\begin{array}{l}\text { Total OTU } \\
\text { counts in } \\
\text { sample }\end{array}$ & $\begin{array}{c}19934 \\
4\end{array}$ & $\begin{array}{c}14513 \\
0\end{array}$ & $\begin{array}{c}14547 \\
7\end{array}$ & $\begin{array}{c}1404 \\
99\end{array}$ & $\begin{array}{c}13284 \\
6\end{array}$ & $\begin{array}{c}22172 \\
8\end{array}$ & $\begin{array}{c}19744 \\
4\end{array}$ & $\begin{array}{c}20842 \\
9\end{array}$ & $\begin{array}{c}18989 \\
6\end{array}$ & $\begin{array}{c}19466 \\
9\end{array}$ & $\begin{array}{c}18320 \\
5\end{array}$ & $\begin{array}{c}20069 \\
1\end{array}$ \\
\hline
\end{tabular}




\section{JF980498.1.1419 sequence:}

CTGGTTGATCCTGCCGGCGGCCACCGCTATAGGAATTCGATTAAGACATGCGAGTCGAGAGTCG TAATGGACTCGGCGGA

CTGCTCAGTAACACGTGGATAACGTGCCCTTAAGTGGAGGATAATCTCGGGAAATTGAGGATAAT ACTCCATAGATCATG

ACACCTGGAATGAGTCATGGTTCAAAGTTCCGGCGCTTAAGGATCGGTCTGCGGCCTATCAGGTA GTAGTGGGTGTAATG

TACCTACTAGCCTATGACGGGTATGGGCCTTGAGAGAGGGAGCCCAGAGTTGGATTCTGAGACA CGAATCCAGGCCCTAC

GGGGCGCAGCAGTCGCGAAAACTTCACAATGGGCGCAAGCCCGATGAGGGAACTCCTAGTGCTA GCACTTTTTKTGTTAG

CTITTCTTCAGCGTAGATAACTGAAGGAATAAGGGCTGGGTAAGACGGGTGCCAGCCGCCGCGG TAATACCTGCAGCCCA

AGTGGTGGTCGATTTTATTGAGTCTAAAACGTTCGTAGCCGGTCTGGTAAATCCTTGGGTAAATC GGAAAGCTTAACTTT

CCGAATTCCGAGGAGACTGCCAGACTTGGGACCGGGAGAGGCTAGAGGTACTTCTGGGGTAGG GGTAAAATCCTGTAATC

CTAGAAGGACCACCGGTGGCGAAGGCGTCTAGCTAGAACGGATCCGACGGTGAGGGACGAAGC CCTGGGTCGCAAACGGG

ATTAGATACCCCGGTAGTCCAGGGTGTAAACGCTGCAGACTTGGTGTTGGAGATCCTTCGAGGG TATTCAGTGCCGGAGA

GAAGTTGTTAAGTCTGCTACTTGGGGAGTACGTCCGCAAGGATGAAACTTAAAGGAATTGGTGG GGGAGCACCGCAACGG

GAGGAGCGTGCGGTTTAATTGGATTCAACACCGGAAAACTCACCAGGGGAGACTGTTACATGAA AGCCAGGCTAATGACC

TTGCTCGATTTTCAGAGAAGTGGTGCATGGCCGTCGTCAGTTCGTACCGTAAGGCGTTCTCTTAA GTGAGATAACGAACG

AGACCCTCACTAATATTTGCTACTCCGTTCTCCGGAACGGAGGCACATTATTGGGACCGCTGGCG CTAAGCCAGAGGAAG

GAGAGGTCAACGGTAGGTCAGCATGCCCTGAATCTCCTGGGCTACACGCGCGCTACAAAGGGCG GGACAATGGGTTCCGA

CACCGAAAGGTGAAGGTAATCTCGAAACCCGTCCGTAGTTCGGATTGAGGGTTGTAACTCACCCT CATGAAGCTGGATTC

CGTAGTAATCGCGAATCAACAACTCGCGGTGAATATGCCCCTGCTCCTTGCACACACCGCCCGTC AAACCATCCGAGTTG

GGTITCAGTGAAGCTGCCTCTAACTAGGGTTGTTGAACTGAGATTTAGCAAGGAAGGTT 


\section{Supporting Information - Chapter 4}

Branched Medium Chain Fatty Acids: iso-caproate formation from isobutyrate broadens the product spectrum for microbial chain elongation

Kasper D. de Leeuw, Cees J. N. Buisman, David P. B. T. B. Strik 


\section{Materials and Methods}

\section{Reactor set-up and operation}

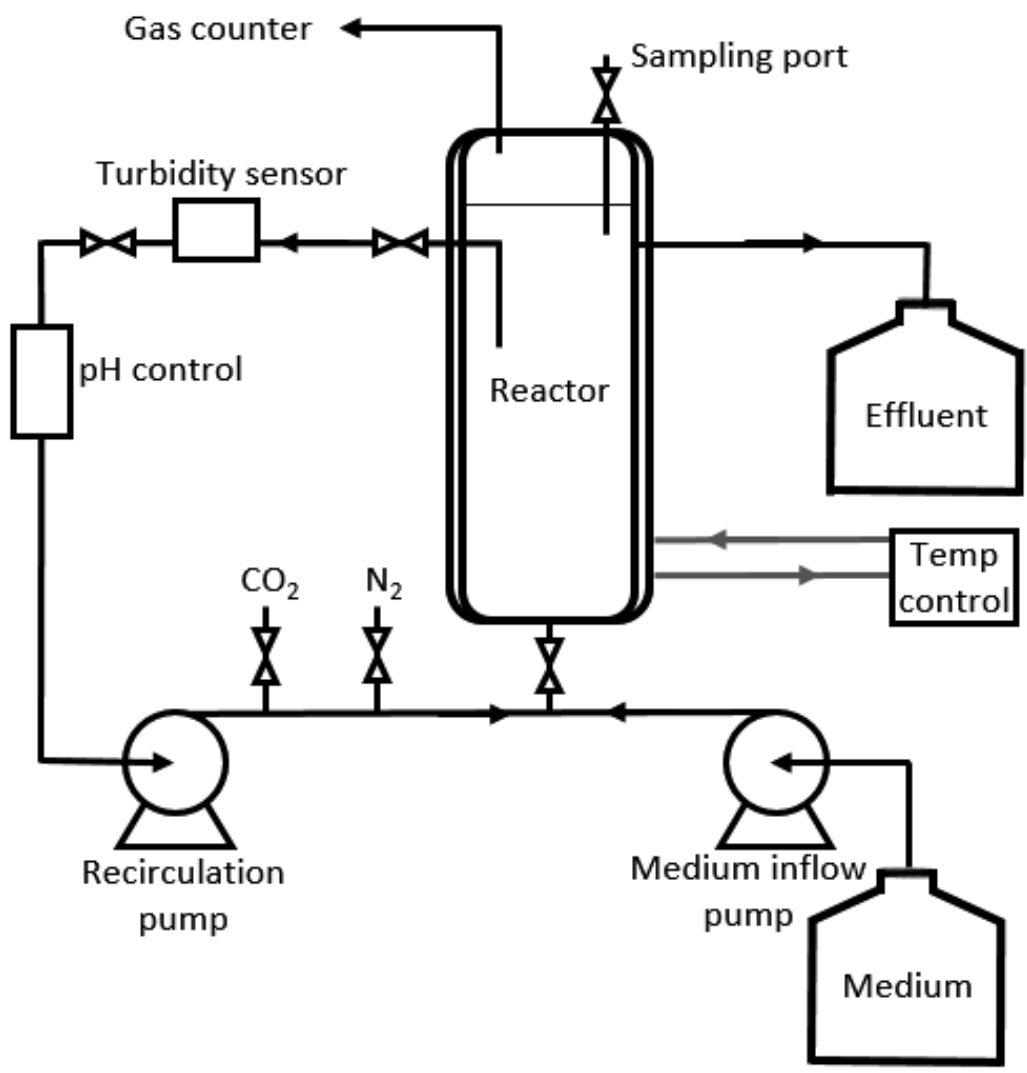

Figure S1. Schematic depiction of the reactor setup that was used for the experiment. 


\section{Medium considerations for $B_{12}$ variations and initial methanol addition}

A high ( $\left.0.375 \mathrm{mg} \mathrm{l}^{-1}\right) \mathrm{B}_{12}$ concentration was used because the previous study on isobutyrate formation used this amount to ensure sufficient availability required for isomerization of butyate ${ }^{35}$. In the initial stage of the experiment it was unclear if branched $\mathrm{C}_{6}$ would be formed via similar reciprocal isomerization ${ }^{95}$ (which would lead to 2-methyl pentanoic acid) or $\mathrm{i}-\mathrm{C}_{4}$ elongation (which would lead to 4-methyl pentanoic acid). Lowering the $B_{12}$ concentration in phase III was performed to investigate if an elevated $B_{12}$ concentration in regard to branched $\mathrm{C}_{6}$ formation was necessary.

Methanol was initially included in the medium because (i) it stimulated branched $\mathrm{C}_{4}$ formation $^{35}$ (It was unclear if branched $C_{6}$ would be formed in a similar fashion.) and (ii) its effect on ethanol based chain elongation should be investigated in case methanol based chain elongation would be used as prior fermentation step to produce an i- $\mathrm{C}_{4}$ substrate stream.

\section{Sampling and measurement}

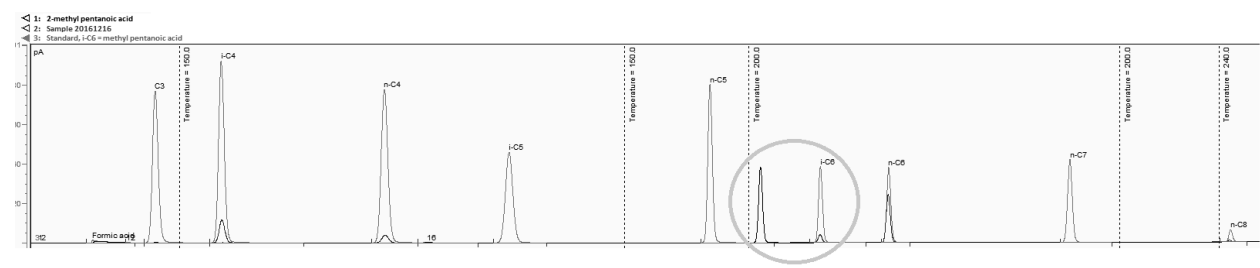

Figure S2. The three chromatograms in the figure show that the compound identified in the sample (blue) as 4-methyl pentanoic acid matches the retention time of 4-methyl pentanoic acid (i-C6) in the standard (pink). A prepared solution of 2-methyl pentanoic acid (black) was also analysed and shows clearly different retention time, indicating that this method is suitable to distinguish between isoforms of six carbon monocarboxylic acids. Chromeleon software was used to generate this figure.

\section{Microbial community analysis - QIIME 16s rRNA gene amplicon analysis}

The paired-end MiSeq reads were merged based on the overlap between the two reads. Only merged sequences were used in subsequent analyses and the non-overlapping pairs were discarded. The $16 \mathrm{~S}$ rRNA gene amplicon analyses were performed using QIIME $^{99}$ version 1.9.1. The primer sequences were removed from the merged sequence reads and then place in a single fasta file using the add_qiime_labels.py script with the options 'cutadapt $-\mathrm{m} \quad 100-\mathrm{u} \quad 17-\mathrm{u}-21^{\prime}$. OTU picking was performed with the script pick_open_reference_otus.py using the SILVA version $128^{209} 16 \mathrm{~S}$ reference database and uclust $^{101}$. The RDP classifier (version 2.2) ${ }^{289}$ was trained with the same SILVA reference database and subsequently used to classify the OTUs. The QIIME script core_diversity_analyses.py was used to calculate alpha- and beta-diversity statistics of the samples (see Figures S8-S13). 


\section{Results and Discussion}

Table S1. Thermodynamic calculations of observed bioprocesses under different conditions indicated with superscript 1-4. The coupled bioprocess shows how reactant and product ratios of the respective alcohol and carboxylate species can drive or inhibit carboxylate reduction in the absence of sufficient hydrogen partial pressures. Values of $\Delta \mathrm{G}$ are given in $\mathrm{kJ}$ per reaction. Formation Gibbs energies were taken from Kleerebezem and Van Loosdrecht $(2010)^{28}$.

\begin{tabular}{|c|c|c|c|c|c|}
\hline \# & Bioprocess & Reaction & $\Delta \mathbf{G}^{01}$ & $\Delta \mathbf{G}^{\mathbf{1}}$ & $\Delta \mathbf{G}^{2}$ \\
\hline 1 & $\begin{array}{l}\text { Ethanol based chain elongation } \\
\text { (acetate) }\end{array}$ & $6 \mathrm{CH}_{3} \mathrm{CH}_{2} \mathrm{OH}+4 \mathrm{CH}_{3} \mathrm{COO}^{-} \rightarrow 5 \mathrm{C}_{3} \mathrm{H}_{7} \mathrm{COO}^{-}+2 \mathrm{H}_{2}+4 \mathrm{H}_{2} \mathrm{O}+\mathrm{H}^{+}$ & -183.4 & -206.2 & -240.4 \\
\hline $1 a$ & $\begin{array}{l}\text { Reverse beta-oxidation } \\
(5 x)\end{array}$ & $5 \mathrm{CH}_{3} \mathrm{CH}_{2} \mathrm{OH}+5 \mathrm{CH}_{3} \mathrm{COO}^{-} \rightarrow 5 \mathrm{C}_{3} \mathrm{H}_{7} \mathrm{COO}^{-}+5 \mathrm{H}_{2} \mathrm{O}$ & -193.0 & -193.0 & -193.0 \\
\hline $1 b$ & $\begin{array}{l}\text { Coupled ethanol oxidation } \\
(1 x)\end{array}$ & $\mathrm{CH}_{3} \mathrm{CH}_{2} \mathrm{OH}+\mathrm{H}_{2} \mathrm{O} \rightarrow \mathrm{CH}_{3} \mathrm{COO}^{-}+2 \mathrm{H}_{2}+\mathrm{H}^{+}$ & 9.6 & -13.2 & -47.4 \\
\hline 2 & $\begin{array}{l}\text { Hydrogenogenic ethanol } \\
\text { oxidation }\end{array}$ & $\mathrm{CH}_{3} \mathrm{CH}_{2} \mathrm{OH}+\mathrm{H}_{2} \mathrm{O} \rightarrow \mathrm{CH}_{3} \mathrm{COO}^{-}+2 \mathrm{H}_{2}+\mathrm{H}^{+}$ & 9.6 & -13.2 & -47.4 \\
\hline 3 & Ethanol oxidation with $\mathrm{HCO}^{-}$ & $2 \mathrm{CH}_{3} \mathrm{CH}_{2} \mathrm{OH}+2 \mathrm{HCO}_{3}^{-} \rightarrow 3 \mathrm{CH}_{3} \mathrm{COO}^{-}+2 \mathrm{H}_{2} \mathrm{O}+\mathrm{H}^{+}$ & -85.2 & -85.2 & -85.2 \\
\hline 4 & $\begin{array}{l}\text { Ethanolotrophic } \\
\text { methanogenesis }\end{array}$ & $2 \mathrm{CH}_{3} \mathrm{CH}_{2} \mathrm{OH}+\mathrm{HCO}_{3}^{-} \rightarrow 2 \mathrm{CH}_{3} \mathrm{COO}^{-}+\mathrm{CH}_{4}+\mathrm{H}_{2} \mathrm{O}+\mathrm{H}^{+}$ & -116.2 & -116.2 & -116.2 \\
\hline 5 & Beta-oxidation & $\mathrm{C}_{3} \mathrm{H}_{7} \mathrm{COO}^{-}+2 \mathrm{H}_{2} \mathrm{O} \rightarrow 2 \mathrm{CH}_{3} \mathrm{COO}^{-}+2 \mathrm{H}_{2}+\mathrm{H}^{+}$ & 48.2 & 25.4 & -8.8 \\
\hline 6 & $\begin{array}{l}\text { Hydrogenotrophic } \\
\text { methanogenesis }\end{array}$ & $\mathrm{HCO}_{3}^{-}+\mathrm{H}^{+}+4 \mathrm{H}_{2} \rightarrow \mathrm{CH}_{4}+3 \mathrm{H}_{2} \mathrm{O}$ & -135.5 & -89.8 & -21.3 \\
\hline 7 & Hydrogenotrophic acetogenesis & $2 \mathrm{HCO}_{3}^{-}+\mathrm{H}^{+}+4 \mathrm{H}_{2} \rightarrow \mathrm{CH}_{3} \mathrm{COO}^{-}+4 \mathrm{H}_{2} \mathrm{O}$ & -104.4 & -58.8 & 9.7 \\
\hline 8 & $\begin{array}{l}\text { Hydrogenotrophic carboxylate } \\
\text { reduction }\end{array}$ & $\mathrm{C}_{3} \mathrm{H}_{7} \mathrm{COO}^{-}+2 \mathrm{H}_{2}+\mathrm{H}^{+} \rightarrow \mathrm{C}_{3} \mathrm{H}_{7} \mathrm{CH}_{2} \mathrm{OH}+\mathrm{H}_{2} \mathrm{O}$ & -16.4 & 6.4 & 40.6 \\
\hline 9 & $\begin{array}{l}\text { Methanol based chain } \\
\text { elongation }\end{array}$ & $\mathrm{CH}_{3} \mathrm{OH}+2 \mathrm{C}_{3} \mathrm{H}_{7} \mathrm{COO}^{-} \rightarrow \mathrm{C}_{3} \mathrm{H}_{7} \mathrm{COO}^{-}+2 \mathrm{H}_{2} \mathrm{O}$ & -106.8 & -106.8 & -106.8 \\
\hline 10 & $\begin{array}{l}\text { Methanolotrophic acetate } \\
\text { formation }\end{array}$ & $4 \mathrm{CH}_{3} \mathrm{OH}+2 \mathrm{HCO}_{3}^{-} \rightarrow 3 \mathrm{C}_{3} \mathrm{H}_{7} \mathrm{COO}^{-}+4 \mathrm{H}_{2} \mathrm{O}+\mathrm{H}^{+}$ & -221.6 & -221.6 & -221.6 \\
\hline 11 & $\begin{array}{l}\text { Methanolotrophic } \\
\text { methanogenesis }\end{array}$ & $4 \mathrm{CH}_{3} \mathrm{OH} \rightarrow 3 \mathrm{CH}_{4}+\mathrm{HCO}_{3}^{-}+\mathrm{H}_{2} \mathrm{O}+\mathrm{H}^{+}$ & -314.7 & -314.7 & -314.7 \\
\hline 12 & $\begin{array}{l}\text { Hydrogen-methanol } \\
\text { methanogenesis }\end{array}$ & $\mathrm{CH}_{3} \mathrm{OH}+\mathrm{H}_{2} \rightarrow \mathrm{CH}_{4}+\mathrm{H}_{2} \mathrm{O}$ & -112.6 & -101.1 & -84.0 \\
\hline \multirow[t]{2}{*}{13} & Acetoclastic methanogenesis & $\mathrm{CH}_{3} \mathrm{COO}^{-}+\mathrm{H}_{2} \mathrm{O} \rightarrow \mathrm{CH}_{4}+\mathrm{HCO}_{3}^{-}$ & -31.05 & -31.05 & -31.05 \\
\hline & $\begin{array}{l}\text { Alcohol formation (14) as } \\
\text { combined process ( } 2 \& 8 \text { ) } \\
\text { without } \mathrm{H} 2\end{array}$ & Reaction & $\Delta \mathbf{G}^{01}$ & $\Delta \mathbf{G}^{3}$ & $\Delta \mathbf{G}^{4}$ \\
\hline 2 & $\begin{array}{l}\text { Hydrogenogenic ethanol } \\
\text { oxidation }\end{array}$ & $\mathrm{CH}_{3} \mathrm{CH}_{2} \mathrm{OH}+\mathrm{H}_{2} \mathrm{O} \rightarrow \mathrm{CH}_{3} \mathrm{COO}^{-}+2 \mathrm{H}_{2}+\mathrm{H}^{+}$ & 9.6 & -1.8 & 21.1 \\
\hline 8 & $\begin{array}{l}\text { Hydrogenotrophic carboxylate } \\
\text { reduction }\end{array}$ & $\mathrm{C}_{3} \mathrm{H}_{7} \mathrm{COO}^{-}+2 \mathrm{H}_{2}+\mathrm{H}^{+} \rightarrow \mathrm{C}_{3} \mathrm{H}_{7} \mathrm{CH}_{2} \mathrm{OH}+\mathrm{H}_{2} \mathrm{O}$ & -16.4 & -27.9 & -5.0 \\
\hline 14 & $\begin{array}{l}\text { Carboxylate-to-alcohol } \\
\text { reduction }\end{array}$ & $\mathrm{C}_{3} \mathrm{H}_{7} \mathrm{COO}^{-}+\mathrm{CH}_{3} \mathrm{CH}_{2} \mathrm{OH} \rightarrow \mathrm{C}_{3} \mathrm{H}_{7} \mathrm{CH}_{2} \mathrm{OH}+\mathrm{CH}_{3} \mathrm{COO}^{-}$ & -6.8 & -29.6 & 16.0 \\
\hline
\end{tabular}

$\Delta \mathbf{G}^{01}$

$\Delta \mathbf{G}^{\mathbf{1}}$

$\Delta \mathbf{G}^{2}$

$\Delta \mathbf{G}^{3}$

$\Delta \mathbf{G}^{\mathbf{4}}$

biological standard conditions $(\mathrm{pH} 7)$
hydrogen partial pressure $=1 \mathrm{kPa}$
hydrogen partial pressure $=1 \mathrm{~Pa}$
reactants to products ratio $=100$
reactants to products ratio $=0.01$

biological standard conditions $(\mathrm{pH} 7)$

hydrogen partial pressure $=1 \mathrm{~Pa}$

reactants to products ratio $=0.01$

* For reactants and products only the concentrations of the respective alcohols and carboxylates were changed according to indicated ratios. Proton concentration ( $\mathrm{pH} 7$ ) was kept constant. Hydrogen partial pressure was kept constant at 1 bar. Water activity was set at 1 . 
Table S2. This table shows the averaged molar ratios of isomeric compounds formed (i-C6, $\mathrm{i}-\mathrm{C} 4 \mathrm{OH}$ \& $\mathrm{i}-\mathrm{C} 6 \mathrm{OH}$ ) divided by the amount of $\mathrm{i}-\mathrm{C} 4$ comsumed for each phase during steady state. In all but phase I the produced compounds more or less fit with the amount of consumed iso-butyrate. For phase I the discrepancy could be due to the instable i-C6 production, relatively low i-C6 concentration and relatively high $\mathrm{i}-\mathrm{C} 4$ concentration. Small measurement errors in particular for $\mathrm{i}-\mathrm{C} 4$ concentration would have a big impact on this ratio.

\begin{tabular}{lllllll}
\hline Phases & $I$ & $I I$ & $I I I$ & $I V$ & $V$ & $V I$ \\
\hline \hline i-C6 formed / i-C4 consumed & $51 \%$ & - & $98 \%$ & $111 \%$ & $112 \%$ & $74 \%$ \\
i-C4OH \& i-C6OH formed / i-C4 consumed & - & - & - & - & - & $38 \%$ \\
total & $51 \%$ & - & $98 \%$ & $111 \%$ & $112 \%$ & $112 \%$ \\
\hline \hline
\end{tabular}


Table S3. Averaged conversion rates, $\mathrm{C}$ molar concentrations (liquid) and partial pressures (headspace gas, $\mathrm{kPa}$ ) during each of the six phases.

\begin{tabular}{|c|c|c|c|c|c|c|c|c|c|c|c|c|}
\hline & \multicolumn{2}{|c|}{ Phase I } & \multicolumn{2}{|c|}{ Phase II } & \multicolumn{2}{|c|}{ Phase III } & \multicolumn{2}{|c|}{ Phase IV } & \multicolumn{2}{|c|}{ Phase V } & \multicolumn{2}{|c|}{ Phase VI } \\
\hline Discription & \multicolumn{2}{|c|}{$\begin{array}{l}\mathrm{i}-\mathrm{C} 4 / \mathrm{n}-\mathrm{C} 4 \\
\text { ratio: } 1 / 1\end{array}$} & \multicolumn{2}{|c|}{$\begin{array}{l}\text { i-C4 was } \\
\text { replaced by n- } \\
\text { C4 }\end{array}$} & \multicolumn{2}{|c|}{$\begin{array}{l}\mathrm{n}-\mathrm{C} 4 \text { was } \\
\text { replaced by i- } \\
\text { C4 \& vit B12 } \\
\text { from } 375 \mu \mathrm{g} / \mathrm{L} \\
\text { to } 12.5 \mu \mathrm{g} / \mathrm{L}\end{array}$} & \multicolumn{2}{|c|}{$\begin{array}{c}\text { Vit B12 from } \\
12.5 \mu \mathrm{g} / \mathrm{L} \text { to } \\
375 \mu \mathrm{g} / \mathrm{L}\end{array}$} & \multicolumn{2}{|c|}{$\begin{array}{l}\text { Removed } \\
\text { MeOH from } \\
\text { feed }\end{array}$} & \multicolumn{2}{|c|}{$\begin{array}{c}\text { Lowered } \\
\text { acetate } \\
\text { concentration }\end{array}$} \\
\hline $\begin{array}{l}\text { Phase } \\
\text { period }\end{array}$ & \multicolumn{2}{|c|}{ day 1 to 38} & \multicolumn{2}{|c|}{ day 38 to 112} & \multicolumn{2}{|c|}{ day 112 to 190} & \multicolumn{2}{|c|}{ day 190 to 262} & \multicolumn{2}{|c|}{ day 262 to 297} & \multicolumn{2}{|c|}{ day 297 to 388} \\
\hline $\begin{array}{l}\text { Steady } \\
\text { state } \\
\text { period }\end{array}$ & \multicolumn{2}{|c|}{ day 16 to 36} & \multicolumn{2}{|c|}{ day 52 to 65} & \multicolumn{2}{|c|}{ day 157 to 181} & \multicolumn{2}{|c|}{ day 225 to 254} & \multicolumn{2}{|c|}{ day 269 to 290} & \multicolumn{2}{|c|}{ day 330 to 358} \\
\hline \multicolumn{13}{|c|}{ Volumetric C molar conversion rates (mmolC L-1 day-1) } \\
\hline $\mathrm{C} 2$ & -30.6 & \pm 5 & -36.2 & \pm 3 & -52.9 & \pm 2.1 & -56.0 & \pm 2 & -56.4 & \pm 2 & 4.8 & \pm 1 \\
\hline $\mathrm{i}-\mathrm{C} 4$ & -8.6 & \pm 1.9 & 1.4 & \pm 1 & -21.5 & \pm 2.2 & -18.4 & \pm 1.2 & -17.8 & \pm 4 & -39.6 & \pm 3 \\
\hline$n-C 4$ & 34.8 & \pm 16 & -51.5 & \pm 6 & 51.4 & \pm 2.2 & 55.0 & \pm 2.3 & 54.6 & \pm 3 & 18.3 & \pm 1 \\
\hline i-C6 & 6.6 & \pm 1.3 & - & - & 31.8 & \pm 2.4 & 30.5 & \pm 0.5 & 30.0 & \pm 2 & 44.2 & \pm 6 \\
\hline$n-C 6$ & 171.4 & \pm 18.4 & 240.8 & \pm 22 & 186.8 & \pm 5.8 & 197.3 & \pm 3.8 & 191.7 & \pm 8 & 123.1 & \pm 3 \\
\hline$n-C 8$ & 2.6 & \pm 1.0 & 6.7 & \pm 0.6 & 4.9 & \pm 0.4 & 6.6 & \pm 0.4 & 6.9 & \pm 0.7 & 4.2 & \pm 0.4 \\
\hline $\mathrm{MeOH}$ & -14.8 & \pm 4.1 & -24.0 & \pm 7 & -10.9 & \pm 1.8 & -9.9 & \pm 1.0 & - & - & - & - \\
\hline EtOH & -186.9 & \pm 10.3 & -200.1 & \pm 13 & -201.8 & \pm 3.9 & -199.2 & \pm 2.3 & -197.4 & \pm 6 & -166.1 & \pm 3 \\
\hline $\mathrm{n}-\mathrm{C} 4 \mathrm{OH}$ & 1.9 & \pm 2 & & & & & & & & & & \\
\hline $\mathrm{i}-\mathrm{C} 4 \mathrm{OH}$ & & & & & & & & & & & 15 & \pm 1.4 \\
\hline $\mathrm{n}-\mathrm{C} 6 \mathrm{OH}$ & 2.9 & \pm 1.9 & & & & & 0.9 & \pm 1.1 & & & 9.9 & \pm 0.2 \\
\hline $\mathrm{i}-\mathrm{C} 6 \mathrm{OH}$ & & & & & & & & & & & & \\
\hline CH4 (gas) & 9.3 & \pm 2.4 & 8.2 & \pm 1.3 & 4.0 & \pm 1.9 & 3.2 & \pm 0.7 & 3.0 & \pm 0.5 & 4.8 & \pm 1.2 \\
\hline CO2 (gas) & -9.1 & \pm 1.9 & -8.6 & \pm 0.3 & -9.0 & \pm 0.4 & -8.7 & \pm 0.2 & -8.7 & \pm 0.1 & -8.7 & \pm 0.2 \\
\hline \multicolumn{13}{|c|}{ C molar concentrations (liquid, mM C) and partial pressures (headspace gas, kPa) } \\
\hline $\mathrm{C} 2$ & 89.9 & \pm 11 & 81.2 & \pm 8 & 53.9 & \pm 6 & 48.6 & \pm 3 & 44.4 & \pm 2 & 21.8 & \pm 2 \\
\hline i-C4 & 115.8 & \pm 4 & 2.5 & \pm 1 & 215.9 & \pm 20 & 233.0 & \pm 2 & 232.8 & \pm 7 & 199.1 & \pm 4 \\
\hline$n-C 4$ & 192.5 & \pm 28 & 173.1 & \pm 9 & 82.1 & \pm 7 & 95.3 & \pm 3 & 98.2 & \pm 7 & 30.1 & \pm 2 \\
\hline i-C6 & 11.6 & \pm 2 & - & - & 50.4 & \pm 6 & 52.9 & \pm 1 & 53.7 & \pm 2 & 72.5 & \pm 7 \\
\hline $\mathrm{n}-\mathrm{C} 6$ & 303.5 & \pm 35 & 420.3 & \pm 20 & 303.9 & \pm 22 & 341.9 & \pm 7 & 344.0 & \pm 10 & 202.0 & \pm 8 \\
\hline $\mathrm{n}-\mathrm{C} 8$ & 4.5 & \pm 2 & 11.7 & \pm 1 & 8.2 & \pm 1 & 11.4 & \pm 1 & 12.3 & \pm 1 & 6.9 & \pm 1 \\
\hline $\mathrm{MeOH}$ & 64.3 & \pm 7 & 49.0 & \pm 9 & 67.3 & \pm 6 & 73.9 & \pm 2 & 0.0 & - & 3.2 & \pm 1 \\
\hline EtOH & 25.3 & \pm 16 & 8.4 & \pm 2 & 18.4 & \pm 3 & 13.7 & \pm 5 & 10.1 & \pm 4 & 85.2 & \pm 9 \\
\hline $\mathrm{n}-\mathrm{C} 4 \mathrm{OH}$ & 4.6 & \pm 3 & Traces & & & & & & & & & \\
\hline $\mathrm{i}-\mathrm{C} 4 \mathrm{OH}$ & & & & & & & & & & & 24.1 & \pm 3 \\
\hline $\mathrm{n}-\mathrm{C} 6 \mathrm{OH}$ & 5.9 & \pm 3 & Traces & & Traces & & 4.8 & \pm 1 & & & 16.3 & \pm 0.3 \\
\hline $\mathrm{i}-\mathrm{C} 6 \mathrm{OH}$ & & & & & & & & & & & Traces & \\
\hline $\mathrm{CH} 4$ (gas) & 80.2 & \pm 2 & 79.3 & \pm 3 & 74.6 & \pm 1 & 47.3 & \pm 17 & 49.4 & \pm 12 & 68.0 & \pm 19 \\
\hline CO2 (gas) & 3.2 & \pm 2.3 & 3.4 & \pm 0.6 & 3.1 & \pm 0.6 & 3.9 & \pm 0.6 & 4.0 & \pm 0.2 & 1.1 & \pm 0.1 \\
\hline
\end{tabular}




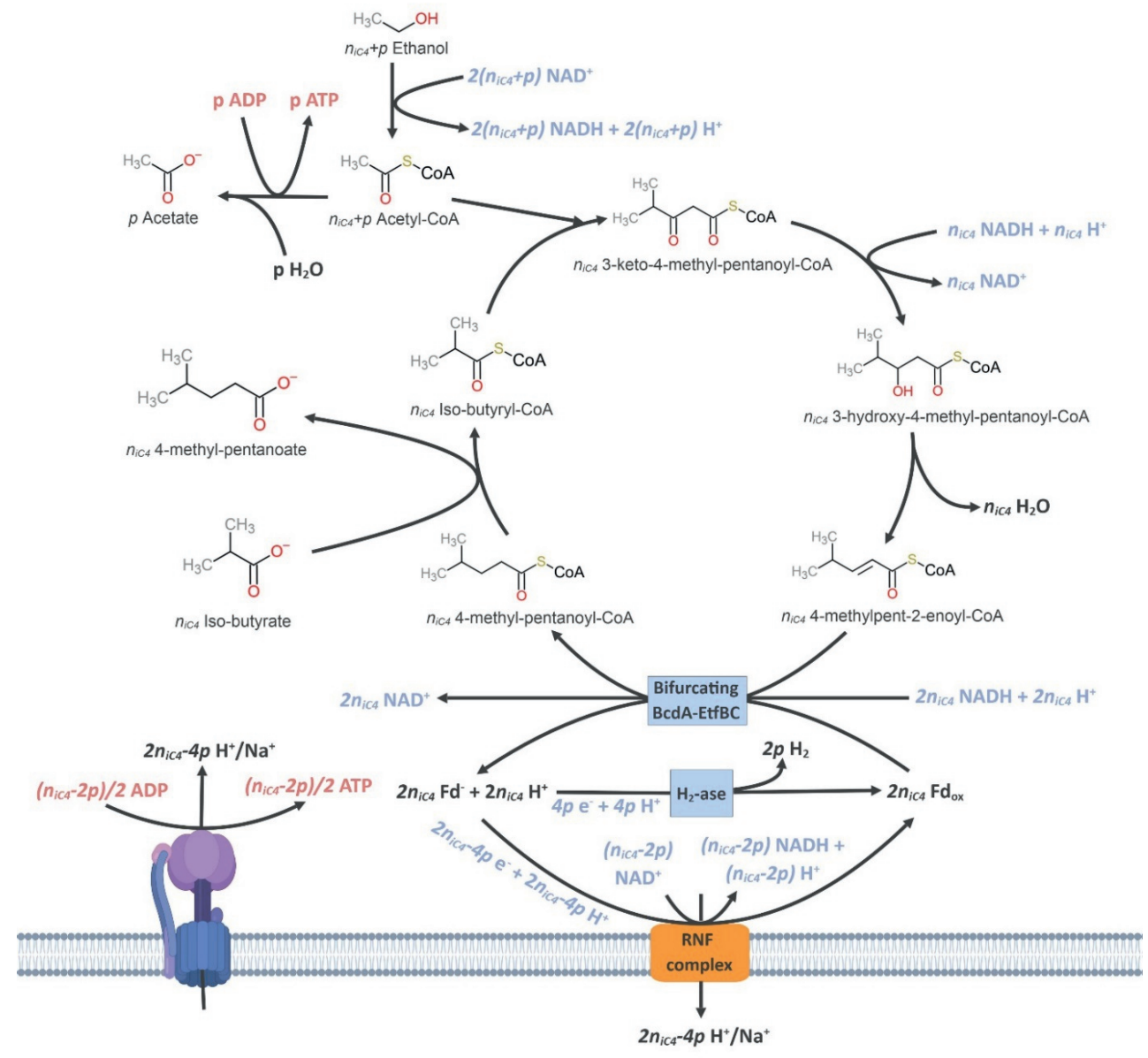

Figure S3.29 The figure shows the proposed mechanism for iso-butyrate elongation to 4-methylpentanoate within the reverse beta-oxidation pathway. Iso-butyryl-CoA is condensed with acetylCoA to form 3-keto-4-methyl-pentanoyl-CoA, which is subsequently reduced to 3-hydroxy-4-methylpentanoyl-CoA, hydrolyzed to 4-methylpent-2-enoyl-CoA and reduced further to 4-methylpentanoyl-CoA (4MPC), before the Coenzym-A is released and the cycle begins anew. The stoichiometry is indicated in terms of $n$ (amount of carboxylates elongated) and $p$ (amount of ethanol oxidized to acetate via substrate-level phosphorylation route). The figure was adjusted from Angenent et al. (2016) ${ }^{22}$. The stoichiometry was suggested so that $n=5$ and $p=1$. It is important to note that if chain elongation of different fatty acids occur simultaneously within the same organism, then $n$ can be seen as the sum of $n_{C 2}, n_{n-C 4}, n_{i-C 4}$, etc. 


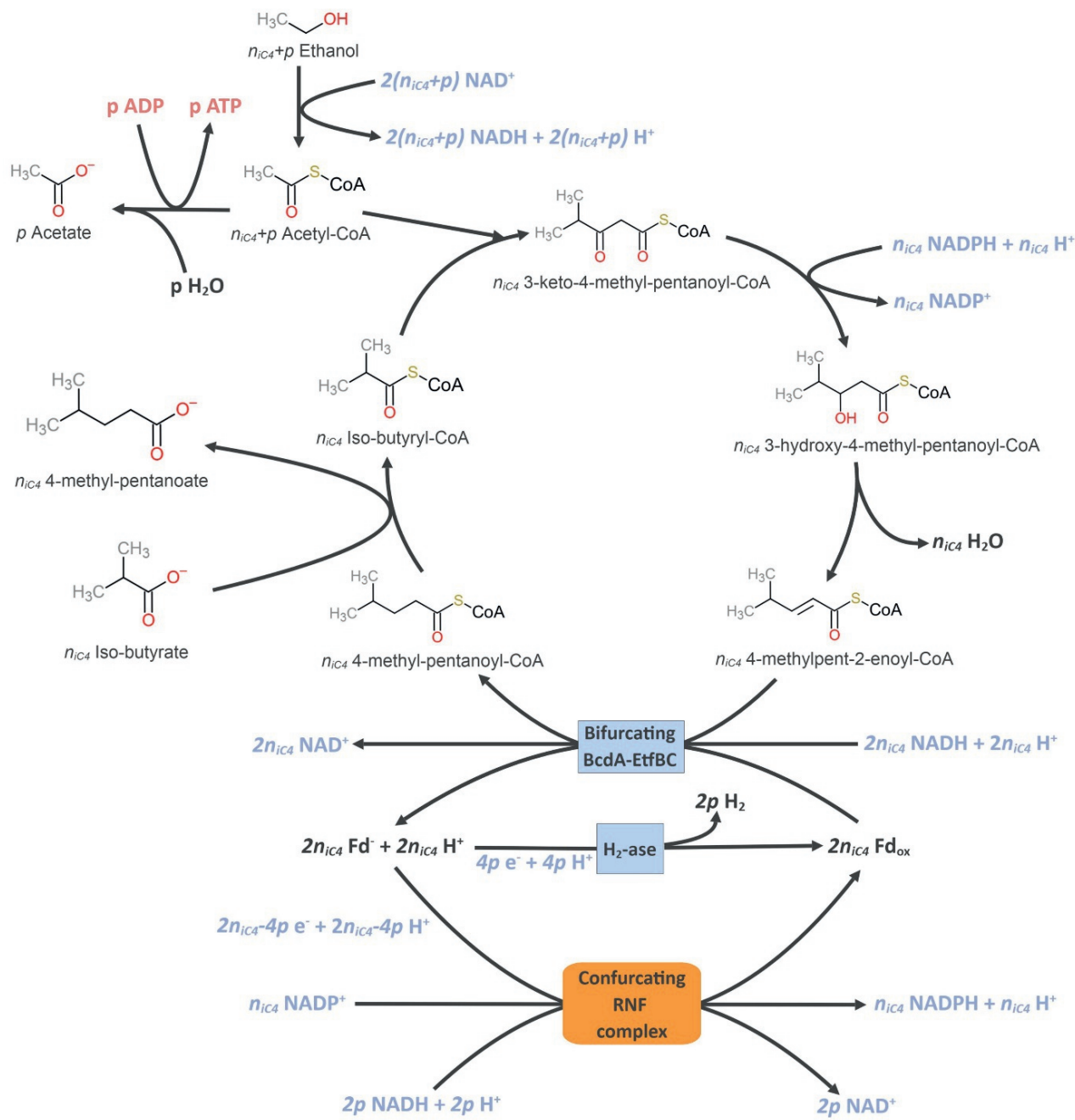

Figure S4. ${ }^{29}$ This figure shows the alternative hypothesized chain elongation mechanism in the case of a confucating RNF complex to form NADPH. The figure was adjusted from Angenent et al. (2016). For the confurcating pathway the stoichiometry was suggested so that $n=4$ and $p=1$. 


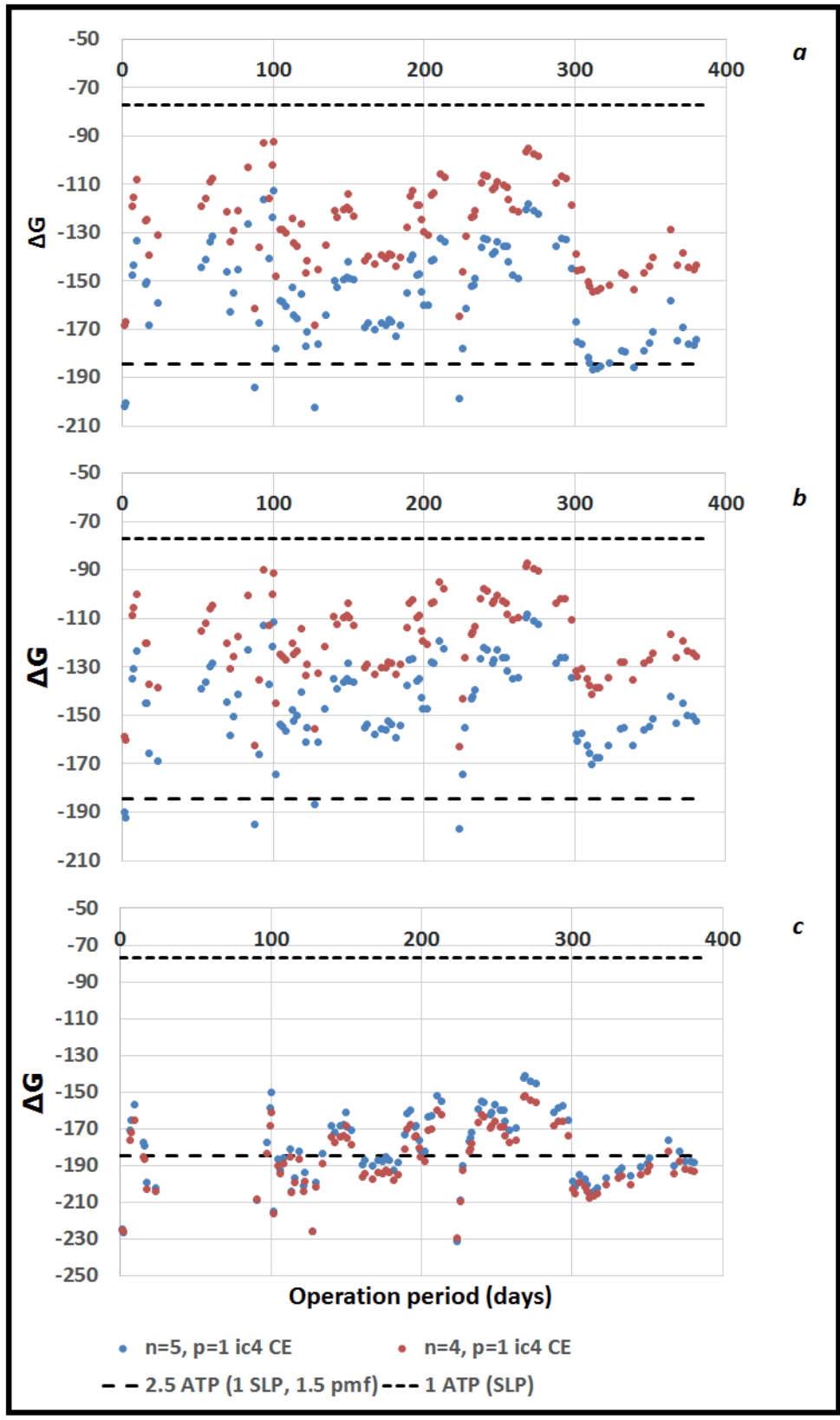

Figure S5. The figure shows the calculated Gibbs energies for chain elongation of a) acetate to nbutyrate, $b$ ) $\mathrm{n}$-butyrate to $\mathrm{n}$-caproate and $c$ ) iso-butyrate to iso-caproate, using the metabolite concentration within the reactor. In blue the stoichiometry is used where $n=5$ and $p=1$ and in red the stoichiometry is used where $n=4$ and $p=1$. The bracketed lines indicate the energy needed for $i$ ) formation of one ATP via substrate level phosphorylation (SLP) and ii) the energy that would be needed for one ATP via SLP and additional 1.5 ATP production via $\mathrm{H}^{+} / \mathrm{Na}^{+}$motive force driven ATPase. (Here indicated with pmf.) 


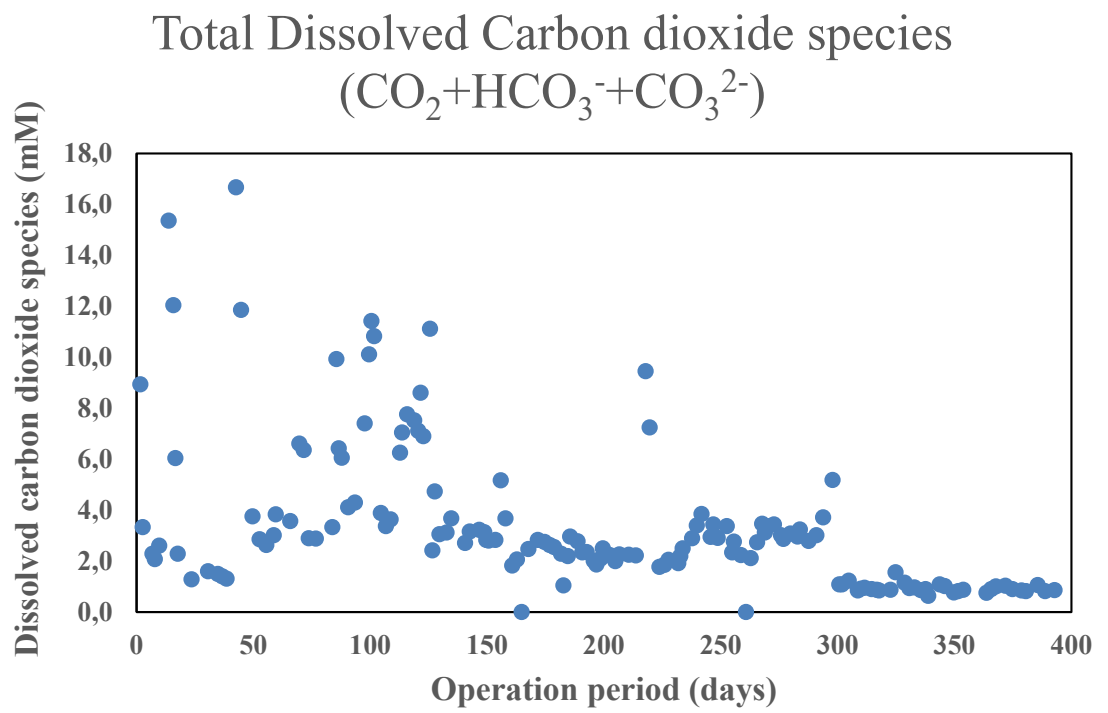

Figure S6. The graphs shows the calculated dissolved carbon dioxide species within the reactor during the whole operation period. Values are calculated from measured $\mathrm{CO} 2$ in the headspace and $\mathrm{pH}$ measurements using the Henderson-Hasselbalch equation for carbon dioxide species within the liquid (pKa 6.35 for $\mathrm{HCO}_{3}{ }^{-}$and pKa 10.33 for $\mathrm{CO}_{3}{ }^{2-}$ ) and a $\left(\mathrm{K}_{\mathrm{H}}\right)$ Henry's constant for $\mathrm{CO}_{2}$ of 29.41 $\mathrm{L} \cdot \mathrm{atm} \cdot \mathrm{mol}^{-1}$. 


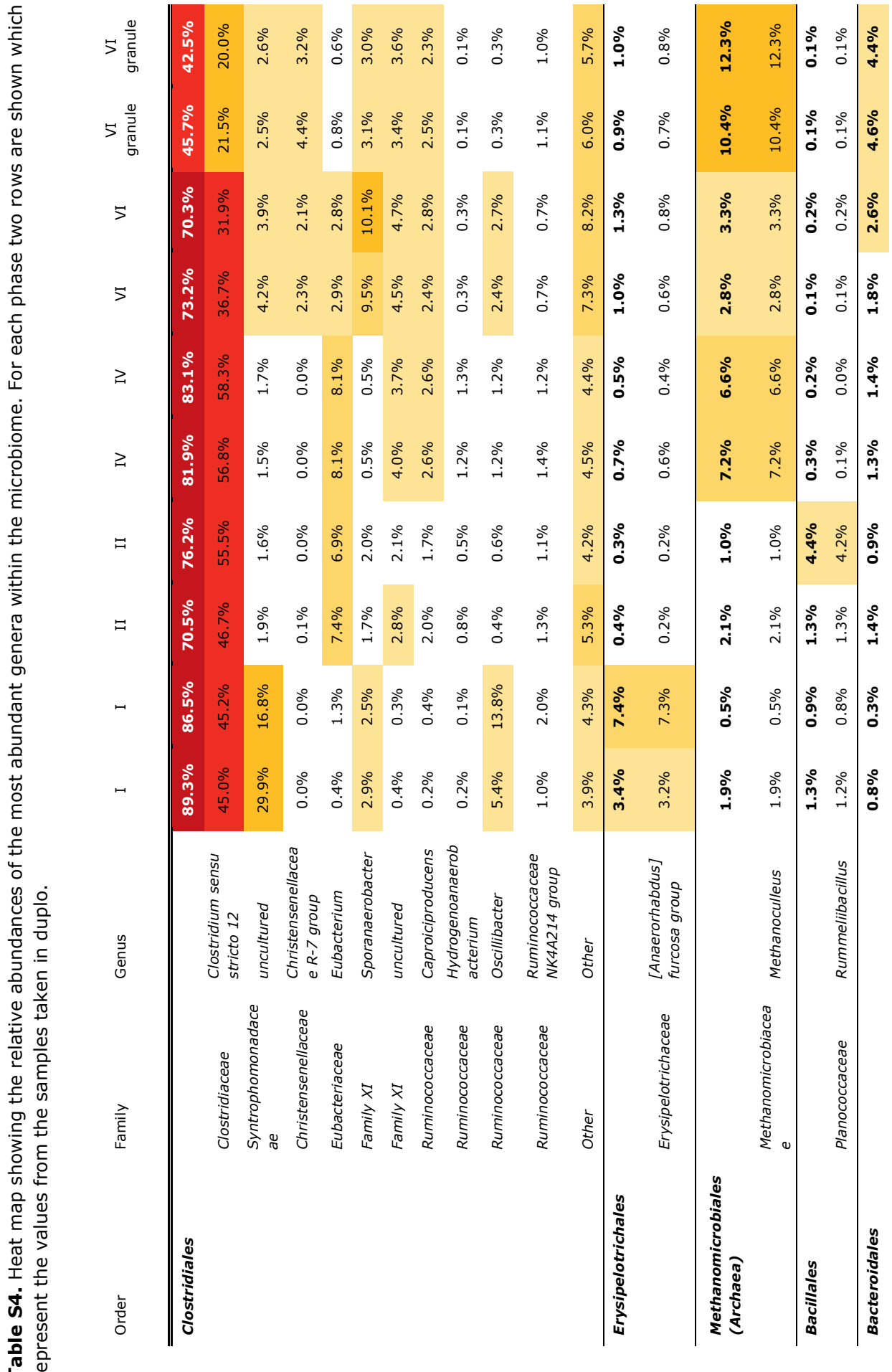




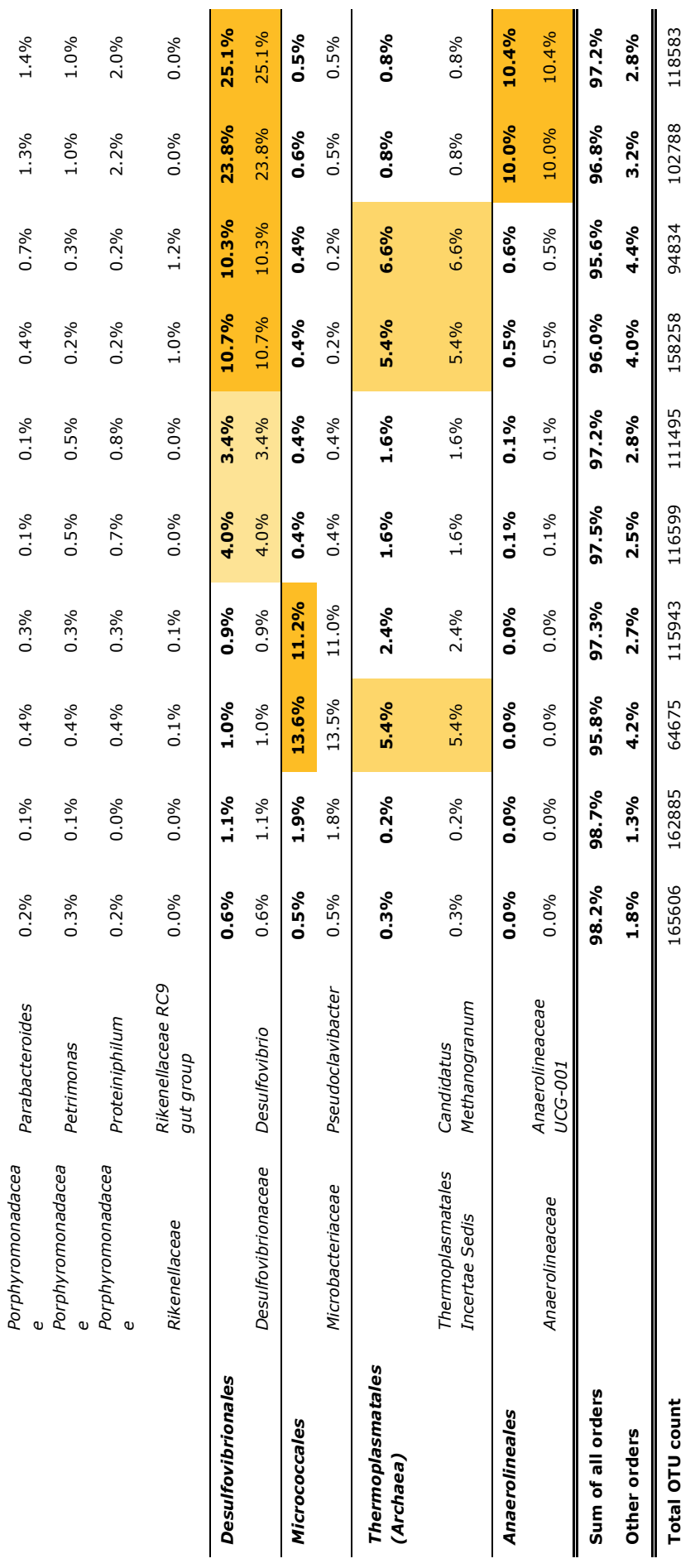




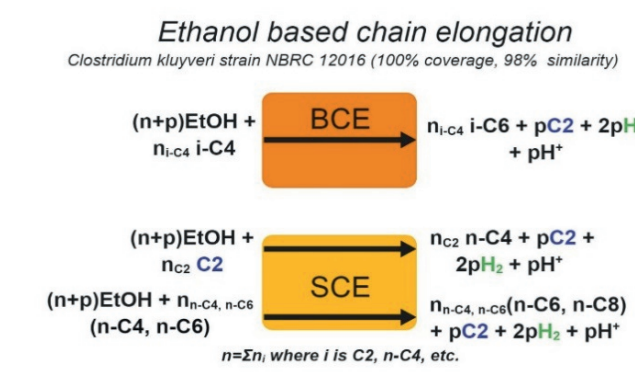

Syntrophic oxidation routes

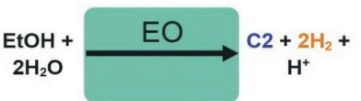

Desulsovibrio legallii strain H1 (95\% coverage, $99 \%$ similarity)

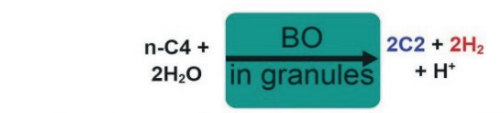

Syntrophomonas zehnderi strain OL-4 (100\% coverage, $96 \%$ similarity)

Desulsovibrio legallii strain $\mathrm{H} 1$ ( $95 \%$ coverage, $99 \%$ similarity) Anaerolineales order

Syntrophic partner reduction routes

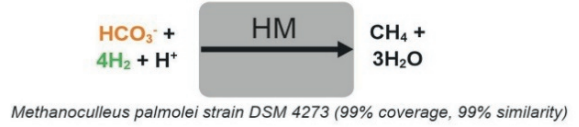

Methanoculleus palmole/ strain DSM $4273(99 \%$ coverage, $99 \%$ similarity)
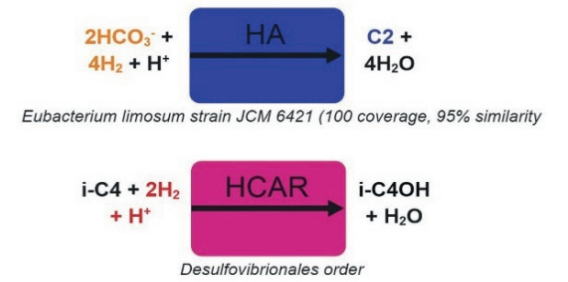

\section{Alternative fermentations}
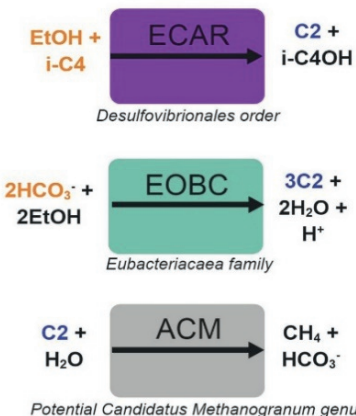

Potential Candidatus Methanogranum genus

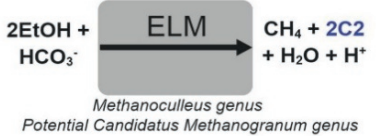

Potential Candidatus Methanogranum genus

$4 \mathrm{MeOH}$
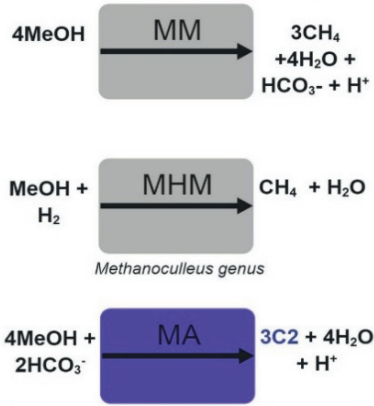

Eubacterium limosum strain JCM 6421 (100 coverage, 95\% similarity

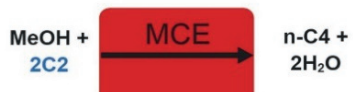

Eubacterium limosum strain JCM 6421 (100 coverage, 95\% similarity

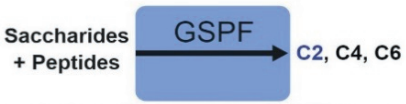

Sporanaerobacter acetigenes strain Lup33 (100\% coverage, 99\% similarity Caproiciproducens galactitolivorans ( $92 \%$ coverage, $97 \%$ similarity)

Figure S7. The figure depicts a collection of different possible functional groups within the microbiome: $\mathrm{BCE}=$ branched chain elongation, $\mathrm{SCE}=$ straight chain elongation, $\mathrm{EO}=$ ethanol oxidation, $\mathrm{BO}=$ beta-oxidation, $\mathrm{HM}=$ hydrogenotrophic methanogenesis, $\mathrm{HA}=$ hydrogenotrophic acetogenesis, $\mathrm{HCAR}=$ hydrogenotrophic carboxylate-to-alcohol reduction, ECAR $=$ Ethanolotrophic carboxylate-to-alcohol reduction, $\mathrm{EOBC}=$ Ethanol oxidation with bicarbonate, $\mathrm{ACM}=$ Acetoclastic methanogenesis, ELM = ethanolotrophic methanogenesis, $\mathrm{MM}=$ methanolotrophic methanogenesis, MHM = methanol-hydrogen methanogenesis, MA = methanolotrophic acetogenesis, $\mathrm{MCE}=$ methanol based chain elongation and GSPF $=$ Generalized saccharide and peptide fermentation. The color of the reducing equivalent, expressed as $\mathrm{H} 2$, indicates its relative thermodynamic feasibility depending on the hydrogen partial pressure. Green indicates that the process can occur in a wide range of hydrogen partial pressures, while orange and red signifiy that a lower (in the case of reduction routes) or higher (in the case of oxidation routes) partial pressure is needed for an energetically feasible bioconversion (see Table S1 for numbers). 
Table S5. OTU counts within the genus Clostridium sensu stricto 12 (Clostridiales order). The $16 \mathrm{~S}$ rRNA gene amplicon sequences of the in grey highlighted OTU identifyers were selected to search the NCBI database using Megablast.

\begin{tabular}{|c|c|c|c|c|c|c|c|c|c|c|}
\hline \multirow[b]{3}{*}{ OTU identifyer } & \multicolumn{10}{|c|}{ OTU counts } \\
\hline & \multicolumn{2}{|l|}{ phase I } & \multicolumn{2}{|c|}{ phase II } & \multicolumn{2}{|c|}{ Phase IV } & \multicolumn{2}{|c|}{ Phase VI } & \multicolumn{2}{|c|}{$\begin{array}{l}\text { Phase } \\
\text { granules }\end{array}$} \\
\hline & $\mathbf{a}$ & b & $\mathbf{a}$ & b & $\mathbf{a}$ & b & $\mathbf{a}$ & b & $\mathbf{a}$ & $\mathbf{b}$ \\
\hline EU307088.1.1475 & 74402 & 73085 & 29896 & 64364 & 65863 & 64755 & 42913 & 19834 & 21710 & 23294 \\
\hline JQ072606.1.1361 & 267 & 178 & 69 & 115 & 136 & 135 & 119 & 52 & 99 & 115 \\
\hline JN650257.1.1438 & 258 & 590 & 111 & 249 & 507 & 467 & 203 & 140 & 128 & 120 \\
\hline New.ReferenceOTU460 & 107 & 108 & 7 & 8 & 37 & 29 & 21 & 5 & 10 & 17 \\
\hline AB509213.1.1431 & 81 & 101 & 709 & 726 & 42 & 54 & 14263 & 9275 & 345 & 316 \\
\hline New.ReferenceOTU538 & 52 & 53 & 15 & 34 & 8 & 14 & 154 & 132 & 12 & 31 \\
\hline JQ072575.1.1354 & 51 & 143 & 10 & 33 & 81 & 119 & 39 & 21 & 33 & 18 \\
\hline JQ072154.1.1365 & 46 & 88 & 14 & 23 & 69 & 101 & 36 & 16 & 26 & 25 \\
\hline $\begin{array}{l}\text { New.CleanUp.ReferenceOT } \\
\text { U36918 }\end{array}$ & 19 & 24 & 22 & 40 & 117 & 102 & 38 & 30 & 45 & 69 \\
\hline GQ487959.1.1446 & 17 & 5 & 0 & 2 & 18 & 35 & 6 & 2 & 78 & 70 \\
\hline New.ReferenceOTU691 & 13 & 13 & 6 & 4 & 31 & 36 & 14 & 5 & 11 & 11 \\
\hline CBXI010000035.40.1552 & 12 & 6 & 0 & 2 & 7 & 5 & 601 & 346 & 3 & 8 \\
\hline Cropped... & $\cdots$ & $\cdots$ & $\cdots$ & $\cdots$ & $\cdots$ & $\cdots$ & $\cdots$ & $\cdots$ & $\cdots$ & $\cdots$ \\
\hline SUM & 75416 & 74651 & 30932 & 65736 & 67262 & 66161 & 58938 & 30277 & 22734 & 24358 \\
\hline Total OTU count in sample & $\begin{array}{l}16560 \\
6\end{array}$ & $\begin{array}{l}16288 \\
5\end{array}$ & 64675 & $\begin{array}{l}11594 \\
3\end{array}$ & $\begin{array}{l}11659 \\
9\end{array}$ & $\begin{array}{l}11149 \\
5\end{array}$ & $\begin{array}{l}15825 \\
8\end{array}$ & 94834 & $\begin{array}{l}10278 \\
8\end{array}$ & $\begin{array}{l}11858 \\
3\end{array}$ \\
\hline $\begin{array}{l}\text { Relative abundance of } \\
\text { genus }\end{array}$ & $45.5 \%$ & $45.8 \%$ & $47.8 \%$ & $56.7 \%$ & $57.7 \%$ & $59.3 \%$ & $37.2 \%$ & $31.9 \%$ & $22.1 \%$ & $20.5 \%$ \\
\hline
\end{tabular}

Table S6. Results of searching the ncbi $16 \mathrm{~S}$ rRNA gene amplicon sequence (Bacteriea and Archaea) database using Megablast (executed in september 2018) with the selected Clostridium sensu stricto 12 OTUs.

\begin{tabular}{|c|c|c|c|c|c|c|c|}
\hline $\begin{array}{l}\text { OTU } \\
\text { identifyer }\end{array}$ & Description & $\begin{array}{l}\text { Max } \\
\text { score }\end{array}$ & $\begin{array}{l}\text { Total } \\
\text { score }\end{array}$ & $\begin{array}{l}\text { Query } \\
\text { cover }\end{array}$ & $\begin{array}{l}\text { E } \\
\text { valu } \\
\text { e } \\
\end{array}$ & $\begin{array}{l}\text { Iden } \\
\mathrm{t}\end{array}$ & Accession \\
\hline \multirow[t]{3}{*}{$\begin{array}{l}\text { EU307088.1. } \\
1475\end{array}$} & $\begin{array}{l}\text { Clostridium kluyveri strain } \\
\text { NBRC } 1201616 \text { S ribosomal } \\
\text { RNA, partial sequence }\end{array}$ & 2553 & 2553 & $100 \%$ & 0 & $98 \%$ & NR_074447.1 \\
\hline & $\begin{array}{l}\text { Clostridium kluyveri strain } \\
\text { DSM } 55516 \mathrm{~S} \text { ribosomal } \\
\text { RNA, partial sequence }\end{array}$ & 2519 & 2519 & $98 \%$ & 0 & $98 \%$ & NR_074165.1 \\
\hline & \begin{tabular}{lrr}
\multicolumn{2}{l}{ Clostridium } & \multicolumn{2}{r}{ luticellarii } \\
strain & FW431 & $16 \mathrm{~S}$ \\
ribosomal & RNA, & partial \\
sequence & &
\end{tabular} & 2252 & 2252 & $99 \%$ & 0 & $94 \%$ & NR_145907.1 \\
\hline
\end{tabular}




\begin{tabular}{|c|c|c|c|c|c|c|c|}
\hline $\begin{array}{l}\text { AB509213.1. } \\
1431\end{array}$ & \begin{tabular}{lrr}
\multicolumn{2}{l}{ Clostridium } & \multicolumn{2}{c}{ luticellarii } \\
strain & FW431 & $16 \mathrm{~S}$ \\
ribosomal & RNA, & partial \\
sequence & &
\end{tabular} & 2623 & 2623 & $100 \%$ & 0 & $99 \%$ & NR_145907.1 \\
\hline \multirow[t]{2}{*}{$\begin{array}{l}\text { JQ072606.1. } \\
1361\end{array}$} & $\begin{array}{l}\text { Clostridium kluyveri strain } \\
\text { DSM } 55516 \mathrm{~S} \text { ribosomal } \\
\text { RNA, partial sequence }\end{array}$ & 2396 & 2396 & $99 \%$ & 0 & $98 \%$ & NR_074165.1 \\
\hline & $\begin{array}{l}\text { Clostridium kluyveri strain } \\
\text { NBRC } 1201616 \text { S ribosomal } \\
\text { RNA, partial sequence }\end{array}$ & 2396 & 2396 & $99 \%$ & 0 & $98 \%$ & NR_074447.1 \\
\hline \multirow[t]{2}{*}{$\begin{array}{l}\text { JN650257.1. } \\
1438\end{array}$} & $\begin{array}{l}\text { Clostridium kluyveri strain } \\
\text { DSM } 55516 \mathrm{~S} \text { ribosomal } \\
\text { RNA, partial sequence }\end{array}$ & 1973 & 1973 & $99 \%$ & 0 & $92 \%$ & NR_074165.1 \\
\hline & $\begin{array}{l}\text { Clostridium kluyveri strain } \\
\text { NBRC } 1201616 \mathrm{~S} \text { ribosomal } \\
\text { RNA, partial sequence }\end{array}$ & 1973 & 1973 & $99 \%$ & 0 & $92 \%$ & NR_074447.1 \\
\hline $\begin{array}{l}\text { CBXI010000 } \\
035.40 .1552\end{array}$ & $\begin{array}{l}\text { Clostridium tyrobutyricum } \\
\text { strain ATCC } 2575516 \mathrm{~S} \\
\text { ribosomal RNA, partial } \\
\text { sequence }\end{array}$ & 2632 & 2632 & $97 \%$ & 0 & $99 \%$ & NR_044718.2 \\
\hline
\end{tabular}

Table S7. OTU counts within the family Syntrophomonadacaea (Clostridiales order). The 16S rRNA gene amplicon sequences of the in grey highlighted OTU identifyers were selected to search the NCBI database using Megablast.

\begin{tabular}{|c|c|c|c|c|c|c|c|c|c|c|}
\hline \multirow[b]{3}{*}{ OTU identifyer } & \multicolumn{10}{|c|}{ OTU counts } \\
\hline & \multicolumn{2}{|l|}{ phase I } & \multicolumn{2}{|c|}{ phase II } & \multicolumn{2}{|l|}{ Phase IV } & \multicolumn{2}{|c|}{ Phase VI } & \multicolumn{2}{|c|}{ Phase VI, granules } \\
\hline & $\mathbf{a}$ & b & $\mathbf{a}$ & b & $\mathbf{a}$ & b & $\mathbf{a}$ & $\mathbf{b}$ & $\mathbf{a}$ & b \\
\hline New.ReferenceOTU249 & 48905 & 26991 & 1258 & 1853 & 1762 & 1873 & 6660 & 3632 & 2568 & 3063 \\
\hline New.ReferenceOTU53 & 1142 & 476 & 34 & 51 & 77 & 62 & 98 & 49 & 76 & 90 \\
\hline JX575888.1.1355 & 426 & 95 & 4 & 10 & 4 & 5 & 24 & 13 & 23 & 30 \\
\hline New.ReferenceOTU593 & 319 & 166 & 11 & 12 & 33 & 24 & 24 & 17 & 15 & 22 \\
\hline HQ904231.1.1514 & 301 & 186 & 8 & 9 & 25 & 13 & 115 & 55 & 8 & 5 \\
\hline Cropped... & $\cdots$ & $\ldots$ & $\cdots$ & $\ldots$ & $\cdots$ & $\cdots$ & $\cdots$ & $\ldots$ & $\cdots$ & $\cdots$ \\
\hline SUM & 51613 & 28362 & 1329 & 1968 & 1936 & 2029 & 7083 & 3922 & 2819 & 3402 \\
\hline Total OTU count in sample & 165606 & 162885 & 64675 & 115943 & 116599 & 111495 & 158258 & 94834 & 102788 & 118583 \\
\hline Relative abundance of genus & $31.17 \%$ & $17.41 \%$ & $2.05 \%$ & $1.70 \%$ & $1.66 \%$ & $1.82 \%$ & $4.48 \%$ & $4.14 \%$ & $2.74 \%$ & $2.87 \%$ \\
\hline
\end{tabular}


Table S8. Results of searching the ncbi 16S rRNA gene amplicon sequence (Bacteriea and Archaea) database using Megablast (executed in september 2018) with the selected Syntrophomonadacaea OTUs.

\begin{tabular}{|c|c|c|c|c|c|c|c|}
\hline OTU identifyer & Description & $\begin{array}{l}\text { Max } \\
\text { score }\end{array}$ & $\begin{array}{l}\text { Total } \\
\text { score }\end{array}$ & $\begin{array}{l}\text { Query } \\
\text { cover }\end{array}$ & E value & Ident & Accession \\
\hline New.ReferenceOTU249 & \begin{tabular}{lr}
\multicolumn{2}{l}{ Syntrophomonas } \\
zehnderi & strain \\
OL-4 & $16 S$ \\
ribosomal & RNA \\
gene, & partial \\
sequence & \\
\end{tabular} & 684 & 684 & $100 \%$ & 0 & $96 \%$ & NR_044008.1 \\
\hline \multirow[t]{3}{*}{ New.ReferenceOTU53 } & 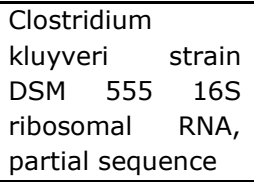 & 527 & 527 & $100 \%$ & $2 \mathrm{E}-149$ & $90 \%$ & NR_074165.1 \\
\hline & $\begin{array}{l}\text { Clostridium } \\
\text { kluyveri strain } \\
\text { NBRC } 1201616 S \\
\text { ribosomal RNA, } \\
\text { partial sequence }\end{array}$ & 527 & 527 & $100 \%$ & $2 E-149$ & $90 \%$ & NR_074447.1 \\
\hline & \begin{tabular}{lr}
\multicolumn{2}{l}{ Syntrophomonas } \\
zehnderi & strain \\
OL-4 & $16 S$ \\
ribosomal & RNA \\
gene, & partial \\
sequence &
\end{tabular} & 507 & 507 & $76 \%$ & $2 \mathrm{E}-143$ & $96 \%$ & NR_044008.1 \\
\hline
\end{tabular}

Table 59. OTU counts within the genus Eubacterium (Clostridiales order). The 16S rRNA gene amplicon sequence of the in grey highlighted OTU identifyer was selected to search the NCBI database using Megablast.

\begin{tabular}{|c|c|c|c|c|c|c|c|c|c|c|}
\hline \multirow[b]{2}{*}{ OTU identifyer } & \multicolumn{10}{|c|}{ OTU counts } \\
\hline & $\mathbf{a}$ & b & $\mathbf{a}$ & b & $\mathbf{a}$ & b & $\mathbf{a}$ & b & $\mathbf{a}$ & b \\
\hline DQ804417.1.1386 & 689 & 2094 & 4685 & 7831 & 9052 & 8575 & 4472 & 2576 & 782 & 700 \\
\hline EU939391.1.1539 & 9 & 34 & 63 & 117 & 172 & 198 & 43 & 15 & 19 & 15 \\
\hline JN698651.1.1409 & 5 & 1 & 1 & 2 & 3 & 5 & 5 & 2 & 3 & 0 \\
\hline Cropped... & $\ldots$ & $\ldots$ & $\cdots$ & $\cdots$ & $\ldots$ & $\ldots$ & $\ldots$ & $\ldots$ & $\ldots$ & $\cdots$ \\
\hline SUM & 712 & 2155 & 4847 & 8190 & 9525 & 9145 & 4649 & 2669 & 819 & 743 \\
\hline Total OTU count in sample & 165606 & 162885 & 64675 & 115943 & 116599 & 111495 & 158258 & 94834 & 102788 & 118583 \\
\hline Relative abundance of genus & $0.43 \%$ & $1.32 \%$ & $7.49 \%$ & $7.06 \%$ & $8.17 \%$ & $8.20 \%$ & $2.94 \%$ & $2.81 \%$ & $0.80 \%$ & $0.63 \%$ \\
\hline
\end{tabular}


Table S10. Results of querying the ncbi 16S rRNA gene amplicon sequence (Bacteriea and Archaea) database using Megablast (executed in september 2018) with the selected Eubacterium OTU.

\begin{tabular}{|c|c|c|c|c|c|c|c|}
\hline OTU identifyer & Description & $\begin{array}{l}\text { Max } \\
\text { score }\end{array}$ & $\begin{array}{l}\text { Total } \\
\text { score }\end{array}$ & $\begin{array}{l}\text { Query } \\
\text { cover }\end{array}$ & $\begin{array}{l}\text { E } \\
\text { value }\end{array}$ & Ident & Accession \\
\hline \multirow[t]{3}{*}{ DQ804417.1.1386 } & $\begin{array}{l}\text { Eubacterium } \\
\text { limosum strain JCM } \\
642116 \mathrm{~S} \text { ribosomal } \\
\text { RNA gene, partial } \\
\text { sequence }\end{array}$ & 2150 & 2150 & $100 \%$ & 0 & $95 \%$ & NR_113248.1 \\
\hline & $\begin{array}{l}\text { Eubacterium } \\
\text { callanderi strain DSM } \\
366216 \mathrm{~S} \text { ribosomal } \\
\text { RNA gene, partial } \\
\text { sequence }\end{array}$ & 2137 & 2137 & $98 \%$ & 0 & $95 \%$ & NR_026330.1 \\
\hline & $\begin{array}{l}\text { Eubacterium } \\
\text { limosum strain ATCC } \\
848616 \mathrm{~S} \text { ribosomal } \\
\text { RNA, partial } \\
\text { sequence }\end{array}$ & 2132 & 2132 & $100 \%$ & 0 & $94 \%$ & NR_044719.2 \\
\hline
\end{tabular}

Table S11. OTU counts within the family Family XI (Clostridiales order). The 16S rRNA gene amplicon sequences of the in grey highlighted OTU identifyers were selected to search the NCBI database using Megablast.

\begin{tabular}{|c|c|c|c|c|c|c|c|c|c|c|}
\hline \multirow[b]{3}{*}{ OTU identifyer } & \multicolumn{10}{|c|}{ OTU counts } \\
\hline & \multicolumn{2}{|l|}{ phase I } & \multicolumn{2}{|c|}{ phase II } & \multicolumn{2}{|l|}{ Phase IV } & \multicolumn{2}{|l|}{ Phase VI } & \multicolumn{2}{|c|}{ Phase VI, granules } \\
\hline & $\mathbf{a}$ & b & $\mathbf{a}$ & b & $\mathbf{a}$ & b & $\mathbf{a}$ & b & $\mathbf{a}$ & b \\
\hline EU887812.1.1471 & 4520 & 3796 & 1052 & 2218 & 551 & 517 & 14703 & 9021 & 2977 & 3284 \\
\hline$A B 742052.1 .1452$ & 616 & 488 & 1785 & 2306 & 4497 & 3894 & 6888 & 4230 & 3394 & 3967 \\
\hline JX575815.1.1501 & 710 & 1055 & 410 & 540 & 24 & 32 & 696 & 530 & 517 & 572 \\
\hline EU888001.1.1399 & 56 & 48 & 21 & 67 & 55 & 41 & 186 & 116 & 190 & 214 \\
\hline New.ReferenceOTU265 & 57 & 47 & 19 & 28 & 15 & 12 & 165 & 119 & 65 & 82 \\
\hline New.ReferenceOTU132 & 18 & 10 & 57 & 104 & 226 & 237 & 163 & 97 & 110 & 151 \\
\hline Cropped... & $\ldots$ & $\ldots$ & $\ldots$ & $\ldots$ & $\ldots$ & $\ldots$ & $\cdots$ & $\cdots$ & $\cdots$ & $\ldots$ \\
\hline SUM & 6302 & 5721 & 3500 & 5549 & 5493 & 4910 & 23817 & 14979 & 7830 & 9060 \\
\hline Total OTU count in sample & 165606 & 162885 & 64675 & 115943 & 116599 & 111495 & 158258 & 94834 & 102788 & 118583 \\
\hline Relative abundance of genus & $3.81 \%$ & $3.51 \%$ & $5.41 \%$ & $4.79 \%$ & $4.71 \%$ & $4.40 \%$ & $15.05 \%$ & $15.79 \%$ & $7.62 \%$ & $7.64 \%$ \\
\hline
\end{tabular}


Table S12. Results of querying the NCBI $16 \mathrm{~S}$ rRNA gene amplicon sequence database (Bacteriea and Archaea) with Megablast (executed in september 2018) with the selected Familiy XI OTUs.

\begin{tabular}{llllllll}
\hline OTU Identifyer & Description & $\begin{array}{l}\text { Max } \\
\text { score }\end{array}$ & $\begin{array}{l}\text { Total } \\
\text { score }\end{array}$ & $\begin{array}{l}\text { Query } \\
\text { cover }\end{array}$ & $\begin{array}{l}\mathrm{E} \\
\text { value }\end{array}$ & Ident & Accession \\
\hline \hline \multirow{2}{*}{ EU887812.1.1471 } & $\begin{array}{l}\text { Sporanaerobacter } \\
\text { acetigenes strain } \\
\text { Lup33 16S ribosomal } \\
\text { RNA, partial sequence }\end{array}$ & 2623 & 2623 & $100 \%$ & 0 & $99 \%$ & NR_025151.1 \\
\hline AB742052.1.1452 & $\begin{array}{l}\text { Sporanaerobacter } \\
\text { acetigenes strain } \\
\text { Lup33 16S ribosomal } \\
\text { RNA, partial sequence }\end{array}$ & 1851 & 1851 & $100 \%$ & 0 & $90 \%$ & NR_025151.1 \\
\hline \hline
\end{tabular}

Table S11. OTU counts within the genus Oscilibacter (Clostridiales order). The 16S rRNA gene amplicon sequences of the in grey highlighted OTU identifyers were selected to search the NCBI database using Megablast.

\begin{tabular}{|c|c|c|c|c|c|c|c|c|c|c|}
\hline \multirow[b]{3}{*}{ OTU identifyer } & \multicolumn{10}{|c|}{ OTU counts } \\
\hline & \multicolumn{2}{|l|}{ phase I } & \multicolumn{2}{|c|}{ phase II } & \multicolumn{2}{|l|}{ Phase IV } & \multicolumn{2}{|c|}{ Phase VI } & \multicolumn{2}{|c|}{ Phase VI, granules } \\
\hline & $\mathbf{a}$ & b & $\mathbf{a}$ & b & $\mathbf{a}$ & b & $\mathbf{a}$ & $\mathbf{b}$ & $\mathbf{a}$ & b \\
\hline HK556666.9.1518 & 8381 & 20994 & 144 & 254 & 1216 & 1093 & 263 & 154 & 58 & 64 \\
\hline New.ReferenceOTU529 & 157 & 262 & 3 & 17 & 28 & 37 & 34 & 14 & 7 & 6 \\
\hline New.CleanUp.ReferenceOTU29092 & 117 & 531 & 3 & 1 & 33 & 35 & 17 & 13 & 2 & 0 \\
\hline EU775103.1.1401 & 72 & 334 & 1 & 3 & 11 & 20 & 7 & 6 & 1 & 1 \\
\hline JQ246090.1.1431 & 46 & 116 & 71 & 267 & 46 & 36 & 993 & 633 & 136 & 133 \\
\hline New.CleanUp.ReferenceOTU127988 & 43 & 63 & 0 & 0 & 0 & 0 & 1 & 3 & 0 & 0 \\
\hline Cropped... & $\ldots$ & $\ldots$ & $\ldots$ & $\ldots$ & $\ldots$ & $\ldots$ & $\ldots$ & $\ldots$ & $\ldots$ & $\ldots$ \\
\hline SUM & 8908 & 22438 & 268 & 654 & 1414 & 1296 & 3835 & 2567 & 305 & 343 \\
\hline Total OTU count in sample & 165606 & 162885 & 64675 & 115943 & 116599 & 111495 & 158258 & 94834 & 102788 & 118583 \\
\hline Relative abundance of genus & $5.38 \%$ & $13.78 \%$ & $0.41 \%$ & $0.56 \%$ & $1.21 \%$ & $1.16 \%$ & $2.42 \%$ & $2.71 \%$ & $0.30 \%$ & $0.29 \%$ \\
\hline
\end{tabular}

Table S12. Results of searching the ncbi 16S rRNA gene amplicon sequence (Bacteriea and Archaea) database using Megablast (executed in september 2018) with the selected Oscilibacter OTUs.

\begin{tabular}{|c|c|c|c|c|c|c|c|}
\hline OTU Identifyer & Description & $\begin{array}{l}\text { Max } \\
\text { score }\end{array}$ & $\begin{array}{l}\text { Total } \\
\text { score }\end{array}$ & $\begin{array}{l}\text { Query } \\
\text { cover }\end{array}$ & $\begin{array}{l}\mathrm{E} \\
\text { value }\end{array}$ & Ident & Accession \\
\hline HK556666.9.1518 & \begin{tabular}{ll}
\multicolumn{2}{l}{ Oscillibacter } \\
valericigenes \\
strain Sjm18-20 \\
$16 S \quad$ ribosomal \\
RNA, complete \\
sequence
\end{tabular} & 2789 & 2789 & $100 \%$ & 0 & $100 \%$ & NR_074793.2 \\
\hline JQ246090.1.1431 & $\begin{array}{l}\text { Oscillibacter } \\
\text { ruminantium strain } \\
\text { GH1 } 16 \text { S ribosomal } \\
\text { RNA gene, partial } \\
\text { sequence }\end{array}$ & 2573 & 2573 & $98 \%$ & 0 & $99 \%$ & NR_118156.1 \\
\hline
\end{tabular}


Oscillibacter

ruminantium strain

GQ243726.1.1415 GH1 16 S ribosomal $2449 \quad 2449 \quad 96 \% \quad 0 \quad 99 \% \quad$ NR_118156.1

RNA gene, partial

sequence

Table S13. OTU counts within the genus Caproiciproducens (Clostridiales order). The 16S rRNA gene amplicon sequences of the in grey highlighted OTU identifyers were selected to search the NCBI database using Megablast.

\begin{tabular}{|c|c|c|c|c|c|c|c|c|c|c|}
\hline \multirow[b]{2}{*}{ OTU identifyer } & \multicolumn{9}{|c|}{ OTU counts } & ranules \\
\hline & $\mathbf{a}$ & b & $\mathbf{a}$ & $\mathbf{b}$ & $\mathbf{a}$ & b & $\mathbf{a}$ & b & $\mathbf{a}$ & b \\
\hline CCNL01000002.384.1925 & 306 & 458 & 1243 & 1890 & 2369 & 2215 & 2089 & 1372 & 1790 & 1920 \\
\hline New.ReferenceOTU280 & 47 & 88 & 13 & 21 & 659 & 588 & 1006 & 639 & 608 & 594 \\
\hline KT337628.1.1480 & 12 & 30 & 1 & 1 & 1 & 0 & 24 & 18 & 12 & 13 \\
\hline New.CleanUp.ReferenceOTU53385 & 10 & 4 & 16 & 22 & 26 & 35 & 11 & 18 & 22 & 21 \\
\hline Cropped... & $\ldots$ & $\ldots$ & $\ldots$ & $\ldots$ & $\cdots$ & $\ldots$ & $\ldots$ & $\ldots$ & $\ldots$ & $\cdots$ \\
\hline SUM & 391 & 603 & 1287 & 1966 & 3114 & 2917 & 3849 & 2628 & 2612 & 2765 \\
\hline Total OTU count in sample & 165606 & 162885 & 64675 & 115943 & 116599 & 111495 & 158258 & 94834 & 102788 & 118583 \\
\hline Relative abundance of genus & $0.24 \%$ & $0.37 \%$ & $1.99 \%$ & $1.70 \%$ & $2.67 \%$ & $2.62 \%$ & $2.43 \%$ & $2.77 \%$ & $2.54 \%$ & $2.33 \%$ \\
\hline
\end{tabular}

Table S14. Results of searching the ncbi 16S rRNA gene amplicon sequence (Bacteriea and Archaea) database using Megablast (executed in september 2018) with the selected Caproiciproducens OTUs.

$\begin{array}{llllll} & & \text { Max } & \text { Total } & \text { Query } & \text { E } \\ \text { OTU Identifyer } & \text { Description } & \text { score } & \text { score } & \text { cover } & \text { value }\end{array}$

\begin{tabular}{|c|c|c|c|c|c|c|c|}
\hline $\begin{array}{l}\text { CCNL0100000 } \\
2.384 .1925\end{array}$ & $\begin{array}{l}\text { Caproiciproducens } \\
\text { galactitolivorans strain BS- } \\
1 \quad 16 \mathrm{~S} \text { ribosomal RNA, } \\
\text { partial sequence }\end{array}$ & 2353 & 2439 & $92 \%$ & 0 & $97 \%$ & $\begin{array}{l}\text { NR_1459 } \\
29.1\end{array}$ \\
\hline $\begin{array}{l}\text { New. Referenc } \\
\text { eOTU280 }\end{array}$ & $\begin{array}{l}\text { Caproiciproducens } \\
\text { galactitolivorans strain BS- } \\
1 \quad 16 \mathrm{~S} \text { ribosomal RNA, } \\
\text { partial sequence }\end{array}$ & 597 & 597 & $100 \%$ & $\begin{array}{l}1 \mathrm{E}- \\
170\end{array}$ & $93 \%$ & $\begin{array}{l}\text { NR_1459 } \\
29.1\end{array}$ \\
\hline
\end{tabular}


Table S15. OTU counts within the genus Christensenellaceaea R-7 group (Clostridiales order). The 16S rRNA gene amplicon sequences of the in grey highlighted OTU identifyers were selected to search the NCBI database using Megablast.

\begin{tabular}{|c|c|c|c|c|c|c|c|c|c|c|}
\hline \multirow[b]{3}{*}{ OTU identifyer } & \multicolumn{10}{|c|}{ OTU counts } \\
\hline & \multicolumn{2}{|l|}{ phase I } & \multicolumn{2}{|c|}{ phase II } & \multicolumn{2}{|l|}{ Phase IV } & \multicolumn{2}{|l|}{ Phase VI } & \multicolumn{2}{|c|}{ Phase VI, granules } \\
\hline & a & b & $\mathbf{a}$ & b & $\mathbf{a}$ & b & a & b & $\mathbf{a}$ & b \\
\hline New.ReferenceOTU382 & 7 & 1 & 11 & 6 & 2 & 5 & 3353 & 1809 & 4086 & 3451 \\
\hline GQ132397.1.1400 & 17 & 37 & 14 & 18 & 1 & 3 & 100 & 77 & 185 & 94 \\
\hline New.CleanUp.ReferenceOTU124159 & 0 & 1 & 0 & 1 & 0 & 0 & 37 & 26 & 69 & 99 \\
\hline Cropped... & $\ldots$ & $\ldots$ & $\ldots$ & $\ldots$ & $\ldots$ & $\ldots$ & $\ldots$ & $\ldots$ & $\ldots$ & $\ldots$ \\
\hline SUM & 30 & 62 & 31 & 37 & 5 & 8 & 3592 & 1997 & 4540 & 3852 \\
\hline Total OTU count in sample & 165606 & 162885 & 64675 & 115943 & 116599 & 111495 & 158258 & 94834 & 102788 & 118583 \\
\hline Relative abundance of genus & $0.02 \%$ & $0.04 \%$ & $0.05 \%$ & $0.03 \%$ & $0.00 \%$ & $0.01 \%$ & $2.27 \%$ & $2.11 \%$ & $4.42 \%$ & $3.25 \%$ \\
\hline
\end{tabular}

Table S16. Results of searching the ncbi $16 \mathrm{~S}$ rRNA gene amplicon sequence (Bacteriea and Archaea) database using Megablast (executed in september 2018) with the selected Christensenellaceae R-7 group OTUs.

\begin{tabular}{|c|c|c|c|c|c|c|c|}
\hline OTU Identifyer & Description & $\begin{array}{l}\text { Max } \\
\text { scor } \\
\text { e }\end{array}$ & $\begin{array}{l}\text { Total } \\
\text { scor } \\
\text { e }\end{array}$ & $\begin{array}{l}\text { Quer } \\
\text { y } \\
\text { cover }\end{array}$ & $\begin{array}{l}\mathrm{E} \\
\text { value }\end{array}$ & $\begin{array}{l}\text { Iden } \\
\mathrm{t}\end{array}$ & Accession \\
\hline $\begin{array}{l}\text { New.Referenc } \\
\text { eOTU382 }\end{array}$ & $\begin{array}{l}\text { Christensenella } \\
\text { massiliensis strain } \\
\text { Marseille-P2438 } 165 \\
\text { ribosomal RNA, partial } \\
\text { sequence }\end{array}$ & 508 & 508 & $\begin{array}{l}100 \\
\%\end{array}$ & $\begin{array}{l}7 \mathrm{E}- \\
144\end{array}$ & $89 \%$ & $\begin{array}{l}\text { NR_144742. } \\
1\end{array}$ \\
\hline \multirow[t]{3}{*}{$\begin{array}{l}\text { GQ132397.1.1 } \\
400\end{array}$} & $\begin{array}{l}\text { Christensenella } \\
\text { massiliensis strain } \\
\text { Marseille-P2438 } 16 \mathrm{~S} \\
\text { ribosomal RNA, partial } \\
\text { sequence }\end{array}$ & 1677 & 1677 & $\begin{array}{l}100 \\
\%\end{array}$ & 0 & $88 \%$ & $\begin{array}{l}\text { NR_144742. } \\
1\end{array}$ \\
\hline & $\begin{array}{l}\text { Christensenella } \\
\text { timonensis } \\
\text { Marseille-P2437 } 165 \\
\text { ribosomal RNA, partial } \\
\text { sequence }\end{array}$ & 1615 & 1615 & $\begin{array}{l}100 \\
\%\end{array}$ & 0 & $88 \%$ & $\begin{array}{l}\text { NR_144743 } \\
1\end{array}$ \\
\hline & $\begin{array}{l}\text { Christensenella minuta } \\
\text { strain YIT } 12065165 \\
\text { ribosomal RNA gene, } \\
\text { partial sequence }\end{array}$ & 1615 & 1615 & $99 \%$ & 0 & $88 \%$ & $\begin{array}{l}\text { NR_112900 } \\
1\end{array}$ \\
\hline
\end{tabular}


Table S17. OTU counts within the genus Anaerolineaceae UCG-001 (Anaerolineales order). The $16 \mathrm{~S}$ rRNA gene amplicon sequence of the in grey highlighted OTU identifyer was selected to search the NCBI database using Megablast.

\begin{tabular}{|c|c|c|c|c|c|c|c|c|c|c|}
\hline \multirow[b]{3}{*}{ OTU identifyer } & \multicolumn{10}{|c|}{ OTU counts } \\
\hline & \multicolumn{2}{|l|}{ phase I } & \multicolumn{2}{|c|}{ phase II } & \multicolumn{2}{|l|}{ Phase IV } & \multicolumn{2}{|l|}{ Phase VI } & \multicolumn{2}{|c|}{ Phase VI, granules } \\
\hline & $\mathbf{a}$ & b & $\mathbf{a}$ & b & $\mathbf{a}$ & b & $\mathbf{a}$ & b & $\mathbf{a}$ & b \\
\hline EF688175.1.1346 & 5 & 4 & 26 & 21 & 103 & 129 & 709 & 510 & 10158 & 12092 \\
\hline New.CleanUp.ReferenceOTU25650 & 0 & 0 & 0 & 0 & 0 & 0 & 0 & 1 & 9 & 21 \\
\hline Cropped... & $\cdots$ & $\cdots$ & $\cdots$ & $\cdots$ & $\cdots$ & $\cdots$ & $\cdots$ & $\cdots$ & $\cdots$ & $\cdots$ \\
\hline SUM & 5 & 4 & 26 & 21 & 103 & 132 & 715 & 521 & 10375 & 12343 \\
\hline Total OTU count in sample & 165606 & 162885 & 64675 & 115943 & 116599 & 111495 & 158258 & 94834 & 102788 & 118583 \\
\hline Relative abundance of genus & $0.00 \%$ & $0.00 \%$ & $0.04 \%$ & $0.02 \%$ & $0.09 \%$ & $0.12 \%$ & $0.45 \%$ & $0.55 \%$ & $10.09 \%$ & $10.41 \%$ \\
\hline
\end{tabular}

Table S18. Results of searching the ncbi $16 \mathrm{~S}$ rRNA gene amplicon sequence (Bacteriea and Archaea) database using Megablast (executed in september 2018) with the selected Anaerolineaceae UCG001 OTU.

\begin{tabular}{lllll}
\hline & & Max & Total & Query \\
OTU Identifyer & Description & score & score & cover value Ident Accession
\end{tabular}

\begin{tabular}{|c|c|c|c|c|c|c|c|}
\hline EF688175.1.1346 & $\begin{array}{lr}\text { Bellilinea } & \\
\text { caldifistulae } & \text { strain } \\
\text { GOMI-1 } & 16 S \\
\text { ribosomal } & \text { RNA } \\
\text { gene, } & \text { partial } \\
\text { sequence } & \end{array}$ & 1810 & 1810 & $99 \%$ & 0 & $91 \%$ & NR_041354.1 \\
\hline & $\begin{array}{lr}\text { Ornatilinea apprima } \\
\text { strain P3M-1 } \\
\text { ribosomal } \\
\text { gene, } \\
\text { sequence }\end{array}$ & 1753 & 1753 & $97 \%$ & 0 & $91 \%$ & NR_109544.1 \\
\hline & $\begin{array}{lr}\text { Longilinea } & \\
\text { arvoryzae } & \text { strain } \\
\text { KOME-1 } & 16 S \\
\text { ribosomal } & \text { RNA } \\
\text { gene, } & \text { partial } \\
\text { sequence } & \end{array}$ & 1749 & 1749 & $99 \%$ & 0 & $90 \%$ & NR_041355.1 \\
\hline & $\begin{array}{lr}\text { Levilinea } & \\
\text { saccharolytica strain } \\
\text { KIBI-1 } & 16 S \\
\text { ribosomal } & \text { RNA } \\
\text { gene, } & \text { partial } \\
\text { sequence } & \end{array}$ & 1749 & 1749 & $97 \%$ & 0 & $91 \%$ & NR_040972.1 \\
\hline & $\begin{array}{l}\text { Pelolinea submarina } \\
\text { strain MO-CFX1 } 16 S \\
\text { ribosomal RNA, } \\
\text { partial sequence }\end{array}$ & 1746 & 1746 & $97 \%$ & 0 & $91 \%$ & NR_133813.1 \\
\hline
\end{tabular}


Table S19. OTU counts within the genus Desulfovibrio (Desulfovibrionales order). The 16S rRNA gene amplicon sequence of the in grey highlighted OTU identifyer was selected to search the NCBI database using Megablast.

\begin{tabular}{|c|c|c|c|c|c|c|c|c|c|c|}
\hline \multirow[b]{3}{*}{ OTU identifyer } & \multicolumn{10}{|c|}{ OTU counts } \\
\hline & \multicolumn{2}{|l|}{ phase I } & \multicolumn{2}{|c|}{ phase II } & \multicolumn{2}{|l|}{ Phase IV } & \multicolumn{2}{|l|}{ Phase VI } & \multicolumn{2}{|c|}{ Phase VI, granules } \\
\hline & $\mathbf{a}$ & b & $\mathbf{a}$ & b & $\mathbf{a}$ & b & $\mathbf{a}$ & b & $\mathbf{a}$ & b \\
\hline EU234111.1.1514 & 978 & 1656 & 629 & 993 & 4270 & 3384 & 15809 & 8896 & 22405 & 27998 \\
\hline EU234116.1.1507 & 35 & 79 & 27 & 42 & 219 & 242 & 838 & 499 & 1657 & 1265 \\
\hline New.ReferenceOTU528 & 7 & 9 & 9 & 17 & 40 & 44 & 90 & 53 & 148 & 182 \\
\hline New.ReferenceOTU520 & 6 & 8 & 2 & 4 & 19 & 33 & 111 & 53 & 159 & 166 \\
\hline New.ReferenceOTU431 & 13 & 13 & 6 & 20 & 79 & 84 & 98 & 49 & 112 & 158 \\
\hline New.ReferenceOTU79 & 0 & 0 & 0 & 0 & 0 & 0 & 15 & 6 & 86 & 115 \\
\hline SUM & 1060 & 1792 & 679 & 1092 & 4688 & 3851 & 17165 & 9771 & 24976 & 30396 \\
\hline Total OTU count in sample & 165606 & 162885 & 64675 & 115943 & 116599 & 111495 & 158258 & 94834 & 102788 & 118583 \\
\hline Relative abundance of genus & $0.64 \%$ & $1.10 \%$ & $1.05 \%$ & $0.94 \%$ & $4.02 \%$ & $3.45 \%$ & $10.85 \%$ & $10.30 \%$ & $24.30 \%$ & $25.63 \%$ \\
\hline
\end{tabular}

Table S20. Results of searching the ncbi 16S rRNA gene amplicon sequence (Bacteriea and Archaea) database using Megablast (executed in september 2018) with the selected Desulfovibrio OTU.

\begin{tabular}{|c|c|c|c|c|c|c|c|}
\hline OTU Identifyer & Description & $\begin{array}{l}\text { Max } \\
\text { score }\end{array}$ & $\begin{array}{l}\text { Total } \\
\text { score }\end{array}$ & $\begin{array}{l}\text { Query } \\
\text { cover }\end{array}$ & $\begin{array}{l}\mathrm{E} \\
\text { value }\end{array}$ & Ident & Accession \\
\hline EU234111.1.1514 & $\begin{array}{lr}\text { Desulfovibrio legallii } \\
\text { strain H1 } \\
\text { ribosomal } \\
\text { gene, } \\
\text { sequence }\end{array}$ & 2562 & 2562 & $95 \%$ & 0 & $99 \%$ & NR_108301.1 \\
\hline
\end{tabular}

Table S21. OTU counts within the genus Anaerohabdus furcosa group (Erysipelotrichales order). The 16S rRNA gene amplicon sequence of the in grey highlighted OTU identifyer was selected to search the NCBI database using Megablast.

\begin{tabular}{|c|c|c|c|c|c|c|c|c|c|c|}
\hline \multirow[b]{3}{*}{ OTU identifyer } & \multicolumn{10}{|c|}{ OTU counts } \\
\hline & \multicolumn{2}{|c|}{ phase I } & \multicolumn{2}{|c|}{ phase II } & \multicolumn{2}{|c|}{ Phase IV } & \multicolumn{2}{|c|}{ Phase VI } & \multicolumn{2}{|c|}{ Phase VI, granules } \\
\hline & a & b & a & b & $\mathbf{a}$ & b & a & b & $\mathbf{a}$ & b \\
\hline FJ660595.1.1491 & 5279 & 11772 & 156 & 234 & 646 & 447 & 937 & 780 & 724 & 992 \\
\hline New.CleanUp.ReferenceOTU98424 & 64 & 172 & 0 & 11 & 15 & 12 & 13 & 7 & 10 & 13 \\
\hline New.CleanUp.ReferenceOTU11853 & 48 & 57 & 1 & 0 & 6 & 7 & 3 & 1 & 1 & 0 \\
\hline New.CleanUp.ReferenceOTU91548 & 21 & 41 & 3 & 2 & 17 & 6 & 6 & 1 & 4 & 3 \\
\hline New.CleanUp.ReferenceOTU106291 & 21 & 21 & 0 & 0 & 2 & 1 & 1 & 1 & 0 & 0 \\
\hline New.CleanUp.ReferenceOTU49871 & 0 & 0 & 0 & 0 & 0 & 0 & 0 & 2 & 0 & 0 \\
\hline New.CleanUp.ReferenceOTU105945 & 0 & 0 & 0 & 0 & 0 & 0 & 2 & 1 & 6 & 6 \\
\hline Cropped... & $\ldots$ & $\ldots$ & $\ldots$ & $\ldots$ & $\ldots$ & $\ldots$ & $\ldots$ & $\ldots$ & $\ldots$ & $\ldots$ \\
\hline
\end{tabular}


Table S22. Results of searching the ncbi 16S rRNA gene amplicon sequence (Bacteriea and Archaea) database using Megablast (executed in september 2018) with the selected Anaerohabdus furcosa group OTU.

$\begin{array}{llllll}\text { OTU Identifyer Description } & \begin{array}{l}\text { Max } \\ \text { score }\end{array} & \begin{array}{l}\text { Total } \\ \text { score }\end{array} & \begin{array}{l}\text { Query } \\ \text { cover }\end{array} & \text { E value Ident Accession }\end{array}$

\begin{tabular}{|c|c|c|c|c|c|c|c|}
\hline \multirow[t]{3}{*}{ FJ660595.1.1491 } & $\begin{array}{l}\text { Holdemania } \\
\text { massiliensis } \\
\text { strain AP2 } \\
16 S \\
\text { ribosomal } \\
\text { RNA gene, } \\
\text { partial } \\
\text { sequence }\end{array}$ & 1389 & 1389 & $99 \%$ & 0 & $84 \%$ & $N R \_125628.1$ \\
\hline & $\begin{array}{l}\text { Erysipelothrix } \\
\text { larvae strain } \\
\text { LV19 16S } \\
\text { ribosomal } \\
\text { RNA, } \\
\text { complete } \\
\text { sequence }\end{array}$ & 1386 & 1386 & $99 \%$ & 0 & $84 \%$ & NR_146813.2 \\
\hline & $\begin{array}{l}\text { Traorella } \\
\text { massiliensis } \\
\text { strain } \\
\text { Marseille- } \\
\text { P3110 16S } \\
\text { ribosomal } \\
\text { RNA, partial } \\
\text { sequence }\end{array}$ & 1378 & 1378 & $99 \%$ & 0 & $84 \%$ & NR_147369.1 \\
\hline
\end{tabular}

Table S23. OTU counts within the genus Pseudoclavibacter (Micrococcales order). The $16 \mathrm{~S}$ rRNA gene amplicon sequences of the in grey highlighted OTU identifyers were selected to search the NCBI database using Megablast.

\begin{tabular}{|c|c|c|c|c|c|c|c|c|c|c|}
\hline \multirow[b]{3}{*}{ OTU identifyer } & \multicolumn{10}{|c|}{ OTU counts } \\
\hline & \multicolumn{2}{|l|}{ phase I } & \multicolumn{2}{|c|}{ phase II } & \multicolumn{2}{|l|}{ Phase IV } & \multicolumn{2}{|c|}{ Phase VI } & \multicolumn{2}{|c|}{ Phase VI, granules } \\
\hline & $\mathbf{a}$ & b & $\mathbf{a}$ & b & $\mathbf{a}$ & b & $\mathbf{a}$ & b & $\mathbf{a}$ & b \\
\hline AB329630.1.1460 & 113 & 1348 & 8344 & 11659 & 393 & 363 & 125 & 99 & 442 & 405 \\
\hline HQ266601.1.1376 & 642 & 1533 & 234 & 709 & 50 & 43 & 137 & 79 & 90 & 113 \\
\hline EU303275.1.1433 & 0 & 3 & 5 & 8 & 0 & 0 & 0 & 3 & 0 & 0 \\
\hline New.CleanUp.ReferenceOTU129543 & 0 & 3 & 10 & 9 & 0 & 0 & 0 & 3 & 4 & 0 \\
\hline New.ReferenceOTU42 & 21 & 58 & 9 & 48 & 4 & 2 & 10 & 2 & 5 & 4 \\
\hline SUM & 776 & 2945 & 8602 & 12433 & 447 & 408 & 272 & 186 & 541 & 522 \\
\hline Total OTU count in sample & 165606 & 162885 & 64675 & 115943 & 116599 & 111495 & 158258 & 94834 & 102788 & 118583 \\
\hline
\end{tabular}


Table S24. Results of searching the ncbi $16 \mathrm{~S}$ rRNA gene amplicon sequence (Bacteriea and Archaea) database using Megablast (executed in september 2018) with the selected Pseudoclavibacter OTUs.

\begin{tabular}{|c|c|c|c|c|c|c|c|}
\hline OTU Identifyer & Description & $\begin{array}{l}\text { Max } \\
\text { score }\end{array}$ & $\begin{array}{l}\text { Total } \\
\text { score }\end{array}$ & $\begin{array}{l}\text { Query } \\
\text { cover }\end{array}$ & $\begin{array}{l}\mathrm{E} \\
\text { value }\end{array}$ & Ident & Accession \\
\hline AB329630.1.1460 & $\begin{array}{l}\text { Pseudoclavibacter } \\
\text { soli strain KP02 } \\
\text { 16S ribosomal RNA } \\
\text { gene, partial } \\
\text { sequence }\end{array}$ & 2697 & 2697 & $100 \%$ & 0 & $100 \%$ & NR_041614.1 \\
\hline & \begin{tabular}{lr}
\multicolumn{2}{l}{ Pseudoclavibacter } \\
alba strain & IFO \\
15616 & $16 \mathrm{~S}$ \\
ribosomal & RNA \\
gene, & partial \\
sequence &
\end{tabular} & 2353 & 2353 & $99 \%$ & 0 & $96 \%$ & NR_024673.1 \\
\hline HQ266601.1.1376 & $\begin{array}{l}\text { Pseudoclavibacter } \\
\text { caeni strain MJ28 } \\
16 \mathrm{~S} \text { ribosomal RNA } \\
\text { gene, partial } \\
\text { sequence }\end{array}$ & 2538 & 2538 & $100 \%$ & 0 & $100 \%$ & NR_109073.1 \\
\hline
\end{tabular}

Table S25. OTU counts within the genus Candidatus Methanogranum (Thermoplasmatales order, Archaea). The 16S rRNA gene amplicon sequence of the in grey highlighted OTU identifyer was selected to search the NCBI database using Megablast.

\begin{tabular}{|c|c|c|c|c|c|c|c|c|c|c|}
\hline \multirow[b]{3}{*}{ OTU identifyer } & \multicolumn{10}{|c|}{ OTU counts } \\
\hline & \multicolumn{2}{|l|}{ phase I } & \multicolumn{2}{|c|}{ phase II } & \multicolumn{2}{|l|}{ Phase IV } & \multicolumn{2}{|l|}{ Phase VI } & \multicolumn{2}{|c|}{ Phase VI, granules } \\
\hline & $\mathbf{a}$ & $\mathbf{b}$ & $\mathbf{a}$ & $\mathbf{b}$ & $\mathbf{a}$ & b & $\mathbf{a}$ & $\mathbf{b}$ & $\mathbf{a}$ & $\mathbf{b}$ \\
\hline JF980498.1.1419 & 475 & 318 & 3532 & 2760 & 1861 & 1738 & 8705 & 6218 & 778 & 1000 \\
\hline New.ReferenceOTU377 & 0 & 0 & 4 & 0 & 3 & 8 & 7 & 4 & 10 & 13 \\
\hline Cropped... & $\cdots$ & $\cdots$ & $\cdots$ & $\cdots$ & $\cdots$ & $\cdots$ & $\cdots$ & $\cdots$ & $\cdots$ & $\cdots$ \\
\hline SUM & 481 & 319 & 3561 & 2782 & 1884 & 1767 & 8750 & 6283 & 796 & 1024 \\
\hline Total OTU count in sample & 165606 & 162885 & 64675 & 115943 & 116599 & 111495 & 158258 & 94834 & 102788 & 118583 \\
\hline Relative abundance of genus & $0.29 \%$ & $0.20 \%$ & $5.51 \%$ & $2.40 \%$ & $1.62 \%$ & $1.58 \%$ & $5.53 \%$ & $6.63 \%$ & $0.77 \%$ & $0.86 \%$ \\
\hline
\end{tabular}


Table S26. Results of searching the ncbi $16 \mathrm{~S}$ rRNA gene amplicon sequence (Bacteriea and Archaea) database using Megablast (executed in september 2018) with the selected Candidatus Methanogranum OTU.

\begin{tabular}{|c|c|c|c|c|c|c|c|}
\hline OTU Identifyer & Description & $\begin{array}{l}\text { Max } \\
\text { scor } \\
\text { e }\end{array}$ & $\begin{array}{l}\text { Total } \\
\text { score }\end{array}$ & $\begin{array}{l}\text { Quer } \\
\text { y } \\
\text { cover }\end{array}$ & $\begin{array}{l}\text { E } \\
\text { valu } \\
\text { e }\end{array}$ & $\begin{array}{l}\text { Iden } \\
\mathrm{t}\end{array}$ & Accession \\
\hline $\begin{array}{l}\text { JF980498.1.141 } \\
9\end{array}$ & $\begin{array}{l}\text { Methanomassiliicocc } \\
\text { us luminyensis strain } \\
\text { B10 16S ribosomal } \\
\text { RNA gene, partial } \\
\text { sequence }\end{array}$ & $\begin{array}{l}165 \\
2\end{array}$ & $\begin{array}{l}165200 \\
\%\end{array}$ & 0.99 & $0 \%$ & 0.88 & $\begin{array}{l}\text { NR_118098. } \\
1\end{array}$ \\
\hline
\end{tabular}

Table S27. OTU counts within the genus Methanoculleus (Methanomicrobiales order, Archaea). The 16S rRNA gene amplicon sequence of the in grey highlighted OTU identifyer was selected to search the NCBI database using Megablast.

\begin{tabular}{|c|c|c|c|c|c|c|c|c|c|c|}
\hline \multirow[b]{3}{*}{ OTU identifyer } & \multicolumn{10}{|c|}{ OTU counts } \\
\hline & \multicolumn{2}{|l|}{ phase I } & \multicolumn{2}{|c|}{ phase II } & \multicolumn{2}{|l|}{ Phase IV } & \multicolumn{2}{|l|}{ Phase VI } & \multicolumn{2}{|c|}{ Phase VI, granules } \\
\hline & $\mathbf{a}$ & b & $\mathbf{a}$ & $\mathbf{b}$ & $\mathbf{a}$ & b & $\mathbf{a}$ & b & $\mathbf{a}$ & b \\
\hline EF112188.1.1002 & 3042 & 803 & 1336 & 1212 & 8343 & 7243 & 4373 & 3060 & 10443 & 14258 \\
\hline EU662678.1.1435 & 80 & 3 & 11 & 6 & 63 & 57 & 31 & 15 & 292 & 356 \\
\hline EU369626.1.1443 & 20 & 4 & 4 & 6 & 38 & 39 & 30 & 24 & 74 & 92 \\
\hline Cropped... & $\ldots$ & $\cdots$ & $\ldots$ & $\ldots$ & $\ldots$ & $\cdots$ & $\ldots$ & $\ldots$ & $\ldots$ & $\ldots$ \\
\hline sum & 3161 & 824 & 1364 & 1242 & 8560 & 7431 & 4488 & 3152 & 10937 & 14883 \\
\hline Total OTU count in sample & 165606 & 162885 & 64675 & 115943 & 116599 & 111495 & 158258 & 94834 & 102788 & 118583 \\
\hline Relative abundance of genus & $1.91 \%$ & $0.51 \%$ & $2.11 \%$ & $1.07 \%$ & $7.34 \%$ & $6.66 \%$ & $2.84 \%$ & $3.32 \%$ & $10.64 \%$ & $12.55 \%$ \\
\hline
\end{tabular}

Table S28. Results of searching the ncbi $16 \mathrm{~S}$ rRNA gene amplicon sequence (Bacteriea and Archaea) database using Megablast (executed in september 2018) with the selected Methanoculleus OTU.

\begin{tabular}{|c|c|c|c|c|c|c|c|}
\hline OTU Identifyer & Description & $\begin{array}{l}\text { Max } \\
\text { score }\end{array}$ & $\begin{array}{l}\text { Total } \\
\text { score }\end{array}$ & $\begin{array}{l}\text { Query } \\
\text { cover }\end{array}$ & $\begin{array}{l}\mathrm{E} \\
\text { value }\end{array}$ & Ident & Accession \\
\hline \multirow[t]{2}{*}{ EF112188.1.1002 } & $\begin{array}{l}\text { Methanoculleus } \\
\text { palmolei strain DSM } \\
427316 S \text { ribosomal } \\
\text { RNA gene, partial } \\
\text { sequence }\end{array}$ & 1779 & 1779 & $99 \%$ & 0 & $99 \%$ & NR_028253.1 \\
\hline & $\begin{array}{l}\text { Methanoculleus } \\
\text { receptaculi strain } \\
\text { ZC-2 } 16 \mathrm{~S} \text { ribosomal } \\
\text { RNA gene, partial } \\
\text { sequence }\end{array}$ & 1729 & 1729 & $99 \%$ & 0 & $98 \%$ & NR_043961.1 \\
\hline
\end{tabular}




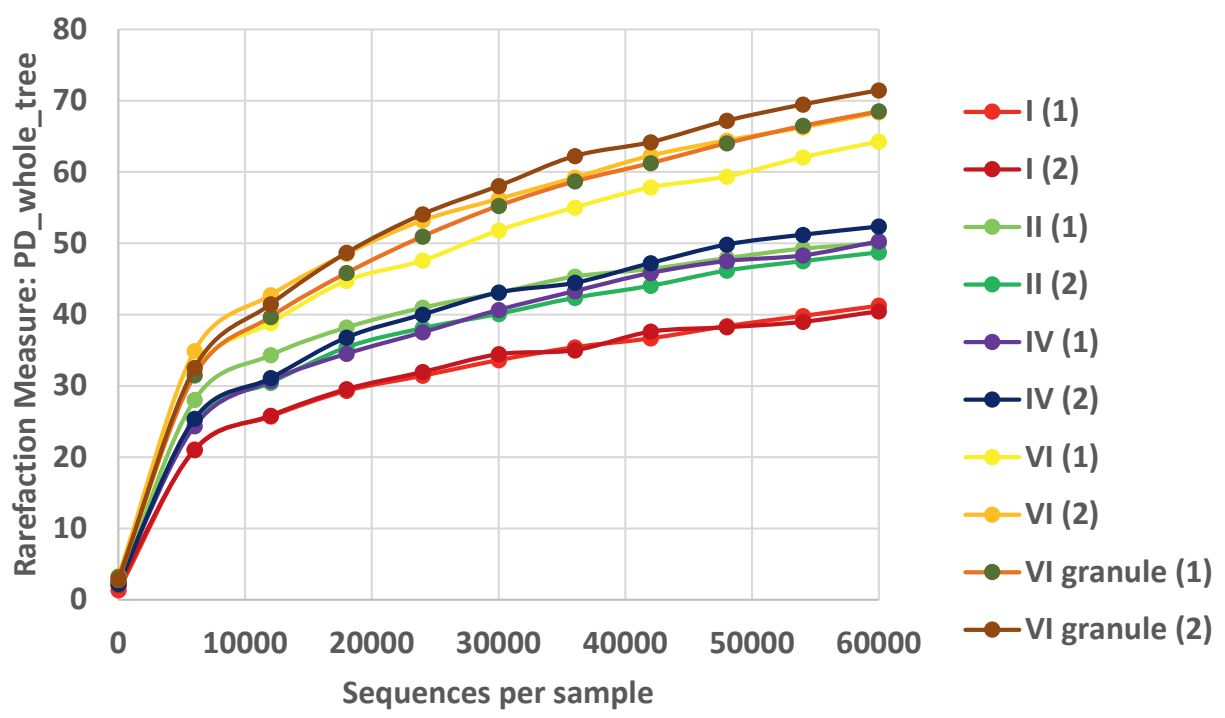

Figure S8. The figure shows the alpha rarefaction: PD_whole_tree of the sample set. Graph only shows values until 60000 sequences. Total OTU counts for each sample are given in Table S4

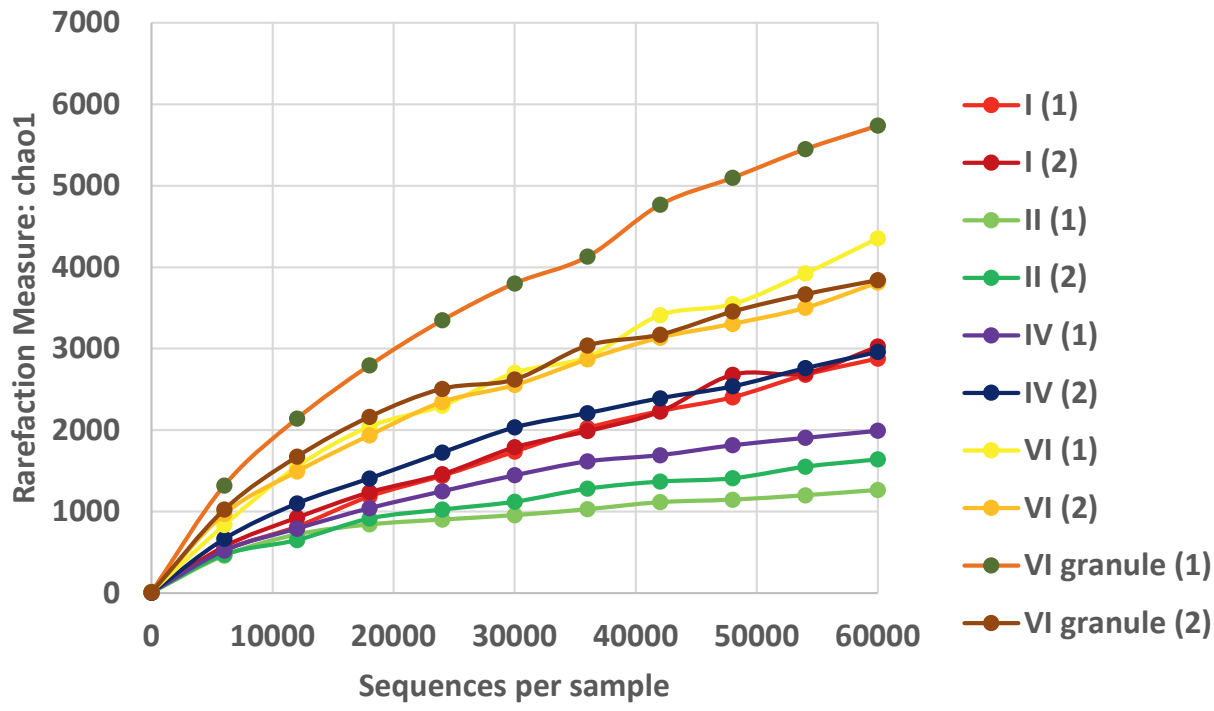

Figure 59. The figure shows the alpha rarefaction: chao 1 of the sample set. Graph only shows values until 60000 sequences. Total OTU counts for each sample are given in Table S4 


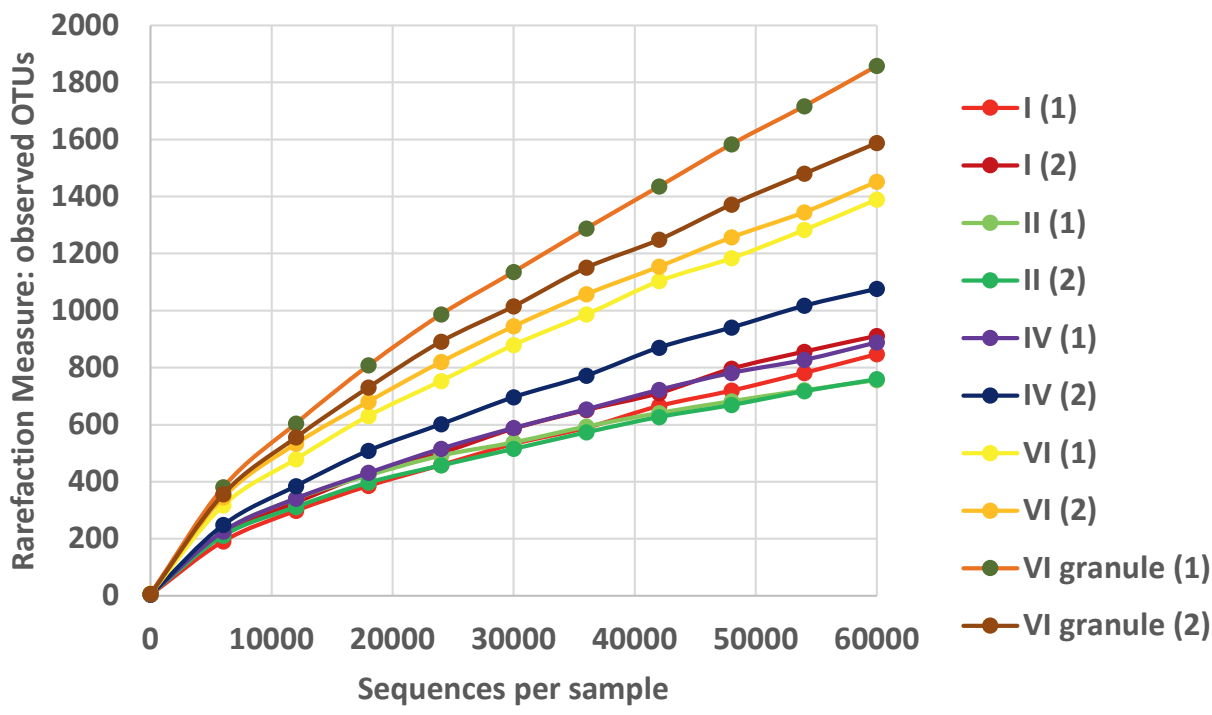

Figure S10. The figure shows the alpha rarefaction: observed OTUs of the sample set. Graph only shows values until 60000 sequences. Total OTU counts for each sample are given in Table S4

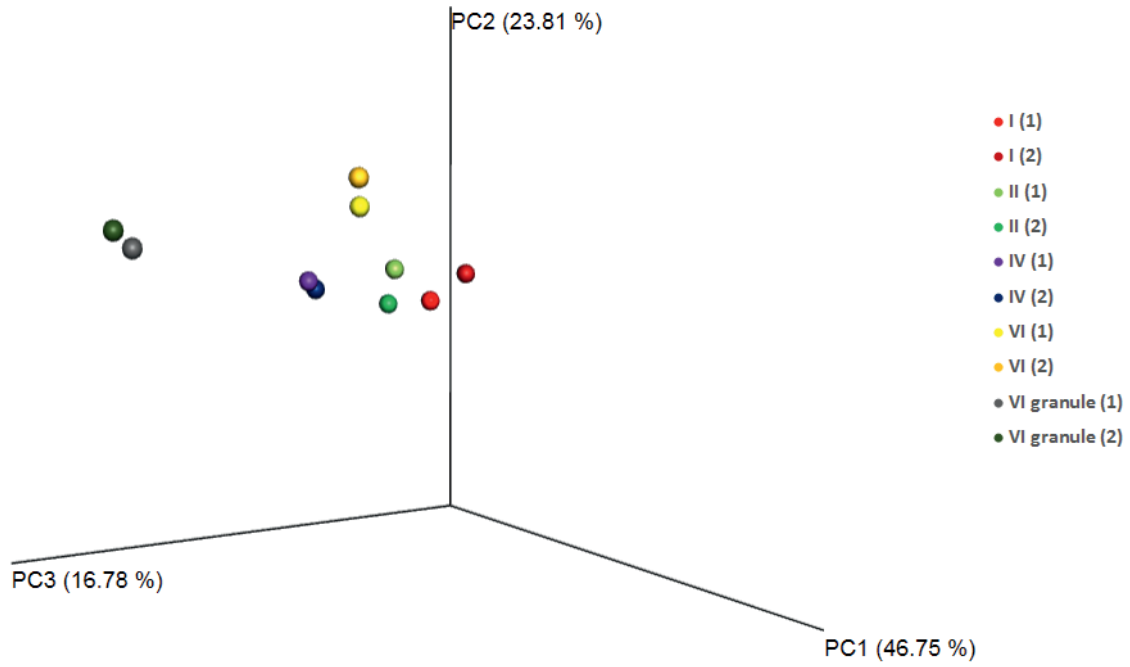

Figure S11. The figure shows the beta-diversity within the sample set. 


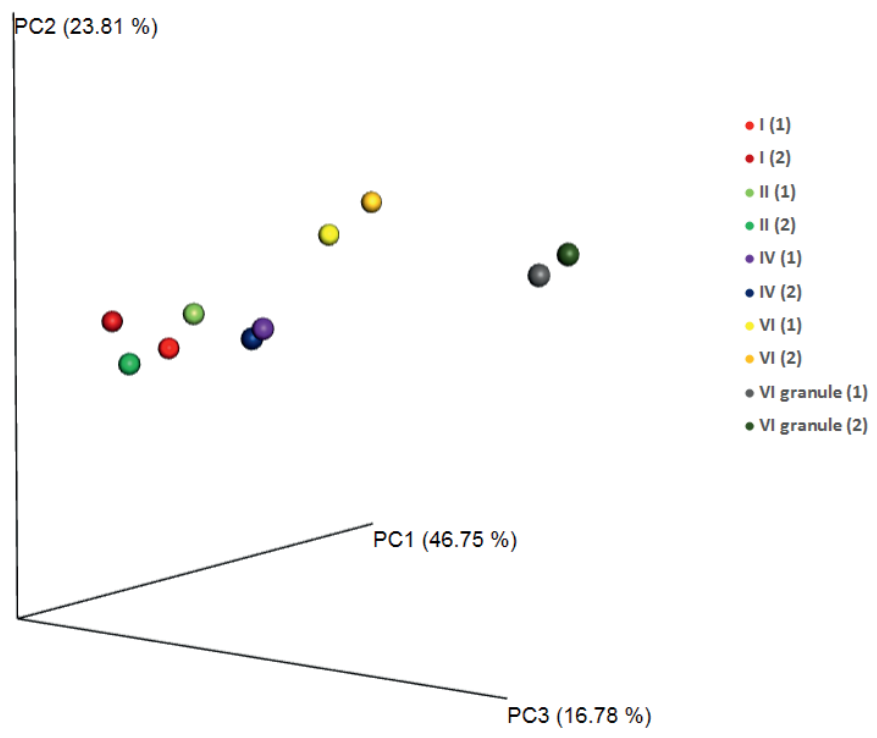

Figure S12. The figure shows the beta-diversity within the sample set.

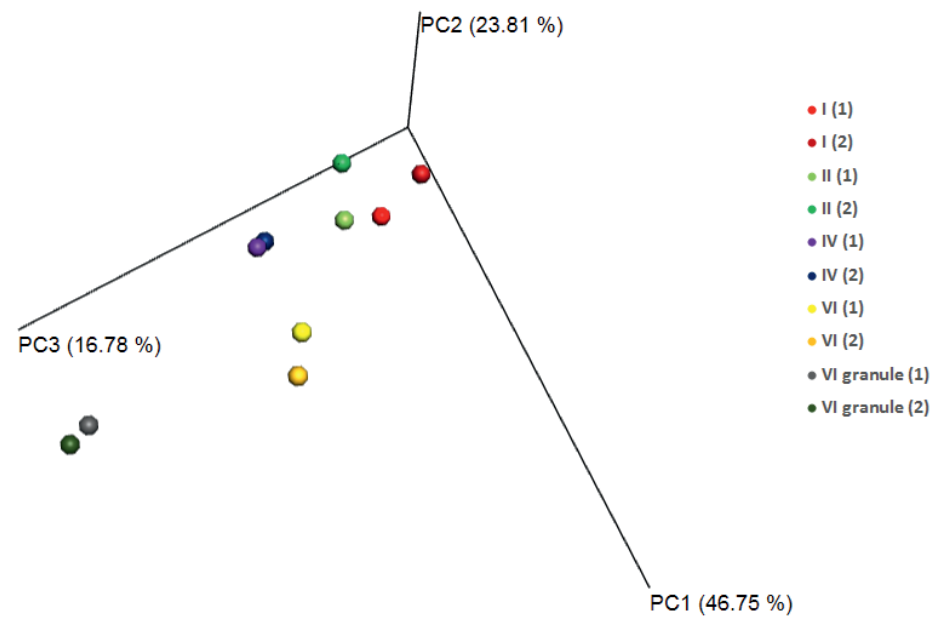

Figure S13. The figure shows the beta-diversity within the sample set. 
Table 529. The Table shows the first results of Qubit measurements to determine DNA concentrations after extraction. Comments say how the samples were handled before measurement. After these measurements some dilution or concentrations steps were performed to achieve a final

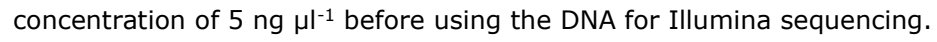

\begin{tabular}{lll}
\hline sample & $\begin{array}{l}\text { DNA concentration } \\
\text { (measurement } \\
\text { ng/ul }\end{array}$ & 1) \\
\hline \hline I (1) & 5.0 & Comments \\
I (2) & 3.5 & after speedvac concentration \\
II (1) & 4.3 & \\
II (2) & 4.6 & \\
IV (1) & 7.9 & \\
IV (2) & 11.5 & \\
VI (1) & 2.0 & after speedvac concentration \\
VI (2) & 2.7 & after speedvac concentration \\
VI granule (1) & 17.9 & diluted 25 times \\
VI granule (2) & 15.3 & diluted 25 times \\
\hline \hline
\end{tabular}




\section{Supporting Information - Chapter 5}

Expanding the Usage of Branched Electron Acceptors for the Formation of Iso-caproate and Iso-heptanoate by Ethanol-based Chain Elongation Reactor Microbiomes.

Kasper D. de Leeuw, Theresa Ahrens, Cees J. N. Buisman, David P. B. T. B. Strik 


\section{Material and Methods}

\section{Medium composition}

The basic feed recipe is shown in Table S1 and remained the same throughout the experiments. The composition of the used stock solutions with vitamin B and trace elements are listed in Table S2, Table S3 and Table S4.

Table S1. Basic feed recipe used for the reactors and with small alterations also for the batch experiments. Composition of the vitamin B and trace element solutions can be found in Table S2, Table S3 and Table S4. Stock solutions I and II were made resulting in the final listed concentrations in the medium.

\begin{tabular}{|c|c|c|}
\hline \multicolumn{2}{|l|}{ Compound } & Concentration $g / l$ \\
\hline \multicolumn{2}{|c|}{ Sodium Acetate trihydrate } & 2.5 \\
\hline \multicolumn{2}{|l|}{ Yeast extract } & 1 \\
\hline \multicolumn{2}{|l|}{$\mathrm{NaOH}$ pellets } & 1.1 \\
\hline \multicolumn{2}{|c|}{ Ethanol (Abs) (liquid) } & 14.96 \\
\hline \multicolumn{2}{|c|}{ i-C4 (liquid) } & 11.9 \\
\hline \multirow{3}{*}{ Stock I (50x) } & $\mathrm{NH}_{4} \mathrm{H}_{2} \mathrm{PO}_{4}$ & 3.6 \\
\hline & $\mathrm{MgCl}_{2} * 6 \mathrm{H}_{2} \mathrm{O}$ & 0.33 \\
\hline & $\mathrm{MgSO}_{4} * 7 \mathrm{H}_{2} \mathrm{O}$ & 0.2 \\
\hline \multirow{2}{*}{ Stock II (50x) } & $\mathrm{CaCl}_{2} * 2 \mathrm{H}_{2} \mathrm{O}$ & 0.2 \\
\hline & $\mathrm{KCl}$ & 0.15 \\
\hline \multicolumn{2}{|c|}{ Vitamin solution } & $1 \mathrm{ml}$ \\
\hline \multicolumn{2}{|c|}{ Trace elements, I \& II } & $0.5 \mathrm{ml}$ \\
\hline
\end{tabular}

Table S2. Composition of the "Vitamin solution" 1000x concentrated

\begin{tabular}{l|l} 
Compound & Concentration $(g / L)$ \\
\hline Biotin & 0.106 \\
Folic acid & 0.005 \\
Pyridoxal-HCl & 0.0025 \\
Lipoic acid & 0.015 \\
Riboflavin & 0.0125 \\
Thiamine HCl & 0.266 \\
Ca-D-Pantothenate & 0.413 \\
Cyanocobalamin (Vit. B12) & 0.0125 \\
P-aminobenzoic acid & 0.0125 \\
Nicotinic acid & 0.0125
\end{tabular}


Table S3. Composition of the "Trace elements I" solution 2000x concentrated

\begin{tabular}{l|l} 
Compound & \multicolumn{1}{l}{ Concentration $(g / L)$} \\
\hline $\mathrm{FeCl}_{2} * 4 \mathrm{H}_{2} \mathrm{O}$ & 30 \\
$\mathrm{HCl}(1 \mathrm{M})$ & 77 \\
$\mathrm{MnCl}_{2} * 4 \mathrm{H}_{2} \mathrm{O}$ & 0.6 \\
$\mathrm{H}_{3} \mathrm{BO}_{3}$ & 6.0 \\
$\mathrm{CoCl}_{2} * 6 \mathrm{H}_{2} \mathrm{O}$ & 4.0 \\
$\mathrm{CuCl}_{2} * \mathrm{H}_{2} \mathrm{O}$ & 0.2 \\
$\mathrm{NiCl}_{2} * 6 \mathrm{H}_{2} \mathrm{O}$ & 0.4 \\
$\mathrm{ZnSO}_{4} * 7 \mathrm{H}_{2} \mathrm{O}$ & 2.0
\end{tabular}

Table S4. Composition of the "Trace elements II" solution 2000x concentrated

Compound

$\mathrm{Na}_{2} \mathrm{MoO}_{4}{ }^{*} \mathrm{H}_{2} \mathrm{O}$

$\mathrm{Na}_{2} \mathrm{SeO}_{3}$

$\mathrm{NaOH}(4 \mathrm{M})$

\section{Concentration $(g / L)$}

0.6
0.2
3.1




\section{Step by step protocol for the batches}

1. Add to a $500 \mathrm{ml}$ beaker in this order (plan for minimal waste):

- $\quad 0.25 \mathrm{~g}$ yeast extract (except batch $1 . \mathrm{H}$ )

- Ethanol, $\mathrm{i}-\mathrm{C}_{5}$, acetate, $\mathrm{i}-\mathrm{C}_{4}$ were added according to Table 1 and Table 2 in the main manuscript: $50 / 25 \mathrm{~mL} 1.6 \mathrm{M}$ ethanol solution, $50 / 25 \mathrm{~mL} 65 \mathrm{mM}$ sodium acetate trihydrate, 100/50 mL $325 \mathrm{mM}(2-)$ / (3-) i-C 5

- $10 \mathrm{ml}$ stock I (see composition in Table S1)

- $10 \mathrm{ml}$ stock II (see composition in Table S1)

- $100 \mathrm{~mL} 50 \mathrm{~g} / \mathrm{L}$ BES solution (except batch $1 . \mathrm{G}$ )

- $0.5 \mathrm{ml}$ vitamin solution (see composition in Table S2)

- $0.25 \mathrm{ml}$ trace element I solution (see composition in Table S3)

- $0.25 \mathrm{ml}$ trace element II solution (see composition in Table S4)

2. Add demi water up to $400 \mathrm{ml}$

3. Set $\mathrm{pH}$ to $6.5(4 \mathrm{M} \mathrm{KOH})$

4. Transfer to a $500 \mathrm{ml}$ flask

5. Add $5 \mathrm{ml}$ re-suspended cells

6. Add demi water up to $500 \mathrm{ml}$

7. Stir shortly

8. Transfer $150 \mathrm{ml}$ to each batch bottles

9. Take a sample from the batch reactors $(5 \mathrm{ml})$

10. Cap the batches

11. Replace the headspace at 1.5 bar with a gas exchanger, composition was $90 \% \mathrm{~N}_{2}$ and $10 \% \mathrm{CO}_{2}$ for the first batch series (Table 1 ) and according to Table 2 for the second batch series. Add one extra bottle in each cycle.

12. Measure the absolute pressure

13. Carry out headspace gas composition analysis of the extra bottle

14. Place the bottles in a $35^{\circ} \mathrm{C}$ shaking incubation cabinet 


\section{Branched $C_{7}$ carboxylate determination}

Standard low conc

5-methylhexanoic acid

Sample batch 2.D

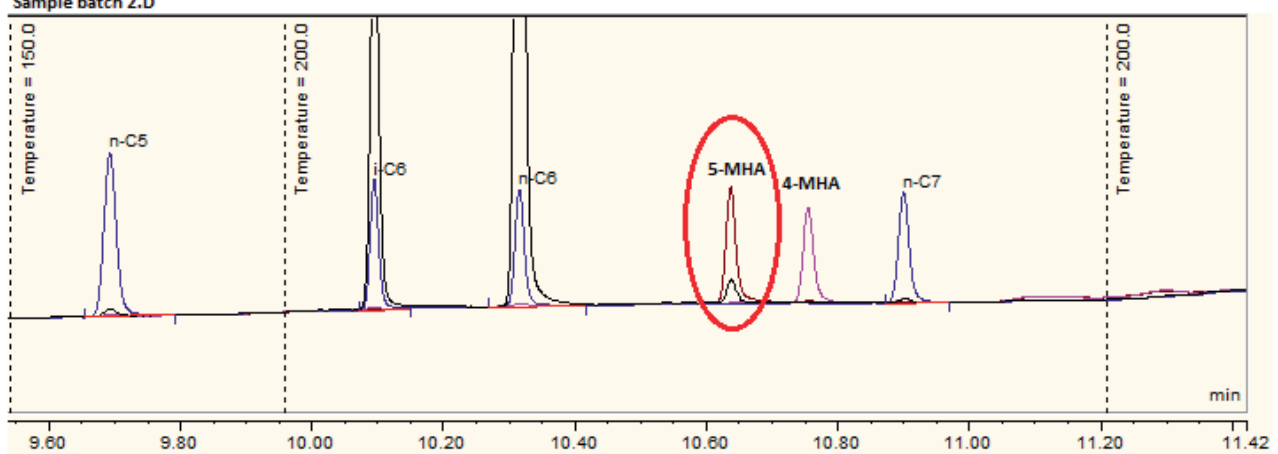

Figure S1. Chromatogram showing the branched heptanoate produced is 5-methylhexanoate (indicated in picture as 5-MHA). 4-MHA clearly has a different retention time and was not observed as product throughout the experiments. 


\section{Results and Discussion}

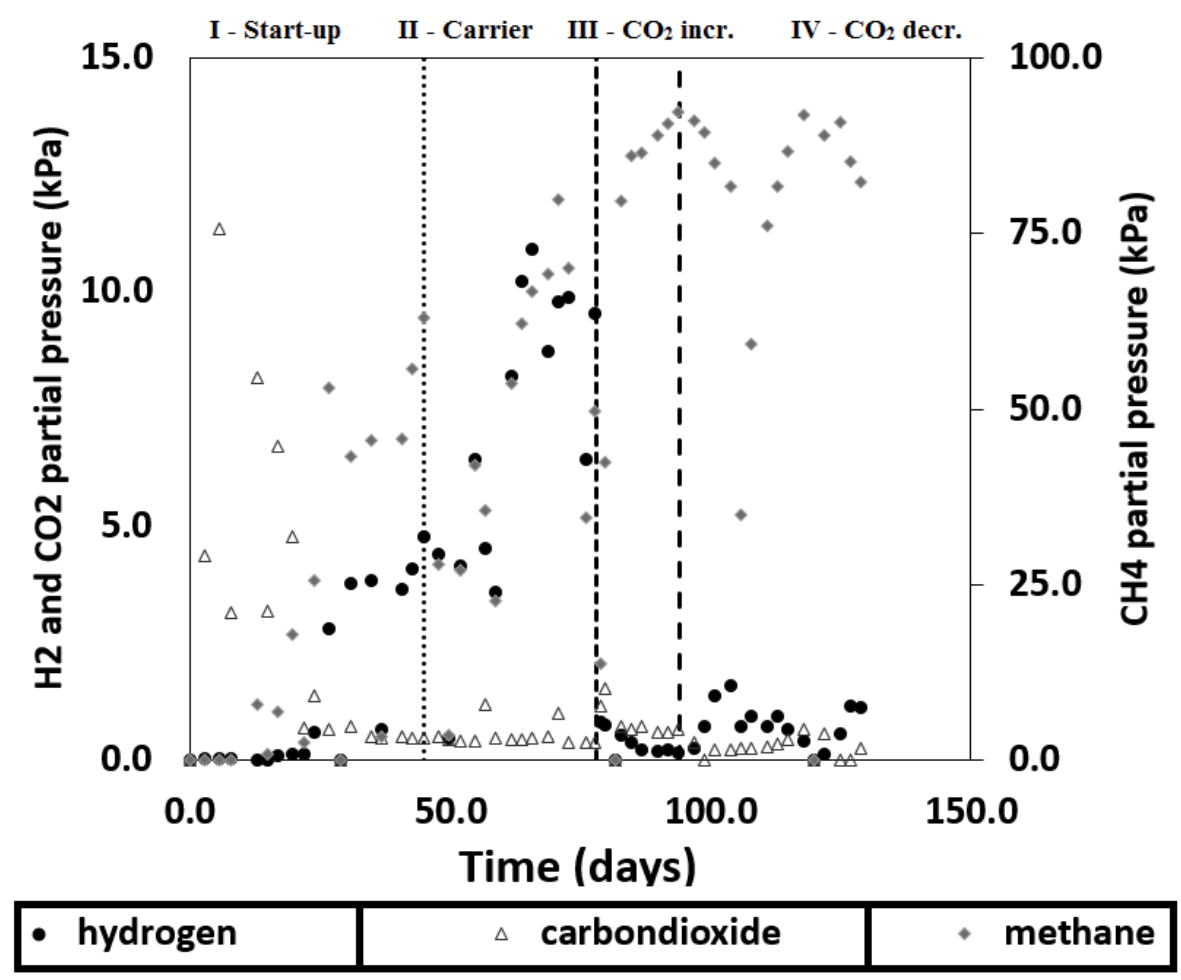

Figure S2. Gas partial pressures within the continuous reactor headspace. 

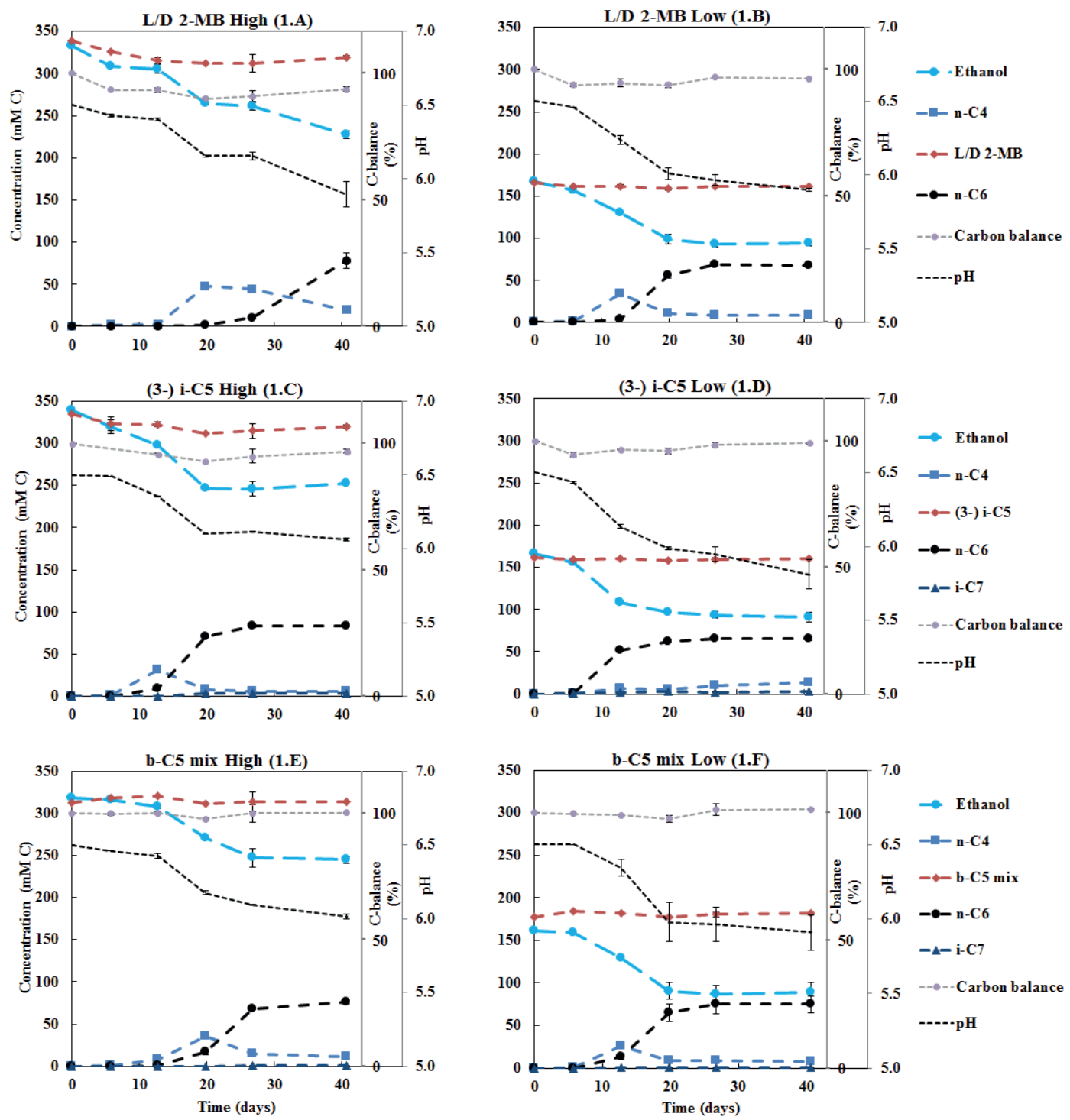

Figure S3. The graphs show the concentration profiles of batch 1.A to 1.F. Concentration profiles of the metabolites that were present in very low concentrations (e.g. acetate, n-hexanol, bpentanol as well as $\mathrm{i}-\mathrm{C}_{7}$ in a range between 0 and $25 \mathrm{mM} \mathrm{C}$ ) are shown in Figure S4. Headspace compositions are shown in Figure S5. Concentration profiles of the control batches are shown in Figure S6. 

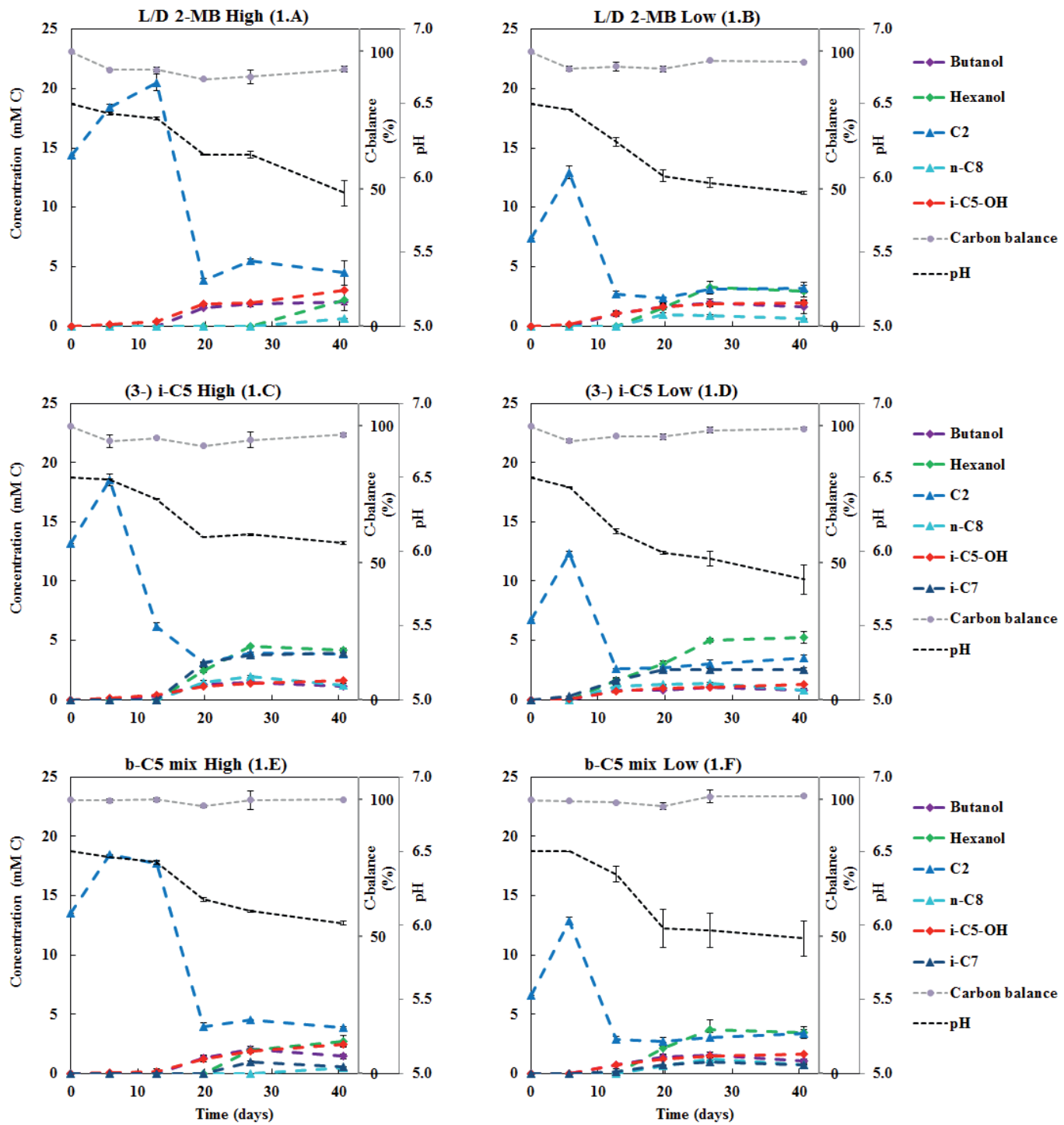

Figure S4. Concentration profiles of the first batch series for metabolites that were present at low concentrations. 

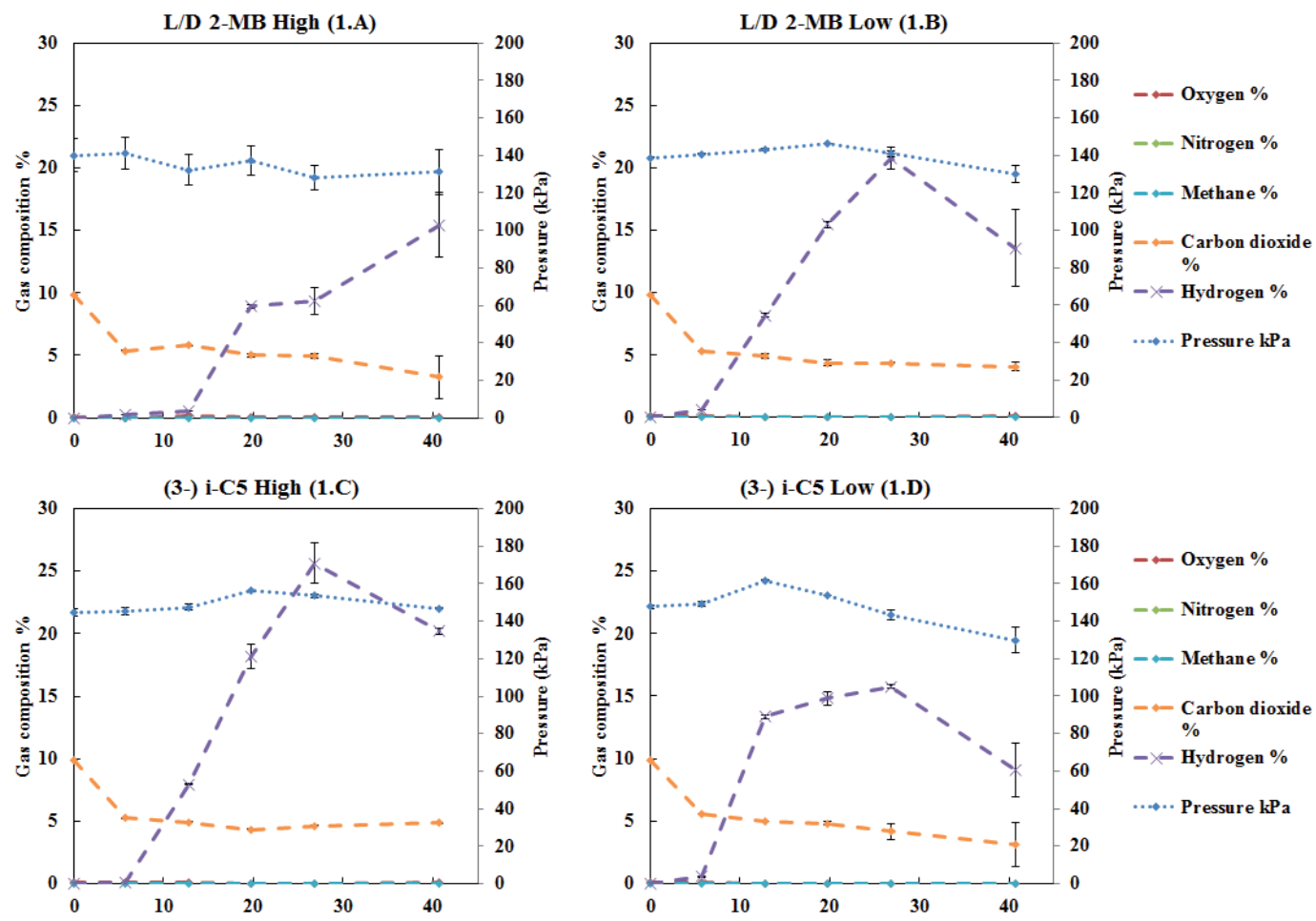

(3-) i-C5 Low (1.D)
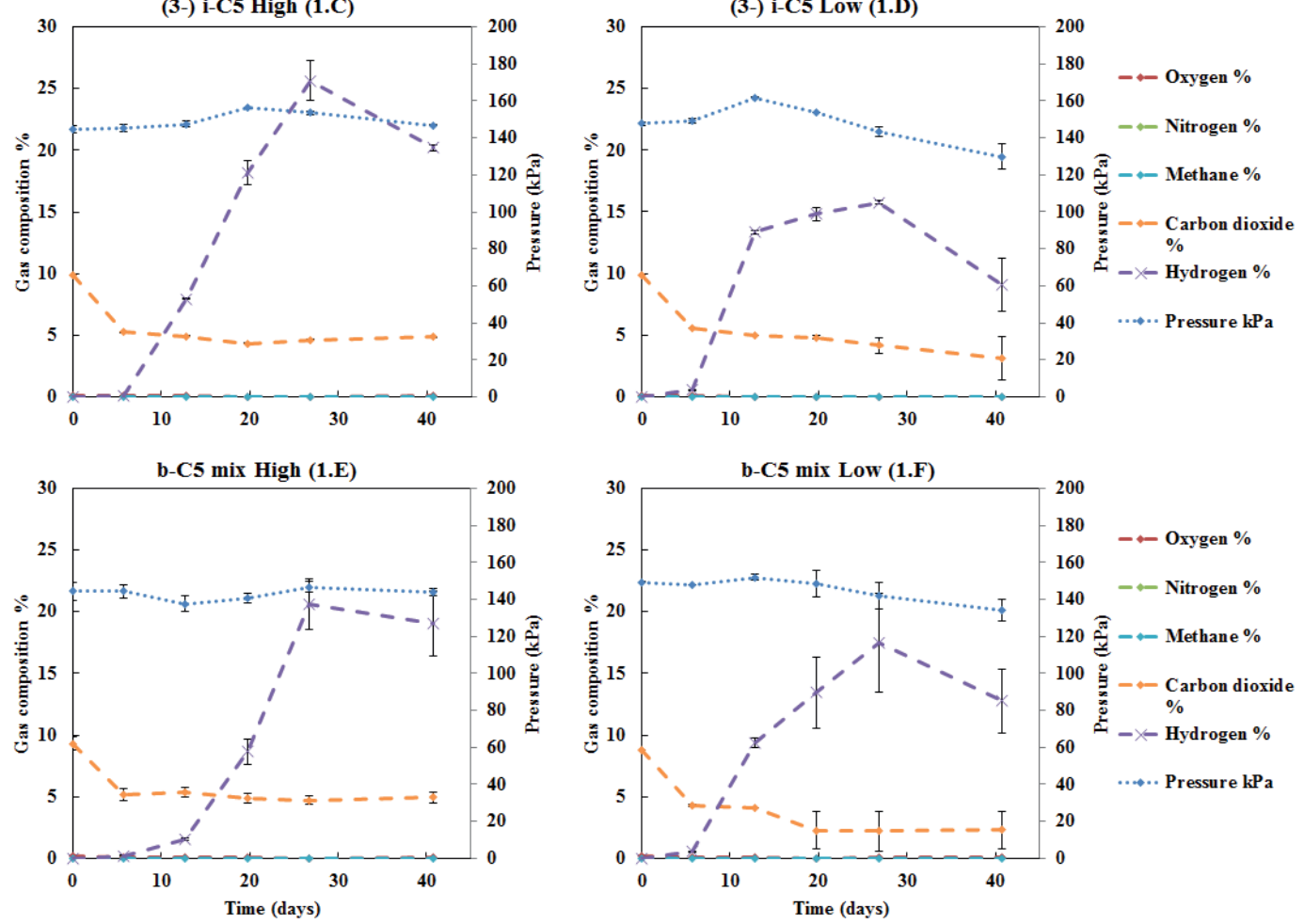

Figure S5. Headspace compositions and pressures of the first batch series. Both methane and oxygen overlap at around $0 \%$. 

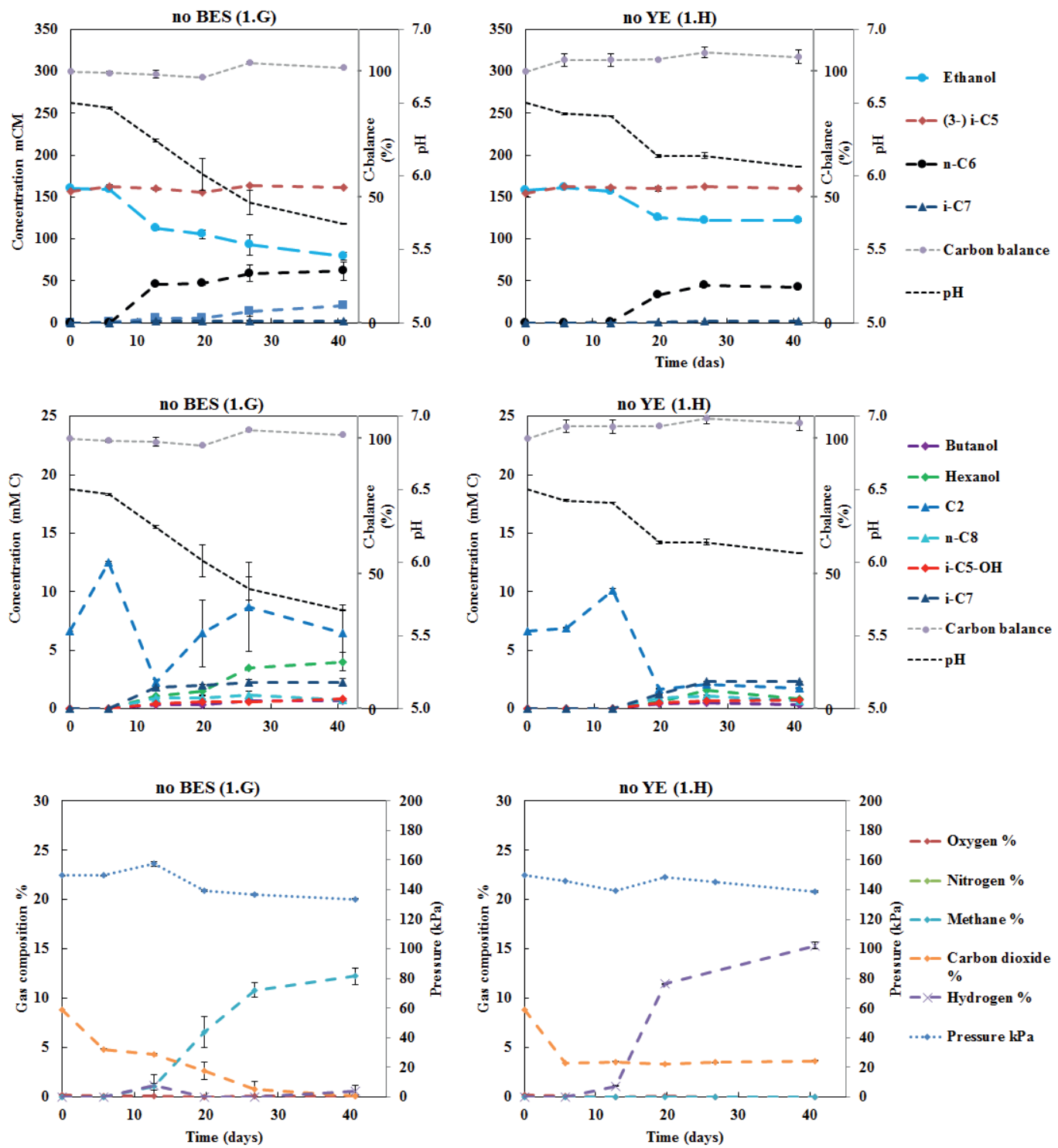

Figure S6. Concentration profiles and headspace compositions of the two negative controls where BES was left out (1.G) and where yeast extract was left out (1.H). 

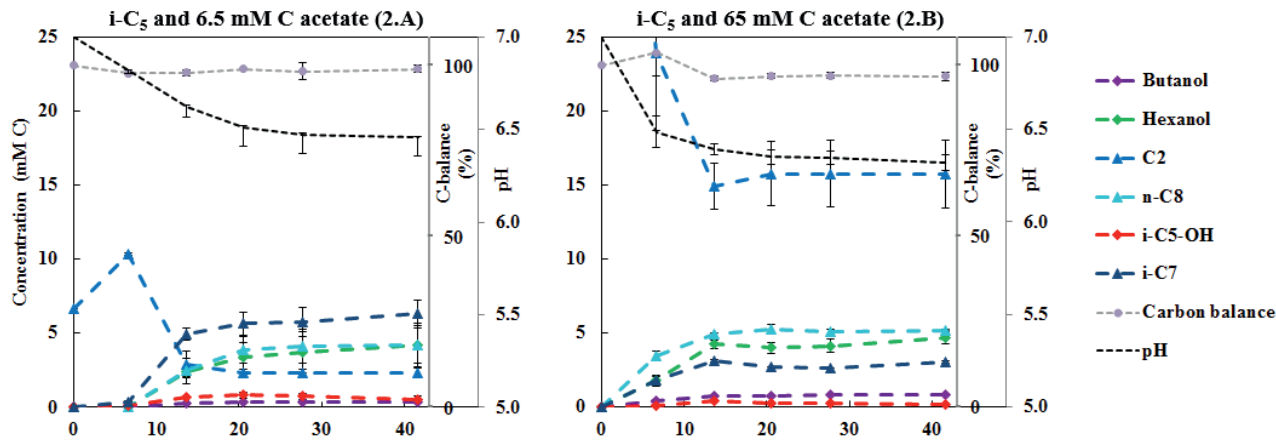

i- $\mathrm{C}_{5}$ and $6.5 \mathrm{mM} \mathrm{C}$ acetate, $80 \%$ initial $\mathrm{H}_{2}$ (2.C)
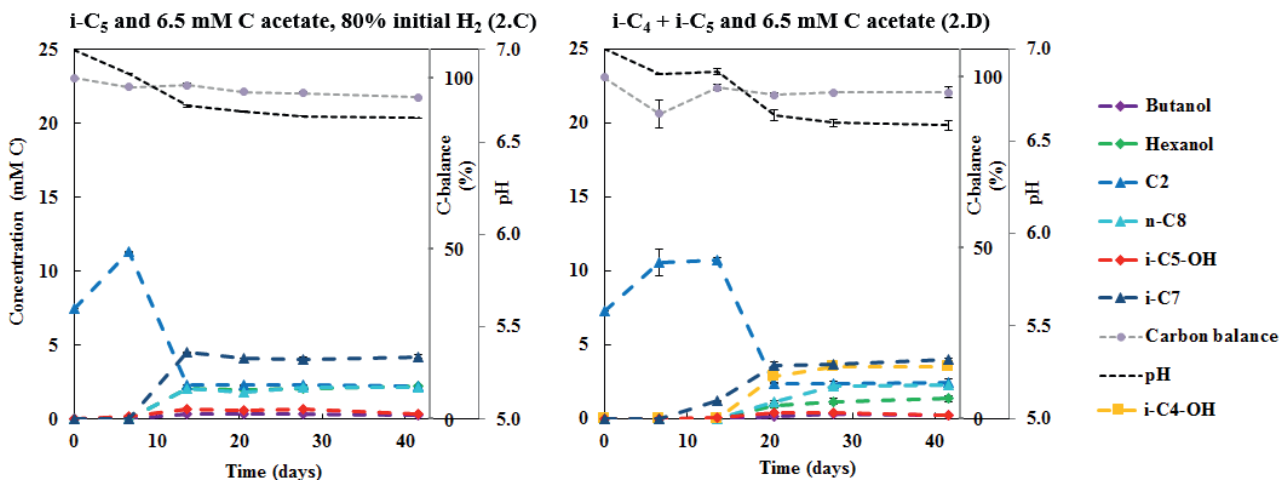

Figure S7. Concentration profiles of the second batch series for metabolites that were present at low concentrations. 

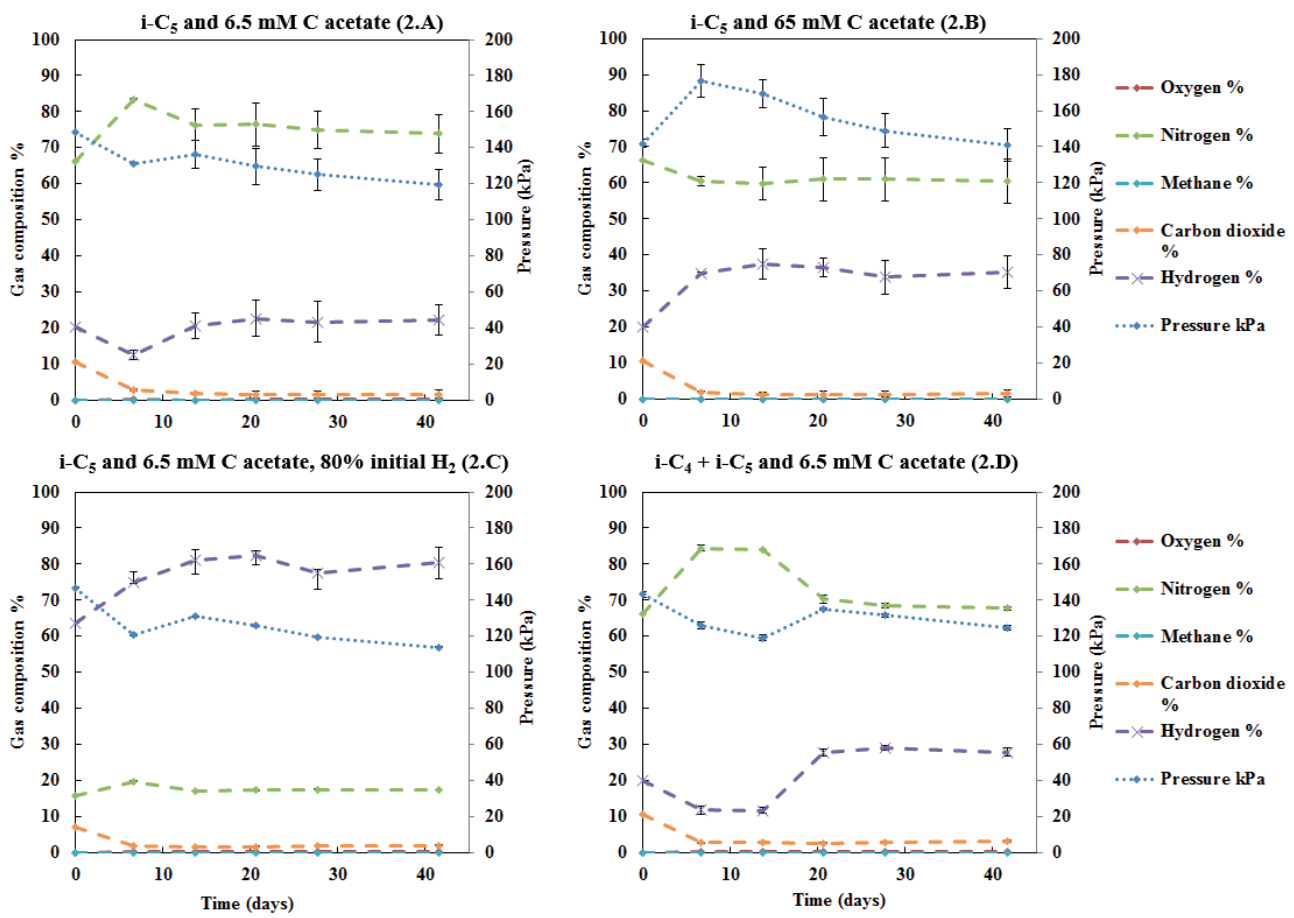

Figure S8. Headspace compositions and pressures of the second batch series. Both methane and oxygen overlap at around $0 \%$. 

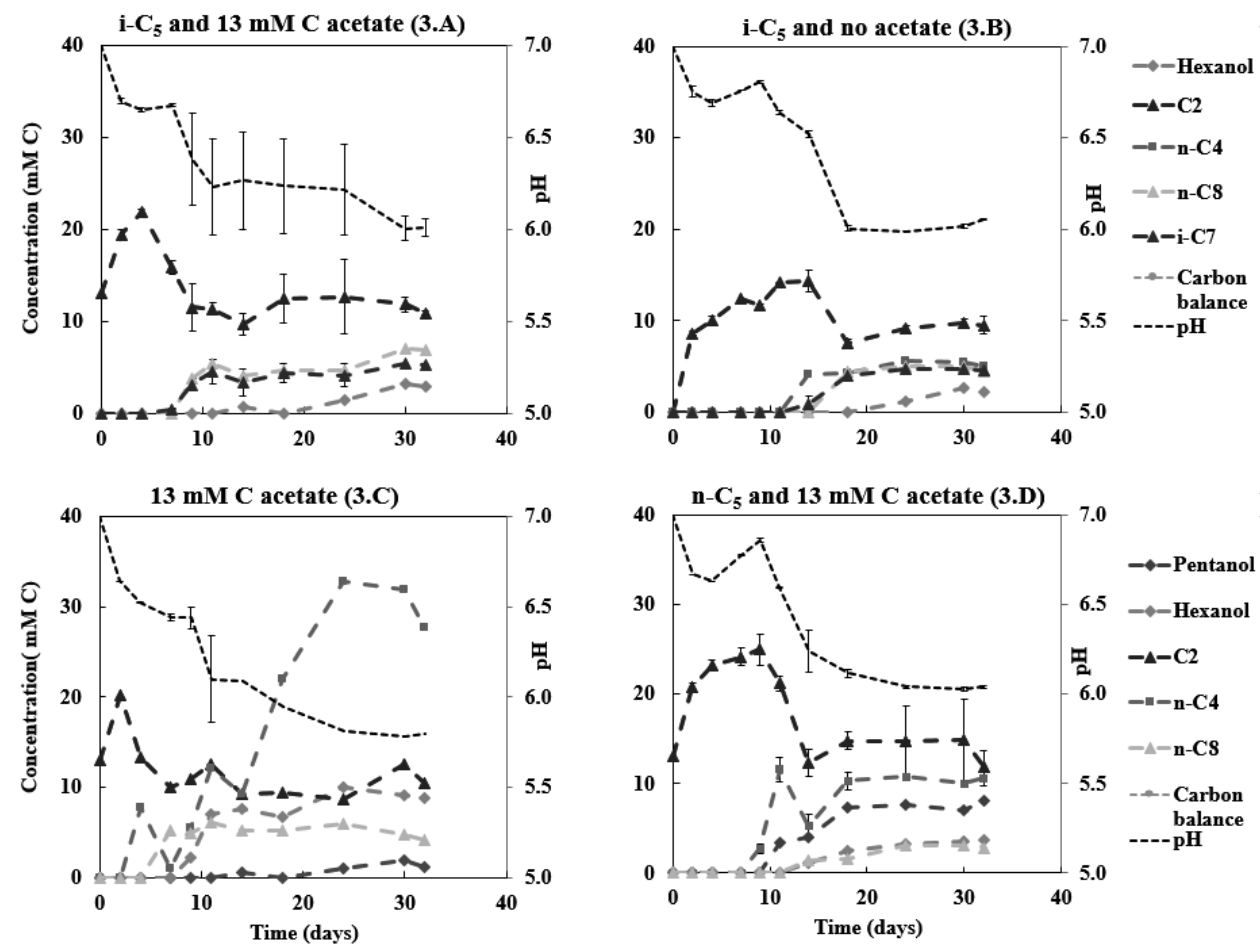

Figure S9. Concentration profiles of the third batch series for metabolites that were present at low concentrations. 


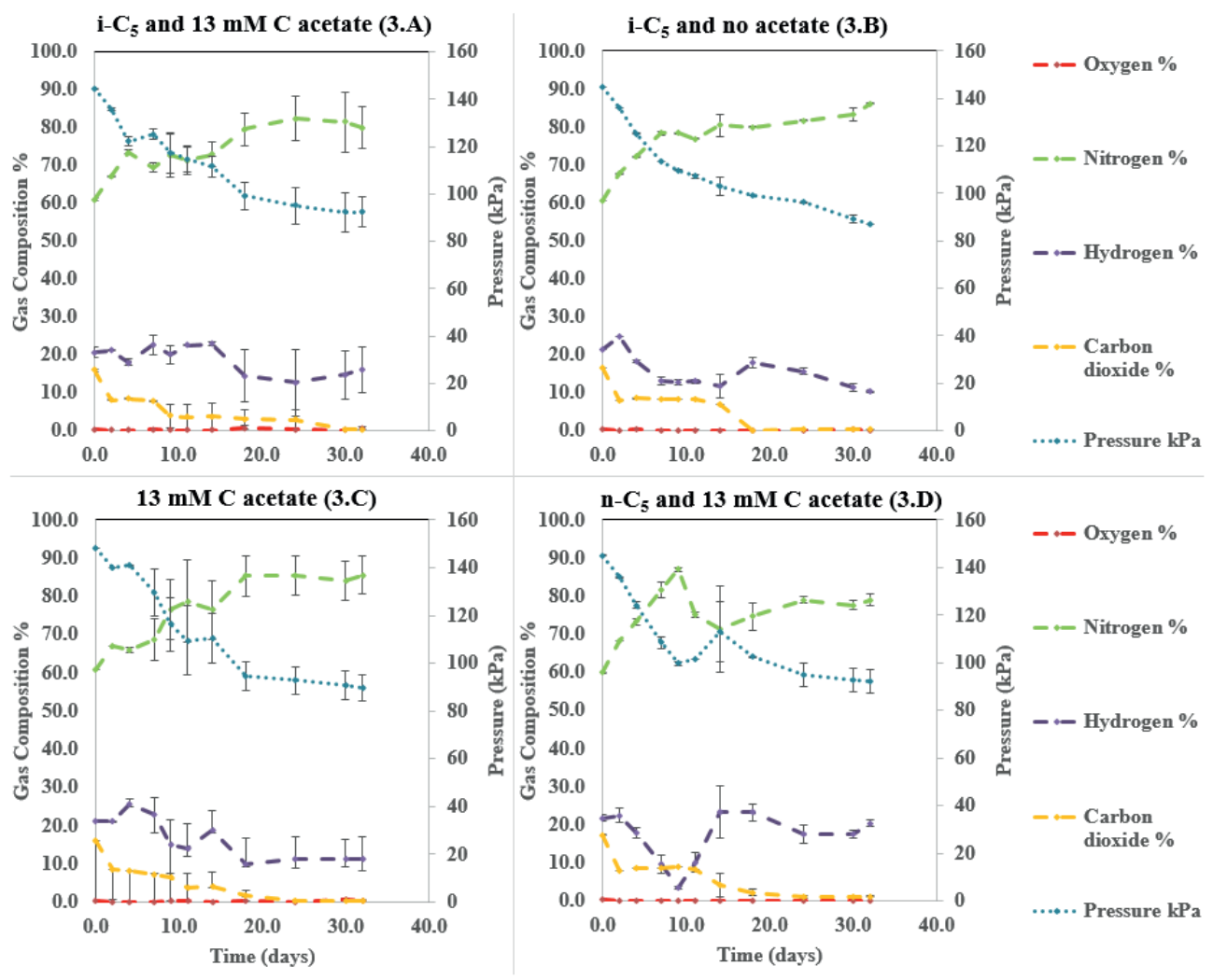

Figure S10. Headspace compositions and pressures of the third batch series. 


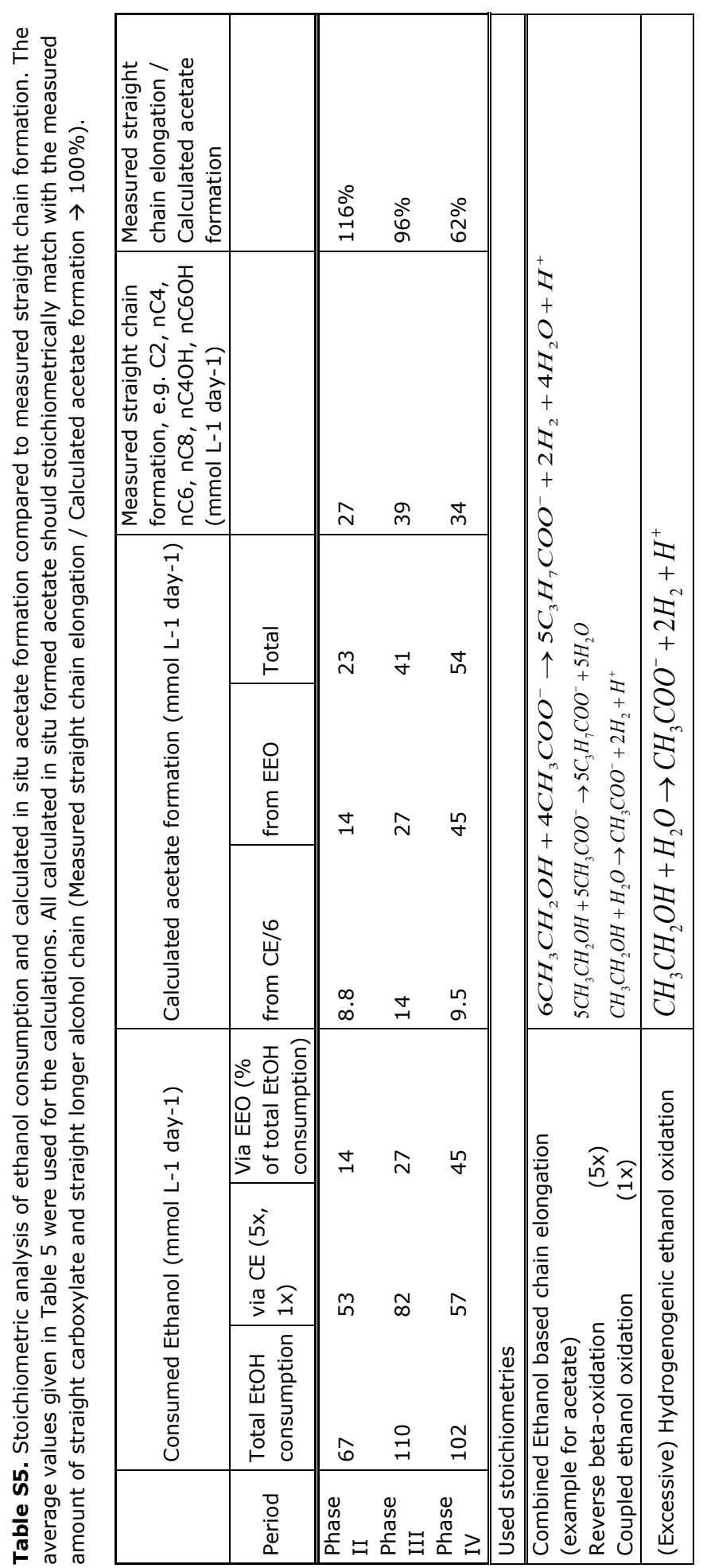

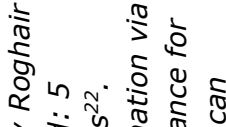

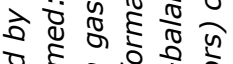

¿

बิ

पू के क्षे

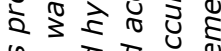

先

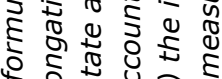

ᄂ के

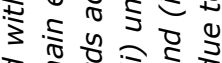

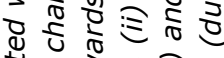

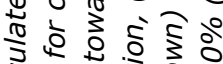

ปัن

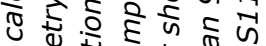

ज ह $\frac{0}{0}$ के

3.

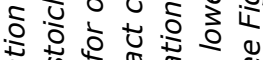

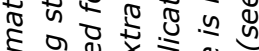

है

\&

ग रें

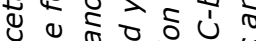

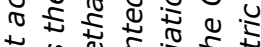

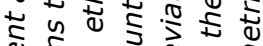

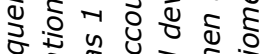

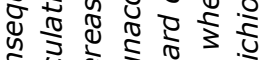

ป

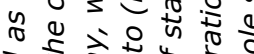

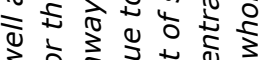

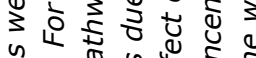

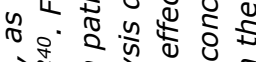

出水 .

出

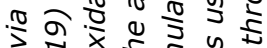

เ సิ

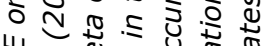

U

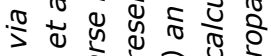

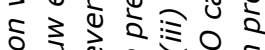

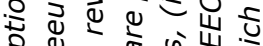

है ป d

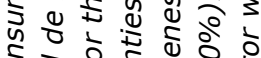

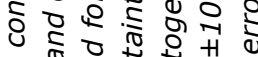

○े

疍

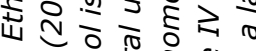

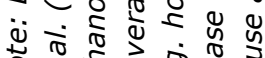

ป 


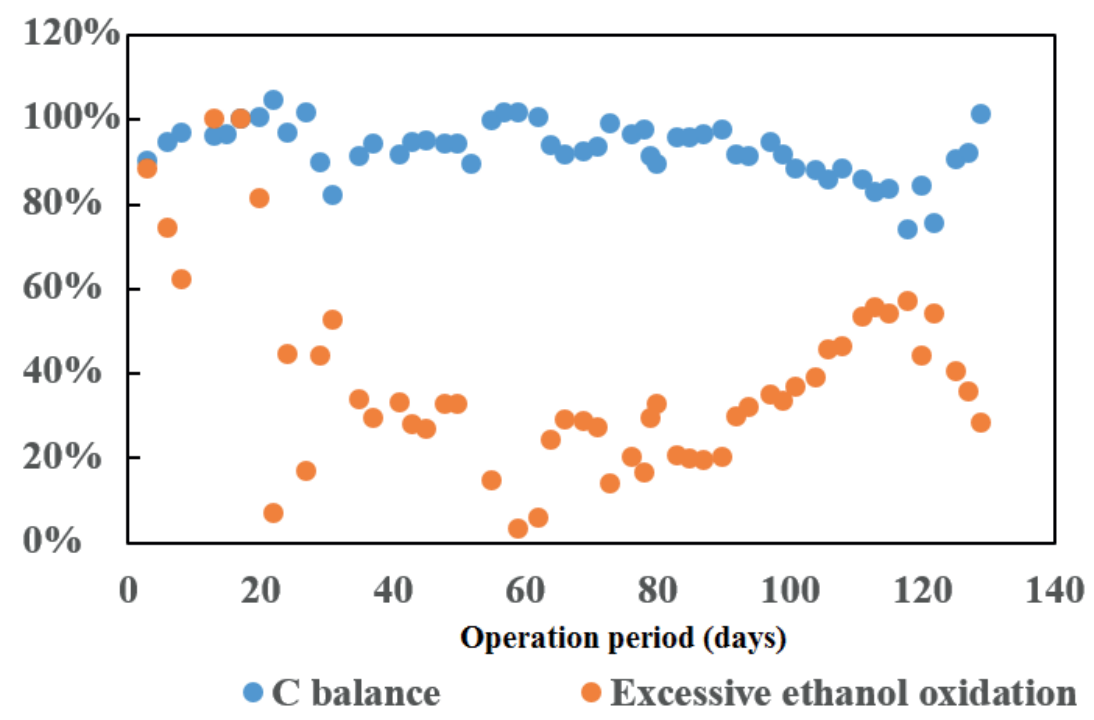

Figure S11. Carbon balance of the continuous reactor and calculated excessive ethanol oxidation. When the carbon balance drops below $~ 90 \%$ around day 100, the calculated excessive ethanol oxidation rises increasingly. The explanation for this overestimated EEO is a mismatch between the measured ethanol and fatty acids compared to what would be expected from the stoichiometry that is used to calculate the EEO. It indicates that concentration measurements (for ethanol in particular) might have been too low for phase IV, leading to an overestimation of EEO and a resulting mismatch in observed CE / calculated C2 formation.

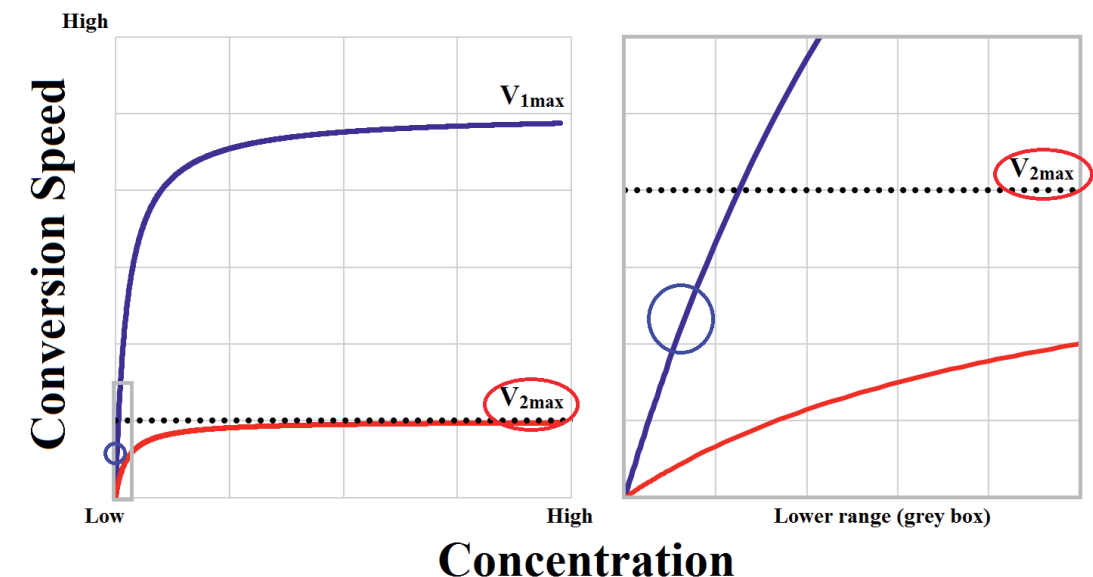

Figure S12. This figure illustrates a simplified interpretation (non-cooperative, Michaelis-Menten) of the possible enzyme kinetics at play when acetate is present at a low concentration $\left(V_{1}\right.$, blue), while a branched carboxylate $\left(V_{2}\right.$, red) is present at a high concentration. Values in this graph are arbitrarily chosen and therefore not shown. The circles show where the conversion speeds would be under acetate limited conditions, $V_{1}$ being severely limited while $V_{2}$ would be operating at maximum rates. At high acetate concentrations the relative speeds would be different leading to a lower branched carboxylate elongation selectivity. 
Table S6. Overview of Gibbs formation energies for components used to calculate the Gibbs reaction energies of the hydroxyl-carboxyl exchange reactions ${ }^{290}$. The Gibbs formation energies are based on chemical group contributions ${ }^{291}$. The formation energies of $n$-hexanol, isohexanoate and isohexanol could not be found. However, during the hydroxyl-carboxyl exchange reactions, the contribution to the reaction Gibbs energy difference comes from the difference between the $\Delta \mathrm{Gf}^{0}$ of acetate/ethanol and the $\Delta \mathrm{G} f^{0}$ of the larger carboxylate/alcohol pairs. The lengths of the hydrated carbon atoms tails for $n$-pentanoate/n-pentanol and $n$-octanoate/n-octanol hardly contribute to the $\Delta \mathrm{Gf}^{0}\left(-177.6\right.$ and $176.6 \mathrm{~kJ} \mathrm{~mol}^{-1}$ respectively) and the resulting $\Delta_{\mathrm{r}} \mathrm{G}^{0}\left(-11.3\right.$ and $-12.3 \mathrm{~kJ} \mathrm{~mol}^{-1}$ respectively). Therefore, the $\Delta_{\mathrm{r}} \mathrm{G}^{0}$ of the $\mathrm{n}$-hexanoate to $\mathrm{n}$-hexanol and the isohexanoate to isohexanol hydroxyl-carboxyl exchange reactions was assumed to be $-11.3 \mathrm{~kJ} \mathrm{~mol}^{-1}$, in line with the hydroxyl-carboxylate exchange reactions for $\mathrm{n}$-pentanoate and n-octanoate.

\begin{tabular}{|c|c|c|c|c|}
\hline Component & $\begin{array}{l}\text { Gfo } \\
k J \mathrm{~mol}^{-1}\end{array}$ & $\begin{array}{l}\Delta G f^{0} \\
k J m o l^{-1}\end{array}$ & Reaction & $\begin{array}{l}\Delta_{r} G^{0} \\
k J \mathrm{~mol} \\
1\end{array}$ \\
\hline $\begin{array}{l}\text { Acetate } \\
\text { Ethanol }\end{array}$ & $\begin{array}{l}-367.9 \\
-179.0\end{array}$ & -188.9 & & \\
\hline $\begin{array}{l}\text { butyrate } \\
n \text {-butanol }\end{array}$ & $\begin{array}{l}-352.6 \\
-171.5\end{array}$ & -181.1 & $\begin{array}{l}\text { hydroxyl-carboxylate exchange } n-\mathrm{C}_{4}+\mathrm{EtOH} \rightarrow n \text { - } \\
\mathrm{C}_{4} \mathrm{OH}+\mathrm{C}_{2}\end{array}$ & -7.8 \\
\hline $\begin{array}{l}\text { i-butyrate } \\
\text { i-butanol }\end{array}$ & $\begin{array}{l}-340.9 \\
-163.3\end{array}$ & -177.6 & $\begin{array}{l}\text { hydroxyl-carboxylate exchange } i-\mathrm{C}_{4}+\mathrm{EtOH} \rightarrow i- \\
\mathrm{C}_{4} \mathrm{OH}+\mathrm{C}_{2}\end{array}$ & -11.3 \\
\hline $\begin{array}{l}n \text {-pentanoate } \\
n \text {-pentanol }\end{array}$ & $\begin{array}{l}-336.6 \\
-159.0\end{array}$ & -177.6 & $\begin{array}{l}\text { hydroxyl-carboxylate exchange } n-C_{5}+E t O H \rightarrow n \text { - } \\
C_{5} \mathrm{OH}+\mathrm{C}_{2}\end{array}$ & -11.3 \\
\hline $\begin{array}{l}\text { n-octanoate } \\
\text { n-octanol }\end{array}$ & $\begin{array}{l}-315.7 \\
-139.1\end{array}$ & -176.6 & $\begin{array}{l}\text { hydroxyl-carboxylate exchange } n-\mathrm{C}_{8}+\mathrm{EtOH} \rightarrow n \text { - } \\
\mathrm{C}_{8} \mathrm{OH}+\mathrm{C}_{2}\end{array}$ & -12.3 \\
\hline n-hexanoate & -329.6 & & & \\
\hline
\end{tabular}




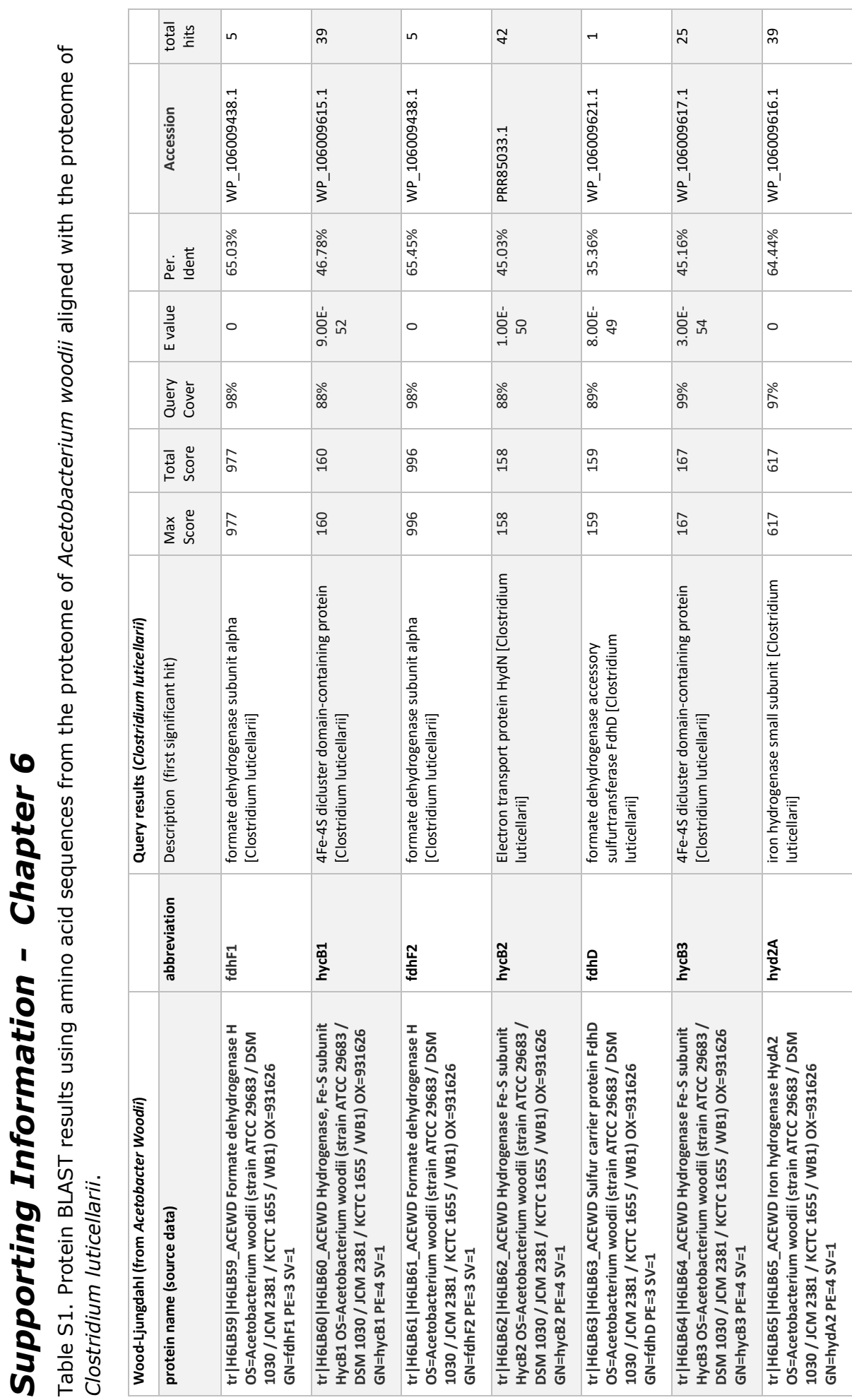




\begin{tabular}{|c|c|c|c|c|c|c|c|c|}
\hline$m$ & $-\gamma$ & $\sim$ & $\approx$ & -1 & H & $\approx$ & 0 & in \\
\hline 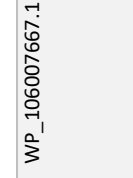 & 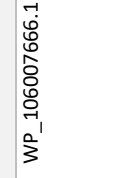 & 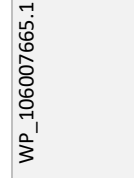 & 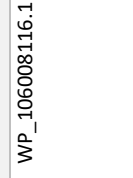 & 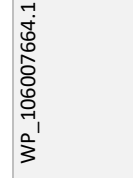 & 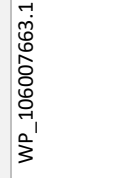 & 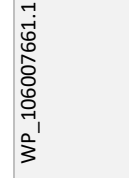 & 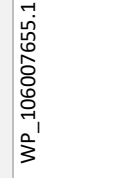 & $\begin{array}{l}\vec{i} \\
0 \\
0 \\
0 \\
0 \\
\stackrel{0}{1} \\
\dot{3}^{\prime}\end{array}$ \\
\hline 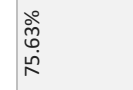 & $\begin{array}{l}\text { ळे } \\
\text { ले } \\
\text { in }\end{array}$ & $\begin{array}{l}\stackrel{\circ}{\circ} \\
\text { مे } \\
\text { on }\end{array}$ & $\begin{array}{l}\stackrel{\circ}{0} \\
\infty \\
\dot{\sigma}\end{array}$ & $\begin{array}{l}\stackrel{\circ}{0} \\
\infty \\
\infty \\
\text { m. }\end{array}$ & $\begin{array}{l}\text { ஓे } \\
\text { gे } \\
\text { gे }\end{array}$ & $\begin{array}{l}\stackrel{े}{0} \\
\stackrel{0}{0}\end{array}$ & 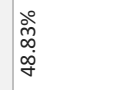 & $\begin{array}{l}\stackrel{\circ}{\circ} \\
\stackrel{\leftrightarrow}{~}\end{array}$ \\
\hline$\circ$ & 岁 & 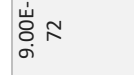 & 岁 § & $\underset{\dot{m}}{\dot{m}} \tilde{m}$ & 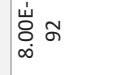 & 岕 & ○ & 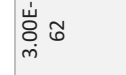 \\
\hline ठ̊ํㅇ & ふิ & ळ & वें & ذి & : & ふें & ఏ̊ำ & まें \\
\hline $\begin{array}{c}\infty \\
\infty \\
\infty\end{array}$ & ণ্ণ & స్తి & 品 & $\stackrel{m}{\vec{F}}$ & $\vec{N}$ & ষ্ল & \& & $\stackrel{9}{\circ}$ \\
\hline $\begin{array}{l}\infty \\
\infty \\
\infty\end{array}$ & 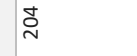 & సి & 尽 & $\stackrel{m}{\vec{F}}$ & $\stackrel{\vec{N}}{ }$ & ৪্ল & \& & $\stackrel{\circ}{\circ}$ \\
\hline 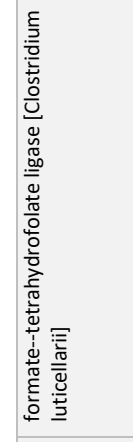 & 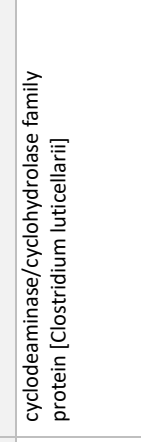 & 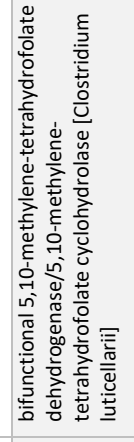 & 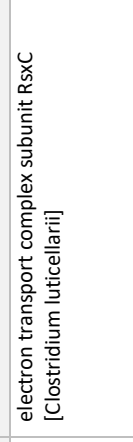 & 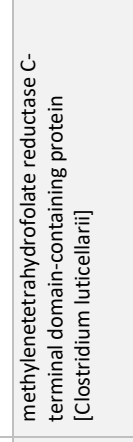 & 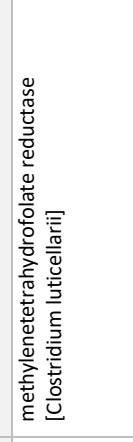 & 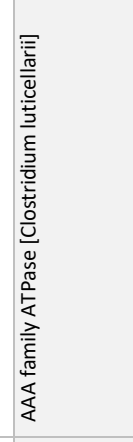 & 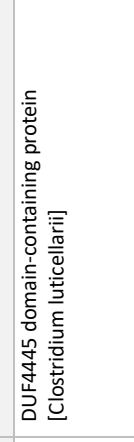 & 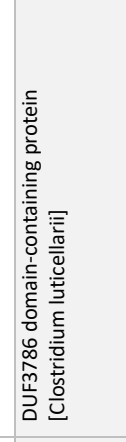 \\
\hline $\overrightarrow{\underline{\underline{v}}}$ & 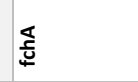 & $\stackrel{2}{\circ}$ & 总 & 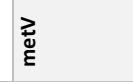 & 岕 & $\stackrel{\square}{\square}$ & ত্] & $\vec{\Xi}$ \\
\hline 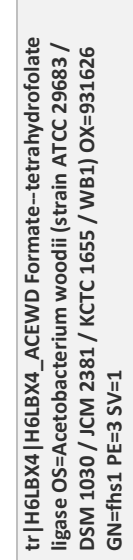 & 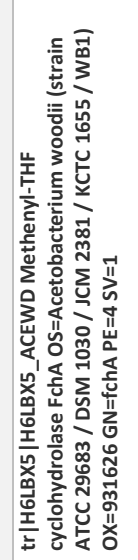 & 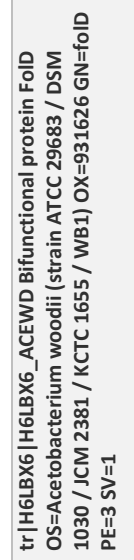 & 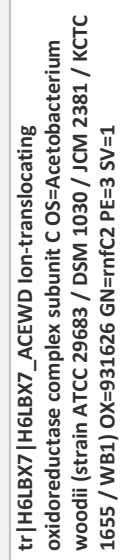 & 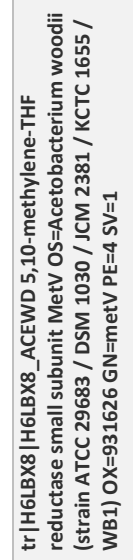 & 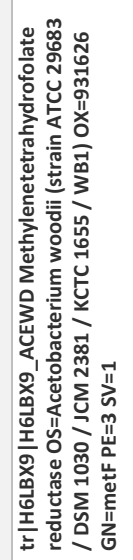 & 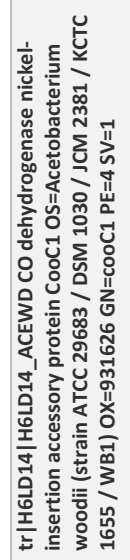 & 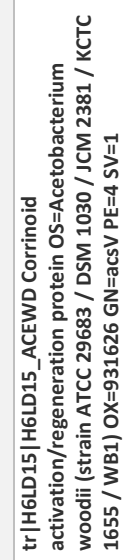 & 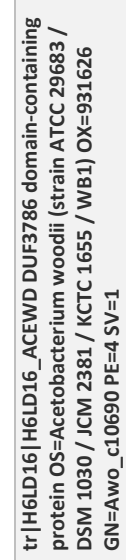 \\
\hline
\end{tabular}




\begin{tabular}{|c|c|c|c|c|c|c|}
\hline$\sim$ & a & $\sim$ & m & in & n & -1 \\
\hline $\begin{array}{l}\text { L } \\
0 \\
0 \\
0\end{array}$ & $\begin{array}{l}\overrightarrow{1} \\
0 \\
0 \\
0 \\
8 \\
0 \\
01 \\
1 \\
3\end{array}$ & $\begin{array}{l}\overrightarrow{1} \\
0 \\
0 \\
0 \\
0 \\
0 \\
0 \\
\vdots \\
3\end{array}$ & $\begin{array}{l}\overrightarrow{0} \\
0 \\
0 \\
0 \\
0 \\
0 \\
0 \\
0 \\
1 \\
\vdots \\
\vdots\end{array}$ & $\begin{array}{l}7 \\
0 \\
0 \\
0 \\
8 \\
0 \\
01 \\
1 \\
3\end{array}$ & $\begin{array}{l}-1 \\
0 \\
0 \\
0 \\
0 \\
0 \\
01 \\
1 \\
3\end{array}$ & 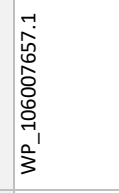 \\
\hline 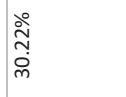 & 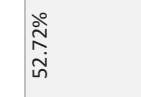 & 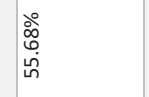 & 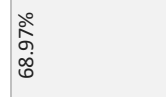 & 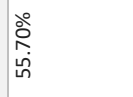 & 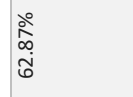 & $\begin{array}{l}\stackrel{\circ}{\infty} \\
ٌ \\
\widetilde{\omega}\end{array}$ \\
\hline 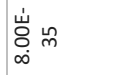 & 岁 & $\circ$ & 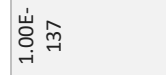 & 。 & 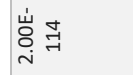 & 。 \\
\hline ळें & ळे & ळ్ & @্ণ & ذ্̊日 & ळ̊ & ஓे \\
\hline$\stackrel{乛}{*}$ & $\underset{m}{\mathbb{m}}$ & iे & $\stackrel{\infty}{\infty}$ & $\stackrel{\infty}{n}$ & $\underset{m}{~}$ & ঃ̆ \\
\hline$\approx$ & $\underset{ల}{\mathbb{N}}$ & हे & $\stackrel{\check{m}}{m}$ & $\stackrel{\infty}{n}$ & 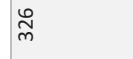 & ঃ̊ \\
\hline 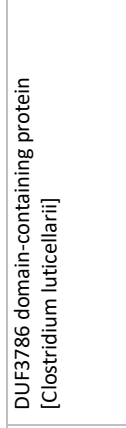 & 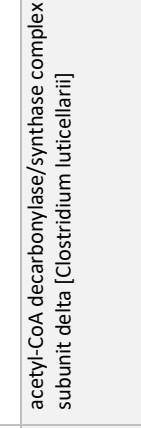 & 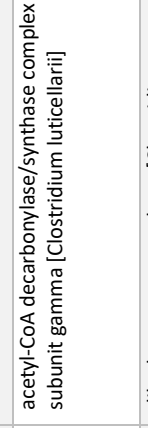 & 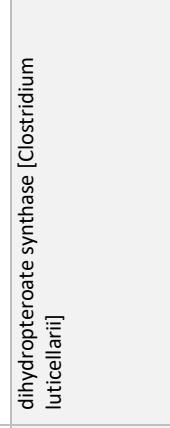 & 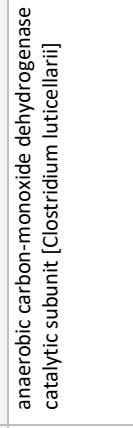 & 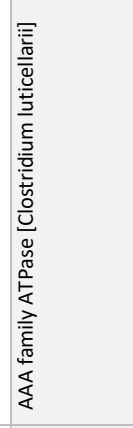 & 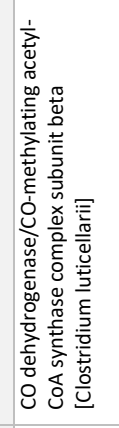 \\
\hline$\tilde{\tilde{z}}$ & 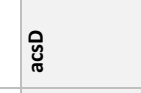 & 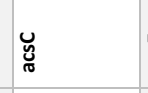 & 崙 & 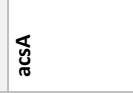 & ฮั & $\begin{array}{l}\overrightarrow{0} \\
\text { 岕 }\end{array}$ \\
\hline 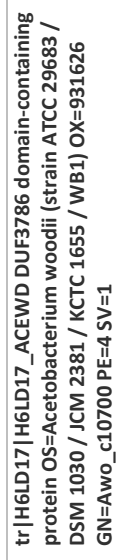 & 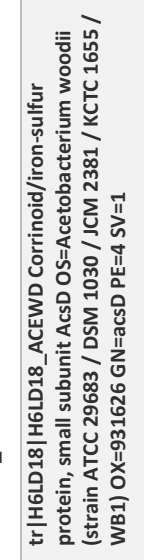 & 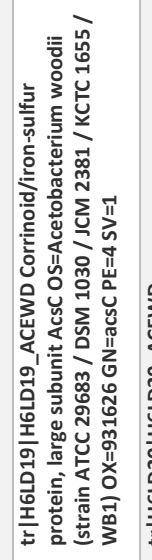 & 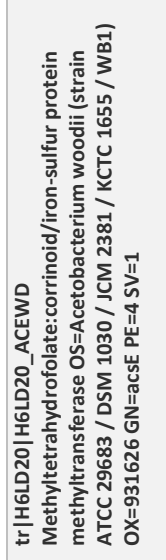 & 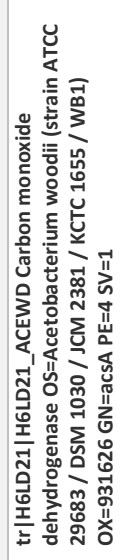 & 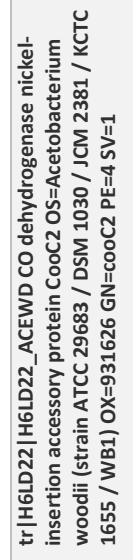 & 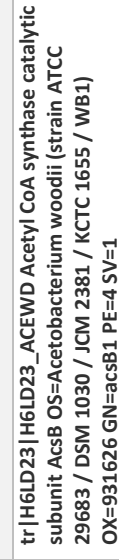 \\
\hline
\end{tabular}




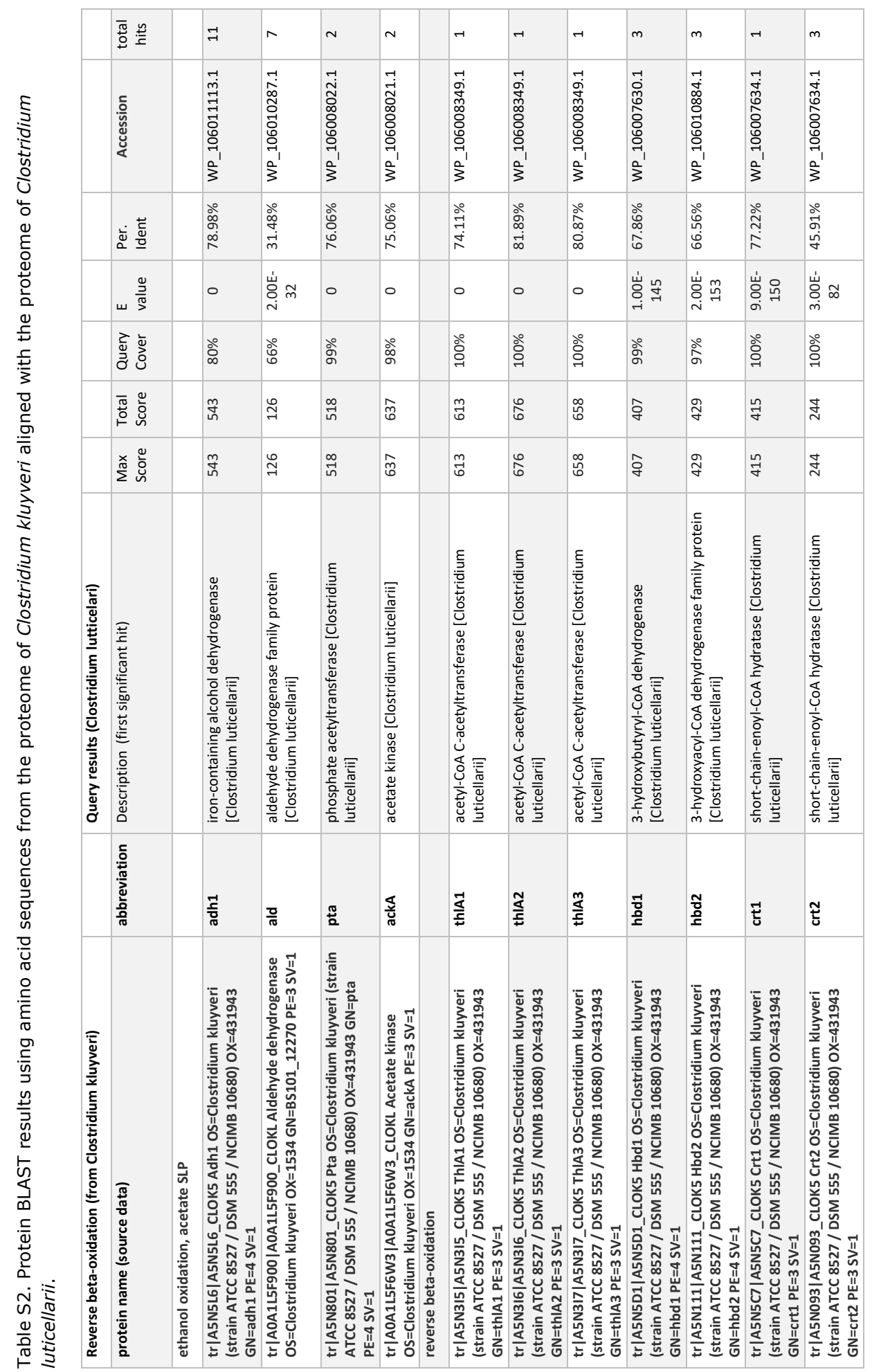




\begin{tabular}{|c|c|c|c|c|c|c|c|c|c|c|c|c|}
\hline m & $\sim$ & 6 & r & $\sigma$ & $\sigma$ & $\sim$ & $r$ & $m$ & 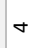 & q & $\dot{m}$ & $\rightarrow$ \\
\hline $\begin{array}{l}7 \\
\infty \\
0 \\
0 \\
0 \\
8 \\
0 \\
0 \\
0\end{array}$ & 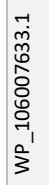 & $\begin{array}{l}F \\
\overrightarrow{0} \\
0 \\
0 \\
0 \\
0 \\
\overbrace{1}^{\prime}\end{array}$ & 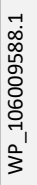 & 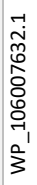 & 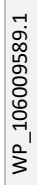 & 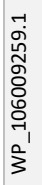 & 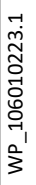 & 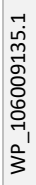 & $\begin{array}{l}\vec{j} \\
\overrightarrow{1} \\
0 \\
\stackrel{0}{0} \\
\stackrel{0}{0} \\
\overrightarrow{3} \\
\overrightarrow{3}\end{array}$ & 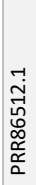 & 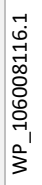 & 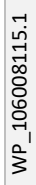 \\
\hline $\begin{array}{l}\stackrel{\circ}{\circ} \\
\stackrel{2}{0}\end{array}$ & $\begin{array}{l}\text { ふे } \\
\text { సं } \\
\text { g. }\end{array}$ & 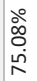 & 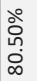 & 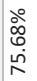 & $\begin{array}{l}\text { วे } \\
\text { के } \\
\text { İ }\end{array}$ & $\begin{array}{l}\grave{0} \\
\text { ஸి } \\
\infty\end{array}$ & 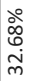 & 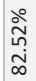 & $\begin{array}{l}\text { ஸे } \\
\text { مَ } \\
\text { مे }\end{array}$ & 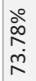 & $\begin{array}{l}\stackrel{\circ}{\circ} \\
\tilde{D}_{\infty}\end{array}$ & 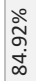 \\
\hline
\end{tabular}

\begin{tabular}{|c|c|c|c|c|c|c|c|c|c|c|c|c|}
\hline 0 & 0 & 0 & 0 & 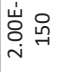 & 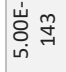 & 0 & 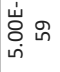 & ○ & 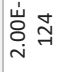 & 岁 꼬ํ & 0 & 0 \\
\hline ふั & ذ̊ำ & ふั & ڤ̊ & هัم & ুે & ふั้ & ஓे & ふे & ুั & ठ̊ำ & ڤั & ळ \\
\hline
\end{tabular}

\begin{tabular}{|c|c|c|c|c|c|c|c|c|c|c|c|c|}
\hline$\widehat{్}$ & $\hat{\tilde{\theta}}$ & คे & $\vec{F}$ & )े & 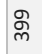 & $\stackrel{\infty}{\infty}$ & 号 & $\stackrel{\swarrow}{\curvearrowright}$ & 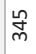 & $\tilde{\vartheta}$ & 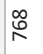 & 员 \\
\hline$\widehat{\widehat{\sigma}}$ & $\hat{\tilde{\theta}}$ & ને & 吕 & $\vec{F}$ & ஓ్ల & $\stackrel{\infty}{\infty}$ & $\stackrel{n}{g}$ & $\stackrel{n}{\circ}$ & 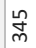 & $\tilde{\mathscr{\gamma}}$ & $\stackrel{\infty}{\circ}$ & 员 \\
\hline
\end{tabular}

\begin{tabular}{|c|c|c|c|c|c|c|c|c|c|c|c|c|c|}
\hline 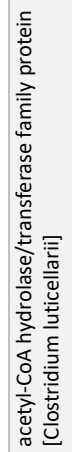 & 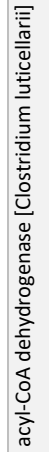 & 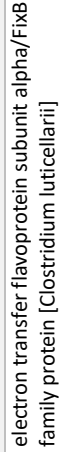 & 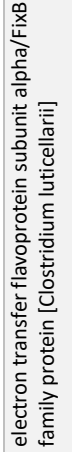 & 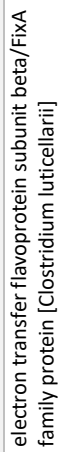 & 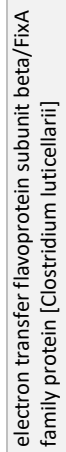 & 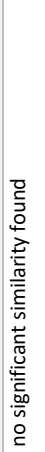 & 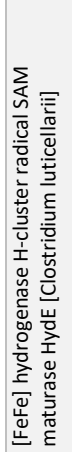 & 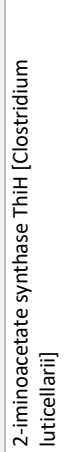 & 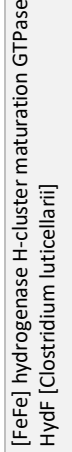 & 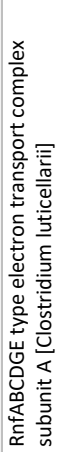 & 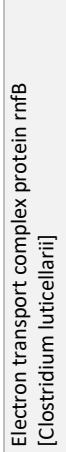 & 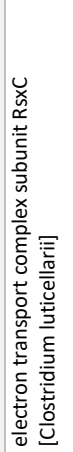 & 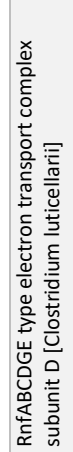 \\
\hline
\end{tabular}

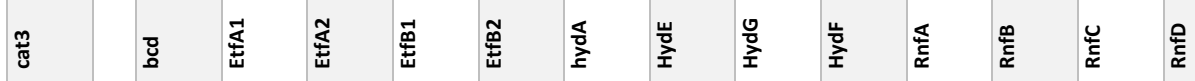

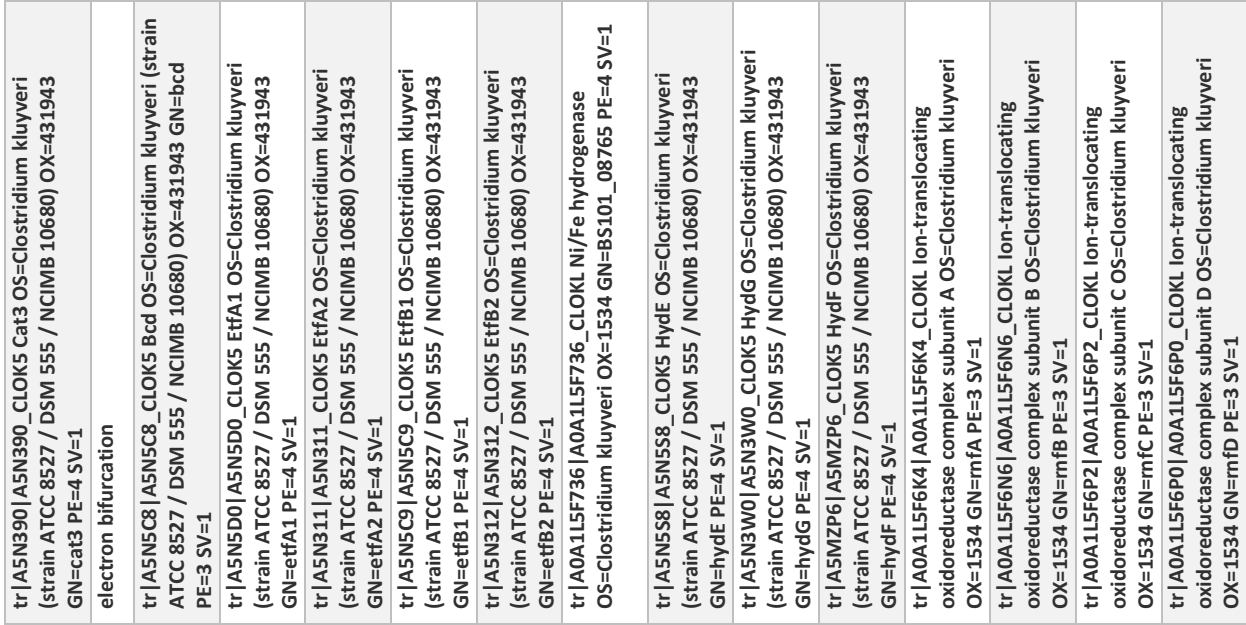




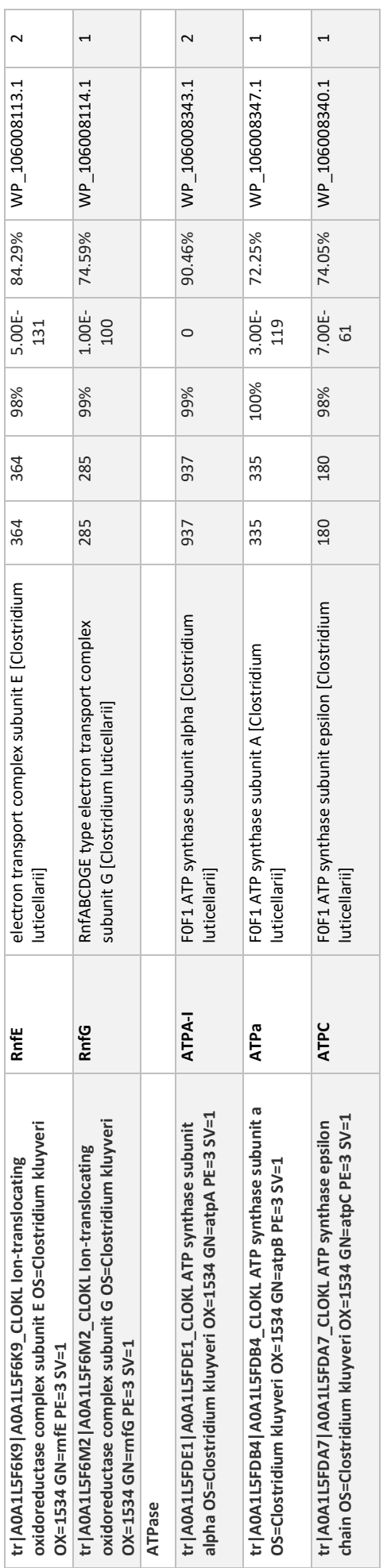




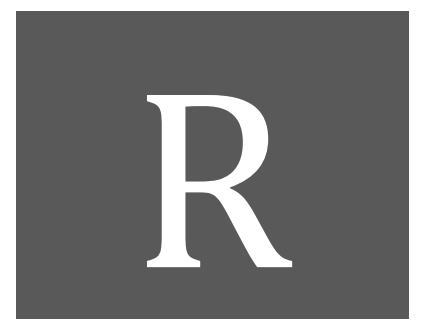

\section{References}




\section{References}

1. Kirchherr, J.; Reike, D.; Hekkert, M., Conceptualizing the circular economy: An analysis of 114 definitions. Resources, Conservation and Recycling 2017, 127, 221-232.

2. Murray, A.; Skene, K.; Haynes, K., The Circular Economy: An Interdisciplinary Exploration of the Concept and Application in a Global Context. Journal of Business Ethics 2017, 140, (3), 369-380.

3. Kirchherr, J.; Piscicelli, L.; Bour, R.; Kostense-Smit, E.; Muller, J.; Huibrechtse-Truijens, A.; Hekkert, M., Barriers to the Circular Economy: Evidence From the European Union (EU). Ecological Economics 2018, 150, 264-272.

4. $\quad$ Bet, B.; Kas, J.; Truijens, D.; Lee, S. v. d.; Broere, J.; Leising, E.; Nuninga, T.; Bose, P.; Ravensberg, E. v.; Francesco, E. d.; Wang, Y.; Hassan, A.; Fanitabasi, F.; Wang, Z., Barriers and Best Practices for the Circular Economy. In 2018.

5. Boyce, J. K., Inequality as a cause of environmental degradation. Ecological Economics 1994, 11, (3), 169-178.

6. Ostolaza, J. M. B.; Orayen, R. E.; Eraso, N. O. In Economic inequality and environmental degradation: An experimental study, XXIII Encuentro de Economía Pública, 2016; Servicio de Publicaciones: 2016; p 17.

7. Liu, Q.; Wang, S.; Zhang, W.; Li, J., Income distribution and environmental quality in China: A spatial econometric perspective. Journal of Cleaner Production 2018, 205, 14-26.

8. $\quad$ Florencio, L., The fate of methanol in anaerobic bioreactors. Florencio: 1994.

9. Madani, K.; Hipel, K. W., Non-Cooperative Stability Definitions for Strategic Analysis of Generic Water Resources Conflicts. Water Resources Management 2011, 25, (8), 1949-1977.

10. Palafox-Alcantar, P. G.; Hunt, D. V. L.; Rogers, C. D. F., The complementary use of game theory for the circular economy: A review of waste management decision-making methods in civil engineering. Waste Management 2020, 102, 598-612.

11. Baas Becking, L. G. M., Geobiologie of inleiding tot de milieukunde. Den Haag : Van Stockum: 1934.

12. McLeay, M.; Radia, A.; Thomas, R., Money creation in the modern economy. Bank of England Quarterly Bulletin 2014, Q1.

13. Jackson, T.; Victor, P. A., Does credit create a 'growth imperative'? A quasi-stationary economy with interest-bearing debt. Ecological Economics 2015, 120, 32-48.

14. Leonard, D.; Treiblmaier, H., Can Cryptocurrencies Help to Pave the Way to a More Sustainable Economy? Questioning the Economic Growth Paradigm. In Business Transformation through Blockchain: Volume II, Treiblmaier, H.; Beck, R., Eds. Springer International Publishing: Cham, 2019; pp 183-205.

15. Baumeister, C. a. B., Luca, Unconventional Monetary Policy and the Great Recession - Estimating the Impact of a Compression in the Yield Spread at the Zero Lower Bound ECB Working Paper No. 1258. 2010.

16. Farhi, E.; Tirole, J., Collective Moral Hazard, Maturity Mismatch, and Systemic Bailouts. American Economic Review 2012, 102, (1), 60-93.

17. Bernoth, K.; König, P.; Beckers, B., ECB asset purchases may affect wealth distribution. DIW Economic Bulletin 2016, 6, (7), 75-81.

18. Greyson, J., An economic instrument for zero waste, economic growth and sustainability. Journal of Cleaner Production 2007, 15, (13), 1382-1390.

19. Zwitter, A.; Hazenberg, J., Decentralized Network Governance: Blockchain Technology and the Future of Regulation. Frontiers in Blockchain-Blockchain for Good, Forthcoming 2020.

20. Pan, Z.; Pan, J.; Zhang, J., Research on Decentralization of Inclusive Finance based on Blockchain Technology.

21. Myeong, S.; Jung, Y., Administrative reforms in the fourth industrial revolution: the case of blockchain use. Sustainability 2019, 11, (14), 3971.

22. Angenent, L. T.; Richter, H.; Buckel, W.; Spirito, C. M.; Steinbusch, K. J. J.; Plugge, C. M.; Strik, D. P. B. T. B.; Grootscholten, T. I. M.; Buisman, C. J. N.; Hamelers, H. V. M., Chain Elongation with Reactor Microbiomes: Open-Culture Biotechnology To Produce Biochemicals. Environmental Science and Technology 2016, 50, (6), 2796-2810.

23. Kleerebezem, R.; Joosse, B.; Rozendal, R.; Van Loosdrecht, M. C. M., Anaerobic digestion without biogas? Reviews in Environmental Science and Bio/Technology 2015, 14, (4), 787-801.

24. Urban, C.; Xu, J.; Sträuber, H.; dos Santos Dantas, T. R.; Mühlenberg, J.; Härtig, C.; Angenent, L. T.; Harnisch, F., Production of drop-in fuels from biomass at high selectivity by combined microbial and electrochemical conversion. Energy \& Environmental Science 2017, 10, (10), 2231-2244. 
25. Agler, M. T.; Wrenn, B. A.; Zinder, S. H.; Angenent, L. T., Waste to bioproduct conversion with undefined mixed cultures: the carboxylate platform. Trends in biotechnology 2011, 29, (2), 70-78.

26. Verbraeken, H., ChainCraft gaat op grotere schaal biologische vetzuren maken. Financieel Dagblad 2017, Ondernemen.

27. Kleerebezem, R.; van Loosdrecht, M. C. M., Mixed culture biotechnology for bioenergy production. Current Opinion in Biotechnology 2007, 18, (3), 207-212.

28. Kleerebezem, R.; Van Loosdrecht, M. C. M., A Generalized Method for Thermodynamic State Analysis of Environmental Systems. Critical Reviews in Environmental Science and Technology 2010, 40, (1), 1-54.

29. Biorender, Created with BioRender.

30. Kremp, F.; Poehlein, A.; Daniel, R.; Müller, V., Methanol metabolism in the acetogenic bacterium Acetobacterium woodii. Environmental Microbiology 2018, 20, (12), 4369-4384.

31. Seedorf, H.; Fricke, W. F.; Veith, B.; Brüggemann, H.; Liesegang, H.; Strittmatter, A.; Miethke, M.; Buckel, W.; Hinderberger, J.; Li, F.; Hagemeier, C.; Thauer, R. K.; Gottschalk, G., The genome of Clostridium kluyveri, a strict anaerobe with unique metabolic features. Proceedings of the National Academy of Sciences 2008, 105, (6), 2128-2133.

32. De Groof, V.; Coma, M.; Arnot, T.; Leak, D. J.; Lanham, A. B., Medium Chain Carboxylic Acids from Complex Organic Feedstocks by Mixed Culture Fermentation. Molecules (Basel, Switzerland) 2019, 24, (3), 398.

33. Kallscheuer, N.; Polen, T.; Bott, M.; Marienhagen, J., Reversal of $\beta$-oxidative pathways for the microbial production of chemicals and polymer building blocks. Metabolic Engineering 2017, 42, 33-42.

34. Haapalainen, A. M.; Meriläinen, G.; Wierenga, R. K., The thiolase superfamily: condensing enzymes with diverse reaction specificities. Trends in Biochemical Sciences 2006, 31, (1), 64-71.

35. Chen, W.-S.; Huang, S.; Strik, D. P.; Buisman, C. J. N., Isobutyrate biosynthesis via methanol chain elongation: converting organic wastes to platform chemicals. J. Chem. Technol. Biotechnol. 2017, 92, (6), 13701379.

36. Lee, I.; Johnson, L. A.; Hammond, E. G., Use of branched-chain esters to reduce the crystallization temperature of biodiesel. Journal of the American Oil Chemists' Society 1995, 72, (10), 1155-1160.

37. Atsumi, S.; Hanai, T.; Liao, J. C., Non-fermentative pathways for synthesis of branched-chain higher alcohols as biofuels. Nature 2008, 451,86 .

38. Zhang, Z. C.; Dery, M.; Zhang, S.; Steichen, D., New process for the production of branched-chain fatty acids. Journal of Surfactants and Detergents 2004, 7, (3), 211-215.

39. Cracan, V.; Banerjee, R., Novel B12-Dependent Acyl-CoA Mutases and Their Biotechnological Potential. Biochemistry 2012, 51, (31), 6039-6046.

40. Roghair, M.; Liu, Y.; Strik, D. P. B. T. B.; Weusthuis, R. A.; Bruins, M. E.; Buisman, C. J. N., Development of an Effective Chain Elongation Process From Acidified Food Waste and Ethanol Into n-Caproate. Frontiers in Bioengineering and Biotechnology 2018, 6, (50), DOI: 10.3389/fbioe.2018.00050.

41. Kerby, R.; Niemczura, W.; Zeikus, J. G., Single-carbon catabolism in acetogens: analysis of carbon flow in Acetobacterium woodii and Butyribacterium methylotrophicum by fermentation and $13 \mathrm{C}$ nuclear magnetic resonance measurement. J Bacteriol 1983, 155, (3), 1208-18.

42. Heijthuijsen, J. H. F. G.; Hansen, T. A., Interspecies hydrogen transfer in co-cultures of methanolutilizing acidogens and sulfate-reducing or methanogenic bacteria. FEMS Microbiology Letters 1986, 38, (1), 5764.

43. Lindley, N. D.; Loubière, P.; Pacaud, S.; Mariotto, C.; Goma, G., Novel Products of the Acidogenic Fermentation of Methanol during Growth of Eubacterium limosum in the Presence of High Concentrations of Organic Acids. Microbiology 1987, 133, (12), 3557-3563.

44. Pacaud, S.; Loubiere, P.; Goma, G., Methanol metabolism byEubacterium limosum B2: Effects of pH and carbon dioxide on growth and organic acid production. Current Microbiology 1985, 12, (5), 245-250.

45. Chen, W. S.; Ye, Y.; Steinbusch, K. J. J.; Strik, D. P. B. T. B.; Buisman, C. J. N., Methanol as an alternative electron donor in chain elongation for butyrate and caproate formation. Biomass and Bioenergy 2016, 93, 201-208.

46. de Smit, S. M.; de Leeuw, K. D.; Buisman, C. J. N.; Strik, D. P. B. T. B., Continuous n-valerate formation from propionate and methanol in an anaerobic chain elongation open-culture bioreactor. Biotechnology for Biofuels 2019, 12, (1), 132.

47. Westphal, L.; Wiechmann, A.; Baker, J.; Minton, N. P.; Müller, V., The Rnf Complex Is an Energy-Coupled Transhydrogenase Essential To Reversibly Link Cellular NADH and Ferredoxin Pools in the Acetogen <span class="named-content genus-species" id="named-content-1" $>$ Acetobacterium woodii $</$ span $>$. Journal of Bacteriology 2018, 200, (21), e00357-18. 
48. Barker, H. A., The production of caproic and butyric acids by the methane fermentation of ethyl alcohol. Archiv für Mikrobiologie 1937, 8, (1), 415-421.

49. Buckel, W.; Thauer, R. K., Energy conservation via electron bifurcating ferredoxin reduction and proton/Na+ translocating ferredoxin oxidation. Biochimica et Biophysica Acta (BBA) - Bioenergetics 2013, 1827, (2), 94-113.

50. Müller, V.; Chowdhury, N. P.; Basen, M., Electron Bifurcation: A Long-Hidden Energy-Coupling Mechanism. Annual Review of Microbiology 2018, 72, (1), 331-353.

51. Morris, B. E. L.; Henneberger, R.; Huber, H.; Moissl-Eichinger, C., Microbial syntrophy: interaction for the common good. FEMS Microbiology Reviews 2013, 37, (3), 384-406.

52. Br, V.; Shetty, V.; Saidutta, M. B., Characterisitcs of biofilms in bioreactors-A review. 2013.

53. Tomlinson, N.; Barker, H. A., Carbon dioxide and acetate utilization by clostridium kluyveri. I. Influence of nutritional conditions on utilization patterns. Journal of Biological Chemistry 1954, 209(2), 585-95.

54. Shin, J.; Cho, S.-K.; Lee, J.; Hwang, K.; Chung, J. W.; Jang, H.-N.; Shin, S. G., Performance and microbial community dynamics in anaerobic digestion of waste activated sludge: Impact of immigration. Energies 2019, 12, (3), 573.

55. Okada, D. Y.; Costa, R. B.; Garcia, C. d. C. B.; Pozzi, E.; Souza, T. S.; Foresti, E., Anoxic Microbial Community Robustness Under Variation of Hydraulic Retention Time and Availability of Endogenous Electron Donors. Applied biochemistry and biotechnology 2020.

56. Sindhu, R.; Rajesh, R.; Godan, T.; Binod, P.; Pandey, A., Bioengineering advancements, innovations and challenges on green synthesis of 2, 5-furan dicarboxylic acid. 2019.

57. Roghair, M.; Liu, Y.; Adiatma, J. C.; Weusthuis, R. A.; Bruins, M. E.; Buisman, C. J. N.; Strik, D. P. B. T. B., Effect of $\mathrm{n}$-Caproate Concentration on Chain Elongation and Competing Processes. ACS Sustainable Chemistry \& Engineering 2018, 6, (6), 7499-7506.

58. Florencio, L.; Field, J. A.; Lettinga, G., Substrate competition between methanogens and acetogens during the degradation of methanol in UASB reactors. Water Research 1995, 29, (3), 915-922.

59. Infantes, D.; González del Campo, A.; Villaseñor, J.; Fernández, F. J., Kinetic model and study of the influence of $\mathrm{pH}$, temperature and undissociated acids on acidogenic fermentation. Biochemical Engineering Journal 2012, 66, 66-72.

60. Heijnen, J. J.; Kleerebezem, R., Bioenergetics of microbial growth. Encyclopedia of Industrial Biotechnology: Bioprocess, Bioseparation, and Cell Technology 2009, 1-66.

61. Hanselmann, K., Microbial energetics applied to waste repositories. Experientia 1991, 47, (7), 645-687.

62. Smith, D. P.; McCarty, P. L., Energetic and rate effects on methanogenesis of ethanol and propionate in perturbed CSTRs. Biotechnol. Bioeng. 1989, 34, (1), 39-54.

63. Bertsch, J.; Siemund, A. L.; Kremp, F.; Müller, V., A novel route for ethanol oxidation in the acetogenic bacterium Acetobacterium woodii: the acetaldehyde/ethanol dehydrogenase pathway. Environ Microbiol 2016, 18, (9), 2913-22.

64. Frimmer, U.; Widdel, F., Oxidation of ethanol by methanogenic bacteria. Archives of Microbiology 1989, $152,(5), 479-483$

65. Jimenez-Diaz, L.; Caballero, A.; Segura, A., Pathways for the Degradation of Fatty Acids in Bacteria. In Aerobic Utilization of Hydrocarbons, Oils and Lipids, Rojo, F., Ed. Springer International Publishing: Cham, 2017; pp 1-23.

66. Breznak, J. A.; Kane, M. D., Microbial $\mathrm{H} 2 / \mathrm{CO} 2$ acetogenesis in animal guts: nature and nutritional significance. FEMS Microbiology Letters 1990, 87, (3), 309-313.

67. Wuhrmann, K., Ecology of methanogenic systems in nature. In New Trends in Research and Utilization of Solar Energy through Biological Systems, Mislin, H.; Bachofen, R., Eds. Birkhäuser Basel: Basel, 1982; pp 121126.

68. Lovley, D. R.; Klug, M. J., Methanogenesis from methanol and methylamines and acetogenesis from hydrogen and carbon dioxide in the sediments of a eutrophic lake. Applied and environmental microbiology 1983, 45, (4), 1310-1315.

69. Kurade, M. B.; Saha, S.; Salama, E.-S.; Patil, S. M.; Govindwar, S. P.; Jeon, B.-H., Acetoclastic methanogenesis led by Methanosarcina in anaerobic co-digestion of fats, oil and grease for enhanced production of methane. Bioresource Technology 2019, 272, 351-359.

70. Di Maio, F.; Rem, P. C.; Baldé, K.; Polder, M., Measuring resource efficiency and circular economy: A market value approach. Resources, Conservation and Recycling 2017, 122, 163-171.

71. Saswattecha, K.; Kroeze, C.; Jawjit, W.; Hein, L., Assessing the environmental impact of palm oil produced in Thailand. Journal of Cleaner Production 2015, 100, 150-169. 
72. Bagby, M. O.; Johnson Jr, R. W.; Daniels, R. W.; Contrell, R. R.; Sauer, E. T.; Keenan, M. J.; Krevalis, M. A.; Updated By, S., Carboxylic Acids. John Wiley \& Sons, Inc (Ed.): 2003.

73. De Groof, V.; Coma, M.; Arnot, T.; Leak, D. J.; Lanham, A. B., Medium Chain Carboxylic Acids from Complex Organic Feedstocks by Mixed Culture Fermentation. Molecules 2019, 24, (3), 398.

74. Steinbusch, K. J.; Hamelers, H. V.; Plugge, C. M.; Buisman, C. J., Biological formation of caproate and caprylate from acetate: fuel and chemical production from low grade biomass. Energy \& Environmental Science 2011, 4, (1), 216-224.

75. Han, W.; He, P.; Shao, L.; Lü, F., Road to full bioconversion of biowaste to biochemicals centering on chain elongation: A mini review. Journal of Environmental Sciences (China) 2019, 86, 50-64.

76. Yang, D.; Zhu, Q.; Chen, C.; Liu, H.; Liu, Z.; Zhao, Z.; Zhang, X.; Liu, S.; Han, B., Selective electroreduction of carbon dioxide to methanol on copper selenide nanocatalysts. Nature Communications 2019, $10,(1), 677$.

77. Demirbas, A., Biomethanol Production from Organic Waste Materials. Energy Sources, Part A: Recovery, Utilization, and Environmental Effects 2008, 30, (6), 565-572.

78. Balan, V.; Chiaramonti, D.; Kumar, S., Review of US and EU initiatives toward development, demonstration, and commercialization of lignocellulosic biofuels. Biofuels, Bioproducts and Biorefining 2013, 7, (6), 732-759.

79. Chen, W.-S.; Huang, S.; Plugge, C. M.; Buisman, C. J. N.; Strik, D. P. B. T. B., Concurrent use of methanol and ethanol for chain-elongating short chain fatty acids into caproate and isobutyrate. Journal of Environmental Management 2020, 258, 110008.

80. Vassilev, I.; Hernandez, P. A.; Batlle-Vilanova, P.; Freguia, S.; Krömer, J. O.; Keller, J.; Ledezma, P.; Virdis, B., Microbial Electrosynthesis of Isobutyric, Butyric, Caproic Acids, and Corresponding Alcohols from Carbon Dioxide. ACS Sustainable Chemistry \& Engineering 2018, 6, (7), 8485-8493.

81. Vassilev, I.; Kracke, F.; Freguia, S.; Keller, J.; Krömer, J. O.; Ledezma, P.; Virdis, B., Microbial electrosynthesis system with dual biocathode arrangement for simultaneous acetogenesis, solventogenesis and carbon chain elongation. Chemical Communications 2019, 55, (30), 4351-4354.

82. Zhang, K.; Woodruff, A. P.; Xiong, M.; Zhou, J.; Dhande, Y. K., A Synthetic Metabolic Pathway for Production of the Platform Chemical Isobutyric Acid. ChemSusChem 2011, 4, (8), 1068-1070.

83. Lang, K.; Zierow, J.; Buehler, K.; Schmid, A., Metabolic engineering of Pseudomonas sp. strain VLB120 as platform biocatalyst for the production of isobutyric acid and other secondary metabolites. Microbial cell factories 2014, 13, 2-2.

84. Ruppert, W.; Siegert, H.-J., Method for making isobutyric acid. In Google Patents: 1993.

85. Zhang, Z. C.; Dery, M.; Zhang, S.; Steichen, D., New process for the production of branched - chain fatty acids. Journal of surfactants and detergents 2004, 7, (3), 211-215.

86. Perez, J. M.; Richter, H.; Loftus, S. E.; Angenent, L. T., Biocatalytic reduction of short-chain carboxylic acids into their corresponding alcohols with syngas fermentation. Biotechnol. Bioeng. 2013, 110, (4), 1066-1077.

87. Rodríguez, J.; Lema, J. M.; Kleerebezem, R., Energy-based models for environmental biotechnology. Trends in Biotechnology 2008, 26, (7), 366-374.

88. Phillips, J. R.; Klasson, K. T.; Clausen, E. C.; Gaddy, J. L., Biological production of ethanol from coal synthesis gas - Medium development studies. Applied Biochemistry and Biotechnology 1993, 39-40, (1), 559571.

89. de Leeuw, K.; Buisman, C. J.; Strik, D. P., Branched Medium Chain Fatty Acids: iso-caproate formation from iso-butyrate broadens the product spectrum for microbial chain elongation. Environmental Science \& Technology 2019.

90. Cresson, R.; Escudié, R.; Steyer, J.-P.; Delgenès, J.-P.; Bernet, N., Competition between planktonic and fixed microorganisms during the start-up of methanogenic biofilm reactors. Water Research 2008, 42, (3), 792800 .

91. Angelidaki, I.; Ahring, B. K., Isomerization ofn- andi-butyrate in anaerobic methanogenic systems. Antonie van Leeuwenhoek 1995, 68, (4), 285-291.

92. Tholozan, J.-L.; Samain, E.; Grivet, J.-P., Isomerization between n-butyrate and isobutyrate in enrichment cultures. FEMS Microbiology Ecology 1988, 4, (3-4), 187-191.

93. Oude Elferink, S. J. W. H.; Lens, P. N. L.; Dijkema, C.; Stams, A. J. M., Isomerization of butyrate to isobutyrate by Desulforhabdus amnigenus. FEMS Microbiology Letters 1996, 142, (2), 237-241.

94. Wu, W.-M.; Jain, M. K.; Zeikus, J. G., Anaerobic Degradation of Normal- and Branched-Chain Fatty Acids with Four or More Carbons to Methane by a Syntrophic Methanogenic Triculture. Applied and Environmental Microbiology 1994, 60, (7), 2220-2226. 
95. Matthies, C.; Schink, B., Reciprocal Isomerization of Butyrate and Isobutyrate by the Strictly Anaerobic Bacterium Strain WoG13 and Methanogenic Isobutyrate Degradation by a Defined Triculture. Appl Environ Microbiol 1992, 58, (5), 1435-1439.

96. Pacaud, S.; Loubière, P.; Goma, G.; Lindley, N. D., Organic acid production during methylotrophic growth of Eubacterium limosum B2: displacement towards increased butyric acid yields by supplementing with acetate. Applied Microbiology and Biotechnology 1986, 23, (5), 330-335.

97. Jourdin, L.; Raes, S. M.; Buisman, C. J.; Strik, D. P., Critical biofilm growth throughout unmodified carbon felts allows continuous bioelectrochemical chain elongation from $\mathrm{CO} 2$ up to caproate at high current density. Frontiers in Energy Research 2018, 6, 7.

98. Takahashi, S.; Tomita, J.; Nishioka, K.; Hisada, T.; Nishijima, M., Development of a prokaryotic universal primer for simultaneous analysis of Bacteria and Archaea using next-generation sequencing. PloS one 2014, 9, (8), e105592.

99. Caporaso, J. G.; Kuczynski, J.; Stombaugh, J.; Bittinger, K.; Bushman, F. D.; Costello, E. K.; Fierer, N.; Peña, A. G.; Goodrich, J. K.; Gordon, J. I.; Huttley, G. A.; Kelley, S. T.; Knights, D.; Koenig, J. E.; Ley, R. E.; Lozupone, C. A.; McDonald, D.; Muegge, B. D.; Pirrung, M.; Reeder, J.; Sevinsky, J. R.; Turnbaugh, P. J.; Walters, W. A.; Widmann, J.; Yatsunenko, T.; Zaneveld, J.; Knight, R., QIIME allows analysis of high-throughput community sequencing data. Nature methods 2010, 7, (5), 335-336.

100. Quast, C.; Pruesse, E.; Yilmaz, P.; Gerken, J.; Schweer, T.; Yarza, P.; Peplies, J.; Glöckner, F. O., The SILVA ribosomal RNA gene database project: improved data processing and web-based tools. Nucleic Acids Res 2013, 41, (Database issue), D590-D596.

101. Edgar, R. C., Search and clustering orders of magnitude faster than BLAST. Bioinformatics 2010, 26, (19), 2460-2461.

102. Duboc, P.; Schill, N.; Menoud, L.; Van Gulik, W.; Von Stockar, U., Measurements of sulfur, phosphorus and other ions in microbial biomass: influence on correct determination of elemental composition and degree of reduction. Journal of Biotechnology 1995, 43, (2), 145-158.

103. Yu, H.-Q.; Mu, Y.; Fang, H. H. P., Thermodynamic analysis of product formation in mesophilic acidogenesis of lactose. Biotechnology and Bioengineering 2004, 87, (7), 813-822.

104. Van Brabant, P.; Ganigué, R.; Rabaey, K., Understanding bio-isomerisation during methanol fermentation. MSc thesis, Universiteit Gent 2019.

105. Zellner, G.; Messner, P.; Winter, J.; Stackebrandt, E., Methanoculleus palmolei sp. nov., an irregularly coccoid methanogen from an anaerobic digester treating wastewater of a palm oil plant in North-Sumatra, Indonesia. International Journal of Systematic and Evolutionary Microbiology 1998, 48, (4), 1111-1117.

106. Iino, T.; Tamaki, H.; Tamazawa, S.; Ueno, Y.; Ohkuma, M.; Suzuki, K.-i.; Igarashi, Y.; Haruta, S., $<\mathrm{i}>$ Candidatus $</ \mathrm{i}>$ Methanogranum caenicola: a Novel Methanogen from the Anaerobic Digested Sludge, and Proposal of $<i>$ Methanomassiliicoccaceae $</ i>$ fam. nov. and $<i>$ Methanomassiliicoccales $</ i>$ ord. nov., for a Methanogenic Lineage of the Class $<i>$ Thermoplasmata</i>. Microbes Environ 2013, 28, (2), 244-250.

107. Kröninger, L.; Gottschling, J.; Deppenmeier, U., Growth Characteristics of Methanomassiliicoccus luminyensis and Expression of Methyltransferase Encoding Genes. Archaea (Vancouver, B.C.) 2017, 2017, 2756573-2756573.

108. Florencio, L. The fate of methanol in anaerobic bioreactors. Florencio, S.I., 1994.

109. Florencio, L.; Nozhevnikova, A.; van Langerak, A.; Stams, A. J. M.; Field, J. A.; Lettinga, G., Acidophilic degradation of methanol by a methanogenic enrichment culture. FEMS Microbiology Letters 1993, 109, (1), 1-6.

110. Florencio, L.; Field, J. A.; Lettinga, G., High-rate anaerobic treatment of alcoholic wastewaters. Brazilian Journal of Chemical Engineering 1997, 14.

111. Staley, B. F.; de Los Reyes, F. L., 3rd; Barlaz, M. A., Effect of spatial differences in microbial activity, $\mathrm{pH}$, and substrate levels on methanogenesis initiation in refuse. Applied and environmental microbiology 2011, 77, (7), 2381-2391

112. Poehlein, A.; Bremekamp, R.; Lutz, V. T.; Schulz, L. M.; Daniel, R., Draft Genome Sequence of the Butanoic Acid-Producing Bacterium Clostridium luticellarii DSM 29923, Used for Strong Aromatic Chinese Liquor Production. Genome Announc. 2018, 6, (18), e00377-18.

113. Tholozan, J.-L.; Samain, E.; Grivet, J.-P., Isomerization between n-butyrate and isobutyrate in enrichment cultures. FEMS Microbiology Letters 1988, 53, (3), 187-191.

114. Wu, W.-M.; Jain, M. K.; Hickey, R. F.; Zeikus, J. G., Perturbation of syntrophic isobutyrate and butyrate degradation with formate and hydrogen. Biotechnology and Bioengineering 1996, 52, (3), 404-411.

115. Sousa, D. Z.; Smidt, H.; Alves, M. M.; Stams, A. J. M., Syntrophomonas zehnderi sp. nov., an anaerobe that degrades long-chain fatty acids in co-culture with Methanobacterium formicicum. International Journal of Systematic and Evolutionary Microbiology 2007, 57, (3), 609-615.

116. Narihiro, T.; Nobu, M. K.; Tamaki, H.; Kamagata, Y.; Sekiguchi, Y.; Liu, W.-T., Comparative Genomics of Syntrophic Branched-Chain Fatty Acid Degrading Bacteria. Microbes Environ 2016, 31, (3), 288-292. 
117. Amador-Noguez, D.; Brasg, I. A.; Feng, X.-J.; Roquet, N.; Rabinowitz, J. D., Metabolome Remodeling during the Acidogenic-Solventogenic Transition in Clostridium acetobutylicum. Applied and Environmental Microbiology 2011, 77, (22), 7984-7997.

118. Chohnan, S.; Furukawa, H.; Fujio, T.; Nishihara, H.; Takamura, Y., Changes in the size and composition of intracellular pools of nonesterified coenzyme $A$ and coenzyme $A$ thioesters in aerobic and facultatively anaerobic bacteria. Applied and environmental microbiology 1997, 63, (2), 553-560.

119. Vadali, R. V.; Bennett, G. N.; San, K.-Y., Cofactor engineering of intracellular CoA/acetyl-CoA and its effect on metabolic flux redistribution in Escherichia coli. Metabolic Engineering 2004, 6, (2), 133-139.

120. Wiesenborn, D. P.; Rudolph, F. B.; Papoutsakis, E. T., Coenzyme A transferase from Clostridium acetobutylicum ATCC 824 and its role in the uptake of acids. Appl Environ Microbiol 1989, 55, (2), 323-329.

121. Cracan, V.; Padovani, D.; Banerjee, R., IcmF Is a Fusion between the Radical B 12 Enzyme IsobutyrylCoA Mutase and Its G-protein Chaperone. The Journal of biological chemistry 2009, 285, 655-66.

122. Wilbanks, B.; Trinh, C. T., Comprehensive characterization of toxicity of fermentative metabolites on microbial growth. Biotechnology for Biofuels 2017, 10, (1), 262.

123. Xiang, T.-X.; Anderson, B. D., Influence of Chain Ordering on the Selectivity of Dipalmitoylphosphatidylcholine Bilayer Membranes for Permeant Size and Shape. Biophysical Journal 1998, 75, (6), 2658-2671.

124. Roghair, M.; Strik, D. P. B. T. B.; Steinbusch, K. J. J.; Weusthuis, R. A.; Bruins, M. E.; Buisman, C. J. N., Granular sludge formation and characterization in a chain elongation process. Process Biochemistry 2016, $51,(10), 1594-1598$

125. Tamis, J.; Joosse, B. M.; Loosdrecht, M. C. M. v.; Kleerebezem, R., High-rate volatile fatty acid (VFA) production by a granular sludge process at low pH. Biotechnol. Bioeng. 2015, 112, (11), 2248-2255.

126. Atasoy, M.; Owusu-Agyeman, I.; Plaza, E.; Cetecioglu, Z., Bio-based volatile fatty acid production and recovery from waste streams: Current status and future challenges. Bioresource Technology 2018, 268, 773 786.

127. Tilman, D., Global environmental impacts of agricultural expansion: the need for sustainable and efficient practices. Proceedings of the National Academy of Sciences 1999, 96, (11), 5995-6000.

128. Foley, J. A.; Ramankutty, N.; Brauman, K. A.; Cassidy, E. S.; Gerber, J. S.; Johnston, M.; Mueller, N. D.; O'Connell, C.; Ray, D. K.; West, P. C.; Balzer, C.; Bennett, E. M.; Carpenter, S. R.; Hill, J.; Monfreda, C.; Polasky, S.; Rockstrom, J.; Sheehan, J.; Siebert, S.; Tilman, D.; Zaks, D. P., Solutions for a cultivated planet. Nature 2011, 478, (7369), 337-42.

129. Ricci, A.; Allende, A.; Bolton, D.; Chemaly, M.; Davies, R.; Herman, L.; Koutsoumanis, K.; Lindqvist, R.; Nørrung, B.; Robertson, L.; Ru, G.; Sanaa, M.; Simmons, M.; Skandamis, P.; Snary, E.; Speybroeck, N.; Kuile, B. T.; Threlfall, J.; Wahlström, H.; Girones, R.; Alvarez Ordoñez, A.; Griffin, J.; Correia, S.; Fernández Escámez, P., Evaluation of the application for a new alternative processing method for animal by - products of Category 3 material (ChainCraft B.V.). EFSA Journal 2018, 16, (6).

130. Spirito, C. M.; Richter, H.; Rabaey, K.; Stams, A. J.; Angenent, L. T., Chain elongation in anaerobic reactor microbiomes to recover resources from waste. Current opinion in biotechnology 2014, 27, 115-122.

131. Jourdin, L.; Raes, S. M. T.; Buisman, C. J. N.; Strik, D. P. B. T. B., Critical Biofilm Growth throughout Unmodified Carbon Felts Allows Continuous Bioelectrochemical Chain Elongation from $\mathrm{CO} 2$ up to Caproate at High Current Density. Frontiers in Energy Research 2018, 6.

132. Angenent, L. T.; Richter, H.; Buckel, W.; Spirito, C. M.; Steinbusch, K. J.; Plugge, C. M.; Strik, D. P.; Grootscholten, T. I.; Buisman, C. J.; Hamelers, H. V., Chain elongation with reactor microbiomes: open-culture biotechnology to produce biochemicals. Environmental science \& technology 2016, 50, (6), 2796-2810.

133. Coma, M.; Vilchez-Vargas, R.; Roume, H.; Jauregui, R.; Pieper, D. H.; Rabaey, K., Product diversity linked to substrate usage in chain elongation by mixed-culture fermentation. Environmental science \& technology 2016, 50, (12), 6467-6476.

134. Apanel, G.; Johnson, E., Direct methanol fuel cells - ready to go commercial? Fuel Cells Bulletin 2004, 2004, (11), 12-17.

135. Kim, J.; Henao, C. A.; Johnson, T. A.; Dedrick, D. E.; Miller, J. E.; Stechel, E. B.; Maravelias, C. T., Methanol production from $\mathrm{CO} 2$ using solar-thermal energy: process development and techno-economic analysis. Energy \& Environmental Science 2011, 4, (9).

136. Leduc, S.; Lundgren, J.; Franklin, O.; Dotzauer, E., Location of a biomass based methanol production plant: A dynamic problem in northern Sweden. Applied Energy 2010, 87, (1), 68-75.

137. Fong, W.-C. F.; Wilson, R. F., Gasification process combined with steam methane reforming to produce syngas suitable for methanol production. In Google Patents: 1996.

138. Banerjee, R.; Ragsdale, S. W., The many faces of vitamin B12: catalysis by cobalamin-dependent enzymes. Annual review of biochemistry 2003, 72, 209-47. 
139. LaBelle, E. V.; May, H. D., Energy Efficiency and Productivity Enhancement of Microbial Electrosynthesis of Acetate. Frontiers in microbiology 2017, 8, 756

140. Gonzalez-Garcia, R.; McCubbin, T.; Navone, L.; Stowers, C.; Nielsen, L.; Marcellin, E., Microbial Propionic Acid Production. Fermentation 2017, 3, (2).

141. Bajracharya, S.; ter Heijne, A.; Dominguez Benetton, X.; Vanbroekhoven, K.; Buisman, C. J.; Strik, D. P.; Pant, D., Carbon dioxide reduction by mixed and pure cultures in microbial electrosynthesis using an assembly of graphite felt and stainless steel as a cathode. Bioresour Technol 2015, 195, 14-24.

142. Lange, J. P.; Price, R.; Ayoub, P. M.; Louis, J.; Petrus, L.; Clarke, L.; Gosselink, H., Valeric biofuels: a platform of cellulosic transportation fuels. Angewandte Chemie International Edition 2010, 49, (26), 4479-4483.

143. Chan-Thaw, C. E.; Marelli, M.; Psaro, R.; Ravasio, N.; Zaccheria, F., New generation biofuels: $y-$ valerolactone into valeric esters in one pot. RSC Adv. 2013, 3, (5), 1302-1306.

144. Luengo, J. M.; Garćia, B.; Sandoval, A.; Naharro, G.; Olivera, E. a. R., Bioplastics from microorganisms. Current Opinion in Microbiology 2003, 6, (3), 251-260.

145. Law Jr, C. G.; Fedkiw, P. S.; Hicks, M. T., Kolbe electrolysis in a polymer electrolyte membrane reactor. In Google Patents: 2001.

146. Moscoviz, R.; Trably, E.; Bernet, N.; Carrère, H., The environmental biorefinery: state-of-the-art on the production of hydrogen and value-added biomolecules in mixed-culture fermentation. Green Chemistry 2018, 20, (14), 3159-3179.

147. Chen, W. S.; Huang, S.; Strik, D. P. B. T. B.; Buisman, C. J. N., Isobutyrate biosynthesis via methanol chain elongation: converting organic wastes to platform chemicals. Journal of Chemical Technology and Biotechnology 2017, 92, (6), 1370-1379.

148. Rode, L.; Genthner, B. S.; Bryant, M., Syntrophic association by cocultures of the methanol-and CO2$\mathrm{H} 2$-utilizing species Eubacterium limosum and pectin-fermenting Lachnospira multiparus during growth in a pectin medium. Applied and environmental microbiology 1981, 42, (1), 20-22.

149. Lindley, N.; Loubiere, P.; Pacaud, S.; Mariotto, C.; Goma, G., Novel products of the acidogenic fermentation of methanol during growth of Eubacterium limosum in the presence of high concentrations of organic acids. Microbiology 1987, 133, (12), 3557-3563.

150. Florencio, L.; Field, J.; Lettinga, G., Importance of cobalt for individual trophic groups in an anaerobic methanol-degrading consortium. Applied and environmental microbiology 1994, 60, (1), 227-234.

151. Royce, L. A.; Liu, P.; Stebbins, M. J.; Hanson, B. C.; Jarboe, L. R., The damaging effects of short chain fatty acids on Escherichia coli membranes. Applied microbiology and biotechnology 2013, 97, (18), 8317-8327.

152. Bainotti, A. E.; Yamaguchi, K.; Nakashimada, Y.; Nishio, N., Kinetics and energetics of Acetobacterium $\mathrm{sp}$. in chemostat culture on methanol-CO2. Journal of fermentation and bioengineering 1998, 85, (2), 223-229.

153. Florencio, L.; Field, J.; Lettinga, G., Substrate competition between methanogens and acetogens during the degradation of methanol in UASB reactors. Water Research 1995, 29, (3), 915-922.

154. Infantes, D.; del Campo, A. G.; Villaseñor, J.; Fernández, F., Kinetic model and study of the influence of $\mathrm{pH}$, temperature and undissociated acids on acidogenic fermentation. Biochemical engineering journal 2012, 66, 66-72.

155. Roghair, M.; Liu, Y.; Strik, D.; Weusthuis, R. A.; Bruins, M. E.; Buisman, C. J. N., Development of an Effective Chain Elongation Process From Acidified Food Waste and Ethanol Into n-Caproate. Frontiers in bioengineering and biotechnology 2018, 6, 50.

156. de Leeuw, K. D.; de Smit, S. M.; van Oossanen, S.; Moerland, M. J.; Buisman, C. J. N.; Strik, D. P. B. T. B., Methanol-Based Chain Elongation with Acetate to $n$-Butyrate and Isobutyrate at Varying Selectivities Dependent on pH. ACS Sustainable Chemistry \& Engineering 2020, 8, (22), 8184-8194.

157. Quast, C.; Pruesse, E.; Yilmaz, P.; Gerken, J.; Schweer, T.; Yarza, P.; Peplies, J.; Glockner, F. O., The SILVA ribosomal RNA gene database project: improved data processing and web-based tools. Nucleic acids research 2013, 41, (Database issue), D590-6.

158. Edgar, R. C., Search and clustering orders of magnitude faster than BLAST. Bioinformatics 2010, 26, (19), 2460-1.

159. Wang, Q.; Garrity, G. M.; Tiedje, J. M.; Cole, J. R., Naive Bayesian classifier for rapid assignment of rRNA sequences into the new bacterial taxonomy. Appl Environ Microbiol 2007, 73, (16), 5261-7.

160. Caporaso, J. G.; Kuczynski, J.; Stombaugh, J.; Bittinger, K.; Bushman, F. D.; Costello, E. K.; Fierer, N.; Pena, A. G.; Goodrich, J. K.; Gordon, J. I., QIIME allows analysis of high-throughput community sequencing data. Nature methods 2010, 7, (5), 335.

161. Kouzuma, A.; Tsutsumi, M.; Ishii, S. i.; Ueno, Y.; Abe, T.; Watanabe, K., Non-autotrophic methanogens dominate in anaerobic digesters. Scientific Reports 2017, 7, (1), 1510. 
162. Conrad, R.; Klose, M.; Claus, P., Phosphate inhibits acetotrophic methanogenesis on rice roots. Applied and environmental microbiology 2000, 66, (2), 828-831.

163. Boone, D. R.; Bryant, M. P., Propionate-degrading bacterium, Syntrophobacter wolinii sp. nov. gen. nov., from methanogenic ecosystems. Applied and Environmental Microbiology 1980, 40, (3), 626-632.

164. Dong, X.; Plugge, C. M.; Stams, A. J., Anaerobic degradation of propionate by a mesophilic acetogenic bacterium in coculture and triculture with different methanogens. Applied and environmental microbiology 1994, $60,(8), 2834-2838$.

165. Games, L. M.; HayesRobert, J.; Gunsalus, P., Methane-producing bacteria: natural fractionations of the stable carbon isotopes. Geochimica et Cosmochimica Acta 1978, 42, (8), 1295-1297.

166. Breznak, J. A.; Kane, M. D., Microbial H2/CO2 acetogenesis in animal guts: nature and nutritional significance. FEMS microbiology reviews 1990, 7, (3-4), 309-313.

167. AMES, J. M.; LEOD, G. M., Volatile components of a yeast extract composition. journal of Food Science 1985, 50, (1), 125-131.

168. Allison, M. J., Production of branched-chain volatile fatty acids by certain anaerobic bacteria. Applied and environmental microbiology 1978, 35, (5), 872-877.

169. Florencio, L.; Nozhevnikova, A.; Van Langerak, A.; Stams, A.; Field, J.; Lettinga, G., Acidophilic degradation of methanol by a methanogenic enrichment culture. FEMS Microbiology Letters 1993, 109, (1), 1-6.

170. Iino, T.; Tamaki, H.; Tamazawa, S.; Ueno, Y.; Ohkuma, M.; Suzuki, K.-i.; Igarashi, Y.; Haruta, S., Candidatus Methanogranum caenicola: a novel methanogen from the anaerobic digested sludge, and proposal of Methanomassiliicoccaceae fam. nov. and Methanomassiliicoccales ord. nov., for a methanogenic lineage of the class Thermoplasmata. Microbes and environments 2013, 28, (2), 244-250.

171. Dridi, B.; Fardeau, M. L.; Ollivier, B.; Raoult, D.; Drancourt, M., Methanomassiliicoccus luminyensis gen. nov., sp. nov., a methanogenic archaeon isolated from human faeces. Int J Syst Evol Microbiol 2012, 62, (Pt 8), 1902-7.

172. Wang, Q.; Wang, C. D.; Li, C. H.; Li, J. G.; Chen, Q.; Li, Y. Z., Clostridium luticellarii sp. nov., isolated from a mud cellar used for producing strong aromatic liquors. Int J Syst Evol Microbiol 2015, 65, (12), $4730-3$.

173. Ragsdale, S. W.; Pierce, E., Acetogenesis and the Wood-Ljungdahl pathway of $\mathrm{CO} 2$ fixation. Biochimica et Biophysica Acta (BBA)-Proteins and Proteomics 2008, 1784, (12), 1873-1898.

174. Buckel, W.; Thauer, R. K., Energy conservation via electron bifurcating ferredoxin reduction and proton/Na+ translocating ferredoxin oxidation. Biochimica et Biophysica Acta (BBA)-Bioenergetics 2013, 1827, (2), 94-113.

175. Seedorf, H.; Fricke, W. F.; Veith, B.; Brüggemann, H.; Liesegang, H.; Strittmatter, A.; Miethke, M.; Buckel, W.; Hinderberger, J.; Li, F., The genome of Clostridium kluyveri, a strict anaerobe with unique metabolic features. Proceedings of the National Academy of Sciences 2008, 105, (6), 2128-2133.

176. Chatellard, L.; Trably, E.; Carrère, H., The type of carbohydrates specifically selects microbial community structures and fermentation patterns. Bioresource technology 2016, 221, 541-549.

177. Abreu, A.; Alves, J.; Pereira, M.; Sousa, D.; Alves, M., Strategies to suppress hydrogen - consuming microorganisms affect macro and micro scale structure and microbiology of granular sludge. Biotechnology and bioengineering 2011, 108, (8), 1766-1775.

178. Albuquerque, M.; Eiroa, M.; Torres, C.; Nunes, B.; Reis, M., Strategies for the development of a side stream process for polyhydroxyalkanoate (PHA) production from sugar cane molasses. Journal of biotechnology 2007, 130, (4), 411-421.

179. Marshall, C. W.; Ross, D. E.; Handley, K. M.; Weisenhorn, P. B.; Edirisinghe, J. N.; Henry, C. S.; Gilbert, J. A.; May, H. D.; Norman, R. S., Metabolic Reconstruction and Modeling Microbial Electrosynthesis. Sci Rep 2017, 7, (1), 8391.

180. Beenackers, A.; van Swaaij, W. In Methanol production from biomass, Bioenergy 84. Proceedings of conference 15-21 June 1984, Goteborg, Sweden. Volume I. Bioenergy state of the art, 1984; Elsevier Applied Science Publishers: 1984; pp 236-275.

181. Fernández, I.; Vázquez-Padín, J. R.; Mosquera-Corral, A.; Campos, J. L.; Méndez, R., Biofilm and granular systems to improve Anammox biomass retention. Biochemical Engineering Journal 2008, 42, (3), 308313.

182. Hulshoff Pol, L. W.; de Castro Lopes, S. I.; Lettinga, G.; Lens, P. N., Anaerobic sludge granulation. Water Res 2004, 38, (6), 1376-89.

183. Di Maio, F.; Rem, P. C.; Baldé, K.; Polder, M., Measuring resource efficiency and circular economy: A market value approach. Resources, Conservation and Recycling 2017, 122, (Supplement C), 163-171.

184. Sauer, M.; Porro, D.; Mattanovich, D.; Branduardi, P., Microbial production of organic acids: expanding the markets. Trends in Biotechnology 2008, 26, (2), 100-108. 
185. Steinbusch, K. J. J.; Hamelers, H. V. M.; Plugge, C. M.; Buisman, C. J. N., Biological formation of caproate and caprylate from acetate: fuel and chemical production from low grade biomass. Energy \& Environmental Science 2011, 4, (1), 216-224.

186. Lin, C. S. K.; Pfaltzgraff, L. A.; Herrero-Davila, L.; Mubofu, E. B.; Abderrahim, S.; Clark, J. H.; Koutinas, A. A.; Kopsahelis, N.; Stamatelatou, K.; Dickson, F.; Thankappan, S.; Mohamed, Z.; Brocklesby, R.; Luque, R., Food waste as a valuable resource for the production of chemicals, materials and fuels. Current situation and global perspective. Energy \& Environmental Science 2013, 6, (2), 426-464.

187. Spirito, C. M.; Richter, H.; Rabaey, K.; Stams, A. J. M.; Angenent, L. T., Chain elongation in anaerobic reactor microbiomes to recover resources from waste. Current Opinion in Biotechnology 2014, 27, 115-122.

188. Liu, Q.; Wang, C.; Guo, G.; Huo, W. J.; Zhang, Y. L.; Pei, C. X.; Zhang, S. L.; Wang, H., Effects of branched-chain volatile fatty acids supplementation on growth performance, ruminal fermentation, nutrient digestibility, hepatic lipid content and gene expression of dairy calves. Animal Feed Science and Technology 2018, 237, 27-34.

189. Research, T. M., Report Review: Palm Kernel Oil and Coconut Oil Based Natural Fatty Acids Market for Detergents, Personal Care, Plastics, Rubber, and Other End-users - Global Industry Analysis, Size, Share, Growth, Trends and Forecast, 2015 - 2023. Chemicals \& Materials 2016, Palm Kernel Oil Coconut Oil Based Natural Fatty Acids Market, Rep Id : TMRGL8617.

190. Turpeinen, A.; Merimaa, P.; Saarela, M., 16 - Functional fats and spreads. In Functional Foods (Second Edition), Woodhead Publishing: 2011; pp 383-400.

191. Agler, M. T.; Spirito, C. M.; Usack, J. G.; Werner, J. J.; Angenent, L. T., Chain elongation with reactor microbiomes: upgrading dilute ethanol to medium-chain carboxylates. Energy \& Environmental Science 2012, 5, (8), 8189-8192.

192. Grootscholten, T. I. M.; Steinbusch, K. J. J.; Hamelers, H. V. M.; Buisman, C. J. N., High rate heptanoate production from propionate and ethanol using chain elongation. Bioresource Technology 2013, 136, 715-718.

193. Kucek, L. A.; Spirito, C. M.; Angenent, L. T., High n-caprylate productivities and specificities from dilute ethanol and acetate: chain elongation with microbiomes to upgrade products from syngas fermentation. Energy \& Environmental Science 2016, 9, (11), 3482-3494.

194. Xiong, M.; Deng, J.; Woodruff, A. P.; Zhu, M.; Zhou, J.; Park, S. W.; Li, H.; Fu, Y.; Zhang, K., A BioCatalytic Approach to Aliphatic Ketones. Scientific Reports 2012, 2, 311.

195. Levy, P. F.; Sanderson, J. E.; Cheng, L. K., Kolbe Electrolysis of Mixtures of Aliphatic Organic Acids. Journal of The Electrochemical Society 1984, 131, (4), 773-777.

196. Perdih, A.; Perdih, F., Chemical Interpretation of Octane Number. Acta Chimica Slovenica. 2006, 53. , (306.).

197. Yang, X.; Liu, X.; Chen, S.; Liu, G.; Wu, S.; Wan, C., Volatile Fatty Acids Production from Codigestion of Food Waste and Sewage Sludge Based on $\beta$-Cyclodextrins and Alkaline Treatments. Archaea 2016, 2016, 1698163.

198. Barker, H. A., Amino Acid Degradation by Anaerobic Bacteria. Annual Review of Biochemistry 1981, 50, (1), 23-40.

199. Parawira, W.; Murto, M.; S Read, J.; Mattiasson, B., Volatile fatty acid production during anaerobic mesophilic digestion of solid potato waste Journal of Chemical Technology \& Biotechnology: 2004; Vol. 79, p 673677.

200. Wang, Q.; Kuninobu, M.; Ogawa, H. I.; Kato, Y., Degradation of volatile fatty acids in highly efficient anaerobic digestion. Biomass and Bioenergy 1999, 16, (6), 407-416.

201. Chen, W. S., Microbial chain elongation based on methanol. Doctoral dissertation 2017, Retrieved from Wageningen University \& Research database (Record No. 2214219).

202. Roghair, M.; Liu, Y.; Strik, D. P. B. T. B.; Weusthuis, R. A.; Bruins, M. E.; Buisman, C. J. N., Development of an Effective Chain Elongation Process From Acidified Food Waste and Ethanol Into n-Caproate. Frontiers in Bioengineering and Biotechnology 2018, 6, (50).

203. Kenealy, W. R.; Waselefsky, D. M., Studies on the substrate range of Clostridium kluyveri; the use of propanol and succinate. Archives of Microbiology 1985, 141, (3), 187-194.

204. Jungermann, K.; Thauer, R. K.; Decker, K., The Synthesis of One-Carbon Units from CO2 in Clostridium kluyveri. European Journal of Biochemistry 1968, 3, (3), 351-359.

205. Tomlinson, N., Carbon Dioxide and Acetate Utilization by Clostridium Kluyveri: II. Synthesis of Amino Acids. Journal of Biological Chemistry 1954, 209, (2), 597-603.

206. R. Phillips, J.; Klasson, K.; C. Clausen, E.; L. Gaddy, J., Biological production of ethanol from coal synthesis gas - Medium development studies. 1993; p 559-571. 
207. Jourdin, L.; Raes, S. M. T.; Buisman, C. J. N.; Strik, D. P. B. T. B., Critical Biofilm Growth throughout Unmodified Carbon Felts Allows Continuous Bioelectrochemical Chain Elongation from $\mathrm{CO} 2$ up to Caproate at High Current Density. Frontiers in Energy Research 2018, 6, (7), DOI: 10.3389/fenrg.2018.00007.

208. Caporaso, J. G.; Kuczynski, J.; Stombaugh, J.; Bittinger, K.; Bushman, F. D.; Costello, E. K.; Fierer, N.; Peña, A. G.; Goodrich, J. K.; Gordon, J. I.; Huttley, G. A.; Kelley, S. T.; Knights, D.; Koenig, J. E.; Ley, R. E.; Lozupone, C. A.; McDonald, D.; Muegge, B. D.; Pirrung, M.; Reeder, J.; Sevinsky, J. R.; Turnbaugh, P. J.; Walters, W. A.; Widmann, J.; Yatsunenko, T.; Zaneveld, J.; Knight, R., QIIME allows analysis of high-throughput community sequencing data. Nature Methods 2010, 7, 335.

209. Quast, C.; Pruesse, E.; Yilmaz, P.; Gerken, J.; Schweer, T.; Yarza, P.; Peplies, J.; Glöckner, F. O., The SILVA ribosomal RNA gene database project: improved data processing and web-based tools. Nucleic Acids Research 2013, 41, (D1), D590-D596.

210. Steinbusch, K. J. J.; Arvaniti, E.; Hamelers, H. V. M.; Buisman, C. J. N., Selective inhibition of methanogenesis to enhance ethanol and $n$-butyrate production through acetate reduction in mixed culture fermentation. Bioresource Technology 2009, 100, (13), 3261-3267.

211. Roghair, M.; Hoogstad, T.; Strik, D. P. B. T. B.; Plugge, C. M.; Timmers, P. H. A.; Weusthuis, R. A.; Bruins, M. E.; Buisman, C. J. N., Controlling Ethanol Use in Chain Elongation by CO2 Loading Rate. Environmental Science \& Technology 2018, 52, (3), 1496-1505.

212. Junicke, H.; van Loosdrecht, M. C. M.; Kleerebezem, R., Kinetic and thermodynamic control of butyrate conversion in non-defined methanogenic communities. Applied Microbiology and Biotechnology 2016, 100, (2), 915-925

213. Steinbusch, K. J. J.; Hamelers, H. V. M.; Buisman, C. J. N., Alcohol production through volatile fatty acids reduction with hydrogen as electron donor by mixed cultures. Water Research 2008, 42, (15), 4059-4066.

214. Diender, M.; Stams, A. J. M.; Sousa, D. Z., Production of medium-chain fatty acids and higher alcohols by a synthetic co-culture grown on carbon monoxide or syngas. Biotechnology for Biofuels 2016, 9, (1), 82.

215. Thauer, R. K.; Jungermann, K.; Hennigner, H.; Wenning, J.; Decker, K., The Energy Metabolism of Clostridium kluyveri. European Journal of Biochemistry 1968, 4, (2), 173-180.

216. Kouzuma, A.; Kato, S.; Watanabe, K., Microbial interspecies interactions: recent findings in syntrophic consortia. Frontiers in microbiology 2015, 6, 477-477.

217. Li, H.; Chang, J.; Liu, P.; Fu, L.; Ding, D.; Lu, Y., Direct interspecies electron transfer accelerates syntrophic oxidation of butyrate in paddy soil enrichments. Environmental Microbiology 2015, 17, (5), 15331547.

218. Shrestha, P. M.; Rotaru, A. E.; Aklujkar, M.; Liu, F.; Shrestha, M.; Summers, Z. M.; Malvankar, N.; Flores, D. C.; Lovley, D. R., Syntrophic growth with direct interspecies electron transfer as the primary mechanism for energy exchange. Environmental Microbiology Reports 2013, 5, (6), 904-910.

219. Kato, S.; Igarashi, K., Enhancement of methanogenesis by electric syntrophy with biogenic iron-sulfide minerals. MicrobiologyOpen 0, (0), e00647.

220. Worm, P.; Müller, N.; Plugge, C. M.; Stams, A. J. M.; Schink, B., Syntrophy in Methanogenic Degradation. In (Endo)symbiotic Methanogenic Archaea, Hackstein, J. H. P., Ed. Springer Berlin Heidelberg: Berlin, Heidelberg, 2010; pp 143-173.

221. Liang, B.; Wang, L.-Y.; Mbadinga, S. M.; Liu, J.-F.; Yang, S.-Z.; Gu, J.-D.; Mu, B.-Z., Anaerolineaceae and Methanosaeta turned to be the dominant microorganisms in alkanes-dependent methanogenic culture after long-term of incubation. AMB Express 2015, 5, (1), 117-117.

222. Liang, B.; Wang, L.-Y.; Zhou, Z.; Mbadinga, S. M.; Zhou, L.; Liu, J.-F.; Yang, S.-Z.; Gu, J.-D.; Mu, B.Z., High Frequency of Thermodesulfovibrio spp. and Anaerolineaceae in Association with Methanoculleus spp. in a Long-Term Incubation of n-Alkanes-Degrading Methanogenic Enrichment Culture. Frontiers in Microbiology 2016, 7, (1431)

223. Thabet, O. B. D.; Wafa, T.; Eltaief, K.; Cayol, J.-L.; Hamdi, M.; Fauque, G.; Fardeau, M.-L., Desulfovibrio legallis sp. nov.: A Moderately Halophilic, Sulfate-Reducing Bacterium Isolated from a Wastewater Digestor in Tunisia. Current Microbiology 2011, 62, (2), 486-491.

224. Sharma, M.; Aryal, N.; Sarma, P. M.; Vanbroekhoven, K.; Lal, B.; Benetton, X. D.; Pant, D., Bioelectrocatalyzed reduction of acetic and butyric acids via direct electron transfer using a mixed culture of sulfate-reducers drives electrosynthesis of alcohols and acetone. Chemical Communications 2013, 49, (58), 6495-6497.

225. Agostino, V.; Rosenbaum, M. A., Sulfate-Reducing ElectroAutotrophs and Their Applications in Bioelectrochemical Systems. Frontiers in Energy Research 2018, 6, (55).

226. Howard, B. H.; Hungate, R. E., Desulfovibrio of the sheep rumen. Applied and environmental microbiology 1976, 32, (4), 598-602.

227. Hockin, S. L.; Gadd, G. M., Linked Redox Precipitation of Sulfur and Selenium under Anaerobic Conditions by Sulfate-Reducing Bacterial Biofilms. Applied and Environmental Microbiology 2003, 69, (12), 7063-7072. 
228. Wang, B.-W.; Shi, A.-Q.; Tu, R.; Zhang, X.-L.; Wang, Q.-H.; Bai, F.-W., Branched-Chain Higher Alcohols. In Biotechnology in China III: Biofuels and Bioenergy, Springer Berlin Heidelberg: Berlin, Heidelberg, 2012; pp 101-118.

229. Grootscholten T.I.M., S. K. J. J., Hamelers H.V.M., Buisman C.J.N., Improving medium chain fatty acid productivity using chain elongation by reducing the hydraulic retention time in an upflow anaerobic filter. Bioresource Technology 2013, 136, 735-738.

230. Chen, W.-S.; Strik, D. P.; Buisman, C. J. N.; Kroeze, C., Production of Caproic Acid from Mixed Organic Waste: An Environmental Life Cycle Perspective. Environmental Science \& Technology 2017, 51, (12), 71597168.

231. Tan, Y. D.; Lim, J. S., Feasibility of palm oil mill effluent elimination towards sustainable Malaysian palm oil industry. Renewable and Sustainable Energy Reviews 2019, 111, 507-522.

232. Ahmad, F. B.; Zhang, Z.; Doherty, W. O.; O'Hara, I. M., The outlook of the production of advanced fuels and chemicals from integrated oil palm biomass biorefinery. Renewable and Sustainable Energy Reviews 2019, $109,386-411$.

233. Zhu, X.; Zhou, Y.; Wang, Y.; Wu, T.; Li, X.; Li, D.; Tao, Y., Production of high-concentration n-caproic acid from lactate through fermentation using a newly isolated Ruminococcaceae bacterium CPB6. Biotechnology for Biofuels 2017, 10, (1), 102.

234. Leng, L.; Nobu, M. K.; Narihiro, T.; Yang, P.; Amy Tan, G.-Y.; Lee, P.-H., Shaping microbial consortia in coupling glycerol fermentation and carboxylate chain elongation for Co-production of 1,3-propanediol and caproate: Pathways and mechanisms. Water Research 2019, 148, 281-291.

235. Yang, P. Omics-based characterization of anaerobic metabolism in methanogenic system and chain elongation process. The Hong Kong Polytechnic University, 2019.

236. Han, W.; He, P.; Shao, L.; Lü, F., Road to full bioconversion of biowaste to biochemicals centering on chain elongation: A mini review. Journal of Environmental Sciences 2019.

237. Chwialkowska, J.; Duber, A.; Zagrodnik, R.; Walkiewicz, F.; Łężyk, M.; Oleskowicz-Popiel, P., Caproic acid production from acid whey via open culture fermentation - Evaluation of the role of electron donors and downstream processing. Bioresource Technology 2019, 279, 74-83.

238. Zhu, X.; Tao, Y.; Liang, C.; Li, X.; Wei, N.; Zhang, W.; Zhou, Y.; Yang, Y.; Bo, T., The synthesis of ncaproate from lactate: a new efficient process for medium-chain carboxylates production. Scientific Reports 2015, 5,14360 .

239. Verbraeken, H., ChainCraft gaat op grotere schaal biologische vetzuren maken. . Financieel Dagblad 2017, Ondernemen.

240. de Leeuw, K. D.; Buisman, C. J. N.; Strik, D. P. B. T. B., Branched Medium Chain Fatty Acids: IsoCaproate Formation from Iso-Butyrate Broadens the Product Spectrum for Microbial Chain Elongation. Environmental Science \& Technology 2019, 53, (13), 7704-7713.

241. Yang, X.; Liu, X.; Chen, S.; Liu, G.; Wu, S.; Wan, C., Volatile Fatty Acids Production from Codigestion of Food Waste and Sewage Sludge Based on \&\#x3b2;-Cyclodextrins and Alkaline Treatments. Archaea 2016, 2016,8 .

242. Atsumi, S.; Hanai, T.; Liao, J. C., Non-fermentative pathways for synthesis of branched-chain higher alcohols as biofuels. nature 2008, 451, (7174), 86.

243. Ghosh, P.; Hickey, K. J.; Jaffe, S. B., Development of a detailed gasoline composition-based octane model. Industrial \& engineering chemistry research 2006, 45, (1), 337-345.

244. Richter, H.; Molitor, B.; Diender, M.; Sousa, D. Z.; Angenent, L. T., A Narrow pH Range Supports Butanol, Hexanol, and Octanol Production from Syngas in a Continuous Co-culture of Clostridium ljungdahlii and Clostridium kluyveri with In-Line Product Extraction. Frontiers in Microbiology 2016, 7, 1773

245. Xin, F.; Wu, Y. R.; He, J., Simultaneous fermentation of glucose and xylose to butanol by Clostridium sp. strain BOH3. Appl Environ Microbiol 2014, 80, (15), 4771-8.

246. Chen, Y.; Zhou, T.; Liu, D.; Li, A.; Xu, S.; Liu, Q.; Li, B.; Ying, H., Production of butanol from glucose and xylose with immobilized cells of Clostridium acetobutylicum. Biotechnology and Bioprocess Engineering 2013, $18,(2), 234-241$.

247. Seitz, H. J.; Schink, B.; Pfennig, N.; Conrad, R., Energetics of syntrophic ethanol oxidation in defined chemostat cocultures. Archives of Microbiology 1990, 155, (1), 82-88.

248. Nozhevnikova, A. N.; Russkova, Y. I.; Litti, Y. V.; Parshina, S. N.; Zhuravleva, E. A.; Nikitina, A. A., Syntrophy and Interspecies Electron Transfer in Methanogenic Microbial Communities. Microbiology 2020, 89, (2), 129-147

249. Grootscholten, T. I. M.; Steinbusch, K. J. J.; Hamelers, H. V. M.; Buisman, C. J. N., Chain elongation of acetate and ethanol in an upflow anaerobic filter for high rate MCFA production. Bioresource Technology 2013, $135,440-445$. 
250. Vogels, G. D.; Keltjens, J. T.; Hutten, T. J.; Van Der Drift, C., Coenzymes of methanogenic bacteria. Zentralblatt für Bakteriologie Mikrobiologie und Hygiene: I. Abt. Originale C: Allgemeine, angewandte und ökologische Mikrobiologie 1982, 3, (2), 258-264.

251. Li, F.; Hinderberger, J.; Seedorf, H.; Zhang, J.; Buckel, W.; Thauer, R. K., Coupled Ferredoxin and Crotonyl Coenzyme A (CoA) Reduction with NADH Catalyzed by the Butyryl-CoA Dehydrogenase/Etf Complex from Clostridium kluyveri. Journal of Bacteriology 2008, 190, (3), 843.

252. Tomlinson, N., Carbon dioxide and acetate utilization by Clostridium kluyveri II. Synthesis of amino acids. Journal of Biological Chemistry 1954, 209, (2), 597-603.

253. Chen, W.; Ye, Y.; Steinbusch, K.; Strik, D.; Buisman, C., Methanol as an alternative electron donor in chain elongation for butyrate and caproate formation. Biomass and Bioenergy 2016, 93, 201-208.

254. Kim, H.; Jeon, B. S.; Sang, B.-I., An Efficient New Process for the Selective Production of Odd-Chain Carboxylic Acids by Simple Carbon Elongation Using Megasphaera hexanoica. Scientific reports 2019, 9, (1), 110.

255. Spirito, C. M.; Marzilli, A. M.; Angenent, L. T., Higher Substrate Ratios of Ethanol to Acetate Steered Chain Elongation toward n-Caprylate in a Bioreactor with Product Extraction. Environmental Science \& Technology 2018, 52, (22), 13438-13447.

256. Barker, H. A.; Taha, S. M., Clostridium kluyverii, an Organism Concerned in the Formation of Caproic Acid from Ethyl Alcohol. J Bacteriol 1942, 43, (3), 347-63.

257. Jackson, B. E.; McInerney, M. J., Anaerobic microbial metabolism can proceed close to thermodynamic limits. Nature 2002, 415, (6870), 454-456.

258. Candry, P.; Ulcar, B.; Petrognani, C.; Rabaey, K.; Ganigué, R., Ethanol:propionate ratio drives product selectivity in odd-chain elongation with Clostridium kluyveri and mixed communities. Bioresource Technology 2020, 313, 123651 .

259. Smith, D. P.; McCarty, P. L., Reduced product formation following perturbation of ethanol- and propionate-fed methanogenic CSTRs. Biotechnol. Bioeng. 1989, 34, (7), 885-895.

260. Schoberth, S.; Gottschalk, G., Considerations on the energy metabolism of Clostridium kluyveri. Archiv für Mikrobiologie 1969, 65, (4), 318-328.

261. Bonk, B. M.; Tarasova, Y.; Hicks, M. A.; Tidor, B.; Prather, K. L. J., Rational design of thiolase substrate specificity for metabolic engineering applications. Biotechnol. Bioeng. 2018, 115, (9), 2167-2182.

262. Clomburg, J. M.; Contreras, S. C.; Chou, A.; Siegel, J. B.; Gonzalez, R., Combination of type II fatty acid biosynthesis enzymes and thiolases supports a functional $\beta$-oxidation reversal. Metabolic Engineering 2018, 45, 11-19.

263. Long, C. P.; Antoniewicz, M. R., High-resolution 13C metabolic flux analysis. Nature Protocols 2019, 14, (10), 2856-2877.

264. Calhoun, S.; Korczynska, M.; Wichelecki, D. J.; San Francisco, B.; Zhao, S.; Rodionov, D. A.; Vetting, M. W.; Al-Obaidi, N. F.; Lin, H.; O'Meara, M. J.; Scott, D. A.; Morris, J. H.; Russel, D.; Almo, S. C.; Osterman, A. L.; Gerlt, J. A.; Jacobson, M. P.; Shoichet, B. K.; Sali, A., Prediction of enzymatic pathways by integrative pathway mapping. Elife 2018, 7, e31097.

265. Boer, H.; Andberg, M.; Pylkkänen, R.; Maaheimo, H.; Koivula, A., In vitro reconstitution and characterisation of the oxidative $\mathrm{D}$-xylose pathway for production of organic acids and alcohols. AMB Express 2019, 9, (1), 48-48.

266. Yin, W.; Wang, Y.; Liu, L.; He, J., Biofilms: The Microbial "Protective Clothing" in Extreme Environments. Int J Mol Sci 2019, 20, (14), 3423.

267. Qureshi, N.; Annous, B. A.; Ezeji, T. C.; Karcher, P.; Maddox, I. S., Biofilm reactors for industrial bioconversion processes: employing potential of enhanced reaction rates. Microb Cell Fact 2005, 4, 24-24.

268. Yang, S.-T.; Huang, H.; Tay, A.; Qin, W.; De Guzman, L.; Nicolas, E. C. S., Chapter 16 - Extractive Fermentation for the Production of Carboxylic Acids. In Bioprocessing for Value-Added Products from Renewable Resources, Yang, S.-T., Ed. Elsevier: Amsterdam, 2007; pp 421-446.

269. Kannengiesser, J.; Sakaguchi-Söder, K.; Mrukwia, T.; Jager, J.; Schebek, L., Extraction of medium chain fatty acids from organic municipal waste and subsequent production of bio-based fuels. Waste Management 2016, 47, 78-83.

270. Saboe, P.; Manker, L.; Michener, W.; Peterson, D.; Brandner, D.; Deutch, S.; Kumar, M.; Cywar, R.; Beckham, G.; Karp, E., In situ recovery of bio-based carboxylic acids. Green Chemistry 2018, 20.

271. Ganigué, R.; Naert, P.; Candry, P.; de Smedt, J.; Stevens, C. V.; Rabaey, K., Fruity flavors from waste: A novel process to upgrade crude glycerol to ethyl valerate. Bioresource Technology 2019, 289, 121574.

272. T. S. Veras, S.; Cavalcante, W.; Gehring, T.; Ribeiro, A.; Ferreira, T.; Kato, M.; Rojas-Ojeda, P.; SanzMartin, J.; Leitão, R., Anaerobic production of valeric acid from crude glycerol via chain elongation. International journal of Environmental Science and Technology 2019. 
273. Hamelinck, C. N.; Suurs, R. A. A.; Faaij, A. P. C., International bioenergy transport costs and energy balance. Biomass and Bioenergy 2005, 29, (2), 114-134.

274. Valerio, O.; Horvath, T.; Pond, C.; Manjusri, M.; Mohanty, A., Improved utilization of crude glycerol from biodiesel industries: Synthesis and characterization of sustainable biobased polyesters. Industrial Crops and Products 2015, 78, 141-147.

275. Khairul Zaman, N.; Law, J. Y.; Chai, P. V.; Rohani, R.; Mohammad, A., Recovery of Organic Acids from Fermentation Broth Using Nanofiltration Technologies: A Review. Journal of Physical Science 2017, 28, 85-109.

276. Tamis, J.; Lužkov, K.; Jiang, Y.; Loosdrecht, M. C. M. V.; Kleerebezem, R., Enrichment of Plasticicumulans acidivorans at pilot-scale for PHA production on industrial wastewater. Journal of Biotechnology 2014, 192, 161-169.

277. Johnson, K., Pha production in aerobic mixed microbial cultures. 2010.

278. Kourmentza, C.; Plácido, J.; Venetsaneas, N.; Burniol-Figols, A.; Varrone, C.; Gavala, H. N.; Reis, M. A. M., Recent Advances and Challenges towards Sustainable Polyhydroxyalkanoate (PHA) Production. Bioengineering (Basel) 2017, 4, (2), 55

279. Boutin, E.; Wang, M.; Lin, J. C.; Mesnage, M.; Mendoza, D.; Lassalle-Kaiser, B.; Hahn, C.; Jaramillo, T. F.; Robert, M., Aqueous Electrochemical Reduction of Carbon Dioxide and Carbon Monoxide into Methanol with Cobalt Phthalocyanine. Angewandte Chemie International Edition 2019, 58, (45), 16172-16176.

280. Albo, J.; Alvarez-Guerra, M.; Castaño, P.; Irabien, A., Towards the electrochemical conversion of carbon dioxide into methanol. Green Chemistry 2015, 17, (4), 2304-2324.

281. Wu, Q.; Feng, X.; Chen, Y.; Liu, M.; Bao, X., Continuous medium chain carboxylic acids production from excess sludge by granular chain-elongation process. Journal of Hazardous Materials 2021, 402, 123471.

282. Woo, H. C.; Kim, Y. H., Eco-efficient recovery of bio-based volatile C2-6 fatty acids. Biotechnology for biofuels 2019, 12, 92-92.

283. Research, W. U. In To explore the potential of nature to improve the quality of life, Mission, 2020; 2020.

284. Folsom, J. P.; Carlson, R. P., Physiological, biomass elemental composition and proteomic analyses of Escherichia coli ammonium-limited chemostat growth, and comparison with iron- and glucose-limited chemostat growth. Microbiology 2015, 161, (8), 1659-1670.

285. Sr, S.; Sincero, G. A., Physical-chemical treatment of water and wastewater. 2002; p 1-832.

286. Sincero, A. P.; Sincero, G. A., Physical-chemical treatment of water and wastewater. CRC press: 2002.

287. Shapley Dissolved Oxygen Carbon Dioxide. http://butane.chem.uiuc.edu/pshapley/GenChem1/L23/web-L23.pdf

288. Heijnen, J., Bioenergetics of microbial growth. In Encyclopedia of bioprocess technology: Fermentation, biocatalysis and bioseparation, Flickinger, M.; Drew, S., Eds. John Wiley \& Sons Ltd: Chichester UK, 1999; pp 267-291.

289. Wang, Q.; Garrity, G. M.; Tiedje, J. M.; Cole, J. R., Naïve Bayesian Classifier for Rapid Assignment of rRNA Sequences into the New Bacterial Taxonomy. Applied and Environmental Microbiology 2007, 73, (16), 5261-5267.

290. Flamholz, A.; Noor, E.; Bar-Even, A.; Milo, R., eQuilibrator-the biochemical thermodynamics calculator. Nucleic Acids Research 2011, 40, (D1), D770-D775.

291. Noor, E.; Haraldsdóttir, H. S.; Milo, R.; Fleming, R. M. T., Consistent Estimation of Gibbs Energy Using Component Contributions. PLOS Computational Biology 2013, 9, (7), e1003098. 
References 


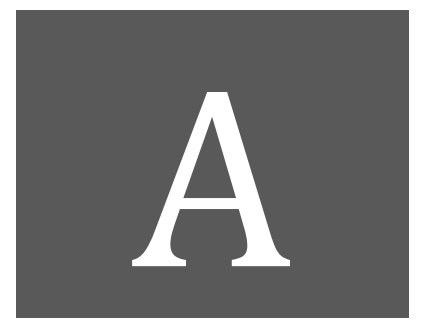

\section{Acknowledgements}




\section{Acknowledgements}

It has been quite a journey the past four years and all the work presented in this thesis has been made possible by the contributions of many. First of all thanks to my co-promotor David Strik. Your endless stream of out-of-the-box ideas were always a delight during brainstorm sessions. Your positivity was stimulating and your patience during the finalization of this thesis is admirable. I really enjoy our collaboration and hope we can come up with many more interesting and impactful research angles. Secondly, a big thanks to my promotor, Cees Buisman, for his renowned sharpness that hones the critical thought of all his PhD students. I appreciate your directness and honesty and always enjoy the talks we have about research and beyond.

Without the help of Merijn, Sanne de Smit, Sabine, Theresa, Victor, Jalees, Bram, Matthijs and Ivo all this work would have been impossible. Many thanks for all your help with performing experiments and interpreting the complexities of our chain elongation microbiomes. It is good to see that some of you liked doing research so much that you decided to start a PhD project of your own!

Sanne Raes, Mark Roghair, Momo, Dandan, David Triana, Ludo and Carlos, I really enjoyed all the inspiring talks we had about the possibilities and mechanistics of chain elongation, albeit electrochemical, via syngas or just using plain carbohydrate-derived electron donors. Dainis, thank you for the countless interesting discussions we have had regarding fermentations in general and the elusive mechanisms behind granulation. Yvonne and Lucia, it was a delight when you shared the office and your conversations with me in the first few years.

Vinnie and Bert, your inexhaustible helpfulness when conceiving / creating / modifying / salvaging / repairing an experimental setup is invaluable in our lab at ETE. I can't count the number of times that both of you saved my experiments from impending doom. Similarly, a huge thanks to everyone from the analytical staff for all the help in acquiring useful data. Katja, your practical knowledge has prevented a lot of errors for me and my students, and your presence always brings a smile to my face.

With the multitude of nice and helpful people working at the Environmental Technology department I have surely forgotten to mention many of you, but thank you nonetheless. Thanks to all people at ETE who contribute to an extremely pleasant working environment. It is a privilege to work beside you all.

I would also like to give thanks to several people outside of ETE. Mirjam, I am very grateful for your meticulous feedback on my first article, from which I learned a great deal. Ton van Gelder from Microbiology, I greatly appreciate your help with handling pure microbial 
cultures. It is always a tricky business to keep cultures clean when you are used to working in a dirty ETE lab. Sven Warris, thank you for your support in performing the bioinformatics that were necessary to interpret the NGS data presented in this thesis. Much thanks also to Niels, Maurice, Robbert, Mark Weijers, Rico and Axel. The enjoyable work I was able to do at your side made it so that I could continue with chain elongation and start this PhD project in Wageningen. And also a very, very big thanks to Bob.

Christa, mijn lief, dankjewel voor alle steun, geduld, positiviteit en voor de onvoorwaardelijke liefde die wij samen delen. 
This research was financed by the Netherlands Organisation for Scientific Research (NWO) with project number ALWGR.2015.8 and with the help of ChainCraft.

Printed by Proefschriftmaken on FSC-certified paper 



\section{SENSE}

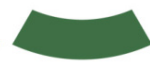

Netherlands Research School for the

Socio-Economic and Natural Sciences of the Environment

\section{I P L O M A}

for specialised PhD training

The Netherlands research school for the

Socio-Economic and Natural Sciences of the Environment

(SENSE) declares that

\section{Kasper Daniël de Leeuw}

born on 9 August 1989, Delft, the Netherlands

has successfully fulfilled all requirements of the educational PhD programme of SENSE.

Wageningen, 3 November 2020
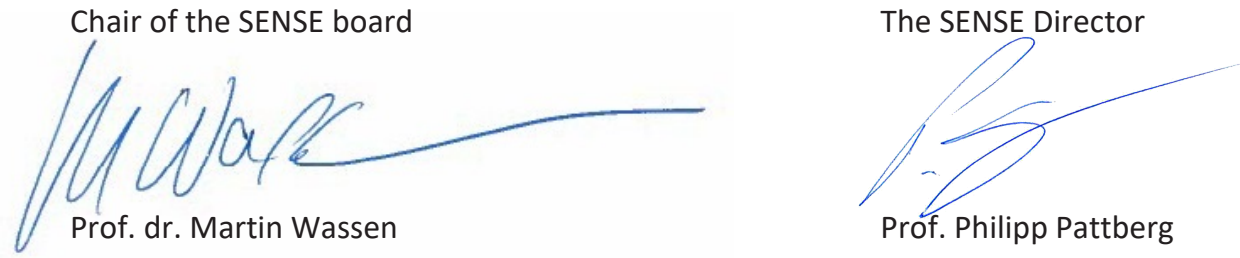

The SENSE Research School has been accredited by the Royal Netherlands Academy of Arts and Sciences (KNAW)

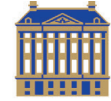

$\begin{array}{llllllllllllllllllllll}K & O & N & I & N & K & L & I & J & K & E & N & E & D & E & R & L & A & N & D & S & E\end{array}$

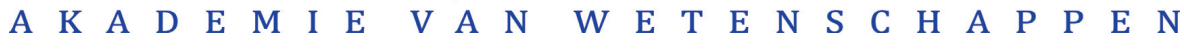




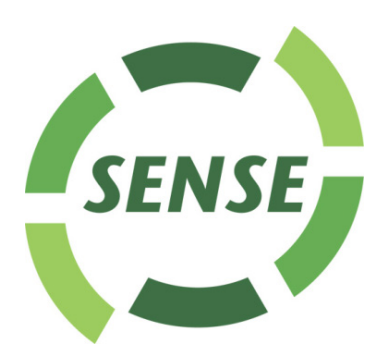

The SENSE Research School declares that Kasper Daniël de Leeuw has successfully fulfilled all requirements of the educational PhD programme of SENSE with a work load of $32.1 \mathrm{EC}$, including the following activities:

\section{$\underline{\text { SENSE PhD Courses }}$}

o Environmental research in context (2016)

- Research in context activity: 'Organize the International Chain Elongation Conference $2020^{\prime}$

\section{Other PhD and Advanced MSc Courses}

- Making an Impact! How to increase the societal relevance of your PhD research, Wageningen Graduate Schools (2016)

- Orientation on teaching for PhD students and Teaching Assistants, Wageningen Graduate Schools (2016)

o Supervising Thesis students Wageningen Graduate Schools (2017)

- Scientific Writing , Wageningen Graduate Schools (2017)

- Communication Styles, Wageningen Graduate Schools (2019)

- Career perspectives course, Wageningen Graduate Schools (2019)

- Brain Training, Wageningen Graduate Schools (2019)

o Bioprocess Design, BioTech Delft (2020)

\section{Management and Didactic Skills Training}

o Supervising six MSc students with thesis (2017-2019)

o Supervising three BSc student with thesis (2017-2020)

- Assisting practicals of the BSc course 'Introduction to Environmental Technology' (20162018)

- Assisting practicals of the BSc course 'Biorefinery' (2016-2018)

o Teaching in the MSc course 'Renewable energy' (2017-2018)

\section{Oral Presentations}

- Enriching Microbiomes for the Production of Branched Carboxylates and Alcohols via Methanol-and Ethanol-based Chain Elongation, International Chain Elongation Conference, 27-28 October 2020, Wageningen, the Netherlands

SENSE coordinator PhD education

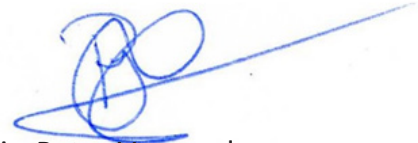

Dr. ir. Peter Vermeulen 


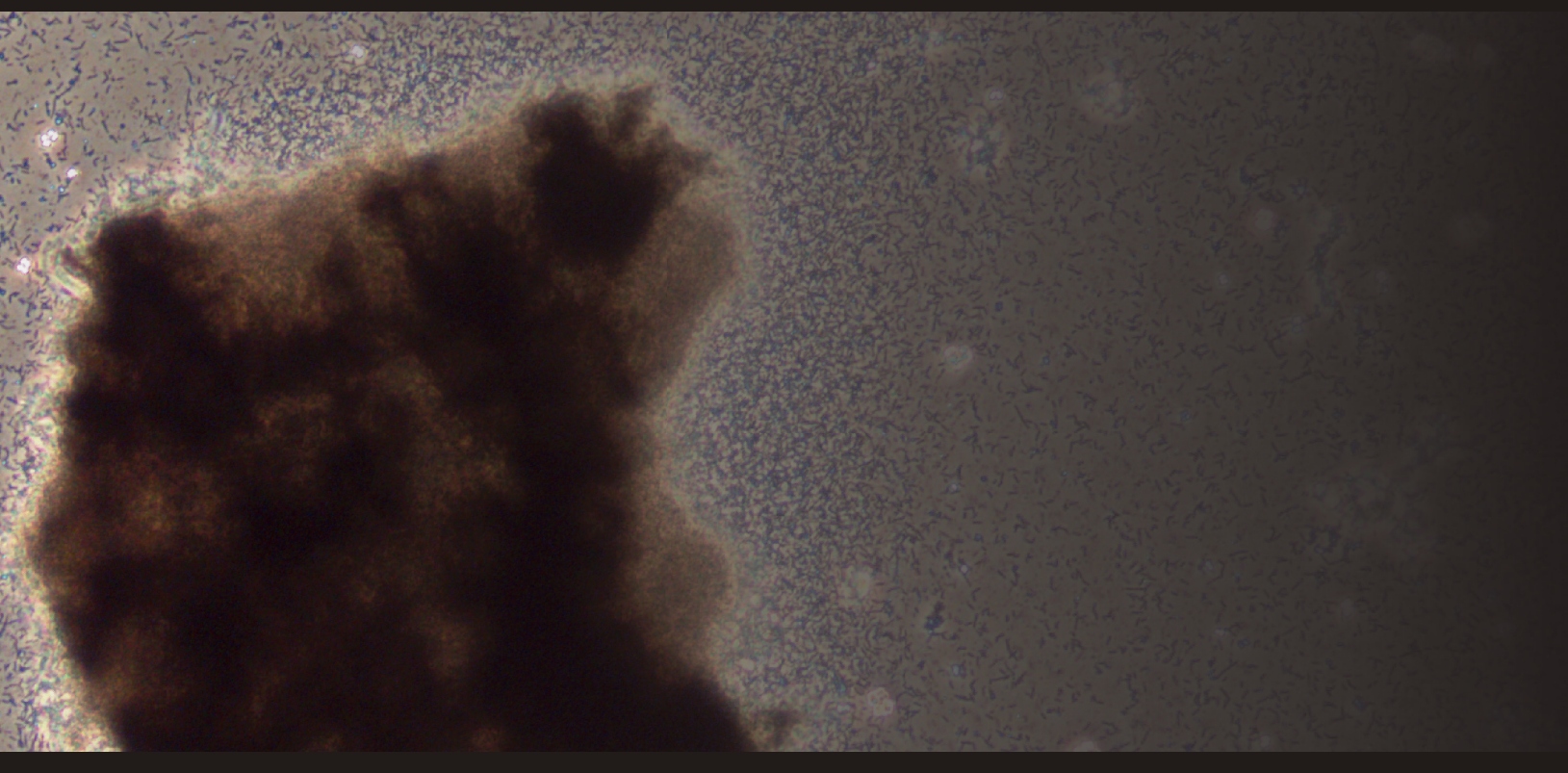

